\title{
Stress Proteins and Myocardial Protection
}

Thesis submitted by

\section{Michael Stephen Marber}

For the degree of

Doctor of Philosophy

in the

Faculty of Physiology

University of London

The Hatter Institute for Cardiovascular Studies

Department of Cardiology

University College London Medical School

London WC1 6AU

August 1993 
ProQuest Number: 10106771

All rights reserved

\title{
INFORMATION TO ALL USERS
}

The quality of this reproduction is dependent upon the quality of the copy submitted.

In the unlikely event that the author did not send a complete manuscript and there are missing pages, these will be noted. Also, if material had to be removed, a note will indicate the deletion.

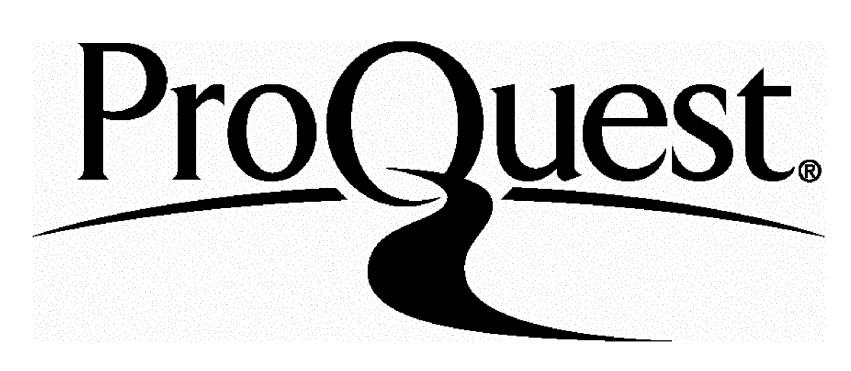

ProQuest 10106771

Published by ProQuest LLC(2016). Copyright of the Dissertation is held by the Author.

All rights reserved.

This work is protected against unauthorized copying under Title 17, United States Code. Microform Edition (c) ProQuest LLC.

\author{
ProQuest LLC \\ 789 East Eisenhower Parkway \\ P.O. Box 1346 \\ Ann Arbor, MI 48106-1346
}


This thesis is dedicated with much love to my wife and daughters. 
(ii)

\section{Abstract}

\section{Stress Proteins and Myocardial Protection}

Background: In the Western World acute myocardial infarction is the most common cause of death in man. Although the mortality of this condition can be halved by therapies designed to promote the return of blood flow to jeopardised myocardium, such treatments must be delivered promptly to be effective and save lives. Procedures that slow the rate of ischaemic damage to the heart will increase the time window available for effective therapy and may thereby be capable of modifying the outcome following acute myocardial infarction.

Aim: $\quad$ This thesis examines the potential of cardiac stress proteins to attenuate and delay ischaemic and other injury to the myocardium.

Methods: $\quad$ The stress protein content of rabbit myocardium was elevated either by whole body heat stress, short episodes of regional ischaemia or mechanical stress. The accumulation of myocardial stress protein 24 hours after each stress was confirmed by densitometric assessment of Western blots. Differing stresses and models relevant to the pathophysiology of cardiac ischaemia and reperfusion were examined. These included hypoxia/reoxygenation and calcium depletion/repletion in the isolated superfused papillary muscle and Langendorff heart, as well as regional ischaemia in the in-situ heart. The endpoints were contractility and infarct size.

Results: Myocardium with a pre-elevated stress protein content was in most instances more resistant to injury than control myocardium, with the resistance to hypoxia/reoxygenation being related to the content of the $72 \mathrm{kDa}$ stress protein. Stress proteins elevated by either whole body heat stress or short episodes of regional cardiac ischaemia were equally effective at reducing infarct size.

Conclusions: The myocardial stress response may offer a future therapeutic avenue capable of favourably influencing the outcome following acute myocardial infarction and preserving viable myocardium during cardiopulmonary bypass and organ preservation prior to transplantation. 
(iii)

\section{Contents}

Abstract $\ldots \ldots \ldots \ldots \ldots \ldots \ldots \ldots \ldots \ldots \ldots \ldots \ldots \ldots \ldots \ldots \ldots \ldots \ldots \ldots$ (ii)

Table of Contents $\ldots \ldots \ldots \ldots \ldots \ldots \ldots \ldots \ldots \ldots \ldots \ldots \ldots \ldots \ldots$ (iii)

List of Figures $\ldots \ldots \ldots \ldots \ldots \ldots \ldots \ldots \ldots \ldots \ldots \ldots \ldots \ldots \ldots \ldots \ldots \ldots$ (xii)

List of Tables $\ldots \ldots \ldots \ldots \ldots \ldots \ldots \ldots \ldots \ldots \ldots \ldots \ldots \ldots \ldots \ldots \ldots \ldots$ (xiii)

Abbreviations $\ldots \ldots \ldots \ldots \ldots \ldots \ldots \ldots \ldots \ldots \ldots \ldots \ldots \ldots \ldots \ldots \ldots \ldots$ (xiv)

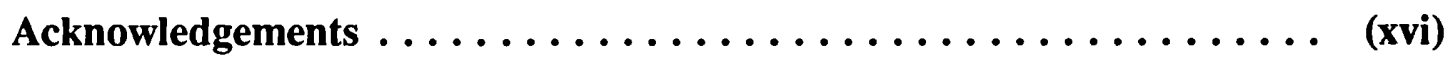

\section{Table of Contents}

Chapter 1 Introduction: Heat stress, stress proteins and the heart.

1 Myocardial Ischaemia and Infarction . . . . . . . . . . . . . . . 2

1.1 The Importance of Attenuating Myocardial Ischaemic Injury $\ldots \ldots \ldots 2$

2 Pathophysiology of Myocardial Ischaemia $\ldots \ldots \ldots \ldots \ldots \ldots \ldots \ldots \ldots \ldots \ldots$

2.1 Myocardial Contractility During Ischaemia $\ldots \ldots \ldots \ldots 2$

2.1.1 Immediate Contractile Failure $\ldots \ldots \ldots \ldots \ldots \ldots \ldots \ldots$

2.1.2 Early Contractile Failure $\ldots \ldots \ldots \ldots \ldots \ldots \ldots \ldots$

2.1.3 Late Contractile Failure $\ldots \ldots \ldots \ldots \ldots \ldots \ldots \ldots$

2.1.4 The Onset of Contracture $\ldots \ldots \ldots \ldots \ldots \ldots \ldots$

2.2 Irreversible Ischaemic Injury and Reperfusion Injury $\ldots \ldots \ldots \ldots \ldots$

2.3 What Determines Cell Death? . . . . . . . . . . . . . 6

3 The Determinants of Myocardial Infarct Size $\ldots \ldots \ldots \ldots \ldots \ldots \ldots$

3.1 Volume at Risk . . . . . . . . . . . . . . . 6

3.2 The Duration of the Ischaemic Insult $\ldots \ldots \ldots \ldots \ldots \ldots \ldots$

3.3 The Degree of Collateral Flow . . . . . . . . . . . . 7

3.4 Metabolic Requirements of the Ischaemic Tissue $\ldots \ldots \ldots \ldots \ldots$

4 Heat Stress and the Stress Response $\ldots \ldots \ldots \ldots \ldots \ldots \ldots \ldots \ldots \ldots$

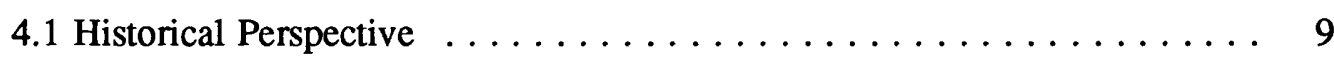

4.2 Defining the Terminology $\ldots \ldots \ldots \ldots \ldots \ldots \ldots \ldots \ldots \ldots \ldots$ 
4.2.1 The 100-110kDa Stress Protein Family . . . . . . . . 10

4.2.2 The $90 \mathrm{kDa}$ Stress Protein Family ............ 12

4.2.3 The 70kDa Stress Proteins Family ............ 13

4.2.4 The $60 \mathrm{kDa}$ Stress Protein Family $\ldots \ldots \ldots \ldots \ldots \ldots \ldots$

4.2.5 The $30 \mathrm{kDa}$ Stress Protein Family $\ldots \ldots \ldots \ldots \ldots \ldots \ldots$

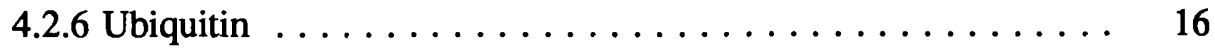

4.3 The Control of the Stress Response $\ldots \ldots \ldots \ldots \ldots \ldots \ldots \ldots \ldots$

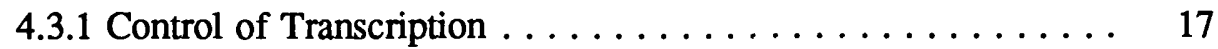

4.3.2 Post-Transcriptional Control $\ldots \ldots \ldots \ldots \ldots \ldots \ldots \ldots \ldots 18$

5 Are Stress Proteins Protective? . . . . . . . . . . . . . . . . . . 19

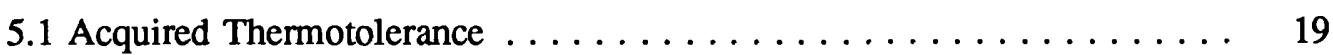

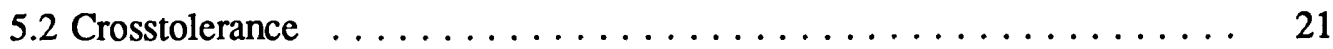

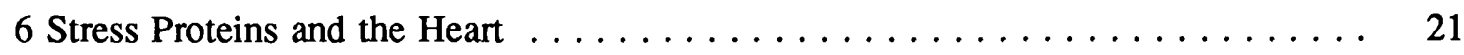

6.1 Heat Stress and Myocardial Protection . . . . . . . . . . 22

6.1.1 The Isolated Heart and Global Ischaemia $\ldots \ldots \ldots \ldots . \ldots 22$

6.1.2 The Isolated Heart and Regional Ischaemia $\ldots \ldots \ldots \ldots 23$

6.1.3 The In Situ Heart and Regional Ischaemia . . . . . . . . . 24

6.2 Heat Stress Proteins and Classical Ischaemic Preconditioning . . . . . 25

6.3 Non-Thermally Induced Stress Proteins and Protection Against Ischaemia . . . . . . . . . . . . . . . . . . . 26

6.4 Protection Against Non-Ischaemic Injury $\ldots \ldots \ldots \ldots \ldots \ldots . \ldots 27$

6.5 Mechanisms of Cardiac Protection by Elevated Temperature . . . . . 28

7 Aims and Scope of the Thesis $\ldots \ldots \ldots \ldots \ldots \ldots \ldots \ldots \ldots$

Chapter 2 Methods: Experimental models and data handling.

1 Thermal Induction of Cardiac Stress Protein $\ldots \ldots \ldots \ldots \ldots \ldots \ldots \ldots . \ldots \ldots$

1.1 Principles of Cardiac Stress Protein Induction $\ldots \ldots \ldots \ldots \ldots . \ldots . \ldots 32$

1.1.2 Rabbit Whole Body Heat Stress $\ldots \ldots \ldots \ldots \ldots \ldots \ldots . \ldots 32$

2 Myocardial Stress Protein Quantitation by SDS-Page and Western Blotting . . . . 33

2.1 Principles of SDS-Page and Western Blotting . . . . . . . . 33

2.2 Preparation of Myocardial Specimens $\ldots \ldots \ldots \ldots \ldots \ldots \ldots \ldots$

2.3 Preparation of SDS-Page Gels . . . . . . . . . . . . . 34

2.4 The Loading of Samples and the One-Dimensional Electrophoretic 
Separation of Proteins in Polyacrylamide Gels $\ldots \ldots \ldots \ldots \ldots$

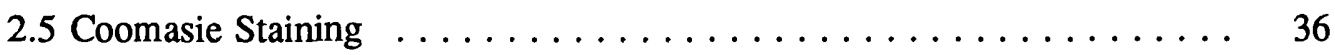

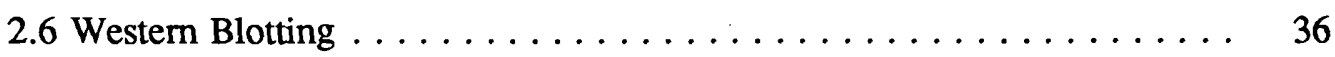

2.7 Immunoprobing Nitrocellulose Membranes with Primary and Secondary

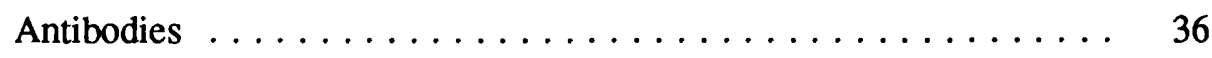

2.7.1 Detection of hsp60 $\ldots \ldots \ldots \ldots \ldots \ldots \ldots \ldots \ldots \ldots$

2.7.2 Detection of the Inducible Form of hsp70 (hsp72) . . . . . 37

2.7.3 Densitometric Quantitation of hsp60 and hsp72 ...... 38

3 The Measurement of Papillary Muscle Contractile Force . . . . . . . . . . . 39

3.1 The Principles of Superfusion $\ldots \ldots \ldots \ldots \ldots \ldots \ldots \ldots \ldots \ldots \ldots$

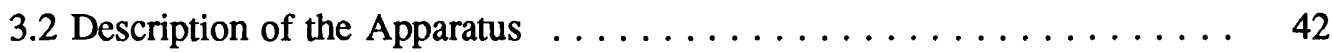

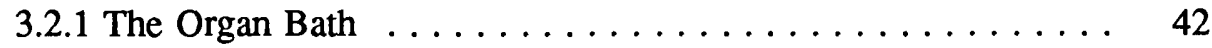

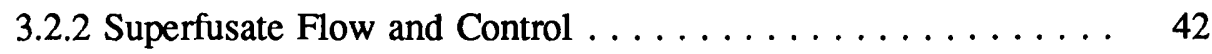

3.2.3 Measurement of Force $\ldots \ldots \ldots \ldots \ldots \ldots \ldots \ldots \ldots . \ldots \ldots \ldots$

3.2.4 Preparation and Suspension of Papillary Muscles ....... 45

3.2.5 Papillary Muscle Stability and Oxygenation . . . . . . . 47

3.2.6 The Force-Interval Protocol and Digital Data Acquisition . . . 47

3.2.7 Programming for the Real-Time Display of Contractile Information, Analysis of the Force Envelope and Chelation of Force Interval Data $\ldots \ldots \ldots \ldots \ldots \ldots \ldots \ldots$

4 The Langendorff Heart $\ldots \ldots \ldots \ldots \ldots \ldots \ldots \ldots \ldots \ldots \ldots \ldots$

4.1 The Principles of Retrograde Perfusion $\ldots \ldots \ldots \ldots \ldots \ldots \ldots \ldots$

4.2 The Preparation of Hearts for Retrograde Perfusion $\ldots \ldots \ldots \ldots$

4.3 Description of the Apparatus $\ldots \ldots \ldots \ldots \ldots \ldots \ldots \ldots \ldots$

4.3.1 Delivery of the Perfusate $\ldots \ldots \ldots \ldots \ldots \ldots \ldots \ldots \ldots$

4.3.2 Assessment of Left Ventricular Pressure . . . . . . . 56

4.3.3 Left Ventricular Monophasic Action Potentials . . . . . . . . 56

4.3.4 Temperature Control . . . . . . . . . . . . . . 56

4.3.5 Control of Heart Rate $\ldots \ldots \ldots \ldots \ldots \ldots \ldots \ldots \ldots$

4.3.6 Oxygen, Carbon Dioxide and $\mathrm{pH}$ Differences Across the

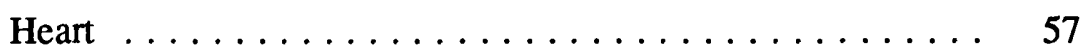

4.3.7 Creatine Phosphokinase within the Coronary Effluent $\ldots \ldots .57$

5 The In-Vivo Model of Infarction $\ldots \ldots \ldots \ldots \ldots \ldots \ldots \ldots \ldots \ldots$

5.1 The Determinants of Infarct Size $\ldots \ldots \ldots \ldots \ldots \ldots \ldots \ldots \ldots$

5.2 Recovery Experiments Involving Ischaemic Pretreatment $\ldots \ldots \ldots \ldots 58$

5.2.1 Anaesthesia for Recovery Experiments Involving Ischaemic Pretreatment . . . . . . . . . . . . . . . . . . . 59 
5.2.2 The Surgical Preparation for Recovery Experiments Involving

Ischaemic Pretreatment . . . . . . . . . . . . 59

5.3 Non-Recovery Experiments Involving Prolonged Ischaemia and

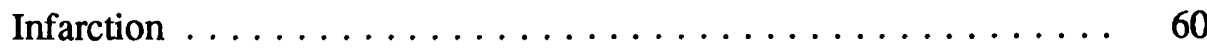

5.3.1 Surgical Preparation for Non-Recovery Experiments Involving

Prolonged Ischaemia and Infarction $\ldots \ldots \ldots \ldots \ldots \ldots 60$

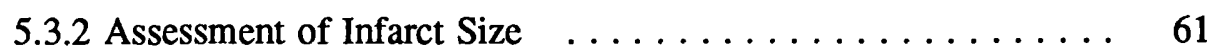

6 Statistical Methods $\ldots \ldots \ldots \ldots \ldots \ldots \ldots \ldots \ldots \ldots \ldots \ldots \ldots \ldots$

Chapter 3 Results: Initial pilot study of papillary muscles subjected to serial stresses.

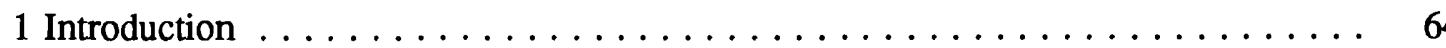

2 Specific Methods $\ldots \ldots \ldots \ldots \ldots \ldots \ldots \ldots \ldots \ldots \ldots \ldots \ldots \ldots$

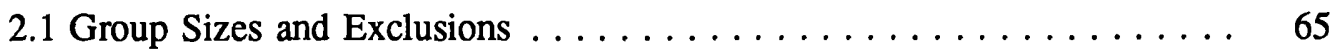

2.2 The Experimental Model . . . . . . . . . . . . . . . 66

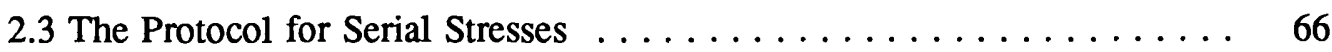

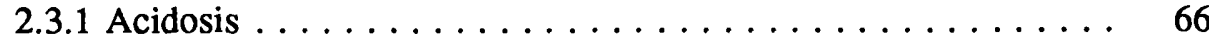

2.3.2 Nominally Calcium-Free $\ldots \ldots \ldots \ldots \ldots \ldots \ldots \ldots 68$

2.3.3 High Flow-Hypoxia without Substrate $\ldots \ldots \ldots \ldots \ldots \ldots 68$

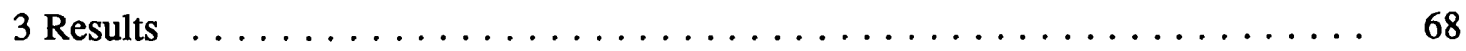

3.1 Myocardial Heat Stress Protein Content . . . . . . . . . . 68

3.2 Baseline Papillary Muscle Characteristics . . . . . . . . . . . . . . . 69

3.3 Changes in Contractility With Serial Stresses . . . . . . . . . 69

3.3.1 General Pattern of Changes in Contractility .......... 69

3.3.2 Papillary Muscle Contraction During Acidosis ......... 73

3.3.3 Papillary Muscle Contraction During and Following Nominally

Calcium-Free Superfusion . . . . . . . . . . 73

3.3.4 Papillary Muscle Contraction During and Following High Flow

Hypoxia Without Substrate $\ldots \ldots \ldots \ldots \ldots \ldots \ldots$

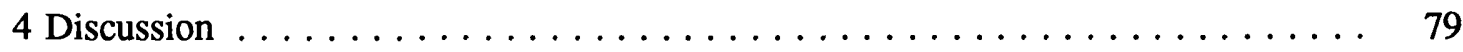

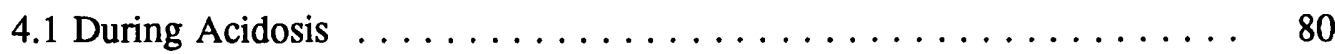

4.2 Following Nominally Calcium-Free Superfusion $\ldots \ldots \ldots \ldots \ldots \ldots$

4.3 Following High Flow Hypoxia without Substrate $\ldots \ldots \ldots \ldots . \ldots . \ldots 8$

4.4 Critique of Methods $\ldots \ldots \ldots \ldots \ldots \ldots \ldots \ldots \ldots \ldots \ldots \ldots$

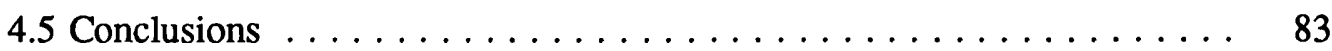


Chapter 4 Results: Papillary muscles, substrate preference and stress protein content. 1 Introduction $\ldots \ldots \ldots \ldots \ldots \ldots \ldots \ldots \ldots \ldots \ldots \ldots \ldots$

2 Specific Methods $\ldots \ldots \ldots \ldots \ldots \ldots \ldots \ldots \ldots \ldots \ldots \ldots$

2.1 Group Sizes and Exclusions $\ldots \ldots \ldots \ldots \ldots \ldots \ldots \ldots \ldots \ldots$

2.2 The Experimental Model $\ldots \ldots \ldots \ldots \ldots \ldots \ldots \ldots \ldots$

2.2.1 Papillary Muscle Stimulation Protocol and Data Acquisition . . 88

2.2.2 Timing of Changes in Papillary Muscle Superfusion . . . . . . 89

2.2.3 Heat Stress Protein Assessment . . . . . . . . . . . 89

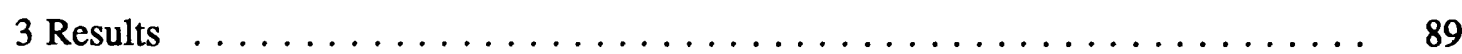

3.1 Baseline Characteristics of Papillary Muscles . . . . . . . . . . . 89

3.2 Contractility During High Flow Hypoxia without Substrate . . . . . . 92

3.3 Contractility Following High Flow Hypoxia without Substrate . . . . . 92

3.4 The Force-Interval Relationship Between Heat Stress and Sham Heat

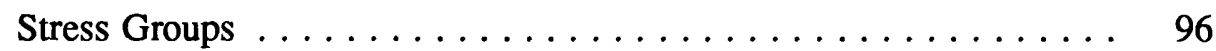

3.5 The Effect of Hypoxia and Reoxygenation on the Force-Interval Relationship ......................... 99

3.6 The 72kDa Heat Stress Protein and Contractile Recovery . . . . . . . 99

3.7 The 60kDa Heat Stress Protein is not Associated with Contractile

Recovery ............................ 104

4 Discussion . . . . . . . . . . . . . . . . . . . . . . . . 104

4.1 Papillary Muscle Contractility and Substrate Preference . . . . . . . . . 104

4.2 Force-Interval Relationships . . . . . . . . . . . . . . . . . . . . . . 109

4.3 Stress Proteins and Myocardial Resistance . . . . . . . . . . . . 109

4.4 Contracture and Substrates $\ldots \ldots \ldots \ldots \ldots \ldots \ldots \ldots \ldots \ldots$

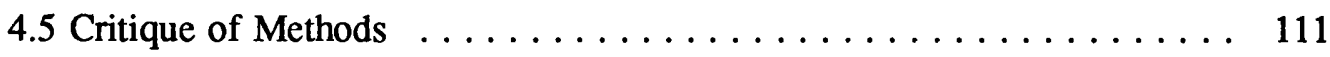

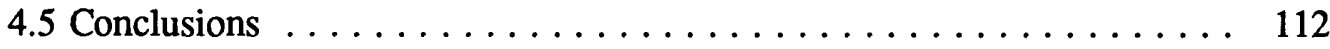

Chapter 5 Results: The isolated heart subjected to a submaximal calcium paradox. 1 Introduction . . . . . . . . . . . . . . . . . . . . . . . . . . . . . . 113

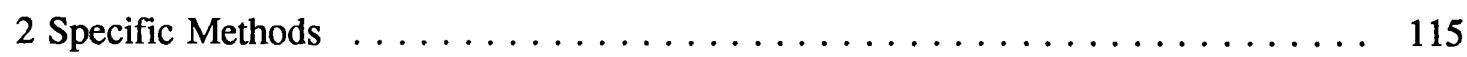

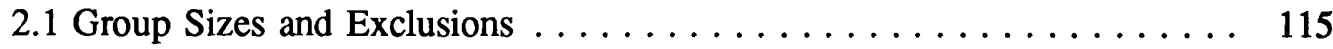

2.2 The Experimental Model $\ldots \ldots \ldots \ldots \ldots \ldots \ldots \ldots \ldots \ldots \ldots$

2.2 The Perfusion Protocol $\ldots \ldots \ldots \ldots \ldots \ldots \ldots \ldots \ldots \ldots$ 


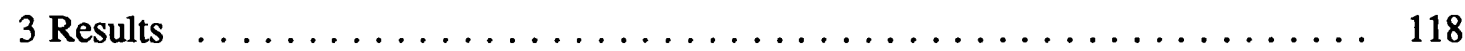

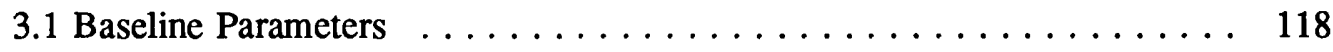

3.2 Temperature Changes During Heat Stress $\ldots \ldots \ldots \ldots \ldots \ldots \ldots . \ldots \ldots$

3.3 Functional Changes During the Calcium Paradox ............. 118

3.4 Left Ventricular and Coronary Perfusion Pressures During the Calcium Paradox .............................. 121

3.5 Oxygen, Carbon Dioxide and pH Changes Across the Heart During the Calcium Paradox ......................... 121

3.6 Creatine Phophokinase and Myoglobin Efflux During the Calcium Paradox ................................ 121

3.7 Changes in Action Potential Duration During the Calcium Paradox .... 125

3.8 The Interdependence of Functional and Metabolic Parameters . . . . . 125

3.9 Stress Protein Analysis $\ldots \ldots \ldots \ldots \ldots \ldots \ldots \ldots \ldots \ldots . .129$

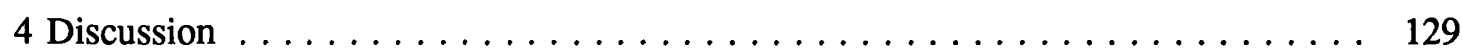

4.1 The Implication of the Functional and Metabolic Preservation Following the Calcium Paradox in Heat Stressed Hearts . . . . . . . . . . 129

4.2 The Mechanism of Resistance to the Calcium Paradox ......... 132

4.3 Critique of Methods $\ldots \ldots \ldots \ldots \ldots \ldots \ldots \ldots \ldots \ldots . \ldots \ldots . \ldots \ldots$

4.4 Conclusion $\ldots \ldots \ldots \ldots \ldots \ldots \ldots \ldots \ldots \ldots \ldots \ldots \ldots, 134$

Chapter 6 Results: The isolated heart subjected to hypoxia and reoxygenation. 1 Introduction ................................. 135

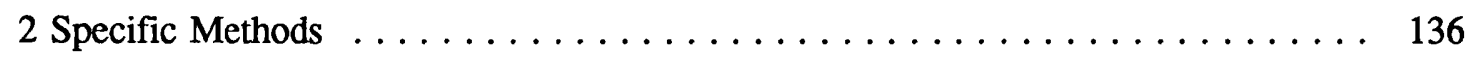

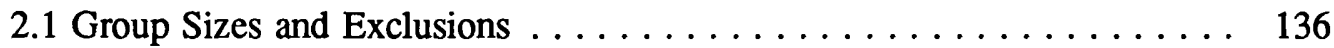

2.2 The Experimental Model $\ldots \ldots \ldots \ldots \ldots \ldots \ldots \ldots \ldots . \ldots \ldots$

2.3 The Experimental Protocol ...................... 137

3 Results ......................................... 137

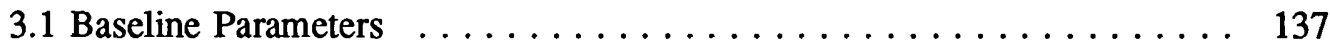

3.2 Temperature Changes During Heat Stress $\ldots \ldots \ldots \ldots \ldots \ldots \ldots .140$

3.2 Changes in Contractility During Hypoxia and Reoxygenation $\ldots \ldots .140$

3.3 Left Ventricular and Coronary Perfusion Pressures During Hypoxia and Reoxygenation. .......................... 140

3.4 Oxygen, Carbon Dioxide and $\mathrm{pH}$ Changes Across the Heart During Hypoxia and Reoxygenation. . . . . . . . . . . . . . . . . . 144

3.5 Creatine Phosphokinase Efflux $\ldots \ldots \ldots \ldots \ldots \ldots \ldots \ldots \ldots . \ldots \ldots$

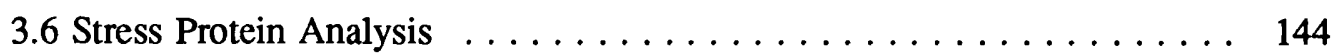


4 Discussion . . . . . . . . . . . . . . . . . . . . . . . 144

4.1 Changes in Left Ventricular and Coronary Perfusion Pressures . . . . . . 147

4.2 The Mechanism of Injury Following Hypoxia and Reoxygenation . . . . 147

4.3 Conclusions . . . . . . . . . . . . . . . . . . . 149

Chapter 7 Results: Infarct size following stress protein elevation by sublethal heat stress or ischaemia.

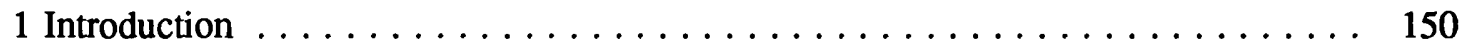

2 Specific Methods $\ldots \ldots \ldots \ldots \ldots \ldots \ldots \ldots \ldots \ldots \ldots \ldots \ldots \ldots$

2.1 Group Sizes and Exclusions $\ldots \ldots \ldots \ldots \ldots \ldots \ldots \ldots \ldots \ldots \ldots \ldots$

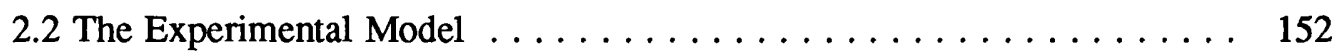

2.2.1 Ischaemic and Sham Ischaemic Pretreatments . . . . . . 152

2.2.2 Heat Stress and Sham Heat Stress Pretreatments . . . . . . 154

2.2 .372 and $60 \mathrm{kDa}$ Stress Protein Estimation . . . . . . . 154

2.2.4 Myocardial Infarction and Infarct Size Assessment $\ldots \ldots \ldots 154$

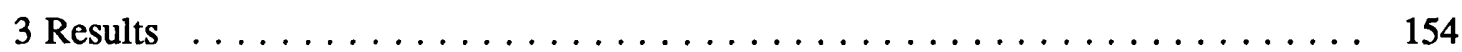

3.1 Temperature Changes During Heat Stress $\ldots \ldots \ldots \ldots \ldots \ldots 154$

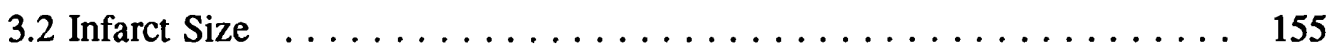

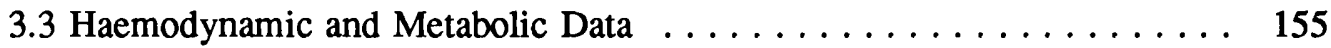

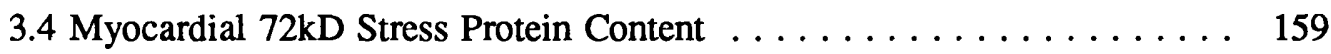

3.5 Myocardial 60kD Stress Protein Content . . . . . . . . . . . . . 162

3.6 Myocardial Specimen Loading for Stress Protein Estimation . . . . . . 162

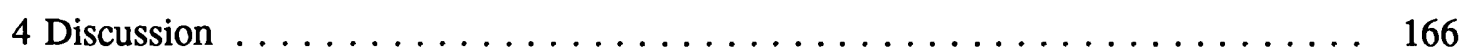

4.1 Whole Body Heat Stress and Resistance to Infarction In-vivo . . . . . 166

4.2 The Basis for the Reduction in Myocardial Infarct Size . . . . . . . 166

4.3 The Relationship between Ischaemic Pretreatment and Ischaemic

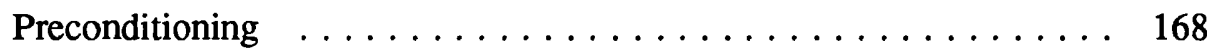

4.4 The Haemodynamic Benefits of Whole Body Heat Stress $\ldots \ldots \ldots . .169$

4.5 Critique of Methods . . . . . . . . . . . . . . . . . 169

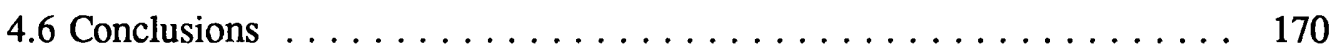


$(\mathrm{x})$

Chapter 8 General discussion and conclusions.

1 Summary of Results . . . . . . . . . . . . . . . . . 172

2 The Mechanism of Myocardial Protection $\ldots \ldots \ldots \ldots \ldots \ldots \ldots \ldots$

3 Ongoing Investigations $\ldots \ldots \ldots \ldots \ldots \ldots \ldots \ldots \ldots \ldots \ldots \ldots \ldots \ldots$

5 The Implications of the Work Described $\ldots \ldots \ldots \ldots \ldots \ldots \ldots \ldots$

Appendix 1 Solutions used for SDS-Page and Western blotting.

1 Sample Buffer (Two-Times) . . . . . . . . . . . . . . . . . . . . . . . . . . 179

2 Acrylamide Gel Solution $\ldots \ldots \ldots \ldots \ldots \ldots \ldots \ldots \ldots \ldots \ldots$

3 TRIS Stock Solutions for SDS-PAGE Gels $\ldots \ldots \ldots \ldots \ldots \ldots \ldots$. . . . . . . 180

3.1 Resolving (Base) Gel . . . . . . . . . . . . . . 180

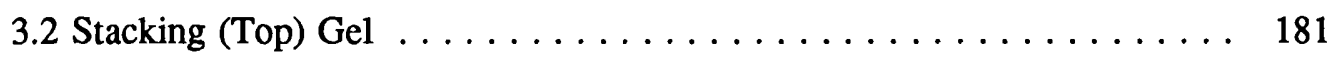

4 SDS-PAGE Running Buffer $\ldots \ldots \ldots \ldots \ldots \ldots \ldots \ldots \ldots \ldots \ldots \ldots$

5 Coomasie Blue Staining Solution $\ldots \ldots \ldots \ldots \ldots \ldots \ldots \ldots \ldots \ldots \ldots$

6 Coomasie Blue De-Staining Solution $\ldots \ldots \ldots \ldots \ldots \ldots \ldots \ldots \ldots \ldots$

7 Ten-Times Blotting Buffer for Western Blotting . . . . . . . . . . . . 182

Appendix 2 Programmes used for the analysis of papillary muscle contraction.

1 MMAQUIS.BAS ................................ 183

1.1 Description of MMAQUIS.BAS $\ldots \ldots \ldots \ldots \ldots \ldots \ldots \ldots \ldots \ldots$

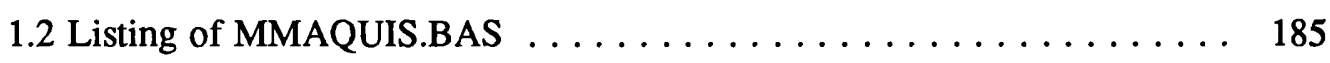

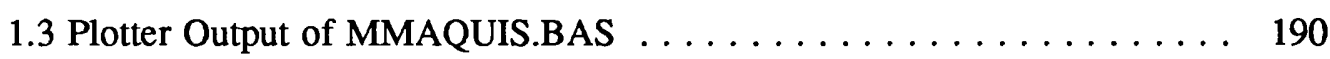

2 MMCALCUL.BAS . . . . . . . . . . . . . . . . . . . . . . . . 193

2.1 Description of MMCALCUL.BAS $\ldots \ldots \ldots \ldots \ldots \ldots \ldots \ldots \ldots$

2.2 Listing of MMCALCUL.BAS $\ldots \ldots \ldots \ldots \ldots \ldots \ldots \ldots \ldots \ldots$

2.3 Plotter Output of MMCALCUL.BAS $\ldots \ldots \ldots \ldots \ldots \ldots 20 \ldots \ldots \ldots$

2.4 Printer Output of MMCALCUL.BAS $\ldots \ldots \ldots \ldots \ldots \ldots \ldots 20 \ldots \ldots$ 


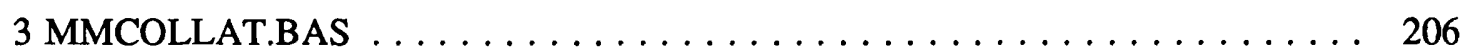

3.1 Description of MMCOLlat.BAS $\ldots \ldots \ldots \ldots \ldots \ldots \ldots \ldots .206$

3.2 Listing of MMCOLLAT.BAS . . . . . . . . . . . . . . . 207

3.3 Output of MMCOLLAT.BAS ................... 209

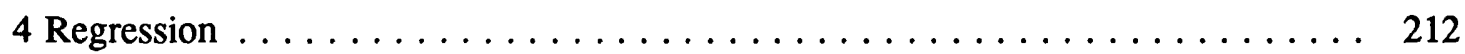

4.1 Description of Regression $\ldots \ldots \ldots \ldots \ldots \ldots \ldots \ldots \ldots \ldots .212$

4.2 Output of Regression ........................ 212

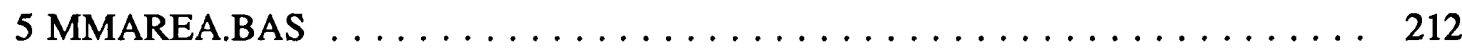

5.1 Description of MMAREA.BAS $\ldots \ldots \ldots \ldots \ldots \ldots \ldots \ldots \ldots, 212$

5.2 Listing of MMAREA.BAS $\ldots \ldots \ldots \ldots \ldots \ldots \ldots \ldots \ldots \ldots \ldots \ldots$

6 MMINFRSK.BAS ............................ 218

6.1 Description of MMINFRSK.BAS $\ldots \ldots \ldots \ldots \ldots \ldots \ldots \ldots \ldots, 218$

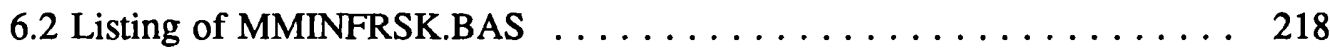

References ............................... $\quad 220-247$

Publications arising from work contained within this Thesis $\ldots \ldots \ldots$ back cover 


\section{List of Figures}

Figure 2.1 Protein loading, Coomasie staining and hsp immunoreactivity . . . 40

Figure 2.2 Protein loading and densitometry $\ldots \ldots \ldots \ldots \ldots \ldots \ldots$

Figure 2.3 Apparatus to measure papillary muscle contractility . . . . . . . 43

Figure 2.4 Organ bath for papillary muscle superfusion . . . . . . . . . . 44

Figure 2.5 Calibration of force transducer and amplifier . . . . . . . . . 46

Figure 2.6 Description of the force-interval relationship . . . . . . . . . 48

Figure 2.7 Calibration of the A/D converter $\ldots \ldots \ldots \ldots \ldots \ldots \ldots \ldots$

Figure 2.8 Computer monitor during force-interval assessment $\ldots \ldots \ldots \ldots 52$

Figure 2.9 The Langendorff apparatus $\ldots \ldots \ldots \ldots \ldots \ldots \ldots$

Figure 2.10 Assessment of infarct and risk zone size $\ldots \ldots \ldots \ldots \ldots$. . . . 62

Figure 3.1 Protocol for serial stresses . . . . . . . . . . . . . . 67

Figure 3.2 Output of MMAQUIS.BAS during experiment . . . . . . $71 \& 72$

Figure 3.3 Force during acidosis and post nominally calcium-free $\ldots \ldots \ldots 74$

Figure 3.4 Force post hypoxia $\ldots \ldots \ldots \ldots \ldots \ldots \ldots \ldots \ldots$

Figure 3.5 Recovery of force post hypoxia . . . . . . . . . . . . 77

Figure 3.6 Resting tension and force-interval rate constants $\ldots \ldots \ldots \ldots \ldots$

Figure 4.1 Protocol for substrate preference $\ldots \ldots \ldots \ldots \ldots \ldots$

Figure 4.2 Assessment of papillary muscle activity during hypoxia $\ldots . . . .990$

Figure 4.3 Papillary muscle size and developed force . . . . . . . . . . . 93

Figure 4.4 Papillary muscle contractility during hypoxia . . . . . . . . . . 13

Figure 4.5 Developed and resting force $\ldots \ldots \ldots \ldots \ldots \ldots \ldots$

Figure 4.6 Timings of papillary muscle contraction $\ldots \ldots \ldots \ldots$. . . . . 97

Figure 4.7 Rates of papillary muscle contraction . . . . . . . . . . . . 98

Figure 4.8 Basal force-interval relationships . . . . . . . . . . . . . 100

Figure 4.9 Force-interval relationship at 90 mins reoxygenation . . . . . . . . 101

Figure 4.10 Effect of hypoxia/reoxia on force-interval in control groups . . . . 102

Figure 4.11 Effect of hypoxia/reoxia on force-interval in heat stress groups . . 103

Figure 4.12 Hsp72 content of papillary muscles . . . . . . . . . . . . . 105

Figure 4.13 Densitometric assessment and relation to contractility of hsp72 . 106

Figure $4.14 \mathrm{Hsp} 60$ and relation to contractility . . . . . . . . . . . . 107

Figure 5.1 Protocol for the calcium paradox . . . . . . . . . . . . 117

Figure 5.2 Experimental trace during the calcium paradox . . . . . . . . 120

Figure 5.3 Left ventricular pressures during the calcium paradox . . . . . . . . 122

Figure 5.4 Coronary perfusion pressure and oxygen extraction $\ldots \ldots \ldots \ldots 123$

Figure 5.5 Carbon dioxide production and $\mathrm{pH}$. . . . . . . . . . . . . . . . 124

Figure $5.6 \mathrm{CPK}$ and myoglbin release $\ldots \ldots \ldots \ldots \ldots \ldots \ldots$ 
Figure 5.7 Action potential duration and hsp 72 content $\ldots \ldots \ldots \ldots \ldots 127$

Figure 5.8 Relationships between function and metabolic parameters . . . . 128

Figure 5.9 Left ventricular hsp content $\ldots \ldots \ldots \ldots \ldots \ldots \ldots \ldots \ldots$

Figure 5.10 Coomasie stain of left ventricular samples . . . . . . . 131

Figure 6.1 Protocol for hypoxia and reoxygenation of Langendorff heart . . 138

Figure 6.2 Experimental trace during hypoxia/reoxygenation $\ldots \ldots \ldots \ldots 141$

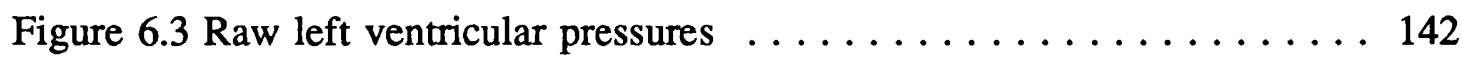

Figure 6.4 Normalised left ventricular pressures $\ldots \ldots \ldots \ldots \ldots \ldots \ldots 143$

Figure $6.5 \mathrm{pH}$ and gasseous exchange across the heart $\ldots \ldots \ldots \ldots \ldots 145$

Figure 6.6 Relationships between function and metabolic parameters . . . . 146

Figure 6.7 Experimental trace during long hypoxia/reoxygenation $\ldots \ldots \ldots 148$

Figure 7.1 Protocol for infarction after ischaemic or thermal stress . . . . 153

Figure 7.2 Risk and infarct volumes after 30 minutes ischaemia $\ldots \ldots \ldots 156$

Figure 7.3 Experimental trace during myocardial infarction . . . . . . 157

Figure 7.4 Mean arterial pressure and rate pressure product $\ldots \ldots \ldots \ldots 158$

Figure 7.5 Realtionship between infarct size and rate pressure product . . . 160

Figure 7.7 Left ventricular hsp72 content after thermal and ischaemic stress . 163

Figure 7.8 Left ventricular hsp60 content after thermal and ischaemic stress . 164

Figure 7.9 Coomasie staining of left ventricular samples $\ldots \ldots \ldots \ldots \ldots 165$

Figure 8.1 Heat stress protein content after aortic cross-clamping . . . . . 175

Figure 8.2 Myocardial antioxidant activity after various stresses $\ldots \ldots \ldots .176$

Figure App2.1 Output of MMAQUIS.BAS during hypoxia/reoxia protocol91 \& 192

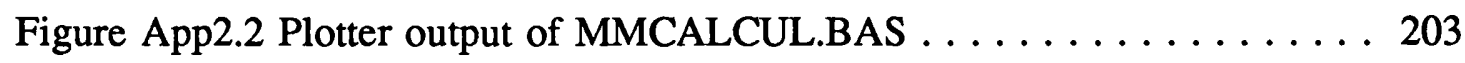

Figure App2.3 Printer output of MMCALCUL.BAS . . . . . . . 204 \& 205

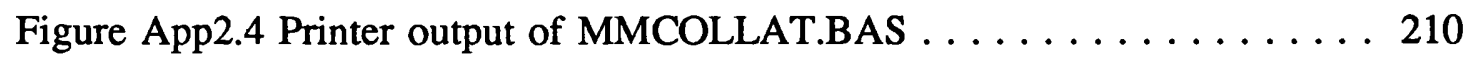

Figure App2.5 Printer output of Regression $\ldots \ldots \ldots \ldots \ldots \ldots .215$ \& 216

\section{List of Tables}

Table 1.1 Stress protein families and function $\ldots \ldots \ldots \ldots \ldots \ldots \ldots . \ldots \ldots$

Table 3.1 Papillary muscle characteristics for serial stresses $\ldots \ldots \ldots \ldots \ldots 70$

Table 4.1 Papillary muscle characterisitics for substrate preference $\ldots . \ldots 91$

Table 5.1 Characteristics of hearts subjected to the calcium paradox . . . . 119

Table 6.1 Characteristics of hearts subjected to hypoxia/reoxygenation . . . . 139

Table 7.1 Haemodynamic changes and blood gasses during infarction $\ldots \ldots 161$ 


\section{Abbreviations}

A/D

ADP

APD

$\mathrm{APD}_{90}$

APS

AMP

ANOVA

ASCII

ATP

CPK

DHFR

DNA

ECG

eIF

FPLSD

FSD

GSH

GS-SG

hsp

HSTF

HSE

IgG

$\mathrm{I} / \mathrm{R}$

IV

$\mathrm{kDa}$

$\mathrm{kPa}$

mRNA

myoD1

OD

PAGE

PBS

PC

$\mathrm{pO}_{2}$

$\mathrm{R}^{\wedge} 2$ or $\mathrm{R}^{2}$

RNA
Analogue to digital converter

Adenosine diphosphate

Action potential duration

Action potential duration to $90 \%$ repolarisation

Ammonium persulphate

Adenosine monophosphate

Analysis of variance

American standard code for information interchange

Adenosine triphosphate

Creatine phosphokinse

Dihydrofolate reductase

Deoxyribonucleic acid

Electrocardiograph

Eukaryotic initiation factor

Fischer's protected least significance difference test

Full scale deflection

Reduced glutathione

Oxidised glutathione

Heat shock protein

Heat shock transcription factor

Heat shock element

Immunoglobulin class $\mathbf{G}$

Infarct to risk ratio

Intravenous

kilodalton

kilopascal

Messenger RNA

Myogenic determination protein 1

Optical density

Polyacrylamide gel electrophoresis

Phophate-buffered saline

Personal computer

Partial pressure of oxygen

Coefficient of determination

Ribonucleic acid 
SDS

$\mathrm{SH}$

SOD

TEMED

TRIS

UV
Sodium dodecyl sulphate

Sulphydryl group

Superoxide Dismutase

NNNN-tetraethylethalinediamine

Tris(hydroxymethyl)methylamine

Ultra-violet 


\section{Acknowledgements}

I am very grateful to a number of individuals who helped me during the period of research that has culminated in this thesis. I am especially grateful to Prof DM Yellon who together with Prof DS Latchman and Dr JM Walker formulated the original ideas that are the basis of this thesis. Without their close supervision my research would undoubtedly have been less productive and certainly much less enjoyable.

I would also like to thank a number of other individuals who provided assistance and companionship. Dr S Iliodromitis and Dr E Pasini are particularly worthy of praise for their encouragement and tolerance to the novice in their midst. Technical assistance from Mr D Farquharson was innovative and of the highest calibre. Software and more general advise from Dr J Downey was greatly appreciated.

I am indebted to the British Heart Foundation for providing the necessary 3 years of financial support to submitt a PhD.

Finally, I would like to thank my wife who was always understanding when research encroached on home life and was a constant source of support and diversion. In addition she made the research period especially fruitful by introducing 2 daughters into the Marber household. It is to my wife and daughters that I dedicate this thesis. 
(xvii)

"One does not discover new lands without consenting to lose sight of the shore for a very long time."

André Gide (1869-1951) French Novelist.

The Counterfeiters 


\section{Chapter 1}

\section{Introduction: Heat Stress, Stress Proteins and the Heart}

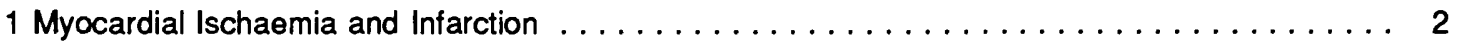

1.1 The Importance of Attenuating Myocardial Ischaemic Injury $\ldots \ldots \ldots \ldots 2$

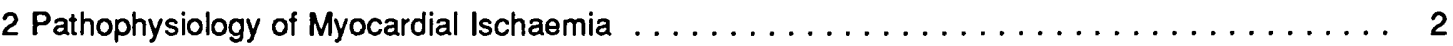

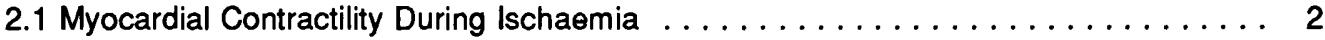

2.1.1 Immediate Contractile Failure ................... 3

2.1.2 Early Contractile Failure $\ldots \ldots \ldots \ldots \ldots \ldots \ldots \ldots \ldots \ldots \ldots$

2.1.3 Late Contractile Failure . . . . . . . . . . . . . . . . . . 3

2.1.4 The Onset of Contracture ....................... 4

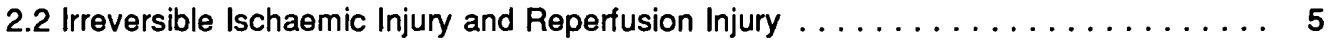

2.3 What Determines Cell Death? . . . . . . . . . . . . . . . . . . . . . . 6

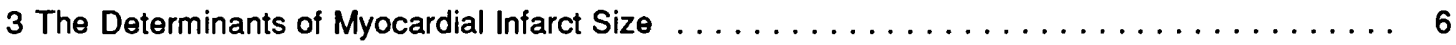

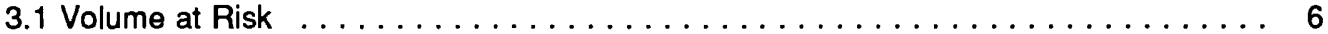

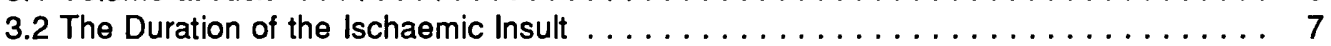

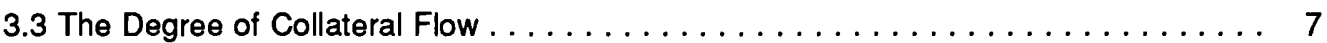

3.4 Metabolic Requirements of the Ischaemic Tissue . . . . . . . . . . . . . 8

4 Heat Stress and the Stress Response $\ldots \ldots \ldots \ldots \ldots \ldots \ldots \ldots \ldots \ldots \ldots \ldots$

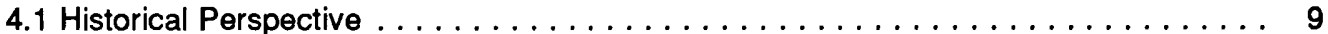

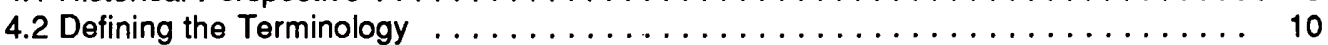

4.2.1 The 100-110kDa Stress Protein Family ................ 10

4.2.2 The 90kDa Stress Protein Family . . . . . . . . . . . . . . . . . . . 12

4.2.3 The 70kDa Stress Proteins Family . . . . . . . . . . . . 13

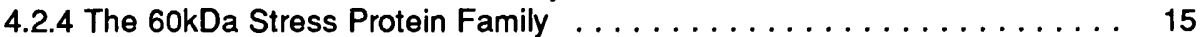

4.2.5 The $30 \mathrm{kDa}$ Stress Protein Family .................. 15

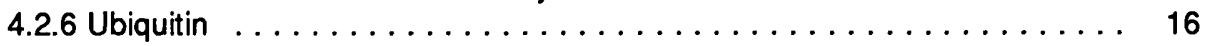

4.3 The Control of the Stress Response $\ldots \ldots \ldots \ldots \ldots \ldots \ldots \ldots \ldots \ldots \ldots \ldots \ldots$

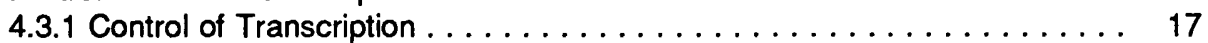

4.3.2 Post-Transcriptional Control .................... 18

5 Are Stress Proteins Protective

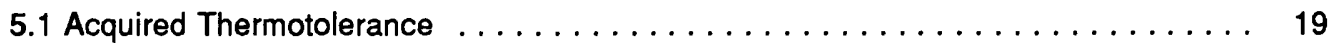

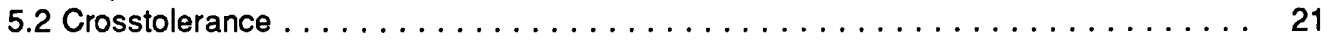

6 Stress Proteins and the Heart . . . . . . . . . . . . . . . . . . . 21

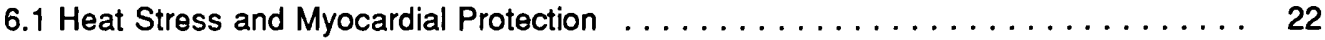

6.1.1 The Isolated Heart and Global Ischaemia $\ldots \ldots \ldots \ldots \ldots \ldots \ldots \ldots \ldots \ldots . \ldots \ldots$

6.1.2 The Isolated Heart and Regional Ischaemia . . . . . . . . . . . 23

6.1.3 The In Situ Heart and Regional Ischaemia . . . . . . . . . . . . . . 24

6.2 Heat Stress Proteins and Classical Ischaemic Preconditioning . . . . . . . . . . . 25

6.3 Non-Thermally Induced Stress Proteins and Protection Against Ischaemia . . . . . 26

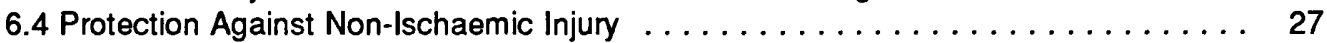

6.5 Mechanisms of Cardiac Protection by Elevated Temperature $\ldots \ldots \ldots \ldots \ldots \ldots$

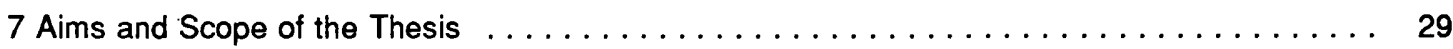




\section{Myocardial Ischaemia and Infarction}

\subsection{The Importance of Attenuating Myocardial Ischaemic Injury}

In the Western world acute myocardial infarction is the most common cause of death in men. In England and Wales during 1990, 90,000 deaths were caused by acute myocardial infarction (OPCS 1991) approximately $20 \%$ of the total all age mortality (DoH 1992). The treatment of acute myocardial infarction is no longer simply supportive, awaiting the complications of ischaemic injury: but has entered a new era where the mortality of acute myocardial infarction can be approximately halved by thrombolytic agents and aspirin (ISIS-2 Collaborative Group 1988), with the greatest benefit seen in those treated aggressively soon after the onset of symptoms (Gusto 1993). The lack of a reduction in mortality when thrombolytic treatment is administered late is most likely due to the fact that the prolonged coronary occlusion has resulted in such severe necrosis of the myocardium that little benefit can be derived by restoring blood flow (Reimer and Jennings 1979). Any intervention that could delay the onset of necrosis could extend the effective time window for thrombolysis. Hence, the widespread use of thrombolytic therapy has increased the need to understand the mechanisms involved in ischaemic myocyte death and acted as an impetus to discover new ways of delaying such injury.

The purpose of this thesis is to determine if a group of proteins, known as the heat shock or stress proteins, offer such potential and are capable of attenuating or delaying myocardial injury. The rationale for examining the myocardial benefits of stress protein induction is discussed in detail in the later part of this chapter after a brief overview of the pathophysiology and assessment of myocardial ischaemia.

\section{Pathophysiology of Myocardial Ischaemia}

Myocardial ischaemia can be defined as a reduction in coronary flow that is so severe that supply of substrates and washout of metabolites is insufficient to meet the needs of the tissue. The important consequences of this are initially a reduction in myocardial contractility with a progression to myocardial cell death if ischaemia is severe and prolonged. 


\subsection{Myocardial Contractility During Ischaemia}

\subsubsection{Immediate Contractile Failure}

When coronary flow is reduced there is an almost immediate reduction in the amplitude of myocardial contraction. Such immediate contractile failure occurring in the first seconds of ischaemia, is secondary to the loss of the stretching effect that an "inflated" coronary vasculature has on the myocyte (Koretsune et al 1991), the so called garden-hose effect.

\subsubsection{Early Contractile Failure}

Beyond the first few seconds of ischaemia contractility is reduced by a number of metabolic and ionic changes occurring within the myocyte (see Poole-Wilson 1992 for review). Lack of oxygen results in a fall in tissue $\mathrm{pO}_{2}$, a cessation of aerobic (mitochondrial) ATP production, and the accumulation of inorganic phosphate and magnesium. At least initially, inorganic phosphate is derived from the hydrolysis of creatine phosphate, creatine not being recharged by the creatine phosphate shunt. The accumulation of inorganic phosphate has a direct inhibitory action on the contractile proteins and reduces the free energy available from ATP hydrolysis, whilst magnesium probably competes with calcium for the calcium specific binding sites on troponin $\mathrm{C}$. These processes account for the early contractile failure during the first 30 seconds to 1 to 3 minutes of ischaemia (Allen and Orchard 1987).

\subsubsection{Late Contractile Failure}

During the next few minutes of ischaemia further changes occur that reduce the strength of contraction.

Glycolysis is initially stimulated, largely as result of AMP and inorganic phosphate increasing the activity of phosphofructokinase, the rate limiting control enzyme of the glycolytic pathway (Opie 1992a, Opie 1992b). Since mitochondrial metabolism is inhibited by the fall in tissue $\mathrm{pO}_{2}$, the end products of enhanced glycolysis are not converted to $\mathrm{CO}_{2}$, and therefore lactate and protons accumulate. During ischaemia, the lack of metabolite washout therefore results in proton and lactate build up and an inhibition of glycolysis by its end products. In contrast during high flow hypoxia where flow continues, glycolysis is not inhibited and may continue 
stimulated several hundred fold compared to baseline aerobic conditions. This situation will continue indefinitely if glucose is present in the perfusion fluid, and if not, until tissue glycogen is depleted, since contractile function is supported by glycolytically derived ATP (Weissler et al 1968). The accumulation of protons during ischaemia acts to reduce the affinity of troponin $\mathrm{C}$ for calcium (Blanchard and Solaro 1984) causing further loss of contractile function. In addition the opening of ATP sensitive potassium channels is associated with potassium efflux which hyperpolarises the cell, reduces the length of the plateau phase of the action potential, and thereby may reduce transarcolemmal calcium entry. This scenario is however difficult to reconcile with the fact that systolic and diastic cytosolic calcium actually increases during early ischaemia (Poole-Wilson 1992).

\subsubsection{The Onset of Contracture}

After approximately 10-15 minutes of ischaemia, ATP levels have fallen sufficiently to prevent the detachment of myosin crossbridges by myosin ATPase (Katz 1992a). This is compounded by the fact that co-operativity of crossbridge formation occurs at low ATP levels (Ford 1991), so that for a given intracellular calcium concentration, crossbridge attachment rates are increased. These effects result in rigor crossbridge formation which causes tonic myocyte shortening, manifesting as an increase in diastolic pressure in the isovolumic Langendorff heart, or an increase in resting tension in isometric isolated muscle. The drop in ATP effects other ATP dependant processes, most notably ionic gradients become disturbed as $\mathrm{Na} / \mathrm{K}$ ATPase, sarcolemmal $\mathrm{Ca}^{2+} \mathrm{ATPase}$ and sarcoplasmic $\mathrm{Ca}^{2+} \mathrm{ATPase}$ activities fall. The failure of these ionic pumps results in an accumulation of calcium and sodium intracellularly (Allen and Orchard 1987). The calcium accumulation further increases rigor crossbridge formation and may additionally compromise mitochondrial function, since mitochondria are freely permeable to calcium which flows down its electrochemical gradient (Crompton et al 1988), predominantly when the mitochondrial proton gradient is recharged upon reperfusion. 


\subsection{Irreversible Ischaemic Injury and Reperfusion Injury}

Beyond a certain critical timepoint the ischaemic injury to the myocyte becomes irreversible, so that even if blood flow, with coincident supply of substrates and washout of metabolites, is restored, there is no recovery. The critical change that occurs within the myocyte that determines if it will survive or die upon reperfusion is not known. The design of experiments to examine the critical changes that result in myocyte death are complicated by the fact that death can really only be determined by the absence of contractile recovery and leakage of intracellular constituents occurring upon reperfusion. However, reperfusion itself may exacerbate preceding ischaemic injury and contribute to cell death, a concept referred to as reperfusion injury (Opie 1989, Yellon and Downey 1990). As a result of these difficulties the concept of reperfusion causing cell death remains controversial despite a decade of intensive investigation. The idea that reperfusion is capable of causing specific types of sublethal myocardial injury such as stunning (contractile dysfunction) or dysrhythmia are however generally accepted (Bolli 1990 and Opie 1989). Whilst the appearance of other forms of injury upon reperfusion such as, enzyme release, myocardial haemorrhage, sarcolemmal rupture, and contraction band necrosis, are likely to reflect reperfusion triggered events occurring in myocytes or areas of myocardium destined to die as a consequence of injury during ischaemia.

Nonetheless, certain biochemical changes occur within the myocyte that are fairly specific to reperfusion. The readmission of oxygen causes the formation of superoxide radicals, hydrogen peroxide, singlet oxygen and the hydroxyl radical (Kukreja and Hess 1993). These species maybe of importance in the genesis of myocardial stunning (Bolli 1990) and reperfusion dysrhythmias (Hearse and Tosaki 1987). Myocardial calcium overload is also associated with reperfusion (Opie 1989). This is largely a result of intracellular $\mathrm{Na}^{+}$and $\mathrm{H}^{+}$accumulation during ischaemia. Upon reperfusion, $\mathrm{Na}^{+}$accumulation leads to $\mathrm{Na}^{+} / \mathrm{Ca}^{2+}$ exchange $\left(\mathrm{Ca}^{2+}\right.$ in for $\mathrm{Na}^{+}$out) and proton accumulation leads to $\mathrm{Na}^{+} / \mathrm{H}^{+}$exchange $\left(\mathrm{Na}^{+}\right.$in for $\mathrm{H}^{+}$out). Thus, inappropriately high intracellular $\mathrm{Na}^{+}$levels are maintained despite extrusion by the $\mathrm{Na}^{+} / \mathrm{K}^{+}$ATPase which becomes active during reperfusion once ATP levels increase (Allen and Orchard 1987). Hence, $\mathrm{H}^{+}$accumulation during ischaemia indirectly worsens the calcium overload upon reperfusion (Lazdunski et al 1985). 


\subsection{What Determines Cell Death?}

Cell viability during ischaemia is unlikely to be determined by a single factor, for example although high energy phosphate content is a major determinant of ionic changes during ischaemia, (either directly of via lactate accumulation), ATP concentration alone does not necessarily reflect cell viability (see Opie 1992a for discussion).

At the molecular level the changes occurring in key proteins during ischaemia are even less fully understood. Cytoskeletal disruption has been described during ischaemia and maybe a key feature of ischaemic injury, causing the appearance of sarcolemmal blebs (Steenberg et al 1987). Such disruption may occur as a result of changes within the proteins that constitute the cytoskeleton such as desmin, actin and vinculin. Actin and desmin are sensitive to changes in $\mathrm{pH}$ and ionic environment, acidosis tending to promote aggregation of these proteins together with alphaB crystallin another cytoskeletal component (Bennardini et al 1992), in fact alphaB crystallin aggregation has been proposed as a molecular marker of ischaemic damage to the heart (Chiesi and Bennardini 1992). Other molecular changes include the oxidation of protein thiol groups upon reperfusion, which may cause the specific changes observed in the activity of the key branch point enzyme mitochondrial carnitine palmitoyltransferase responsble for the shift away from free fatty acid as the preferred substrate during ischaemia (Pauly et al 1991).

\section{The Determinants of Myocardial Infarct Size}

When the flow within a coronary artery ceases, the area of myocardium supplied by that coronary artery is rendered ischaemic and the functional and biochemical changes described above occur within the myocytes. Even if flow is returned, a portion of the area of myocardium may still die and myocardial infarction result. The amount of tissue that infarcts as a result of a transient occlusion is determined by a number of factors. 


\subsection{Volume at Risk}

Intuitively for a given ischaemic insult, the degree of infarction will be a function of the volume of tissue subjected to ischaemia. Hence, the ligation or occlusion of a major coronary artery will result in a larger infarct than the occlusion of a smaller coronary artery for the same period of time. From the experimental viewpoint, since infarct size is directly proportional to the size of the ischemic zone (Miura et al 1987) it is conventional to express the volume of infarct tissue, as a percentage of the volume of tissue rendered ischaemic by coronary ligation (this relationship becomes distorted when risk volumes are small see Chapter 7 Section 3.2. The evaluation of such ratios of infarct to risk zone, reduces the variation in infarct volume that would result from differences in coronary anatomy, and the exact position of the coronary ligature from animal to animal.

\subsection{The Duration of the Ischaemic Insult}

As the duration of ischaemia increases, the proportion of myocardium at risk that progresses to infarction increases. Thus in the rabbit, (little or no coronary collateral flow), at normal body temperature the percentage of the volume at risk that progresses to infarction after a 30 minute coronary occlusion is approximately $50 \%$ and this increases to $55 \%$ after a 45 minute, $68 \%$ after a 60 minute and $85 \%$ after a 90 minute coronary occlusion (Maxwell et al 1989).

\subsection{The Degree of Collateral Flow}

The severity of ischaemia can be attenuated if the volume of tissue at risk of infarction is supplied by blood from more than one coronary artery. In this situation coronary occlusion will not reduce myocardial blood flow to zero and a percentage of normal resting flow will remain. The degree of coronary collateralisation will determine what percentage of normal flow will continue in the volume of tissue at risk during occlusion and becomes a critical determinant of infarct size. Within a particular species, for a given duration of myocardial ischaemia, the higher the degree of collateralisation the smaller the resulting infarct volume as percentage of volume at risk (Miura et al 1987), ie collateral flow is inversely correlated to infarct size. In 
addition, as well as variations within a species, the degree of coronary collateralisation varies between species, and may partially account for the inter-species variation in suceptability to infarction (Maxwell et al 1987).

\subsection{Metabolic Requirements of the Ischaemic Tissue}

Although the factors that determine myocyte death during ischaemia are poorly understood, tissue ATP content is one of the factors thought to be of importance (Jennings and Steenbergen 1985). Therefore any increase in myocardial oxygen demand will tend to increase the relative severity of an ischaemic insult and sensitise to infarction (Schaper and Schaper 1988). Myocardial oxygen demand is related to cardiac work which in turn can be measured in terms of cardiac output (stroke volume multiplied by heart rate) and mean aortic root pressure. In practise the contribution of stroke volume is often ignored and rate pressure product is used to estimate cardiac work. Conversely, agents which tend to reduce myocardial oxygen consumption such as a reduction in myocardial temperature (hypothermia) or a cessation of contractile and electrical activity (cardioplegia) will attenuate the effects of ischaemia.

In addition, some areas of the heart seem to be particularly prone to ischaemic damage. Thus in the canine heart (Reimer and Jennings 1979, Downey 1984) infarction tends to start in the subendocardial region of the volume at risk and spread progressively to the epicardium so as to ultimately cause transmural infarction as the period of coronary occlusion lengthens.

\section{Heat Stress and the Stress Response}

All organisms respond at the cellular level to heat and other stresses by the preferential synthesis of a group of proteins known as the stress or heat shock proteins. The process whereby general protein synthesis is inhibited whilst the synthesis of stress proteins is enhanced is known as the stress response. Studies have demonstrated the importance of these proteins in both the stressed and the unstressed cell, where they perform functions that seem essential to the maintenance of cell viability. Recent interest in this group of proteins has grown with the recognition that manipulation of stress protein content may enhance cell survival following injury. In particular studies suggest that they may be capable of delaying ischaemic injury to the 
myocardium (see editorials anonymous 1991, Black and Lucchesi 1993) and offer a possible future approach to increase the effective timewindow for thrombolysis. Before reviewing the evidence that heat stress and the stress response alter resistance to myocardial ischaemia, some of the general properties of stress proteins will be discussed.

\subsection{Historical Perspective}

The earliest description of the effect of heat on gene expression was by Goldschmidt (1935), who described developmental abnormalities, known as phenocopies within Drosophila heat stressed during embryonal development. The genetic basis for these observations was indicated by the studies of Ritossa (1962, 1963,1964), a geneticist working in Naples. Ritossa reported that when Drosphila (D. buskii or D. melanogaster) larvae incubated at $25^{\circ} \mathrm{C}$, were exposed to temperatures of $30-32^{\circ} \mathrm{C}$ for 30 minutes several new puffs appeared on the giant chromosomes of their salivary glands. He also reported that puffs visible prior to heat stress disappeared following heat stress. In addition these changes were not confined to salivary tissue, but were also visible in cells derived from hindgut and midgut. The heat shock puffing pattern could also be induced by exposing excised salivary glands to elevated temperatures, 2,4-dinitrophenol, sodium salicylate, sodium azide, dicoumarol or reoxygenation following anoxia (Ritossa 1962). Later experiments with radioactive nucleotide precursors showed that these heat induced puffs were sites of intense transcriptional activity. Thus, several of the important features of the heat stress response were with hindsight described in these early studies, namely that the transcription of certain genes are specifically enhanced by heat stress whilst others (the chromosomal puffs that disappear) are inhibited.

Tissières et al (1974) were later able to identify on polyacrylamide gels the protein products, directed from the mRNA's transcribed at the heat shock puff sites. These investigators demonstrated seven new protein species following heat shock, whereas many proteins, made constitutively before the stress was applied regressed, and in some experiments disappeared altogether. Similar results were obtained from different Drosophila tissues including, salivary gland, brain, Malpighian tubes and wing marginal discs at several stages of development (Tissières et al 1974). Later 
work by Linquist-McKenzie et al (1975) demonstrated that following heat shock in Drosophila-derived cells, polysomes (strings of ribosomes on mRNA) would disappear rapidly and this would be followed by the build up of new larger polysomes. The newly made RNA comprising these polysomes was of a size sufficient to code for hsp70 and hybridized in situ to chromosomal DNA at the sites of the two largest puffs. Spradling et al (1977), using a slightly different strategy demonstrated that labelled RNA from cells grown at $25^{\circ} \mathrm{C}$ (normal temperature) hybridised with about 50 different chromosomal bands, whilst labelled RNA isolated from heat shocked cells hybridized to 7 bands at a different location to the 50 previously seen. These seven bands corresponded to the heat shock puffs. Moreover when the purified fraction of such mRNA from heat shocked cells was added to an in vitro translation system, heat shock proteins were translated (Linquist-McKenzie and Meselson 1977, Spradling et al 1977, Mirault et al 1978). Taken together these experiments clearly indicated that heat shock mRNA's made at heat shock puff sites were translated into heat shock proteins.

\subsection{Defining the Terminology}

To understand the possible protective benefits of stress proteins, a brief description of their function is necessary. Constitutive stress proteins are expressed in the basal, unstressed state, but will, by definition, be synthesized at higher levels during stress. Alternatively, stress proteins may be exclusively inducible, being present only in the stressed organism.

Stress proteins are grouped into families with differing functions (Linquist 1986, Welch 1990, Hightower 1991) and they are classified primarily according to molecular weight (see table 1.1 for summary). Specifically, the stress protein families include those in the range $100-200 \mathrm{kDa} ; 80-100 \mathrm{kDa} ; 70 \mathrm{kDa} ; 58-65 \mathrm{kDa} ; 25-30 \mathrm{kDa}$ and ubiquitin.

\subsubsection{The 100-110kDa Stress Protein Family}

This is probably the most poorly characterized group of stress proteins. They are present in most, if not all eukaryocytic cells and are located within the nucleolus in both unstressed and heat stressed cells (Subjeck et al 1983, Sanchez and Linquist 


\section{Table 1.1: Stress Protein Families and Function}

$=$

Family
$100-110 \mathrm{kDa}$
$90 \mathrm{kDa}$
$70 \mathrm{kDa}$
$60 \mathrm{kDa}$
30kDa
Ubiquitin

\section{Functions}

Poorly defined may be responsible for acquired thermotolerance in yeast (Sanchez and Linquist 1990)

? Involved in nucleolar RNA processing (Subjeck et al 1983)

Bind actin probably under calcium/calmodulin control (Koyasu 1989)

Transport molecules between organelles whilst maintaining structure (Kang et al 1990)

Able to promote correct protein refolding in vitro (Weich et al 1992)

Inducible member most frequently used marker of stress response

Bind and hydrolyse ATP may promote protein folding (Flaherty et al 1990)

Post-translational protein import into mitochondria and

endoplasmic reticulum (Horwich 1990)

Facilitate protein refolding in mitochondrial matrix (Osterman et al 1989)

Protect proteins from denaturation (Martin et al 1992)

Diverse group, antioxidant (Kim et al 1989) and

structural members (Chiesi and Bernnardini 1992)

Tags proteins for degradation (Hershko 1988) 
1990). Their synthesis in rat soleus muscle can be enhanced by exercise as well as heat stress (Locke et al 1990).

In terms of function, hsp104 is thought to be essential in some organisms in conferring thermotolerance. For example, in yeast a mutation within the HSP104 gene allows normal growth in the unstressed state but not at elevated temperatures (Sanchez and Linquist 1990). The exact function of this family of proteins is at present conjecture but they seem to be involved in ribosomal RNA processing since they can be released by RNAase (Subjeck et al 1983).

\subsubsection{The 90kDa Stress Protein Family}

This family of constitutive proteins are highly conserved between prokaryotes and eukaryotes (Bardwell and Craig 1987).

The $90 \mathrm{kDa}$ family bind actin, a phenomenon that is modulated by both calcium and calmodulin (Koyasu 1989). Their location is predominantly cytoplasmic and they are thought to act as chaperones (Ellis 1990), being able to assist the transport of molecules through their interaction with actin filaments whilst also offering protection against inappropriate or premature molecular interactions. For example the glucocorticoid receptor is found in two forms in the cell, a 7-10S inactive form and a smaller 4S DNA-binding (active) form (Beaulieu 1987, Sherman and Stevens 1984). In the absence of an appropriate agonist the receptor is inactive (the heavier 7-10S form) and bound to actin via hsp90 (Koyasu et al 1989). When steroid hormone binds, hsp90 dissociates, and the receptor becomes capable of binding DNA and activating transcription, since without hsp90, DNA binding of the receptor occurs in the absence of steroid hormone, it is thought that hsp90 acts to hold the receptor in an "inactive" state until the appropriate hormonal signal is given (Sanchez et al 1987). It probably achieves this inactive (non-DNA-binding) state by altering the conformation of the steroid receptor (Picard et al 1988).

Since hsp90 seems to regulate protein phosphorylation by influencing the activity of a number of kinases this family probably has a far more reaching and general function in the control of protein synthesis. For example, protein translation is dependant on eukaryocyte initiation factors, one such factor eIF- $2 \alpha$ is activated by phosphorylation and the activity of the relevant kinase is enhanced when bound to 
hsp90 (Rose et al 1989). Such association with, and control of, kinases seems to be a general property of this family, since hsp90 interacts with several viral oncogene products that display tyrosine kinase activity and are capable of influencing cell growth and/or mitotic rate (Brugge et al 1981, Opperman et al 1981, Lipsich et al 1982, Ziemiecki et al 1986).

The nature of the association of hsp90 with putative target proteins has been demonstrated by two elegant studies. MyoD1 is a myogenic determination protein, it achieves this by binding to specific DNA sequences that are the enhancer elements controlling the transcription of a number of muscle specific proteins. Activation of myoD1 occurs following a transient association with hsp90, this association is dependant on the C-terminal 194 amino acids of hsp90 (determined by selecting deletion mutants), and does not require ATP (Shaknovitch et al 1992). Interestingly, hsp90 seems to be "consumed" in the association leading to the speculation that the conformational energy that resides within the tertiary structure of hsp90 is used to alter the tertiary structure of myoD1 in such a way as to increase it's sequence specific DNA binding. It is therefore possible that ATP is required to refold "spent" hsp90. Similarly in an in vitro system hsp90 was found to increase the yield (once again independent of ATP) of functioning citrate synthase and monoclonal antibody Fab fragment following guanidine denaturation (Weich et al 1992). These are structurally very different proteins, citrate synthase comprising helices held together by hydrogen bonds, and the Fab fragment beta-pleated sheets held together by disulphide bridges. An ability to correctly reconstitute and prevent aggregation of such differing structures, suggests that protein refolding maybe a general property of the hsp90 family.

\subsubsection{The 70kDa Stress Proteins Family}

This is perhaps the best characterised family of stress proteins and from the cardiac viewpoint the most extensively investigated. The importance of this family of proteins is indicated by the fact that they are the most highly conserved proteins known (greater than actin), sequence homology between E.coli and man being better than 50\% (Hunt and Morimoto 1985). There are relatively abundant constitutive members of this family found in the nucleus, nucleolus and endoplasmic reticulum. 
Monoclonal antibodies to both constitutive and inducible members of this family are commercially available.

The $70 \mathrm{kDa}$ family bind and hydrolyse ATP, their release from polypeptide chains being an energy dependent process. ATP binding activity resides in the Nterminus of the protein which exhibits higher conservation than the polypeptide binding C-terminus (Flaherty et al 1990). The ATP binding domain has a bilobed structure that resembles the ATPase cleft of hexokinase (Flaherty et al 1990). The authors postulate (Flaherty et al 1990) that the energy made available by ATP hydrolysis causes a conformational change in the ATP binding domain that may be transmitted to the substrate recognition domain, forcing a conformation change within the bound substrate.

The hsp70 family have been implicated in a number of processes requiring protein folding. For example they are involved in post-translational import of proteins into endoplasmic reticulum and mitochondria (Horwich 1990). Only a small portion of mitochondrial protein is encoded on mitochondrial DNA and translated on mitochondrial ribosomes, the remainder is nuclear encoded and transcripts are translated on cytoplasmic ribosomes. Such nuclear encoded proteins are imported into mitochondria through specific pores, the dimensions of which are such that proteins must first be unfolded, and then correctly refolded once admitted into the mitochondrial matrix. Hsp70 is thought to be essential as both the unfoldase on the cytoplasmic side of the outer mitochondrial membrane and the refoldase within the matrix (Rothman and Kornberg 1986, Hutchinson et al 1989, Horwich 1990, Cheng et al 1990, Kang et al 1990). More generally it has been proposed that hsp70 is able to recognize improperly folded and unfolded proteins (Pelham 1986). It is not known what would regulate this function, recognition of exposed hydrophobic regions of unfolded proteins has been suggested. However, this cannot be the only consideration because hsp70 binds to many synthetic peptides without showing preference for hydrophobic regions (Flynn et al 1989), more likely, a subtle change occurs within a protein that increases the probability of hsp binding. A scenario similar to this seems to occur with clatharin triskelions which aggregate to form cages around coated endocytotic vesicles (Ungewickell and Branton 1981). Soon after coated vesicles form, the clatharin triskelions are released (Heuser and Reese 1973) an ATP consuming 
process, mediated by hsp70 binding to the light chain component of the triskelion (Schmidt and Rothman 1985). The affinity of hsp70 for the clathrin light chain is altered by the presence of $\mathrm{Ca}^{2+}$ and/or $\mathrm{K}^{+}$which change the conformation of the light chain in such a way as to make the hsp70-clatharin light chain interaction less likely (DeLuca-Faherty et al 1990).

The $72 \mathrm{kDa}$ stress protein, a member of this multigene family, is highly inducible and the most frequently used marker of the cardiac stress response.

\subsubsection{The 60kDa Stress Protein Family}

The $60 \mathrm{kDa}$ family of proteins are predominantly mitochondrial, and are essential, together with hsp70, in facilitating the refolding of proteins imported from the cytoplasm. Nuclear encoded mitochondrial proteins are translocated via specific contact sites between the outer and inner mitochondrial membranes, before proteolytic processing and interaction with mitochondrial hsp70 (mhsp70) and hsp60 in the matrix. The role of hsp60 is illustrated by the processing of dihydrofolate reductase (DHFR) (Ostermann et al 1989). When linked to a signal sequence for mitochondrial protein import, this protein is imported into mitochondria the signal sequence is removed, and the enzyme folds into a compact protease resistant form. However this folding is dependant on the presence of hsp60 and ATP. In the absence of ATP, hsp60 is associated with the enzyme, however when ATP is added DHFR is released and folds normally. In addition a temperature dependant association between DHFR and hsp60 has been demonstrated (Martin et al 1992) which prevents thermal denaturation of DHFR a process during which hsp70 may act in a cooperative fashion (Martin et al 1992). Interestingly many components of the inner mitochondrial membrane such as cytochrome reductase and components of the assembled F1-ATPase complex contain hsp60 proteins which may have dual roles controlling both, mitochondrial ATP synthesis and facilitating the correct refolding of imported proteins (Luis et al 1990).

\subsubsection{The 30kDa Stress Protein Family}

This family is the most divergent of the stress protein families with little sequence homology across species. There are nevertheless related to one another having a conserved sequence near the $\mathrm{C}$-terminus as well as conserved amino acids 
at various other positions (Linquist and Craig 1988). In addition, they all tend to form large polymeric particles known as heat shock granules (Arrigo and Welch 1987). One such low molecular stress protein found in abundance in cardiac tissue is alphacrystallin. The B subunit of alpha-crystallin is associated with the cytoskeleton and alters the physical properties of actin and desmin. The binding of alphaB-crystallin to actin and desmin is increased at acidic $\mathrm{pH}$ and also following heat shock. The association of alphaB-crystallin with these cytoskeletal proteins decreases their propensity to form aggregates at acidic $\mathrm{pH}$, leading to the suggestion that may play a role in preventing filament aggregation and irreversible structural damage during ischaemia (Bennardini et al 1992). In fact alphaB-crystallin has been proposed as a molecular marker of myocardial ischaemic damage (Chiesi and Bennardini 1992). A similar protein has been isolated from skeletal muscle where it acts as a barbed end capping protein preventing F-actin polymerisation (Miron et al 1991) and may form polymeric complexes with other small molecular weight heat shock proteins (Kato et al 1992).

Other low molecular weight stress proteins are induced by oxidant stress and seem to have direct anti-oxidant activity (Kim et al 1989), one such protein induced in human tissue has tyrosine phosphatase activity, and may therefore play a role in coordinating the stress response (Keyse and Emslie 1992).

\subsubsection{Ubiquitin}

This a highly conserved 76 amino acid protein which is ubiquitous to all eukaryotic cells and plays an integral role in the selective degradation of both shortlived and abnormal proteins (Hershko 1988). Ubiquitin is linked to proteins via its terminal glycine a process probably involving three other intermediate proteins (E1, E2 and E3). Ubiquitin is bound first to E1 and then E2 which probably exists in a number of forms, those forms of E2 involved with protein degradation require the target protein first to be bound to E3 prior to linkage with ubiquitin and targeting to proteosomes for proteolysis (see Hershko 1988 for review). Although covalent linkage to ubiquitin seems to tag proteins destined for degradation the system has further poorly understood complexities, for example certain stable proteins are reversibly linked to ubiquitin such as histones (Busch 1984) and membrane receptors (Leung et 
al 1987). Leading to the suggestion that ubiquitin is involved in cell cycle control (Goebl et al 1988), DNA repair (Jentsh et al 1987) and receptor modulation (Leung et al 1987).

\subsection{The Control of the Stress Response}

Heat, heavy metals, metabolic poisons, amino acid analogues, hypoxia and ischaemia are amongst the many stresses known to cause stress protein expression. How do such varying stresses act to trigger the stress response?

Although the stress response is universal, its quality and magnitude vary widely. In general eukaryotes possess at least two copies of heat shock genes, one under heat shock/stress regulation and the other(s) under constitutive control. However prokaryotes possess only a single copy of each heat shock gene. This fact, coupled with the essential function of these genes necessitates the constitutive expression of most prokaryote heat shock genes. Consequently, control processes for eukaryote and prokaryote heat stress gene transcription differ, in this thesis only the eukaryote stress response will be discussed further.

\subsubsection{Control of Transcription}

The induction of eukaryotic heat shock genes in response to an increase in temperature is mediated by the binding of a transcriptional activator, heat shock factor, (HSTF) to a short highly conserved DNA sequence known as the heat shock element (HSE). Heat shock elements are contiguous arrays, of variable numbers, of the 5 base pair sequence nGAAn or nTTCn which are arranged in alternating head to head and tail to tail alignment, with strict spacing requirements (Perisic et al 1989). Heat shock factor, a trimeric protein binds to these elements, each subunit is thought to bind to a single nGAAn unit in a cooperative fashion (Xiao et al 1991) and probably a number of such elements must be bound to activate transcription (Bienz and Pelham 1986). It is feasible that heat shock factor binding could act to alter the tertiary structure of DNA by holding nGAAn sequences in adjacent strands in close proximity, a rearrangement that may promote gene transcription. Upon heat shock, a pre-existing pool of unactivated heat shock factor is converted into a form capable of efficiently stimulating transcription. This conversion may be secondary to temperature dependant 
phosphorylation (Sorger and Pelham 1988) of the heat shock factor that results in binding to the heat shock element (Goldenberg et al 1988). In common with thermal stress, exposure of mammalian myocardial cells to a hypoxic environment activates DNA binding of heat shock factor, a process independent of new protein synthesis (Benjamin et al 1990). In a more recent study, the induction of hsp72 mRNA in neonatal cardiocytes in response to hypoxia precedes the intracellular depletion of ATP, whilst induction in response to metabolic inhibitors is more closely related to ATP depletion (Iwaki et al 1993). In addition, hsp70 mRNA levels increased prior to apparent binding of HSTF to the HSE suggesting alternative "sensing" mechanisms maybe responsible for transcriptional activation in this model (Iwaki et al 1993).

The presence of denatured or damaged proteins within the cytoplasm may be a common feature of signalling pathways by which diverse stresses increase heat stress gene transcription, since microinjection of denatured protein leads to activation of the heat shock factor (Ananthan et al 1986). In addition this activation can be modelled in cell-free systems. Cytosolic extracts from unstressed HeLa cells exhibit HSEbinding activity in vitro only after exposure to elevated temperature, protein denaturing agents or profound acidosis (Mosser et al 1990). In addition in cultured myogenic cells DNA binding of heat shock factor is induced by hypoxia, inhibitors of oxidative phosphorylation and by acidosis, leading to the speculation that depletion of high energy phosphate may also be a contributing determinant of heat shock factor activation (Benjamin and Williams 1990) although this is not compatible with the more recent findings presented above (Iwaki et al 1993).

In addition to the heat shock element, other cis acting elements control stress protein gene transcription. The control of one of the human hsp70 genes for example involves promotor elements that confer, interleukin 2, forskolin, viral (adenovirus and herpes simplex), tissue and development specific gene activation (Morimoto and Milarski 1990).

\subsubsection{Post-Transcriptional Control}

As well as an increase in gene transcription, other changes occur to facilitate stress protein synthesis during the stress response. Most hsp70 genes for example, lack introns making RNA splicing unnecessary (Yost et al 1990). In addition compared to 
other mRNA transcripts, mRNAs encoding heat stress proteins exhibit increased stability during stress conditions (Petersen and Linquist 1988). Whereas protein synthesis of other mRNA species declines during heat stress, mRNAs encoding stress proteins continue to be translated efficiently. The binding of a $25 \mathrm{kDa}$ translation factor to the proximal nucleotides of hsp70 mRNA has been postulated to have a role in the preferential translation of this message (Yost et al 1990). Whilst a 17kDa stress protein found in cardiac muscle acts to inhibit general protein synthesis, by inhibiting $80 \mathrm{~S}$ ribosomal initiation of low molecular weight peptides (Havre and Hammond 1988). In addition heat shock mRNAs have a relative paucity of secondary structure in the $5^{\prime}$ untranslated region and therefore are expected to have a limited requirement for ATP-dependent RNA unwinding during translation (Yost et al 1990).

\section{Are Stress Proteins Protective?}

The ability of stress proteins to interact with other proteins and; (1) alter their conformation (eg hsp70 and mitochondrial protein import); (2) protect them from inappropriate and premature interactions (eg hsp90 and the steroid receptor); (3) protect them from thermal denaturation (hsp60 and DHFR); (4) encourage the correct refolding of denatured proteins (hsp90 and Fab or citrate synthase); (5) aid the degradation of denatured proteins (eg ubiquitin); (6) "sense" subtle changes in the conformation of other proteins (hsp70 and clatharin triskelions); (7) stabilise the cytoskeleton (alphaB-crystallin interacting with actin and desmin); all suggest that stress proteins maybe capable of increasing cell viability both during and following denaturing stresses. What is the evidence that this is indeed the case?

\subsection{Acquired Thermotolerance}

When prokaryotic, or eukaryotic cells, or even intact multicellular organisms, are exposed abruptly to temperatures several degrees above their normal ambient temperature, the cells or animals will die within a predictable period of time. However if the cells are exposed first to the elevated temperature for a shorter time period and allowed to recover at normal temperatures, they can survive a subsequent period of otherwise lethal heat stress. This universal phenomenon is known as Acquired Thermotolerance (Gerner and Schneider 1975). Stress proteins induced by the first 
period of sublethal thermal stress are attractive candidates to explain the acquisition of thermotolerance.

The evidence that stress proteins can mediate thermotolerance is strong. For example with regard to hsp70 in eukaryotic cell lines; thermal tolerance and stress protein induction are temporally related ( $\mathrm{Li}$ and Werb 1982); the degree of stress protein expression following stable transfection correlates with the degree of thermotolerance (Li et al 1991); cell lines expressing abnormal hsp70 with a missing ATPase domain retain thermotolerance ( $\mathrm{Li}$ et al 1992); cells microinjected with a monoclonal antibody recognising a shared epitope on constitutive and inducible forms of hsp70 are thermosensitive (Riabowol et al 1988); mutants selected for multiple copies of the hsp70 promotor region are thermosensitive (Johnston and Kucey 1988) (in this study copies of the hsp70 regulatory region presumably competed with the endogenous hsp70 encoding genes for factors that activate hsp70 expression, since hsp70 induction was reduced by $90 \%$ ); and stress proteins incorporating amino-acid analogues (ie defunct stress proteins) result in thermal sensitisation ( $\mathrm{Li}$ and Laszlo 1985). Other stress proteins may also be important since rodent cell lines stably transfected with hsp27 (homologous to alphaB crystallin) are "naturally" thermotolerant (Landry et al 1989), whilst cells with an hsp104 heat stress protein deletion are unable to acquire thermotolerance (Sanchez and Lindquist 1990). In addition transfection with antisense RNA for hsp90 (to specifically hybridise with, and prevent the translation of native hsp90 mRNA) also prevents the acquisition of thermotolerance (Bansal et al 1991).

However, it is not certain whether thermotolerance represents better recovery following heat stress or actual attenuation of injury during the thermal stress. Laszlo (1992) has demonstrated that protein synthesis and RNA synthesis are similarly depressed following heat stress of thermotolerant and naive fibroblasts, however recovery of both processes was enhanced in cells overexpressing hsp70 (either by transfection or by prior treatment with sublethal thermal stress or sodium arsenite). In contrast other investigators (Burgman and Konings 1992) have found evidence for protection during thermal stress since conformational changes within proteins, detected by both $\mathrm{SH}$ group targeted electron spin resonance and mobility by thermal gel analysis, occur at a higher temperature in thermotolerant eukaryotic cell lines. Clearly 
protection may occur both during and following the thermal denaturing stress and these mechanisms are not mutually exclusive.

The arguments are further complicated by the fact that, thermotolerance in some situations can occur in the absence of stress protein induction, leading to the speculation that two types of thermotolerance exist, stress proteins being responsible for the acquisition of long but not short-lasting thermotolerance (see discussion in Black and Subjeck 1991).

\subsection{Crosstolerance}

If sublethal heat stress induces stress proteins that protect against subsequent lethal heat shock, can other non-thermal stresses that also induce stress proteins give rise to similar thermal tolerance? Interestingly, this does seem to be the case. However, of more relevance to experimental cardiology thermally induced stress proteins seem capable of protecting against stresses which themselves induce stress protein synthesis. Such cross-protection is known as crosstolerance.

There are numerous studies that support the concept of crosstolerance first suggested by Li and Hahn (1978), who demonstrated that a hamster cell line could be rendered resistant to both adriamycin and heat toxicity by pretreatment with ethanol. Later studies have demonstrated similar findings but with very different stresses. For example; whole body heat stress in rats protects retinal pigment cells from light injury, protection being temporally dependant on hsp70 (Barbe et al 1988); pretreatment with hydrogen peroxide protects against subsequent oxidative stress (Christman et al 1985); heat stress protects against subsequent oxidative stress in a number of models (see Polla et al 1991 for review); heat stressed human breast cancer cells are rendered resistant to doxorubicin an effect that seems related to hsp70 and hsp27 cell content (Ciocca et al 1992) and heat stressed neuronal cells are resistant to the excito-toxic effects of glutamate an effect dependant on protein synthesis and related to hsp70 (Lowenstein et al 1991, Rordorf et al 1991).

\section{Stress Proteins and the Heart}

Stress proteins have been detected in the myocardium of a variety of mammalian species. Their synthesis has been shown to be increased by whole body 
temperature elevation (Currie and White 1983, Currie 1987, Iwaki et al 1989, Yellon et al 1992) and other stressful stimuli including ischaemia (Mehta et al 1988), brief ischaemia and reperfusion (Knowlton et al 1990a), anoxia (Tuijl et al 1991), hypoxia (Howard and Geoghegan 1986), pressure or volume overload (Delcayre et al 1988), mechanical stretch (Knowlton et al 1991b), cytokines (Löw-Friedrich et al 1992) and drugs such as vasopressin (Moalic et al 1989), isoproterenol (White and White 1986), hydrogen peroxide (Löw et al 1989), L-type (slow) calcium channel blockers (LöwFriedrich and Schoeppe 1991) and other cardiotoxic drugs and heavy metals (Tuijl et al 1991 and Löw-Friedrich et al 1991). The implication of these findings is that stress proteins may play an important role in the cardiac stress response. Although many of these stimuli induce members of the $70 \mathrm{kDa}$ family of stress proteins, there appear to be differences in the pattern of stress protein induction when other families of stress proteins are considered. For example, cardiotoxic drugs preferentially induce a $30 \mathrm{kDa}$ stress protein (Löw-Friedrich et al 1991). It is possible that each stress induces its own subtly differing profile of stress proteins best suited to meet the cellular consequences of that particular stress.

\subsection{Heat Stress and Myocardial Protection}

Following the suggestion that, (1) tissues with thermally pre-elevated stress proteins are resistant to stresses that normally induce stress proteins (crosstolerance), and (2) cardiac ischaemia causes stress protein induction (Dillmann et al 1986, White and White 1986, Knowlton et al 1991a), investigators have been prompted to examine whether myocardial tissue entering ischaemia with a pre-elevation of stress proteins is resistant to infarction (Currie et al 1988, Donnelly et al 1992, Liu et al 1992, Yellon et al 1992, Currie et al 1993). The investigation of stress protein expression and their possible involvement in cardioprotection is an evolving area of research, consequently many of the studies are preliminary and in abstract form only.

\subsubsection{The Isolated Heart and Global Ischaemia}

Currie et al (1988) were the first investigators to show that temperature elevation to $42^{\circ} \mathrm{C}$ in rats resulted in concomitant cardiac stress protein and catalase induction, and an attenuation of ischaemia/reperfusion injury. Using an isolated heart 
model these investigators demonstrated that following global ischaemia/reperfusion, contractile function is enhanced whilst creatine kinase release is dramatically reduced in heat stress compared with control hearts. These findings have been confirmed by Yellon's group in both the rat (Pasini et al 1991) and the rabbit (Yellon et al 1992a). Moreover, these authors have observed improvements in additional parameters of protection in the heat stressed rabbit heart post-ischaemia (Yellon et al 1992a). These include preservation of high energy phosphates, a reduction in oxidative stress during reperfusion (as measured by lower levels of oxidized glutathione) and significant mitochondrial preservation following ischaemia. There does, however, appear to be some species variation in the metabolic changes associated with protection following heat stress. In the rabbit for example, higher levels of high energy phosphates mirror the enhanced contractile activity of heat stressed hearts during reperfusion (Yellon et al 1992a). In the rat, however, the enhanced contractile activity following ischaemia in the heat stressed groups is not associated with differences in high energy phosphate content between heat stressed and control hearts (Pasini et al 1991, Currie and Karmazyn 1990).

More recently, preliminary studies by other investigators have shown that similar protection is seen in the hypertrophied rat heart (Snoeckx at al 1993, Cornelussen et al 1993). These investigators have shown that the benefits of whole body heat stress are even more marked in the hypertrophied than in the normal heart. In this model protection was evident as both a reduction in reperfusion arrhythmias and $\mathrm{LDH}$ release.

Not all studies have found heat stress to be cardioprotective. For example, preliminary studies by one group (Wall et al 1990) have been unable to demonstrate any protection against "no-flow" ischaemia following heat stress in the isolated unpaced working rat heart, with rate pressure product and cardiac output as endpoints. These investigators, although following an established protocol known to enhance cardiac stress protein synthesis, did not measure stress proteins directly.

\subsubsection{The Isolated Heart and Regional Ischaemia}

The studies summarized to this point have demonstrated protection expressed in terms of myocardial contractility and metabolic state. The ability of whole body 
heat stress to reduce the extent of myocardial infarction as examined by triphenyl tetrazolium staining has also been examined. In a preliminary study in the rat model infarct size is reduced following 37.5 minutes of regional ischaemia, with protection being temporally related to elevated stress protein levels at 24-96 hours following whole body heat stress (Loesser et al 1992). In addition, Walker et al (1993) have demonstrated a similar reduction in infarct size following 45 minutes of regional ischaemia and 2 hours reperfusion in the buffer perfused rabbit heart removed 24 hours after whole body heat stress.

\subsubsection{The In Situ Heart and Regional Ischaemia}

Interestingly, in contrast to isolated heart studies, controversy surrounds the ability of whole body heat stress to reduce infarct size in vivo. In the rabbit, heat stress 24 hours prior to ischaemia was unable to reduce infarct size following a 45 minute coronary occlusion (Yellon et al 1992b) although protection was found following a 30 minute occlusion by Currie et al (1993). A similar dependence on the length of coronary occlusion is seen in the rat. Donnelly and coworkers (1992) have demonstrated a reduction in infarct size in the rat following a 35 minute, but not a 45 minute, coronary occlusion initiated 24 hours after whole body heat stress. Moreover in this model the reduction in infarct size, following a graded heat stress procedure, is related to the degree of stress protein induction (Hutter et al 1993). The apparent dependency of protection on the length of ischaemic insult is difficult to explain. One possibility is that the protection conferred by heat stress is only moderate, and that as the severity of the ischaemic insult increases the protection becomes less evident. A similar phenomenon occurs with ischaemic preconditioning in dogs where a marked reduction in infarct size occurs with 60 minutes of coronary occlusion, but not with 90 minutes (Nao et al 1990). Another apparent anomaly is the fact that although infarct size is reduced after a 30 minute coronary occlusion twenty-four hours following whole body heat stress in the rabbit, no protection is seen at 48 hours after heat stress at a time when cardiac stress protein content is still increased (Currie et al 1993).

Other investigators have been able to demonstrate in-vivo protection following hot blood cardioplegia of the pig heart (Liu et al 1992). In another rather "novel" 
approach (Schott et al 1990), a microwave diathermy probe was applied to the canine heart. Although such regional hyperthermia was shown to increase stress protein mRNA expression, there was no concomitant protection against infarction. However, the time course of this study was such that appreciable stress protein accumulation was unlikely to have occurred in the one hour between thermal stress and coronary ligation. In addition, the spatial relationship between heat-treated myocardium and the subsequent area of hypoperfusion during coronary ligation was not documented.

The cause for the discrepancy between in vivo and in vitro studies is not clear. However recent observations from our laboratory suggest that whole body heat stress may activate a blood borne component that overrides the beneficial effect of cardiac stress protein induction. This inference was made after noticing that blood from a heat stressed support rabbit; when used to perfuse an isolated rabbit heart, significantly increased infarct size (Walker et al 1993). It may be that whole body heat stress, although conferring myocardial protection causes confounding physiological changes which have negative effects on infarct size. This is consistent with the finding that cytotoxic T-cells directed against myocardial heat shock proteins are induced in rats by stresses that elevate myocardial heat stress protein content (Huber 1992), and that these cells are cytotoxic in vitro to heat stressed myocytes from the same species. In addition, the possibility remains that the duration of recovery after heat stress and length of the ischaemic insult may also influence the ultimate infarct size and appearance of cardioprotection.

\subsection{Heat Stress Proteins and Classical Ischaemic Preconditioning}

Acquired thermotolerance, where sublethal temperature elevation protects against subsequent lethal hyperthermia is similar in concept to ischaemic preconditioning, with sublethal ischaemia protecting against subsequent lethal ischaemia. One could speculate that stress proteins synthesised in response to the first brief episode of preconditioning ischaemia are involved in the protection against the subsequent injury. In agreement with this idea Knowlton et al (1990a) demonstrated that brief bursts of ischaemia, such as those used in preconditioning protocols, can induce hsp70 mRNA and protein accumulation. The mechanism by which stress proteins are induced by short episodes of ischaemia may be secondary to the free 
radical stress induced by reperfusion, since in the isolated rat heart stress protein induction following a $15 \mathrm{~min}$ infusion of xanthine plus xanthine oxidase is quantitatively similar to that induced by ischaemia with reperfusion (Kukreja et al 1992). However, in both the study by Knowlton et al (1990a) and work from Yellon's laboratory (Kucukoglu et al 1991), elevated levels of the hsp70 protein were only manifest at 2-24 hours after the ischaemic insult. In contrast the protective effect of preconditioning is lost approximately 1 hour after the initial brief ischaemic episode (Van Winkle et al 1991).

The involvement of stress protein in ischaemic preconditioning has been further questioned by a study (Thornton et al 1990) which indicates that the protective effect of preconditioning can be observed under conditions where de novo protein synthesis has been almost entirely inhibited. Thus, it is unlikely that stress proteins are involved in classical ischaemic preconditioning. However, the changes in mRNA coding for stress proteins indicate an adaptive response to ischaemia which may predict a delayed protection dependent on stress protein synthesis.

\subsection{Non-Thermally Induced Stress Proteins and Protection Against Ischaemia}

The fact that stress protein induction by ischaemia is not temporally related to classical ischaemic preconditioning has prompted some investigators to examine whether preconditioning ischaemia is associated with a delayed, as well as an early phase of myocardial protection. Preliminary reports in the dog (Yamashita et al 1992 and Hoshida et al 1992) as well as our studies in the rabbit presented later in this thesis (see Chapter 7), suggest that a second phase of protection exists 24 hours after preconditioning with 4 repeated, 5 minute episodes of ischaemia. Interestingly, a similar phenomenon appears to occur within the brain, where ischaemic pretreatment with 2, repeated episodes of 2 minute bilateral carotid occlusions is capable of limiting the neuronal cell loss that follows a subsequent more prolonged bilateral carotid occlusion (Kitagawa et al 1990, Kitagawa et al 1991, Liu et al 1992). For this protective effect to be manifest, the short occlusions must precede the long occlusion by at least 24 hours, a time interval known to result in cerebral heat stress protein accumulation in an identical model (see Nowak 1990 for review). Similarly whole 
body heat stress in the rat offers similar protection against ischaemic neuronal damage (Chop et al 1989). It is interesting to speculate if myocardial adaptation to ischaemia may explain the apparent benefit of a 7 day or more history of angina prior to myocardial infarction (Muller et al 1990), although likely collateral vessel formation and concomitant medication make any definite conclusions impossible. In contrast other attempts to induce myocardial protection by ischaemic pretreatment have been unsuccessful. For example, Donelly et al (1992) compared the protective benefit of heat stress with 24 hours of recovery to 20 minutes of ischaemia with 8 hours of reperfusion. Following a subsequent 35 minute occlusion in the rat, heat stress pretreatment reduced infarct size, whilst ischaemic pretreatment did not. However, as heat stress resulted in more marked stress protein induction, the authors concluded that ischaemic pretreatment failed because of insufficient stress protein accumulation.

Other methods of stress protein induction have included immobilization stress which has also been shown to protect against subsequent ischaemia/reperfusion injury assessed by CPK leakage, contractile function and reperfusion arrhythmias (Meerson and Malyshev 1989, Meerson et al 1992).

\subsection{Protection Against Non-Ischaemic Injury}

The observations that cardiac tissues synthesize stress proteins in response to a variety of stresses has encouraged investigators to explore the breadth of protection that follows heat stress. In this regard Meerson (Meerson et al 1991a, Meerson et al 1991b) has demonstrated that stress protein induction by either heat or immobilization protects the isolated rat and rabbit heart against a subsequent calcium paradox.

The fact that oxidant stress is capable of inducing cardiac stress proteins (Ananthan et al 1986, Löw et al 1989) has prompted Su et al (1992) to examine the protective benefits of prior heat stress with exposure to $\mathrm{H}_{2} \mathrm{O}_{2}$ as the final stress. In a rat myocyte culture model, heat stress is capable of inducing acquired thermotolerance and limiting myocyte injury on subsequent $\mathrm{H}_{2} \mathrm{O}_{2}$ exposure. More importantly, transfecting the same culture system so as to overexpress the human inducible hsp70 (hsp72) causes cells to become more tolerant to simulated ischaemia (Mestril et al 1992). This provides the most direct evidence to date that stress proteins per se, rather than the non-specific effects of heat stress, are responsible for myocardial protection. 


\subsection{Mechanisms of Cardiac Protection by Elevated Temperature}

All studies using heat to elevate stress protein synthesis result in a large number of physiological perturbations (Gisolfi et al 1991) which may in themselves have cardioprotective properties. For example, Currie's group (Currie et al 1988 and Karmazyn et al 1991) have shown that heat stress also results in an increase in the endogenous levels of the anti-oxidant enzyme catalase. Moreover, they have demonstrated (Karmazyn et al 1991) that inactivating catalase with 3-AT (3aminotriazole) results in an abolition of the protective effect normally observed at 24 and 48 hours after heat stress. This protective role of catalase would be dependent upon its ability to minimize the damage caused by secondary free radical generation to sulphydryl containing enzymes, DNA and lipids (Ceconi et al 1988) by catalyzing the conversion of $\mathrm{H}_{2} \mathrm{O}_{2}$ to water. Following a period of prolonged ischaemia the importance of catalase is increased since there is a marked reduction in the activity of SOD as well as in the ratio of reduced to oxidized glutathione (Ceconi et al 1988). In agreement with such a mechanism, we have observed a reduction in the levels of oxidized glutathione in the coronary effluent following ischaemia/reperfusion in heat stressed hearts, suggesting that a second, alternative line of antioxidant defence exists in these hearts (Yellon et al 1992a). The picture however becomes more complicated since cardiac mRNA levels for catalase are not increased by heat stress (Currie and Tanguay 1991). The increase in catalase may therefore result, from post-translational mechanisms. An alternative suggestion is that stress proteins may modulate the catalytic activity of catalase by direct interaction with the enzyme (Kukreja and Hess 1992).

Other evidence suggests that stress proteins may be able to limit myocardial damage independent of an antioxidant effect. One recent report suggests that the injury occurring during the calcium paradox can be influenced by procedures that cause stress protein synthesis (Meerson et al 1991a). The precise mode by which the calcium paradox damages the heart is a matter of controversy, but free-radical production is probably not involved (Ferrari et al 1989). It is thought that during the period of low calcium exposure changes occur in the structural proteins of the myocyte so as to increase fragility, and, on calcium repletion, the return of contractile activity causes myocyte mechanical disruption (Altschuld et al 1991). A similar process involving 
cytoskeletal disruption may also occur during ischaemia (Steenberg et al 1987). Heat stress proteins are known to alter the physical properties of actin and desmin (Bennardini et al 1992) and may themselves form an integral part of the cytoskeleton (Green and Liem 1989). Yet another possible mechanism of protection is that during heat stress, protein synthesis (apart from the stress proteins) is inhibited. A similar response has been noted during other forms of stress in cardiac tissue (Hammond et al 1983) and it has been postulated that such a response allows the cell to redirect energy into more vital cell processes during and following times of stress (Yellon et al 1993). A $17 \mathrm{kDa}$ stress protein, isolated from cardiac tissue and expressed in response to heat and pressure overload, inhibits protein translation and maybe the mechanism by which general protein synthesis is inhibited (Havre and Hammond 1988).

Although the specific changes that result in myocyte death during ischaemia are poorly understood alterations in the structural conformation of proteins will inevitably occur secondary to, changes in $\mathrm{pH}$, ionic concentration and free radical stress. The general protective properties of stress proteins may be able to attenuate or correct these changes (see Section 5 of this Chapter). A more specific example involves the structural changes known to occur in the mitochondrial enzyme carnitine palmitoyltransferase which may explain the alterations in fatty acid oxidation during reperfusion (Pauly et al 1991). Since hsp60 is presumably involved in the initial folding of this enzyme it is possible that it may also promote its correct refolding.

\section{Aims and Scope of the Thesis}

Previous attempts to limit myocardial infarct size pharmacologically have been largely unsuccessful (Bache 1984). A more mechanistic and novel approach would be to try to understand the cardiac response to ischaemia and determine whether any aspects of this response represent an adaptation capable of limiting ischaemic injury. Ischaemia triggers the cardiac stress response and a number of the investigations discussed above suggest that this response is itself associated with resistance to subsequent ischaemia. The purpose of the studies contained within this thesis is to investigate more fully the possible protective role of the cardiac stress proteins.

The studies described in this thesis were designed to answer a number of 
specific questions namely:

-Does whole body heat stress raise cardiac stress proteins?

-To which components of ischaemic injury if any, is myocardial tissue with an elevated stress protein content resistant?

-Is the resistance to injury dependant on enhanced anti-oxidant defences?

-What is the metabolic basis for the protection that follows heat stress?

-Is the protection that follows heat stress dependant upon stress proteins?

-Are non-thermally elevated stress proteins also protective?

-What is the physiological relevance of the cardiac stress response? 


\section{Chapter 2}

\section{Methods: Experimental Models and Data Handling}

1 Thermal Induction of Cardiac Stress Protein $\ldots \ldots \ldots \ldots \ldots \ldots \ldots \ldots \ldots \ldots \ldots \ldots \ldots \ldots$

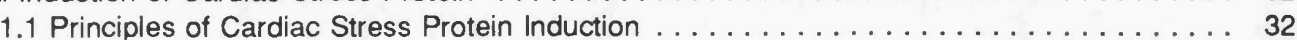

1.1.2 Rabbit Whole Body Heat Stress $\ldots \ldots \ldots \ldots \ldots \ldots \ldots \ldots \ldots \ldots \ldots \ldots$

2 Myocardial Stress Protein Quantitation by SDS-Page and Western Blotting . . . . . . . . 33

2.1 Principles of SDS-Page and Western Blotting ................... 33

2.2 Preparation of Myocardial Specimens . . . . . . . . . . . . . . . . . 33

2.3 Preparation of SDS-Page Gels . . . . . . . . . . . . . . . . . . . . . . . 34

2.4 The Loading of Samples and the One-Dimensional Electrophoretic Separation of

Proteins in Polyacrylamide Gels . . . . . . . . . . . . . . . . . . . 35

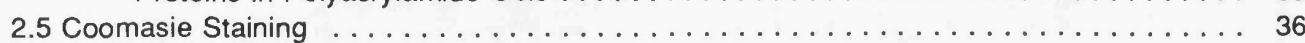

2.6 Western Blotting . . . . . . . . . . . . . . . . . . . . . . 36

2.7 Immunoprobing Nitrocellulose Membranes with Primary and Secondary Antibodies . . . . . 36

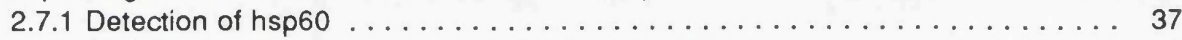

2.7.2 Detection of the Inducible Form of hsp70 (hsp72) . . . . . . . . . . 37

2.7.3 Densitometric Quantitation of hsp 60 and hsp72 ............ 38

3 The Measurement of Papillary Muscle Contractile Force . . . . . . . . . . . . . . 39

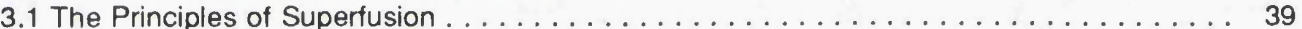

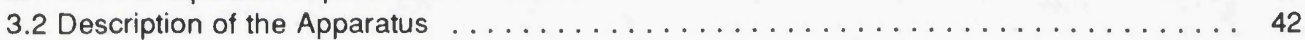

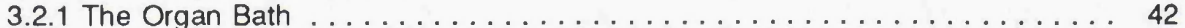

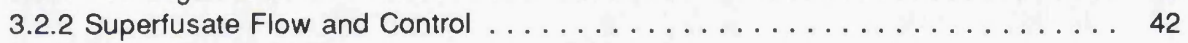

3.2.3 Measurement of Force ... . . . . . . . . . . . . . . . . . . . . . . . 42

3.2.4 Preparation and Suspension of Papillary Muscles $\ldots \ldots \ldots \ldots \ldots \ldots \ldots . \ldots 45$

3.2.5 Papillary Muscle Stability and Oxygenation . . . . . . . . . . . . . . . 47

3.2.6 The Force-Interval Protocol and Digital Data Acquisition . . . . . . . . . . . 47

3.2.7 Programming for the Real-Time Display of Contractile Information, Analysis of the Force Envelope and Chelation of Force Interval Data . . . . . . . 49

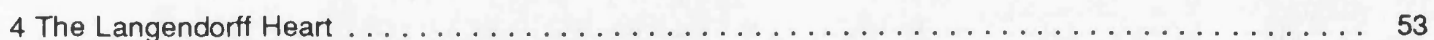

4.1 The Principles of Retrograde Perfusion . . . . . . . . . . . . . 53

4.2 The Preparation of Hearts for Retrograde Perfusion . . . . . . . . . . . . . . . . . 54

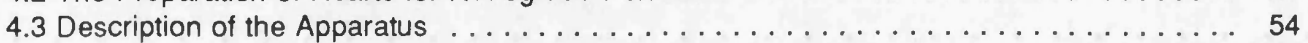

4.3.1 Delivery of the Perfusate . . . . . . . . . . . . . . . . . . . 54

4.3 .2 Assessment of Left Ventricular Pressure . . . . . . . . . . . . . . 56

4.3.3 Left Ventricular Monophasic Action Potentials . . . . . . . . . . . . . 56

4.3.4 Temperature Control . . . . . . . . . . . . . . . . . . . . 56

4.3.5 Control of Heart Rate . . . . . . . . . . . . . . . . . . 57

4.3.6 Oxygen, Carbon Dioxide and pH Differences Across the Heart . . . . . . . 57

4.3.7 Creatine Phosphokinase within the Coronary Effluent . . . . . . . . . 57

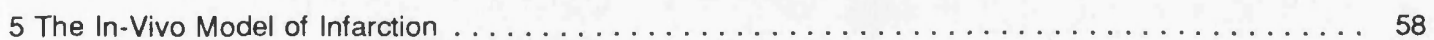

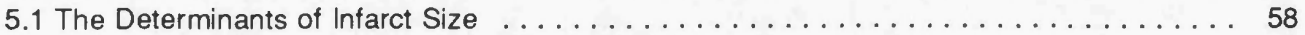

5.2 Recovery Experiments Involving Ischaemic Pretreatment .............. 58

5.2.1 Anaesthesia for Recovery Experiments Involving Ischaemic Pretreatment . . . 59

5.2.2 The Surgical Preparation for Recovery Experiments Involving Ischaemic

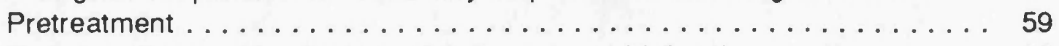

5.3 Non-Recovery Experiments Involving Prolonged Ischaemia and Infarction . . . . . . 60

5.3.1 Surgical Preparation for Non-Recovery Experiments Involving Prolonged Ischaemia and Infarction ........................ 60

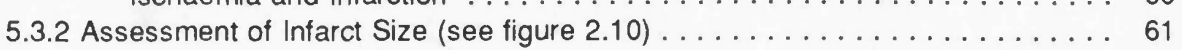

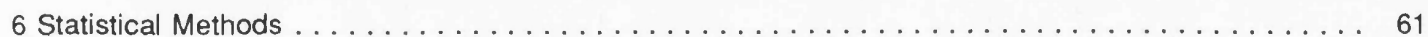




\section{Thermal Induction of Cardiac Stress Protein}

\subsection{Principles of Cardiac Stress Protein Induction}

Whole body heat stress in the rat reproducibly causes hsp72 induction within the heart (Currie et 1988). The trigger for stress protein expression in these circumstances is probably a combination of cardiac stresses, with components due to direct temperature elevation (see Chapter 1 Section 6) and cardiac volume overload secondary to vasodilation (see Chapter 1 Section 6). A heat stress regime identical to that of Currie et al (1988) was used to induce cardiac stress proteins in our studies.

\subsubsection{Rabbit Whole Body Heat Stress}

All rabbits were outbred, housed under similar conditions and given a normal diet. Following cannulation of a marginal ear vein with a 21 gauge $(0.8 \mathrm{~mm})$ Butterfly needle (Venisystems, Abbott, Ireland) rabbits were anaesthetised by administering pentobarbitone (Sagatal, May and Baker, Dagenham, England) at a dose of $40 \mathrm{mg} / \mathrm{kg}$ $(0.6 \mathrm{ml} / \mathrm{kg})$ intravenously. Once an adequate level of anaesthesia had been reached (no spontaneous movement, no withdrawal on foot pad pressure), a type $\mathrm{K}$ thermocouple (RS components, Northants, England) was introduced into the rectum and connected to a calibrated digital temperature indicator (Digitron 1408K, RS components, Northants, England). Basal temperature was recorded, and the rabbit wrapped (including ears) in a pre-warmed domestic electric underblanket. Temperature was recorded at 5 minute intervals and maintained above $42^{\circ} \mathrm{C}$ for 15 minutes. This was achieved by unwrapping the warming blanket when the rectal temperature reached $41.6^{\circ} \mathrm{C}$, so that only the undersurface of the rabbit remained in contact with the blanket. After the temperature had remained above $42^{\circ} \mathrm{C}$ for 15 minutes, the underblanket was removed and the rabbits placed on a cool surface to recover before returning them to the animal house.

Sham heat stress rabbits were treated in an identical manner but were not wrapped in the warming blanket. Heat stress and sham rabbits were treated simultaneously, care being taken to ensure the total dose of anaesthetic was similar between rabbits.

All rabbits were recovered for 24 hours prior to further experimentation or removal of hearts for stress protein estimation. 
Chapter 2

2 Myocardial Stress Protein Quantitation by SDS-Page and Western Blotting

\subsection{Principles of SDS-Page and Western Blotting}

Proteins are separated according to molecular mass and charge by electrophoresis through polyacrylamide gels. The relative distance that a protein migrates is dependant on: (i) The effective pore size of the polyacrylamide gel. Pore size decreases as the concentration of acrylamide is increased (Hames 1990). (ii) The native charge of the protein. (iii) The effective radius of the protein which is determined by its quaternary structure. The migration distance can become more dependant on molecular weight, and less dependant on the native charge and quaternary structure of proteins by denaturing the protein with a reducing agent in the presence of excess SDS. This acts to unfold the protein which than binds SDS in a constant weight ratio so that different proteins have essentially identical negative charge densities and hence will migrate in polyacrylamide gels of the correct porosity according to size (Hames 1990). Once proteins have been separated spatially within the gel, they are transferred to nitrocellulose by applying an electric field perpendicular to the plane of the gel. The proteins then become immobilised within the nitrocellulose matrix, whilst epitopes still remain accessible to immunoglobulins allowing immunodetection.

\subsection{Preparation of Myocardial Specimens}

New Zealand white rabbits were deeply anaesthetised by a administering IV pentobarbitone (Sagatal, May and Baker, Dagenham, England) at a dose of $60 \mathrm{mg} / \mathrm{kg}$ $(0.6 \mathrm{ml} / \mathrm{kg})$ and 500 units of heparin via the marginal ear vein. The heart was removed by elevating the sternum and washed in cold saline to remove blood from the ventricular cavities prior to retrograde aortic perfusion to rinse blood from the coronary circulation. Once the coronary perfusate was no longer blood stained, the right ventricular free wall and atria were trimmed off and left ventricles blotted dry and frozen in liquid nitrogen. At a latter date heart samples were crushed to a powder in a stainless steel pestle and mortar cooled in liquid nitrogen. $50 \mathrm{mg}$ of crushed heart was added to $500 \mu$ l of two-times sample buffer (see Appendix 1 Section 1) in an 
eppendorf tube and dounce homogenized (Eppendorff Homogeniser, Biomedix, Pinner, England). Following centrifugation at approximately $1000 \mathrm{~g}$ (micro centuar, MSE, Leicester, England) the supernatant was removed and protein concentration determined with the Pierce BCA reagent (modified Lowry technique) (Pierce, Illinois, USA) using bovine serum albumin at differing concentrations to construct a standard curve. Samples were further diluted with two-times sample buffer where necessary to equalise protein concentrations between specimens. 2-mercaptoethanol (reducing agent) was then added to a final concentration of $20 \%$ and samples were placed in a boiling water bath for 5 minutes. Following boiling samples were centrifuged once again at $1000 \mathrm{~g}$ for 5 minutes and bromophenol blue dye added to the supernatant. Samples were then stored at $-70^{\circ} \mathrm{C}$ until needed.

\subsection{Preparation of SDS-Page Gels}

The polacrylamide gels were prepared between glass plates separated by $1 \mathrm{~mm}$ spacers. Two gels of differing acrylamide concentration and $\mathrm{pH}$ were poured between the same glass plates. The upper "stacking" gel had indentations or wells to load the protein samples, which then migrated into the lower "resolving" gel.

The lower resolving gel ( $\mathrm{pH} 8.8$ ) was made by mixing $30 \%$ acrylamide solution, Tris buffered (to $\mathrm{pH}$ 8.8) SDS solution, and distilled water in varying proportions (see Appendix 1 Sections 2 and 3) to give either a $12.5 \%$ acrylamide or a $10 \%$ acrylamide gel mixture. Before pouring the gel mixture between the glass plates, ammonium persulphate (APS) and NNNN-tetraethylethalinediamine (TEMED) were added, to promote acrylamide polymerisation and crosslinking. Water saturated butanol was floated on the upper meniscus formed between the glass plates by the resolving gel solution, to prevent drying during polymerisation.

Once the lower resolving gel had polymerised, the upper stacking gel solution was poured after discarding the butanol layer. The upper stacking gel (pH 6.8) was prepared by mixing, $30 \%$ acrylamide solution, TRIS buffered (to $\mathrm{pH} \mathrm{6.8)} \mathrm{SDS}$ solution, and distilled water in proportions to give a $5 \%$ acrylamide gel mixture which was polymerised by adding APS and TEMED (see Appendix 1 Sections 2 and 3). Lane wells were formed by placing a toothed plastic well former between the glass plates in the stacking gel solution. After polymerisation the well former was removed 
to leave rectangular indentations in the polyacrylamide to load samples.

\subsection{The Loading of Samples and the One-Dimensional Electrophoretic}

\section{Separation of Proteins in Polyacrylamide Gels}

The polymerised stacking and resolving gels sandwiched between the glass plates were placed in an electrophoresis apparatus (BRL, Maryland, USA) so that the lower and upper margins of the gel were in contact with running buffer (see Appendix 1 Section 4) in 2 separate upper and lower chambers. The only communication between these chambers was through the gel. The upper chamber in contact with the upper margin of the stacking gel was connected to the cathode and the lower chamber in contact with the lower margin of the resolving gel was connected to the anode. The voltage gradient across the gel therefore tended to cause the negatively charged SDSbound proteins to migrate towards the anode at the lower margin of the resolving gel.

Samples were loaded into the wells formed in the stacking gel using a $10 \mu \mathrm{l}$ Hamilton syringe. Sample buffer has a high glycerol content, therefore samples were heavier than the running buffer in the upper reservoir and sank to the bottom of their individual wells. Once samples had been loaded, the anode and cathode were connected to their respective terminals of a power pack (BRL, Maryland, USA). A current of $40 \mathrm{~mA}$ was delivered through each gel, initially this corresponded to a voltage of $200 \mathrm{~V}$, as the protein samples migrated through the polyacrylamide the resistance of the gel and therefore the voltage increased. In order to prevent gel heating, secondary to this rise in resistance, the power pack was set to deliver a constant current of $40 \mathrm{~mA}$ until the voltage reached $400 \mathrm{~V}$ and then to maintain a constant voltage of $400 \mathrm{~V}$. Under these conditions a potential difference would be applied across both $10 \%$ and $12.5 \%$ acrylamide gels until the "dye front", the fastest migrating portion of the samples, reached the bottom of the resolving gel. The gel plates were then removed from the apparatus, glass plates separated and stacking gel discarded. The resolving gel would then be used either for Western blotting or visualisation of proteins by Coomasie staining. 


\subsection{Coomasie Staining}

Coomasie blue R-250 (BDH, Dorset, England) binds to proteins and can detect bands containing as little as $0.2-0.5 \mu$ g of protein and remains quantitative up to $15 \mu \mathrm{g}$ of protein (Hames 1990). The resolving gels containing the electrophoresed samples were gently agitated overnight in Coomasie blue staining solution (see Appendix 1 Section 5). The following day, the background non-specific Coomasie stain was washed out of the gel by destaining with methanol (see Appendix 1 Section 6) and the gel containing the resolved protein bands preserved in $7 \%$ acetic acid.

\subsection{Western Blotting}

The proteins separated according to molecular weight by polyacrylamide gel electrophoresis, were transferred to nitrocellulose membranes (Hybond C, Amersham, Bucks, England) by Western blotting. This was done by laying the nitrocellulose membrane "en face" over the polyacrylamide gel under blotting buffer (see Appendix 1 Section 7), taking care to exclude air bubbles between gel and membrane. The membrane and gel were then sandwiched in a plastic cassette within a blotting tank (BioRad, Herts, England) containing the same blotting buffer. A current was applied orthogonal to the plane of the gel and membrane, with the anode on the nitrocellulose side of the gel, forcing the negatively charged proteins to migrate out of the gel and into the matrix of the nitrocellulose. Gels were blotted overnight using a current of $210 \mathrm{~mA}$. The following day the polyacrylamide gels were discarded and the nitrocellulose membranes used to immunolocalise heat stress protein.

In the initial pilot studies, the polyacrylamide gels were stained with Coomasie blue following Western blotting. Directly visualised protein transfer was complete apart from very high molecular weight proteins close to the resolving gel, stacking gel junction.

\subsection{Immunoprobing Nitrocellulose Membranes with Primary and}

\section{Secondary Antibodies}

Initially, a number of concentrations of primary and secondary antibodies were used for the detection of stress proteins before the binding conditions were optimised. 
In addition early protocols relied upon ${ }^{125} \mathrm{I}$ labelled anti-mouse Fab fragments (Amersham, Bucks, England) for primary antibody detection. These preliminary protocols will not be described.

\subsubsection{Detection of hsp60}

The nitrocellulose membranes containing the immobilised proteins were agitated for 1 hour at room temperature in phosphate buffered saline (PBS) containing $5 \%(\mathrm{w} / \mathrm{v}$ ) dried skimmed milk powder (Marvel) and $0.05 \%$ Tween 20 (Sigma, Mousourri, USA). This acts to block non-specific protein binding sites on the nitrocellulose. Following blocking, membranes were washed for 3, 5 minute periods in PBS containing $0.05 \%$ Tween 20 and $0.1 \%(\mathrm{w} / \mathrm{v})$ dried skimmed milk powder. A mouse IgG monoclonal antibody prepared in-house (Shariff et al 1992) against the human hsp60 protein (P1) was added to PBS/0.05\% Tween 20 at 1 in 2000 dilution. The nitrocellulose membranes were exposed to this monoclonal antibody and agitated on a mechanical shaker to ensure that the whole membrane surface was wetted. After 1 hour of agitation membranes were washed for 3, 5 minute periods in PBS $0.05 \%$ Tween 20 and then exposed for 1 hour to horse radish peroxidase conjugated rabbit IgG directed against mouse IgG (Dakopatts, Glostrup, Denmark) at a concentration of 1 in 1000 in PBS $0.05 \%$ Tween 20 . The non-specifically bound horse radish peroxidase conjugated antibody was washed off the nitrocellulose membrane by 3,5 minute rinses with PBS $0.05 \%$ Tween 20 . Membranes were then developed using an enhanced chemiluminescence technique (ECL Western Blotting Detection System, Amersham, Bucks, England). This system detects the peroxidase activity within the membrane which is used to catalyse the oxidation of luminol in the presence of hydrogen peroxide, immediately after oxidation, luminol is in an excited state and emits light whilst decaying to the ground state. This light emission could be both seen with the naked eye and recorded on photographic film.

\subsubsection{Detection of the Inducible Form of hsp70 (hsp72)}

The procedure to detect the inducible form of the hsp70 family (variously called hsp72 or hsp70i) differed slightly to that described above for hsp60. Nitrocellulose membranes were blocked with $0.1 \%$ skimmed milk protein in PBS with 
0.05\% Tween 20. Commercially available anti-hsp72 (Amersham, Bucks, England or Stress Gen, Sidney, Canada) was diluted 1 in 1000 in PBS with $0.05 \%$ Tween 20 and $0.1 \%$ Marvel, and exposed to the nitrocellulose membrane for 1 hour at $37^{\circ} \mathrm{C}$. Subsequent washes, exposure to secondary antibody, and visualisation of hsp72 were as described for hsp60.

\subsubsection{Densitometric Quantitation of hsp60 and hsp72}

A method developed by other investigators within the laboratory, based on the densitometric assessment of specific protein bands was used to quantitate myocardial stress proteins (Norton and Latchman 1987).

Protein bands visualised by chemiluminescence, and recorded on photographic film, were scanned using a white light source and absorption data digitised and transferred to personal computer for subsequent analysis (Biorad model 620 video densitometer, Hemel Hempstead, Herts England). When bands were not homogeneously dense, the band was scanned at a number of points along its length to calculate a mean absorption value.

Although the total protein content of samples was equalised on the basis of protein assessment with the Pierce BCA reagent, this was done prior to boiling and final centrifugation. The final protein concentrations of the samples therefore varied slightly and identically loaded SDS-PAGE gels were also prepared for Coomasie staining to control for this variability. Samples on the gel for stress protein visualisation by Western blotting and protein visualisation by Coomasie staining were loaded simultaneously and run on opposite sides of the same gel electrophoresis apparatus. The reproducibility for dispensing sample volumes in the range 6 to $10 \mu \mathrm{l}$ with a $10 \mu \mathrm{l}$ Hamilton syringe was better than $5 \%$ (determined spectrophotometrically by absorption at $560 \mathrm{~nm}$ after dispensing Coomasie blue dye in a constant volume of distilled water). Since the protein transfer from SDS-PAGE gel to nitrocellulose is nearly complete, the protein loading on the nitrocellulose will be virtually identical to that of the Coomasie stained gel. The actin band on these identically loaded Coomasie stained gels was also scanned and absorption digitised.

The absorption pattern of the stress protein bands and the actin band of the Coomasie stained gel were displayed on a personal computer and area under the 
relevant peak integrated to give a total absorption value (Biorad Analyst 2 version 3.1 software, Biorad, Herts, England). For each lane the absorption value for the stress protein band was divided by the absorption value for the actin Coomasie band, the resultant ratio was independent of protein loading (see figures 2.1 and 2.2B). In addition the absorption values for both the coomasie actin band and the stress protein bands were linear over a range of loading conditions (see figure 2.2B).

\section{The Measurement of Papillary Muscle Contractile Force}

\subsection{The Principles of Superfusion}

Muscle specimens with a small diameter, superfused in an organ bath containing oxygenated physiological buffer, can remain viable without arterial perfusion. Such specimens must be small enough to allow free diffusion of metabolic substrates (oxygen, glucose and pyruvate) and metabolites (carbon dioxide and perhaps lactate). In principle the rates at which individual substrates are utilised, and metabolites produced by the muscle must be matched by their rates of diffusion. In practise the diffusion of oxygen is the factor limiting contractility (Paradise et al 1981). The rate of oxygen delivery to cells along the central axis or core, of the isolated muscle specimen is determined by, (i) the difference between the partial pressure of oxygen in the external solution and that at the muscle core (the oxygen difference), (ii) the diffusion distance for oxygen (muscle radius). AV Hill (1928) from purely biophysical considerations described a method to determine the critical distance for the diffusion of oxygen. Assuming an oxygen consumption of approximately $1.4 \mathrm{ml} / \mathrm{min} / 100 \mathrm{~g}$ of active muscle bathed in Ringer's solution and ignoring any contributions from myoglobin, a critical radius of $0.466 \mathrm{~mm}$ has been suggested (Prasad and Callaghan 1969). This has been confirmed more directly by progressively decreasing the partial pressure of oxygen in the superfusate until a decrease is observed in the force developed by muscles of varying radius. Such studies suggest the critical diameter in superfused cat papillary muscle, stimulated at $0.5 \mathrm{~Hz}$ at $37^{\circ} \mathrm{C}$ is $0.96 \mathrm{~mm}$ decreasing to $0.71 \mathrm{~mm}$ at $1 \mathrm{~Hz}$ (Paradise et al 1981). 


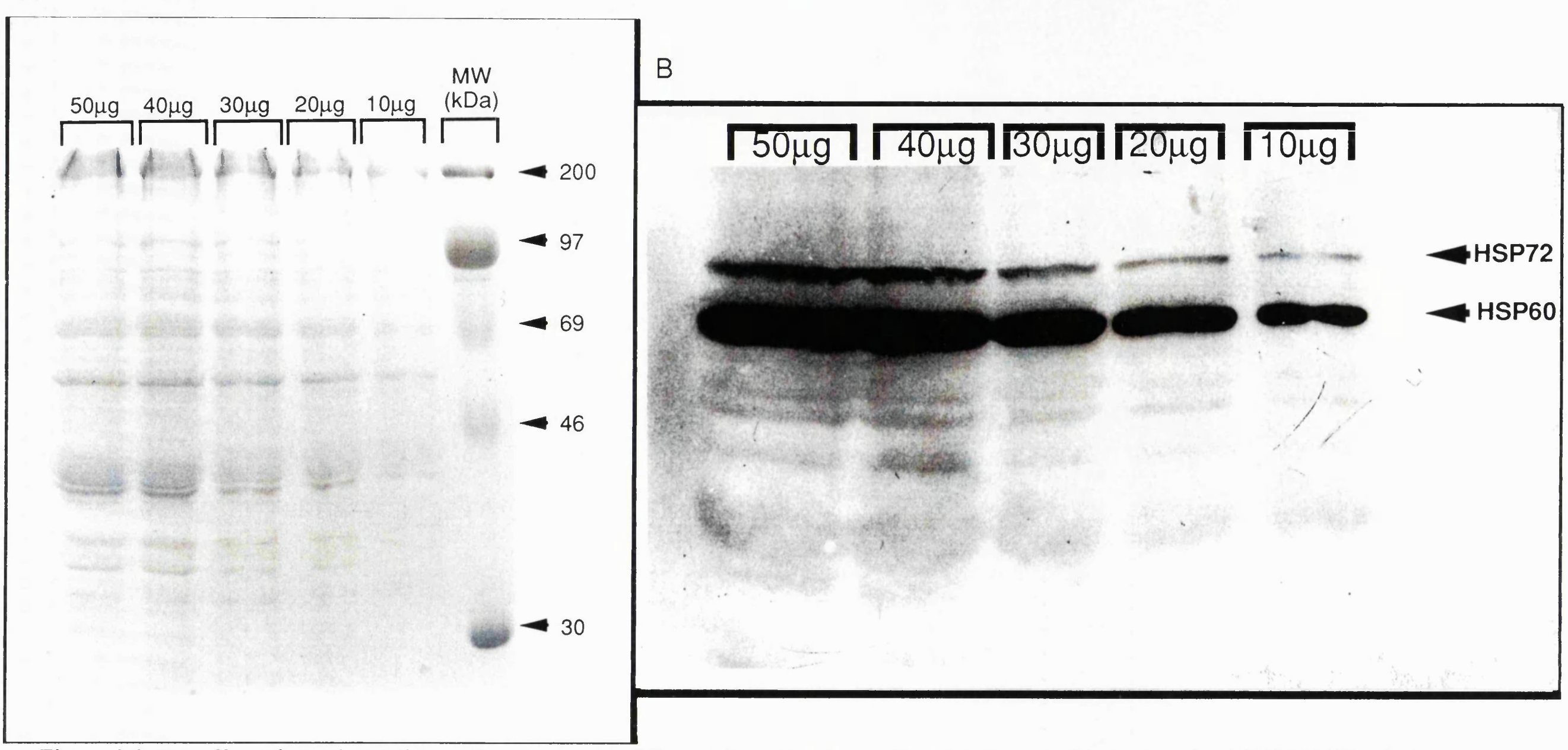

Figure 2.1: The effect of protein loading on the appearance of Coomasie and hsp bands. Panel A shows a Coomasie stained SDS-PAGE gel after running myocardial samples with increasing amounts of total protein in each of the 5 lanes. The actin band runs at approximately $40 \mathrm{kDa}$. Panel B shows an

over-exposed immunoblot probed with anti-hsp60. The previous day the nitrocellulose membrane had been probed with anti-hsp72 and weak $\frac{\pi}{\delta}$ immunoreactivity remains. The pattern of protein loading is as in panel A. 
A

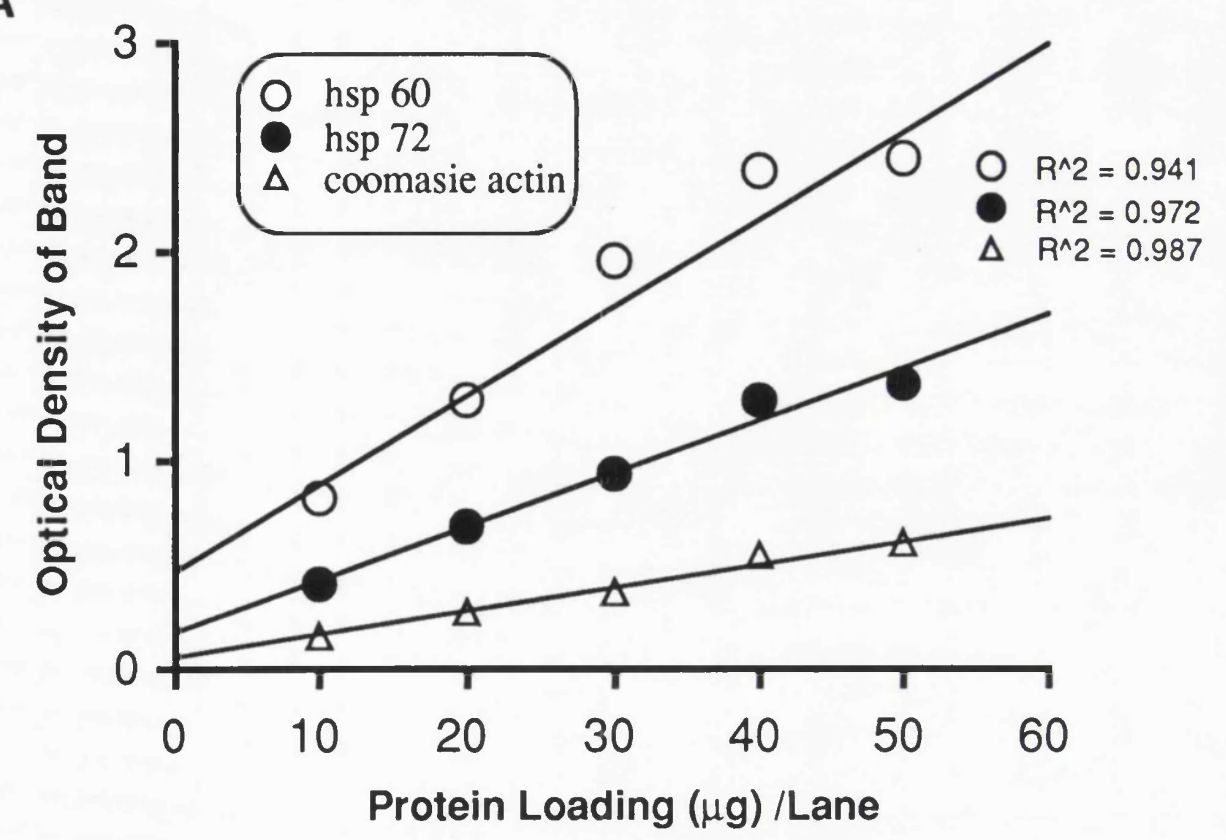

B

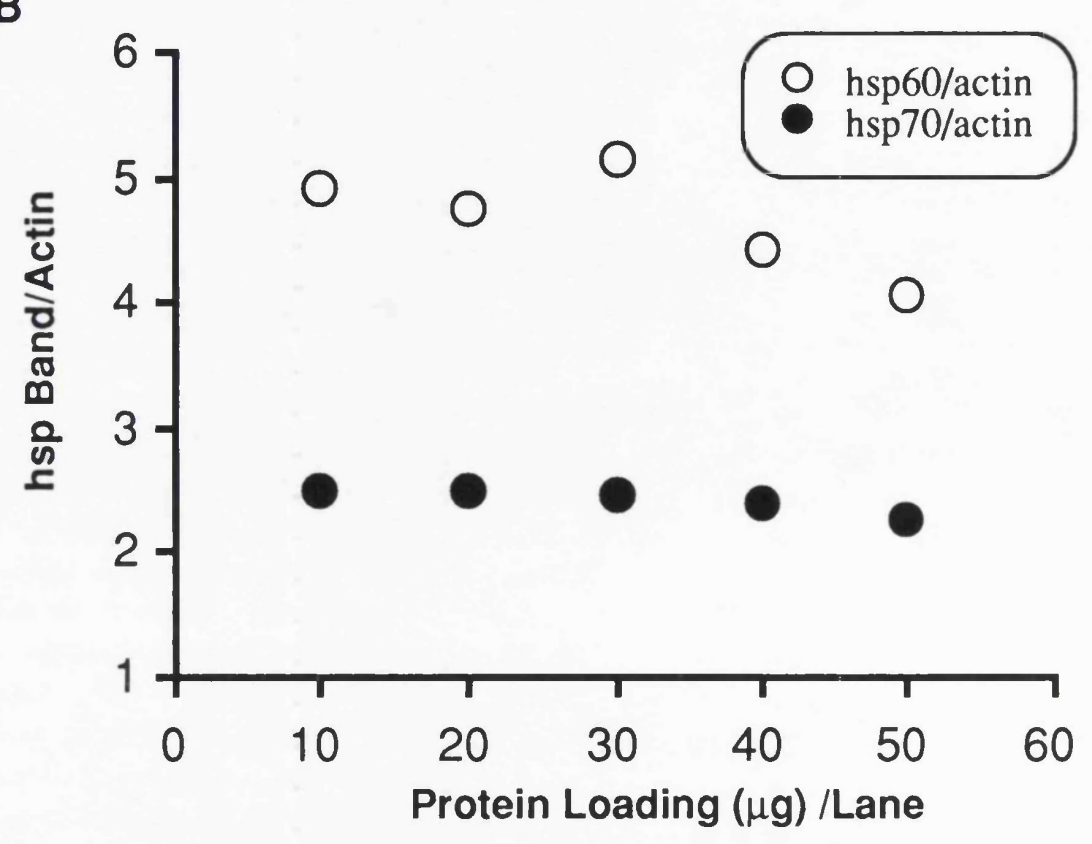

Figure 2.2: The relationship between optical densities of Coomasie and stress protein bands with varying amounts of total protein loaded per lane of SDS-PAGE gel. The densities are derived from the bands depicted in figure 2.1. Graph A: The optical densities of hsp70, hsp60 on immunoblots, and actin band on Coomasie stained gel, are compared to loading of total protein. The optical densities of hsp60 and 70 bands on immunoblotting and the actin band on coomasie stained SDS-PAGE gels are proportional to protein loading over the relevant loading ranges. Graph B: When the optical densities of the specific hsp60 and 70 bands on immunoblots, are normalised using the Coomasie actin band density, the resultant ratio is independant of protein loading. 


\subsection{Description of the Apparatus}

The apparatus was manufactured from glass, polythene/silicone tubing and nontoxic perspex (see figures 2.3 and 2.4 ).

\subsubsection{The Organ Bath}

The organ bath was manufactured by the physiology workshop at UCL to the design illustrated in figure 2.4 . The capacity of the bath was approximately $4 \mathrm{ml}$ and perfusate flow was $8 \mathrm{ml} / \mathrm{min}$. The entry well served a dual purpose, being used to accommodate a pH meter (Jencons, Beds, England) and damp the pulsatile perfusate flow delivered by the peristaltic pump (Watson-Marlow 502S, Cornwall, England). Two parallel bores within the body of the bath were used to circulate the warm water used in the jacketing of the system, in order to maintain organ bath temperature. Superfusate temperature and $\mathrm{pH}$ were monitored continuously using a thermocouple (type K, Radiospares, Northants, England) placed close to the papillary muscle and a $\mathrm{pH}$ electrode at the in-flow well. $\mathrm{pO}_{2}$ and $\mathrm{pCO}_{2}$ tensions were monitored intermittently in the organ bath effluent (ABL2 automated blood gas analyzer, Radiometer Copenhagen, Copenhagen, Denmark).

\subsubsection{Superfusate Flow and Control}

Superfusates were oxygenated using a gassing stick with either, $95 \% \mathrm{O}_{2} / 5 \% \mathrm{CO}_{2}$, or $85 \% \mathrm{O}_{2} / 10 \% \mathrm{~N}_{2} / 5 \% \mathrm{CO}_{2}$, or $90 \% \mathrm{O}_{2} / 10 \% \mathrm{CO}_{2}$ or $95 \% \mathrm{~N}_{2} / 5 \% \mathrm{CO}_{2}$ (all from British Oxygen Company, London, England). Different superfusates could be drawn via a three-way tap selector from different jacketed reservoirs and then passed through the tubing of the peristaltic pump to a jacketed heat exchanger to supply the organ bath. The temperature of the superfusate was kept at $37 \pm 0.2^{\circ} \mathrm{C}$ by water circulation through jackets surrounding the, reservoir, pre-organ bath heat exchanger and organ bath (Techne C-85a Circulator, Cambridge UK).

\subsubsection{Measurement of Force}

Papillary muscles were suspended from a force transducer (Gould Statham UCT2, Ohio, USA) and field stimulated via parallel flattened platinum electrodes using an isolated stimulator (Digitimer DS2, Hertfordshire, England) triggered by a 
Figure 2.3 The Apparatus Used to Assess Papillary Muscle Contractility A

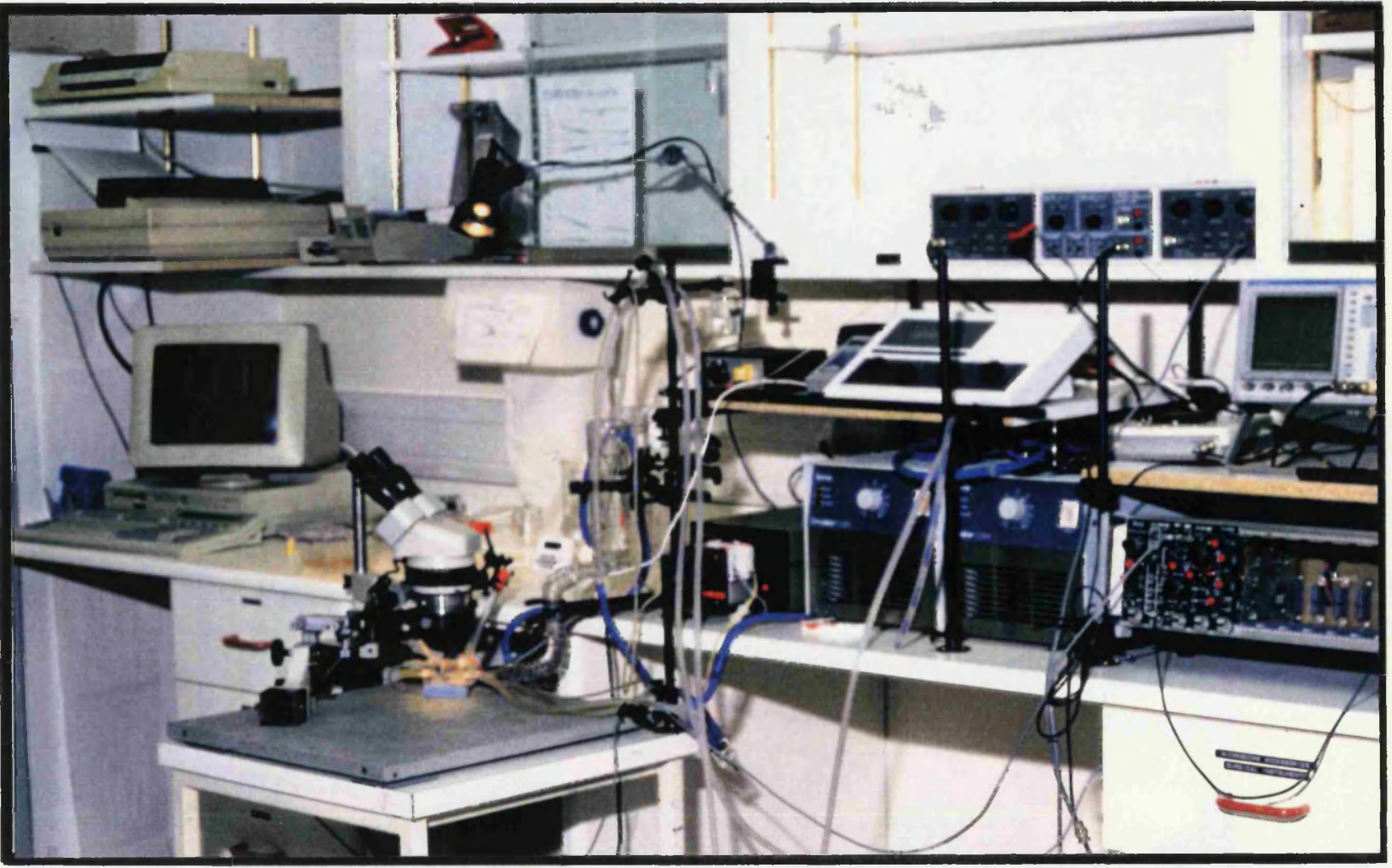

B

Schematic Representation of the Apparatus used to Stimulate and Record Force from Isolated Papillary Muscles

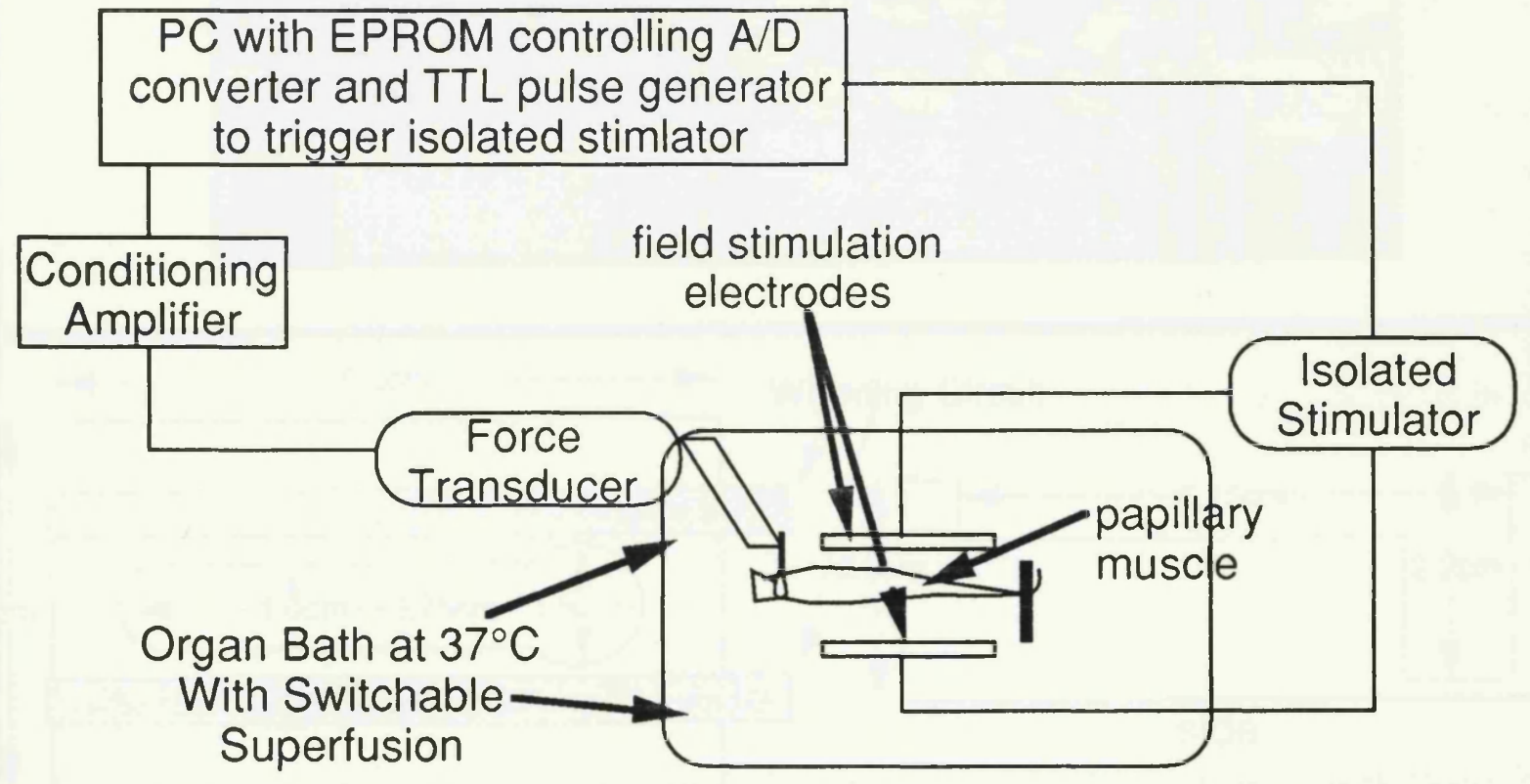

Figure 2.3: Panel A: is a photograph of the equipment used to asses papillary contractility. In the left off-centre foreground is the low vibration table with organ bath and force transducer. Panel B: is a schematic representation of the equipment shown in panel $\mathrm{A}$. 


\section{The Organ Bath for Papillary Muscle Superfusion}

B
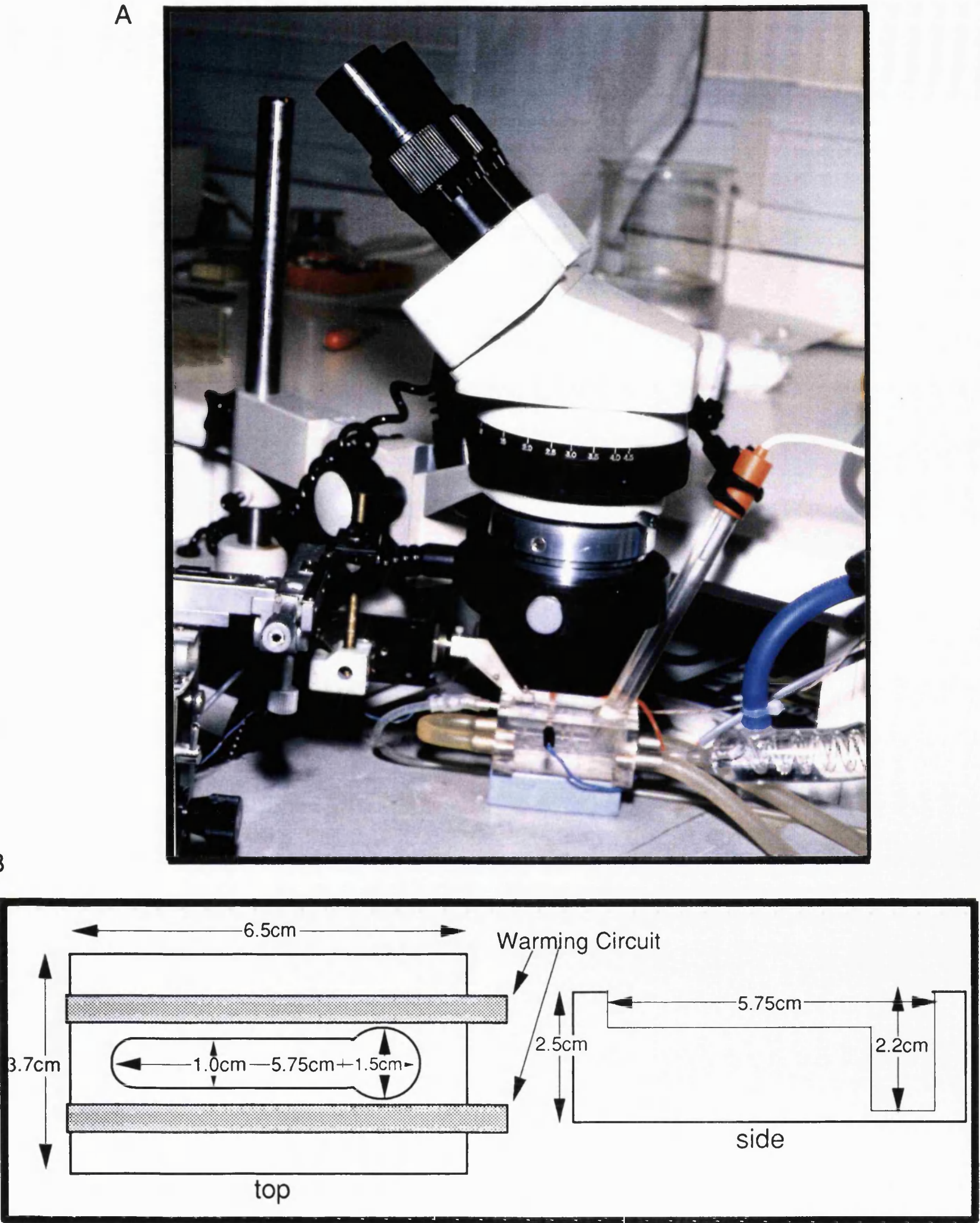

Figure 2.4: Panel A: Shows the organ bath with $\mathrm{pH}$ meter in entry well. The force transducer is seen immediately below the stereoscopic microcope, to the left of the organ bath is the superfusate heat exchanger, the circuits to warm the organ bath can be seen on either side of the heat exchanger. Panel B: is a schematic diagram of the organ bath viewed from above and the side with relevant dimensions. 
computerized clock, the pulse amplitude being set at twice threshold with a fixed pulse duration of $2 \mathrm{~ms}$. The force transducer was attached to a micromanipulator (Prior, Cambridge, England) allowing precise three dimensional positioning. The excitation voltage for the force transducer was provided by a DC bridge amplifier (Digitimer, Neurolog recorder amplifier NL 107). The output of the force transducer was "conditioned" using the same amplifier and recorded on paper at slow paper speed (5mm/min) (Gould RS3400 chart recorder, Ohio, USA), and also simultaneously digitized at $250 \mathrm{~Hz}$ for on-line analysis. The force transducer has a flat frequency response to $60 \mathrm{~Hz}$ and a displacement of $\pm 0.06 \mathrm{~mm}$ at the upper limits of its sensing range $\pm 30 \mathrm{~g}$. The recorder amplifier has a frequency response giving less than a $3 \mathrm{~dB}$ reduction in signal at $1 \mathrm{kHz}$. The frequency response of the transducer and its mechanical linkage has been previously determined as flat to $50 \mathrm{~Hz}$ (Walker 1983). The force transducer was calibrated by suspending varying axial weights and measuring the voltage output of the recorder amplifier on an autocalibrating digital oscilloscope (Gould 1602, Ohio, USA). The varying weights comprised of differing lengths of coiled lead solder which had themselves been weighed on a calibrated 5 figure electronic balance (Mettler AJ50, Leicester, England). The linearity of the transducer at varying degrees of amplification (full scale deflection of NL107 set at 1,2 or 5 ) is shown in figure $2.5 \mathrm{~A}$. In addition the linearity of the zero suppression offset of the recorder amplifier (NL107) was determined at each full scale deflection (figure 2.5B).

\subsubsection{Preparation and Suspension of Papillary Muscles}

New Zealand White rabbits (1.5-3.0kg) were deeply anaesthetised by administering IV pentobarbitone (Sagatal, May and Baker, Dagenham, England) at a dose of $60 \mathrm{mg} / \mathrm{kg}(1.0 \mathrm{ml} / \mathrm{kg})$ followed by 500 units of heparin via the marginal ear vein. The heart removed by elevating the sternum was washed in cold oxygenated saline. Hearts were then immobilised in a Petri dish of continuously oxygenated physiological saline (composition as for experimental protocol see Chapters 3 and 4).

Under 10x magnification (Prior stereoscopic microscope, Cambridge, England, and a Schott, fibre-optic light source, W. Germany) the anterior wall of the right ventricle was laid open by cutting down through the pulmonary artery, through the 


\section{Calibration of Force Transducer and Recording Amplifier}
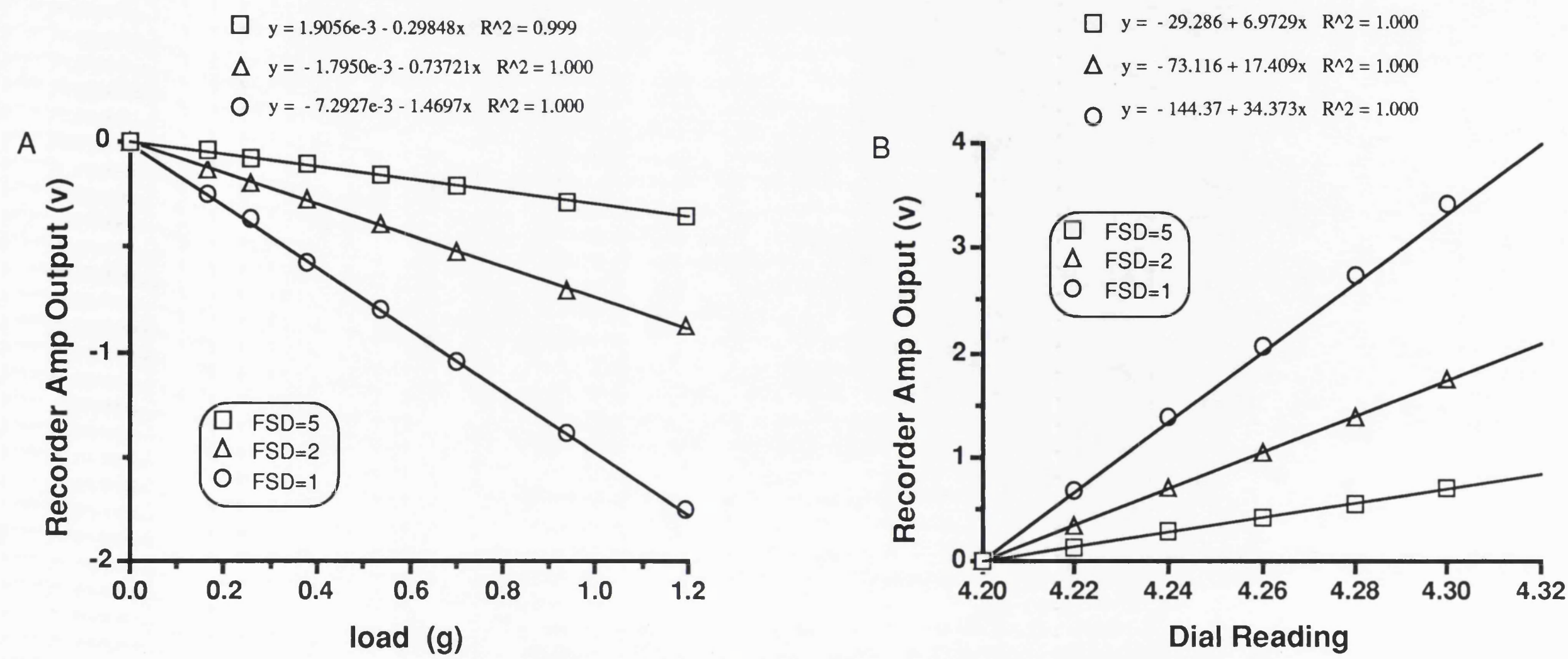

Figure 2.5 The calibration of force transducer and recording amplifier at each of the amplification settings appropriate to the amplitude of force developed by papillary muscles. Graph A: Describes the relationship between load on transducer in grammes and voltage output from the recorder amplifier presented to the analogue to digital converter. This relationship was determined for each of the amplification settings. The slope of the relationships was used by the program MMCALCUL.BAS to calculate force from the ASC II value returned by the analogue to digital converter. Graph B: Describes the relationship between vernier reading on the offset dial of the recorder amplifier and recorder amplifier voltage output presented to the analogue to digital converter. This relationship was also determined for each of the amplification settings. The slopes of these relationships were used by the program MMCALCUL.BAS to calculate force from the ASC II value returned by the analogue to digital converter (see Appendix 2 Section 2). 
infundibulum and along the interventricular groove. One of the papillary muscles exposed by this approach would be chosen for superfusion. An ideal muscle would be thin (diameter $\approx 0.6 \mathrm{~mm}$ ), unbranched and long (length $\approx 3.0 \mathrm{~mm}$ ). Braided $6 / 0$ silk (Pearsalls Sutures, England) was passed gently under the chosen muscle and tied around the chordae tendinae before cutting the distal chordae. The base of the papillary muscle was undercut so that the entire muscle together with a "wedge of either right ventricular free wall or interventricular septum was removed and placed in the organ bath. Remaining papillary muscles with adjoining ventricular wall were either discarded or frozen in liquid nitrogen for stress protein estimation.

Once transferred to the organ bath, the silk attached to the chordae was tied to the post in the organ bath, whilst the waist between the body of the papillary muscle and the ventricular septal wedge was used to snare the papillary muscle and attach it to the force transducer. Thirty minutes after the papillary muscles were suspended in the organ bath they were stretched to a length developing $90 \%$ of maximal active force and the pacing threshold determined. This was followed by a further 30 minute stabilization period before entering an experimental protocol. Throughout this time the stimulation rate was maintained at $1 \mathrm{~Hz}$.

\subsubsection{Papillary Muscle Stability and Oxygenation}

Initial pilot experiments with rabbit right ventricular papillary muscles demonstrated that for 10-45 minutes after suspension developed and resting force were unstable, thereafter force would remain stable for upto 8 hours. Providing muscles were adequately stabilised and below $1.0-1.2 \mathrm{~mm}$ in unloaded diameter there was no decrease in developed force when superfusion was switched to physiological saline equilibrated with $85 \% \mathrm{O}_{2} / 10 \% \mathrm{~N}_{2} / 5 \% \mathrm{CO}_{2}$, suggesting oxygenation was adequate (see Chapter 3 Section 4.4 for criticism).

\subsubsection{The Force-Interval Protocol and Digital Data Acquisition}

The timing of papillary muscle field stimulation was by a computer clock resident on a standalone board constructed and programmed in Forth by $\mathrm{Mr} \mathrm{Jim}$ Johnson, Dept of Medicine, John Radcliffe Infirmary, Oxford. This board also housed an analogue to digital (A/D) converter. The timing of the pacing protocols was 


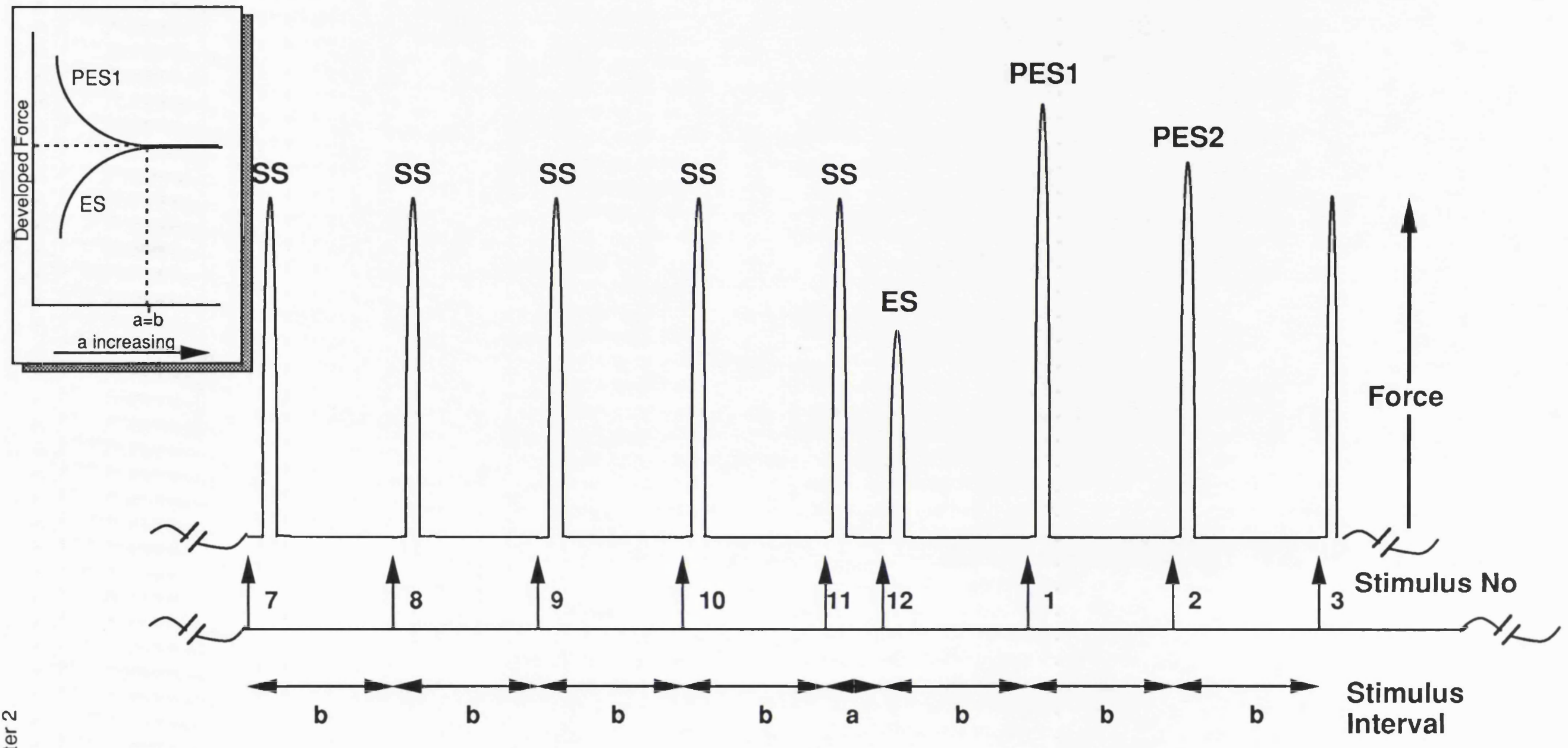

Figure 2.6: The effect of a sudden change in interbeat interval on developed force. "b" is the steady state interbeat interval, "a" is the extrasystolic interval $(200 \mathrm{~ms} \leq \mathrm{a} \leq 10,000 \mathrm{~ms}$ and $\mathrm{b}=1000 \mathrm{~ms}$ in all the experiments descibed in this thesis). When a $\leq \mathrm{b}$ then the extrasystolic beat (ES) is weaker than the steady state beat (SS) and the post extrasystolic beats (PES1 and PES2) are stronger than SS. As "a" becomes longer every 12th beat and approaches "b" then the force of ES increases whilst that of PES decreases. A schematic diagram of this relationship is shown inset. 
versatile so that single "extrasystolic" beats could be interpolated in a train of steady state beats.

Papillary muscles were stimulated at $1 \mathrm{~Hz}$, apart from when an assessment of force generation in response to extra-systolic beats was performed. Each of these assessments was identical and consisted of a single extra-systolic beat with an initial coupling interval of $200 \mathrm{~ms}$ to the preceding beat. Every 12 beats this interval was increased by $25 \mathrm{~ms}$, until the coupling interval reached $800 \mathrm{~ms}$, the increment then was set at $500 \mathrm{~ms}$ until the coupling interval reached $1000 \mathrm{~ms}$ and finally the increment was set at $1000 \mathrm{~ms}$ until the extrasystolic beat followed the preceding beat by $10,000 \mathrm{~ms}$, concluding the protocol (see figure 2.6). The force envelope of (i) the steady state beat immediately preceding the extra-systolic beat, (ii) the extra-systolic beat, (iii) the beat following the extra-systolic beat, (the first post extra-systolic beat) and (iv) the second beat after the extrasystolic beat, (the second post extra-systolic beat), were recorded digitally for subsequent analysis. This entire force interval protocol was recorded over a 15 minute period. The 44 digitized steady state beats recorded during this protocol were averaged to calculate means and standard errors for resting force, developed force, maximum rate of rise of force, maximum rate of fall of force, time to peak force and time to $90 \%$ relaxation.

The force developed by the extrasystolic and post extra-systolic beats was normalised by dividing by the force developed by the preceding steady state beat. Normalised beat force was recorded at each extra-systolic interval to derive forceinterval curves. The force-interval relationships were then either, individually fitted to a biexponential expression, or collated to derive force-interval curves comprising of data from one whole experimental groups; at each extra-systolic interval a mean and standard error of normalised beat force for extra-systolic and post-extra-systolic beats was obtained and these averaged curves fitted to a biexpontential expression.

\subsubsection{Programming for the Real-Time Display of Contractile Information,} Analysis of the Force Envelope and Chelation of Force Interval Data

The A/D converter on the Forth board was characterised using steady state voltages and "peeking" at the value of the ASC II character returned. The A/D converter had a 2 volt range which was encoded in one byte to give a resolution of 
$\approx 0.008$ volts (see figure 2.7), this corresponded to a force of $4.2 \mathrm{mg}$ at $\mathrm{FSD}=1$ and $22.2 \mathrm{mg}$ at $\mathrm{FSD}=5$. The $\mathrm{A} / \mathrm{D}$ converter triggered by the stimulation pulse, would digitise voltage for a period of $400 \mathrm{mS}$ at $4 \mathrm{~ms}$ intervals following the trigger. A file containing the 100 ASC II characters (or bytes) describing the voltage over the $400 \mathrm{mS}$ period would be transferred to the RS232 (Com 1) port of a PC for analysis and display (see figure 2.8).

The slopes of the relationships between transducer load in grammes and recorder amplifier output in volts for each FSD setting (together with the recorder amplifier output at zero load, the so called zero load constant) was used to derive the transducer load for any particular ASC II value. Hence, the transducer load or papillary muscle developed force could be determined at $4 \mathrm{mS}$ intervals for each stimulated papillary muscle force envelope. A suite of programmes was written in Microsoft G-W basic to analyse the ASC II characters returned by the A/D converter and derive contractile information. The programme listings together with more information about the programmes appears in Appendix 2.

The 4 programmes used were.

1) MMAQUIS.BAS (Appendix 2 Section 1): This acquisition program, would plot the force envelopes of steady state beat, extrasystolic beat and first and second extrasystolic beats on the computer screen in real time. In addition the program determined the highest ASC II value for each envelope and plotted these against extrasystolic interval to derive a force-interval relationship on screen (see figure 2.8) and hard copy on plotter. The ASC II (raw data) provided by the A/D converter was written to disc in sequential files for latter analysis.

2) MMCALCUL.BAS (Appendix 2 Section 2): This analysis programme converted the ASC II strings describing the papillary muscle twitch envelopes, to force in grammes and calculated for the 44 steady state beats means \pm SE for, peak force (g), maximum rate of contraction $(\mathrm{g} / \mathrm{s})$, maximum rate of relaxation $(\mathrm{g} / \mathrm{s})$, time to peak force (s) and time to $90 \%$ relaxation (s). In addition output files were written to disc for both extrasystolic and first post extrasystolic beats for each of the following, extrasystolic interval versus (i) peak force (ii) maximum, and (iii) minimum rates of rise of force, the option was also available to output this information to a plotter. 


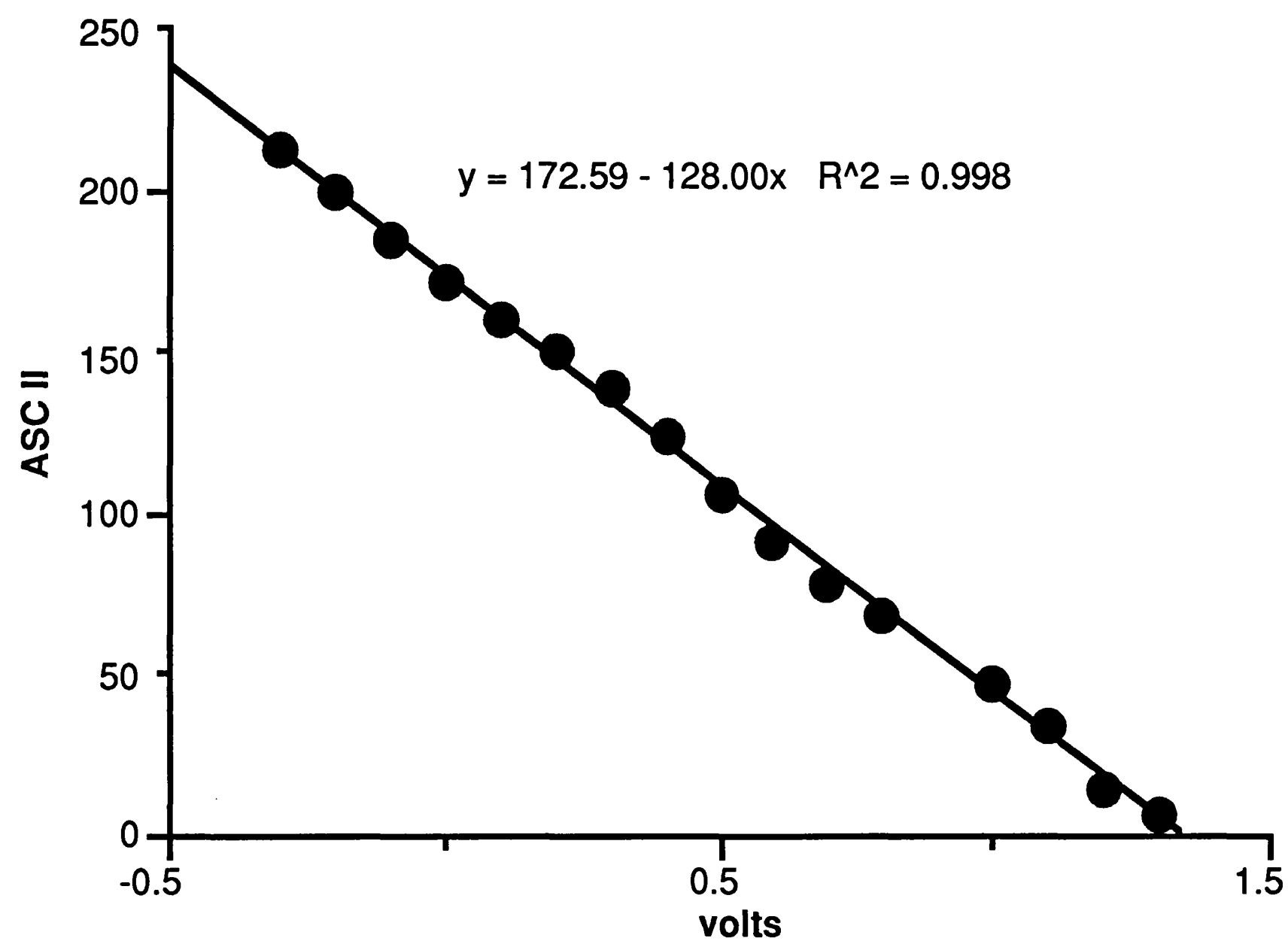

Figure 2.7: The relationship between voltage input and digital output of the analogue to digital converter. The voltage presented to the A/D converter was increased in 0.1 volt steps and the ASC II value of the character returned by the A/D converter interrogated by the personal computer. The slope and intercept of this relationship was used by the program MMCALCUL.BAS to convert ASC II values into force in grams (see Appendix 2 Section 2). 
Appearance of the Computer Monitor at the End of a Papillary Muscle Force-Interval Assessment

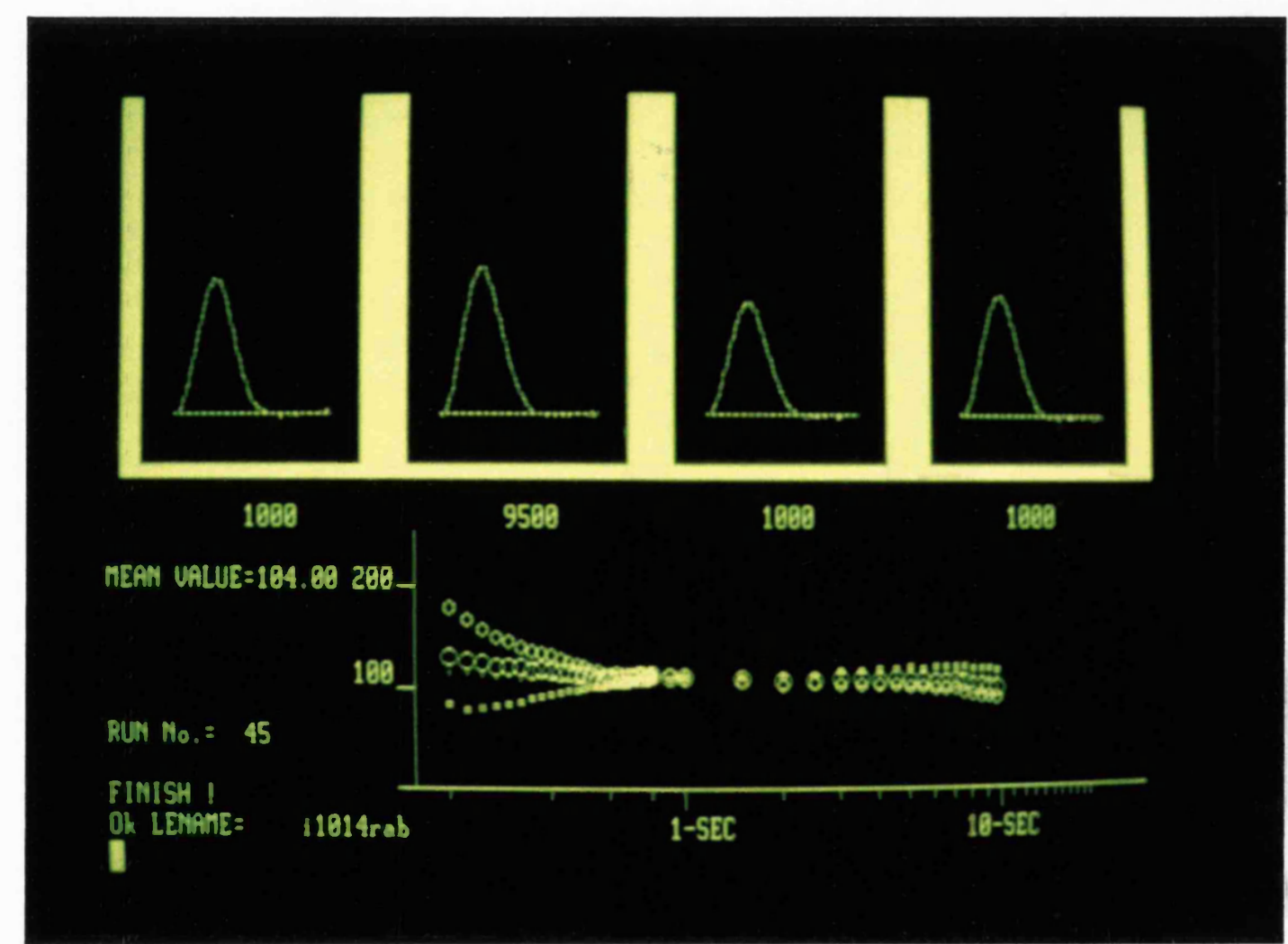

Figure 2.8: The screen output of the program MMAQUIS.BAS. This program generated both screen and plotter outputs. The screen output above shows the 4 data acquisition windows uppermost, displaying from left to right, steady state, extrasystolic, first post-extrasystolic and second post-extrasystolic beats. The inter-beat interval preceeding the relevant force envelope is shown in milliseconds immediately below the window. The lower portion of the screen is used to plot a force interval relationship for each of the 4 beats displayed in the acquisition windows. The ordinate is in ASC II units. The upper portion of the screen is replotted for each run (change in extra-systolic interval), the force amplitude of the new beats is then added to the lower graph. 
3) MMCOLLAT.BAS (Appendix 2 Section 3): This analysis program would divide peak force by steady state force at each extra-systolic interval over any number of experiments outputted by MMCALCUL.BAS. It was thus possible to derive a single collated force-interval relationship for each experimental group. Regression (see below) could then be used to fit a biexponential expression to the force-interval data.

4) Regression (Appendix 2 Section 4): This commercially available software package (Blackwell Scientific, Oxford, England) was used to fit a biexponential expression to the force-interval data output of MMCALCUL.BAS.

5) MMAREA.BAS (Appendix 2 Section 5): A bit pad (Summa Graphics, Summa Sketch II, Connecticut, USA) was used to trace the papillary muscle force envelopes at slow paper speed to provide a crude measure of papillary muscle activity during hypoxia. A program based on Simpson's rule was used to calculate this area on the basis of stylus position information received from the bit pad.

\section{The Langendorff Heart}

\subsection{The Principles of Retrograde Perfusion}

Early physiologists attempted to isolate the mammalian heart to examine it $\mathrm{s}$ metabolic requirements, but realised that unlike the thin walled frog heart, the thick walled mammalian heart had a specialised circulatory system that required a steady flow to maintain cardiac contraction (Opie 1992a). The first practical description of a simple apparatus to maintain the isolated heart was made by Oscar Langendorff (1895).

In the Langendorff preparation the heart is retrogradely perfused by tying the ascending aorta to a cannula through which the perfusate enters the aortic root. The perfusate is unable to enter the left ventricular cavity providing the aortic valve is intact and the pressure in the aortic root is greater than that in the left ventricle or outflow tract. The only orifices in the ascending aorta by which perfusate may escape are those of the right and left coronary arteries which lie in the coronary sinuses. Perfusate is forced through the coronary circulation, myocardial capillaries and then to the right atrium and ventricle via the coronary sinus (a small proportion also enters 
the left ventricle through thebesian drainage). Coronary vascular resistance can be measured by perfusing the heart in constant pressure mode and measuring flow or perfusing in constant flow mode and measuring pressure. If the perfusate contains the essential substrates and its flow rate is sufficient to supply these substrates and remove metabolites then the myocardium can remain viable and contract for many hours. In addition, by comparing the composition of the perfusate above (in the cannula) and below (in the right ventricle) the heart, it is possible to measure substrate extraction and metabolite production.

\subsection{The Preparation of Hearts for Retrograde Perfusion}

Twenty-four hours following either whole body heat or sham heat stress rabbit hearts were removed for further experimentation.

New Zealand white rabbits (1.5-3.5kg) were deeply anaesthetised by a administering IV pentobarbitone (Sagatal,May and Baker, Dagenham, England) at a dose of $60 \mathrm{mg} / \mathrm{kg}(1.0 \mathrm{ml} / \mathrm{kg})$ followed by 500 units of heparin via the marginal ear vein. The hearts were removed by elevating the sternum and washed in cold oxygenated saline. The aorta was threaded onto the perfusion cannula under a meniscus of cold saline to prevent entry of air. Once the aorta was tied to the cannula, perfusion was started. The time between removal of heart and perfusion on the Langendorff apparatus (ie the cold ischaemic time) was never more than 2 minutes. Once perfused, the hearts were trimmed of pericardium, the pulmonary artery opened to allow drainage of perfusate and placement of right ventricular temperature probes and cannulae, and the left atrial appendage removed.

\subsection{Description of the Apparatus}

\subsubsection{Delivery of the Perfusate}

A schematic diagram of the apparatus appears in figure 2.9. Perfusates were oxygenated in a jacketed reservoir from which fluid was drawn off by a variable rate peristaltic pump (Watson-Marlow 502S/R, Cornwall, England). The perfusate was then pumped through a $10 \mu \mathrm{m}$ filter and heat exchanger to a bubble trap. A rubber membrane over the vent of the bubble trap as well as air within the bubble trap dampened the pulsations from the peristaltic pump. The hearts were suspended from 


\section{The Langendorff Apparatus}

另

Balloon: to dampen pressure fluctuations

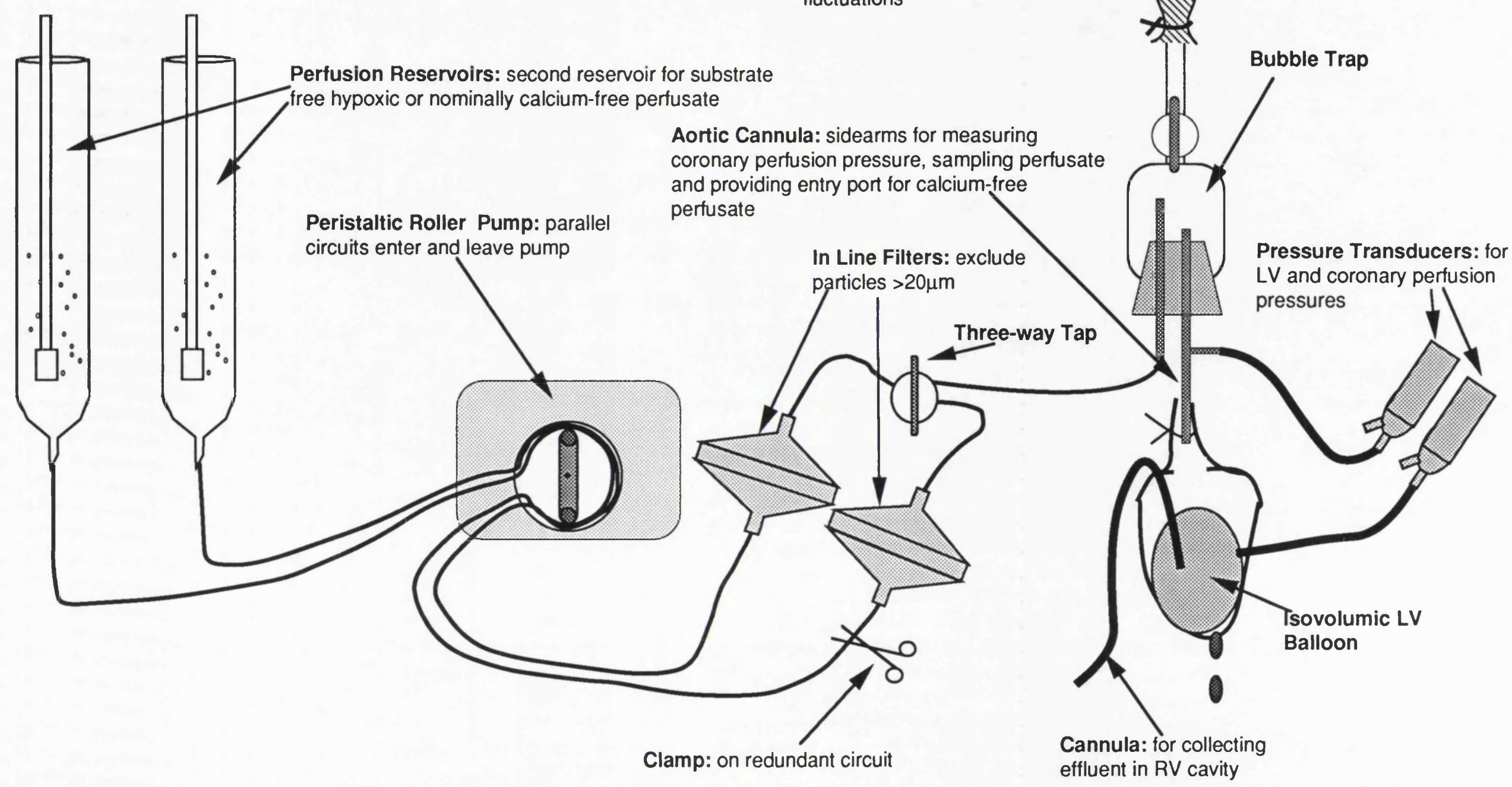

Figure 2.9: Schematic diagram of Langendorff perfusion apparatus. Parallel circuits draw perfusate from two reservoirs through the same peristaltic pump with the same diameter tubing. In line filters exclude large particulate contamination. All solutions are prefiltered to $2 \mu \mathrm{m}$. When the apparatus was used for calcium free perfusion the calcium free circuit entered at the level of the aortic cannula having passed through a "dedicated" bubbletrap. The apparatus was surrounded by a water jacket to maintain perfusate in the right ventricle at $37^{\circ} \mathrm{C}$. This has been excluded for clarity. 
a cannula draining the bubble trap. The cannula had a polythene sidearm for collection of perfusate and measurement of coronary pressure using a pressure transducer (P23XL, Gould Electronics, Ohio, USA) and appropriate amplifier (transducer amplifier, Gould electronics).

\subsubsection{Assessment of Left Ventricular Pressure}

Left ventricular developed pressure was measured by passing a deflated latex balloon through the mitral valve after excising the left atrial appendage. Once the balloon was in the left ventricular cavity it was filled with water. A pressure transducer (P23XL, Gould Electronics, Ohio, USA) linked by a short length of rigid polythene tubing to the latex balloon was used to measure isovolumic left ventricular pressure. After the balloon and connecting tubing had been purged of air, the balloon volume was adjusted so give a left ventricular end-diastolic pressure of between 5 and $10 \mathrm{mmHg}$.

The pressure transducers and respective bridge amplifiers used to measure developed and coronary perfusion pressures were balanced daily and calibrated using a mercury sphygmomanometer.

\subsubsection{Left Ventricular Monophasic Action Potentials}

Left ventricular monophasic action potentials (MAP) were measured using a purpose built $\mathrm{Ag} / \mathrm{AgCl}$ suction electrode applied to the epicardial surface of the heart. (The MAP electrode was a gift from Prof Max Lab, Department of Physiology, Westminster and Charing Cross Hospital). Reference and recording electrodes resident within the MAP electrode were connected to a "Universal" amplifier (Gould Electronics, Ohio, USA).

Appropriately amplified coronary and left ventricular pressure traces and MAP's were displayed on a chart recorder (RS3400, Gould Electronics, Ohio, USA).

\subsubsection{Temperature Control}

Temperature was measured in the right ventricular cavity with a type $\mathrm{K}$ thermocouple (Radiospares, Northants, England) and output sensed by a calibrated digital thermometer (Digitron, Northants, England). The temperature of the heart was 
kept at $37 \pm 0.2^{\circ} \mathrm{C}$ by water circulating within the jacketing surrounding the perfusion reservoir, heat exchanger and bubble trap (Techne C-85a Circulator, Cambridge UK).

\subsubsection{Control of Heart Rate}

In order to reduce variability in substrate demand all hearts were paced, via the right atrium at 180 beats/min, throughout the experiments using an isolated stimulator (Digitimer DS2, Hertfordshire, UK) triggered by a pulse generator (Digitimer DG2). Square wave pulse amplitude was set at twice threshold with a pulse duration of $2 \mathrm{mS}$.

\subsubsection{Oxygen, Carbon Dioxide and pH Differences Across the Heart}

Perfusates were collected simultaneously from the sidearm of the aortic perfusion cannula and from a cannula dwelling in the right ventricle. The syringes containing the samples were capped and $\mathrm{pH}, \mathrm{pO}_{2}$ and $\mathrm{pCO}_{2}$ measured using an automated blood gas analyser (ABL2 Radiometer Copenhagen, Copenhagen, Denmark). By subtracting values obtained from perfusates taken above and below the heart, $\mathrm{O}_{2}$ consumption and, $\mathrm{CO}_{2}$ and $\mathrm{pH}$ production could be estimated.

\subsubsection{Creatine Phosphokinase within the Coronary Effluent}

Creatine phosphokinase (CPK) exists within mitochondria and cytosol where it plays a role in the phosphocreatine-creatine energy shuttle (Katz 1992). The purpose of this enzyme is to transfer a high energy phosphate from ATP to creatine (mitochondrial) or from creatine phosphate to ADP (cytosolic in region of contractile apparatus). Damage to mitochondrial and sarcolemmal membranes allows the leakage of cellular constituents including creatine phophokinase which can be used as a marker of myocyte damage.

In the Langendorff heart, CPK leakage into the coronary effluent can be measured and augments changes in contractility as a measure of myocardial injury. A commercially available assay kit (CK NAC-activated system, Boehringer Mannheim, Meylan, France) was used to measure CPK. The assay relies on creatine phosphokinase present in the effluent reacting to form ATP from ADP and creatine phosphate, the ATP is used in subsequent reactions (ATP being the rate limiting substrate) to form gluconate-6-phosphate. The rate of accumulation of this compound, 
an indirect indicator of CPK activity, was measured by spectrophotometric absorption at 365nm (PU8700 Spectrophotometer, Phillips, Holland).

\subsubsection{Myoglobin Absorption at 280nm}

Myoglobin an intracellular constituent with a relatively low molecular weight $(17 \mathrm{kDa})$ can be used as indicator of sarcolemmal damage (Yamashita et al 1993). The content within the coronary effluent can be estimated in crude fashion by measuring the absorption of the globin moiety at 280nm (Busselen 1985).

\section{The In-Vivo Model of Infarction}

\subsection{The Determinants of Infarct Size}

The principle determinants of infarct size have already been discussed (Chapter 1 Section 3). The duration of ischaemia in the rabbit model of infarction used in this thesis was 30 minutes with 120 minutes of reperfusion. Body temperature and rate pressure product were closely monitored to ensure that the metabolic requirements between experimental groups were similar. Collateral flow in the rabbit is close to zero and therefore should not be a cause for inter-experimental variability (Maxwell et al 1987). The contribution of the volume at risk of infarction to overall infarct size was controlled by expressing infarct volume as a percentage of the volume at risk of infarction (although this well recognised technique does seem to have some drawbacks, see Chapter 7 Section 3.2).

\subsection{Recovery Experiments Involving Ischaemic Pretreatment}

The rationale behind setting up a surgical recovery model in the rabbit was to examine the induction of myocardial stress proteins in response to ischaemia. Four, 5 minute episodes of ischaemia followed by 24 hours of reperfusion have been shown to result in cardiac stress protein induction (Knowlton et al 1991a), and an identical protocol was adopted for the purposes of our investigations. 


\subsubsection{Anaesthesia for Recovery Experiments Involving Ischaemic Pretreatment}

Rabbits were anaesthetised with intramuscular fentanyl $100 \mu \mathrm{g} / \mathrm{kg}$ and fluanisone $3 \mathrm{mg} / \mathrm{kg}$ (Janssen pharmaceuticals, Oxford, UK), followed by intraperitoneal diazepam $2 \mathrm{mg} / \mathrm{kg}$. Anaesthesia was maintained by fentanyl and fluanisone, administered every 30 minutes. Once the rabbits were adequately anaesthetized, they were orally intubated using a wire introducer (endotracheal intubation stylet, Portex, Kent, England) and uncuffed endotracheal tube (Portex size 3.0, 3.5 or $4.0 \mathrm{~mm}$ ) and paediatric Laryngoscope (Penlon laryngoscope with Wisconsin no 0 or no 1 blade, Penlon, Oxford, England). The skin over scapulae and ilia was shaved with a scalpel blade and limb lead ECG electrodes (Medicotest, Rugmarken, Denmark) were attached. The rabbits were mechanically ventilated with $100 \%$ oxygen, at a tidal volume of $5 \mathrm{ml} / \mathrm{kg}$ delivered at a rate of $1 \mathrm{~Hz}$ (Harvard small animal ventilator, Kent, England). A marginal ear vein was cannulated to administer fluids and drugs and $30 \mathrm{mg}$ of intramuscular amoxycillin was given prophylactically.

\subsubsection{The Surgical Preparation for Recovery Experiments Involving Ischaemic Pretreatment}

The heart was exposed through a median sternotomy taking care to avoid the pleura and internal mammary arteries. Once the chest was open, the pericardium was cut and a coronary artery (usually an anterolateral branch of the circumflex), underrun with $3 / 0$ silk suture on a tapered needle. The free ends of the suture were passed through a soft vinyl tube so as to form a snare to occlude the artery. Rabbits then received 1000 units of heparin prior to the first 5 minute coronary artery ligation. Successful ligation was confirmed by myocardial cyanosis with bulging and changes in the amplified ECG signal (ECG amplifier and series 3000 recorder, Gould Inc, Ohio, USA). Reperfusion was confirmed by a myocardial blush and resolution of ECG changes. Following four, 5 minute coronary ligations separated by 10 minutes of reperfusion, the vinyl occluder was removed, the coronary suture was left in situ whilst the sternotomy was closed by suturing muscle and then skin layers. Animals were given $10 \mathrm{ml} / \mathrm{kg}$ of $0.9 \%$ saline intravenously, allowed to breathe spontaneously and eventually extubated.

The surgical preparation for the sham ischaemic pre-treatment group was 
identical to that described above. However, although the pericardium was opened, a coronary artery was neither underrun nor occluded, thus avoiding the mechanical manipulation of the myocardium which may in itself act as a trigger for stress protein induction (Knowlton et al 1991b).

\subsection{Non-Recovery Experiments Involving Prolonged Ischaemia and Infarction}

\subsubsection{Surgical Preparation for Non-Recovery Experiments Involving Prolonged Ischaemia and Infarction}

Ischaemically pre-treated and heat stressed rabbits were recovered for 24 hours and then underwent infarct size assessment following a 30 minute coronary occlusion.

New Zealand White rabbits were anaesthetised by administering IV pentobarbitone (Sagatal, May and Baker, Dagenham, England) at a dose of $40 \mathrm{mg} / \mathrm{kg}$ $(0.6 \mathrm{ml} / \mathrm{kg})$ and then shaved to allow attachment of ECG electrodes as described above. The fur over the sternum and anterior aspect of the neck was also shaved. The skin over the trachea was then infiltrated with $2-3 \mathrm{mls}$ of $1 \%$ lignocaine solution using a $22 \mathrm{G}$ needle. After further IV pentobarbitone, the trachea was exposed through an anterior cervical incision and intubated with a $3 \mathrm{~mm}$ endotracheal tube following a tracheotomy. Rabbits were mechanically ventilated at $1 \mathrm{~Hz}$ with a reduced tidal volume of $4 \mathrm{ml} / \mathrm{kg}$ in keeping with the reduction in ventilatory dead space. The right common carotid artery was exposed in the peritracheal fascia and cannulated with a short rigid polythene cannula attached to a pressure transducer (Gould P23XL), for continuous recording of arterial pressure and intermittent arterial blood gas estimations (ABL2, Radiometer, Copenhagen, Denmark). Throughout the procedure rectal temperature was monitored (Type $\mathrm{K}$ thermocouple and calibrated Digital thermometer, Radiospares, Northants, England) and maintained between $38.5^{\circ} \mathrm{C}$ and $39.0^{\circ} \mathrm{C}$ using an electric warming pad. The chest was opened via a midline sternotomy and a coronary artery identified. Rabbits were given 1000units of heparin intravenously and the coronary artery ligated as described above. In the case of the ischaemic pretreatment group, the existing coronary tie was re-used to ensure that the same coronary bed was rendered ischaemic as on the previous day. In the sham ischaemia group identification of a suitable coronary vessel was often difficult since the surface 
of the heart was encased in a thin fibrinous exudate.

Following a 30 minute coronary occlusion, reperfusion was confirmed by the appearance of a myocardial blush. Experiments were continued for 120 minutes of reperfusion during which haemodynamic data was recorded every 15 minutes and blood gases analysed every 30 minutes.

\subsubsection{Assessment of Infarct Size (see figure 2.10)}

At the end of the 120 minute reperfusion period, a further 1000 units of heparin was given, and hearts were removed and retrogradely perfused with $0.9 \%$ saline. After blood had been washed out of the coronary vasculature the coronary snare was retightened and fluorescent microspheres (Zinc Cadmium-Sulfide 1-10 $\mu \mathrm{m}$ particles, Duke Scientific, California, USA) infused via the aortic cannula. Demarcation of the myocardial surface at risk (zone without spheres) was confirmed under UV light (see figure 2.10). Hearts were then frozen at $-18^{\circ} \mathrm{C}$ overnight. The following day, whilst still frozen, hearts were sliced at $2 \mathrm{~mm}$ intervals at right angles to their long axis. The slices were then incubated at $37^{\circ} \mathrm{C}$ in triphenyltetrazolium chloride (Sigma Chemicals, Mousourri, USA) at a concentration of $10 \mathrm{mg} / \mathrm{ml}$ of phosphate buffer at $\mathrm{pH} 7.4$. Once the viable myocardium had stained, slices were placed in $10 \%$ formaldehyde solution. Approximately 24 hours later heart slices were placed caudal surface upwards and compressed between two glass plates separated by $2 \mathrm{~mm}$ spacers. Risk areas, viewed under UV light, and areas of infarction were traced, photographically enlarged and planimetered (Summa Graphics, Summa Sketch II, Connecticut, USA, Jumpers modified for ASC II output) using dedicated software based on Simpson's Rule (see program MMINFRSK.BAS in Appendix 2 Section 6). The area of infarction (no tetrazolium staining), was expressed as a percentage of the area at risk of infarction (no fluorescent microspheres). The volume of myocardial tissue at risk and the volume of infarction were calculated by multiplying the corresponding areas by the depth ( $2 \mathrm{~mm}$ ) of the tissue slices (see figure $2.10 \mathrm{~B}$ ).

\section{Statistical Methods}

All values are expressed as means \pm SEM. All comparisons involving more than 2 groups were assessed for significance with a one-way analysis of variance (ANOVA), 


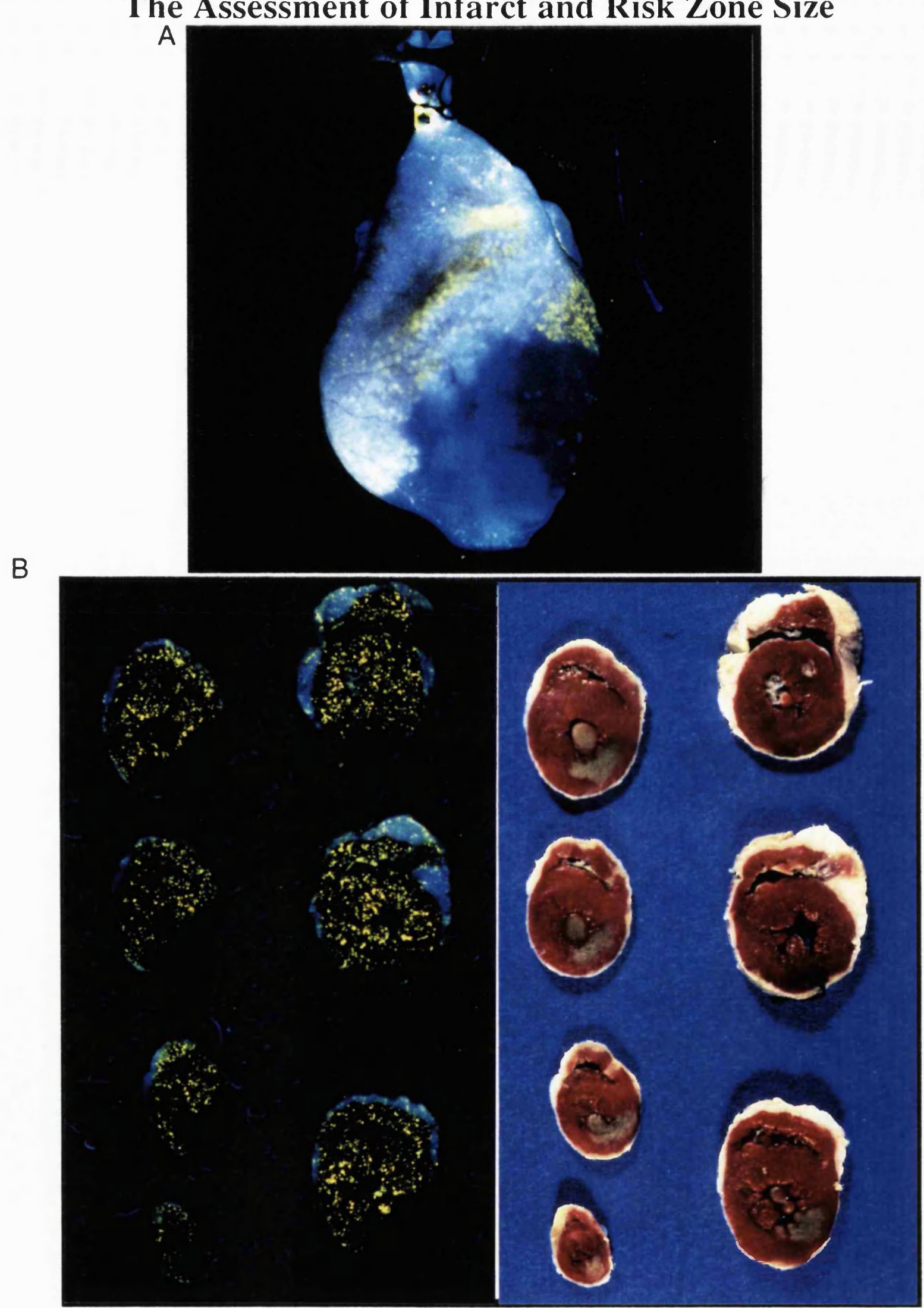

Figure 2.10: The use of fluorescent particles and triphenyl tetrazolium to measure infarct size. Panel A: shows the appearance of a whole heart after the coronary artery has been re-ligated and flourecent particles retrogradely perfused. Under ultraviolet light $(366 \mathrm{~nm})$ the area at risk appears dark whilst the area of perfusion fluoresces brightly. Panel B: Shows a heart treated as shown in panel A then frozen and 24 hours later sliced at right angles to its long axis and incubated in triphenyltetrazolium. The slices on the left are viewed under ultraviolet light and those on the right are the same slices viewed under white light. The slices on the left show a clear risk area, the corresponding area viewed under white light contains red staining and pale tissue. The pale tissue represents infarction. 
Fisher's Protected Least Significance Difference (FPLSD) method being used for comparisons within the ANOVA table (Armitage and Berry 1987). Changes in haemodynamic parameters within groups over the course of the experiment were compared by two-way analysis of variance (Wallenstein et al 1980) with a post-hoc paired $t$-test for comparison of variables at baseline and the end of an experiment. An unpaired $t$-test was used to compare means when comparisons were confined to 2 experimental groups for example between heat stress and sham heat stress groups. Associations between rate pressure product and infarct size, papillary muscle diameter and developed force, and stress protein content and contractile recovery, were tested by the Spearman Rank Correlation method (Armitage and Berry 1987). The relationships between risk area and areas of infarction between contemporaneous experiments was determined by analysis of covariance. Statistical comparisons were made with commercially available software (Statview version 3.0 Cherwell Scientific Publishing, Oxford, England and Minitab version 8, Clecom, Birmingham, England). Statistical significance was defined as $\mathrm{p} \leq 0.05$. 


\section{Chapter 3}

\section{Results: Initial Pilot Study of Papillary Muscles Subjected to Serial Stresses}

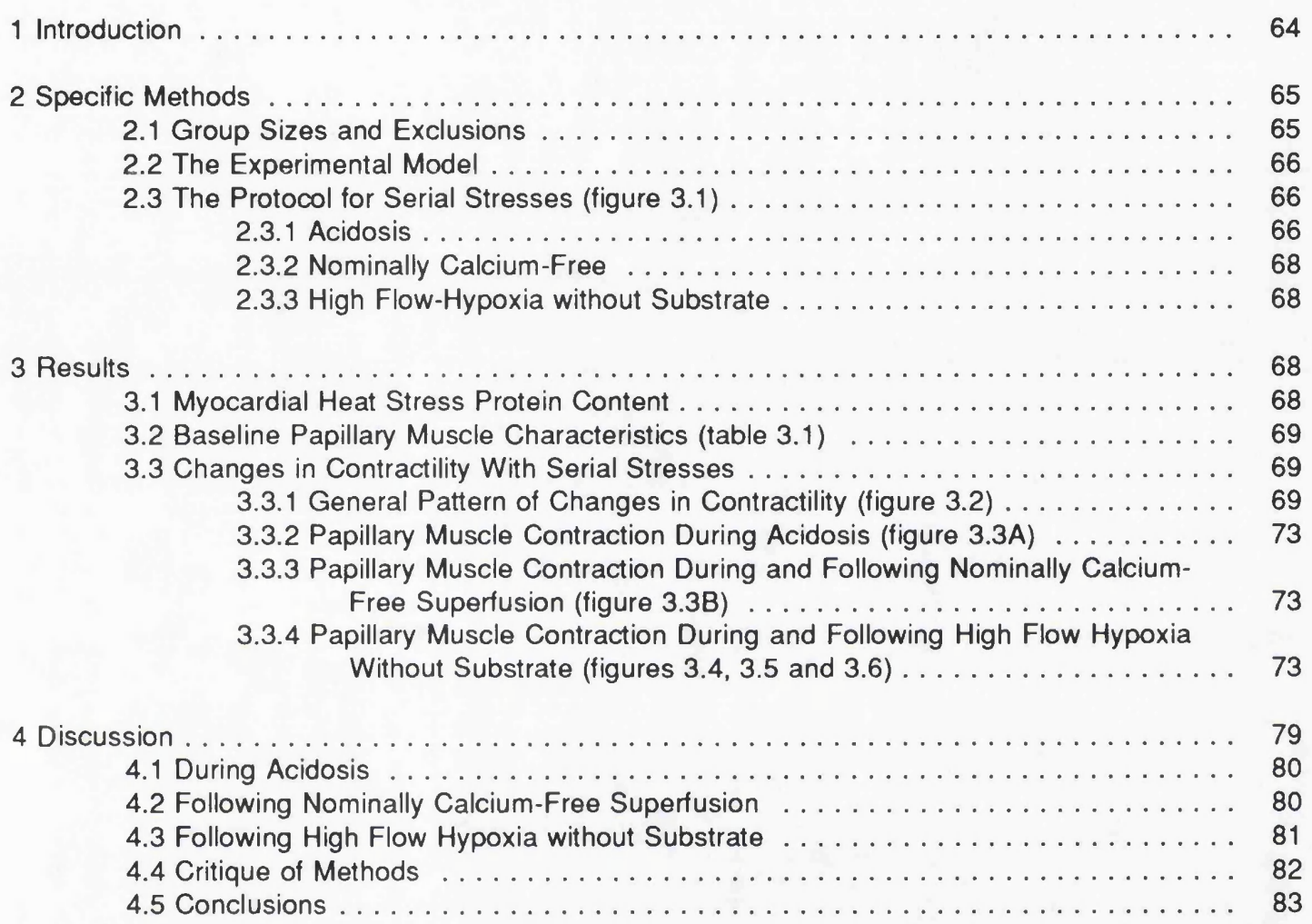

\section{Introduction}

Whole body heat stress is known to increase cardiac stress protein synthesis and protect the isolated rat heart against ischaemia (Currie et al 1988). The purpose of this initial pilot study was to examine the protection afforded by whole body heat stress against a number of components of ischaemia/reperfusion thought to be involved in the injury process. By such methods we hoped to gain a greater understanding of the basis for the protection following heat stress. Among the components of ischaemia/reperfusion thought to result in myocardial injury are (i)acidosis, (ii)calcium overload, (iii)depletion of high energy phosphates and (iv)free radical stress at reperfusion (see Chapter 1 Section 2). For the purposes of this study, acidosis was simulated by hypercapnia; calcium overload by low calcium superfusion 
followed by calcium repletion (the Calcium Paradox); high energy phosphate depletion by hypoxia without substrate and free-radical stress by re-oxygenation following hypoxia.

The laboratory in which this work was carried out had already demonstrated that the benefits of whole body heat stress, which are seen in the rat, are also present in the rabbit (Yellon et al 1992a). It was therefore decided to use the rabbit for this and subsequent studies. Papillary muscle specimens rather than whole hearts were used because of a number of theoretical advantages. In the whole heart, measurement of contractile function during and following ischaemia is complicated by alterations in cavity geometry associated with ischaemic contracture, tissue oedema and the effects of changes in coronary perfusion pressure, the "garden hose effect" (Chappell et al 1985). This makes it difficult for practical purposes to be certain if changes in the contractile function of a Langendorff heart, during or following an intervention are secondary to alterations in coronary perfusion pressure/flow or left ventricular enddiastolic pressure (Vogel et al 1985). In addition the papillary muscle model has the further advantage of allowing force interval measurements to be made (see Chapter 2 Section 3.2.6), independent of loading conditions and intrinsic pacemaker activity.

\section{Specific Methods}

\subsection{Group Sizes and Exclusions}

Rabbit right ventricular papillary muscles from heat stress $(n=12)$ and sham heat stress rabbits $(n=10)$ were used for this study. Two experiments in the heat stress group were excluded. One rabbit died during the heat stress procedure secondary to an inadvertent anaesthetic overdose. The second exclusion from the same group was due to alternans of papillary muscle developed force despite normal temperature and $\mathrm{pH}$ of the Tyrode solution. Consequently 10 papillary muscles from sham heat stressed (control) and 10 papillary muscles from heat stressed (heat stress) rabbits were used for the analysis of contractility. Rabbits were assigned to heat stress or control groups on alternate days apart from when a technical failure required repetition of an experiment. 


\subsection{The Experimental Model}

The rabbit whole body heat stress procedure (Chapter 2 Section 1.1.2) and preparation (Chapter 2 Section 3.2.4), suspension (Chapter 2 Section 3.2.4), superfusion (Chapter 2, Section 3.2.2), field stimulation (Chapter 2 Section 3.2.3) and force-interval timings (Chapter 2 Section 3.2.6) of rabbit right ventricular papillary muscles were as previously described. The acquisition of contractile information was similar to that discussed (see Chapter 2 Section 3.2.6) apart from the fact that analysis of tension envelopes was by an earlier version of the MMCALCUL.BAS programme to that listed in Appendix 2 Section 2. No information was therefore available on, maximum rate of contraction, maximum rate of relaxation, the time to peak tension or the time to $90 \%$ relaxation for the steady state beats.

\subsection{The Protocol for Serial Stresses (figure 3.1)}

Heat stress and control muscles were treated identically. After harvesting papillary muscles were stabilised in Tyrode solution of the following composition, $\mathrm{NaCl} 118 . \mathrm{mM}, \mathrm{KCl} 4.0 \mathrm{mM}, \mathrm{MgCl}_{2} \cdot 6 \mathrm{H}_{2} \mathrm{O} 1.0 \mathrm{mM}, \mathrm{NaHCO}_{3} 24.0 \mathrm{mM}, \mathrm{NaH}_{2} \mathrm{PO}_{4} \cdot 2 \mathrm{H}_{2} \mathrm{O}$ $0.4 \mathrm{mM}, \mathrm{CaCl}_{2} \cdot 2 \mathrm{H}_{2} \mathrm{O} 1.8 \mathrm{mM}$, Glucose $6.5 \mathrm{mM}$ and Napyruvate $5.0 \mathrm{mM}$, equilibrated with $95 \% \mathrm{O}_{2} / 5 \% \mathrm{CO}_{2}$. After 30 minutes of stabilisation muscles were stretched to a length giving $90 \%$ of maximal active force and pacing threshold determined. The order of exposure to serial stresses had to take into account the degree of papillary muscle injury that each stress would cause. For this reason it was decided that acidosis should be followed by exposure to nominally calcium-free superfusate, the final and most injurious stress being high flow hypoxia without substrate.

\subsubsection{Acidosis}

After stabilisation muscles were superfused for 30 minutes with the same Tyrode's solution (as above) equilibrated with $90 \% \mathrm{O}_{2} / 10 \% \mathrm{CO}_{2}$, resulting in a shift in organ bath pH of approximately 0.27 units (Heat stress group 7.49 \pm 0.00 to $7.23 \pm 0.01$, Control group $7.49 \pm 0.01$ to $7.22 \pm 0.01$, means $\pm S E M, n=10$ for each group). Force interval assessments were performed at regular intervals, including during acidosis as indicated in figure 3.1 . 


\section{Protocol for Serial Stresses}

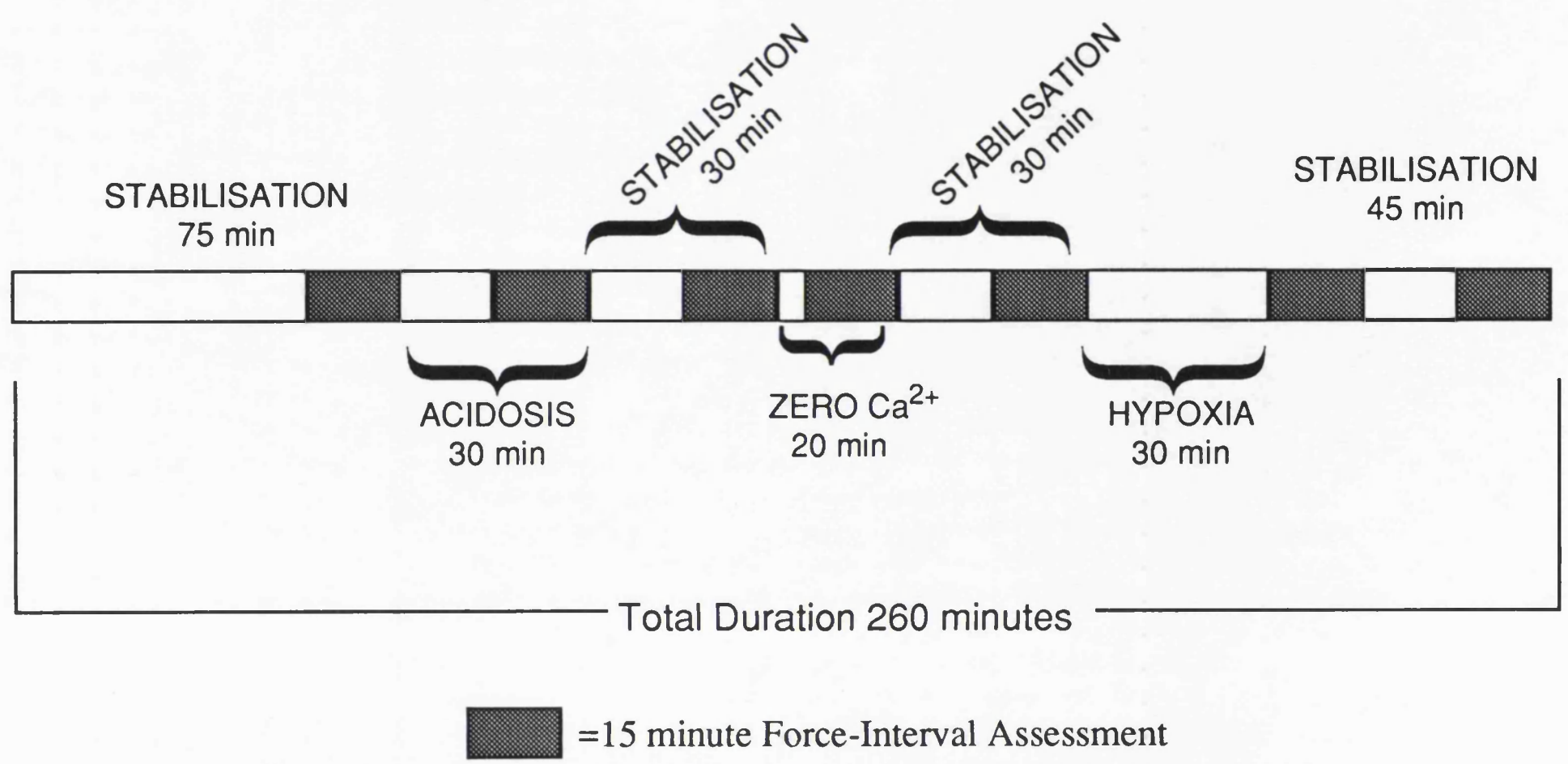

Figure 3.1: Rabbit right ventricular papillary muscles were subjected to the serial stresses of (i) acidosis (10\% carbon dioxide), (ii)no added calcium and (iii)hypoxia (partial pressure of oxygen $<6.0 \mathrm{kPa}$ ) without substrate. Force-Interval assessments at various points during the protocol allowed the measurement of changes in contractility in response to each of the stresses. 


\subsubsection{Nominally Calcium-Free}

Following acidosis papillary muscle force returned to pre-acidosis baseline values in both groups. Muscles were then exposed Tyrode solution from which $\mathrm{CaCl}_{2}$ had been omitted. After 20 minutes of superfusion with nominally calcium-free solution contractile activity ceased without any noticeable change in resting force. In similar experiments the calcium concentration in such nominally calcium-free solution was measured by atomic absorption spectrophotometry (PYE, Unicam SP9, England) using lanthanum chloride in $\mathrm{HCl}$ to prevent the formation of calcium phosphate. The estimated calcium concentration of these solutions was $6.1 \pm 1.7 \mu \mathrm{M}$ (mean $\pm \mathrm{SE} n=10$ ).

\subsubsection{High Flow-Hypoxia without Substrate}

Following calcium-free perfusion muscles were stabilised and force-interval data collected prior to superfusion with glucose and pyruvate free (substrate free) Tyrode's solution bubbled with $95 \% \mathrm{~N}_{2} / 5 \% \mathrm{CO}_{2}$. The reservoir used to de-oxygenate the superfusate was covered with clingfilm and over-filled so as to wet the clingfilm/glass interface and exclude free communication with room air. A similar meniscus was formed between the upper surface of the organ bath and a glass coverslip to prevent room air oxygenation of the superfusate bathing the papillary muscle. The hypoxic superfusate was bubbled with the nitrogen mixture for 20 minutes prior to use. During high flow hypoxia without substrate contracture developed and contractile activity ceased in all muscles. At the end of the hypoxic period oxygen tension measured in the organ bath effluent was less than $6.0 \mathrm{kPa}$. Upon re-oxia with return of substrates, contracture became less marked and contractility increased. Force interval assessments were performed at two time points during reoxygenation as shown in figure 3.1 .

\section{Results}

\subsection{Myocardial Heat Stress Protein Content}

The whole body heat stress procedure used for this protocol results in an approximate 4 to 8 fold increase in both left and right ventricular hsp72 without an appreciable change in hsp60 (see Yellon et al 1992a and Chapter 4 Sections 3.6 and 3.7 and Chapter 5 Section 3.9). 


\subsection{Baseline Papillary Muscle Characteristics (table 3.1)}

Papillary muscle dimensions and weight were similar between groups. The papillary muscle volume was calculated on the basis of length and diameter assuming a cylindrical shape. The calculated volume was approximately two-thirds of that expected on the basis of weight given that muscle density is slightly greater than that of water. The probable explanation for this discrepancy is the fact that papillary muscles were weighed together with the attached wedge of septal or right ventricular free wall tissue. Basal resting force after tensioning the muscles was similar between groups as was developed force. Developed force was calculated as peak force minus resting force. Force was not expressed in terms of tension (force/unit crossectional area) since there was no correlation between papillary muscle diameter and developed force (an example of the lack of dependence of muscle diameter on force is seen in Chapter 4 Section 3.1).

\subsection{Changes in Contractility With Serial Stresses}

\subsubsection{General Pattern of Changes in Contractility (figure 3.2)}

An example of the changes in contractility during the serial stress protocol is seen in figure 3.2 which is an example of the plotter output of MMAQUIS.BAS for a control papillary muscle. The first stress used, namely acidosis depressed contractility without altering the shape of either the restitution or decay of potentiation curves and recovery post acidosis was complete. In the example shown steady state beat developed force was approximately 55 ASC II units, dropping to 40 during acidosis and returning to 55 post-acidosis. Contractile activity ceased during the second stress of calcium-free superfusion with complete recovery to approximately 55 ASC II units on calcium repletion. This recovery occurred without an appreciable change in the shape of the force interval relationship. After the third stress of high flow hypoxia without substrate, contractile activity was markedly depressed but some recovery in developed force was seen even during the 15 minute force interval assessment. Thirty minutes post-hypoxia recovery is more complete with a steady state developed force of 35 ASC II units (recovery compared to pre-hypoxia therefore about $65 \%$ ). In addition the shape of both the restitution and decay of potentiation curves is altered. 


\begin{tabular}{l|c|c|c} 
& Heat Stress & Control & t-test \\
\hline Muscle Diameter (mm) & $0.84 \pm 0.07$ & $0.86 \pm 0.09$ & $p=N S$ \\
Muscle Length (mm) & $3.28 \pm 0.25$ & $3.65 \pm 0.27$ & $p=N S$ \\
Calculated Volume* $\left(\mathrm{mm}^{\wedge} 3\right)$ & $2.01 \pm 0.39$ & $2.42 \pm 0.52$ & $p=N S$ \\
Muscle Wet Weight (g) & $3.80 \pm 0.61$ & $3.83 \pm 0.44$ & $p=N S$ \\
Baseline Resting Force (g) & $0.31 \pm 0.10$ & $0.47 \pm 0.11$ & $p=N S$ \\
Baseline Developed Force (g) & $0.85 \pm 0.18$ & $0.94 \pm 0.10$ & $p=N S$
\end{tabular}

* Muscle volume was calculated on the basis of diameter and length assuming a cylinder.

\section{Table 3.1:}

Unloaded muscle length and diameter were determined at the end of the experimental protocol.

Comparisons between control and heat stress groups were made by two-tailed unpaired t-test. 


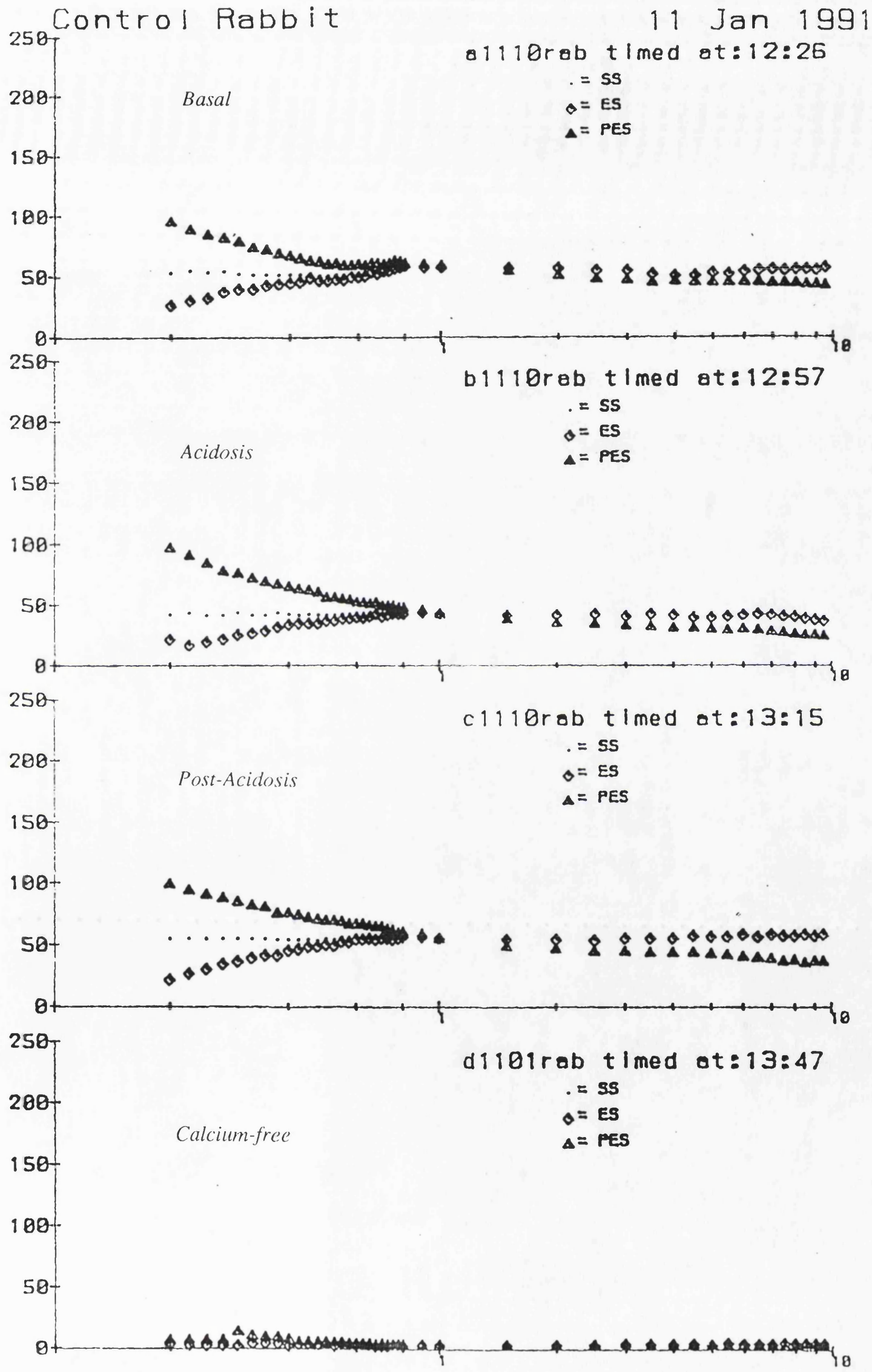




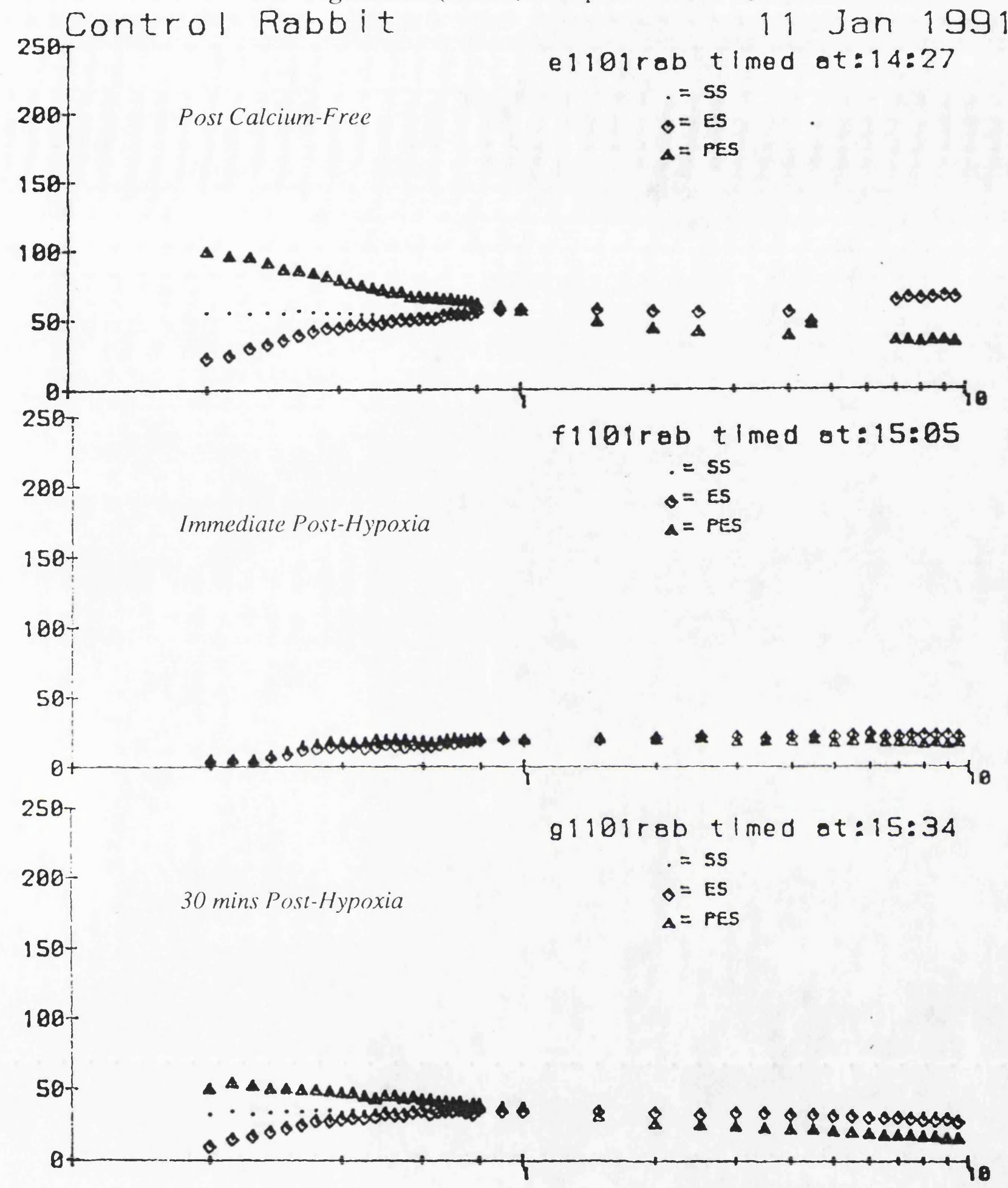

Figure 3.2: The output of MMAQUIS.BAS for the entire serial stress protocol. The $y$-axis of each force-interval curve is in ASC II units, the x-axis in log seconds. Each curve is plotted out in real time at the end of the data collection (when extrasystolic interval=10sec), the steady state beats are averaged and used to calculate developed and resting force. $\mathrm{SS}=$ steady state beat, $\mathrm{ES}=$ extrasystolic beat and PES=postextrasystolic beat.

The force-interval protocol post calcium-free perfusion was interupted as the data disc had to be changed and hence is incomplete. 


\subsubsection{Papillary Muscle Contraction During Acidosis (figure 3.3A)}

The degree of acidosis resulting from superfusate equilibration with $10 \% \mathrm{CO}_{2}$ was similar for control and heat stress muscles (7.22 \pm 0.01 and 7.23 \pm 0.01 respectively). Figure 3.3A illustrates the changes in papillary muscle developed force 15 minutes after switching to low $\mathrm{pH}$ superfusion. Developed force in figure $3.3 \mathrm{~A}$ is expressed as a percentage of baseline developed force (see table 3.1). Developed force was similarly depressed in heat stress (72.5\% of basal) and control (79.4\% of basal) papillary muscles. Resting force and the force interval relationship were not significantly altered during or following acidosis.

\subsubsection{Papillary Muscle Contraction During and Following Nominally Calcium- Free Superfusion (figure 3.3B)}

During calcium-free superfusion papillary muscle contraction ceased, upon reintroduction of calcium there was a prompt return of contraction and this was measured after 15 minutes of calcium repletion as shown in figure 3.3B. Surprisingly, there was no increase in resting force on return of calcium (increase in resting force pre- $v$ post- zero calcium for control group $0.08 \pm 0.03 \mathrm{~g}$ and for heat stress group $0.08 \pm 0.03 \mathrm{~g}$ ) but there was a mild though significant degree of contractile impairment seen only in the control papillary muscles. Once again developed force was expressed as a percentage of developed force prior to calcium-free superfusion (from the postacidosis force-interval relationship). Even though developed force was depressed upon calcium repletion there was no significant alteration in the force-interval relationship (see figure 3.2 for example).

\subsubsection{Papillary Muscle Contraction During and Following High Flow Hypoxia Without Substrate (figures 3.4, 3.5 and 3.6)}

Within the first few minutes of the switch to the hypoxic superfusate, without substrate papillary muscle developed force diminished, but then as resting force (contracture) increased papillary muscle developed force would also often transiently increase (see Chapter 4 Section 3.2). However at the end of the 30 minute hypoxic period papillary muscle contraction had ceased in all muscles despite attempts to capture contraction by increasing the stimulation amplitude and pulse width. With the 
A Developed Force During Acidosis

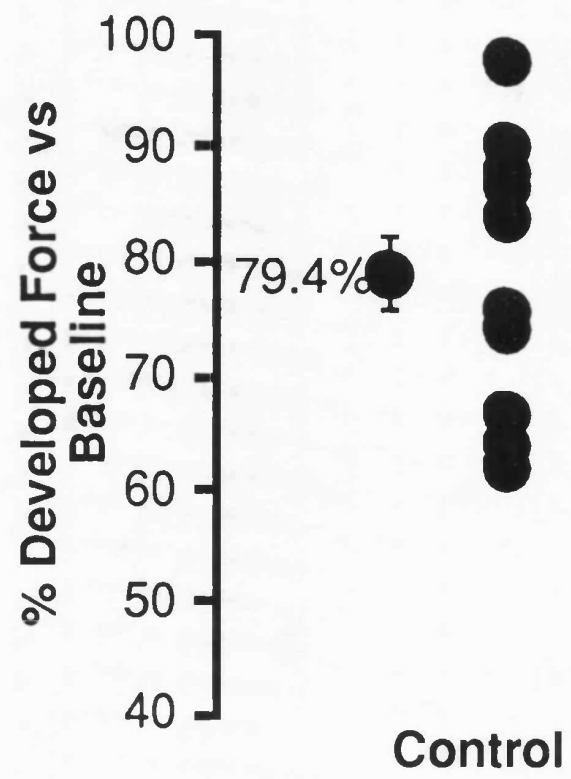

Heat Stress
B Developed Force 15-30 mins After Nominally Calcium Free Superfusion

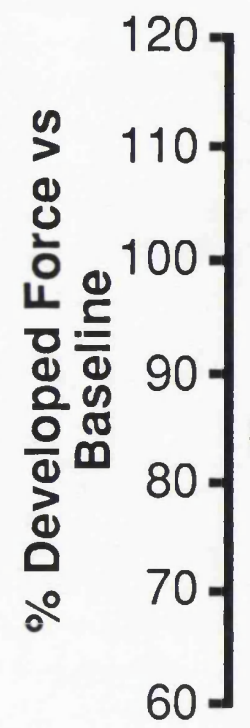

\section{$72.5 \%$}

$p=0.26$

601

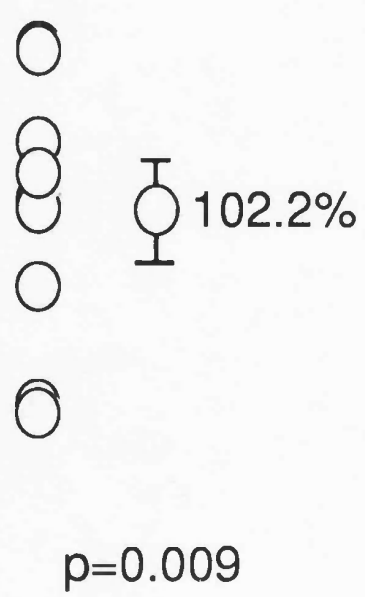

Heat Stress

Figure 3.3: Papillary muscle developed force during acidosis and after nominally calcium free superfusion. For each papillary muscle developed force during acidosis was expressed as a percentage of developed force during baseline stabilisation. Similarly developed force post-"no added calcium" was expressed as a percentage of developed force prior to no added calcium. $n=10$ for each group. Error bars represent $+/$ - SEM. Comparisons between heat stress and control groups were by unpaired, two tailed t-tests. 
re-introduction of, oxygen, glucose and pyruvate, contractions would return and contracture partially normalise. Interestingly, upon re-oxygenation there would often be a very prompt return of contraction in the first 2-3 minutes which would rapidly decrease over approximately 30 seconds and then beat strength would slowly increase once again. This transient burst of contractile activity, which occurred in both experimental groups, resembles the initial hyper-active segmental shortening seen after a short coronary occlusion before classical stunning becomes apparent. (A poor example of this effect is seen in figure 3.2 , in the immediate post-hypoxia force interval trace which shows a mild hump in the force of the steady state beat between extrasystolic intervals of 200 to $800 \mathrm{mS}$ ).

In the first 15 minutes of recovery after hypoxia (figure 3.4A), the average developed force was similar in control and heat stress groups at approximately onethird of the force developed prior to hypoxia. During reoxygenation developed force in both groups increased to differing degrees, so that when measured between 30 and 45 minutes of reoxygenation the developed force was significantly greater in the heat stressed muscles as shown in figure 3.4B. The greater rate of contractile recovery in the heat stressed group is shown in figure 3.5 which shows that the percentage increase in contractile recovery for each papillary muscle during the first $\mathbf{4 5}$ minutes of reoxygenation is more in heat stressed than in control muscles.

The increase in resting force occurring as result of hypoxia is illustrated in figure 3.6A and 3.6B. Resting force for each muscle prior to hypoxia was subtracted from resting force following hypoxia. Resting force was similarly increased in both control and heat stressed groups both immediately and 30 minutes after reoxygenation and return of substrates. Although there was a tendency for the contracture to be less marked in heat stressed muscles at both timepoints this was not significant. Interestingly resting force was still significantly elevated 30 minutes after reoxygenation suggesting a degree of irreversible injury and contracture in both experimental groups.

The analysis of the fast components of the decay of potentiation and restitution curves both before and 30 minutes after hypoxia no substrate are shown in figure 3.6C. The curves immediately post-hypoxia could not be analysed because their shape was too distorted by the rapid recovery in contractile strength in the first minutes of 


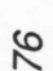

B

\section{Developed Force 30-45 mins Following \\ High Flow Hypoxia/No Substrate}
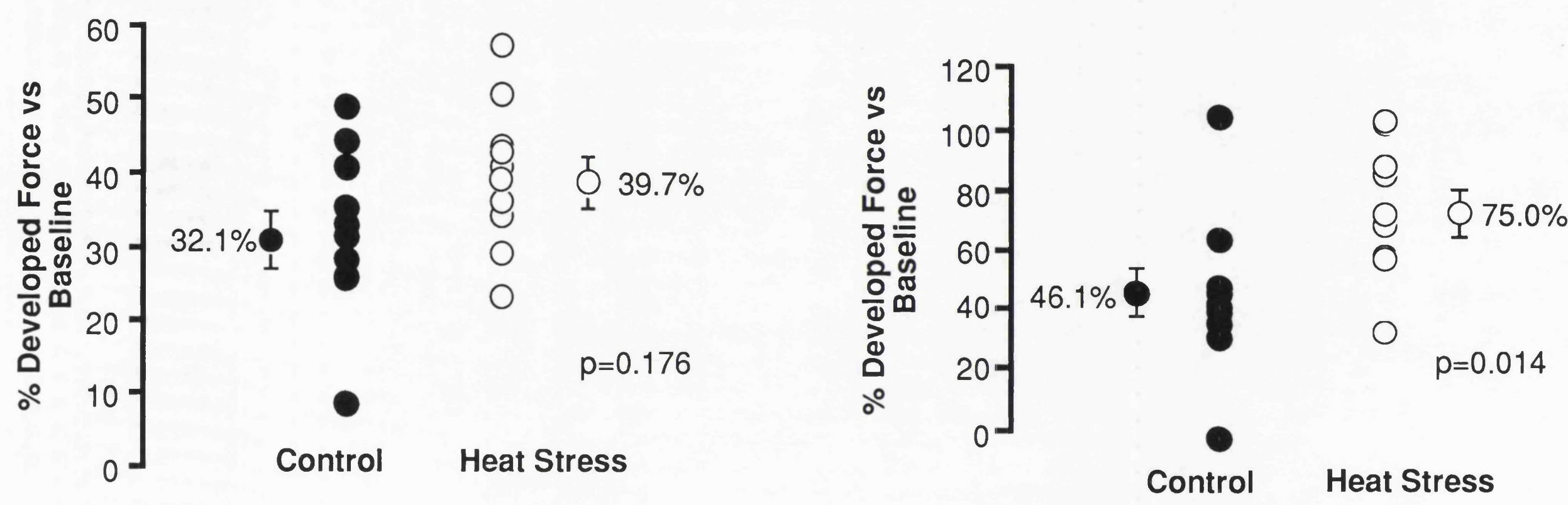
of developed force prior to hypoxia. $n=10$ for each group. Error bars represent $+/$ - SEM. Comparisons between heat stress and control groups were by unpaired, two tailed t-tests. 

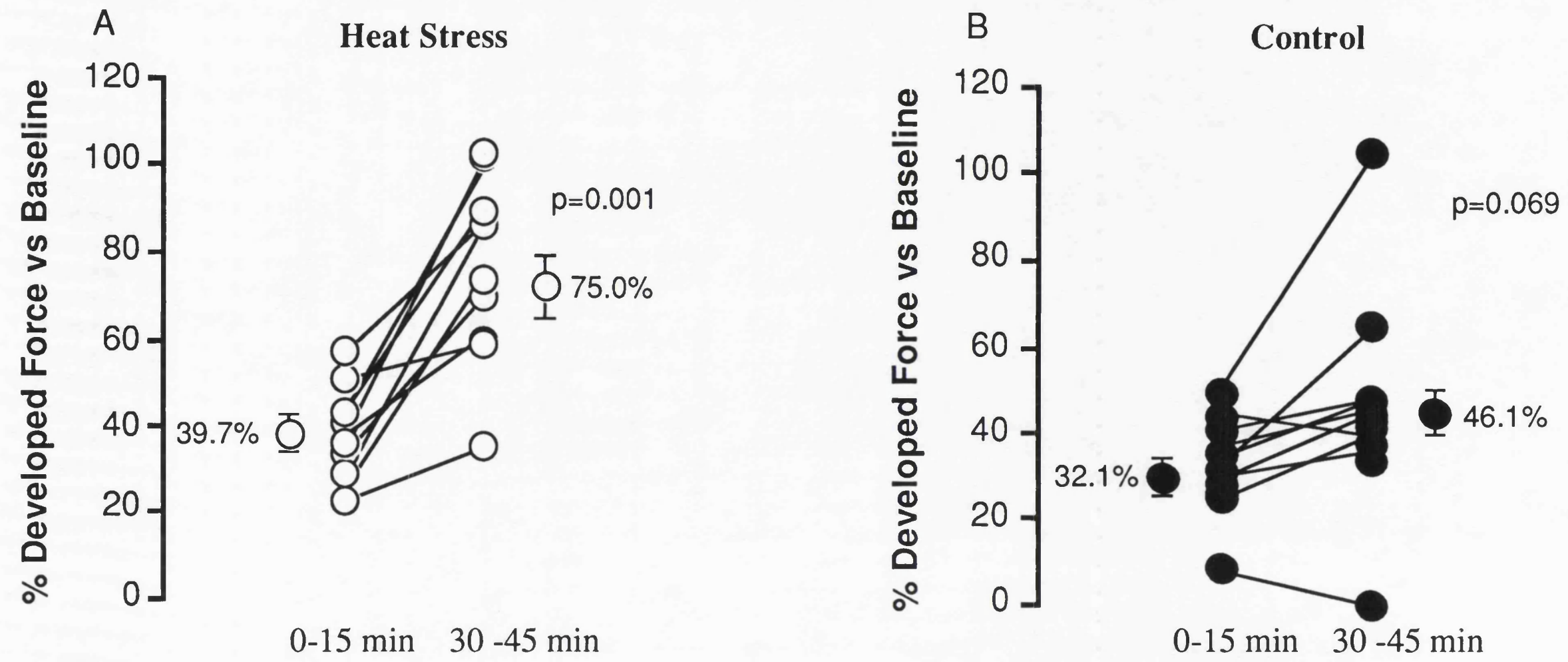

Figure 3.5: Recovery of developed force post-hypoxia expressed as a percentage of developed force prior to hypoxia. Recovery of force 5 minutes post-hypoxia is compared to that 30 minutes post hypoxia for both heat stressed and control papillary muscles. There was a significant recovery during re-oxia in heat stressed but not control muscles. $n=10$ for each group. Error bars represent $+/-$ SEM. Comparisons within heat stress and control groups were by paired, two tailed t-tests. 
A

$\stackrel{n}{\infty}$

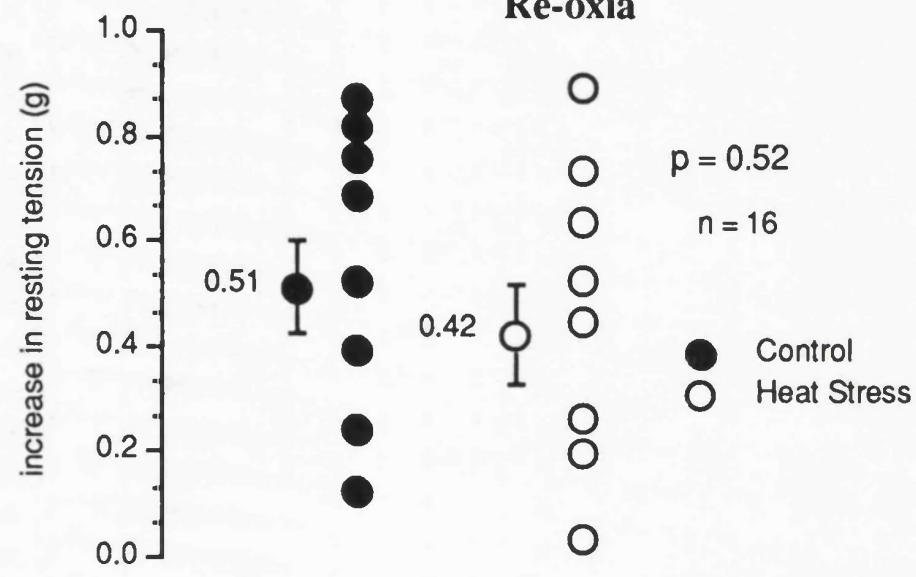

B

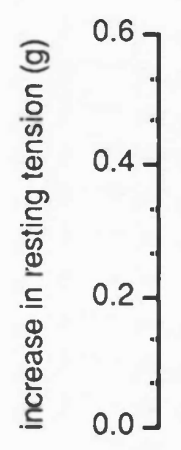

Increase in Resting Force 30-45 mins Re-Oxia

Figure 3.6: Resting force and force-interval time constants after high flow hypoxia without substrate. Figures A and B show the rise in resting force in grammes after, compared to before high flow hypoxia no substrate. No significant differences were observed between heat stress and control groups. Figure $\mathrm{C}$, shows the mean values for Tau 1 derived by fitting restitution and decay of potentiation curves for each individual experiment to an equation of the form: Force $=a+b \cdot \exp (-$ time/tau1) $+c \cdot \exp (-$ time/tau2). Standard errors are large because of the wide variability in rate constants see text for details. $\mathrm{n}=10$ for each group.

Error bars represent $+/$ - SEM. Comparisons between heat stress and control groups were by unpaired, two tailed t-tests.
C Tau 1 Time Constants for Extra-systolic and Post Extra-systolic Beats Before and 30mins after High
$800-\quad$ Flow Hypoxia/No Substrate

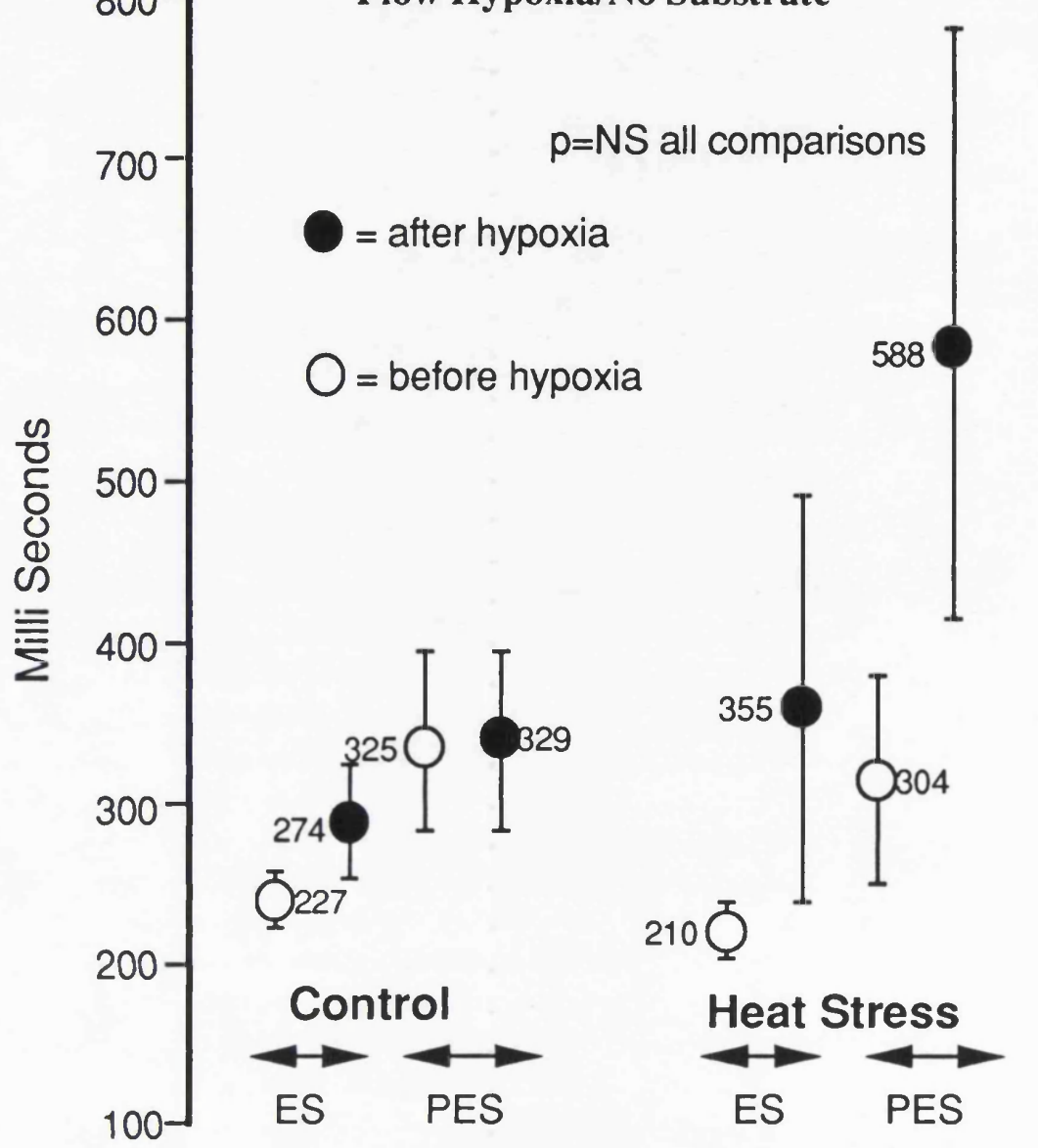


reoxygenation. There were a number of problems encountered with the curve fitting used to derive the rate constants of the force interval relationship. The behaviour of the fitting algorhythm, and the non-linear nature of the force-interval curves, meant that there was an appreciable range of fast and an even larger range of slow rate constants. Consequently there were no significant differences in rate constants between heat stress and control muscles either at baseline or following hypoxia, although there was a tendency towards faster restitution and decay of potentiation post-hypoxia in the heat stress group.

The slow components (tau 2) of the restitution and decay of potentiation curves exhibited even greater variability, if the second rate constant was greater than 5 seconds (it could be many hundreds of seconds) it was given the value of 5 seconds. Such a treatment is partially justified by the fact that substitution of a value of 5 seconds for a much greater value of fitted tau 2, increases the residual differences on the new curve fit, by less than $10 \%$. In addition the distribution of rate constants remained positively skewed after more orthodox forms of transformation. When the values for tau2 were "transformed" in this manner, the rate constants (tau 2) for the

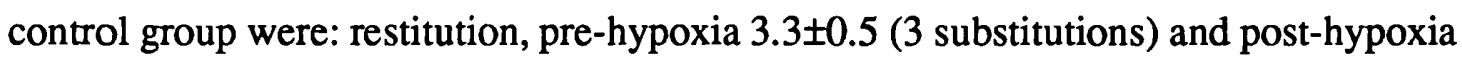
$3.9 \pm 0.45$ ( 3 substitutions) decay of potentiation, pre-hypoxia $3.2 \pm 0.5$ ( 2 substitutions) and post hypoxia 3.6 \pm 0.5 (3 substitutions) and corresponding values for the heat stress group were: $2.7 \pm 0.5$ (2 substitutions) and $2.8 \pm 0.4$ (2 substitutions), $3.0 \pm 0.3$ (no substitutions) and $3.4 \pm 0.4$ (3 substitutions). There were no significant differences between the values of tau 2 between heat stress and control groups compared by t-test on the transformed data or by the Mann-Whitney $U$ test on the positively skewed raw data.

\section{Discussion}

The results of this initial pilot study indicate that papillary muscles from heat stressed rabbits are more resistant to some, but not other components of ischaemia. Resistance is increased to superfusion with nominally calcium-free and hypoxic substrate free solutions. In contrast, there is no difference in contractile function at low $\mathrm{pH}$ between heat stress and control papillary muscles. The advantages in the force developed by heat stress muscles are not accompanied by clear differences in the force 
interval relationships between groups, implying that calcium handling on a beat to beat basis is unaltered.

\subsection{During Acidosis}

A drop in intracellular $\mathrm{pH}$ reduces contractility by altering the affinity of troponin $\mathrm{C}$ for calcium, effectively shifting the calcium-force relationship to the right (Fabiato and Fabiato 1978, Blanchard and Solaro 1984). The change in intracellular $\mathrm{pH}$ that will result from a shift in extracellular $\mathrm{pH}$ depends on the intrinsic buffering capacity of the myocyte, and its ability to extrude protons by $\mathrm{Na}^{+} / \mathrm{H}^{+}$ion exchange and by $\mathrm{HCO}_{3}{ }^{-}$influx (various mechanisms $\mathrm{Na}^{+} / \mathrm{HCO}_{3}{ }^{-}$symport, probably $\mathrm{HCO}_{3} / \mathrm{Cl}^{-}$antiport and $\mathrm{HCO}_{3}^{-}$equivalents by $\mathrm{CO}_{2}$ efflux). As a result of these mechanisms, a given drop in external $\mathrm{pH}$ produces a much smaller drop in internal $\mathrm{pH}$. In the ferret ventricular muscle for example, a change in external $\mathrm{pH}\left(\mathrm{pH}_{\mathrm{o}}\right)$ of 1 unit (down to $\mathrm{pH}_{\mathrm{o}}$ of 6.8) results in a change in internal $\mathrm{pH}\left(\mathrm{pH}_{\mathrm{i}}\right)$ of $0.085 \mathrm{pH}$ units (Blatter and McGuigan 1991). The observation that developed force was equally depressed by low $\mathrm{pH}_{\mathrm{o}}$ in both control and heat stressed groups implies that the net effectiveness of the mechanisms responsible for the control of $\mathrm{pH}_{\mathrm{i}}$ are probably not significantly altered by prior heat stress. In addition heat stress is unlikely to alter the calcium-force relationship. The alternative explanation that heat stress did alter the ability of the myocyte to control $\mathrm{pH}_{\mathrm{i}}$, but also altered the calcium force relationship in a reverse direction so the net effect was unchanged seems far less plausible.

We found that the force-interval relationship was unchanged during acidosis, interestingly other investigators examining the mechanism of mechanical alternans during acidosis have demonstrated that the process of mechanical restitution is slowed (Orchard et al 1991). However the $\mathrm{pH}_{\mathrm{o}}$ in this study of ferret papillary muscle at $30^{\circ} \mathrm{C}$ was $6.85\left(15 \% \mathrm{CO}_{2}\right)$, so that differences between models is the most likely explanation for discrepant findings.

\subsection{Following Nominally Calcium-Free Superfusion}

In the whole heart (and other organs) just a brief period of calcium-free perfusion causes profound injury with contracture and release of intracellular constituents on calcium repletion, the so called calcium paradox (Zimmerman and 
Hülsmann 1966). The aetiology of this injury is unknown (see Chapter 5 Section 4.2 for a more in-depth discussion). In small muscle specimens the injury observed following low/zero calcium exposure is less marked, more difficult to produce, and may depend on different mechanisms (Bhojani and Chapman 1990). However the end result of low calcium super/perfusion in both isolated muscle and whole heart is intracellular calcium overload, allowing similarities to be made between this form of injury and that of the oxygen paradox and ischaemia/reperfusion (Hearse et al 1978).

The results of nominally calcium-free superfusion are difficult to interpret. In the first case the degree of injury which was non-existent in heat stress muscles, was very mild in control muscles and not associated with contracture (rise in resting force). The only explanation for these findings is that a full calcium paradox certainly did not occur, and that the injury seen was the result of a more gentle sub-total paradox. The fact that the injury induced was mild is not surprising since it would have taken many minutes for the calcium in the organ bath to be washed out by the nominally calciumfree solution $\left(\left[\mathrm{Ca}^{2+}\right] \approx 6 \mu \mathrm{m}\right)$, and it is known that the paradox is absent whilst the calcium concentration remains above 50 $\mu \mathrm{m}$ (Kirkels et al 1989). This difficulty could have been resolved by more rapid superfusion or the introduction of a calcium chelating agent, however the nature of the protocol required contractile recovery in order to assess the response to high flow hypoxia.

Since the enhanced resistance to nominally calcium-free superfusion in heat stress muscles was difficult to interpret, this study was repeated and findings confirmed in the whole heart (see Chapter 5). An increased resistance to the calcium paradox suggests that prior heat stress is capable of protecting the myocardium independent of the proposed increase in anti-oxidant defenses that follows heat stress (Karamazyn et al 1990) an interpretation discussed in more detail in Chapter 5.

\subsection{Following High Flow Hypoxia without Substrate}

During hypoxia all muscles stopped contracting and developed contracture. Unfortunately contractility during hypoxia could not be examined in detail since a chart recorder only became available during the latter half of this pilot study (heat stress and control papillary muscle contractility during high flow hypoxia is discussed in detail in Chapter 4 Section 3.2). 
Immediately following high flow hypoxia papillary muscle developed force was similarly depressed and resting force similarly elevated suggesting that the degree of hypoxic injury was comparable in the two groups. After 30 minutes of reoxygenation developed force was significantly greater in the heat stress group because of the greater rate of contractile recovery in this group. It therefore seems likely that prior heat stress enhances the ability of papillary muscles to recover after hypoxia without necessarily increasing resistance to hypoxia. This situation is analogous to the debate that surrounds recovery from thermal injury in thermotolerant cell lines (see Chapter 1 Section 5.1). In this situation stress protein induction seems to both attenuate thermal injury and enhance recovery.

The force-interval analysis failed to show any significant differences in the processes of restitution and decay of potentiation between heat stress and control groups post-hypoxia. This means that it is unlikely that the enhanced recovery seen is a function of alterations in calcium handling by the sarcoplasmic reticulum. The basis for the protection observed in the heat stressed muscles is not clear and was therefore studied in greater depth in the next Chapter.

\subsection{Critique of Methods}

The major concern with the papillary muscle model is the adequacy of oxygenation. In cat papillary muscle stimulated at $1 \mathrm{~Hz}$ at $37^{\circ} \mathrm{C}$, the critical diameter at which a drop in $\mathrm{pO}_{2}$ from 650 to $550 \mathrm{mmHg}$ produced a change in developed force was $0.71 \mathrm{~mm}$. Since this is below the mean papillary muscle diameter in our preparation it is likely that the muscle cores in the experiments described above were hypoxic. Further evidence in favour of inadequate oxygenation is the fact that papillary muscle diameter did not correlate with developed force, suggesting that there was no extra contractile contribution from thick muscles. In our preliminary experiments switching superfusion to Tyrode's equilibrated with $85 \% \mathrm{O}_{2} / 5 \% \mathrm{CO}_{2} / 10 \% \mathrm{~N}_{2}$ did not appreciably alter developed force, however the interpretation of this is complicated by the fact that even with hypoxic superfusion developed force may initially paradoxically increase. In addition the papillary muscles used for the serial stress protocol were not examined in this way because of the theoretical concern that it may result in hypoxic preconditioning of a portion of the 
muscle (Shizukida et al 1992). Given that a hypoxic core existed, what influence would this have on our experimental findings? Since papillary muscle dimensions are constant the hypoxic core would remain hypoxic throughout the whole experimental protocol. Since the first assessment of contractile function was made 60 minutes after papillary muscle suspension, at a time when developed force was stable, it seems unlikely that the hypoxic core would influence any of the assessments of contractility but may contribute a constant fraction to resting force. In essence, the information derived from the analysis of the serial stresses is relevant only to a rim of viable myocardium close to the muscle surface.

Another possible drawback of this study is the fact that the degree of recovery from one stress may influence recovery from subsequent stresses. Certainly recovery of contractility was complete after acidosis and almost complete after calcium-free superfusion, making carry over artifacts unlikely. It is however possible, for example, that the viable myocardium in the control group following low calcium superfusion had been sub-lethally injured and sensitised to damage by the subsequent hypoxia. Supporting such a concept is the related observation that myocardium sub-lethally damaged by ischaemia becomes sensitised to the calcium paradox (Kirkels et al 1989). The enhanced recovery seen post-hypoxia in heat stressed muscle may therefore have been a carry over effect of differences in susceptibility to a mild calcium paradox. The issue of whether heat stress is capable of attenuating hypoxic myocardial injury or attenuating the calcium paradox is examined in more detail in later Chapters.

\subsection{Conclusions}

The results of this pilot study, despite a number of drawbacks, suggests that papillary muscles from heat stressed rabbits are resistant to injury following nominally calcium-free and low-oxygen substrate-free superfusion. The basis for this protection is unknown, but is associated with stress protein induction within the papillary muscle and occurs in the absence of changes in the force interval relationship. There were no similar differences in contractility between groups during superfusion at low $\mathrm{pH}$. These findings prompted us to further investigate the influence of prior heat stress on myocardial resistance to hypoxic and nominally calcium-free perfusion. 


\section{Chapter 4}

\section{Results: Papillary Muscles, Substrate Preference and Stress Protein Content}

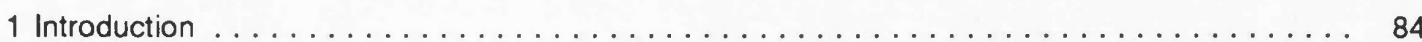

2 Specific Methods . . . . . . . . . . . . . . . . . . . . . . 86

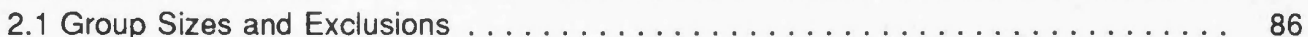

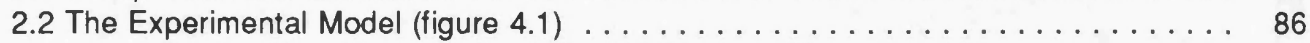

2.2.1 Papillary Muscle Stimulation Protocol and Data Acquisition . . . . . . . . 88

2.2.2 Timing of Changes in Papillary Muscle Superfusion (figure 4.1) . . . . . . 89

2.2.3 Heat Stress Protein Assessment . . . . . . . . . . . . . . . . . 89

3 Results . . . . . . . . . . . . . . . . . . . . . . . . . . . 89

3.1 Baseline Characteristics of Papillary Muscles (table 4.1 and figure 4.3) . . . . . 89

3.2 Contractility During High Flow Hypoxia without Substrate (figure 4.4) . . . . . . . 92

3.3 Contractility Following High Flow Hypoxia without Substrate (figures 4.5, 4.6 and

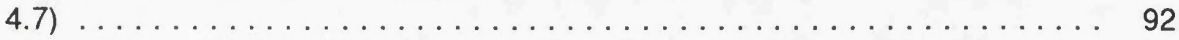

3.4 The Force-Interval Relationship Between Heat Stress and Sham Heat Stress Groups (figures 4.8 and 4.9 ) $\ldots \ldots \ldots \ldots \ldots \ldots \ldots \ldots \ldots \ldots \ldots$

3.5 The Effect of Hypoxia and Reoxygenation on the Force-Interval Relationship (figures 4.10 and 4.11 ) ........................ 99

3.6 The 72kDa Heat Stress Protein and Contractile Recovery (figures 4.12 and 4.13) . . 99

3.7 The $60 \mathrm{kDa}$ Heat Stress Protein is not Associated with Contractile Recovery (figures

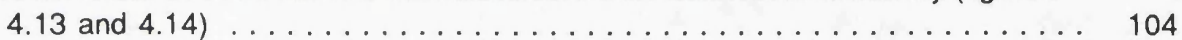

4 Discussion ........................................ 104

4.1 Papillary Muscle Contractility and Substrate Preference . . . . . . . . . . . 104

4.2 Force-Interval Relationships . . . . . . . . . . . . . . . . . . . . . . . . 109

4.3 Stress Proteins and Myocardial Resistance .................. 109

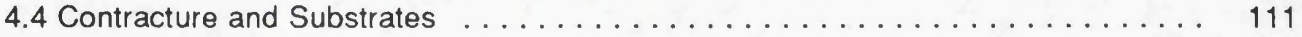

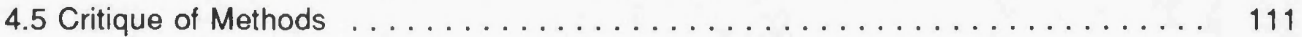

4.5 Conclusions .............................. 112

\section{Introduction}

Studies using heat to elevate the stress protein content of myocardial tissue at the onset of ischaemia have demonstrated both, enhanced recovery of contractile function, and resistance to infarction following ischaemia (Currie et al 1988, Karmazyn et al 1990, Donnelly 1992). However it remains uncertain if the observed protection is secondary to stress protein induction or is a non-specific effect of whole body temperature elevation.

In previous studies no attempt has been made to correlate the myocardial stress protein content within individual hearts, to the degree of observed protection. The advantage of such a comparison is that it would help define the role of stress proteins 
in the myocardial protection observed following heat stress. The main difficulty with such an approach is that the stress protein content of the tissue needs to be estimated prior to the onset of a subsequent stress to ensure that the pattern of induction is not perturbed by the experimental conditions (Knowlton et al 1991a, Knowlton et al 1991b). By using an isolated papillary muscle model we were able to obtain several similar myocardial specimens from each heart. Whilst one such specimen was used for the assessment of contractile function, the remaining specimens could be used for stress protein analysis.

In the rabbit, preserved mitochondrial activity, increased levels of high energy phosphates, diminished enzyme release, as well as enhanced functional recovery have been reported following ischaemia/reperfusion of heat stressed hearts (Karamazyn et al 1990, Currie et al 1993). Similarly in the rat, the enhanced functional recovery that follows heat stress is associated with preservation of mitochondrial ultrastructure (Currie et al 1988). Heat stress proteins are known to play an important role in mitochondrial protein metabolism and energy production (see Chapter 1 Section 4), and are also known to enhance the activity of glycolytic enzymes in yeasts (Piper et al 1986, Iida and Yahara 1985). However, the relative contribution of mitochondrial and glycolytic pathways to the post-ischaemic recovery seen with heat stress is not known. In addition, studies in non-cardiac tissue suggest that heat stress proteins are able to influence the activity of diverse protein kinases (Rose et al 1989, Chapter 1 Section 4.2.2) as well as bind calmodulin (Stevenson and Calderwood 1990) and thereby may effect the uptake and release of calcium from the sarcoplasmic reticulum. For these reasons changes in myocardial calcium handling may also contribute to the improved functional recovery that follows ischaemia in heat stress hearts.

In this chapter we have attempted to address these issues by comparing the contractile performance of heat stressed and non-heat stressed cardiac muscle using substrates with differing mitochondrial and glycolytic availabilities, whilst assessing calcium handling within the sarcoplasmic reticulum by means of force interval parameters (Seed and Walker 1988). 


\section{Specific Methods}

\subsection{Group Sizes and Exclusions}

Forty-nine papillary muscles from 49 rabbits ( 25 heat stressed and 24 controls) were used in this study and allocated to 4 groups. Group 1 , heat stress pyruvate $(n=12)$, in which rabbits were heat stressed and the papillary muscle superfused with pyruvate as substrate. Group 2, heat stress glucose $(n=13)$, in which rabbits were heat stressed and papillary muscles superfused with glucose as substrate. Group 3, control pyruvate $(n=12)$, in which papillary muscles from control rabbits were superfused with pyruvate as substrate. Group 4, control glucose $(n=12)$, rabbits were controls and papillary muscles were superfused with glucose as substrate. There was one exclusion from the heat stress glucose group as a result of spontaneous force development following high flow hypoxia. Each group therefore contained 12 papillary muscles. Although three papillary muscles, one from each of the heat stressed pyruvate, control glucose and control pyruvate groups suddenly failed to contract, secondary to a rise in pacing threshold during the late hypoxic period, they were still included in the final analysis.

\subsection{The Experimental Model (figure 4.1)}

Male New Zealand White rabbits, weighing 1.8-2.5 kg, underwent sham $(n=24)$ or true heat stress $(n=25)$ as described previously (Chapter 2 Section 1.1). The following day hearts were removed and papillary muscles harvested and suspended as previously described (Chapter 2 Section 3). The sister papillary muscles remaining after the chosen muscle had been suspended in the organ bath were frozen in liquid nitrogen for stress protein analysis.

Papillary muscle dissection and perfusion was performed in oxygenated modified Krebs solution with the following composition; $\mathrm{NaCl} 118.5 \mathrm{mM}, \mathrm{NaHCO}_{3} 24.8 \mathrm{mM}$, $\mathrm{KCl} 4.8 \mathrm{mM}, \mathrm{KH}_{2} \mathrm{PO}_{4} 1.2 \mathrm{mM}, \mathrm{CaCl}_{2} 2.0 \mathrm{mM}, \mathrm{MgSO}_{4} 1.4 \mathrm{mM}$. To this was added either glucose $15 \mathrm{mM}$ or sodium pyruvate $10 \mathrm{mM}$, (sodium pyruvate from Sigma Chemicals, Missouri, USA; all other reagents ANALAR grade from BDH Chemicals, Poole, England). During hypoxia when the perfusate contained no substrate, $10 \mathrm{mM}$ Choline Chloride was substituted for either glucose or pyruvate to maintain the osmolarity. Solutions were continuously bubbled with either $95 \%$ oxygen plus $5 \%$ carbon dioxide or $95 \%$ nitrogen plus $5 \%$ carbon dioxide, to provide a $\mathrm{pH}$ in the organ 


\section{Experimental Protocol for Glucose and Pyruvate Papillary Muscle Superfusion}

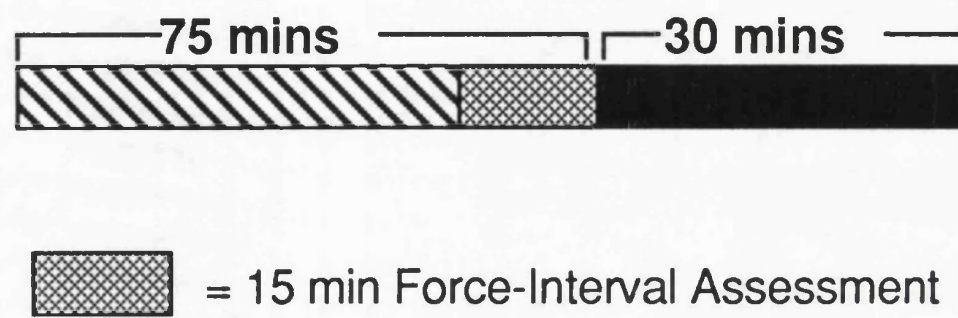

90 mins

NTWV

= Glucose or Pyruvate as Substrate

Period of High Flow Hypoxia

Without Substrate $\left(\mathrm{pO}_{2}<6.0 \mathrm{kPa}\right)$

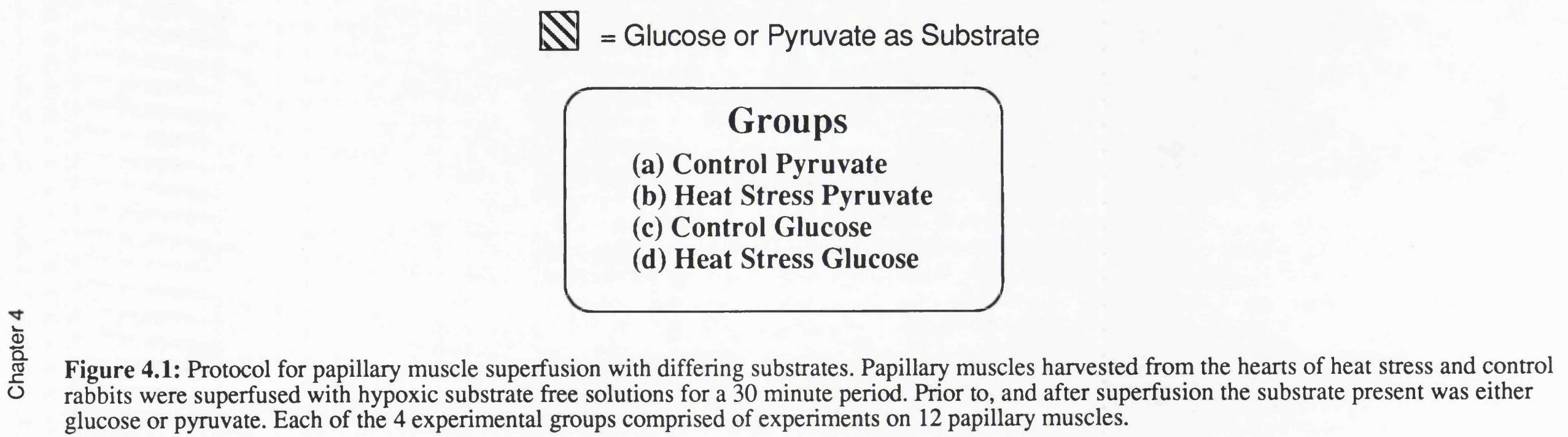

glucose or pyruvate. Each of the 4 experimental groups comprised of experiments on 12 papillary muscles. 
bath of 7.35-7.45 during the entire procedure. The corresponding oxygen tensions for these gas mixtures in the bath effluent were $50.5-60.2 \mathrm{kPa}$ and less than $6.0 \mathrm{kPa}$ respectively.

\subsubsection{Papillary Muscle Stimulation Protocol and Data Acquisition}

Thirty minutes after the papillary muscles were fixed in the organ bath they were stretched to a length developing $90 \%$ of maximal active force and the pacing threshold determined. This was followed by a further 30 minute stabilization period. Throughout this time the stimulation rate was maintained at $1 \mathrm{~Hz}$.

Papillary muscle stimulation and data acquisition in response to extra-systolic beats were as previously described (see Chapter 2 Section 3.2.6).

In Chapter 3 each of the force-interval curves from each individual experiment were fitted to biexponential expressions and means and standard errors obtained for the population of rate constants derived for each experimental group. The non-linearity of the iterative fitting procedure resulted in large standard errors and a failure of normalisation even after logarithmic transformation making it difficult to exclude a type II error as the cause for our negative findings. These difficulties prompted us to develop a different analytical approach for this study.

The force developed by the extrasystolic and post extra-systolic beats was normalised by dividing by the force developed by the preceding steady state beat. Normalised beat force was recorded at each extra-systolic interval, these measurements were then collated for each of the groups to derive force-interval curves comprising of data from 12 individual experiments. For each of the four experimental groups, at each extrasystolic interval examined, a mean and standard error value was derived based on normalised force from 12 experiments. Normalised extra-systolic and post extra-extrasystolic beats were then plotted against extra-systolic interval (appropriate programs are described in Chapter 2 Section 3.2.7 and listed in Appendix 2) allowing a visual comparison of chelated force-interval data. 


\subsubsection{Timing of Changes in Papillary Muscle Superfusion (figure 4.1)}

Following the 75 minute stabilization period, ( 60 minutes plus 15 minute forceinterval assessment), muscles were rendered hypoxic and deprived of metabolic substrate. Organ bath flow during this 30 minute period was maintained with Krebs buffer bubbled with $95 \%$ nitrogen and 5\% carbon dioxide in which the glucose or pyruvate had been replaced with choline chloride. This 30 minute period of hypoxia was followed by re-introduction of oxygen and substrate for a further 90 minutes. The substrate present during baseline stabilisation was either pyruvate or glucose, the same substrate was returned following reoxygenation. The period of hypoxia without substrate resulted in contracture and complete loss of developed force in all groups. Muscle activity during high flow hypoxia was measured, using a digitizing tablet (Summa Graphics, Summa Sketch II, Connecticut USA), by tracing the area of developed force for the 30 minutes hypoxic period recorded at slow paper speed (5mm/min) (program MMAREA.BAS see Appendix 2 Section 5). Muscle force during hypoxia was expressed as a percentage of force developed during the stabilisation period (see figure 4.2).

Following 90 minutes of re-oxygenation the papillary muscle relaxed (unloaded) length and diameter were measured using a stage mounted graticule (Gradicules Ltd, Kent, England), muscles were then blotted dry and weighed.

\subsubsection{Heat Stress Protein Assessment}

Right ventricular papillary muscles, remaining after the chosen sister papillary muscle had been successfully suspended in the organ bath, were frozen in liquid nitrogen. At a later date the stress protein content of these muscles was measured as previously described (see Chapter 2 Section 2).

\section{Results}

\subsection{Baseline Characteristics of Papillary Muscles (table 4.1 and figure} 4.3)

The baseline characteristics of the papillary muscles prior to high flow hypoxia without substrate, were similar in all groups (table 4.1). Prior heat stress or choice of substrate did not influence the resting or developed force, the maximum rate of rise 


\section{The Assessment of Papillary Muscle Activity During Hypoxia}

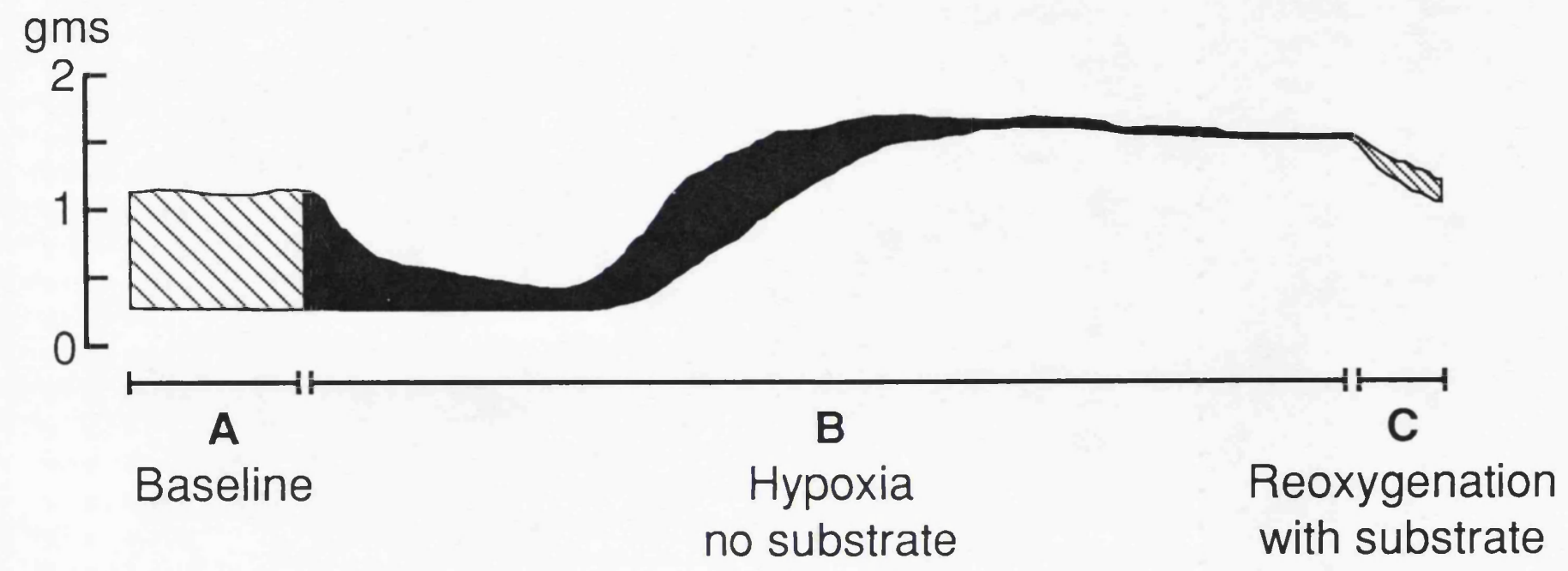

Figure 4.2: The method for assessing papillary muscle contractility during hypoxia. The illustration above is a scan of the paper trace recorded at slow speed during superfusion with hypoxic substrate free buffer. The trace illustrates the increase in developed force that occurs as the resting force increases. The time interval "A" is 5 minutes and the interval "B" is 30 minutes. The area above "A" ( $\mathrm{A}$ ) and the area above "B" (JB) were planimetered using a bit pad and the areas calculated using the program MMAREA.BAS (see Appendix 2 Section 5). A normalised index of papillary muscle activity $\left(\int \mathrm{act}\right)$ during hypoxia was derived by the following simple formula: $\left.\int \mathrm{act}=\int \mathrm{B} /(6 \mathrm{x}] \mathrm{A}\right)$. This calculation was performed for each of the 48 individual experiments comprising this study. The mean values of Jact with their respective SEM's were used to draw figure 4.4A. 


\section{Papillary Muscle Characteristics}

$\bar{a}$

\begin{tabular}{|c|c|c|c|c|c|}
\hline & Control Pyruvate & Heat Stress Pyruvate & Control Glucose & Heat Stress Glucose & ANOVA \\
\hline Unloaded Diameter (mm) & $0.87 \pm 0.04$ & $0.87 \pm 0.05$ & $0.89 \pm 0.06$ & $1.00 \pm 0.07$ & $p=N S$ \\
\hline Muscle Length (mm) & $3.67 \pm 0.10$ & $3.93 \pm 0.13$ & $3.73 \pm 0.19$ & $3.53 \pm 0.19$ & $p=N S$ \\
\hline Muscle Wet Weight (g) & $3.93 \pm 0.27$ & $4.01 \pm 0.25$ & $4.38 \pm 0.76$ & $4.36 \pm 0.73$ & $p=N S$ \\
\hline Developed Force (g) & $0.74 \pm 0.09$ & $0.64 \pm 0.09$ & $0.64 \pm 0.10$ & $0.79 \pm 0.11$ & $p=N S$ \\
\hline Resting Force (g) & $0.14 \pm 0.02$ & $0.18 \pm 0.03$ & $0.10 \pm 0.04$ & $0.17 \pm 0.04$ & $p=N S$ \\
\hline Time to Peak Force (mS) & $106.3 \pm 2.6$ & $94.4 \pm 4.4$ & $103.9 \pm 4.4$ & $97.8 \pm 4.1$ & $p=N S$ \\
\hline Time to $90 \%$ Relaxation $(\mathrm{mS})$ & $193.4 \pm 5.8$ & $183.2 \pm 9.5$ & $186.2 \pm 6.1$ & $174.8 \pm 6.6$ & $p=N S$ \\
\hline Max Rate of Contraction $(\mathrm{g} / \mathrm{s})$ & $13.3 \pm 1.6$ & $13.1 \pm 1.6$ & $12.6 \pm 2.0$ & $15.4 \pm 2.2$ & $p=N S$ \\
\hline Max Rate of Relaxation (g/s) & $11.1 \pm 1.4$ & $10.5 \pm 1.2$ & $11.8 \pm 1.7$ & $15.2 \pm 2.0$ & $p=N S$ \\
\hline
\end{tabular}

\section{TABLE 4.1:}

Unloaded muscle length and diameter were determined at the end of the experimental protocol. Values represent means \pm standard error. $n=12$ for each group. 
or fall of force, the time to peak force or the time to $90 \%$ relaxation.

Papillary muscle developed force measured at the end of the stabilisation period was not significantly related to papillary muscle diameter (see figure 4.3). Since force was not greatest with the thickest muscles there was no advantage to expressing contractility in terms of developed tension.

\subsection{Contractility During High Flow Hypoxia without Substrate (figure}

\section{4)}

Individual papillary muscle responses to high flow hypoxia were variable with some muscles increasing their developed force above that of baseline during the early hypoxic period. Developed force fell to zero in all muscles at the end of the hypoxic period. During hypoxia developed force appeared better sustained in the heat stressed papillary muscles. The total developed force during hypoxia as a percentage of developed force over the same time period of oxygenation revealed that with either pyruvate or glucose as substrate, the heat stress muscles tended to developed more force than their respective controls (heat stress pyruvate $28.8 \pm 3.8 \%$ v control pyruvate $17.8 \pm 2.2 \% \mathrm{p}=\mathrm{ns}$ and heat stress glucose $42.8 \pm 4.4 \% \mathrm{v}$ control glucose 27.7 $\pm 4.17 \mathrm{p}<0.05$ ) see figure $4.4 \mathrm{~A}$.

In addition the presence of glucose prior to high flow hypoxia was associated with a marked delay in both the time to onset of initial contracture (see figure 4.4B) and the time taken for the level of contracture to reach $25 \%$ of developed force (figure 4.4C). In contrast to the effect of substrate, there was no effect on time to contracture between heat stress and control groups with the same substrate (figure 4.4B and C). Interestingly, although marked differences existed for development of contracture (resting force) between substrate groups, this was not reflected by similar differences of integrated developed force (comparing figure $4.4 \mathrm{~A}$ with $4.4 \mathrm{~B}$ or $4.4 \mathrm{C}$ )

\subsection{Contractility Following High Flow Hypoxia without Substrate} (figures 4.5, 4.6 and 4.7)

Figure 4.5A shows that following re-oxygenation and re-introduction of substrate the developed force was similar in all groups, however by 60 minutes of 


\section{Papillary Muscle Size and Developed Force}

ฒุ
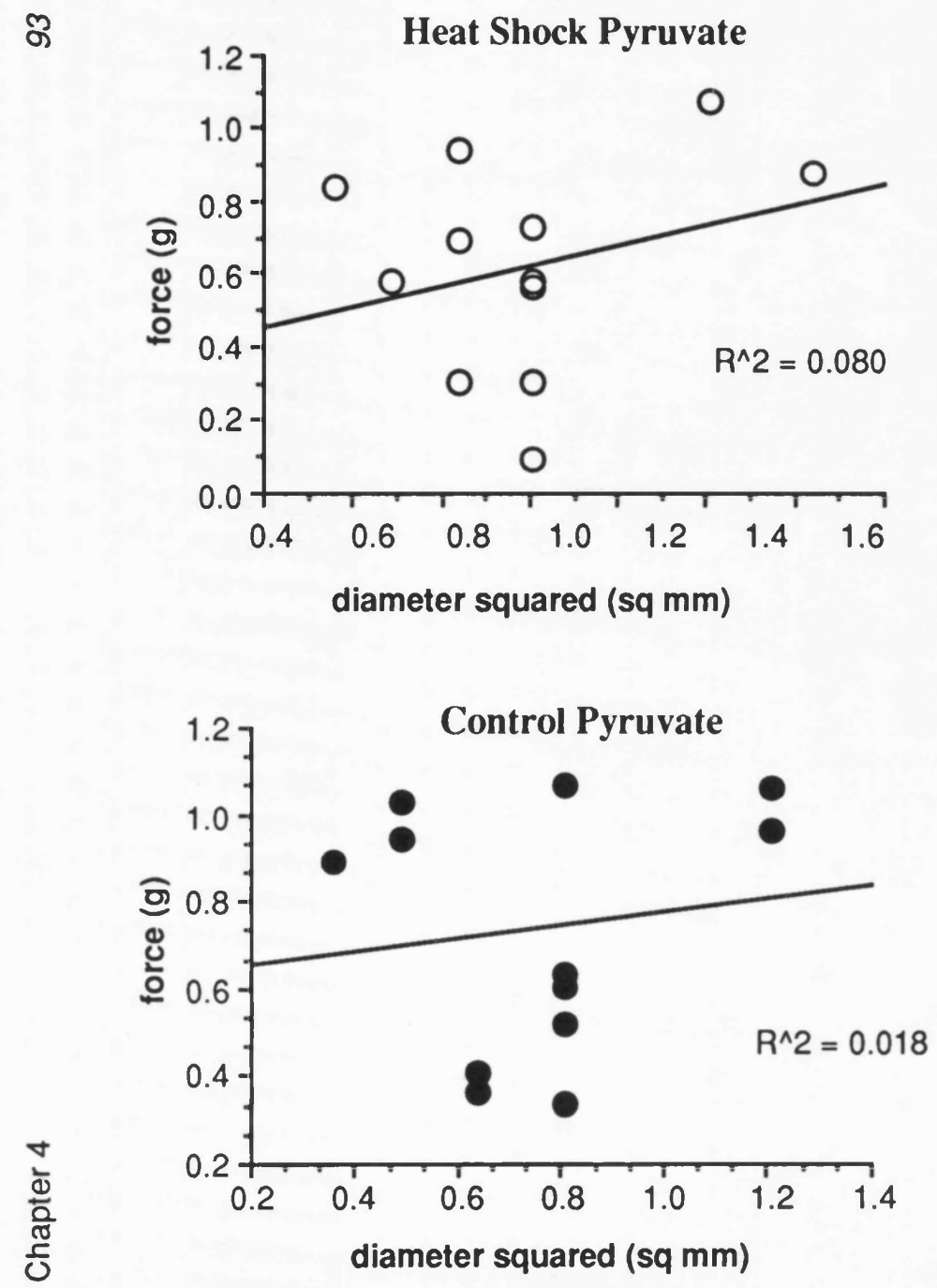

Heat Shock Glucose
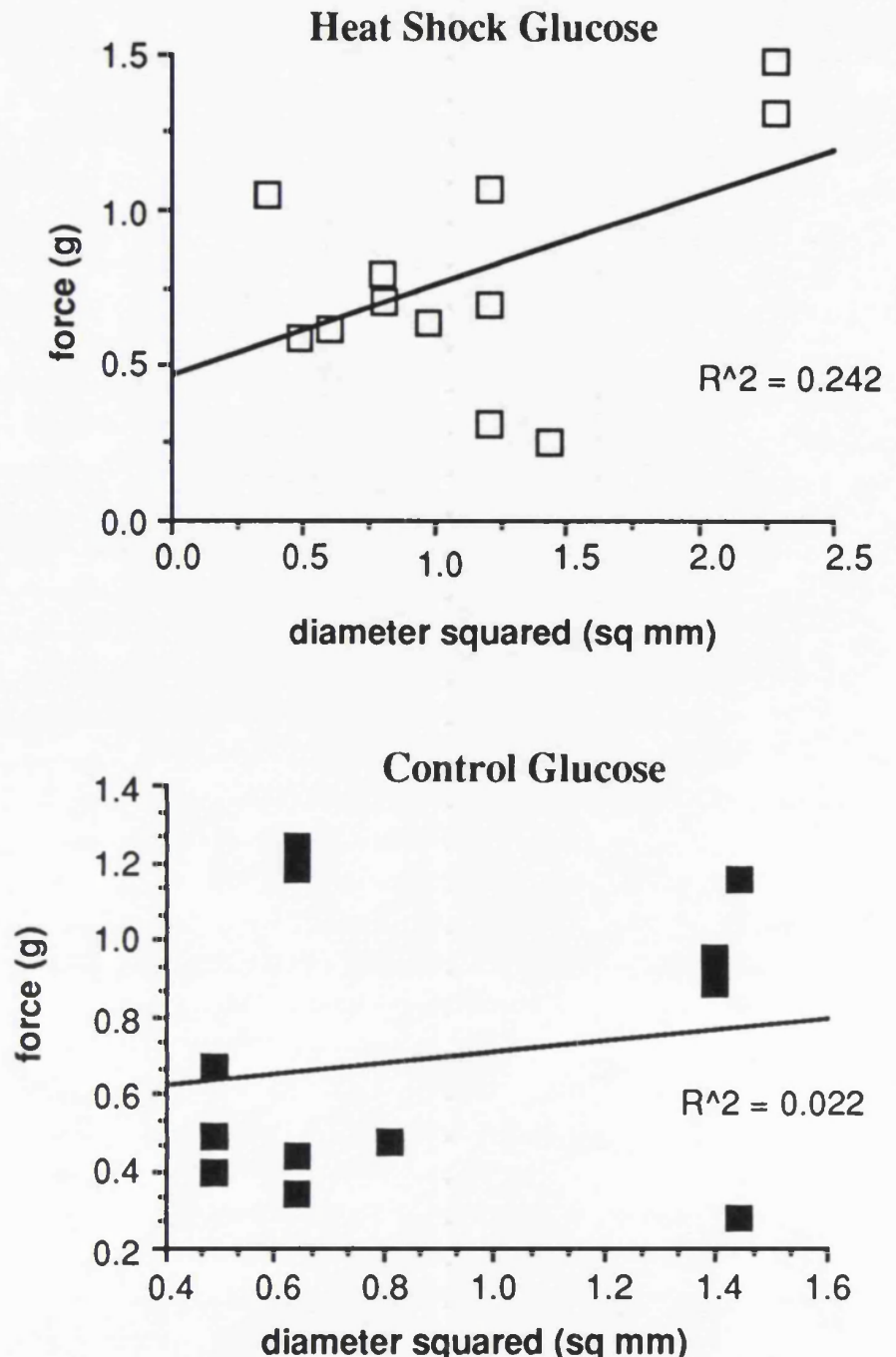

Figure 4.3: The relationship between papillary muscle crossectional area (estimated by diameter squared) and developed force. For each experimental group coefficient of determination was calculated by the Spearman Rank Correlation method. $p=N S$, $n=12$ for each group. 


\section{Papillary Muscle Contractility During Hypoxia}

C

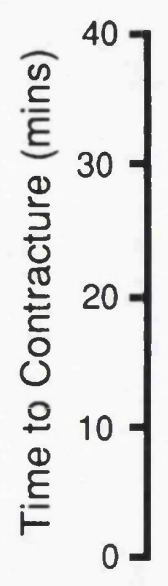

Time for Contracture to Reach $25 \%$ Developed Force

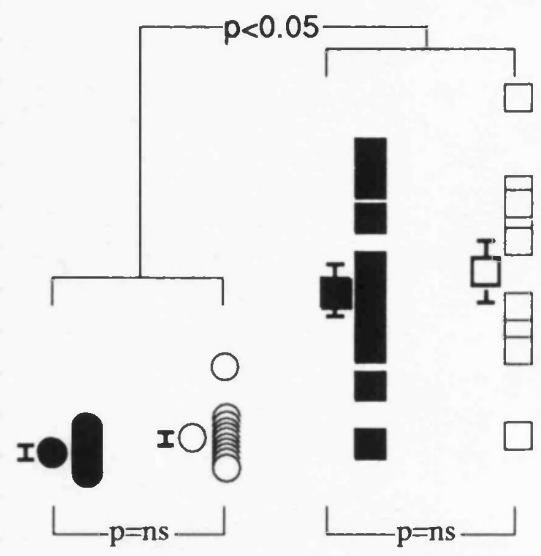

B

Time to Initial Onset of Contracture

Figure 4.4: Description of papillary muscle contractility during hypoxia. Figure A shows the area of the developed force envelope during hypoxia expressed as a percentage of developed force over a similar time period during stabalisation. Figures $\mathrm{B}$ and $\mathrm{C}$ show the time to onset of initial contracture and time for contracture to reach $25 \%$ of force developed at baseline, respectively. Comparisons between groups was by ANOVA with a post-hoc FPLSD, $n=12$ for each group. Bars represent \pm SEM. 


\section{Developed and Resting Force}

A

ॐ

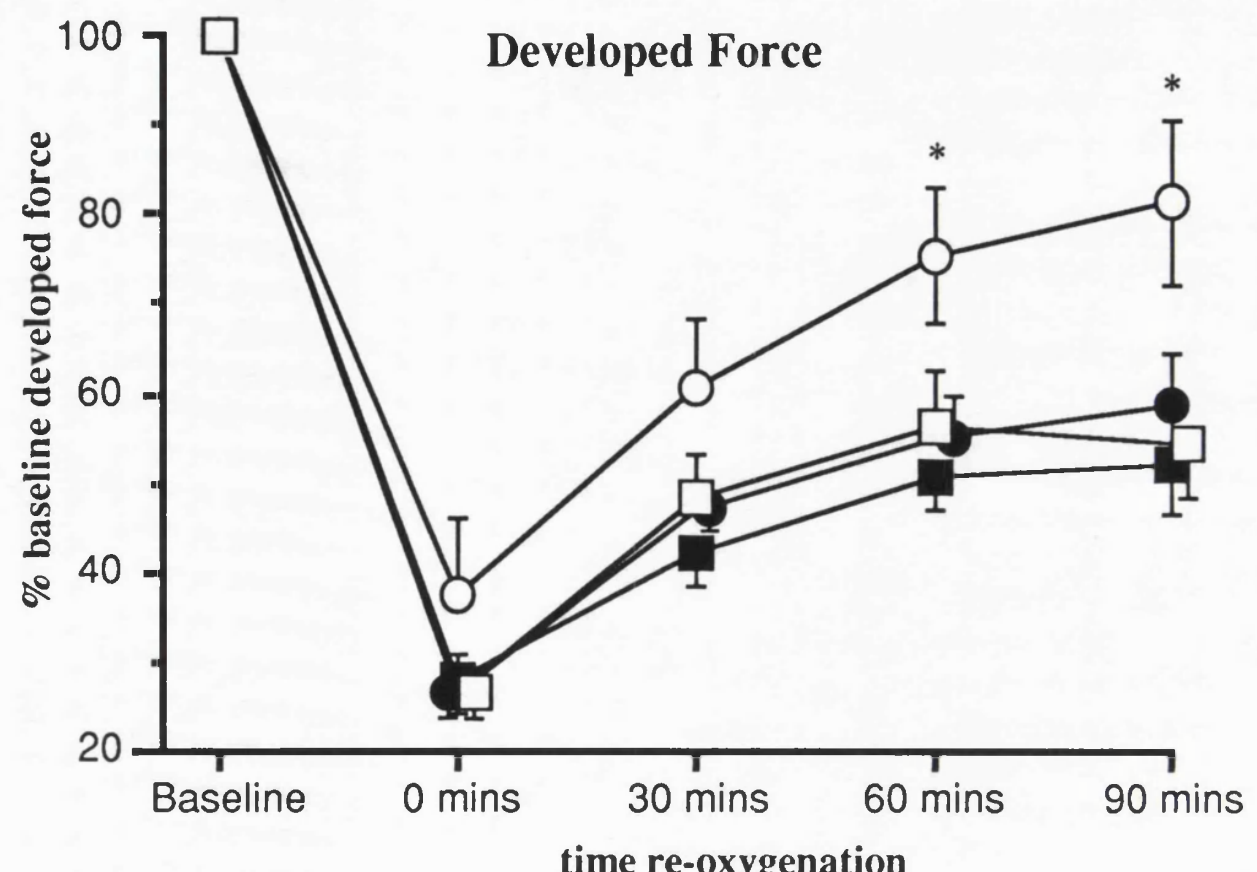

B

$$
\begin{aligned}
& \longrightarrow \text { - Control Pyruvate } \\
& \longrightarrow \text { - Heat Stress Pyruvate } \\
& \longrightarrow-\text { Control Glucose } \\
& -\square-\text { Heat Stress Glucose }
\end{aligned}
$$

$\checkmark \quad$ Figure 4.5: Developed force and resting force for papillary muscles in each of the four groups. Graph A: The developed force of each muscle is

¿ expressed as a percentage of developed force at baseline (pre-hypoxia). Note the time on the $x$-axis denotes the start of a force-interval assessment, the force recorded being the mean value for steady state beats over a 14 minute period. Graph B: Papillary muscle resting force at baseline is subtracted from $\mathcal{5}$ resting force at each timepoint during re-oxygenation. Comparisons between groups was by ANOVA. ${ }^{*} \mathrm{p} \leq 0.05$ heat stress pyruvate $v$ all other groups (FPLSD). $\mathrm{n}=12$ for each group. Bars represent $t$ SEM. 
reoxygenation the developed force appeared greatest in the heat stress group when pyruvate was used as substrate $(p<0.05$ heat stress pyruvate $v$ all other groups by ANOVA). This difference was maintained through to the end of the re-oxygenation period.

Despite a delay in the times to contracture during high flow hypoxia in muscles superfused with glucose the degree of contracture at the end of hypoxia and during early reoxygenation was similar in all groups (see figure 4.5B). During reoxygenation resting force (contracture) returned towards baseline in all groups with no significant difference between groups, however at every timepoint there is a trend towards less contracture in the heat stressed groups over their respective controls.

Figure 4.6A demonstrates that the better preservation of developed force in the heat stress pyruvate group is also associated with a significantly greater time to peak force in this group, suggesting a more complete return to the shape of the pre-hypoxia force envelope in this group. However in contrast there are no clear differences in time to $90 \%$ relaxation (figure $4.6 \mathrm{~B}$ ). This suggests that the rate of contraction maybe similar between groups whilst the rate of relaxation may not. A suggestion that is confirmed in figures $4.7 \mathrm{~A}$ and $4.7 \mathrm{~B}$, the inference being that the heat stressed muscles superfused with pyruvate have a more minor disturbance of post-hypoxic relaxation.

In summary, the results indicate that the protection against hypoxia and reoxygenation seen following heat stress, is most marked when pyruvate is used as substrate both before and following hypoxia. The parameters that are best preserved in this group are developed force and the rate of relaxation of force.

\subsection{The Force-Interval Relationship Between Heat Stress and Sham}

\section{Heat Stress Groups (figures 4.8 and 4.9)}

The entire family of force-interval relationships obtained during an experiment and plotted by MMAQUIS.BAS is illustrated in Appendix 2 Section 1.1.3. These traces show the variation in developed force during the early reoxygenation period.

When the force-interval relationships between control and heat stressed groups are examined at baseline and at 90 minutes of re-oxygenation (figures 4.8 and 4.9) there is little separation of data points and the relationships appear similar. 


\section{Timings of Papillary Muscle Contraction Envelope}

ลे
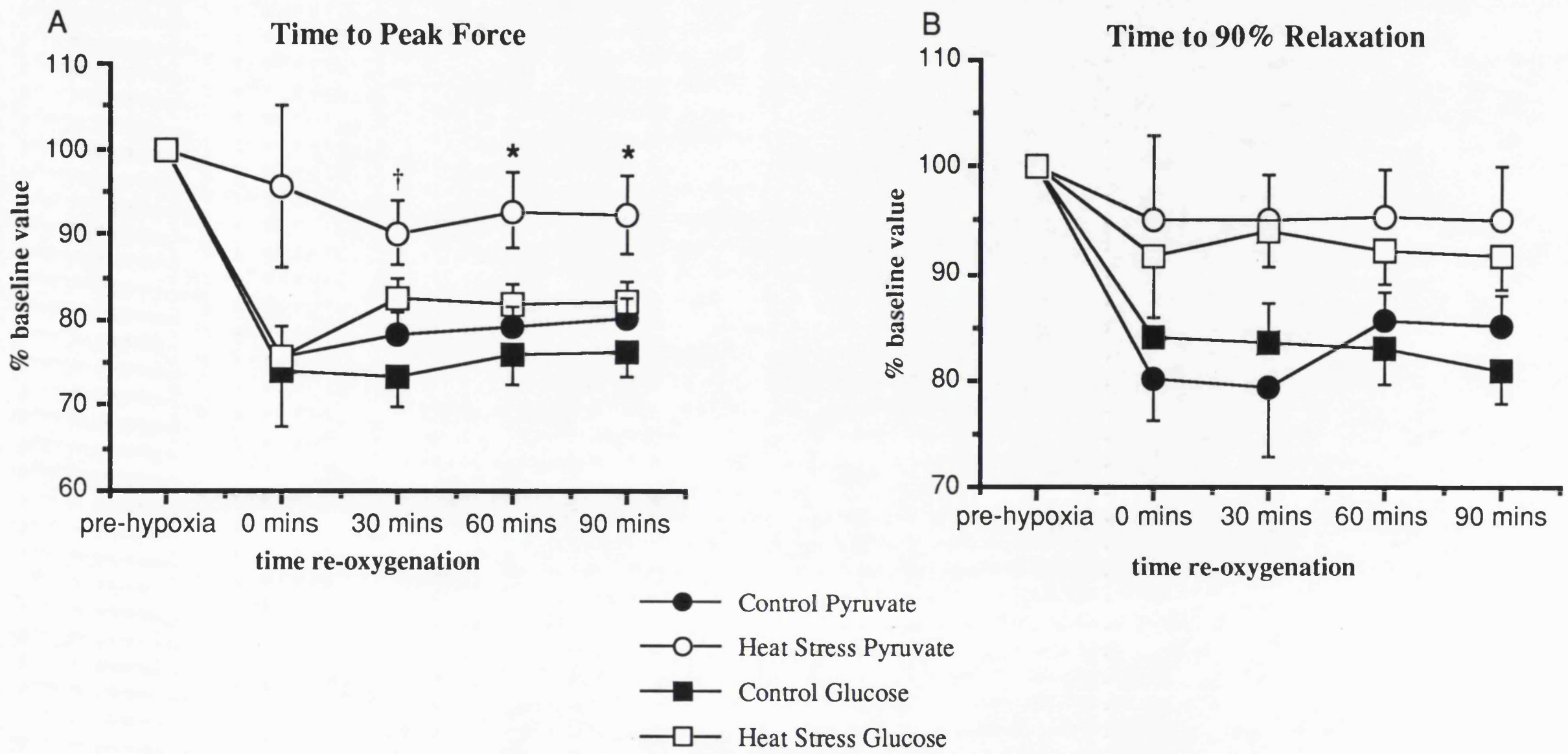

Figure 4.6: Time to peak force and $90 \%$ relaxation for papillary muscles in each of the four groups. Timings for each muscle are expressed as a percentage of the timing at baseline (pre-hypoxia). Comparisons between groups was by ANOVA. ${ }^{*} \mathrm{p} \leq 0.05 \mathrm{v}$ all other groups, $\dagger \mathrm{p}<0.05 \mathrm{v}$ control glucose and control pyruvate (FPLSD), $\mathrm{n}=12$ for each group. Bars represent + SEM. 


\section{Maximum and Minimum Rates of Papillary Muscle Contraction}

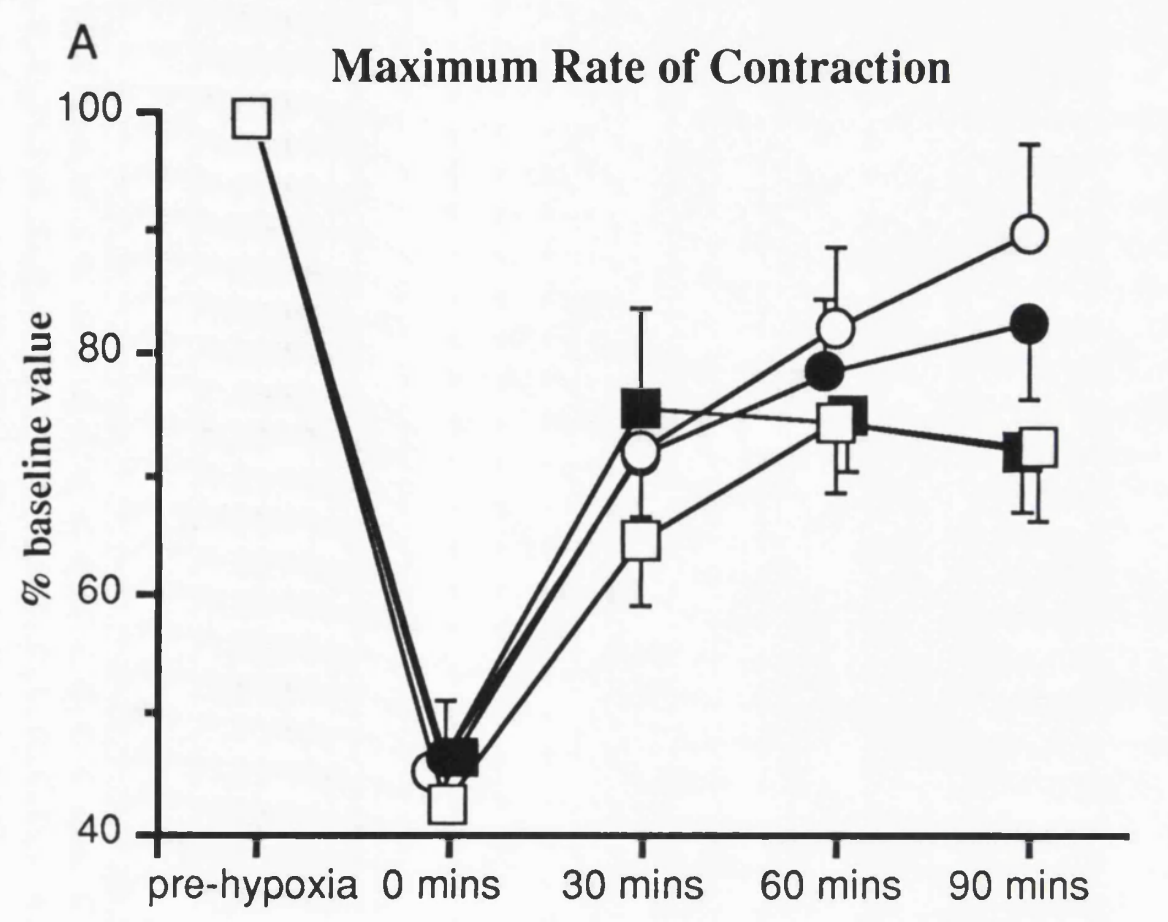

time re-oxygenation
B

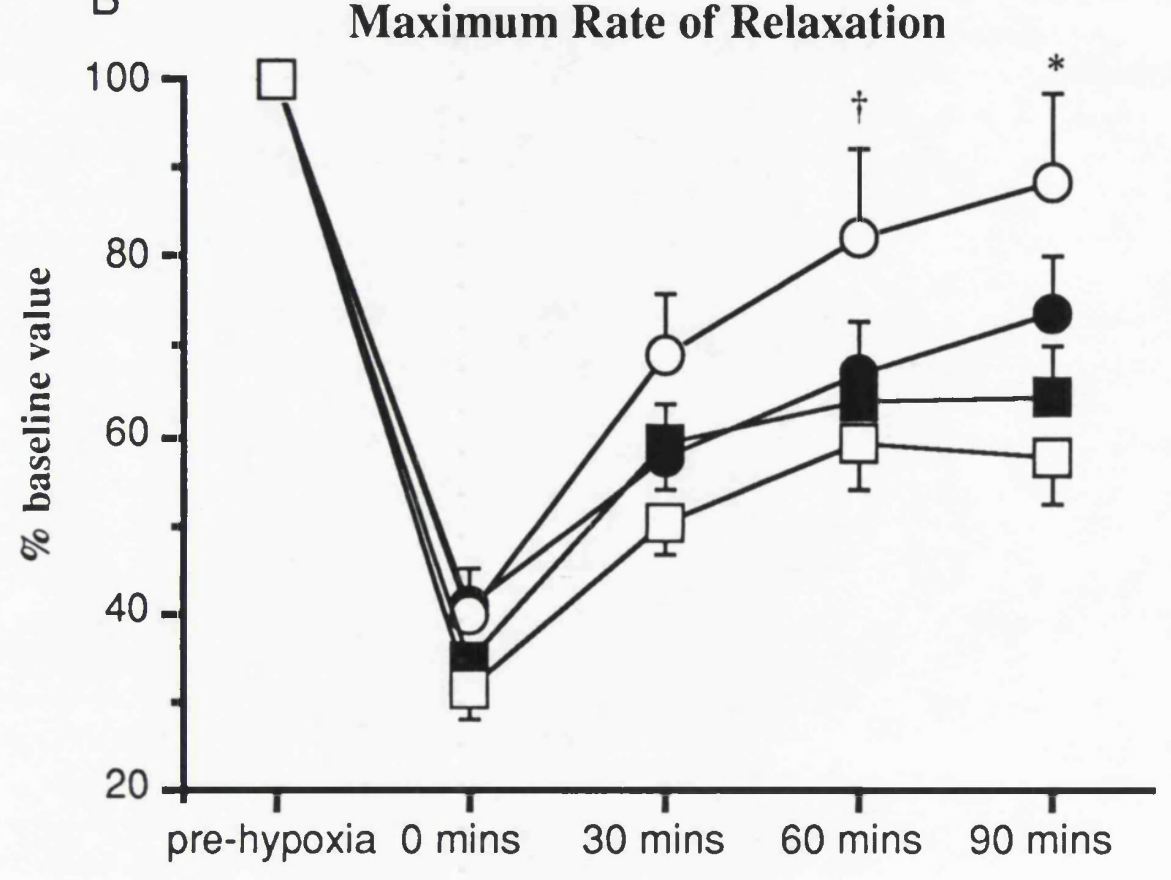

time re-oxygenation

$$
\begin{aligned}
& -1 \text { - Control Pyruvate } \\
& - \text { - Heat Stress Pyruvate } \\
& -\square-\text { Control Glucose } \\
& -\square \text { - Heat Stress Glucose }
\end{aligned}
$$

Figure 4.7: Maximum rates of contraction and relaxation for papillary muscles in each of the four groups. Rates for each muscle are expressed as a percentage of the rate at baseline (pre-hypoxia). Comparisons between groups was by ANOVA. ${ }^{*} \mathrm{p} \leq 0.05$ for heat stress pyruvate $v$ control and heat stress glucose, $\uparrow \mathrm{p} \leq 0.05$ heat stress pyruvate $\mathrm{v}$ control pyruvate and control glucose (FPLSD), $\mathrm{n}=12 \mathrm{for}$ each group. Bars represent + SEM. 
The curves shown are each derived from 12 experiments. At baseline there is a significantly greater potentiation in the heat stress pyruvate group over control at the shortest extra-systolic interval of $200 \mathrm{mS}$, however the separation of curves at this single point probably has no biological significance. Since the heat stress pyruvate group has the greatest contractile recovery, the most relevant comparison is between heat stress and control groups superfused with pyruvate at 90 minutes of reoxygenation. At this timepoint (figure 4.9) both restitution and decay of potentiation are similar between these groups making it improbable that changes in the cycling of calcium within the myocyte, in particular calcium handling of the sarcoplasmic reticulum are responsible for the enhanced contractile recovery in the heat stress pyruvate group.

\subsection{The Effect of Hypoxia and Reoxygenation on the Force-Interval} Relationship (figures 4.10 and 4.11)

The effect of hypoxia and reoxygenation on force-interval parameters in control groups is shown in figure 4.10, and in heat stress groups in figure 4.11. In contrast to whole body heat stress, hypoxia and reoxygenation does seem to have a significant effect on either the process of restitution or decay of potentiation. In each of the four experimental groups the rate of restitution and the rate of decay of potentiation is increased following hypoxia and reoxygenation a finding in keeping with previous observations (see Seed and Walker 1988 for review).

\subsection{The 72kDa Heat Stress Protein and Contractile Recovery (figures}

\subsection{2 and 4.13)}

Basal expression of the $72 \mathrm{kD}$ protein was seen in all control specimens examined (see figure 4.12). However, prior heat stress resulted in a variable increase in stress protein content, when blots were examined densitometrically there was an approximate four-fold increase in the content of the $72 \mathrm{kD}$ protein 24 hours following heat stress (heat stress $6.2 \pm 0.7 \mathrm{v}$ control $1.7 \pm 0.3$ units $p=0.003$ see figure $4.13 \mathrm{~A}$ )).

When the stress protein content of papillary muscle harvested from heat stressed hearts was compared to the contractile performance of sister papillary muscles 


\section{Baseline Force-Interval Relationships}
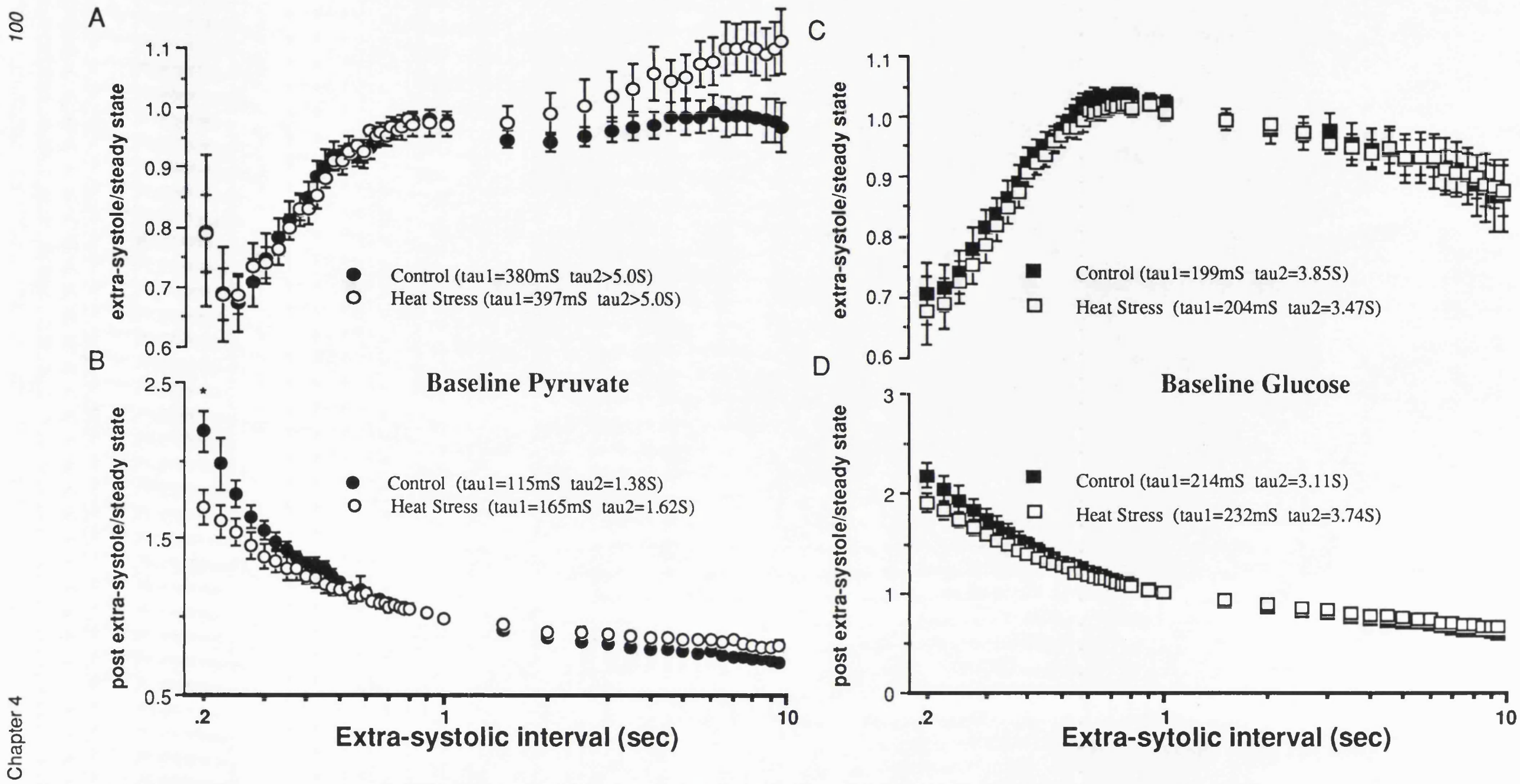

Figure 4.8: Force-interval relationships at baseline prior to hypoxia, with glucose and pyruvate as substrates. No consistent differences exist between heat stress and control groups for either extra-systolic or post-extrasystolic beats with either glucose or pyruvate as substrates. $\mathrm{n}=12 \mathrm{for}$ each group. * $\leq 0.05$ by unpaired $t$-test. Bars represent \pm SEM. 


\section{Force-Interval Relationships at 90 mins Re-oxygenation}
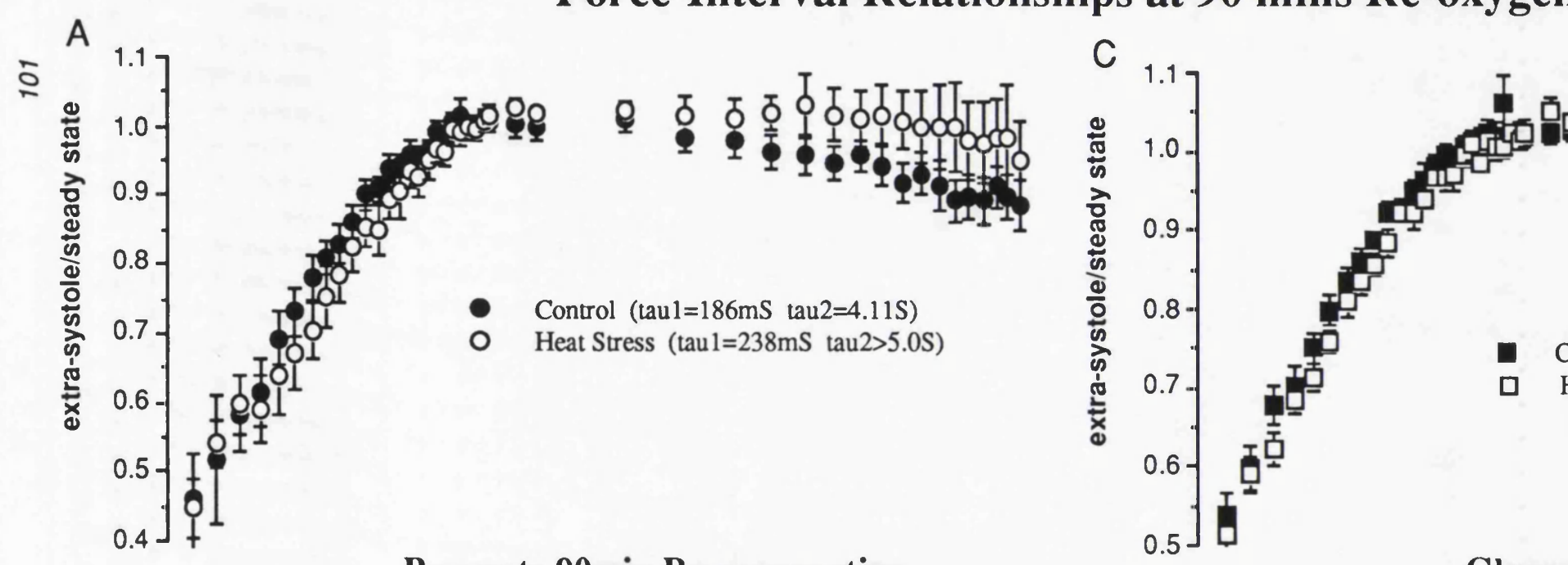

Control $(\operatorname{tau} 1=186 \mathrm{mS}$ tau2 $=4.11 \mathrm{~S}$ )

Heat Stress (taul $=238 \mathrm{mS}$ tau $2>5.0 \mathrm{~S}$ )
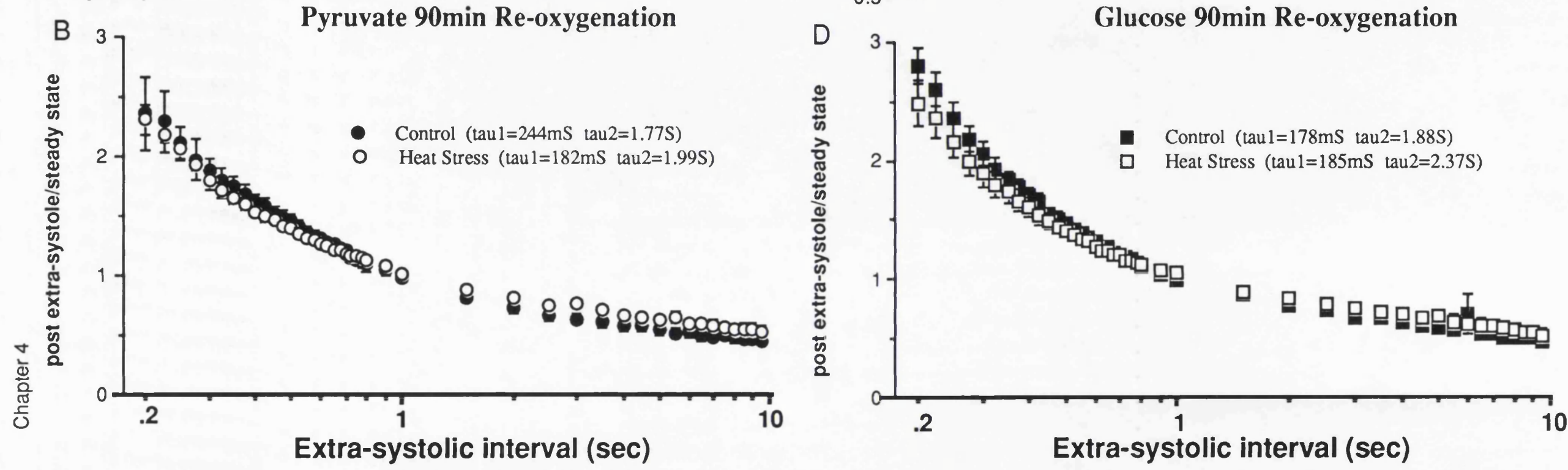

Figure 4.9: Force-interval relationships at 90 minutes of reoxygenation with glucose and pyruvate as substrates. No significant differences exist between heat stress and control groups for either extra-systolic or post-extrasystolic beats with either glucose or pyruvate as substrates. $\mathrm{n}=12 \mathrm{for}$ each group. Bars represent \pm SEM. 


\section{Effect of Hypoxia/Reoxygenation on the Force-Interval Relationship for Control Groups}

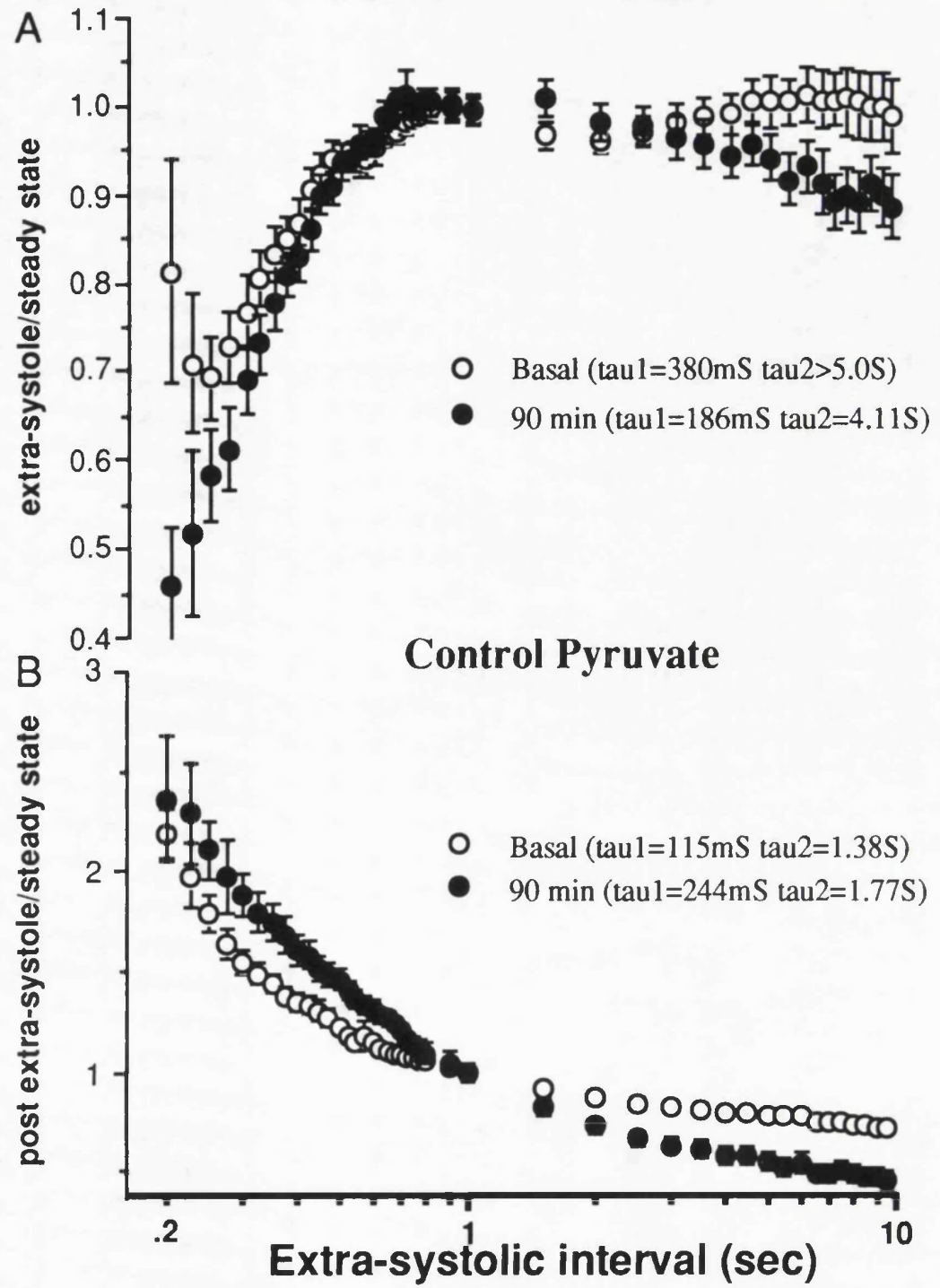

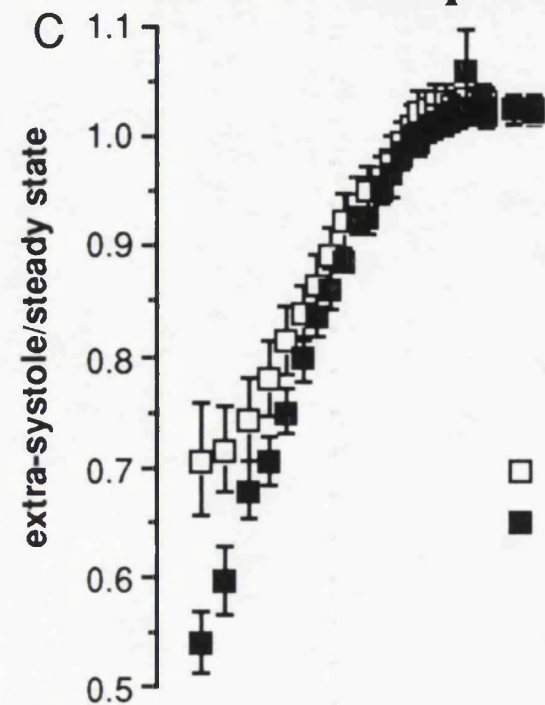

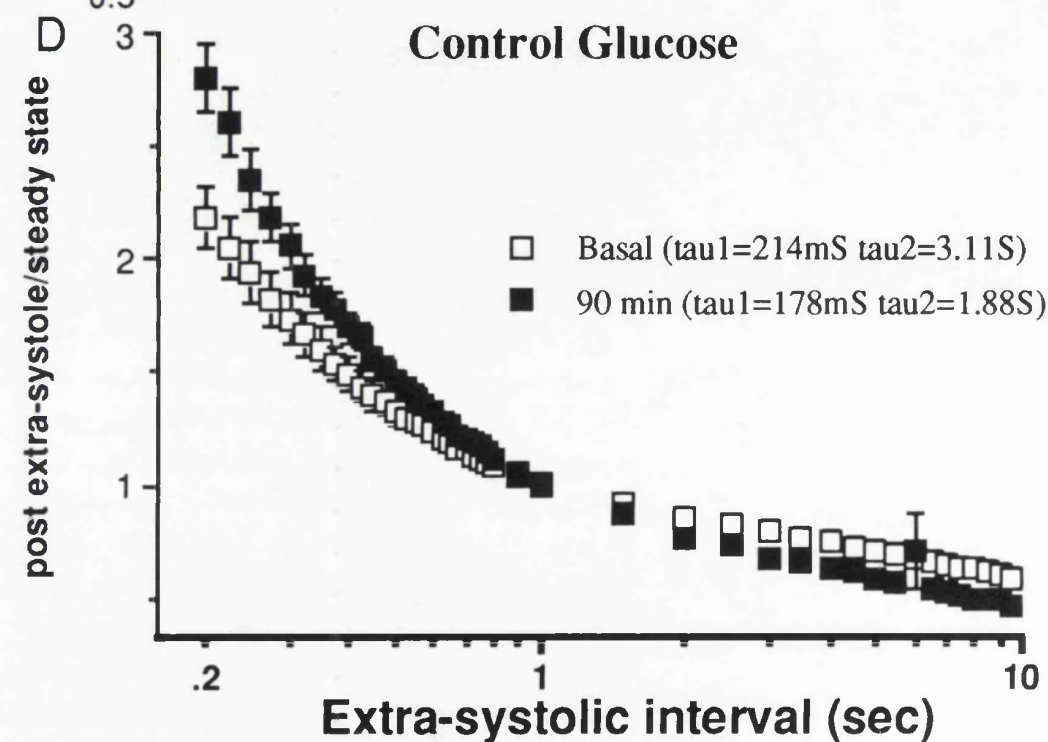

Figure 4.10: Force interval relationships prior to, and after high flow hypoxia. Differences exist between groups with faster restitution and decay of potentiation post high flow hypoia without substrate. $n=12$ for each group. Bars represent \pm SEM. 


\section{Effect of Hypoxia/Reoxygenation on the Force-Interval Relationship for Heat Stress Groups}
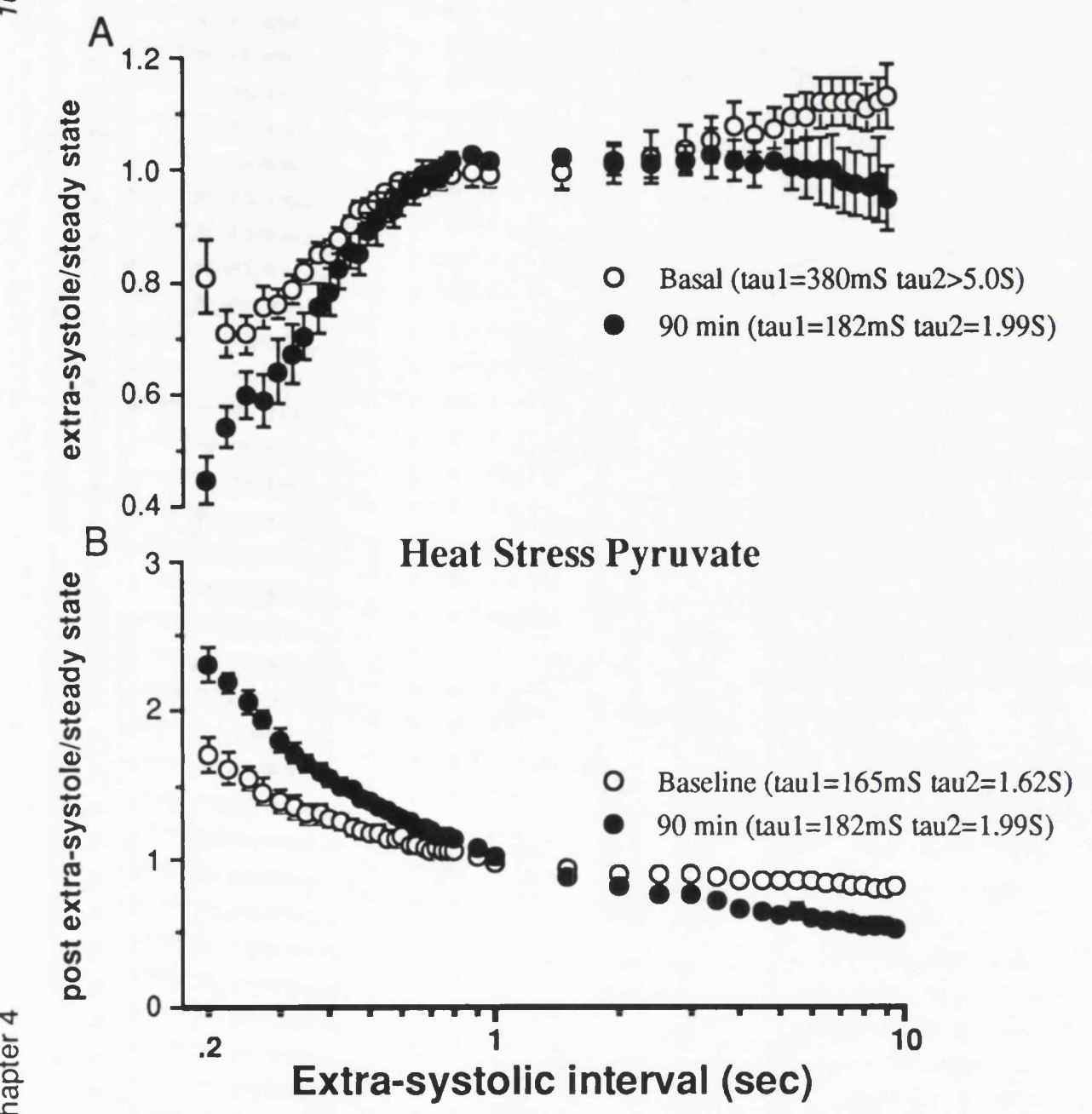
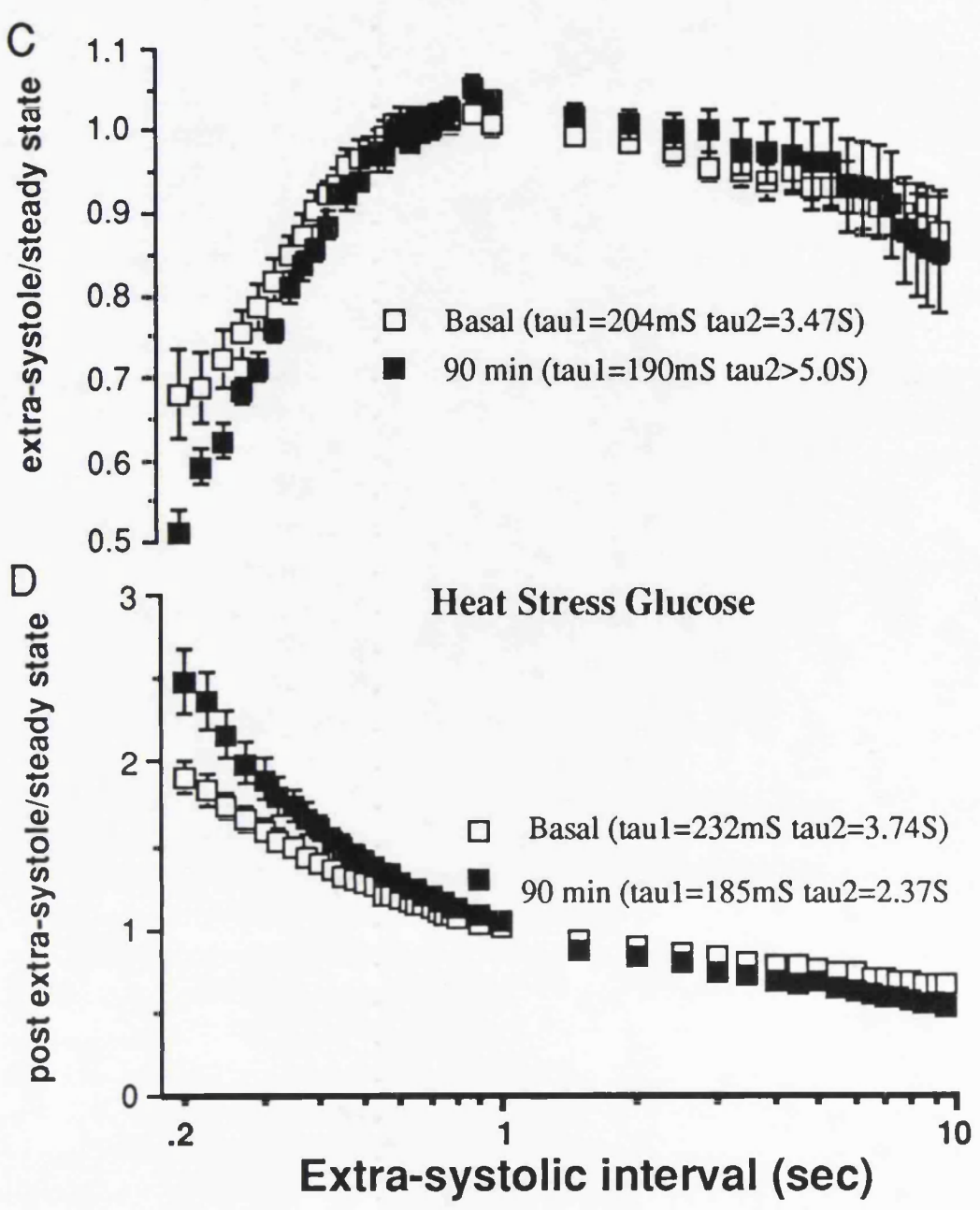

Figure 4.11: Force-interval relationships prior to, and after high flow hypoxia. Differences exist between groups with faster restituion and decay of potentiation post highflow hypoxia without substrate. $n=12$ for each group. Bars represen $t$ SEM. 
superfused with pyruvate, a significant correlation was found $\left(\mathrm{R}^{2}=0.56, \mathrm{p}=0.01\right)$. This indicates that those papillary muscles with a high stress protein content tended to be those muscles with the greatest resistance to hypoxia and reoxygenation (figure 4.13B). Although 12 sister papillary muscles to those in the heat stress pyruvate group were taken for the analysis of stress protein content, because of technical problems only 10 samples were available for comparison (1 sample inadequate tissue, 1 sample lost during boiling in water bath).

\subsection{The 60kDa Heat Stress Protein is not Associated with Contractile} Recovery (figures 4.13 and 4.14)

In contrast to the $72 \mathrm{kDa}$ heat shock protein, the content of the $60 \mathrm{kDa}$ heat shock protein was not markedly elevated 24 hours following heat stress (see figure 4.14A). When control and heat stress samples were compared densitometrically the $60 \mathrm{kD}$ protein was raised only approximately 1.5 fold following heat stress (heat stress $4.0 \pm 0.41 \mathrm{v}$ control $2.9 \pm 0.28$ units $\mathrm{p}=0.14)$. In addition there was no correlation between papillary muscle $60 \mathrm{kDa}$ stress protein content and the resistance of sister papillary muscles to hypoxia and reoxygenation $\left(R^{2}=0.14 p=0.3\right)$ figure $4.14 B$.

\section{Discussion}

Prior heat stress has been shown to protect against injury in a number of biological systems (Barbe et al 1988, Chopp et al 1989, Brown et al 1992). This study confirms that a similar protection against hypoxia and reoxygenation is seen within rabbit right ventricular papillary muscles. These findings extend the observations of other investigators whom have shown that prior heat stress is able to limit the injury associated with ischaemia and reperfusion of the isolated and in situ heart (Currie et al 1988, Karmazyn et al 1990, Donnelly et al 1992, Liu et al 1992, Yellon et al 1992a, Currie et al 1993).

\subsection{Papillary Muscle Contractility and Substrate Preference}

Papillary muscle physical dimensions as well as amplitude and rates of change of developed force were similar in all groups prior to hypoxia. Differences in 
A 72kDa Stress Protein Content of Papillary Muscles

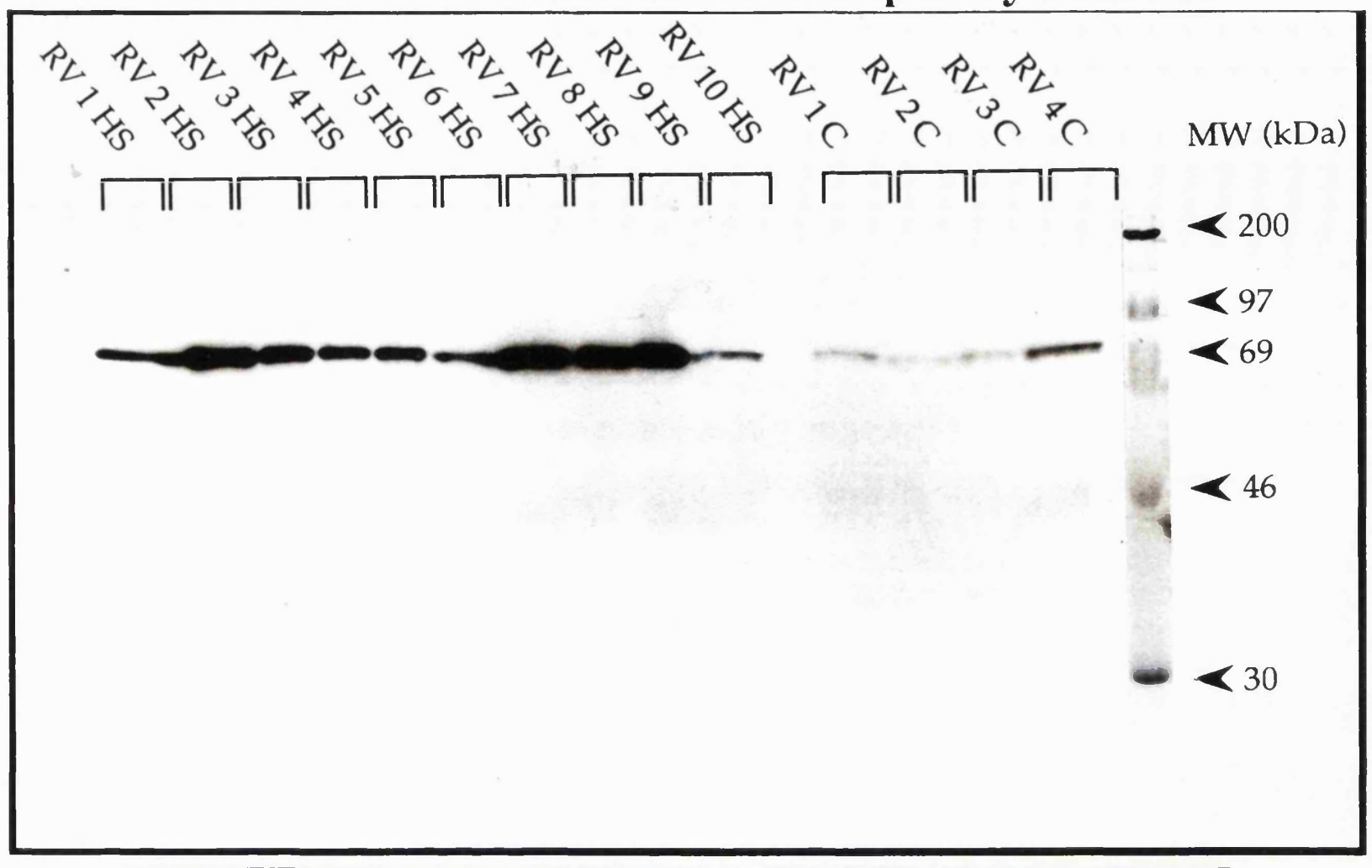

B

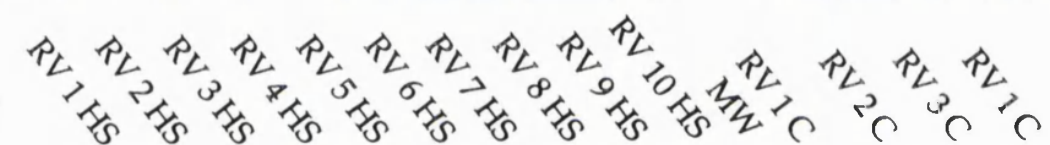

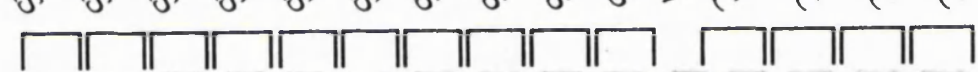
$\mathrm{MW}(\mathrm{kDa})$

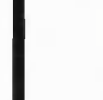

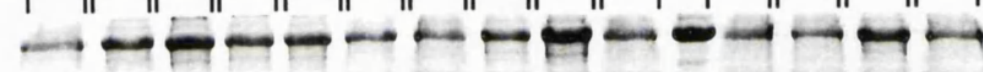
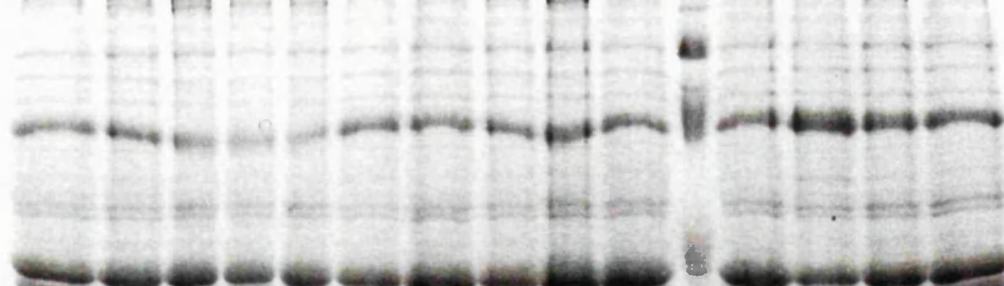

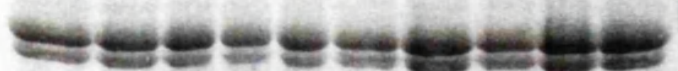
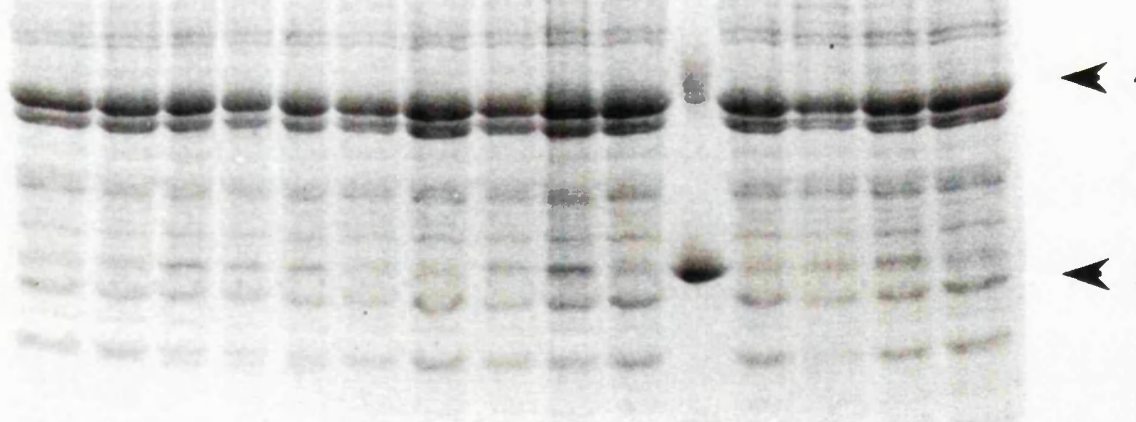

Figure 4.12: The $72 \mathrm{kDa}$ stress protein content of papillary muscle and adjoining right ventricular tissue 24 hours following whole body heat stress. Panel A: Shows the hsp 72 immunoreactivity. The lanes on the left are prepared from papillary muscles from heat stressed hearts where sister muscles were subsequently superfused with pyruvate as substrate. The lanes on the right were prepared form control papillary muscles. Panel B: Shows the Coomasie stain of an SDS-PAGE gel loaded with the same samples, at the same volumes, as the immunoblot in panel A. Despite the variation in density of the hsp 72 band, protein loading is relatively constant indicating that variations in hsp72 concentration exist between samples. 


\section{Analysis of Immunoblots of Right Ventricular Specimens for 72kDa Stress Protein Content}
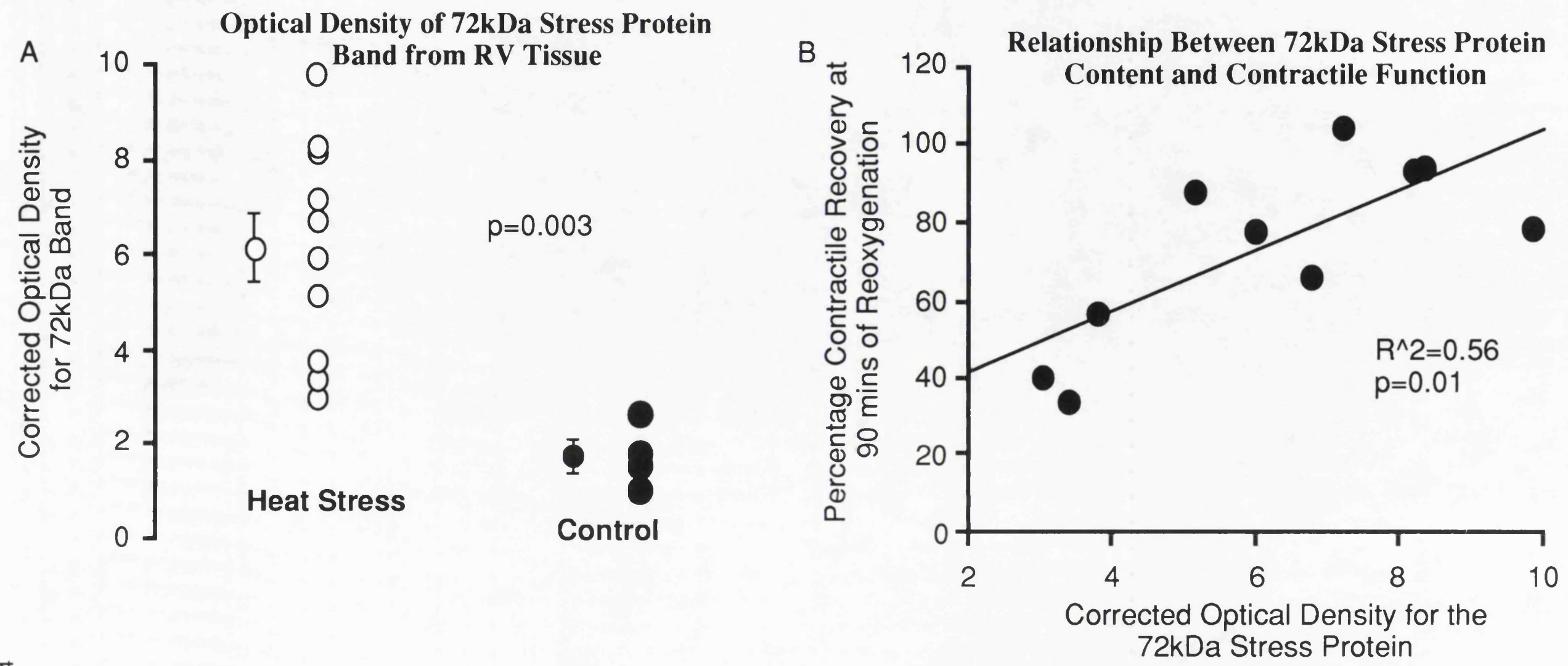

Figure 4.13: Stress protein content of heat stress and control pyruvate groups, and the relationship between $72 \mathrm{kDa}$ stress protein content and contractile recovery of "sister" papillary muscles. Comparisons between heat stress and control groups was by unpaired student t-test. The correlation between papillary muscle contractile recovery in the heat stress pyruvate group was by the Spearman Rank Correlation method. $n=10$ for heat stress pyruvate group $\mathrm{n}=4$ for control pyruvate group, bars represent \pm SEM. 


\section{Papillary Muscle 60kDa Stress Protein Content and Resistance to Hypoxia}

A
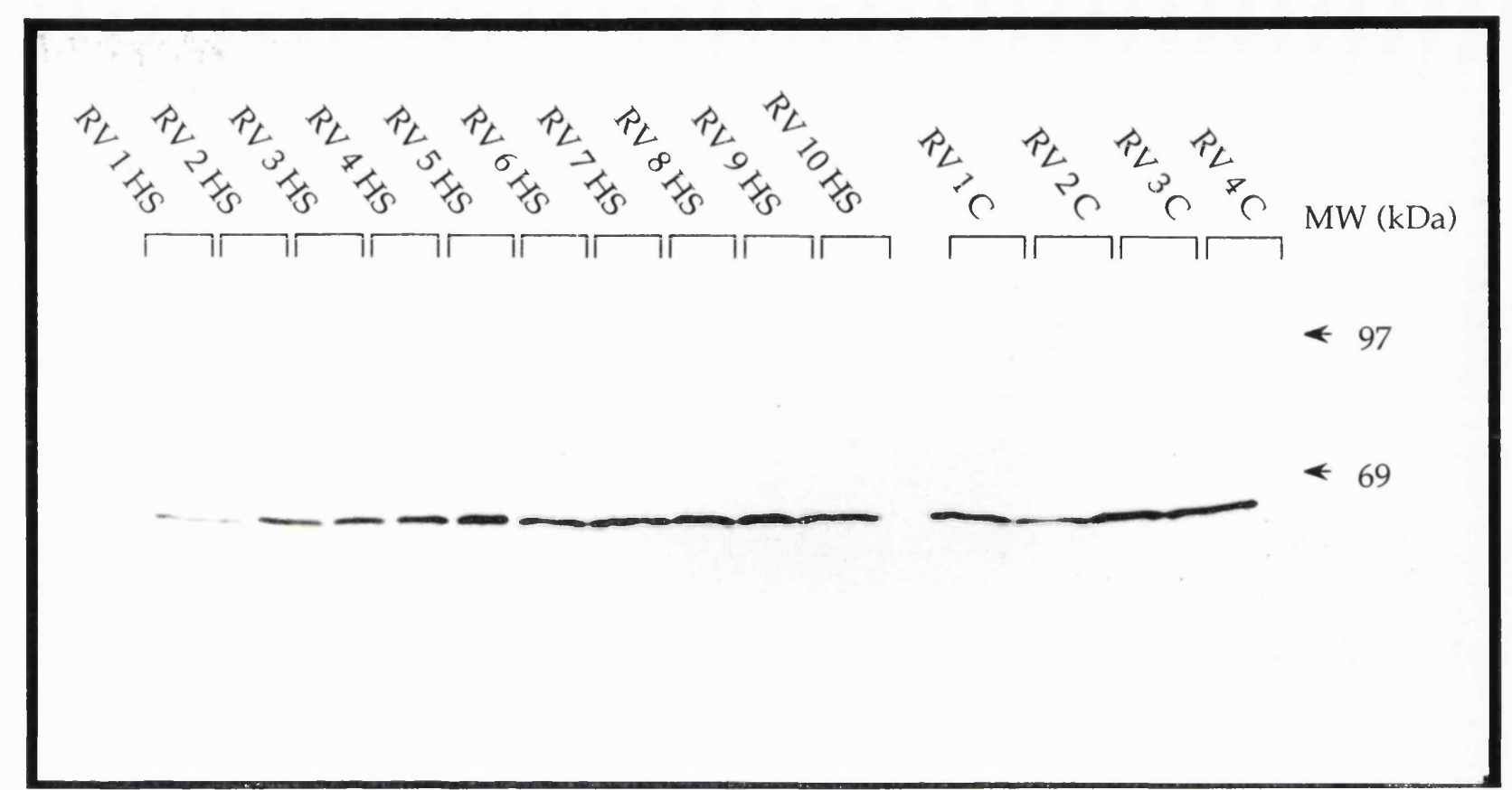

$\leftarrow 97$

$\leftarrow 69$

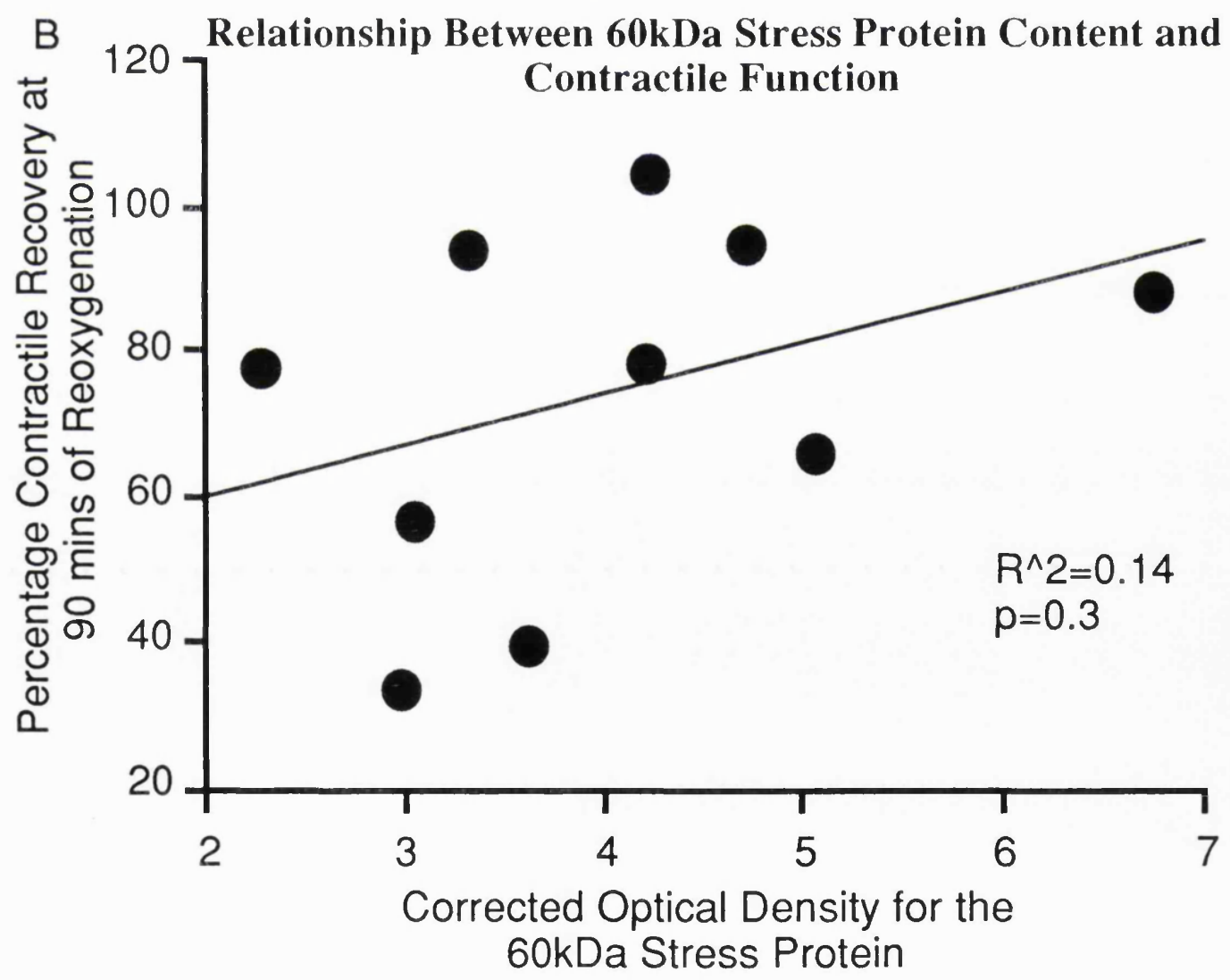

Figure 4.14: Papillary muscle hsp60 content and resistance to hypoxia. Panel A: Shows the hsp60 immunoreactivity, the lane arrangement and sample loading volumes of the blot are identical to those in figure 4.12. Hsp60 band density is similar between heat stress and control myocardium. Graph B: shows that that there is no significant association between optical density of the hsp60 bands normalised for protein loading and recovery 90 minutes following hypoxia for sister heat stressed papillary muscles superfused with pyruvate. 
subsequent performance are not therefore the result of any chance differences in papillary muscle characteristics between the groups. During hypoxia, papillary muscle activity was assessed by integration of the force envelope. The activity of muscles in the heat stressed groups with either pyruvate or glucose as substrate, tended to be greater than in their respective control groups. The importance of this observation lies in the fact that contractile activity during hypoxia is inversely related to ultimate recovery upon reoxygenation (Lewis et al 1979). It would therefore be expected that upon reoxygenation the heat stressed muscles would recover to the same or a lesser degree than their corresponding controls. In fact with both substrates the opposite was found and this reversal of the expected findings was most marked with pyruvate. Advantages to reperfusion with pyruvate have been described previously in isolated (non-heat stressed) hearts (van Bilsen et al 1988, Cavallini et al 1990). Hypothetically, if heat stress were to enhance the capabilities of the mitochondria, this would explain the greater functional recovery seen in the heat stress pyruvate group. This benefit may not be apparent with glucose as substrate if damage to the glycolytic pathway were to limit mitochondrial substrate availability to such a degree as to obviate any effect of enhanced mitochondrial function. In addition, the suggestion that the enhanced contractility that follows heat stress is a result of improved mitochondrial function is supported indirectly by the finding that both in vitro mitochondrial function and morphology are preserved following ischaemia and reperfusion of heat stressed hearts (Currie et al 1988, Yellon et al 1992a). An alternative explanation of the enhanced recovery in the heat stressed pyruvate group, could be that heat stress enhances pyruvate uptake by the myocyte. This, however seems less likely as there is no difference in function between the heat stress and control pyruvate groups at baseline.

The mitochondria are thought to be a target for re-oxygenation injury and therefore heat stress, by preserving mitochondrial function, may be capable of limiting this injury. Indirect evidence for reduced oxidative stress following ischaemia and reperfusion in heat stressed rabbit hearts, is provided by the finding of increased tissue levels of reduced glutathione (GSH), protein-SH groups and reduced levels of oxidized glutathione (GS-SG) (Yellon et al 1992a). In this study enhanced mitochondrial activity was coincident with the seemingly lower levels of tissue oxidant stress. 


\subsection{Force-Interval Relationships}

The changes occurring in the processes of restitution and decay of potentiation following hypoxia are characteristic of the findings seen by other investigators, where various forms of myocardial damage are generally associated with an increase in the degree of post extrasystolic potentiation which decays more rapidly than usual (Seed and Walker 1988). These phenomena appear to be related to beat to beat variability in cytosolic calcium within the myocardial cell, since active tension generated at varying interbeat intervals is related to the calcium transient (Weir and Yue 1986). In particular the process of mechanical restitution seems to depend upon the kinetics of the calcium release channel of sarcoplasmic reticulum (Benijamali 1991). Using these indirect measures of sarcoplasmic reticulum function, no clear differences exist between heat stress and control pyruvate groups at 90 minutes of reoxygenation. However, since clear differences exist at this timepoint in terms of developed force between these groups, these findings suggest that changes in the calcium handling behaviour of the sarcoplasmic reticulum are not the cause for the enhanced recovery in the heat stress pyruvate group.

\subsection{Stress Proteins and Myocardial Resistance}

Although a number of investigators have demonstrated that heat stress limits myocardial injury following ischaemia and that similar heat treatment in other animals is associated with an elevation in stress protein content, it is not clear whether these two observations are causally linked. Indeed Currie and coworkers (Currie et al 1988, Karamazyn et al 1990) have shown that in the rat, endogenous catalase activity is increased following heat stress and that if catalase activity is inhibited, the protection previously noted is lost. The currently favoured hypothesis therefore, is that heat stress increases endogenous myocardial antioxidant defences which thereby act to limit the injury associated with free-radical formation upon reperfusion.

The results presented in this chapter suggest that heat stress may act by preserving mitochondrial function, however the mechanisms by which stress protein induction or other aspects of heat stress could influence mitochondrial performance are at present tentative. Both the $60 \mathrm{kDa}$ and $72 \mathrm{kDa}$ family of stress proteins are known to have essential roles in allowing correct unfolding and refolding of nuclear 
encoded mitochondrial proteins as they enter the mitochondria from the cytoplasm (see Chapter 1 Sections 4.2.3 and 4.2.4) and so maybe able to correctly refold mitochondrial enzymes or structural proteins altered during ischaemia and reperfusion. In our study the $70 \mathrm{kDa}$ stress protein is variably induced following heat stress and correlates with the variable papillary muscle resistance to hypoxia and reoxygenation. Such a correlation provides circumstantial evidence for a direct role of this protein in recovery following hypoxia. However, papillary muscles that have responded to heat stress with the highest stress protein response may also respond with the greatest increase in endogenous antioxidant activity. Papillary muscle catalase activity was not measured in this study but transfection studies in rat myocytes suggest that hsp72 is directly able to enhance viability following anoxia/reoxia (Mestril et al 1992). In addition in the rat variable degrees of hsp72 induction by differing severities of heat stress result in an inverse relationship between stress protein content and infarct size (Hutter et al 1993). Although the interpretation of this study is complicated by the fact, that in contrast to the experimental design in this chapter, infarct size and stress protein content were measured in different hearts.

We also analysed the papillary muscle samples for the $60 \mathrm{kDa}$ stress protein. The $60 \mathrm{kDa}$ family of proteins, which are predominantly mitochondrial, are found in association with essential components of the ATP synthesis pathway (namely cytochrome c and F1-ATPase) and are therefore prime candidates for heat stress mediated mitochondrial protection. When the content of the $60 \mathrm{kDa}$ stress protein was measured, it was only mildly elevated 24 hours following heat stress, and there was little variation between samples. In addition no correlation was found between papillary muscle $60 \mathrm{kDa}$ stress protein content and contractile recovery.

There are 2 possible interpretations for the preferential mitochondrial preservation inferred in this study. The mitochondria could themselves be more resistant to the changes in the cellular internal environment during hypoxia and reoxygenation (intrinsic mitochondrial protection), alternatively the protection could be the result of a more controlled cellular environment in heat stressed hearts during the stress (extrinsic mitochondrial protection). The second possibility is favoured by the finding that mitochondrial calcium overload is less in heat stressed hearts at reperfusion (Yellon et al 1992a), and since mitochondrial calcium uptake is a passive 
process, occurring down an electrochemical gradient, this implies that cytosolic calcium is better controlled in these hearts compared to controls (ie: protection is extrinsic).

The results of this study are consistent with the hypothesis that stress proteins are the mediators of the myocardial protection that follows heat stress and indicates for the first time that some families of stress proteins may be more important than others. The hypothesis that stress proteins may directly influence the resistance of the heart to ischaemia has major implications, and would suggest that myocardial stress proteins raised by non-thermal means should also be protective (see Chapter 7 ).

\subsection{Contracture and Substrates}

The increase in resting force during high flow hypoxia is probably secondary to rigor bond formation. As the ATP levels drop during hypoxia the activity of actin myosin ATPase falls and crossbridges remain attached. At a later stage the activity of calcium ATPases falls and cytosolic calcium rises (an effect attributed to the higher $\mathrm{K}_{\mathrm{m}}$ for ATP for actin myosin ATPase $v$ surface membrane $\mathrm{Ca}^{2+}$ ATPase, as discussed by Allen and Orchard 1987). The greater delay in the onset of contracture in papillary muscles perfused with glucose therefore implies these muscles had a higher energy charge during early hypoxia than those perfused with pyruvate. This is likely to imply that glycolytic flux was greater in these muscles, either because of glucose contamination of the organ bath due to incomplete substrate washout at the onset of hypoxia, or conversely and perhaps more likely, because of inhibition of glycolysis by pyruvate. The inhibitory effect of pyruvate is probably secondary to an increase in citrate that inhibits the activity of phosphofructokinase (Mochizuki and Neely 1980).

\subsection{Critique of Methods}

The drawbacks of the papillary muscle model in terms of adequacy of oxygenation which have been discussed in Chapter 3 Section 4.4 are reinforced in this study where once again there is no significant correlation between papillary muscle diameter and developed force. In addition pyruvate is traditionally held as a better substrate than glucose for papillary muscle studies (Daut and Elzinga 1989), yet 
papillary muscle contractile force at baseline was no different in heat stress and control muscle with pyruvate compared to glucose. The most likely explanation for the lack of advantage with pyruvate was that the substrate limiting papillary muscle contractility was neither glucose nor pyruvate, but oxygen.

The interpretation of the study may also be complicated by the observation that pyruvate alone may have antioxidant activity with an ability to enhance hydrogen peroxide breakdown (Varma and Morris 1988).

\subsection{Conclusions}

In conclusion, following 30 minutes of hypoxia with substrate deprivation, the recovery of rabbit papillary muscle contractile activity appears greatest in heat stressed muscles when pyruvate is used as substrate. We postulate this reflects an increase in mitochondrial resistance to hypoxia and reoxygenation following heat stress. The lack of an effect of heat stress on force-interval behaviour of muscle suggests that the enhanced contractility occurs independently of alterations within the calcium release and re-uptake process of the sarcoplasmic reticulum. The finding that papillary muscle contractile recovery is closely correlated with the content of the $72 \mathrm{kDa}$ stress protein implies that the myocardial protection that follows heat stress may be mediated by an increase in myocardial stress protein content. 


\section{Chapter 5}

\section{Results: The Isolated Heart Subjected to a Submaximal Calcium Paradox}

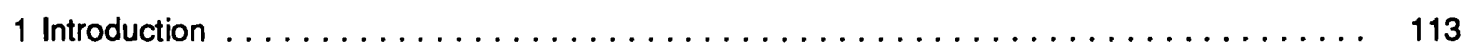

2 Specific Methods .................................... 115

2.1 Group Sizes and Exclusions $\ldots \ldots \ldots \ldots \ldots \ldots \ldots \ldots \ldots \ldots \ldots \ldots . \ldots \ldots$

2.2 The Experimental Model . . . . . . . . . . . . . . . . . . . . . . . 115

2.2 The Perfusion Protocol (figure 5.1) $\ldots \ldots \ldots \ldots \ldots \ldots \ldots \ldots \ldots \ldots \ldots \ldots$

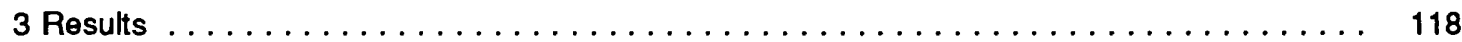

3.1 Baseline Parameters (table 5.1) $\ldots \ldots \ldots \ldots \ldots \ldots \ldots \ldots \ldots \ldots \ldots, 118$

3.2 Temperature Changes During Heat Stress .................. 118

3.3 Functional Changes During the Calcium Paradox (figure 5.2) . . . . . . . 118

3.4 Left Ventricular and Coronary Perfusion Pressures During the Calcium Paradox

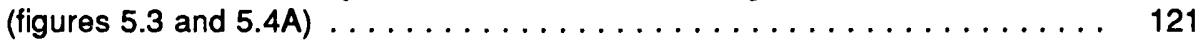

3.5 Oxygen, Carbon Dioxide and pH Changes Across the Heart During the Calcium

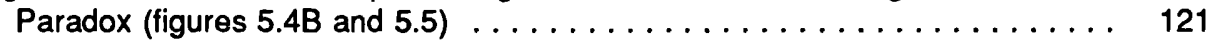

3.6 Creatine Phophokinase and Myoglobin Efflux During the Calcium Paradox (figure

5.6) ................................ 121

3.7 Changes in Action Potential Duration During the Calcium Paradox (figure 5.7) . . . 125

3.8 The Interdependence of Functional and Metabolic Parameters (figure 5.8) . . . . . 125

3.9 Stress Protein Analysis (figures 5.7B, 5.9 and 5.10) . . . . . . . . . . 129

4 Discussion . . . . . . . . . . . . . . . . . . . . . . . . . . . . . . . . 129

4.1 The Implication of the Functional and Metabolic Preservation Following the Calcium

Paradox in Heat Stressed Hearts ............................ 129

4.2 The Mechanism of Resistance to the Calcium Paradox . . . . . . . . . . . . . . 132

4.3 Critique of Methods . . . . . . . . . . . . . . . . . . . . . 133

4.4 Conclusion . . . . . . . . . . . . . . . . . . . . . . . . . . 134

\section{Introduction}

The majority of studies suggest that heat stress is able to limit subsequent ischaemic damage in the isolated heart (Currie et al 1988, Karmazyn et al 1990, Yellon et al 1992a, Steare et al 1993). It is however unclear whether this protection is a consequence of thermal induction of stress proteins or is mediated by some other change within the myocardium following whole body heat stress. A number of investigators have shown that heat stress is followed by changes in the levels of endogenous antioxidants such as catalase in the rat (Currie et al 1988, Steare et al 1993) and superoxide dismutase in the pig (Liu et al 1992). In addition, in both the rat and rabbit there is indirect evidence of enhanced anti-oxidant defences, since oxidised glutathione release into the coronary effluent is diminished upon reperfusion 
of isolated hearts harvested 24 hours after whole body heat stress (Yellon et al 1992a, Pasini et al 1991). More direct evidence that the protection following heat stress may involve an increase in endogenous antioxidants is provided by the study of Karmyzyn et al (1990). These investigators have shown the whole body heat stress approximately doubles endogenous catalase activity and that pre-treatment with the 3-aminotriazole, a catalase inhibitor, reduces catalase activity and abolishes the protection seen these hearts. However the interpretations of this study are complicated by the fact that 3aminotriazole did not alter recovery of the control hearts and other investigators have found 3-aminotriazole to cause paradoxical protection in a similar model (Steare et al 1993). In addition the role of free radicals and oxidant stress as mediators of lethal myocardial injury remains controversial (see Chapter 1 Section 2.2), with scanty evidence that exogenous antioxidants can limit infarction (see Yellon and Downey 1990 for discussion). Further evidence against a primary role for endogenous antioxidants is the observation that a number of cardiac stresses which do not involve oxidant stress also induce stress protein synthesis (Chevalier et al 1989, Delcayre et al 1988, Dillmann et al 1986, Löw et al 1989, Moalic et al 1989, White and White 1986) suggesting that some portion of the stress protein response represents a form of cardiac adaptation that is able to limit myocyte injury independent of anti-oxidant mechanisms.

The aim of this chapter was to examine the hypothesis that whole body heat stress is capable of limiting myocardial injury independent of any increase in endogenous antioxidants. To achieve this we examined the influence of cardiac stress protein elevation by whole body heat stress on a subsequent calcium paradox. The calcium paradox, describes the injury initiated by the re-introduction of calcium following a period of zero or low calcium perfusion (Zimmerman and Hülsmann 1966). The advantage of this pure form of injury is that it is not associated with oxidant stress and therefore enhanced antioxidant defences would not be capable of influencing the degree of damage on calcium repletion. The absence of free-radical involvement in the calcium paradox is indicated by, (i)the lack of changes in protein SH groups or oxidized to reduced glutathione ratio in hearts severely damaged by the calcium paradox (Ferrari et al 1989) (ii)the observation that the calcium paradox occurs with anoxic calcium depletion/repletion (Duncan 1990) and (iii)perfusion with 
superoxide dismutase, catalase and various scavengers and inhibitors of oxygen metabolites fail to attenuate even a mild calcium paradox in the rat heart (Duncan 1990).

\section{Specific Methods}

\subsection{Group Sizes and Exclusions}

Thirty rabbits were used for the purpose of this study. Fifteen rabbits were heat stressed and recovered for 24 hours, of these 4 rabbits were used for stress protein estimation and 11 for contractile assessment in response to a calcium paradox. Fifteen sham rabbits were allocated in an identical fashion. There was one exclusion in the heat stress group because of calcium contamination of the nominally calcium free perfusion solution, the matched control heart was also excluded. Therefore in each group 4 hearts were used for heat stress protein estimation and 10 hearts subjected to a calcium paradox.

\subsection{The Experimental Model}

Male New Zealand White rabbits $(\mathrm{n}=30)$, weighing $2.0-2.5 \mathrm{~kg}$, were anaesthetized and underwent either sham or true heat stress as previously described (see Chapter 2 Section 1.1.2). Following either sham or true heat stress the animals were allowed to recover for 24 hours prior to removal of hearts for stress protein estimation (see Chapter 2 Section 2) or Langendorff perfusion (see Chapter 2 Section 4).

Langendorff hearts were perfused in a constant flow mode with modified Tyrode solution $\left(\mathrm{NaCl} 118.5 \mathrm{mM}, \mathrm{NaHCO}_{3} 24.8 \mathrm{mM}, \mathrm{KCl} 4.8 \mathrm{mM}, \mathrm{KH}_{2} \mathrm{PO}_{4} 1.2 \mathrm{mM}\right.$, $\mathrm{CaCl}_{2} 1.30 \mathrm{mM}, \mathrm{MgSO}_{4} 1.44 \mathrm{mM}$, Glucose $10 \mathrm{mM}$, Pyruvate $2 \mathrm{mM}$; sodium pyruvate from Sigma Chemicals, Missouri, USA; all other reagents ANALAR grade from BDH Chemicals, Poole, UK), equilibrated with $95 \%$ oxygen and $5 \%$ carbon dioxide to $\mathrm{pH}$ 7.35-7.45. The solution for low calcium perfusion was prepared identically but with $\mathrm{CaCl}_{2}$ omitted. Solutions were prepared freshly each day using water with a resistivity greater than 20MS.cm (Elgastat UHP, Elga, Bucks, UK) and prefiltered to exclude

particles of greater than $2 \mu \mathrm{m}$ diameter. The calcium concentration of identically prepared but non-contemporaneous batches of nominally calcium-free Tyrode's 
solution was $6.1 \pm 1.7 \mu \mathrm{M}$ (mean $\pm \mathrm{SE} \mathrm{n}=10$ ). The calcium concentration of these solutions was estimated by atomic absorption spectrophotometry (PYE, Unicam SP9) using lanthanum chloride in $\mathrm{HCl}$ to prevent the formation of calcium phosphate. Matched heat stressed and control hearts were perfused on the same day and shared the same batches of solutions.

Hearts were paced via the right atrium at 180 beats/min whilst left ventricular monophasic action potential (MAP), left ventricular pressure and coronary perfusion pressure were measured (see Chapter 2 Section 4.3.3). Coronary perfusate entering and leaving the heart was analysed to calculate gas exchange and enzyme efflux from the heart (see Chapter 2 Section 4.3.6).

\subsection{The Perfusion Protocol (figure 5.1)}

The degree of injury triggered by calcium repletion is determined by, the duration of the calcium free perfusion period, the actual level of calcium during calcium free perfusion and the coronary flow rate during calcium free perfusion (Oksendal et al 1985). In preliminary experiments, with a calcium chelator present, no recovery was seen in either heat stress or control hearts. Subsequently, a variety of perfusion protocols with varying flow rates and durations for low calcium perfusion were applied until the conditions were found that would consistently result in a subtotal paradox.

During the 15 minute stabilisation period coronary perfusion pressure was set at a mean of $35 \mathrm{mmHg}$ by altering the coronary flow rate $(5 \mathrm{O} 2 \mathrm{~S} / \mathrm{R}$ peristaltic pump, Watson-Marlow, Cornwall, UK), thereafter coronary flow rate was unaltered. Initial experiments at this coronary perfusion pressure, demonstrated that 5 minutes perfusion without a calcium chelator, but with no added calcium (magnesium at $1.44 \mathrm{mM}$, sodium at $143.3 \mathrm{mM})$, resulted in approximately a $50 \%$ recovery of developed pressure on the re-introduction of calcium (1.3mM). This degree of injury was thought appropriate to determine the potential protective benefits of heat stress.

After a 15 minute stabilisation period coronary perfusion was switched, via a parallel circuit entering a sidearm of the coronary cannula, to a perfusate with no added calcium (see figure 5.1). Following 5 minutes of no added calcium perfusion, calcium was re-introduced abruptly, and perfusion continued for a further 10 minutes, 


\section{$\cong$ Experimental Protocol for Induction of the Calcium Paradox in the Isolated Rabbit Heart}

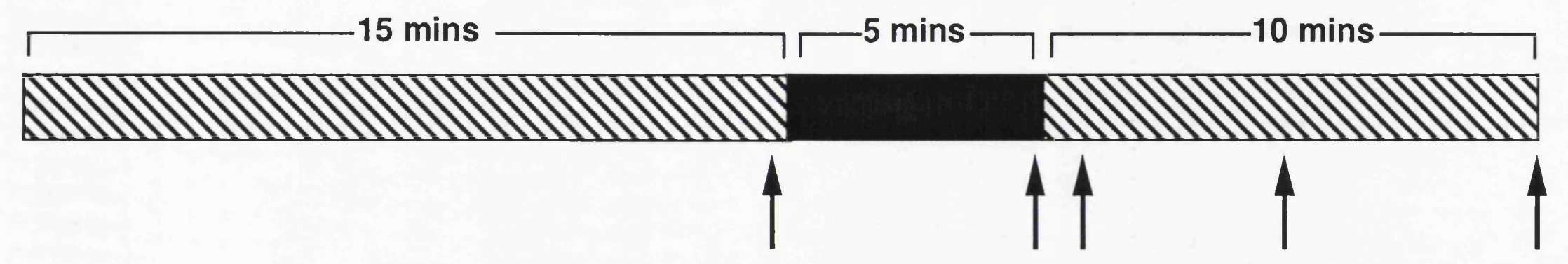

\footnotetext{
MIIII = Perfusion with Tyrode's containing Calcium at $1.3 \mathrm{mM}$
}

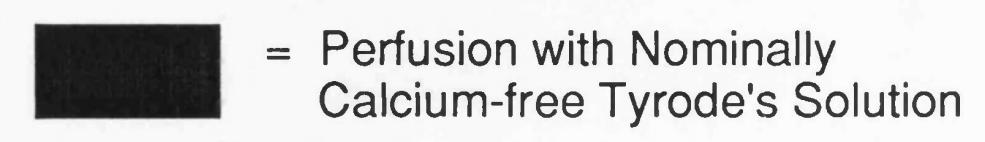

= Timepoints for recording left ventricular and coronary perfusion pressures, LV monophasic action potentials, CPK efflux and oxygen, $\mathrm{pH}$ and carbon dioxide changes across the heart.

Figure 5.1: Protocol used to induce a submaximal calcium paradox within the rabbit heart. Hearts were harvested twenty-four hours after either sham $(n=10)$ or true heat stress $(n=10)$. After a 15 minute stabilisation period, hearts were perfused for 5 minutes with nominally calcium-free solution before calcium repletion. 
after which time hearts were removed for wet weight determination. The hearts were then dried in a hot air oven at $70^{\circ} \mathrm{C}$ overnight for dry weight determination.

\section{Results}

\subsection{Baseline Parameters (table 5.1)}

Prior to zero calcium perfusion, monophasic action potential duration, left ventricular developed pressure, mean coronary perfusion pressure and coronary perfusate flow were similar in heat stressed and control groups (table 5.1). The left ventricular end-diastolic pressure (LVEDP) was by coincidence significantly greater at baseline in the heat stressed group (table 5.1), however, since the difference between the means was small it was not thought that this would have a significant influence on subsequent injury. Heart wet and dry weights measured at the end of the experimental protocol were similar (table 5.1), although the higher wet:dry ratio in the control group suggests there was a tendency for the control hearts to be more oedematous. There was no CPK activity detectable in the coronary effluent prior to the onset of zero calcium perfusion.

\subsection{Temperature Changes During Heat Stress}

The baseline temperature was $39.0 \pm 0.03$ in the heat stress group and $38.9 \pm 0.01$ in the control group. In the heat stress group peak temperature was $42.3 \pm 0.09$ and time to peak temperature $45.0 \pm 4.8$ minutes.

\subsection{Functional Changes During the Calcium Paradox (figure 5.2)}

During the calcium-free perfusion period contractile activity ceased and monophasic action potential morphology altered in all hearts, on the re-introduction of calcium there was variable recovery of left ventricular developed pressure and an increase in diastolic pressure. The pattern of changes in an individual heart are shown in the experimental traces of figure 5.2. 


\section{Characteristics of Hearts Subjected to the Calcium Paradox}

\begin{tabular}{l|c|c|c} 
& Heat Stress & Control & t-test \\
\hline At Baseline & $155.5 \pm 2.5$ & $153.0 \pm 8.3$ & $p=N S$ \\
APD90 (ms) & $50.7 \pm 4.9$ & $49.0 \pm 3.0$ & $p=N S$ \\
LV Developed Pressure (mmHg) & $10.7 \pm 1.2$ & $8.4 \pm 0.6$ & $p=N S$ \\
LV End Diastolic Pressure (mmHg) & $36.5 \pm 1.8$ & $36.4 \pm 1.1$ & $p=N S$ \\
Coronary Perfusion Pressure (mmHg) & $39.2 \pm 3.4$ & $37.3 \pm 2.6$ & $p=N S$ \\
Coronary Perfusate Flow (ml/min) & & & \\
After 10 mins calcium repletion & $8.63 \pm 0.24$ & $9.86 \pm 0.86$ & $p=N S$ \\
Wet Weight (g) & $1.44 \pm 0.05$ & $1.51 \pm 0.14$ & $p=N S$ \\
Dry Weight (g) & $6.05 \pm 0.23$ & $6.62 \pm 0.24$ & $p=N S$ \\
Wet:Dry Ratio & & &
\end{tabular}

Table 5.1:

Baseline values were obtained immediately before nominally calcium-free perfusion.

Heart wet weight was measured at the end of the calcium repletion period.

Hearts dry weight was measured after drying at $70^{\circ} \mathrm{C}$ overnight in a hot air oven.

$\mathrm{n}=10$ for each group. Values represent means \pm SEM. Comparisons were by two-tailed $\mathrm{t}$-test. 


\section{Example of Experimental Trace During Calcium Paradox}

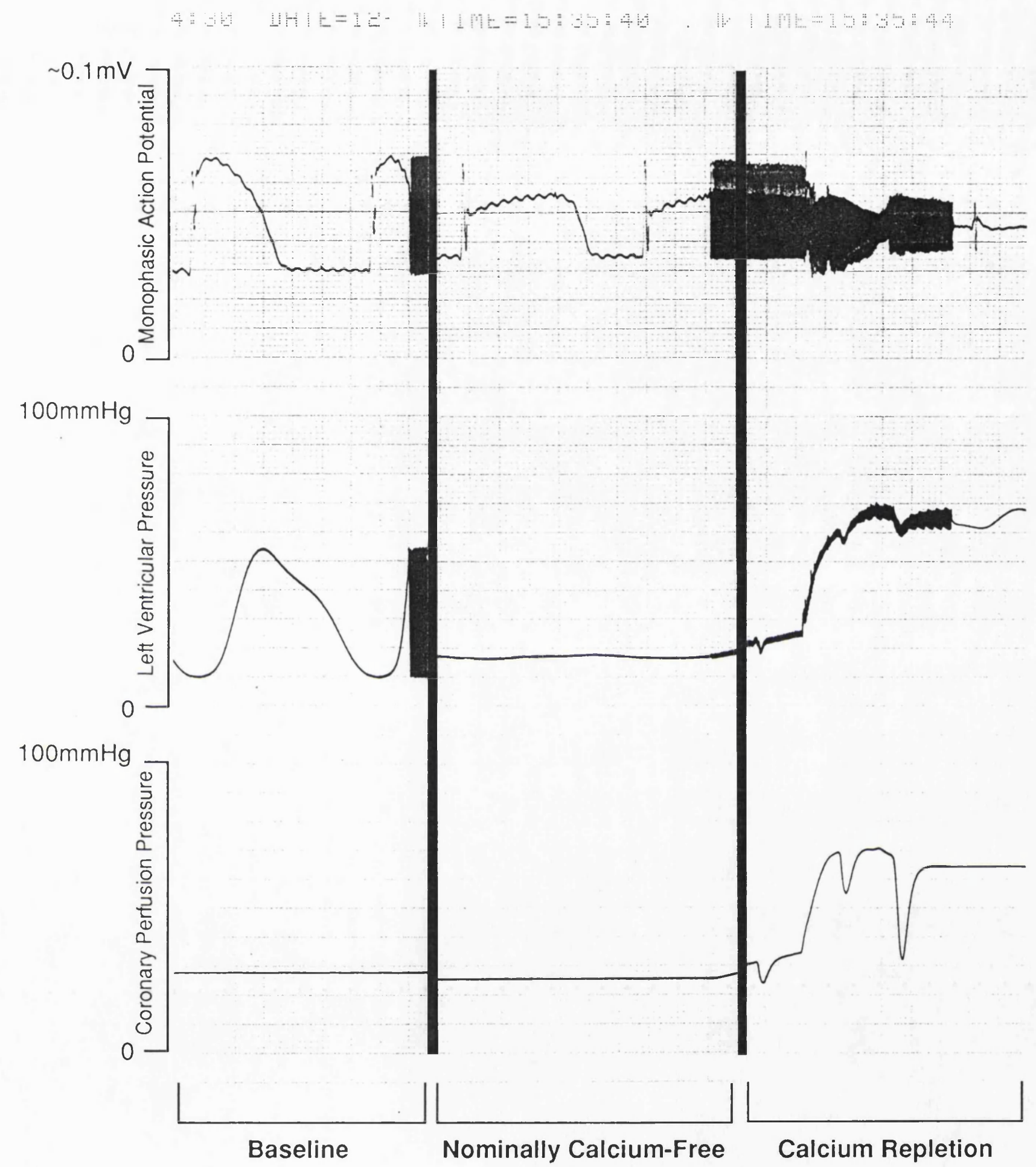

0.5 Seconds

Figure 5.2: The appearance of the monophasic action potential, left ventricular pressure and coronary perfusion pressure during the experimental protocol. This example from a control heart shows marked injury on calcium repletion with contracture and little contractile recovery. 


\subsection{Left Ventricular and Coronary Perfusion Pressures During the}

\section{Calcium Paradox (figures 5.3 and 5.4A)}

Contractile recovery on calcium repletion was significantly greater in the heat stressed group. The better preservation of left ventricular developed pressure (figure 5.3A) was associated with a lesser degree of contracture in this group (figure 5.3B). Similar temporal changes were seen between the groups in terms of the rise in coronary perfusion pressure (figure 5.4A) although these failed to reach significance.

\subsection{Oxygen, Carbon Dioxide and pH Changes Across the Heart During the Calcium Paradox (figures 5.4B and 5.5)}

The differences in the, partial pressures of oxygen and carbon dioxide and $\mathrm{pH}$ of perfusate entering and leaving the heart at various timepoints are shown in figures 5.4B and 5.5. The data comprising these graphs have not been normalised for coronary flow or heart weight since tension and $\mathrm{pH}$ changes are not linearly related to content. During nominally calcium-free perfusion oxygen consumption and carbon dioxide production diminish, presumably secondary to the cessation of contractile activity. Upon calcium repletion metabolic activity increases in both groups and although no significant differences exist between groups, the decrease in activity (compared to baseline) is significantly greater in the control group consistent with the diminished contractile activity in this group.

\subsection{Creatine Phophokinase and Myoglobin Efflux During the Calcium} Paradox (figure 5.6)

The content of creatine phosphokinase (CPK) and myoglobin (absorption at $280 \mathrm{nM}$ ) in the coronary effluent at different timepoints during the calcium paradox is shown in figure 5.6. No CPK and only a low level of myoglobin appears during calcium free perfusion, upon calcium repletion there is a marked increase in CPK and myoglobin efflux reflecting myocyte membrane disruption. The CPK release from control hearts is significantly greater than from heat stressed hearts, although trends exist in the same direction for myoglobin release they are not significant. The standard errors of these measurements are large because two control hearts developed a full 

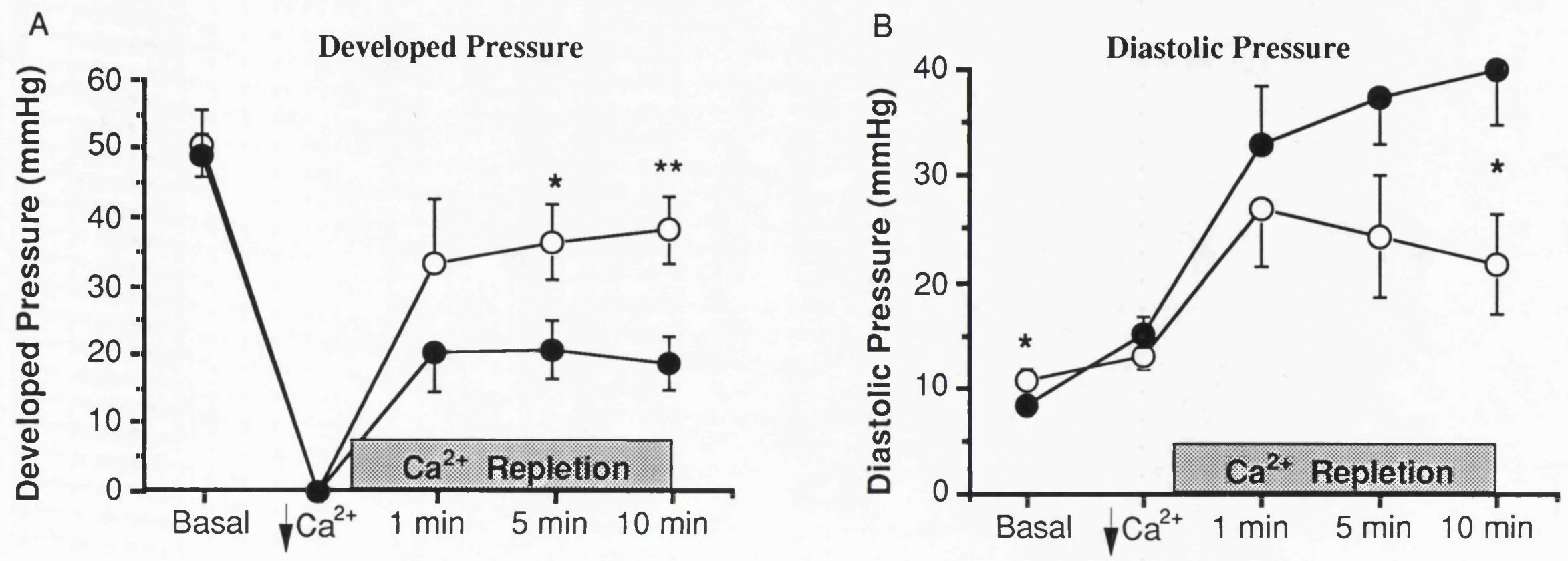

Figure 5.3: Left ventricular developed and diastolic pressures in heat stressed and control rabbit hearts during a submaximal calcium paradox. Isovolumic left ventricular pressure was measured with a fluid filled balloon. ${ }^{*} p \leq 0.05{ }^{* *} p \leq 0.01$ heat stress v control groups, comparisons by unpaired $t$-test, $n=10$ for each group. Bars represent $t$ SEM. 
Coronary Perfusion Pressure and Oxygen Extraction During the Calcium Paradox
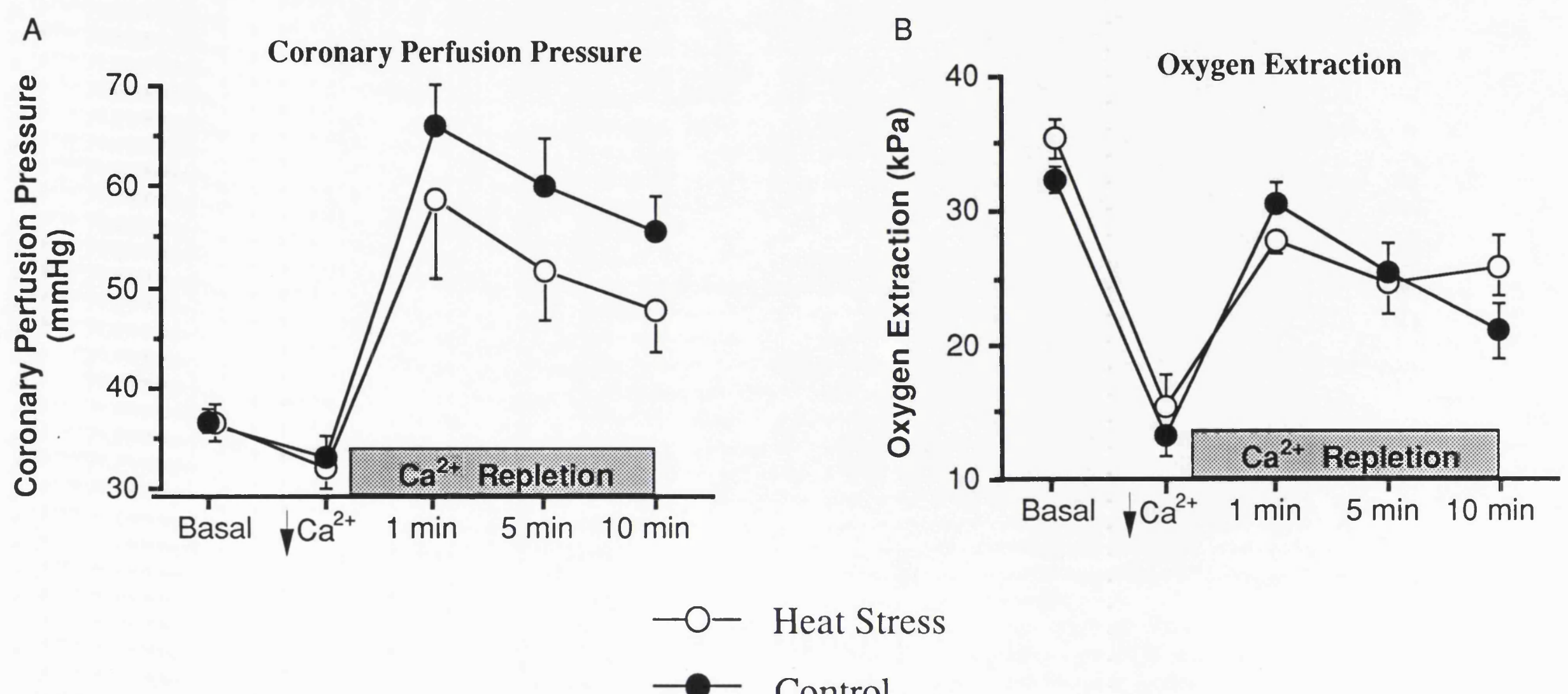


\section{Carbon Dioxide Production and pH Drop During the Calcium Paradox}

A

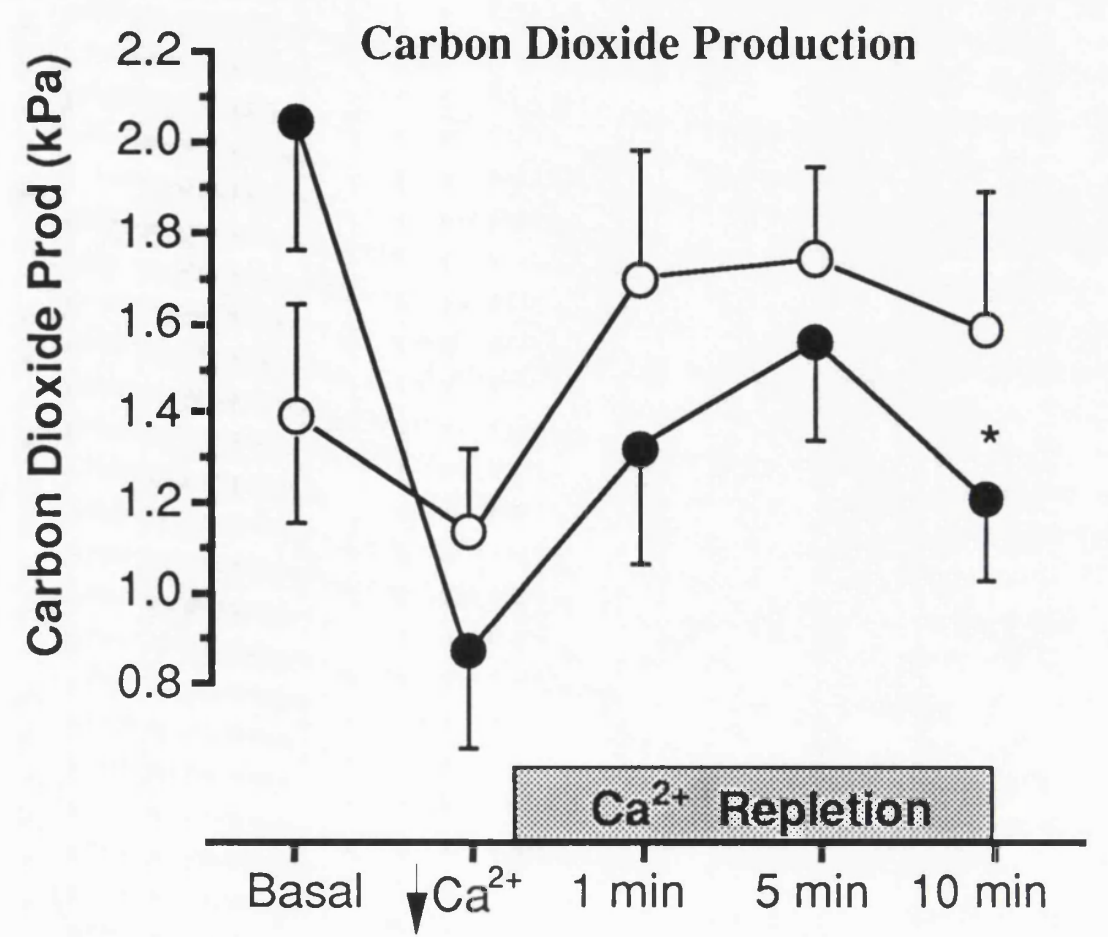

B

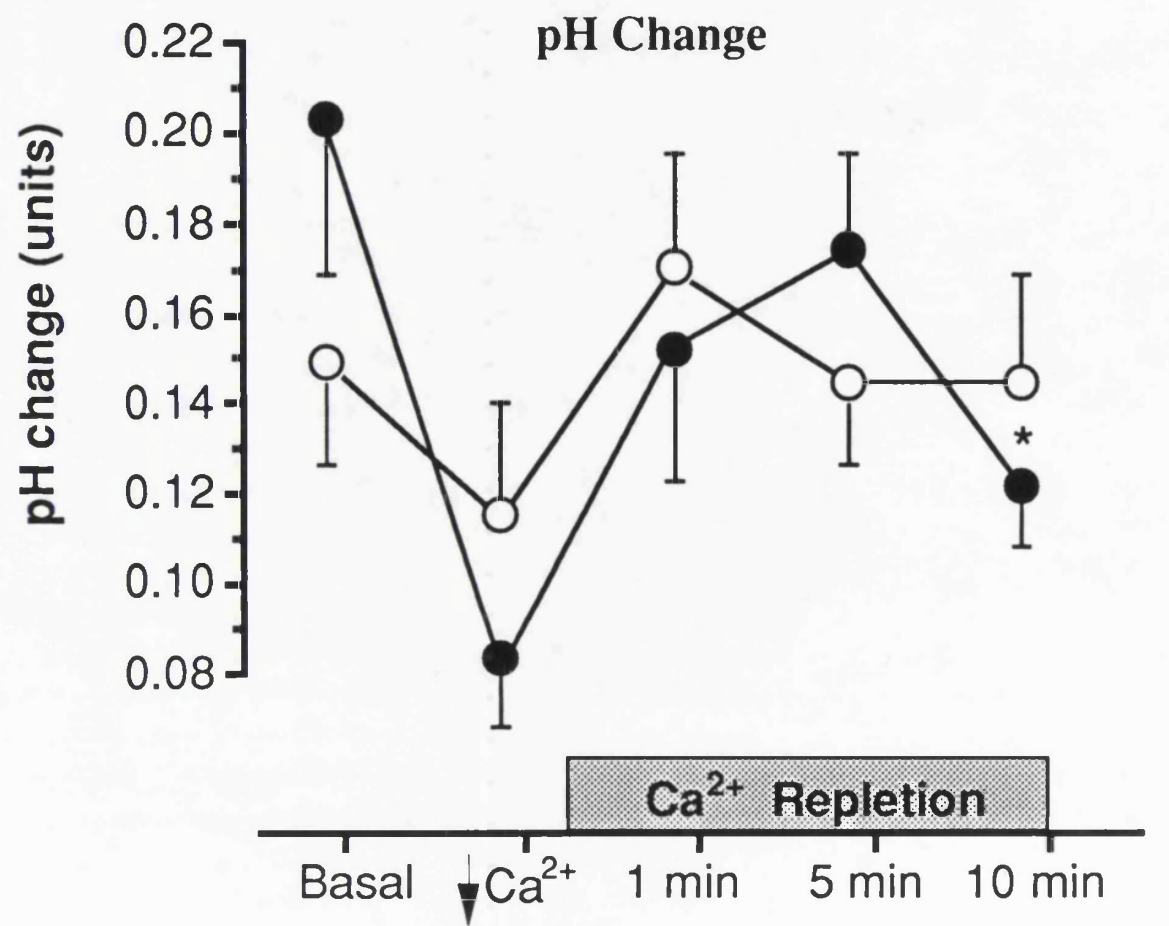
hearts only. ${ }^{*} \mathrm{p} \leq 0.05$ basal $v 10 \mathrm{~min}$ calcium repletion value by paired $\mathrm{t}$-test, $\mathrm{n}=10$ for each group. Bars represent \pm SEM. 
calcium paradox with a pink tinge to the coronary effluent and very high CPK activities.

\subsection{Changes in Action Potential Duration During the Calcium Paradox} (figure 5.7)

Action potential duration measured from onset to $90 \%$ repolarisation $\left(\mathrm{APD}_{90}\right)$ was similar at baseline between groups. In both heat stress and control groups the action potential became markedly prolonged during nominally calcium-free perfusion but shortened on calcium repletion. The assessment of APD became difficult on calcium repletion as amplitude was markedly diminished making accurate assessments difficult. In the two hearts that developed a full calcium paradox action potentials as well as contractile activity ceased on calcium repletion.

\subsection{The Interdependence of Functional and Metabolic Parameters} (figure 5.8)

Graph 5.8A shows the relationship across each heart between change in $\mathrm{pH}$ and change in carbon dioxide tension. The correlation between these two parameters implies that the fall in $\mathrm{pH}$ as perfusate passes through the coronary vasculature is dependant on carbon dioxide content, implying that metabolic acids are not being produced. In turn (graph 5.8B) as expected carbon dioxide production is related to oxygen consumption. Interestingly (graph 5.8D) oxygen consumption correlates extremely well with developed pressure for heat stressed hearts but not for control hearts, this may imply an uncoupling of oxygen consumption and high energy phosphate production in the control group. Graph 5.8C demonstrates that diastolic pressure and coronary perfusion pressure are correlated and reinforces the difficulty experienced in attempting to separate the contribution of the erectile component of coronary artery filling on diastolic tone from the effects of diastolic wall stress on coronary vascular resistance (Vogel et al 1985). 


\section{Appearance of Intracellular Constituents in the Coronary Effluent During the Calcium Paradox}
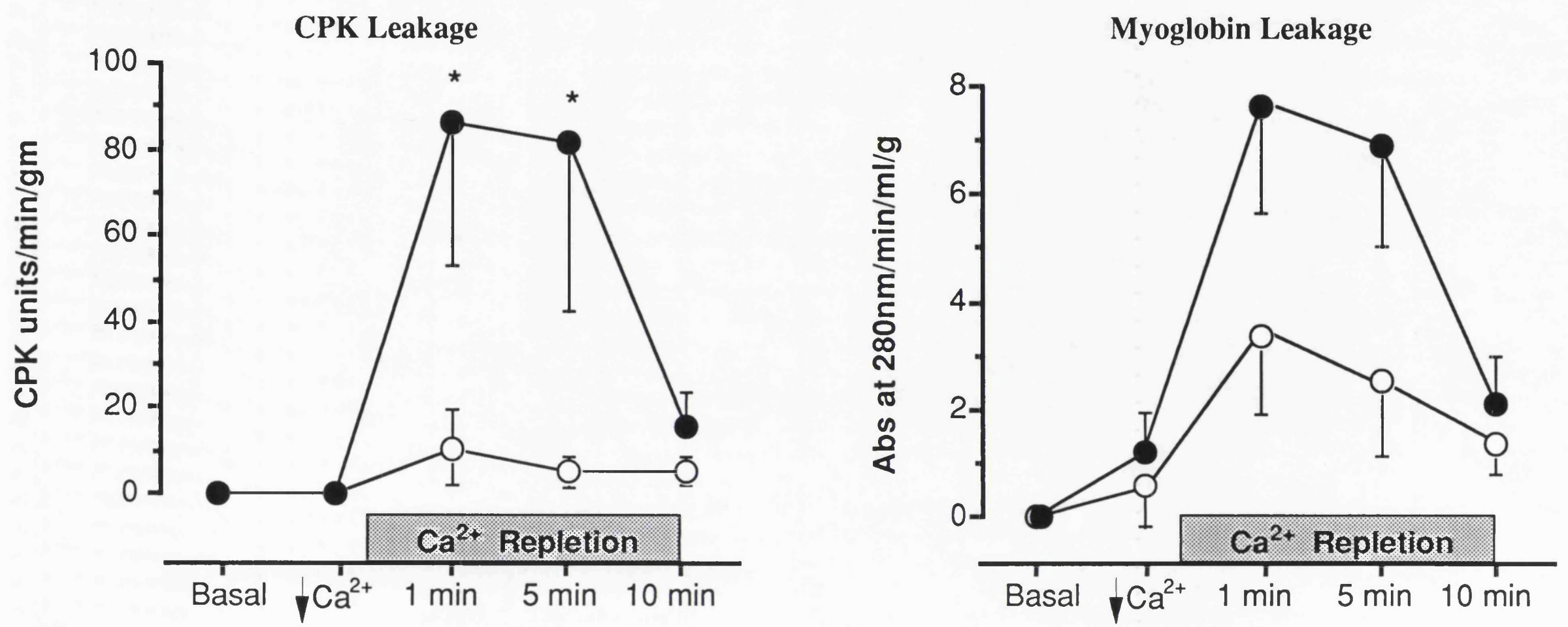

$-\mathrm{O}-$ Heat Stress

- Control

Figure 5.6: The appearance of myoglobin and CPK in the coronary effluent of heat stressed and control rabbit hearts subjected to a submaximal calcium paradox. CPK was measured using a commercial kit, myoglobin release was estimated by measuring the absorption of the coronary effluent at $280 \mathrm{~nm}$. ${ }^{*} \mathrm{p} \leq 0.05$ heat stress $\mathrm{v}$ control groups, comparisons by unpaired $\mathrm{t}$-test, $\mathrm{n}=10$ for each group. Bars represen \pm SEM. 

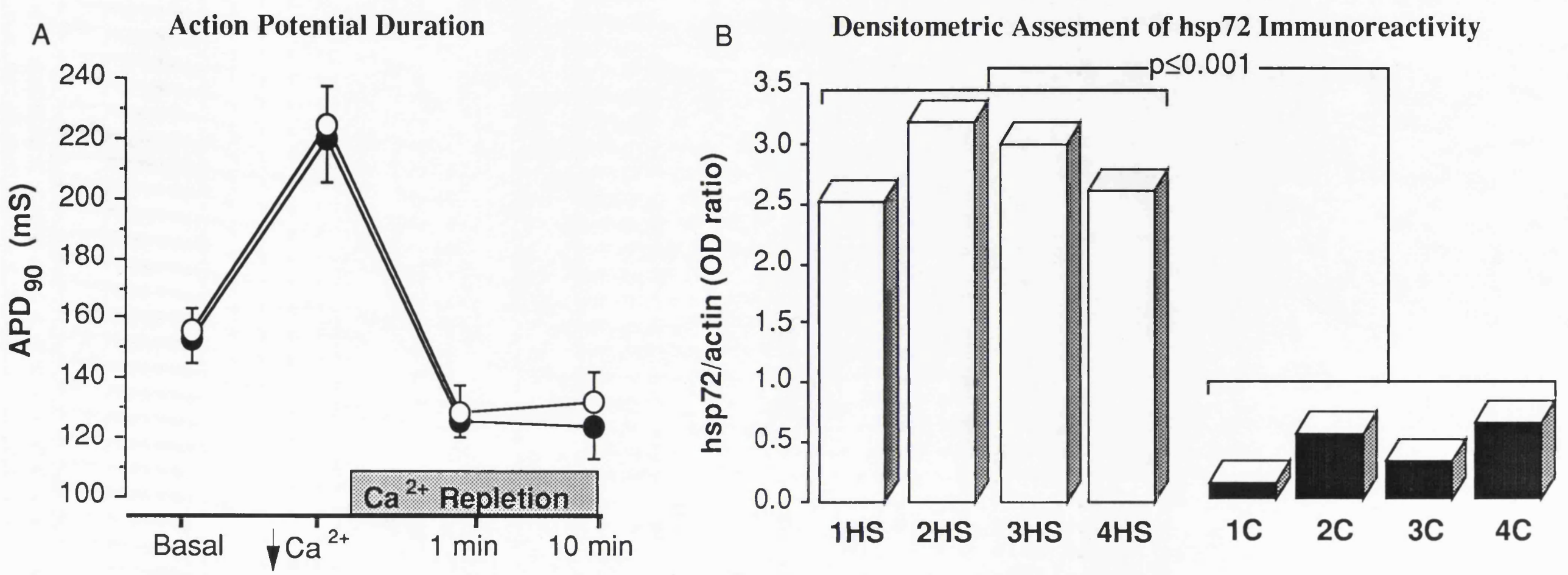

Figure 5.7: Action potential duration and stress protein 72 immunoreactivity. Action potential duration to $90 \%$ repolarisation (APDg0) is similar between groups, $n=10$ for each group at baseline and during nominally calcium-free perfusion and $n=6$ for the control group and $n=8$ for the heat stress group during calcium repletion no significant differences exist between groups. The optical density of the hsp72 band of figure 5.9A was divided by the optical density of the actin band on an identically loaded Coomasie stained gel figure 5.9B. Heat stress followed by 24 hours of recovery was associated with an approximate 8 fold induction of $h \mathrm{sp} 72$. $n=4$ for each group comparison by unpaired $t$-test. 
The Relationships Between Functional and Metabolic Parameters after 10 Minutes of
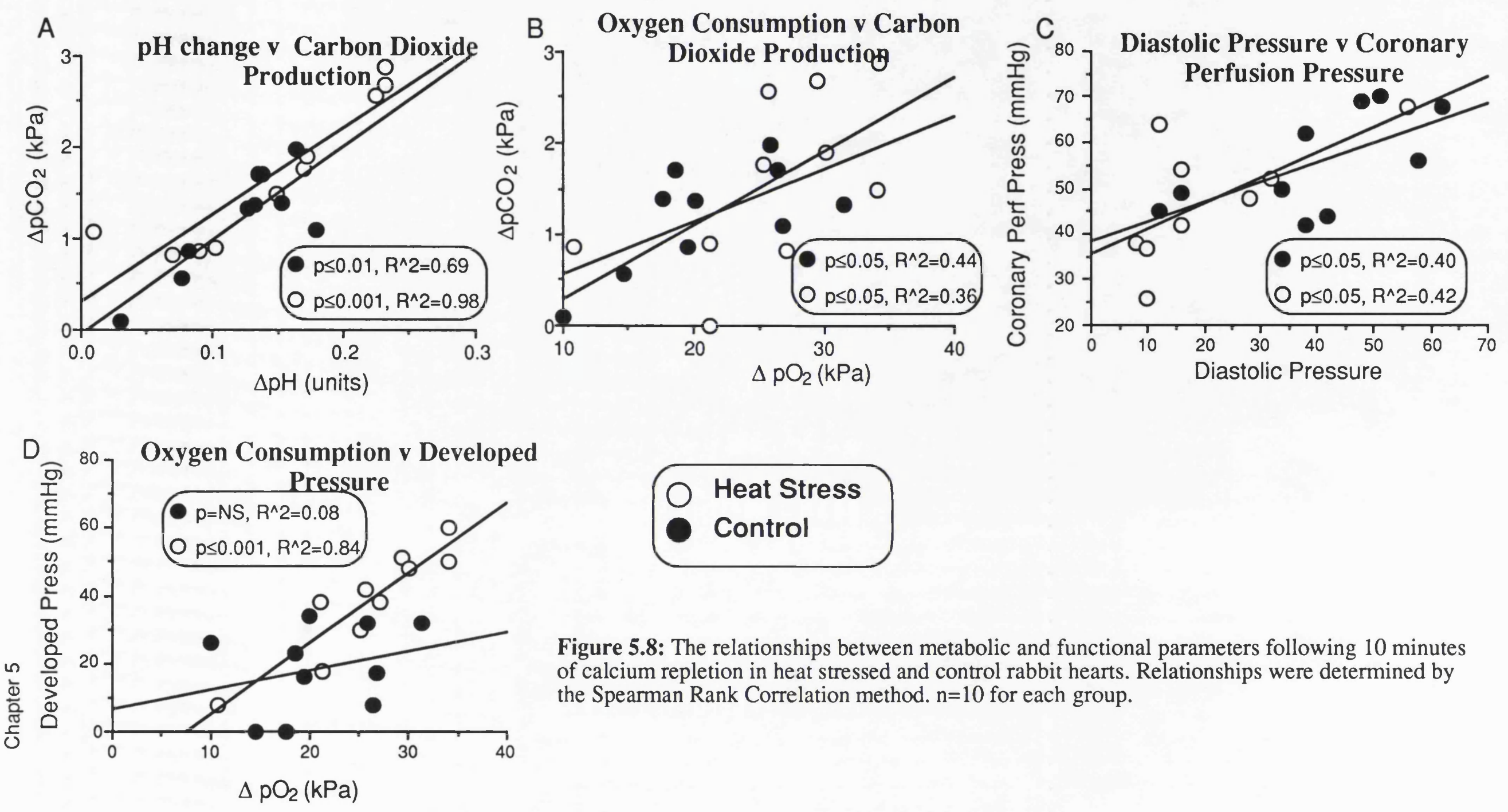

Figure 5.8: The relationships between metabolic and functional parameters following 10 minutes of calcium repletion in heat stressed and control rabbit hearts. Relationships were determined by the Spearman Rank Correlation method. $n=10$ for each group. 


\subsection{Stress Protein Analysis (figures 5.7B, 5.9 and 5.10)}

The inducible $70 \mathrm{kDa}$ stress protein was present at a low level in all control hearts (figure 5.9A), however, heat stress resulted in an approximate 8 fold increase in this level of expression. Formal quantitation by densitometry of immunoblot and correspondingly loaded coomasie stained gel (figure 5.10B) revealed optical density ratios for the control and heat stressed groups of $0.4 \pm 0.1$ and $2.8 \pm 0.2$ respectively $(\mathrm{p}<0.01$ ) (figure 5.7B). In contrast the $60 \mathrm{kDa}$ mitochondrial stress protein was not appreciably induced in the left ventricle 24 hours following whole body heat stress (figure 5.9B).

\section{Discussion}

The results presented indicate that 24 to 26 hours after whole body heat stress hearts become resistant to a submaximal calcium paradox. At this time-point cardiac expression of the $72 \mathrm{kDa}$ stress protein is increased 8 fold. The enhanced resistance to the injury induced by the calcium paradox is reflected by an enhanced contractile performance, less leakage of intracellular constituents and better metabolic performance.

\subsection{The Implication of the Functional and Metabolic Preservation} Following the Calcium Paradox in Heat Stressed Hearts

Prior heat stress increases developed pressure and lessens contracture on calcium repletion. This enhanced contractile function is associated with significantly less leakage of intracellular constituents implying that the protective benefits seen with heat stress involve structural preservation at the level of the myocyte. An observation that is supported by the better coupling of contractile performance with oxygen consumption in the heat stress group.

The enhanced resistance to the injury induced by the calcium paradox is not mediated by enhanced antioxidant defences and suggests that whole body heat stress is capable of attenuating myocardial injury independent of the known increase in endogenous antioxidants. The mechanism of this protection is unknown but it is tempting to postulate that it is secondary to stress protein induction. 


\section{Left Ventricular Stress Protein Content 24 Hours Following Sham or True Heat Stress}

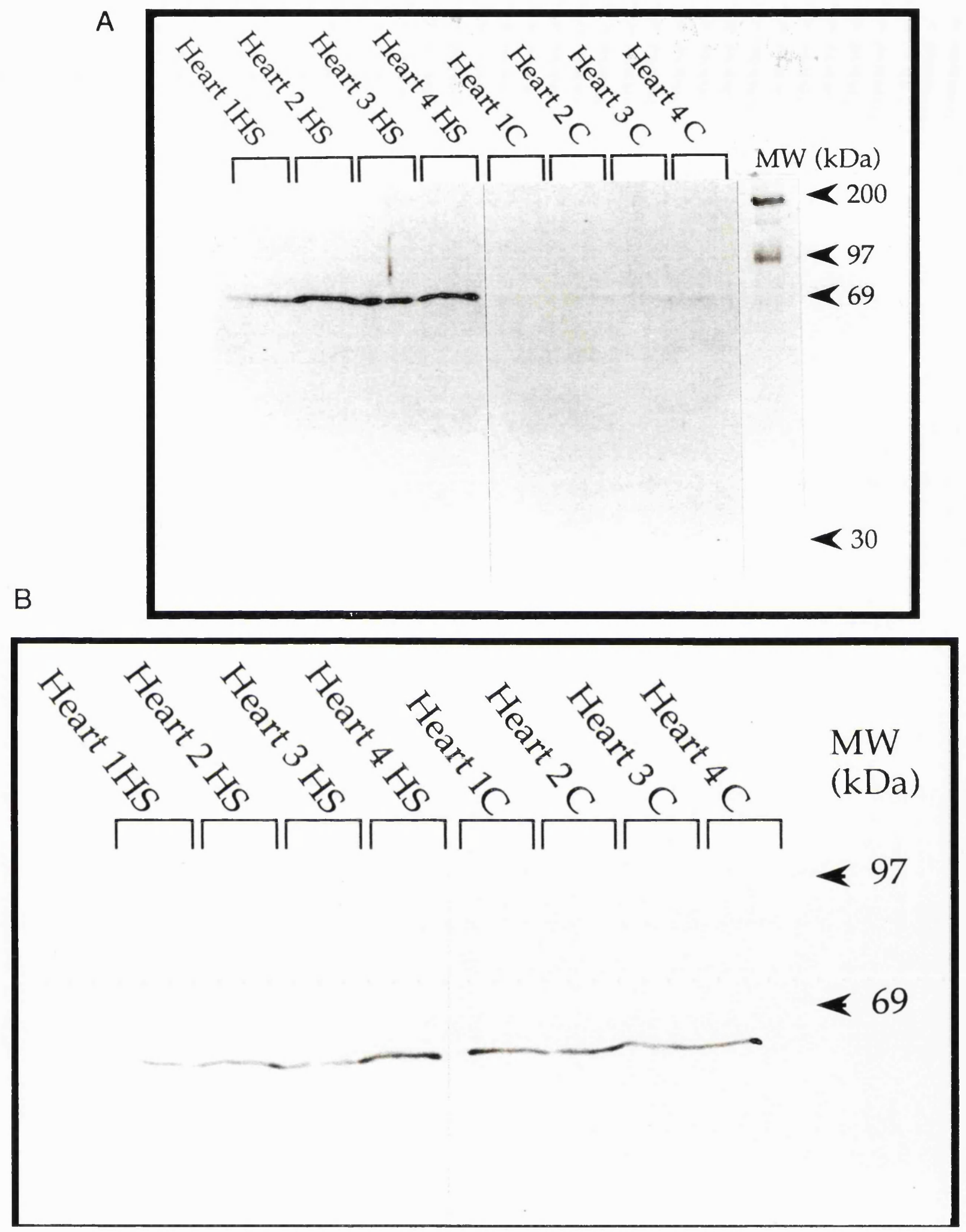

Figure 5.9: Left ventricular hsp 72 and hsp60 immunoreactivity 24 hours following sham and true whole body heat stress. Panel A: Shows hsp72 immunoreactivity with marked induction in the 4 lanes on the left, prepared from hearts harvested 24 hours following heat stress, compared to the 4 lanes on the right, prepared from sham rabbits. Panel B: Shows the hsp60 immunoreactivity, sample loading volumes and lane arrangements are identical to panel A. 


\section{Coomasie Staining of Samples Prepared from Left Ventricular Tissue from Heat Stress and Sham Heat Stress Rabbits}

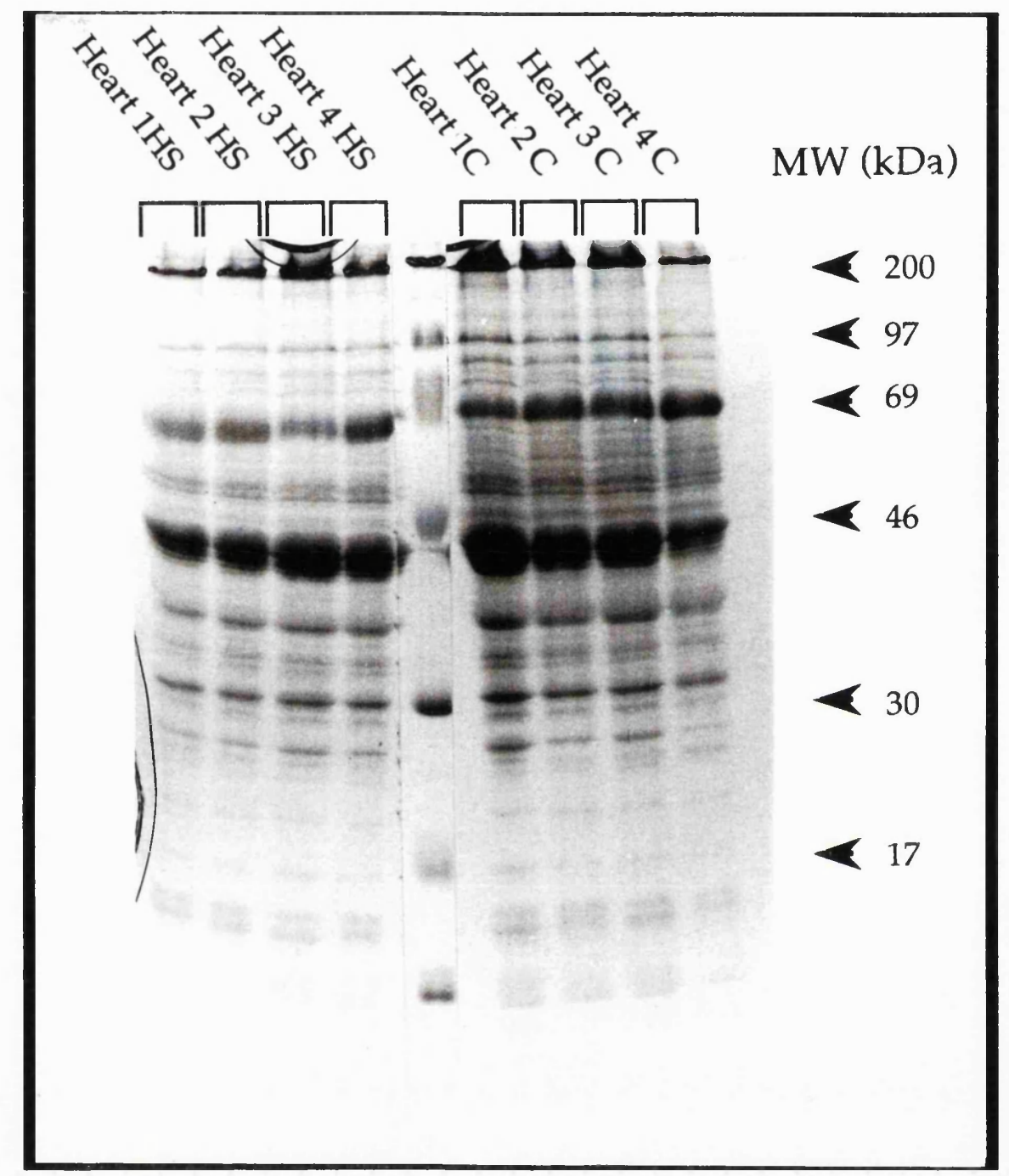

Figure 5.10: Coomasie stain of SDS-Page gel with sample volumes and lane arrangements as depicted in figure 5.9. The Coomasie actin band used to noramlise for slight variations in protein loading is the major band running at approximately $40 \mathrm{kDa}$. Molecular weight markers appear in the middle lane and the numerical values of these markers appears on the right-most portion of the panel. 


\subsection{The Mechanism of Resistance to the Calcium Paradox}

The task of postulating the mechanisms which allow heat stress to attenuate the calcium paradox are necessarily speculative since intense debate surrounds the pathophysiology of this injury (Altschuld et al 1991, Chapman et al 1991, Ruigrok and van Echteld 1991). Any speculation is further confounded by the fact that heat stress has, in addition to its effects on stress protein synthesis and antioxidant activity, other physiological and immunological sequelae that could indirectly influence the heart (Calderwood et al 1989, Gisolfi et al 1991, Huber 1992). However, since in a preliminary report non-thermal means of elevating stress proteins also results in protection against the calcium paradox (Meerson et al 1991a), it is possible that the observations of this study are a direct consequence of stress protein expression, rather than a non-specific effect of heat stress.

Experimental evidence, predominantly in isolated myocytes and small muscle preparations, suggests that the primary event in the induction of the calcium paradox is an influx of sodium during the low calcium perfusion period through L-type calcium channels (Chapman et al 1991). On subsequent re-introduction of calcium, $\mathrm{Na} / \mathrm{Ca}$ exchange results in cellular calcium overload and the damage associated with the calcium paradox. In this model (Chapman et al 1991) the prolongation of the action potential seen during zero calcium perfusion is thought to be a direct result of sodium entry (Tunstall et al 1986). In our experiments $\mathrm{APD}_{90}$ was similarly prolonged in heat stressed and control groups suggesting similar sodium loading during this period. Any difference between these groups must then reside in alterations in the extrusion of cytosolic sodium, for example $\mathrm{Na} / \mathrm{Ca}$ exchange becoming less dominant in heat stressed hearts. Alternatively the consequences of calcium overload may be attenuated in heat stressed hearts. Interestingly it appears that the calcium paradox can be diminished by manoeuvres that reduce mitochondrial calcium uptake (Busselen 1985). We have previously observed a significant reduction in mitochondrial calcium uptake following ischaemia in heat stressed hearts (Yellon et al 1992a) and it may be that heat stress and consequent stress protein-protein interactions are able to decrease the probability of the specific allosteric change necessary to allow mitochondrial pore opening (Crompton et al 1988, Griffiths and Halestrap 1991). 
The other main body of evidence obtained from whole hearts suggests that the damage associated with the calcium paradox is mediated by changes in cytoskeletal and structural proteins of the macula adherens during zero calcium perfusion (Altschuld et al 1991). In this model a structural protein (calcium dependant calhedrin A-CAM) undergoes a conformational change at low calcium concentrations which causes the macula adherens to weaken (Altschuld et al 1991). When contractile activity returns on the repletion of calcium the increase in myocyte fragility results in cell rupture and leakage of intracellular constituents. One could hypothesize that since stress proteins both interact with and constitute the cytoskeleton (Green and Liem 1989, Miron et al 1991) they are able to diminish the degree of sarcolemmal fragility induced by zero calcium perfusion. Further evidence to suggest such an effect is provided by the fact that $\alpha \mathrm{B}$ crystallin, a protein highly homologous to low molecular weight heat stress proteins, is found associated with actin and desmin filaments (Bennardini et al 1992). In addition the physical properties of this association suggest that $\alpha \mathrm{B}$ crystallin has a role in protecting the cytoskeleton during the adverse stresses of ischaemia and temperature elevation (Bennardini et al 1992). Such a process may also limit injury when the myocytes become mechanically loaded on the reintroduction of calcium.

\subsection{Critique of Methods}

In this study although no calcium chelator was used, we did not quantify the calcium concentration in each of our final perfusion solutions. We feel that by perfusing both control and heat stressed hearts with the same solutions any uncertainties due to the presence of small (approximately $6 \mu \mathrm{M}$ ) but variable amounts of calcium do not detract from our findings.

Small changes in the conditions during low calcium perfusion can greatly modify the degree of calcium paradox induced on the re-introduction of calcium (Oksendal 1985). This implies that a submaximal calcium paradox is an unstable form of injury. It is therefore difficult to quantify the degree of protection offered by heat stress in this study, the only possible conclusion being that heat stress has some moderating influence on the processes involved in this form of injury. 
The unstable nature of the injury is probably responsible for the severe degree of injury seen in 2 of the control hearts, if the data are reanalysed excluding these hearts, although differences between means diminish so do standard errors the net result is that statistical significance is unaltered. The hearts have therefore been included in the analysis since they are likely to reflect the extreme end of the spectrum indicating a greater susceptibility to injury in the control group.

The data obtained for oxygen extraction, carbon dioxide production, and $\mathrm{pH}$ change across the heart need to be interpreted with some caution since inferior and superior vena cavae were not ligated and therefore coronary effluent gas exchange with room air was possible in the right atrium, (in practise air is excluded as these structures are collapsed in the isolated heart). In addition variable degrees of myoglobin present in the coronary effluent and the non-linearity of the $\mathrm{pH}$ to $\mathrm{H}^{+}$ion concentration relationship perturb the data.

\subsection{Conclusion}

In conclusion this study demonstrates that heat stress is able to limit the injury associated with a submaximal calcium paradox. The mechanism of this protection is unknown, but we hypothesize that it is a direct effect of stress protein induction and not dependant on a coincident increase in endogenous antioxidant defences. 


\section{Chapter 6}

\section{Results: The Isolated Heart Subjected to Hypoxia and Reoxygenation}

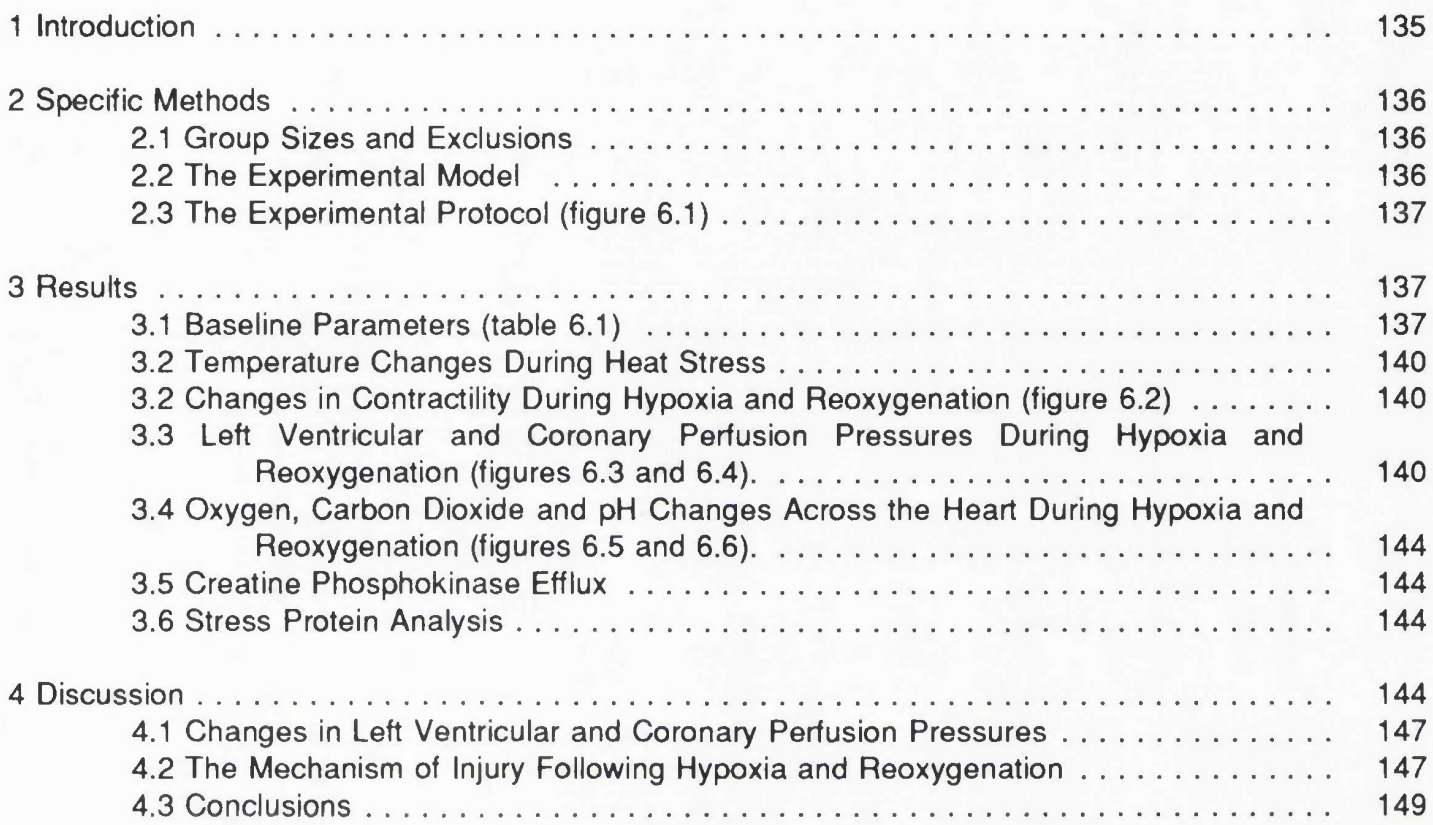

4.1 Changes in Left Ventricular and Coronary Pertusion Pressures . . . . . . . . . . . 147

4.2 The Mechanism of Injury Following Hypoxia and Reoxygenation . . . . . . . . 147

4.3 Conclusions . . . . . . . . . . . . . . . . . . . . . . . . . . . . . . . . . 149

\section{Introduction}

In previous chapters we have demonstrated that 24 hours after whole body heat stress myocardial hsp72 content is increased and at this timepoint the isolated heart or papillary muscle is resistant to a subsequent stress. The mechanism underlying this protection is unknown though an increase in endogenous antioxidant activity has been suggested (Karmazyn et al 1990) and is discussed in preceding chapters.

The evidence that oxidant stress has an important role in the aetiology of lethal myocardial injury is conflicting (see Chapter 1 Section 2.2), in contrast the evidence that oxidant stress is involved in sub-lethal forms of injury such as stunning is compelling. The original observation by Bolli and coworkers (Myers et al 1985) that stunning in the open chest dog can be attenuated by exogenous superoxide dismutase and catalase, has subsequently been confirmed by a number of other independent laboratories (see Bolli 1990 for review). 
Our hypothesis was that if heat stress results in a physiologically meaningful increase in endogenous anti-oxidants then the heat stressed heart should be difficult to stun.

Classically stunning describes a reversible contractile deficit, in the presence of normal coronary flow, occurring following a short ischaemic episode. In pilot studies using the isolated heart we experienced difficulty in maintaining temperature during brief episodes of global ischaemia. In addition since in isolated papillary muscles we were able to demonstrate protection following whole body heat stress against high flow hypoxia, (see Chapter 3 and Chapter 4) we felt a similar model may be appropriate to examine a reversible contractile deficit in the isolated heart.

\section{Specific Methods}

\subsection{Group Sizes and Exclusions}

Sixteen rabbits were used for the purposes of this study, 8 rabbits were heat stressed and 8 rabbits underwent sham heat stress. One rabbit in the sham heat stress group developed sustained ventricular fibrillation on reoxygenation and was excluded from the analysis. The experimental groups therefore comprised of 8 heat stressed and 7 sham heat stressed rabbits. No animals were used for the analysis of stress protein content.

\subsection{The Experimental Model}

Rabbits were heat stressed and sham heat stressed as previously described (see Chapter 2 Section 1.1.2). Twenty-four hours following these procedures animals were sacrificed and hearts removed and retrogradely perfused (see Chapter 2 Section 4.2).

The Langendorff perfusion apparatus has been described previously (Chapter 2 Section 4.3). Hearts were perfused in a constant flow mode with modified Tyrode solution of the following composition $\mathrm{NaCl} 118 \mathrm{mM}, \mathrm{NaHCO}_{3} 24.0 \mathrm{mM}, \mathrm{KCl} 4.0 \mathrm{mM}$, $\mathrm{NaH}_{2} \mathrm{PO}_{4} .2 \mathrm{H}_{2} \mathrm{O} \quad 0.4 \mathrm{mM}, \mathrm{CaCl}_{2} 1.30 \mathrm{mM}, \mathrm{MgCl}_{2} \cdot 6 \mathrm{H}_{2} \mathrm{O} 1.0 \mathrm{mM}$, Glucose $10 \mathrm{mM}$, Pyruvate 2mM; sodium pyruvate from Sigma Chemicals, Missouri, USA; all other reagents ANALAR grade from BDH Chemicals, Poole, UK). The substrate free Tyrode solution was prepared identically with $10 \mathrm{mM}$ choline chloride substituted for 
glucose and pyruvate. For the purposes of this experiment two oxygenation reservoirs were used one containing pre-filtered (excluding particles above $2 \mu \mathrm{m}$ ) substrate free Tyrode's solution equilibrated with $95 \% \mathrm{~N}_{2} / 5 \% \mathrm{CO}_{2}$ and the other containing prefiltered Tyrodes with substrate equilibrated with $95 \% \mathrm{O}_{2} / 5 \% \mathrm{CO}_{2}$.

Hearts were paced at $180 / \mathrm{min}$ via the right atrium. Left ventricular and coronary perfusion pressures were measured throughout the protocol whilst at specific timepoints superfusate was sampled to measure changes in $\mathrm{pH}$, oxygen and carbon dioxide content across the heart and creatine phophokinase leakage (see Chapter 2 Section 4.3).

\subsection{The Experimental Protocol (figure 6.1)}

Initially experiments were performed with hearts perfused with hypoxic substrate free Tyrode solution for a 30 minute period. In these experiments the hypoxic reservoir remained covered and oxygen tensions were reduced to below $8 \mathrm{kPa}$. The resulting injury was severe with irreversible contracture and creatine phosphokinase leakage. The experimental conditions were therefore altered by shortening the hypoxic perfusion period and increasing the oxygen content in the hypoxic buffer by uncovering the reservoir used to equilibrate with the $95 \% \mathrm{~N}_{2} / 5 \% \mathrm{CO}_{2}$ gas mixture.

Following a 15 minute stabilisation period perfusate was switched via a three way tap to hypoxic substrate free Tyrode's solution. Hearts were perfused with this hypoxic buffer for a 20 minute period before reoxygenation and return of substrates. After a 55 minute reoxygenation period hearts were removed blotted dry and weighed immediately and after drying overnight at $70^{\circ} \mathrm{C}$ in a hot air oven.

\section{Results}

\subsection{Baseline Parameters (table 6.1)}

Rabbit weights, heart wet and dry weights, basal coronary flow, coronary flow normalised for heart weight and oxygen tension during hypoxic perfusion were all similar between groups. 


\section{: Experimental Protocol for Hypoxia/Reoxygenation of the Isolated Rabbit Heart}

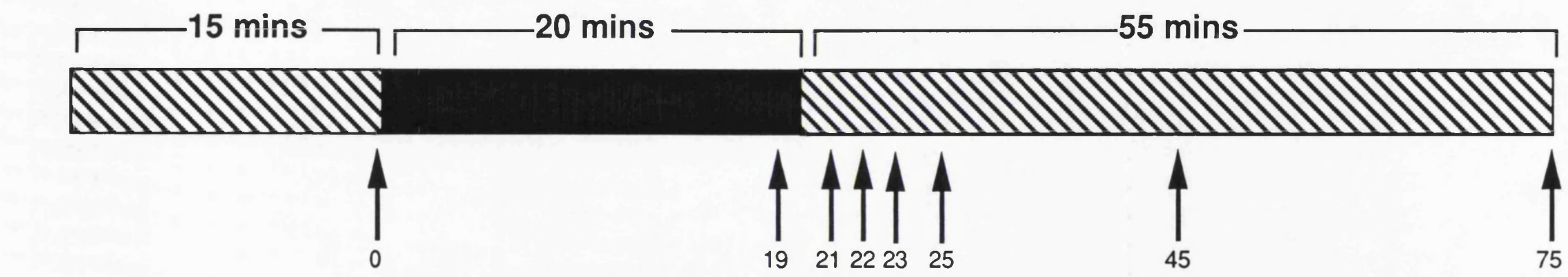

MIIIV = Perfusion with Oxygenated Tyrode's Solution

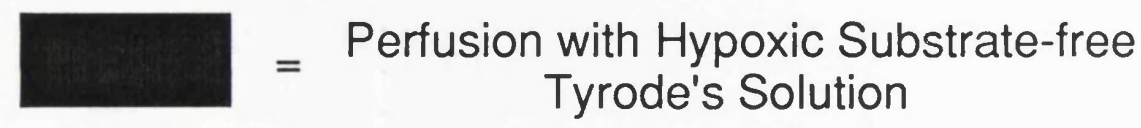

$\Lambda=$ Timepoints in minutes for recording left ventricular and coronary perfusion pressures. Perfusate gases were measures at $0,19,25,45$ and 75 mins. 


\section{Characteristics of Isolated Rabbit Hearts Undergoing Hypoxia and Reoxygenation}

\begin{tabular}{l|c|c|c} 
& Heat Stress (n=8) & Control (n=7) & t-test \\
\hline Rabbit Weight (kg) & $2.56 \pm 0.1$ & $2.53 \pm 0.08$ & $p=N S$ \\
*Heart Wet Weight (g) & $8.58 \pm 0.47$ & $8.92 \pm 0.61$ & $p=N S$ \\
*Heart Dry Weight (g) & $1.48 \pm 0.14$ & $1.60 \pm 0.16$ & $p=N S$ \\
Heart Wet/Dry Weight (units) & $6.10 \pm 0.30$ & $5.80 \pm 0.51$ & $p=N S$ \\
Basal Coronary Flow (ml/min) & $43.3 \pm 0.6$ & $43.7 \pm 2.1$ & $p=N S$ \\
Coronary Flow/g w.wt & $5.15 \pm 0.3$ & $5.10 \pm 0.50$ & $p=N S$ \\
†Oxygen Tension During Hypoxia & $17.1 \pm 0.6$ & $18.2 \pm 1.4$ & $p=N S$
\end{tabular}

TABLE 6.1:

*Weights were determined at the end of the experimental protocol

$\dagger$ Measured in perfusate taken from aortic cannula at 19 minutes of hypoxia

Values shown represent means \pm standard error.

Comparisons between control and heat stress groups were made by two-tailed unpaired t-test. 


\subsection{Temperature Changes During Heat Stress}

Basal rectal temperature was similar between sham heat stress $\left(39.1 \pm 0.01^{\circ} \mathrm{C}\right)$ and heat stress $\left(39.3 \pm 0.02^{\circ} \mathrm{C}\right)$ groups. The temperature in the heat stress group reached $42^{\circ} \mathrm{C}$ after $56.8 \pm 2.5$ minutes and the actual peak temperature was $42.2 \pm 0.0^{\circ} \mathrm{C}$.

\subsection{Changes in Contractility During Hypoxia and Reoxygenation (figure} 6.2)

Figure 6.2 shows the physiological traces obtained from a sham heat stress heart. During hypoxic perfusion diastolic left ventricular pressure increased and the heart failed to pace (19 minutes), on reoxygenation contracture partially reversed, pacing captured and developed pressure increased progressively. The example shown, underwent more marked changes during hypoxia than was typical for the group, the $\mathrm{pO}_{2}$ at the end of hypoxia was $12 \mathrm{kPa}$, well below the group mean. Interestingly the marked increase in diastolic pressure was not accompanied by the expected concomitant increase in coronary perfusion pressure.

\subsection{Left Ventricular and Coronary Perfusion Pressures During Hypoxia and Reoxygenation (figures 6.3 and 6.4).}

The changes in left ventricular pressure are shown in figures 6.3A and 6.3B. The apparent differences in left ventricular developed pressure on reoxygenation are in part a result of the baseline developed pressure being greater in the heat stress hearts. If the data are replotted normalised with respect to baseline developed pressure, the differences become much less marked (see figure 6.4A) and only exist in the first 5 minutes of reoxygenation, ultimate recovery being very similar between groups. When the measurements of diastolic and coronary perfusion pressures are normalised significant differences are apparent during hypoxia but are lost after 2 minutes of reoxygenation. Difficulties exist with the normalisation of diastolic pressure since a spuriously low basal value will perturb subsequent values and may artificially elevate the normalised diastolic pressure at later timepoints. 
Example of Experimental Trace During Hypoxia/Reoxygenation

$100 \mathrm{mmHg}$

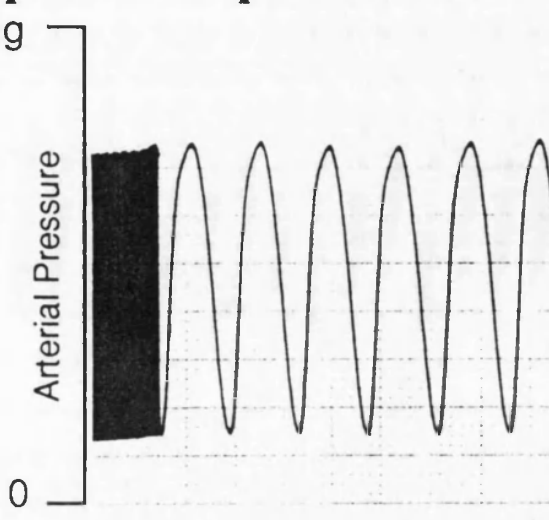

$100 \mathrm{mmHg}$
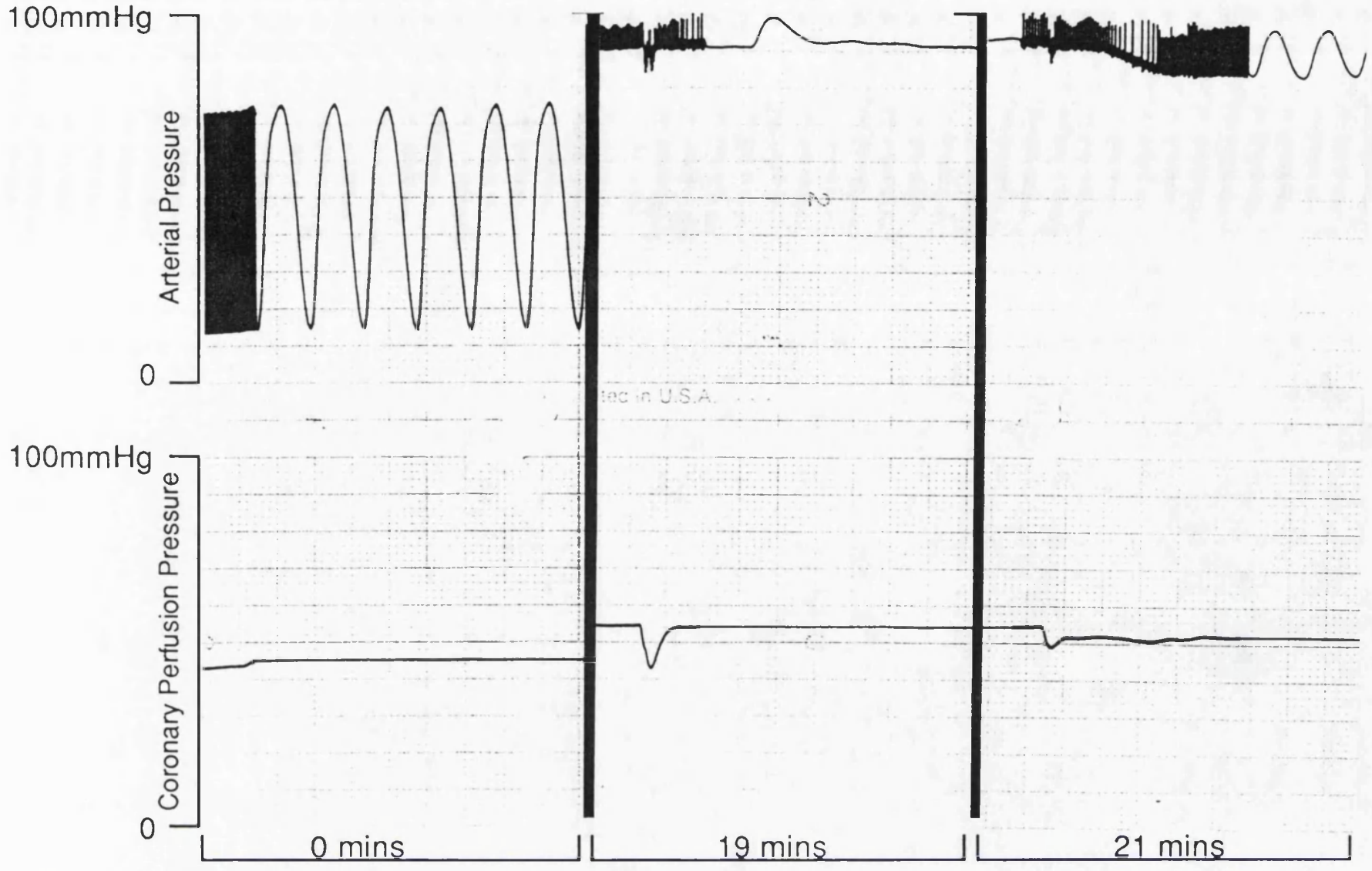

$100 \mathrm{mmHg}$
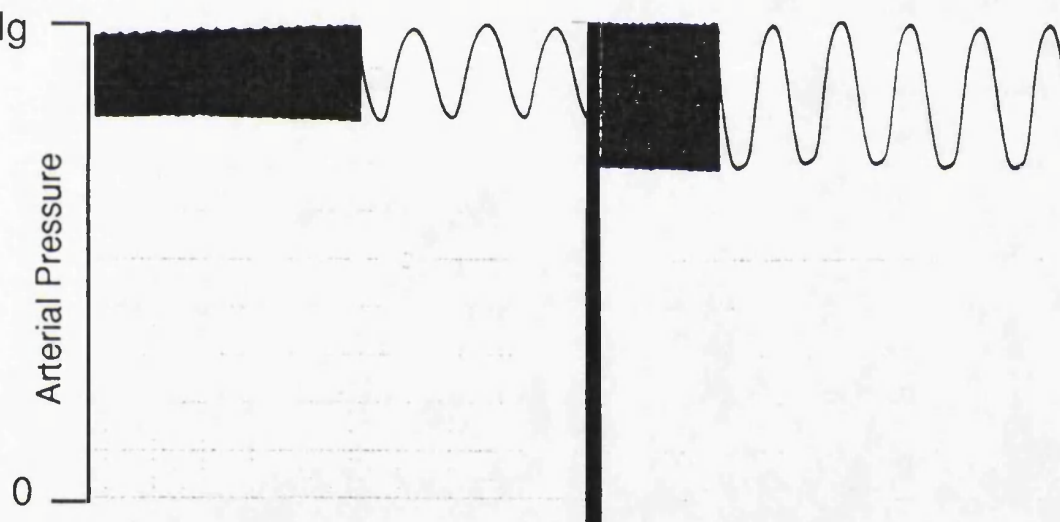

$100 \mathrm{mmHg}$

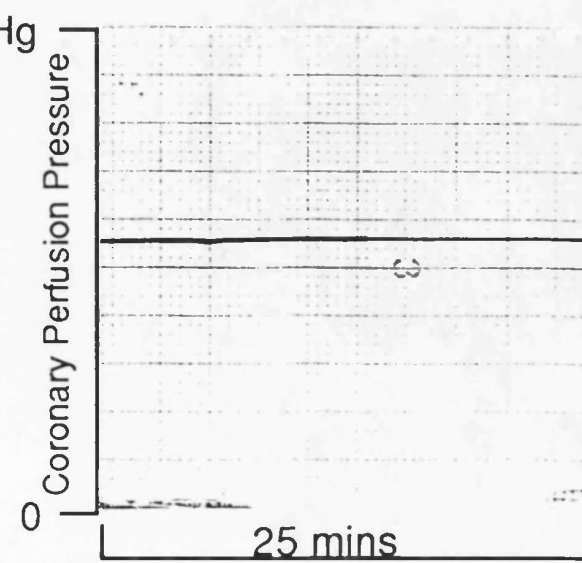

Figure 6.2: The appearance of the left ventricular and mean coronary perfusion pressure traces during the hypoxia and reoxygenation protocol. This example from a sham heat stress heart shows marked contracture during hypoxia ( 0 to 20 minutes) with a rise in diastolic pressure. Intermittent failure to capture occurred towards the end of the hypoxic period. Developed pressure slowly recovered during reoxygenation to approximately $80 \%$ of baseline value. 


\section{Developed, Coronary Perfusion and Diastolic Pressures During Hypoxia and Reoxygenation in the Isolated Heart}
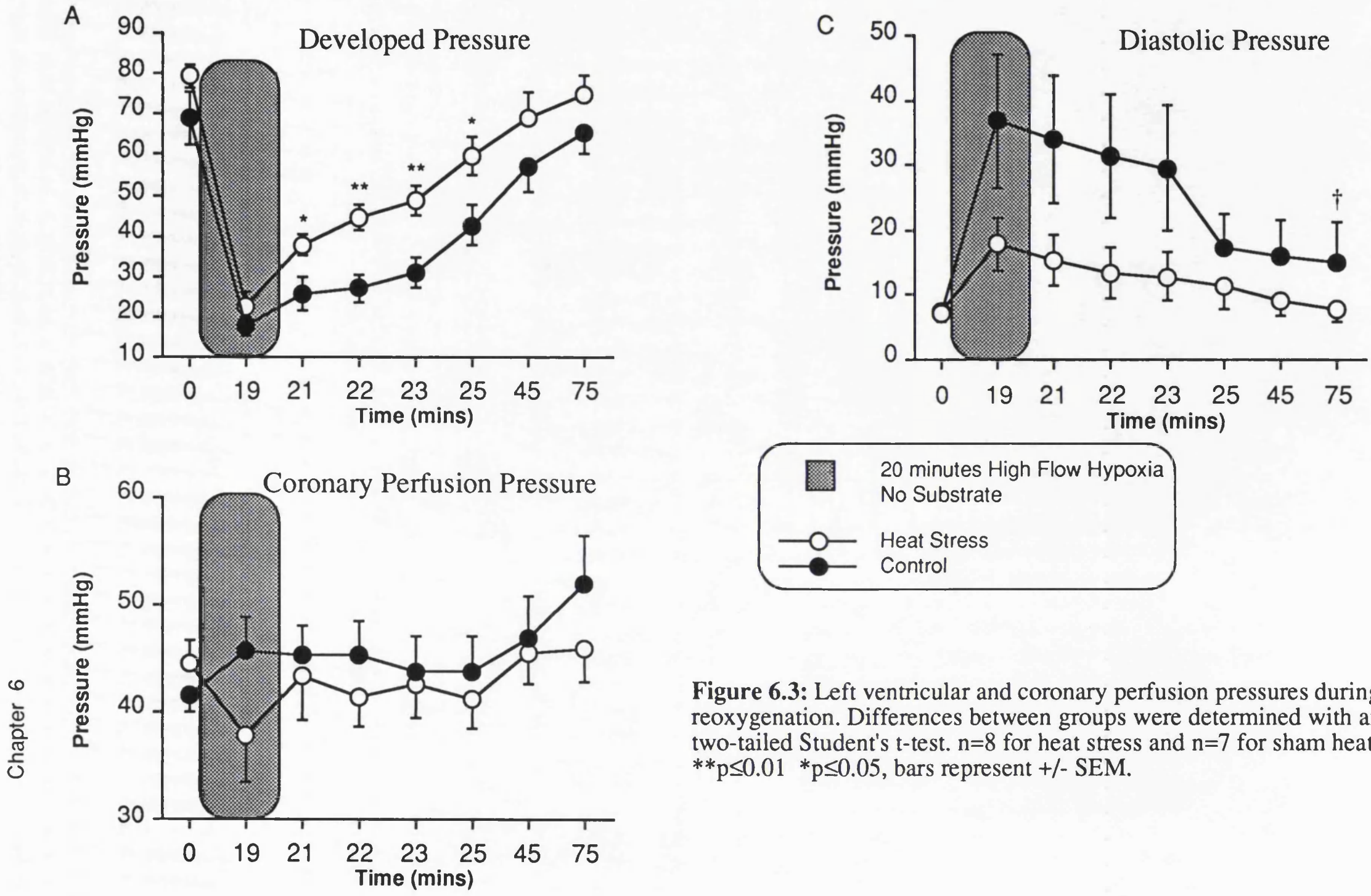

Figure 6.3: Left ventricular and coronary perfusion pressures during hypoxia and reoxygenation. Differences between groups were determined with an unpaired two-tailed Student's t-test. $n=8$ for heat stress and $n=7$ for sham heat stress groups. ${ }^{* *} \mathrm{p} \leq 0.01{ }^{*} \mathrm{p} \leq 0.05$, bars represent $+/$ - SEM. 


\section{Normalised Developed, Coronary Perfusion and Diastolic Pressures During Hypoxia}
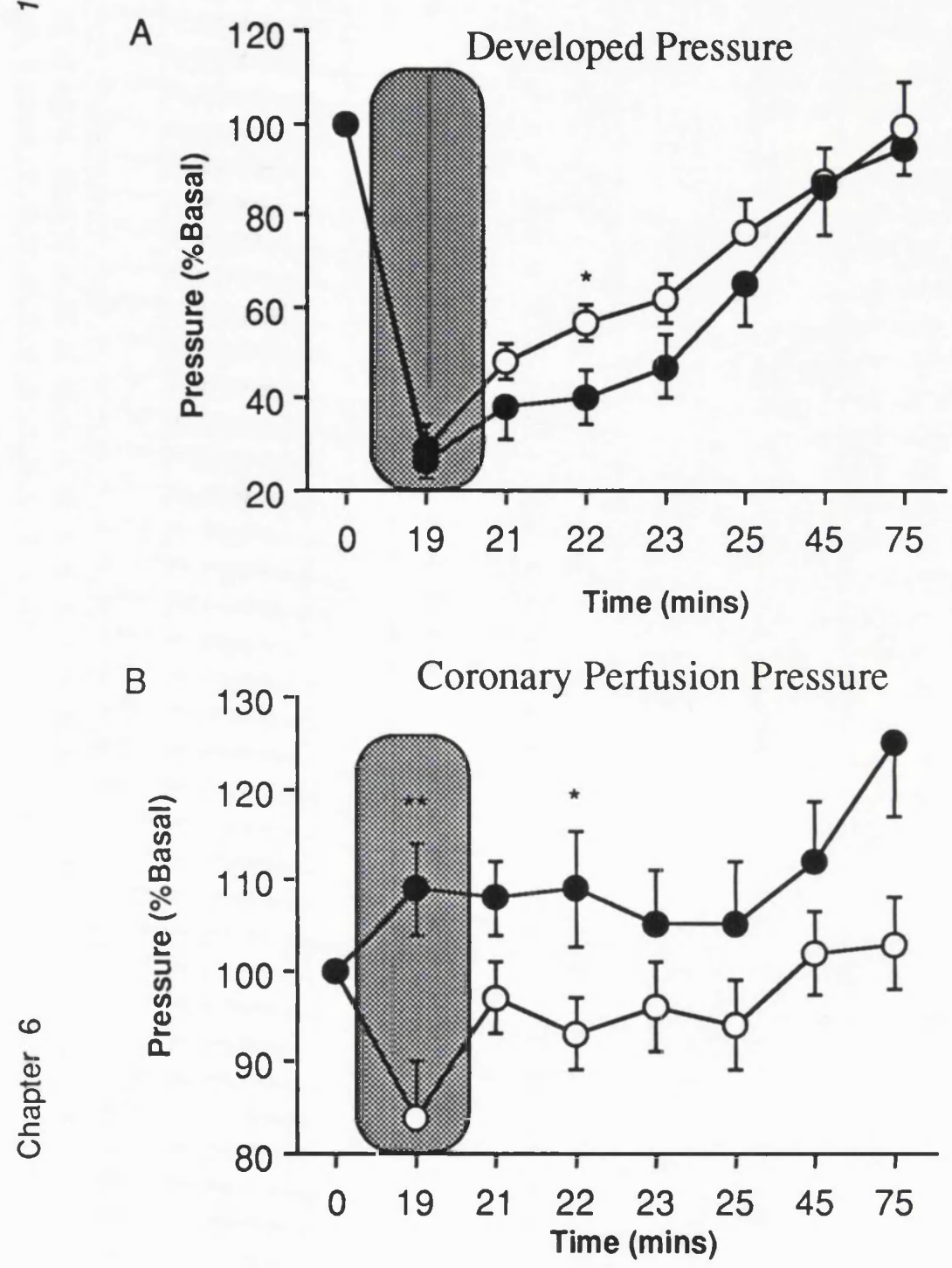
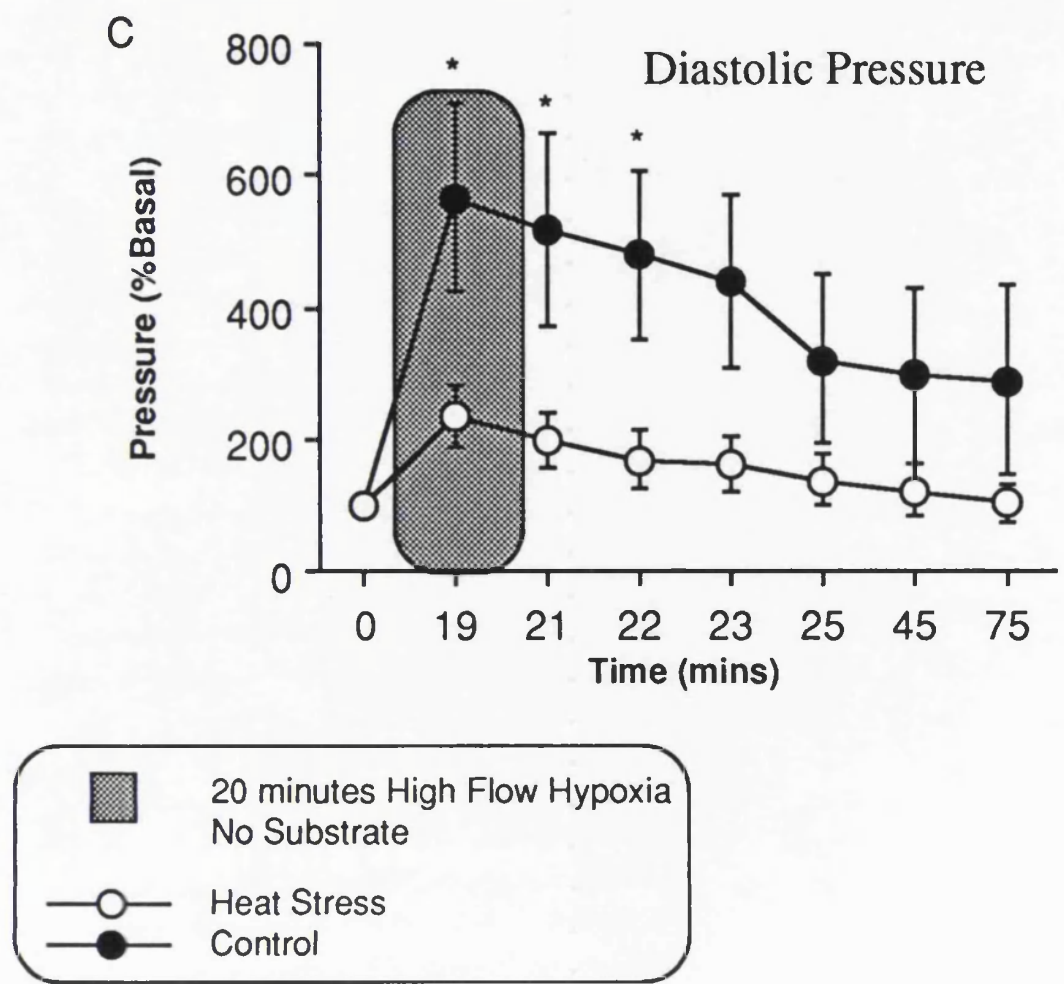

Figure 6.4: Normalised left ventricular and coronary perfusion pressures during hypoxia and reoxygenation. Values are expressed as a percentage of the pressure recorded at 0 mins. Differences between groups were determined with an unpaired two-tailed Student's t-test. $n=8$ for heat stress and $n=7$ for sham heat stress groups. ${ }^{* *} \mathrm{p} \leq 0.01{ }^{*} \mathrm{p} \leq 0.05$. Bars represent $+/-$ SEM. 
3.4 Oxygen, Carbon Dioxide and pH Changes Across the Heart During Hypoxia and Reoxygenation (figures 6.5 and 6.6).

No significant differences exist between groups with respect to $\mathrm{pH}$, carbon dioxide or oxygen changes across the heart. Carbon dioxide production continues during hypoxia in both groups suggesting that oxygen tensions are sufficiently high to support aerobic metabolism. This is supported by the finding of a positive relationship between oxygen tension and carbon dioxide production (figure 6.6). The degree of hypoxia strongly correlates with the rise in diastolic pressure in the heat stress group but not in the sham heat stress group. This lack of correlation maybe secondary to the method used to normalise diastolic pressure or indicate that diastolic pressure in this group rose inappropriately in relation to the degree of hypoxic injury. This later interpretation is supported by the fact that diastolic pressure in the sham group also increased inappropriately in relation to the fall in developed pressure (compare figures $6.3 \mathrm{~A}$ with $6.3 \mathrm{C}$ and $6.4 \mathrm{~A}$ with $6.4 \mathrm{C}$ ).

\subsection{Creatine Phosphokinase Efflux}

No creatine phosphokinase was detected in any of the coronary effluent samples examined (the effluents from the 4 hearts with the worst recovery of contractile function were examined). This implies that the hypoxic insult was mild and did not result in myocyte disruption.

\subsection{Stress Protein Analysis}

Contemporaneous hearts were not prepared for stress protein analysis. However the heat and sham heat stress procedures were identical to those used throughout the thesis and hence similar induction of hsp72 is expected.

\section{Discussion}

This study used a mild insult of high flow hypoxia without substrate followed by reoxygenation to induce a contractile deficit without irreversible myocyte injury. Unfortunately the results are inconclusive and their interpretation complex. No significant differences are observed between heat stress and control groups after the 


\section{Carbon Dioxide Production and pH and Oxygen Change During Hypoxia and Reoxygenation}
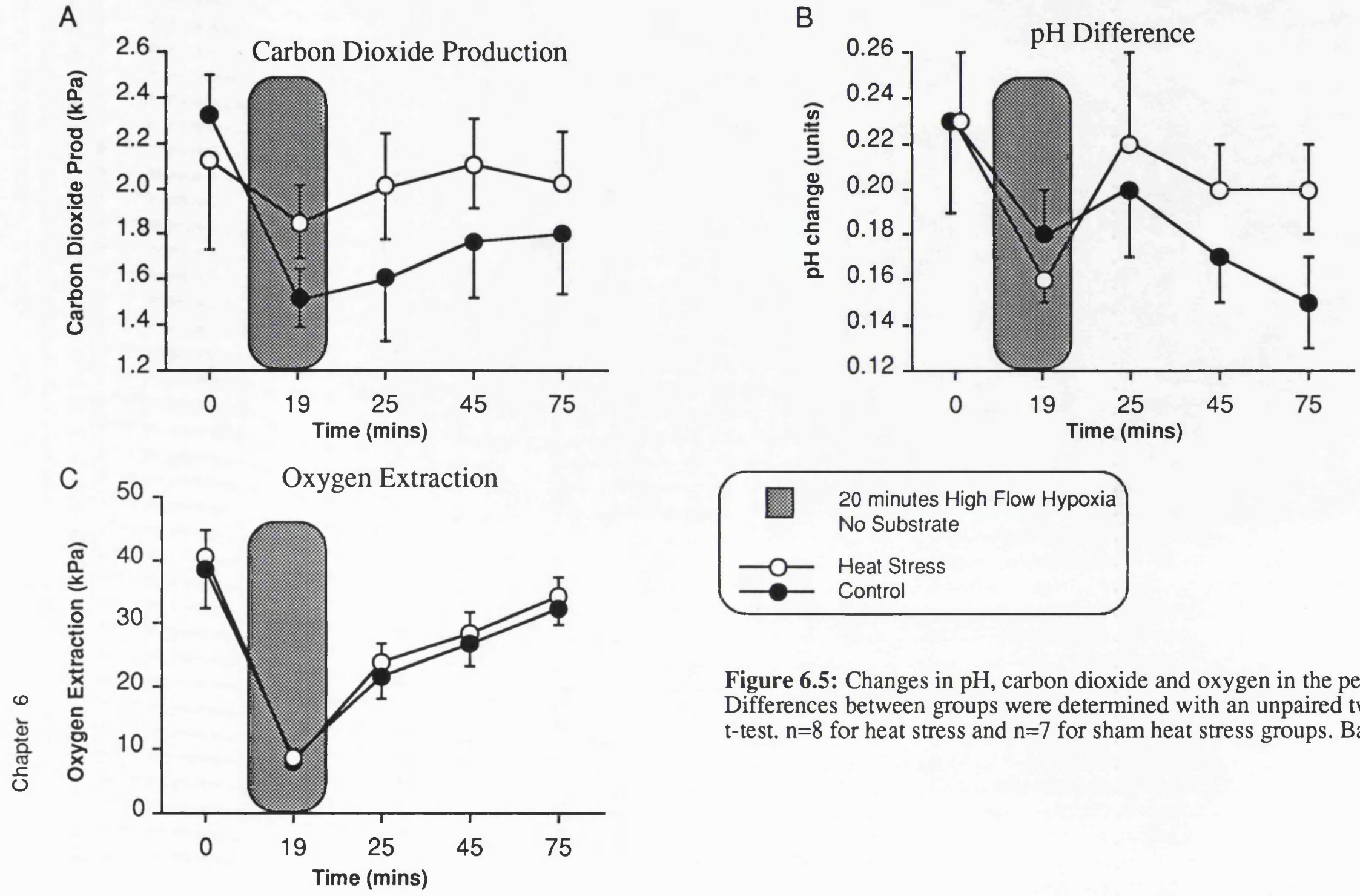

Figure 6.5: Changes in $\mathrm{pH}$, carbon dioxide and oxygen in the perfusate across the heart. Differences between groups were determined with an unpaired two-tailed Student's $t$-test. $n=8$ for heat stress and $n=7$ for sham heat stress groups. Bars represent $+/$ - SEM. 

Production and Diastolic Pressure
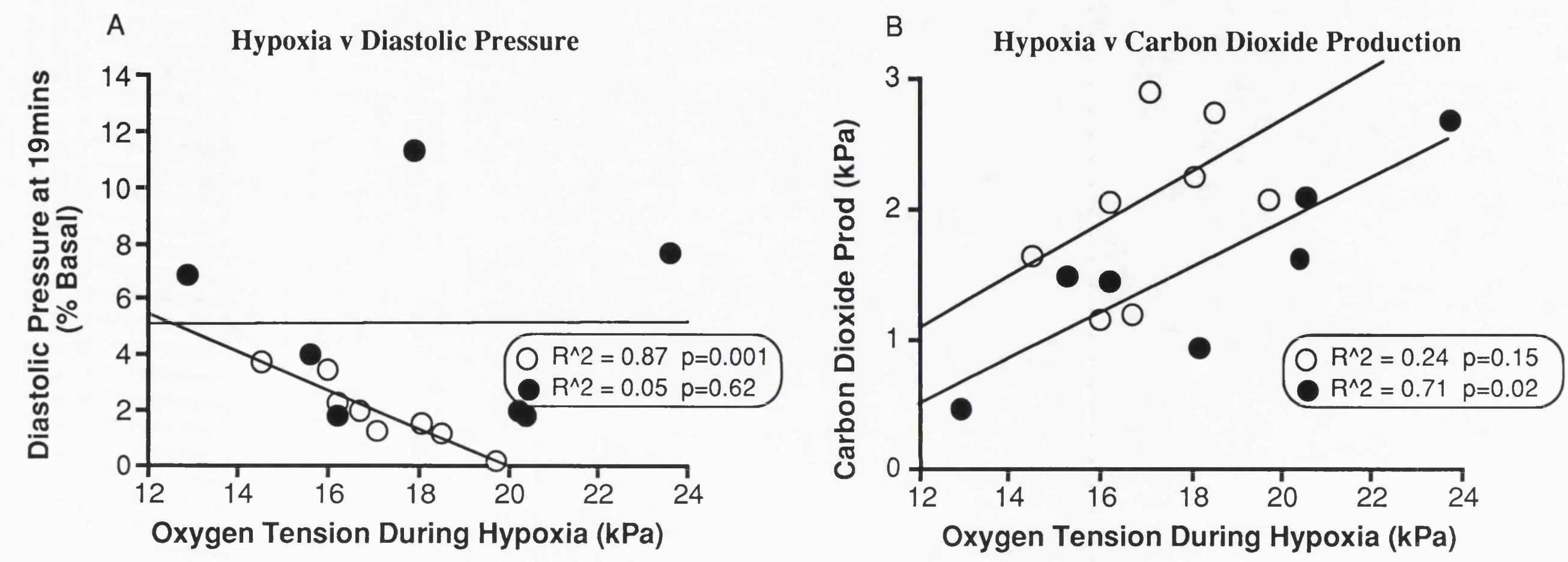

Figure 6.6: The relationship between oxygen tension, contracture and carbon dioxide production after 19 minutes of hypoxia. Relationships were determined by the Spearman Rank Correlation method. $n=8$ for heat stress group and $n=7$ for the sham heat stress group. 
first 3 to 4 minutes of reoxygenation, suggesting that the differences that do exist before this timepoint are not of biological relevance.

\subsection{Changes in Left Ventricular and Coronary Perfusion Pressures}

When the data are normalised no convincing differences exist in developed or coronary perfusion pressures between heat stress and sham groups. The contractile deficit was obtained under constant flow conditions without the need to account for the confounding effects of microvascular stunning or no reflow. Surprisingly the increase in diastolic pressure in the sham group during hypoxia and following reoxygenation is greater than in the heat stress group. However this rise is not associated with an increase in coronary perfusion pressure, ordinarily these pressures are interdependent (see Chapter 5 figure 5.8C and Vogel et al 1985), nor is the rise related to the degree of hypoxia, as occurs in the heat stress group (figure 6.6A). In addition, with the mild degree of injury induced without any creatine phophokinase efflux, one would not expect irreversible rigor crossbridges to form and therefore diastolic pressure should return to baseline during reoxygenation, this did not occur in the sham group. These problems tend to cast some doubt on the relevance of the diastolic pressure rise. In summary there is probably no meaningful difference in the pressures recorded between heat stress and sham heat stress groups.

\subsection{The Mechanism of Injury Following Hypoxia and Reoxygenation}

The injury occurring upon reoxygenation following severe hypoxia is also known as the oxygen paradox, the term being based upon the paradoxical increase in diastolic pressure and enzyme efflux occurring on reoxygenation. Figure 6.7 shows a trace obtained from a preliminary protocol using a longer and more severe hypoxic perfusion period of 30 minutes. In this trace there is no return of contractile activity and diastolic pressure rises upon reoxygenation. It has been proposed that the aetiology of this injury is secondary to the oxidant stress associated with reoxygenation since enzyme efflux can be diminished and contractile activity enhanced by exogenously administered antioxidants (Duncan 1990). In addition other investigators have demonstrated that lipid peroxidation (Gauduel $Y$ et al 1989) and other evidence of free radical stress (Park et al 1991) occurs in this form of injury. It 

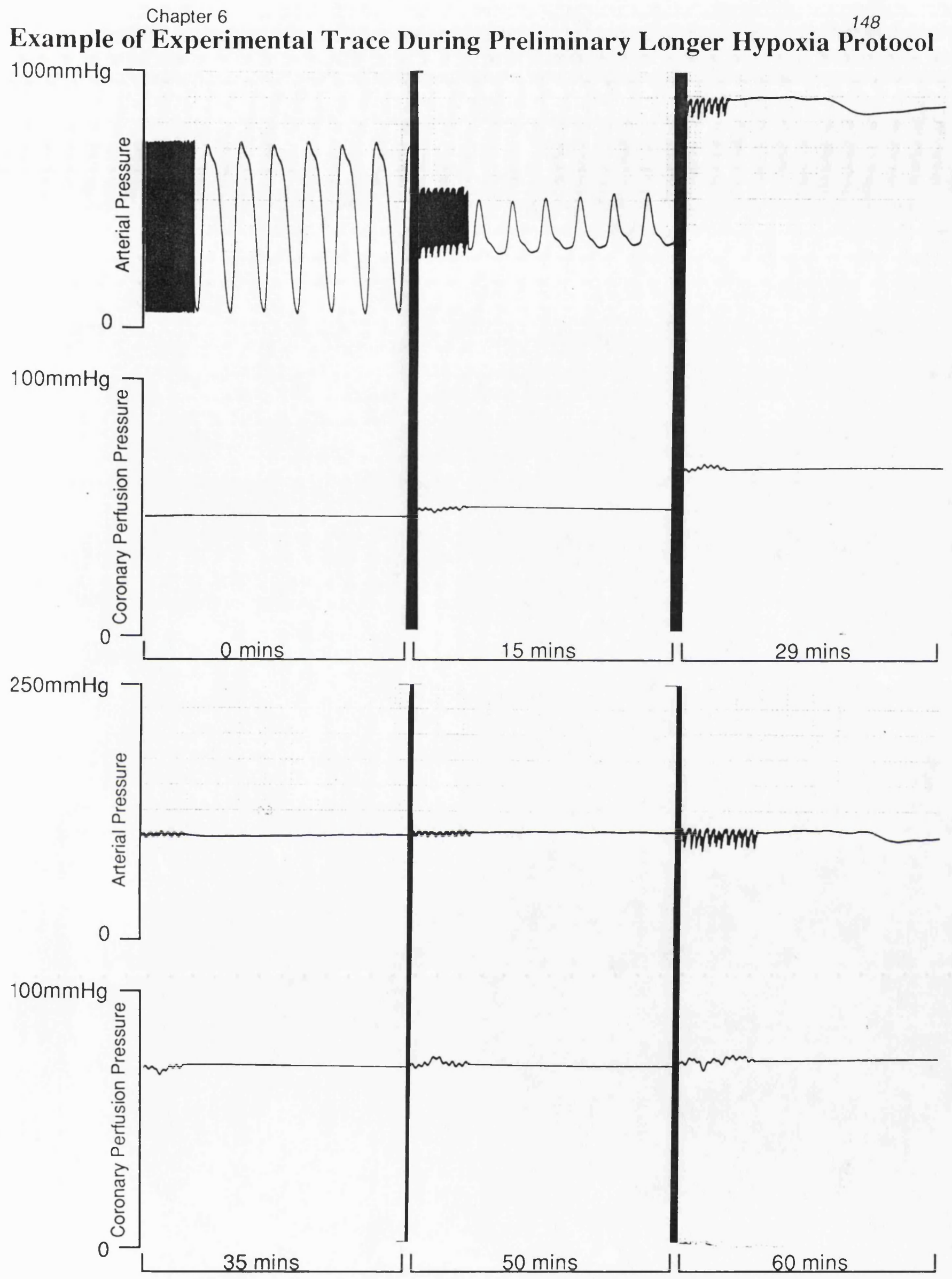

Figure 6.7: The appearance of the left ventricular and mean coronary perfusion pressure traces during a preliminary, longer, 30 minute hypoxia and reoxygenation protocol. This example from a sham heat stress heart shows marked contracture and complete loss of contractile activity between 15 and 30 minutes of hypoxia. Contractile activity fails to return despite an increase in pacing pulse amplitude and duration during reoxygenation. 
is likely that the mild degree of injury seen in this Chapter has similar underlying mechanisms, and hence a relevant increase in endogenous antioxidants should have resulted in protection. However, this interpretation is complicated by contradictory findings suggesting that oxygen centred free-radicals are not involved in the oxygen paradox. For example Van der Heide et al (1987), were unable to show protection following exogenous dimethylthiourea or allopurinol, whilst the protection seen following mannitol was attributed to its effect on reducing cell swelling. Similarly, Darley-Usmar et al (1989) and Timerman et al (1990) were unable to show a shift in oxidised to reduced glutathione in a comparable model. Because of these uncertainties the inability of prior heat stress to protect against this form of injury cannot be used to infer that heat induced increases in endogenous anti-oxidants are of no biological significance.

\subsection{Conclusions}

The results of this study are difficult to interpret but there is probably no meaningful protection following whole body heat stress against a mild form of hypoxia and reoxygenation injury that causes a reversible contractile deficit. These observations suggest that although whole body heat stress confers protection against lethal forms of myocardial injury the same may not be true for more gentle forms of injury not associated with myocyte death. 


\section{Chapter 7}

\section{Results: Infarct Size Following Stress Protein Elevation by Sublethal Heat Stress or Ischaemia.}

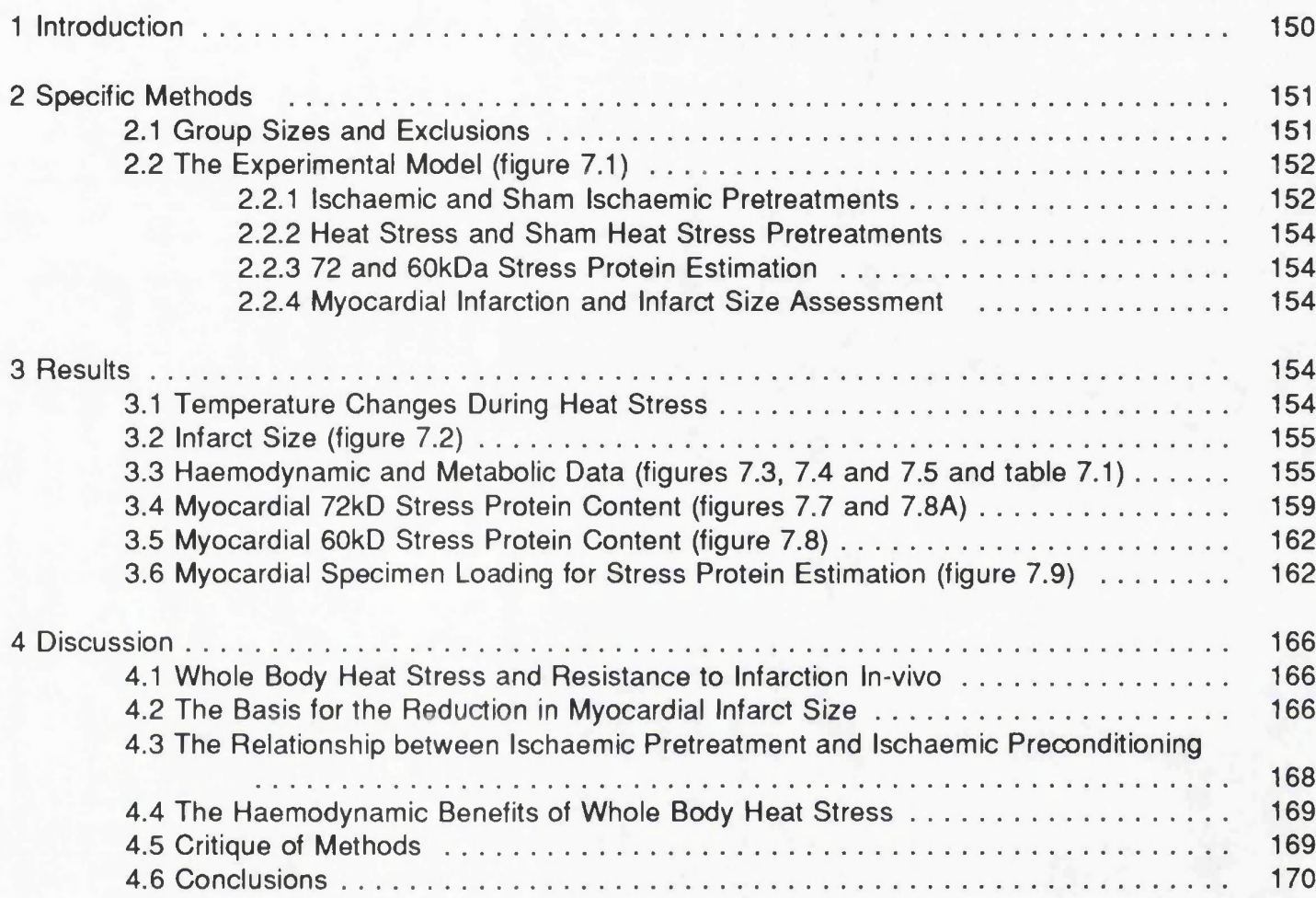

\section{Introduction}

A wealth of evidence suggests that whole body heat stress confers tolerance to ischaemia in-vitro (see Chapter 1 Section 6.1.1 and Chapter 1 Section 6.1.2). Previous chapters of this thesis using differing in-vitro preparations and endpoints have shown similar protection following whole body heat stress. However, the situation in-vivo is less clear since with infarct size as the endpoint, Yellon et al (1992b) were unable to show protection from a 45 minute coronary occlusion of the in situ rabbit heart following whole body heat stress. Furthermore this group of investigators have suggested that the explanation for these disparate findings in-vivo and in-vitro is that whole body heat stress causes extra-cardiac effects that tend to negate the protective benefit seen in-vitro. This conclusion was supported by the observations that infarction was reduced in an isolated rabbit heart harvested from a 
heat stressed rabbit but retrogradely perfused by blood from a non-heat stressed rabbit, but that this protection was abolished if a similar heart was perfused by blood from a heat stressed rabbit (Walker et al 1993). A possible explanation for this deleterious aspect to whole body heat stress is a lymphocyte reaction to stress proteins expressed within the myocyte (Huber 1992).

The purpose of this chapter was to raise stress proteins specifically within the myocardium by sublethal ischaemia and non-specifically by whole body heat stress, the protective benefits of both forms of pretreatment could then be compared using an in-situ infarct size model. The observations cited (Yellon et al 1992b, Walker et al 1993) would suggest that specific myocardial stress protein elevation may offer potential benefits over non-specific elevation by overcoming the deleterious aspects of whole body heat stress.

An established protocol (Knowlton et al 1991a) of repetitive short coronary artery occlusions was used to selectively elevate myocardial stress protein content. Such brief coronary occlusions, unlike thermal stress, are a physiologically relevant stimulus for myocardial stress protein induction having been likened to the clinical situation during unstable angina (Knowlton et al 1991a).

\section{Specific Methods}

\subsection{Group Sizes and Exclusions}

For the purposes of this study a total of 67 New Zealand White rabbits, (2.0$3.0 \mathrm{~kg}$ ) were divided into four experimental groups. Within each group the end-point was either stress protein estimation or infarct size assessment (see protocol, figure 7.1). Experiments were performed sequentially between the groups, except when an experimental exclusion necessitated repetition. Haemodynamic, temperature and arterial blood gas data from experiments that were excluded were not used for subsequent analysis.

Group 1, ischaemic pretreatment, consisted of 15 rabbits. There was 1 exclusion due to respiratory obstruction and death 2 hours following repetitive ischaemia. Four rabbits were used for stress protein estimation and 10 rabbits for infarct size assessment.

Group 2, sham ischaemic pretreatment, consisted of 21 rabbits with 7 
exclusions. In 3 rabbits coronary ligation was unsuccessful, (no risk area evident after microsphere injection), 2 rabbits failed to reperfuse adequately, (as judged by a large area of infarction without intra-myocardial haemorrhage), and 2 animals died during the 30 minute coronary ligation secondary to hypotension and progressive acidosis. Four rabbits were used for stress protein estimation and 10 for infarct size assessment.

Group 3, heat stress, consisted of 16 rabbits, with 2 exclusions. One rabbit died during the 30 minute coronary ligation and 1 experiment had to be excluded because of inadequate staining of viable myocardium with triphenyltetrazolium. Ten rabbits were used for infarct size assessment and 4 rabbits for stress protein estimation.

Group 4, sham heat stress, consisted of 15 rabbits. There was 1 exclusion because of inadequate tissue staining. Ten rabbits were used for infarct size assessment and 4 rabbits for stress protein estimation.

\subsection{The Experimental Model (figure 7.1)}

The study protocol consists of 2 intervention groups with respective controls. Sublethal ischaemic pretreatment consisted of brief repetitive periods of ischaemia followed by 24 hours of recovery, the sham ischaemic group being treated identically but without ischaemia. The preparation for the heat stress and sham heat stress group was identical to previous chapters. Twenty-fours after pretreatments rabbits were either sacrificed, for cardiac stress protein estimation, or after infarct size assessment following a 30 minute coronary occlusion.

\subsubsection{Ischaemic and Sham Ischaemic Pretreatments}

Rabbits in the ischaemic pretreatment group were anesthetised, orally intubated, ventilated and ECG recorded as previously described (Chapter 2 Section 5.2.1). A marginal ear vein was cannulated to administer fluids and drugs and $30 \mathrm{mg}$ of intramuscular amoxycillin was given prophylactically to all rabbits.

The exposure of the heart and isolation of a coronary artery were also as previously described (Chapter 2 Section 5.2.2). Following four, 5 minute coronary ligations separated by 10 minutes of reperfusion the sternotomy was closed, rabbits allowed to breathe spontaneously, and eventually extubated (see Chapter 2 Section 5.2.2 for a more detailed description). 
Protocol for the Assessment of the Stress Protein Response and Resistance to Ischaemia following Thermal and Ischaemic Pretreatments

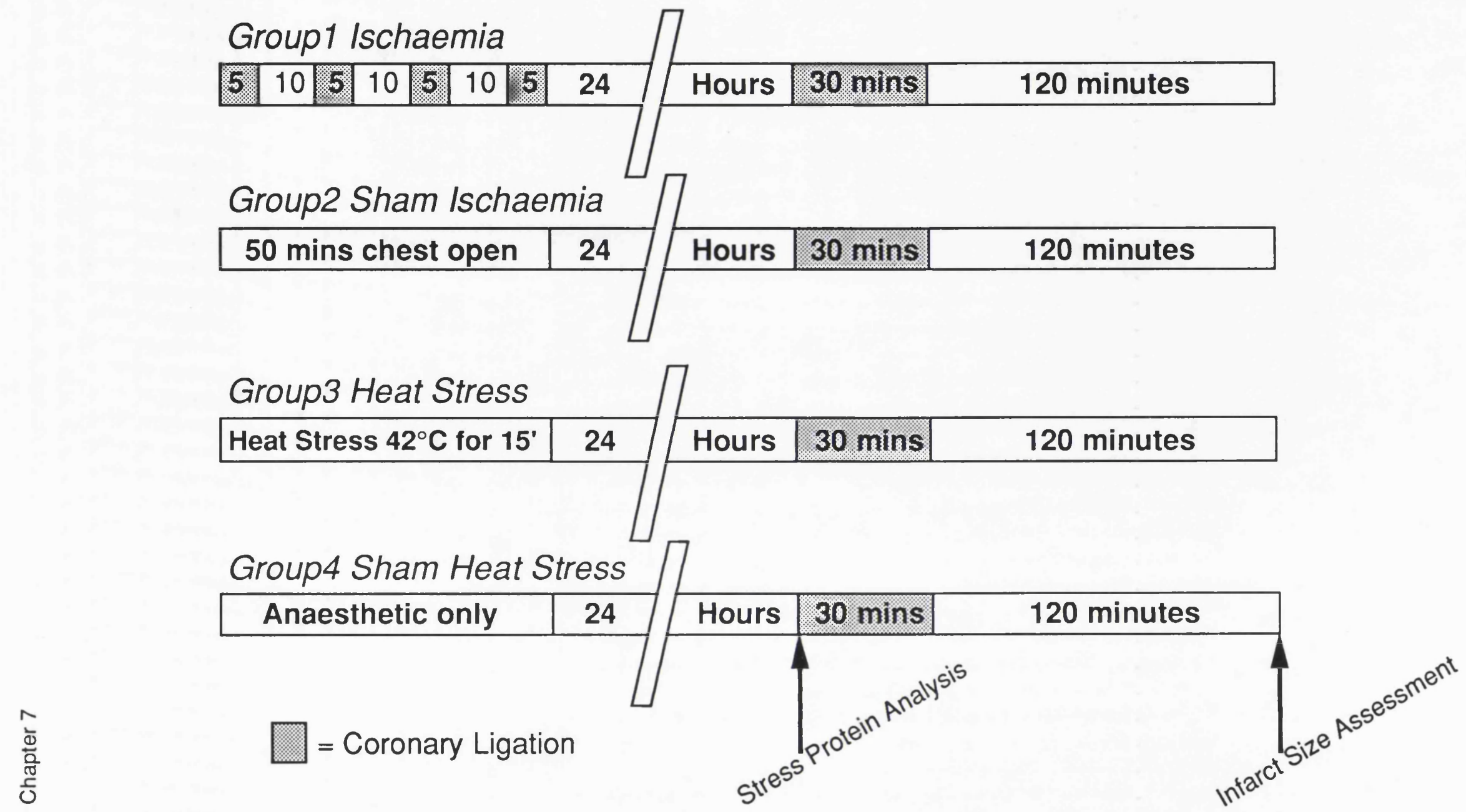

Figure 7.1: Protocol for the measurement of stress protein and infarct size following sublethal ischaemic and thermal pretreatments. In all treatment groups animals are recovered for 24 hours prior either to stress protein estimation or infarct size assessment. 
The surgical preparation for the sham ischaemic pre-treatment group was identical to that described above without coronary artery isolation or ligation (see Chapter 2 Section 5.2 for a more detailed description).

\subsubsection{Heat Stress and Sham Heat Stress Pretreatments}

The whole body heat stress and sham heat stress procedures were identical to those used in previous chapters and described in Chapter 2 Section 1.1.2.

\subsubsection{2 and 60kDa Stress Protein Estimation}

Twenty-four hours following each of the 4 pre-treatment interventions, rabbits were re-anaesthetized and hearts removed for stress protein determination (see figure 7.1) using the methods described previously (Chapter 2 Section 2). In the ischaemic pretreatment group, following mounting on the Langendorff rig, the coronary tie was retightened and Coomasie Brilliant Blue R250 dye (BDH chemicals, Poole, England) introduced into the coronary perfusate. The area without dye (risk zone) was separated from stained (perfused) myocardium and this tissue used for stress protein estimation. In the sham ischaemic pretreatment group the left ventricular apex was taken for analysis (this corresponds to the area of ischaemic pretreatment in most cases). In the heat stress and sham heat stress groups the whole left ventricle was homogenised for stress protein estimation.

\subsubsection{Myocardial Infarction and Infarct Size Assessment}

Approximately twenty-four hours after the above pretreatments, animals were re-anaesthetized and infarct size measured following a 30 minute coronary occlusion and 120 minutes of reperfusion (see Chapter 2 Section 5.3.1 and Chapter 2 Section 5.3.2).

\section{Results}

\subsection{Temperature Changes During Heat Stress}

Prior to warming there was no significant difference between basal rectal temperature within sham or heat stress groups $\left(39.1 \pm 0.1^{\circ} \mathrm{C} n=14\right.$ and $39.0 \pm 0.1^{\circ} \mathrm{C} n=14, p=N S$, respectively). After wrapping the heat stress rabbits in an electric warming blanket an 
average of $42.6 \pm 2.9$ minutes elapsed before the rectal temperature reached $42^{\circ} \mathrm{C}$, the peak temperature recorded was $42.3 \pm 0.1^{\circ} \mathrm{C}$ and the time that the rectal temperature remained above $42^{\circ} \mathrm{C}$ was $17.6 \pm 1.4$ minutes.

\subsection{Infarct Size (figure 7.2)}

Risk volume, infarct volume and percentage of risk area infarcted are shown in figure 7.2A. The volumes of myocardial tissue at risk following coronary ligation although not significantly different between intervention groups tended to be higher in the 2 sham groups, to overcome any confounding effect this may have had on infarct size, data are also presented on the relationship between infarct and risk volumes (figure 7.2B). Thermal or ischaemic pretreatment resulted in both a significant reduction in absolute infarct volume, as well as a reduction in infarct volume expressed as a percentage of the volume of tissue at risk (figure $7.2 \mathrm{~A}$ ). The volume of infarction as a percentage of the volume at risk for ischaemic pretreatment compared with control was $28.8 \pm 5.2 \%$ v $52.0 \pm 5.2 \% \mathrm{p} \leq 0.01$, respectively, and for thermal pretreatment compared with control $32.8 \pm 3.8 \%$ v $56.9 \pm 6.5 \%, \mathrm{p} \leq 0.01$, respectively. Therefore both ischaemic and thermal pretreatment resulted in myocardial protection.

In all treatment groups the infarcted areas exhibited intra-myocardial haemorrhage. The absence of any haemorrhage was rare and usually indicative of a failure to reperfuse (see exclusions).

\subsection{Haemodynamic and Metabolic Data (figures 7.3, 7.4 and 7.5 and table 7.1)}

An example of the physiological traces obtained during the infarction protocol is shown in figure 7.3. On coronary ligation and during ischaemia arterial pressure tends to fall with variable recovery during reperfusion. The ST segment elevation seen during ischaemia usually resolves during reperfusion with loss of $R$ wave amplitude and occasionally, (as in this example), the appearance of $Q$ waves.

Figure 7.4A describes the changes in rate pressure product and figure $7.4 \mathrm{~B}$ the change in mean arterial blood pressure that occur prior to, during, and following the 30 minute coronary occlusion for each group. There were no differences in rate 
Risk and Infarct Volumes Following a 30 minute Coronary Occlusion and 120 minutes of Reperfusion

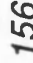

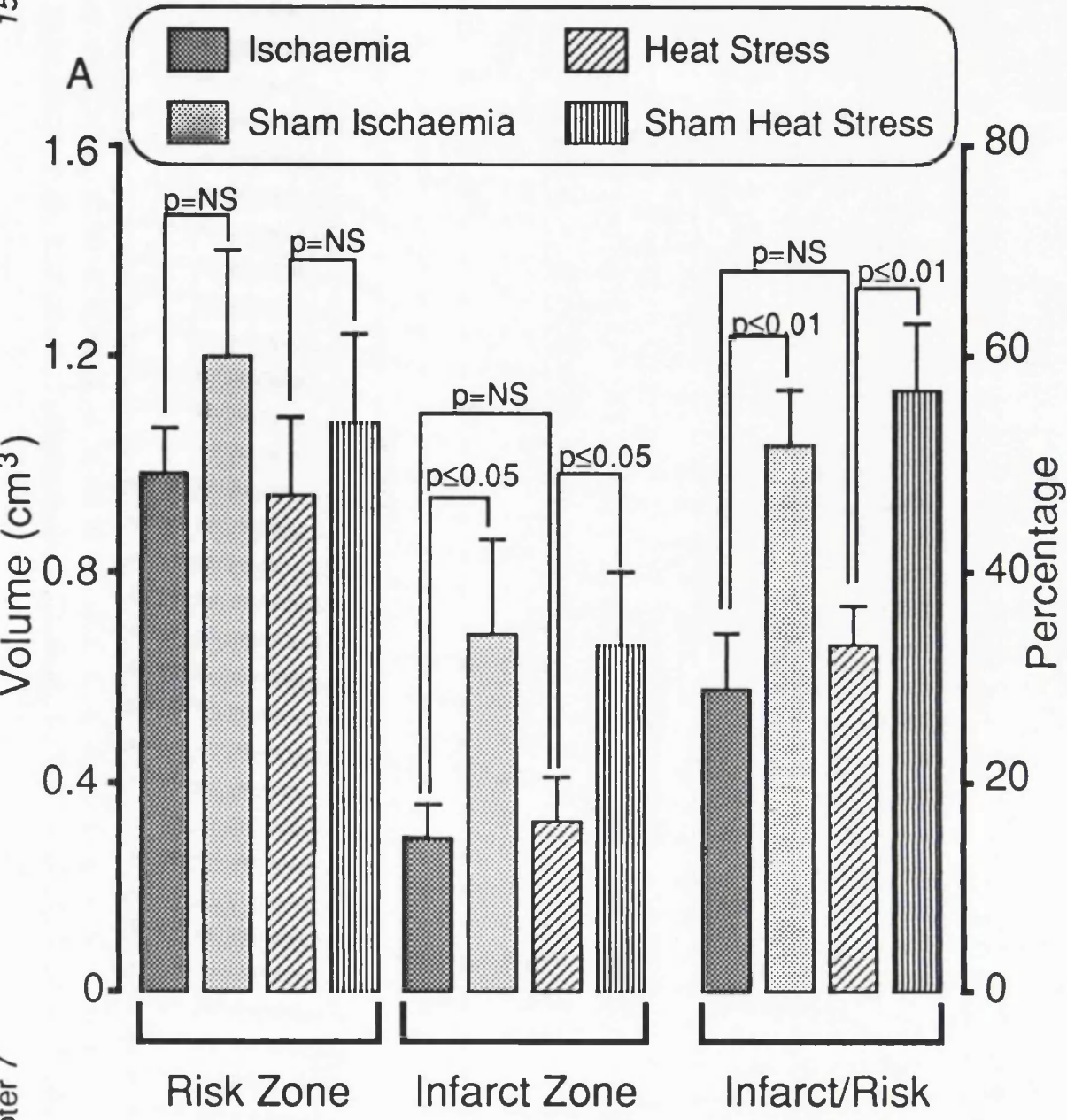

\section{B}

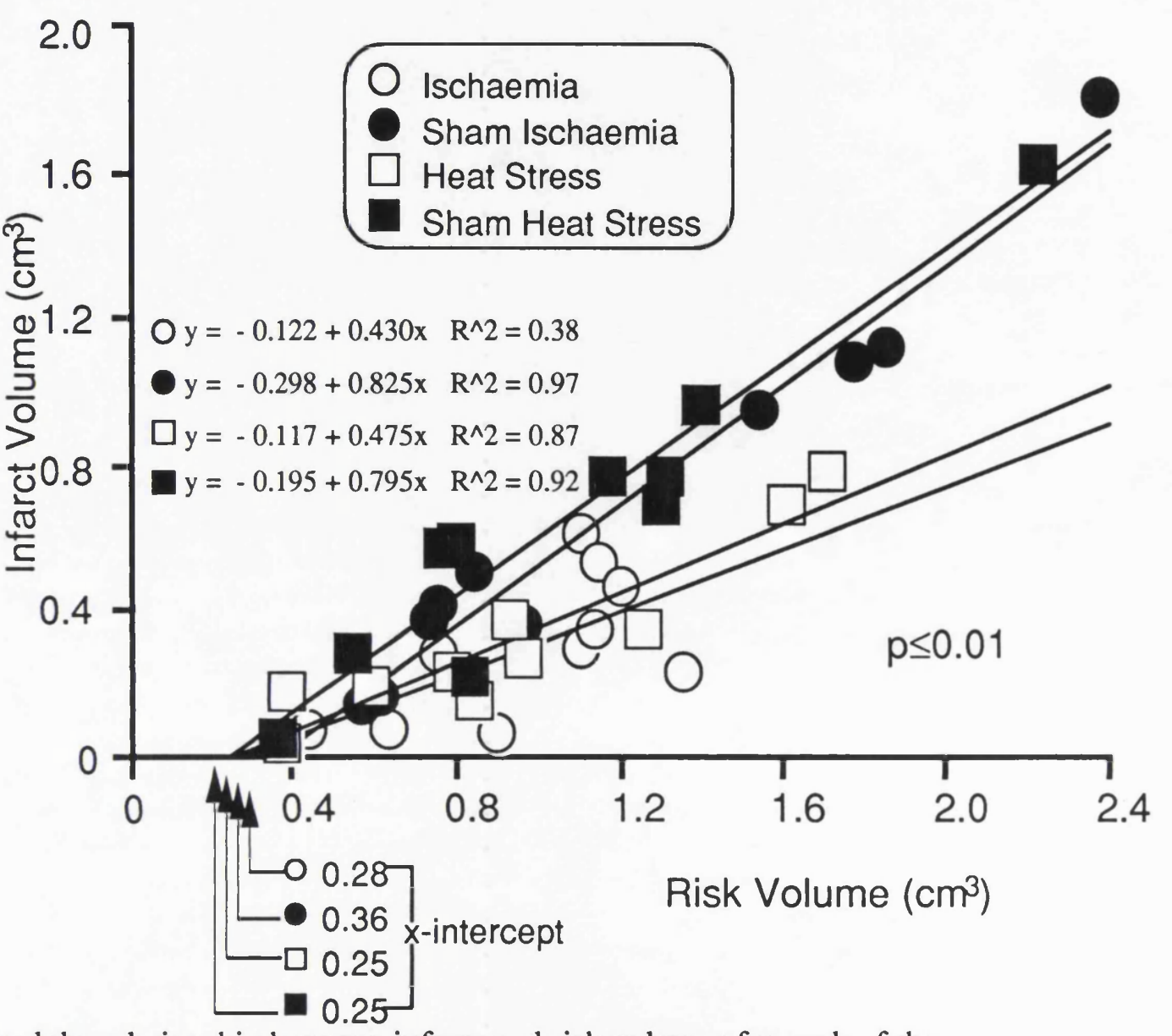

Figure 7.2: Infarct volume expressed as a percentage of the risk volume and the relationship between infarct and risk volumes for each of the

experimental groups. Risk volume was delineated with flourescent microspheres and infarct volume by triphenyltetrazolium. There was a tendancy for risk volume to be smaller in the non-sham pretreatment groups but graph B demonstrates that the protective effect is independant of risk volume size. All comparisons in graph A were by ANOVA followed by a FPLSD test, $n=10$ for each group. The relationships between infarct volume and risk volume in graph B were determined by Analysis of Covariance (ANCOVA). Bars represent \pm SEM. 


\section{Example of Experimental Trace During Myocardial Infarction}
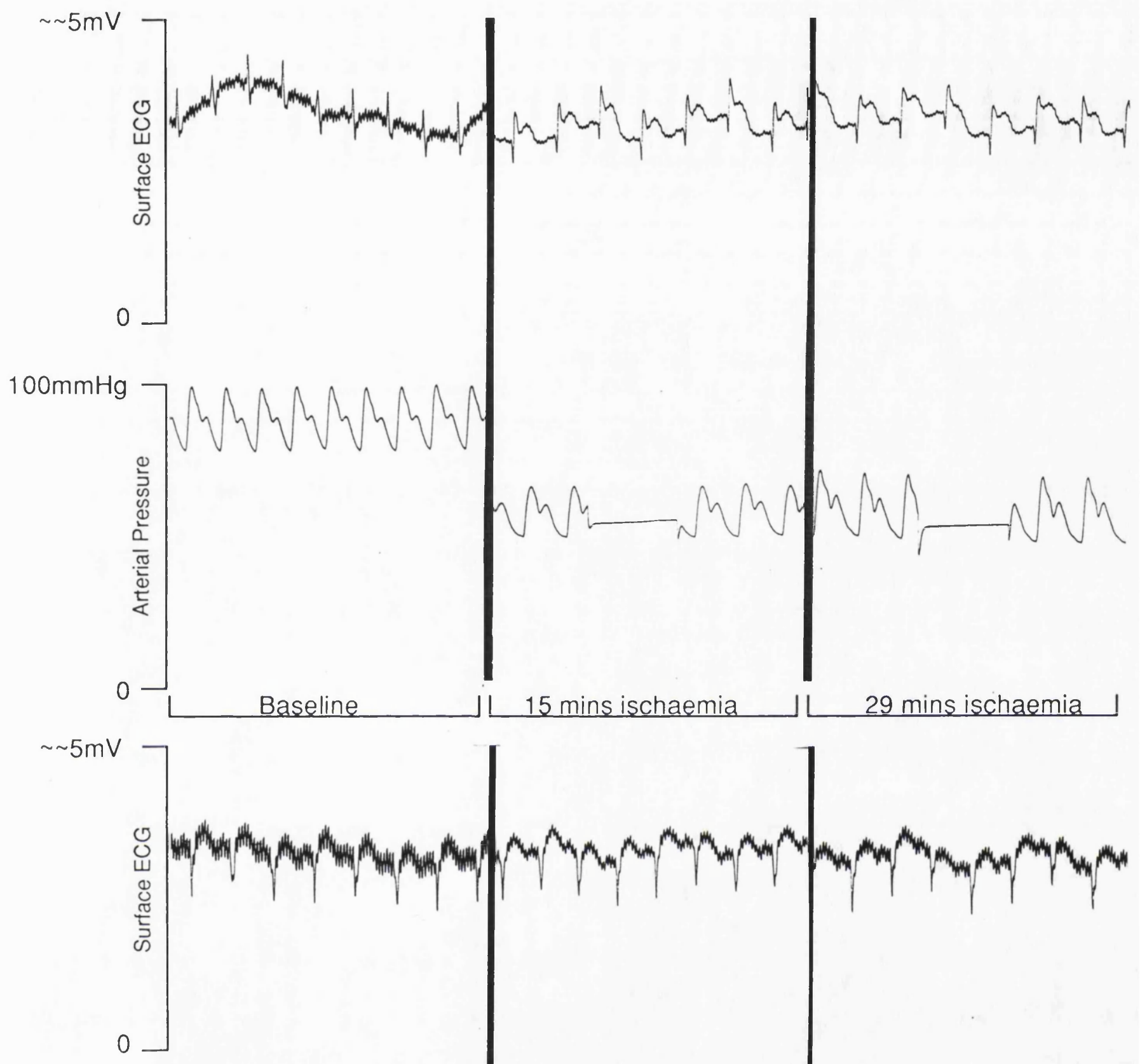

$100 \mathrm{mmHg}$
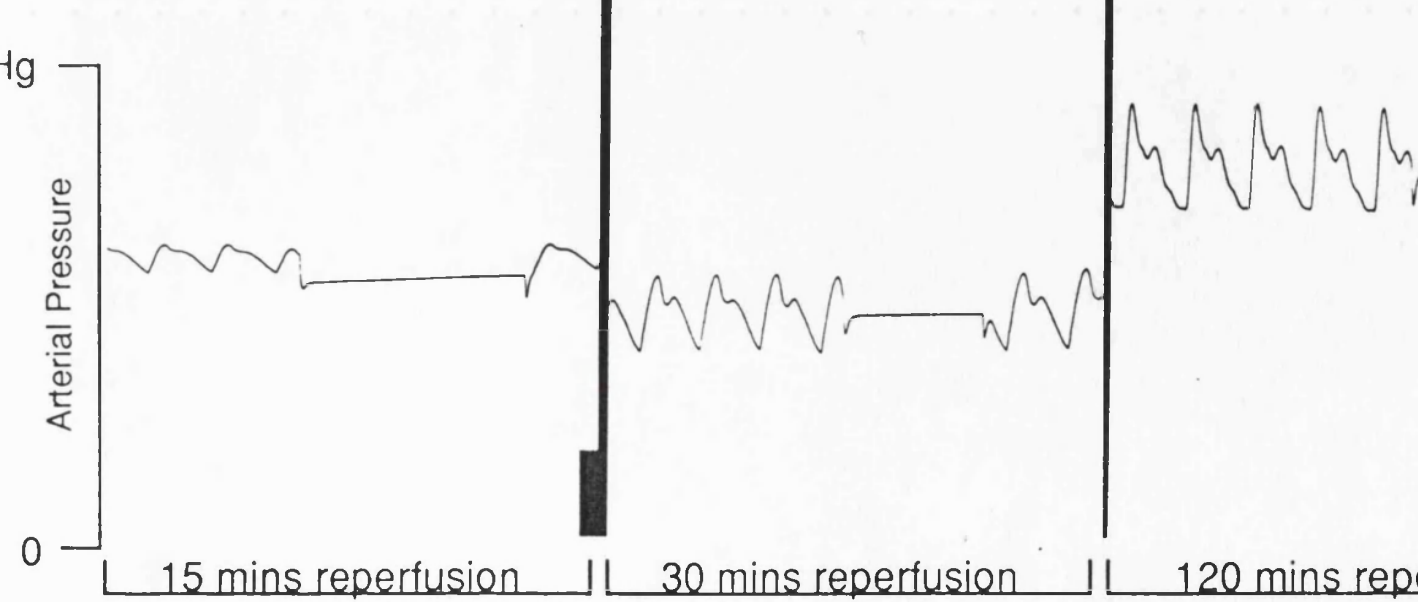

Figure 7.3: The appearance of the surface ECG and carotid artery pressure trace during the infarct size protocol. This example from a sham heat stress heart shows marked ST elevation during ischaemia (0 to 30 minutes) and QS waves during reperfusion. 


\section{Mean Arterial Pressure and Rate Pressure Product During Infarction}
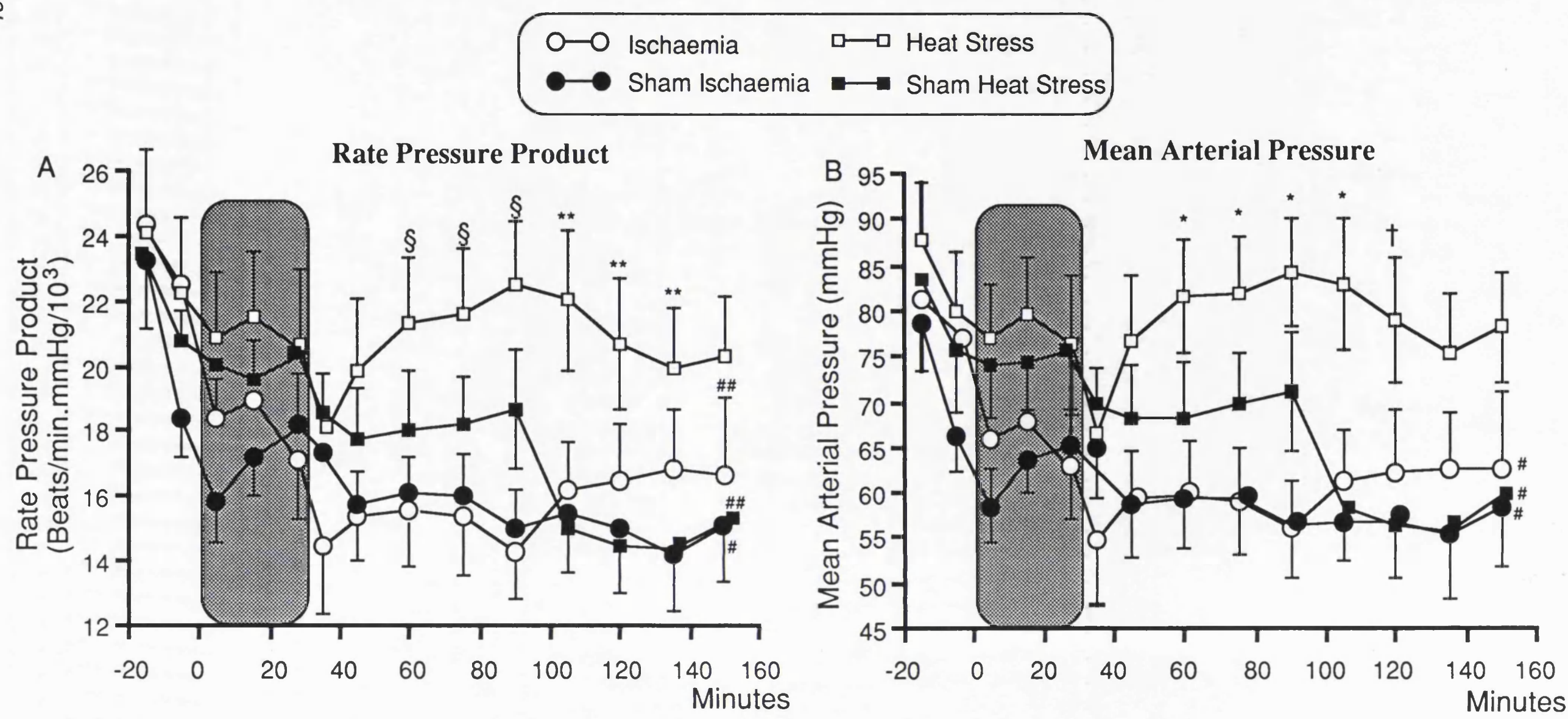

Figure 7.4: Heamodynamic parameters during the experimental protocol. Mean arterial blood pressure and rate pressure product follow a similar timecourse, both are best preserved in the heat stress pretreatment group. ${ }^{*} p \leq 0.05$ heat stress $v$ sham ischaemia and heat stress $v$ ischaemia, $\dagger p \leq 0.05$ heat stress $\mathrm{v}$ sham ischaemia and heat stress $\mathrm{v}$ sham heat stress, $\S p \leq 0.05$ heat stress $\mathrm{v}$ ischaemia, ${ }^{* *} \mathrm{p} \leq 0.05$ heat stress $\mathrm{v}$ sham ischaemia and heat stress v sham heat stress. $n=10$ for each group, all comparisons by one-way ANOVA with a post-hoc FPLSD test. \#p $\leq 0.05$ \#\#p $\leq 0.01$ for -20 minute value compared to 160 minutes (120 minutes of reperfusion) for comparisons within groups two-way ANOVA was followed by a single paired t-test to compare values at -20 minutes to 160 minutes. Bars represent $\pm S E M$. 
pressure product or blood pressure between groups prior to coronary occlusion or during ischaemia, implying that myocardial work and therefore oxygen demand was likely to be similar between groups.

During reperfusion the rate pressure product was consistently higher in the heat stress group and at various timepoints it was significantly different to all other groups. The basis for this preservation was secondary to a higher mean arterial pressure in the heat stress pretreatment group. This preservation of rate pressure product is likely to be independent of infarct size since a similar reduction in infarct size was seen with ischaemic pre-treatment but was not associated with any significant advantage in rate pressure product. In an attempt to further elucidate the reason for this apparent advantage we correlated rate pressure product at the end of reperfusion with infarct volume as a ratio of heart mass (volume : heart weight ratio). As seen in figure 7.5 there was no correlation between infarct size and rate pressure product for any group. This finding suggests, that in this experimental model, surprisingly infarct size does not appear to be the major determinant of cardiac work. In addition there was no relationship between normalised risk area and rate pressure product, indicating that post-ischaemic contractile dysfunction (stunning) of non-infarcted tissue within the risk zone is similarly not the explanation for the difference in rate pressure product between groups (data not shown).

Table 7.1 includes the haemodynamic as well as acid/base balance data for each experimental group. There were no significant differences between groups in terms of acid base balance or heart rate. Prior to coronary occlusion arterial $\mathrm{pH}$ was slightly high since the minute volumes, calculated on the basis of rabbit weight, tended to result in overventillation. These volumes were reduced on the basis of the blood gases so that during ischaemia arterial $\mathrm{pH}$ normalised.

\subsection{Myocardial 72kD Stress Protein Content (figures 7.7 and 7.8A)}

An initial protocol with only 6 hours of recovery following ischaemic pretreatment caused some increase in hsp72, but this was not significantly different from basal expression, and was significantly less than the elevation seen 24 hours following heat stress. The recovery time following ischaemic pretreatment was therefore increased to 24 hours. 
Relationship Between Infarct Size and Rate Pressure Product at 120 Minutes of Reperfusion
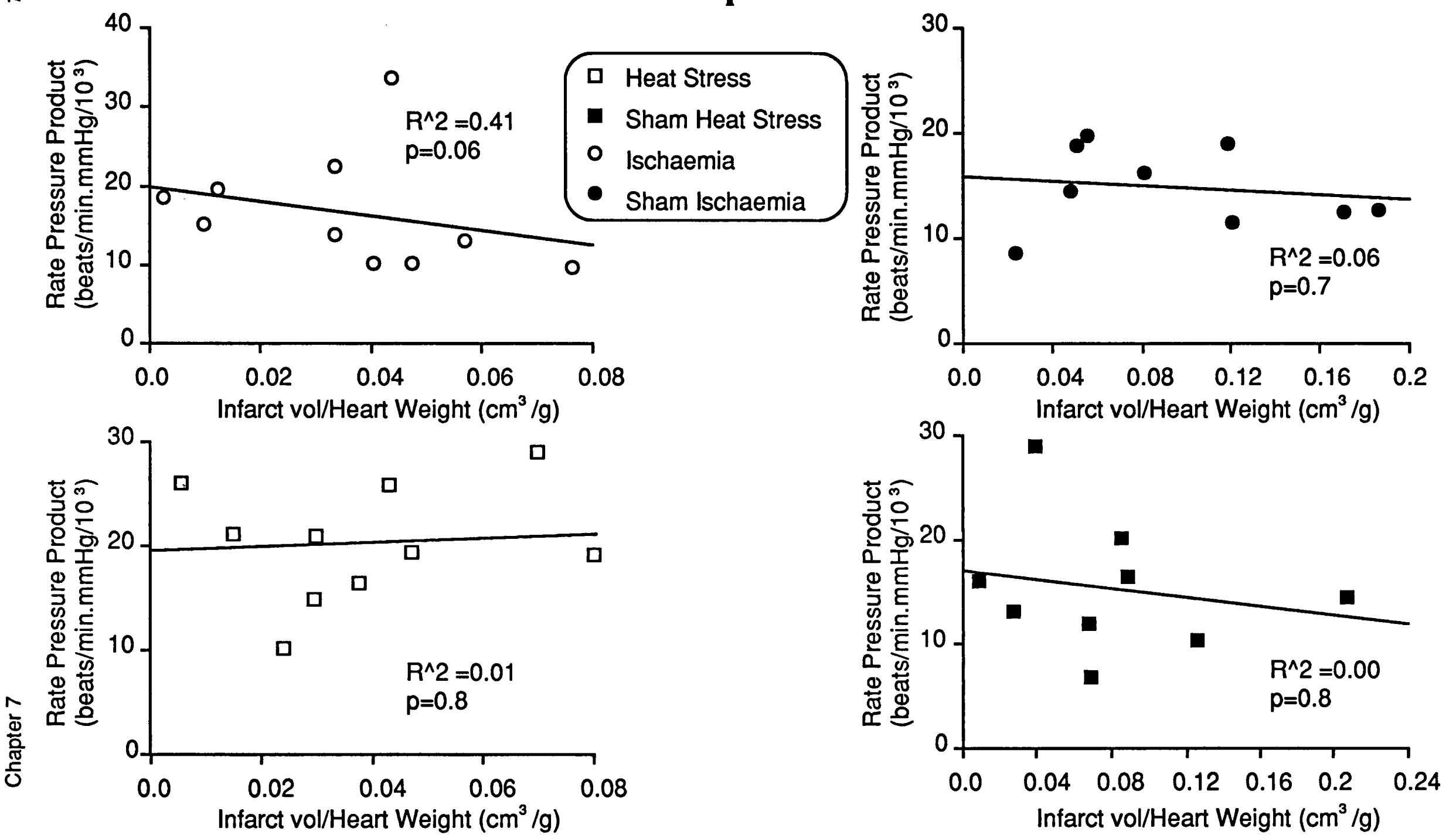

Figure 7.5: The relationship between rate pressure product and normalised infarct volume. Rate pressure product at the end of reperfusion is unrelated to infarct volume. Relationships are determined by the Spearman rank correlation method, $n=10$ for each group. 
Table 7.1. Hemodynamic, Arterial pH and Oxygen Tension Changes During Myocardial Infarction

Period of Coronary Artery Occlusion _P e riod of Myoc a rdial Re perfusion Baseline Pre-occlusion 5 mins 15 mins 30 mins 45 mins 60 mins 90 mins 120 mins 150 mins

\begin{tabular}{|c|c|c|c|c|c|c|c|c|c|c|}
\hline \multicolumn{11}{|l|}{ Heart Rate (bpm) } \\
\hline ischaemia & $297 \pm 6$ & $277 \pm 7$ & $272 \pm 9$ & $278 \pm 6$ & $270 \pm 5$ & $257 \pm 6$ & $258 \pm 7$ & $255 \pm 5$ & $269 \pm 13$ & $271 \pm 11$ \# \\
\hline Sham Ischaemia & $293 \pm 11$ & $277 \pm 9$ & $271 \pm 10$ & $271 \pm 11$ & $284 \pm 15$ & $267 \pm 10$ & $271 \pm 8$ & $268 \pm 10$ & $270 \pm 11$ & $260 \pm 14 \|$ \\
\hline Heat Stress & $273 \pm 9$ & $273 \pm 10$ & $269 \pm 9$ & $269 \pm 8$ & $265 \pm 9$ & $256 \pm 10$ & $259 \pm 10$ & $266 \pm 12$ & $262 \pm 11$ & $264 \pm 11$ \\
\hline Sham Heat Stress & $281 \pm 7$ & $277 \pm 10$ & $270 \pm 13$ & $265 \pm 14$ & $272 \pm 12$ & $263 \pm 11$ & $266 \pm 11$ & $264 \pm 9$ & $261 \pm 10$ & $260 \pm 9 \# \#$ \\
\hline \multicolumn{11}{|c|}{ Art Sys Pressure (mmHg) } \\
\hline ischaemia & $92 \pm 7$ & $90 \pm 7$ & $76 \pm 10$ & $77 \pm 6$ & $74 \pm 6$ & $70 \pm 5$ & $70 \pm 6$ & $67 \pm 6$ & $73 \pm 6$ & $74 \pm 8 \#$ \\
\hline Sham Ischaemia & $91 \pm 6$ & $76 \pm 4$ & $67 \pm 4$ & $72 \pm 3$ & $75 \pm 6$ & $68 \pm 6$ & $68 \pm 5$ & $67 \pm 4$ & $65 \pm 5$ & $71 \pm 6$ \\
\hline Heat Stress & $99 \pm 6$ & $86 \pm 6$ & $83 \pm 6$ & $87 \pm 6$ & $84 \pm 7$ & $85 \pm 6.4^{\star}$ & $89 \pm 6$ & $94 \pm 6^{\star \star}$ & $88 \pm 6+\dagger$ & $87 \pm 6 \dagger$ \\
\hline Sham Heat Stress & $92 \pm 6$ & $86 \pm 5$ & $78 \pm 6$ & $82 \pm 7$ & $81 \pm 5$ & $75 \pm 5$ & $73 \pm 5$ & $78 \pm 5$ & $61 \pm 6$ & $63 \pm 7 \# \#$ \\
\hline \multicolumn{11}{|c|}{ Art Dia Pressure $(\mathrm{mmHg})$} \\
\hline ischaemia & $73 \pm 7$ & $71 \pm 8$ & $59 \pm 9$ & $60 \pm 6$ & $56 \pm 6$ & $52 \pm 5$ & $52 \pm 5$ & $49 \pm 5$ & $55 \pm 6$ & $55 \pm 8 \# \#$ \\
\hline Sham Ischaemia & $73 \pm 6$ & $59 \pm 4$ & $53 \pm 4$ & $58 \pm 3$ & $59 \pm 5$ & $53 \pm 6$ & $52 \pm 5$ & $50 \pm 4$ & $50 \pm 5$ & $52 \pm 6 \#$ \\
\hline Heat Stress & $81 \pm 7$ & $73 \pm 7$ & $70 \pm 6$ & $75 \pm 6$ & $67 \pm 8$ & $69 \pm 7$ & $74 \pm 6^{*}$ & $76 \pm 5^{\star \star}$ & $72 \pm 7$ & $71 \pm 6$ \\
\hline Sham Heat Stress & $78 \pm 7$ & $70 \pm 5$ & $63 \pm 7$ & $68 \pm 8$ & $65 \pm 7$ & $62 \pm 8$ & $57 \pm 7$ & $58 \pm 6$ & $43 \pm 6$ & $49 \pm 6 \# \#$ \\
\hline \multicolumn{11}{|l|}{ Art Blood pH } \\
\hline ischaemia & $7.49 \pm 0.02$ & & $7.50 \pm 0.03$ & & $7.46 \pm 0.02$ & & $7.45 \pm 0.02$ & $7.47 \pm 0.02$ & $7.44 \pm 0.02$ & $7.44 \pm 0.02$ \\
\hline Sham Ischaemia & $7.53 \pm 0.02$ & & $7.48 \pm 0.02$ & & $7.43 \pm 0.04$ & & $7.44 \pm 0.02$ & $7.43 \pm 0.01$ & $7.40 \pm 0.02$ & $7.39 \pm 0.01 \#$ \\
\hline Heat Stress & $7.51 \pm 0.02$ & & $7.53 \pm 0.04$ & & $7.46 \pm 0.02$ & & $7.44 \pm 0.02$ & $7.45 \pm 0.01$ & $7.45 \pm 0.01$ & $7.45 \pm 0.01$ \\
\hline Sham Heat Stress & $7.46 \pm 0.02$ & & $7.45 \pm 0.04$ & & $7.41 \pm 0.03$ & & $7.42 \pm 0.03$ & $7.42 \pm 0.02$ & $7.38 \pm 0.04$ & $7.38 \pm 0.04 \#$ \\
\hline \multicolumn{11}{|c|}{ Art Blood Oxygen (kPa) } \\
\hline ischaemia & $47.9 \pm 6.7$ & & $51.3 \pm 5.8$ & & $48.9 \pm 5.6$ & & $47.2 \pm 6.1$ & $47.8 \pm 4.7$ & $50.7 \pm 5.3$ & $54.2 \pm 4.5$ \\
\hline Sham Ischaemia & $60.3 \pm 3.1$ & & $59.1 \pm 3.1$ & & $55.1 \pm 2.0$ & & $51.4 \pm 4.7$ & $48.4 \pm 4.6$ & $50.1 \pm 3.5$ & $54.9 \pm 3.1$ \\
\hline Heat Stress & $61.5 \pm 4.2$ & & $58.9 \pm 2.3$ & & $56.3 \pm 3.3$ & & $59.4 \pm 1.6$ & $55.9 \pm 4.0$ & $59.6 \pm 2.3$ & $59.5 \pm 1.7$ \\
\hline Sham Heat Stress & $52.3 \pm 5.8$ & & $54.7 \pm 2.0$ & & $50.6 \pm 2.6$ & & $53.1 \pm 2.0$ & $50.5 \pm 3.4$ & $50.5 \pm 5.0$ & $55.0 \pm 6.1$ \\
\hline
\end{tabular}

$\mathrm{n}=10$ for each group, bpm=beats per minute

Baseline data were recorded with the chest closed.

Mean Weights(kg), Ischaemia 2.57 \pm 0.14 , Sham Ischaemia 2.40 \pm 0.12 , Heat Stress $2.58 \pm 0.22$, Sham Heat Stress $2.45 \pm 0.16$

${ }^{*} \mathrm{p} \leq 0.05, * * \leq 0.01$ vs Ischaemia and Sham Ischaemia $\quad \dagger \mathrm{p} \leq 0.05, \dagger \dagger \leq 0.01$ vs Sham Ischaemia and Sham Heat Stress using ANOVA

$\# \mathrm{p} \leq 0.05$, $\leq 0.01$ for each individual group at 150 mins vs baseline using paired $t$ test. 
Figure 7.7A demonstrates that significant induction of the $72 \mathrm{kD}$ stress protein occurred 24 hours following heat stress and ischaemic pretreatments. This protein was detected in all myocardial samples examined (including those from the non-ischaemic area, data not shown). When the blot was examined densitometrically (figure 7.7B), the graded induction of stress protein with differing interventions became more apparent, with thermal pretreatment resulting in marginally greater induction than ischaemic pretreatment. However, the mean stress protein content in each of the pretreatment groups was greater than in corresponding controls. Between the control groups there was a significant induction in the sham ischaemic pretreatment group. The reason for this may lie in the greater surgical trauma occurring with sham ischaemic pretreatment, although other differences exist between these two control groups, including the method of ventilation and anaesthetic regimes. When compared with the sham heat stress group, there was an approximate eight-fold hsp72 induction by thermal, seven-fold by ischaemic, and three-fold by sham ischaemic pretreatments.

\subsection{Myocardial 60kD Stress Protein Content (figure 7.8)}

Figure 7.8A demonstrates that the marked variation seen between experimental groups for the $72 \mathrm{kD}$ stress protein was not apparent when an identically load gel was blotted and probed for the $60 \mathrm{kD}$ stress protein. In this model cardiac hsp60 was not significantly elevated by whole body heat stress. The only intervention associated with any change in hsp60 content was ischaemic pretreatment, which resulted in an approximate 1.5 to 2-fold induction compared with other intervention groups (figure 7.8B). Therefore, differences exist in stress protein inducibility with hsp72 being more inducible than hsp60, whilst hsp60 is preferentially, though only modestly, induced by ischaemia.

\subsection{Myocardial Specimen Loading for Stress Protein Estimation (figure} 7.9)

On the basis of Coomasie staining protein loading of the immunoblots shown in figures 7.7 and 7.8 was equal between lanes. The optical density of the actin band, the major band running at about $40 \mathrm{kDa}$, was used to normalise for slight variations in protein loading. 
$72 \mathrm{kDa}$ stress protein content 24 hours Following

A Each of the Pre-Treatments

MW (kDa)
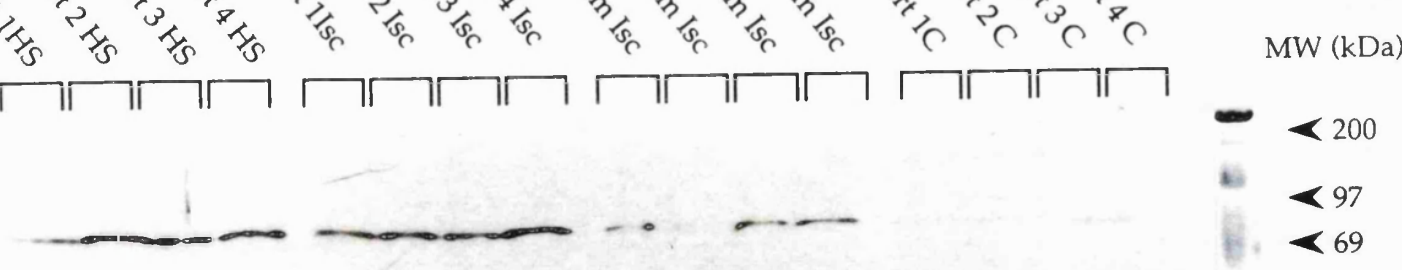

$<46$

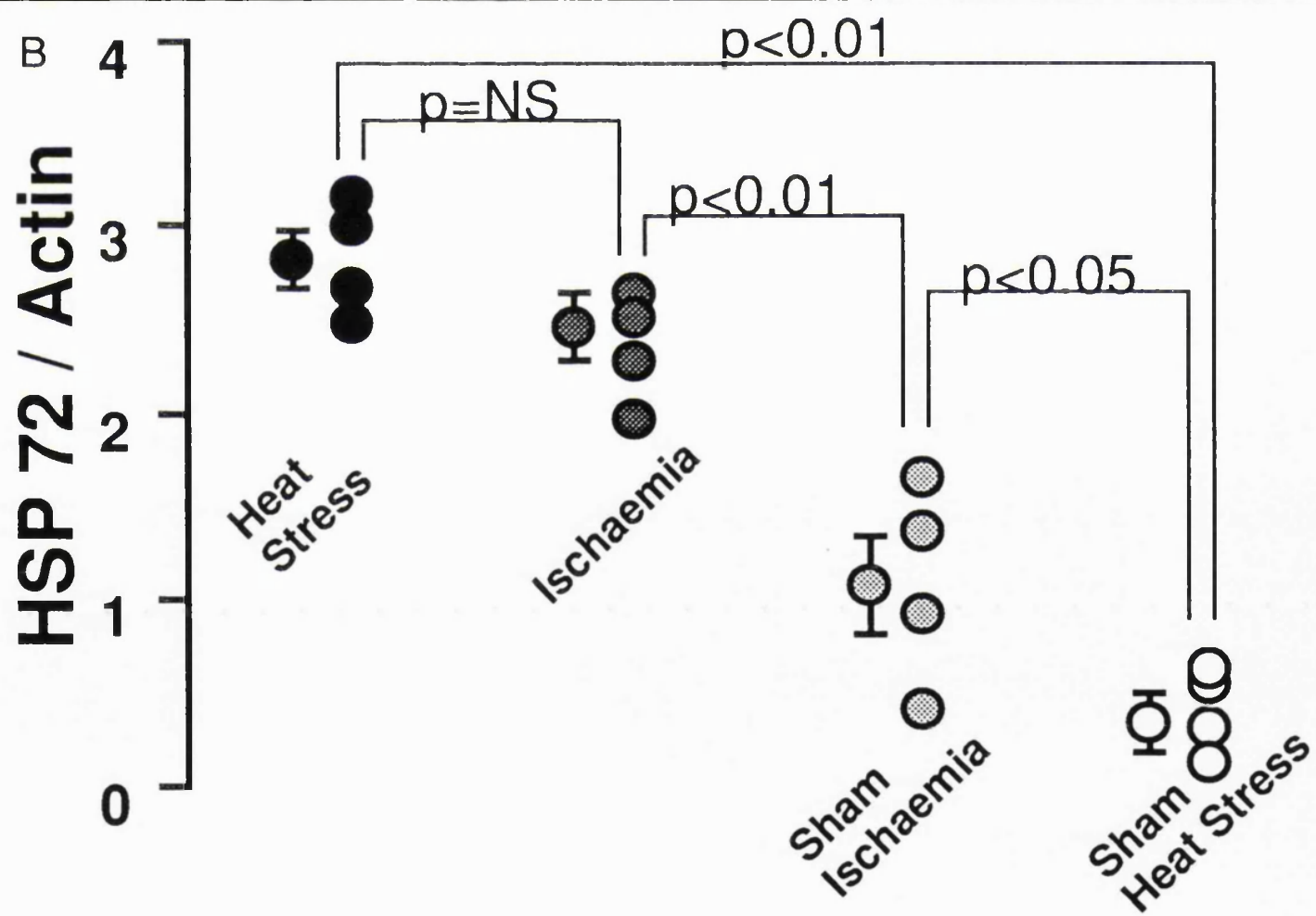

Figure 7.7: $72 \mathrm{kDa}$ stress protein content for each pretreatment group. Panel A: Shows the hsp72 immunoreactivity in 4 hearts from each of the pretreatment groups. Samples for the ischaemic pre-treatment group were prepared from risk zones, samples from the other pretreatment groups were of left ventricular tissue. There is a graded induction of hsp72 moving from heat stress pretreatment group on the left to sham heat stress group on the right. Graph B: Shows that when the hsp72 bands are normalised with respect to the density of the Coomasie actin band (see figure 7.9) the graded induction seen in panel A, is translated into statistically different hsp 72 stress protein concentrations between groups, with heat stress and ischaemic pretreatment groups resulting in significant induction of hsp72. Comparisons are by ANOVA with post-hoc comparisons using the FPLSD method. Bars represent \pm SEM. 


\section{0kDa Stress Protein Content 24 hours Following Each of A the Pre-Treatments}
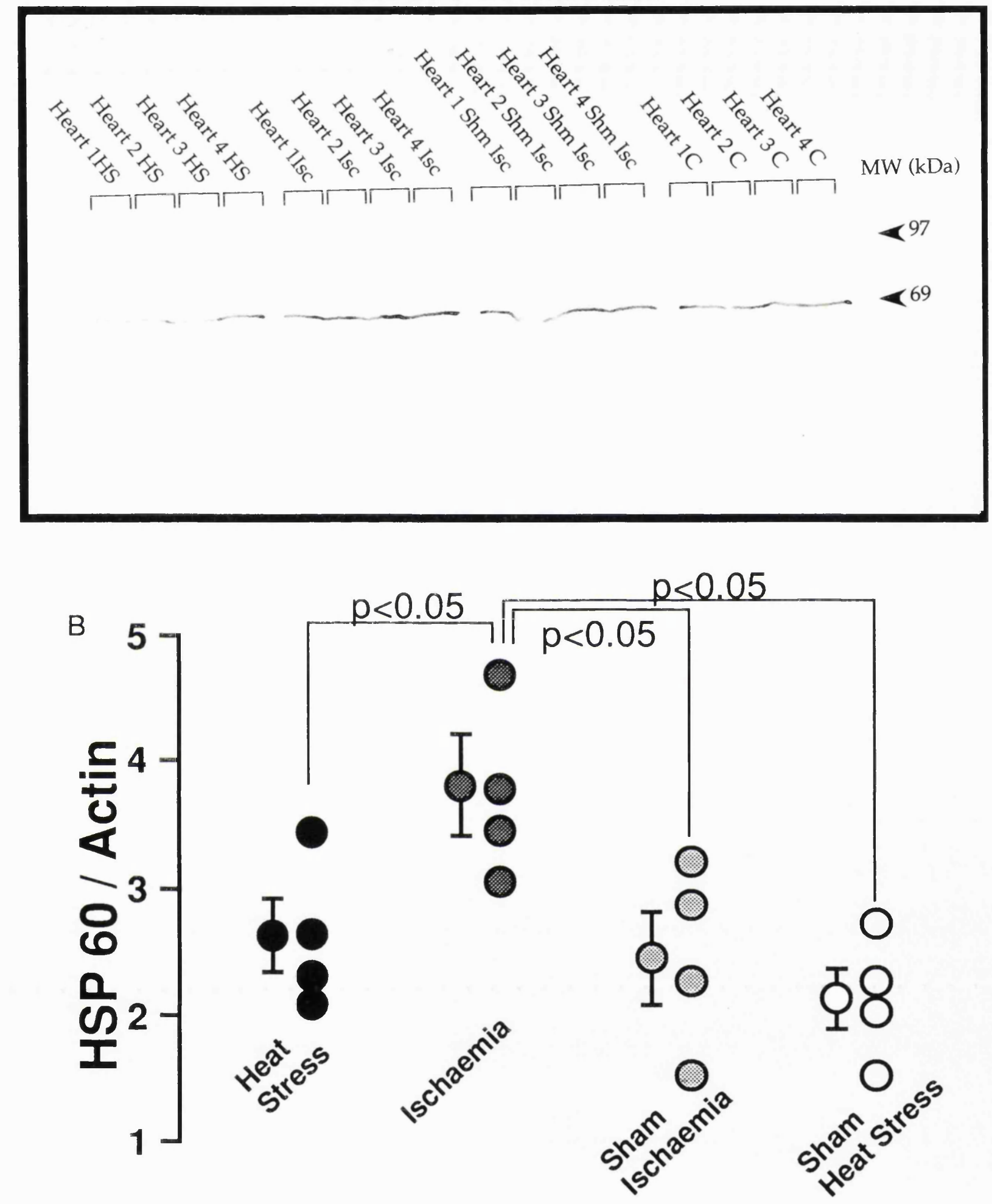

Figure 7.8: $60 \mathrm{kDa}$ stress protein content for each pretreatment group. Panel A: Shows the hsp60 immunoreactivity in 4 hearts from each of the pretreatment groups. Lane arrangements and sample loadings are as in figures 7.7 and 7.9. In contrast to hsp72, hsp60 content is similar between groups. Panel B: Shows the density of the hsp60 bands normalised to actin, there is a slight and significant induction of hsp60 by ischaemic pretreatment. Statistical comparisons are by ANOVA with a post-hoc FPLSD test. Bars represent \pm SEM. 


\section{Coomasie Staining of Samples Prepared from Left Ventricular Tissue Following Heat Stress, Ischaemic, Sham Ischaemic and Sham Heat Stress Pretreatments}

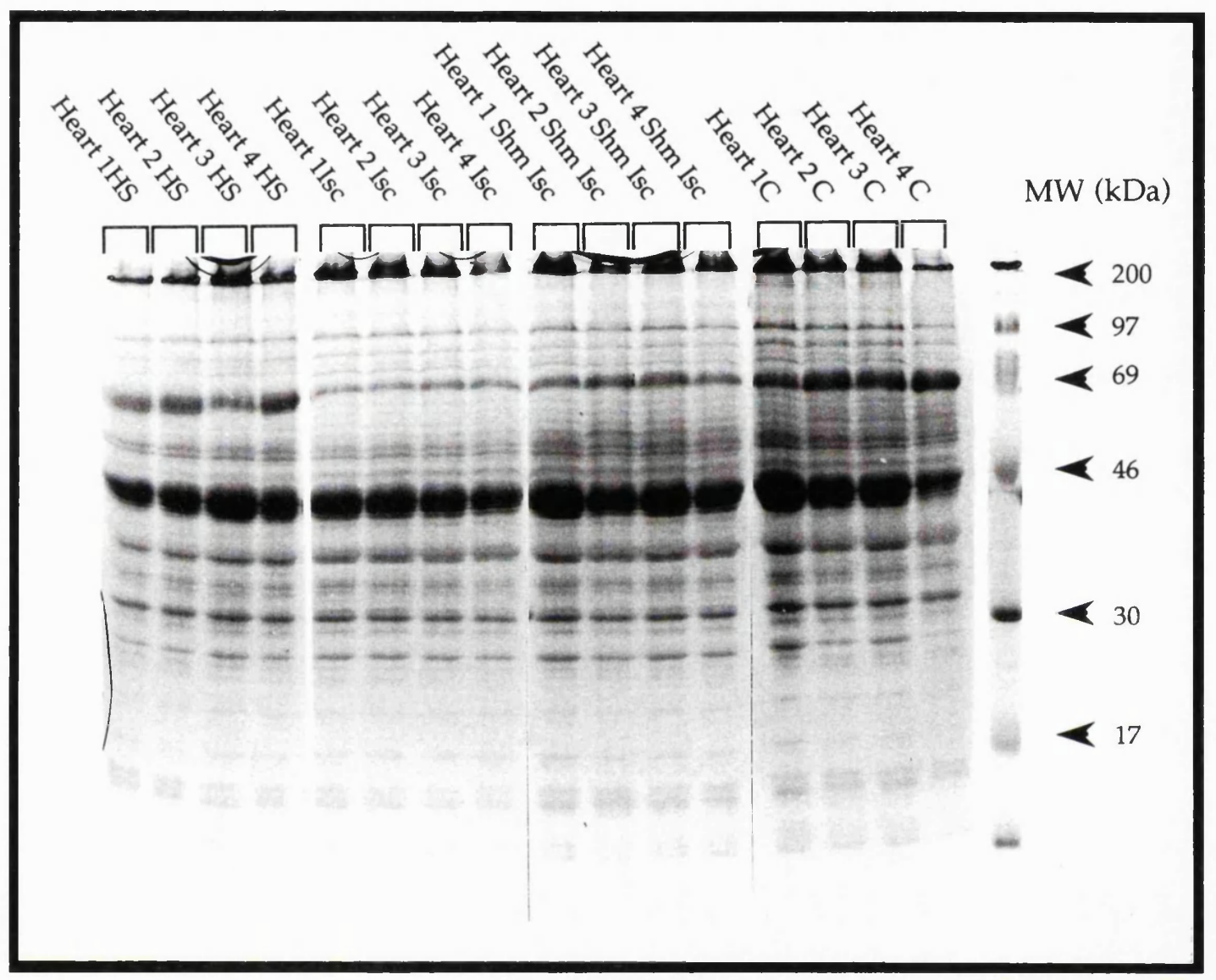

Figure 7.9: Coomasie stain of SDS-Page gel loaded with samples prepared from four hearts from each of the pretreatment groups. The four lanes on the left are from left ventricular tissue harvested 24 hours following whole body heat stress (Heart 1HS to Heart 4HS); the next 4 lanes are prepared from the ischaemic zones of hearts subjected to 4, 5 minute coronary occlusions 24 hours previously (Heart 1 Isc to heart 4 Isc); the next four lanes are loaded with apical tissue from the sham ischaemic group (Heart $1 \mathrm{Shm}$ Isc to Heart $4 \mathrm{Shm}$ Isc): and the last (right) 4 lanes from sham heat stress hearts (Heart 1C to Heart 4C). The molecular weight markers appear on the right, with the Coomasie actin band below the 46 $\mathrm{kDa}$ molecular weight marker. The lane arrangements, samples and sample loading volumes are identical to those of figures 7.7 and 7.8. The density of the actin band on this gel was used to normalise the specific stress protein bands seen in figures 7.7A and 7.8A. 


\section{Discussion}

This study demonstrates that both ischaemic and heat stress pretreatment elevate myocardial hsp72 to a similar extent and are associated with a similar reduction of infarct size. This reduction occurs despite similar rate pressure products prior to and during prolonged coronary artery occlusion, and therefore suggests that the protection is a direct result of enhanced myocardial resistance to infarction. This enhanced resistance to infarction is at least associated with hsp72, and it is tempting to postulate that it occurs as a direct consequence of myocardial stress protein induction.

\subsection{Whole Body Heat Stress and Resistance to Infarction In-vivo}

In a previous report from our laboratory whole body heat stress followed by 24 hours of recovery failed to limit infarction caused by a 45 minute coronary ligation (Yellon et al 1992b), whilst in this study contrary to expectation, in an almost identical model, infarct size was reduced after a 30 minute ligation. Donnelly et al (1992) similarly found a reduction in infarct size with a 35 minute but not a 45 minute coronary occlusion following whole body heat stress in the rat. In addition, very recently Currie et al (1993) reported a significant reduction in infarct size following a 30 minute coronary occlusion 24 hours after whole body heat stress in the rabbit (a model identical to the one used in this study), the protection was however absent 48 hours after whole body heat stress. The evidence that whole body heat stress is capable of limiting infarction resulting from a 30-35 minute ligation is strong and a possible reason underlying the failure to protect against a 45 minute ligation may simply be that the insult is too severe. A similar dependence on the length of coronary occlusion is seen in ischaemic preconditioning in the dog where the marked protection seen after a 60 minute occlusion is absent after a 90 minute occlusion (Nao et al 1990).

\subsection{The Basis for the Reduction in Myocardial Infarct Size}

The design of this study is similar to, and the findings are consistent with those of Donnelly et al (1992). These investigators were able to demonstrate significant infarct size reduction following whole body heat stress, however ischaemic 
pretreatment with a single 20 minute coronary occlusion failed to reduce infarct size, but also failed to increase myocardial hsp72 to the level seen following heat stress. These investigators therefore concluded that the absolute levels of hsp72 maybe important in conferring protection from ischaemic injury. This conclusion is supported and extended by the findings of this study which demonstrate that greater ischaemic elevation of myocardial hsp72 is indeed associated with protection. Moreover a modest elevation of hsp72, as occurred with sham ischaemic pretreatment is not associated with protection. In addition, our study suggests that hsp60 induction appears not to be a pre-requisite for myocardial salvage whilst an appropriate level of hsp72 maybe. However, the relationship between whole body heat stress and subsequent myocardial salvage is complicated by the fact that in the isolated rat heart protection seems to involve an increase in myocardial catalase activity rather than an induction of hsp72, since protection can be abolished by inhibition of myocardial catalase with 3-aminotriazole (Karmazyn et al 1990). In a recent report endogenous superoxide dismutase activity was also increased by repetitive ischaemia and reperfusion (Hoshida et al 1993) and therefore a common mechanism independent of stress proteins may underlie the protection seen with both ischaemic and heat stress pretreatments. Somewhat against this explanation is the observation that heat stress is protective in a number of different biological systems against injuries not obviously involving oxidant stress $(\mathrm{Li}$ and Werb 1982 and Barbe et al 1988). More importantly, other reports suggests that heart cells transfected so as to over express hsp72 become resistant to anoxic injury (Dillmann et al 1992). A possible explanation for these apparently contradictory findings is the suggestion that stress proteins may in some way modify antioxidant activity (Kukreja and Hess 1992). This is supported by the observation that the increase in myocardial catalase following heat stress is not transcriptionally regulated (Currie and Tanguay 1991).

In the ischaemic pretreatment group, the protection observed could be an ischaemia triggered increase in collateral flow or stunning. Both these explanations are unlikely since it is unclear whether the oxygen requirements and susceptibility to infarction are increased, decreased or normal in stunned myocardium (Ohgoshi et al 1991) and in a previous study a pattern of ischaemic pretreatment identical to that used in our experiments, produced only a very mild degree of stunning (Knowlton et 
al 1991a). In addition the rabbit is a species deficient in collateral vessels (Maxwell et al 1987), therefore any increase in collateral blood flow occurring as a result of ischaemic pretreatment would have to be on the basis of new (mitotic) vessel growth and this is unlikely to occur over a 24 hour period.

\subsection{The Relationship between Ischaemic Pretreatment and Ischaemic}

\section{Preconditioning}

The protocol employed in this study and protocols known to trigger "ischaemic preconditioning" are identical (Walker and Yellon 1992). Ischaemic preconditioning however, is a short lived phenomenon with protective benefits waning within 2 hours of reperfusion (van Winkle et al 1992). This study therefore raises the possibility of a delayed, but perhaps longer lasting, "second window of protection" (a biphasic protective effect). Evidence in support of such a phenomenon exists in neuronal tissue, as the stress protein content of neuronal tissue can be elevated by 2 minute, daily episodes of ischaemia a treatment that also seems to increase the neuronal resistance to more prolonged ischaemia (Kitagawa et al 1990, Nowak 1990). Very recent findings from other laboratories confirm a similarly delayed protection at 24 hours following "classical" preconditioning of the dog heart (with repetitive coronary occlusions) (Kuzuya et al 1993); and also 24 hours after rapid pacing of the rabbit heart (Szekeres et al 1993). In the study by Kuzuya et al (1993) the experimental protocol was similar to that described in this chapter with four, 5 minute coronary occlusions followed by variable recovery times before dogs were subjected to a 90 minute coronary occlusion. With 24 hours of recovery following ischaemic pretreatment infarct size as a percentage of the area at risk, was $35.1 \%$ in the sham ischaemic pretreatment group versus $18.8 \%$ in the ischaemic pretreatment group, a protective effect that occurred independent of collateral flow. Interestingly, no protection was evident when infarction was performed 3 or 12 hours after the ischaemic pretreatment. These experimental findings may explain the apparent benefit of a 7 or more day history of angina prior to myocardial infarction (Muller et al 1990), although differences in treatment and collateral vessel formation may complicate the issue. 
The relationship between the early phase of protection following brief episodes of ischaemia (as seen in ischaemic preconditioning), and the later phase of protection (as seen in this study) is speculative. An obvious inconsistency is the fact that multiple coronary occlusions are as effective as a single occlusion in triggering preconditioning (Li et al 1990) but differ in their ability to induce myocardial hsp72 (Knowlton et al 1991). This inconsistency may explain why protection against ischaemic arrhythmia was not seen 24 hours or 72 hours following a single 5 minute coronary occlusion in the rat (Shiki and Hearse 1987).

\subsection{The Haemodynamic Benefits of Whole Body Heat Stress}

During reperfusion, rate pressure product and blood pressure were higher in the heat stress pre-treatment group. This effect seemed to be independent of a reduction in infarct size. It is possible that blood pressure was artifactually depressed in the ischaemic and sham ischaemic groups by a combination of intravascular volume depletion and sepsis secondary to surgical pretreatment. This would not however explain the advantage seen over the sham heat stress pre-treatment group. Since this effect is independent of infarct size it must either be a preferential action of whole body heat stress on non-infarcted myocardium, reducing stunning in the risk zone for example, or a peripheral action that alters arterial tone. Interestingly, Donnelly et al (1992) noted a similar advantage to heat stress pretreatment, and postulated that it may be secondary to a rise in arterial smooth muscle calcium content following whole body heat stress.

\subsection{Critique of Methods}

The volume of myocardium at risk of infarction was greatest in the sham ischaemic pretreatment group. The reason for this was that 24 hours after sternotomy the heart was covered in a fibrinous exudate making visualisation of the coronary vasculature difficult, consequently the marginal branch of the circumflex tended to be ligated closer to the atrioventricular groove where it was often visible close to the marginal vein. This difficulty also resulted in a failure to ligate a coronary artery in 3 rabbits in this group (see this Chapter Section 2.1). The higher risk volume in this group may alter the infarct:risk ratio if the relationship between these variables is non- 
linear. For this reason we plotted risk volume against infarct volume for each experimental group. This plot (figure 7.2B) confirms that the relationship is linear but surprisingly the intercept is not through the origin. The relationship suggests that when risk volume is less than $0.4 \mathrm{~cm}^{3}$ infarction does not occur, presumably since the needs of the myocardium are met by diffusion of substrates and metabolites. The expression of infarct size simply as a volume to volume ratio is not strictly valid and standard errors of the means between groups will be larger than the true biological variation would predict. In fact the standard errors of the mean infarct:risk ratios can halved by subtraction of the $\mathrm{x}$-intercept from each of the risk volumes prior to their use as the denominator in normalising infarct volume. For the purposes of this study this type of analysis confirms that the protection seen following ischaemic pretreatment is not a spurious effect of the larger risk volumes in the corresponding sham group.

The arterial $\mathrm{pH}$ (table 7.1) suggests that rabbits were over ventilated during the early part of the experiment prior to coronary ligation this effect was seen in all groups and therefore cannot account for the differences in infarction found. In addition it would be difficult to postulate how arterial alkalosis could influence infarct size, given that $\mathrm{pH}$ within the risk area will fall rapidly once coronary flow ceases.

Rabbits were ventilated with $100 \%$ oxygen during surgical pretreatment and infarct size assessment. The ischaemic pretreatment protocol induces stress protein expression and the trigger for this event may be the oxidative stress associated with reperfusion. If the enzymes responsible for oxygen centred free radical formation follow first-order kinetics then the degree of oxidative stress will be unphysiologically high in this preparation. The degree of stress protein induction seen in the ischaemic pretreatment group may have been less marked if arterial $\mathrm{pO}_{2}$ had been in the normal range and the protection seen 24 hours following heat stress may therefore have been absent.

\subsection{Conclusions}

The results of this study indicate that substantial myocardial hsp72 induction is possible with either sublethal thermal or ischaemic pretreatment, whilst hsp60 is preferentially elevated by ischaemic pretreatment. This observation is the first indication that myocardial hsp72 maybe more important than other stress proteins, 
since myocardial salvage was seen following a 30 minute coronary occlusion with both ischaemic and thermal pretreatments. In addition to limiting infarct size, whole body heat stress has other non-specific effects which act to maintain rate pressure product.

Our results provide evidence for what may prove to be a clinically relevant myocardial adaptive response, occurring 24 hours after short episodes of cardiac ischaemia, that serves to increase myocardial resistance to infarction. 


\section{Chapter 8:}

\section{General Discussion and Conclusions}

1 Summary of Results ............................ 172

2 The Mechanism of Myocardial Protection .................... 173

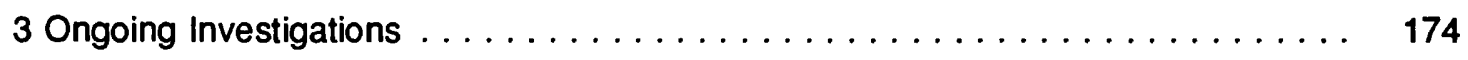

5 The Implications of the Work Described $\ldots \ldots \ldots \ldots \ldots \ldots \ldots \ldots \ldots \ldots$

\section{Summary of Results}

The studies presented in this thesis indicate that subtle changes occur within the rabbit myocardium 24 hours after whole body heat stress. These changes render the myocardium resistant to a variety of different forms of injury including ischaemia, high flow hypoxia without substrate and the calcium paradox. In addition protection is seen in a variety of different models with different endpoints including, superfused right ventricular papillary muscles with contractile recovery, the retrogradely perfused isolated heart with both contractile recovery and intracellular enzyme release, and the in-situ heart with infarct size.

The mechanism of the protection induced by whole body heat stress is not known. The studies using papillary muscles and isolated hearts show that baseline mechanical activity and the timing of mechanical activation are not altered by heat stress, confirming that the protection is not a result of "stunning" with a decreased metabolic requirement for contraction. The papillary muscle studies suggest that the degree of injury during high flow hypoxia is similar in heat stress and control groups, but that prior heat stress increases recovery during reoxygenation, especially with a directly oxidisable substrate such as pyruvate. This preference for an oxidisable substrate was interpreted as relative protection of mitochondrial over glycolytic pathways. In addition the degree of recovery in individual papillary muscles was related to the content of hsp72 but not the mitochondrial stress protein hsp60. 
In the initial papillary muscle studies the protection seen after heat stress against a gentle form of calcium paradox was confirmed in the isolated heart where a more severe degree of injury occurred. This insult, is not thought to involve oxygencentred free radicals and hence indicates that changes within the myocardium following whole body heat stress are capable of attenuating myocardial injury independent of the known increase in endogenous antioxidants.

In the last study described in this thesis sublethal myocardial ischaemia was used to trigger a stress protein response. The benefits of pretreatment with whole body heat stress against pretreatment with sublethal ischaemia were compared in an infarct size model. The two forms of pre-treatment induced similar amounts of hsp72 but differing amounts of hsp60, however a similar reduction of infarct size occurred in both models suggesting that a stress response involving an increase in hsp72 may be necessary for protection whilst one involving hsp60 may not.

In contrast to the studies above, no protection was seen in a model designed to mimic stunning and therefore associated with a mild degree of injury without myocyte death. The interpretation of this study was complex but seemed to indicate that the changes occurring in the myocardium following whole body heat stress may not protect against very mild forms of injury not involving myocyte death.

\section{The Mechanism of Myocardial Protection}

Theoretical considerations as well as evidence from other biological systems suggests that stress proteins are themselves capable of protecting cells from injury (see Chapter 1 Section 5 and Welch 1993). The results presented in this thesis are certainly compatible with this hypothesis, but do not prove that the association between stress protein induction, in particular hsp72, and protection is causal. In particular the evidence presented does not conclusively differentiate between stress proteins and other proposed mechanisms involving enhanced antioxidant defences (Karmazyn et al 1990) or other protective endogenous substances (Parratt 1993). These uncertainties are the subject of ongoing studies. 


\section{Ongoing Investigations}

The fact that thermal and ischaemic pretreatment both trigger stress protein induction and result in protection has prompted us to investigate another completely different form of pretreatment. Knowlton et al (1991b) have demonstrated hsp72 induction following myocardial stretch in the rabbit. A recovery model has been developed where the myocardium is stretched acutely by completely occluding the ascending aorta through a small transverse sternotomy at the level of the second intercostal space. The 10 second aortic occlusions are repeated 4 times, during each occlusion the heart is not ischaemic but remains pink and hyperaemic as the entire left heart cardiac output is directed through the coronary vasculature.

The changes in myocardial hsp72 and hsp60 occurring 24 hours after pretreatment are shown in figure 8.1. Infarction after a 30 minute coronary occlusion has also been assessed in a small number of animals at this timepoint and is $43.6 \pm 3.7$, $\mathrm{n}=6 \mathrm{v} 29.2 \pm 8.9, \mathrm{n}=6, \mathrm{t}=1.51, \mathrm{p}=0.16,(\mathrm{I} / \mathrm{R} \%)$ in the control $\mathrm{v}$ cross-clamp group, respectively. When the data are analysed as plots of infarct volume $v$ risk volume the relationship between the groups is identical (data not shown). Larger numbers are required before any further conclusions can be drawn.

The changes in endogenous antioxidant content within rabbit myocardium 24 hours following each of the pretreatments mentioned in this thesis has been measured with the help of Dr Michaela Mocanu a visiting fellow. The results of these measurements are shown in figure 8.2. In summary heat stress causes a two-fold increase in reduced:oxidised glutathione and a modest increase in superoxide dismutase, whilst ischaemic pretreatment causes a two-fold increase in catalase activity, crossclamp pretreatment causes a minor non-significant increase in catalase alone. Hence the interventions associated with protection do seem to cause an increase in endogenous antioxidants as well as hsp72.

\section{The Design of Future Studies}

The findings presented in this thesis support the need to design experiments to determine the relative role of stress proteins and endogenous antioxidants in the protection occurring following heat stress. The difficulty is that the experimental designs used so far rely on cross-tolerance, namely one stress protecting against a 

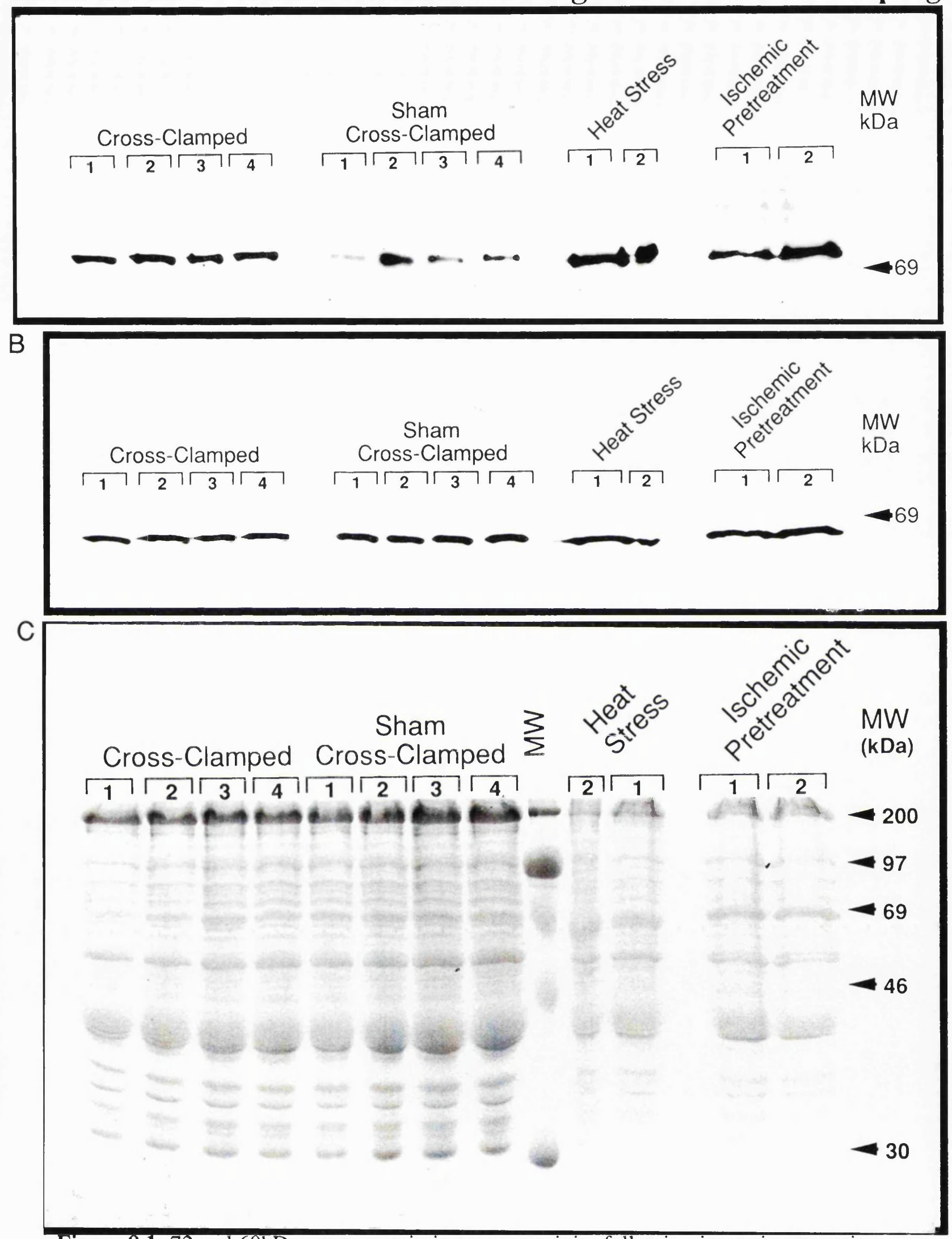

Figure 8.1: 72 and $60 \mathrm{kDa}$ stress protein immunoreactivity following intermittent aortic occlusion. The first 4 lanes were prepared from left ventricular tissue 24 hours following cross-clamping, the next 4 lanes were prepared from left ventricles following sham aortic cross clamping. The heat stress and ischaemic pretreatment samples have been used and described previously (Chapters 5 and 7 respectively). Panel A: Shows hsp72 immunoreactivity with clear induction following 4, 10 second periods of aortic crossclamping. The level of hsp72 induction is similar to that seen following whole body heat stress and ischaemic pretreatment. Panel B: In contrast hsp60 is similar between groups. Panel C: Shows the corresponding Coomasie stained SDS-PAGE gel, the lane arrangement and sample loading are as in panels A and B. 
Endogenous Antioxidant Activity in Rabbit Myocardium Following Different Forms of Pre-treatment $\stackrel{\wp}{ミ}$
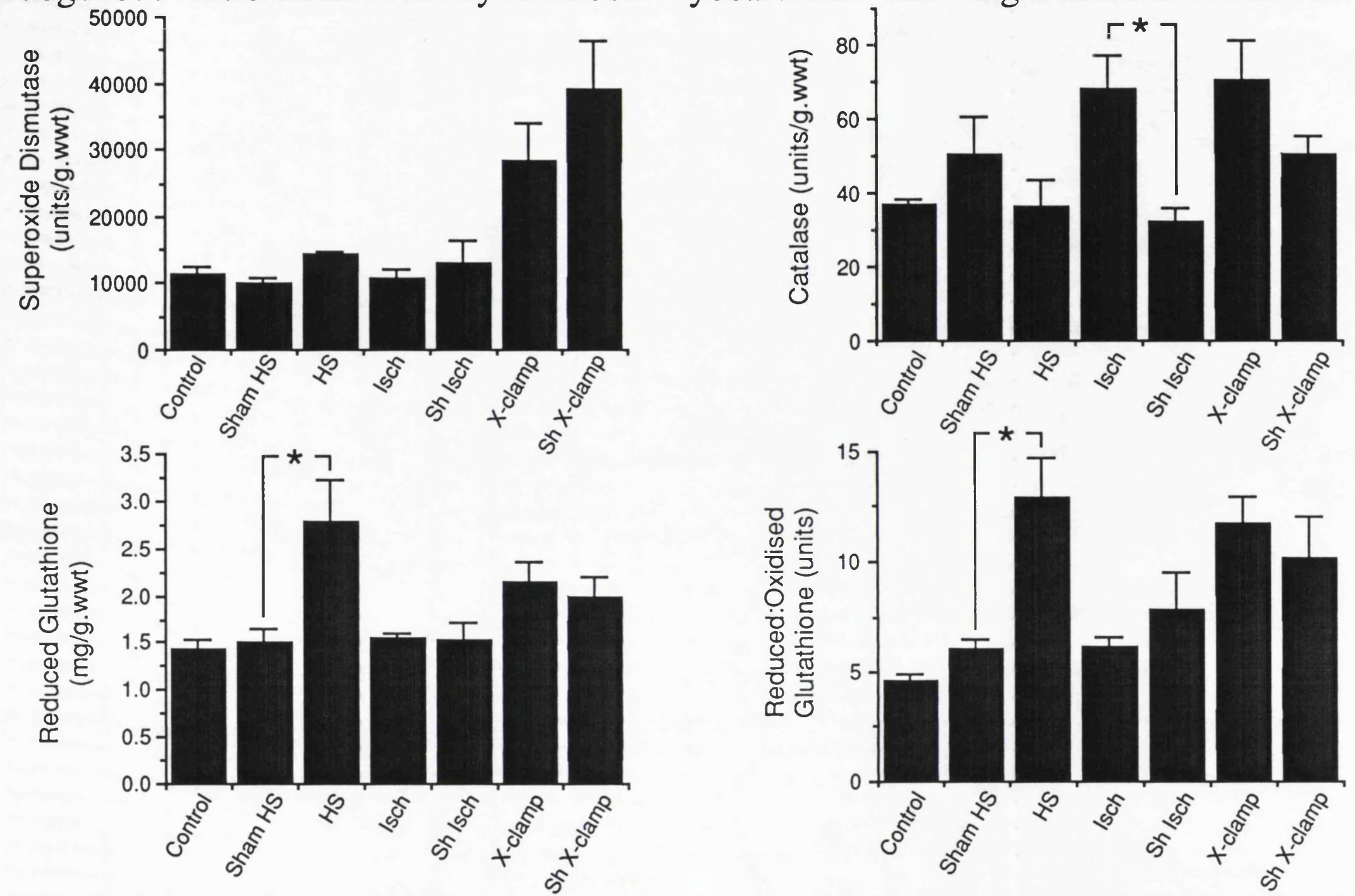

Figure 8.2: Endogenous superoxide dismutase, catalase and glutathione activities following various forms of pretreament. Control=no pretreatment, sham $\mathrm{HS}=$ anaesthetic +24 hours recovery, $\mathrm{HS}=$ heat stress +24 hours recovery, isch $=4 \times 5 \mathrm{~min}$ coronary occlusions +24 hours recovery, $\mathrm{Sh}$

$\mathrm{Isch}=$ anaesthetic with sternotomy +24 hours recovery, $X$-clamp $=4 \times 10$ seconds of complete occlusion of ascending aorta +24 hours recovery, $\mathrm{Sh}$

$\mathrm{X}$-clamp $=$ anaesthetic and sternotomy +24 hours recovery. Bars represent $+/$-sem. $n$ varies between 2 and $6 .{ }^{*} p=\leq 0.05$ by unpaired $t$-test. 
subsequent differing stress. The changes induced by the first stress seem to be numerous and non-specific involving changes in stress protein content, endogenous antioxidants and perhaps several other factors. This drawback can only be overcome by specific manipulation of myocyte stress protein content. This could be achieved by a number of mechanisms. In-vivo gene transfer has been described by direct injection of DNA into the myocardium (von Harsdorf et al 1993) however this technique is inefficient and involves mechanical injury likely to induce the stress response. An alternative would be to transfect myocytes in-vivo using a cardiotropic viral vector such as coxsackie. Once again viral infection acts as a trigger for the stress response at least in the acute phase (Bansal et al 1990). It may however be possible to stably transfect the heart in-vivo over a timeframe that would allow the acute cellular response to viral infection to decay (Stratford-Perricaudet et al 1992).

An alternative strategy would be to use isolated myocytes and transfect either primary cultures or an established dividing myocyte derived cell line. Myocyte cell lines such as the H9c2 embryonal rat line have been stably transfected with hsp72 and transfected cells seem resistant to simulated ischaemia (Mestril et al 1992). The problem with this avenue of investigation is that dividing myocytes are dedifferentiated, the H9c2 line for example more closely resembles skeletal myoblasts than cardiac myocytes (Hescheler et al 1991). Transfecting primary cultures would also present difficulties since these myocytes also tend to de-differentiate with the time needed to confirm stable transfection and expression of the relevant protein. These problems coupled with the complication of, (i)interpreting alterations in isolated myocyte (unloaded) contraction (ii)designing in-vitro stresses to simulate ischaemia and reperfusion (iii)interpreting enzyme efflux and other surrogate endpoints in the absence of cell to cell communication and mechanical coupling, makes the use of these models unattractive.

Perhaps the most attractive strategy would be to develop a transgenic mouse with organ specific and time specific induction of hsp72. This could be achieved by coupling the hsp72 gene to an organ specific promotor such as myosin light chain 2 or by triggering expression through the metalloproteinase promotor (Robbins 1993). However, this model has the disadvantage that in-vivo endpoints of myocardial injury have not been characterised in the mouse. 
Although these molecular manipulative strategies would go someway into determining the relative importance of hsp72, or some other best-bet stress protein, there are intricacies to the stress response that remain difficult to simulate. For example translocation of hsp72 to the nucleus may hold some special importance (Snoeckx et al 1991) as may post-translational modifications that alter hsp72 mobility on 2-dimensional gels (Meerson et al 1992). These subtleties may make the results of genetically engineered increases in single stress proteins difficult to interpret.

\section{The Implications of the Work Described}

The field of myocardial protection has been an area of intensive research for over 2 decades. It's attraction lies in the fact that myocardial infarction is the major cause of death in the Western World and that any manoeuvre that slows or prevents ischaemic cell death would have important clinical applications. The need for "antiinfarct" therapies has now become even more relevant as a possible adjunct to thrombolytic therapy. The results presented in this thesis suggest that the changes occurring within the myocardium following whole body heat stress and sublethal ischaemia are capable of reducing the myocardial injury occurring in response to a number of stresses including lethal ischaemia. At present the mechanisms underlying this protection are unknown, and it is not practical to use either whole body heat stress or sublethal ischaemia in the clinical setting. Future applications must account for the fact that in most instances the onset of myocardial infarction is unpredictable and effective therapies would therefore need to act within the timecourse of thrombolytic therapy, this is unlikely to be tenable. Interestingly, the results presented suggest that endogenous protective mechanisms may already have been triggered in those patients with ischaemic syndromes as a prodrome to infarction.

With further characterisation of the basis for the myocardial protection described in this thesis it may at some future date be possible, by specific and nonabusive means, to increase the heart's endogenous protective mechanisms to alter prognosis in individuals at an increased risk of myocardial ischaemia. 


\section{Appendix 1}

\section{Solutions used for SDS-PAGE and Western Blotting}

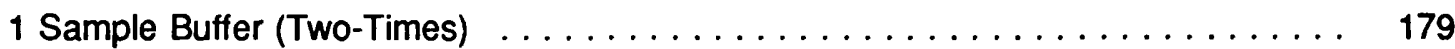

2 Acrylamide Gel Solution $\ldots \ldots \ldots \ldots \ldots \ldots \ldots \ldots \ldots \ldots \ldots \ldots \ldots$

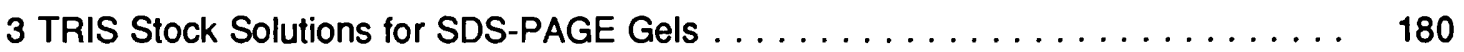

3.1 Resolving (Base) Gel . . . . . . . . . . . . . . . . . . . . . . . . . 180

3.2 Stacking (Top) Gel . . . . . . . . . . . . . . . . . . 181

4 SDS-PAGE Running Buffer $\ldots \ldots \ldots \ldots \ldots \ldots \ldots \ldots \ldots \ldots \ldots \ldots \ldots$

5 Coomasie Blue Staining Solution . . . . . . . . . . . . . . . . . . . . 181

6 Coomasie Blue De-Staining Solution $\ldots \ldots \ldots \ldots \ldots \ldots \ldots \ldots \ldots \ldots$

7 Ten-Times Blotting Buffer for Western Blotting $\ldots \ldots \ldots \ldots \ldots \ldots \ldots \ldots \ldots$

\section{Sample Buffer (Two-Times)}

This solution was used to solublize myocardial specimens, and has a high specific gravity allowing samples to sink into the wells in the stacking gel.

Comprises 20\% Glycerol, 6\% SDS in $0.12 \mathrm{M}$ TRIS at $\mathrm{pH} 6.8$

$\begin{array}{ll}\text { Glycerol } & 20 \mathrm{ml} \\ \text { SDS* } & 6 \mathrm{~g} \\ \text { TRIS } \dagger & 1.4 \mathrm{~g}\end{array}$

the above was made upto $100 \mathrm{ml}$ with $\mathrm{D}$ water.

Prior to use $800 \mathrm{mcl}$ of 2-times buffer is added to $200 \mathrm{mcl}$ of beta- mercaptoethanol and $5 \mu \mathrm{l}$ of Bromophenol Blue dye $8 \%$ in distilled water (w/v).

*SDS = sodium dodecylsulphate (Laural Sulphate, Sigma chemicals)

†TRIS = tris(hydroxymethyl)methylamine (Sigma chemicals, Mousourri, USA) 


\section{Acrylamide Gel Solution}

The gels were prepared from varying proportions of acrylamide $30 \%(\mathrm{w} / \mathrm{v}$ in dist water), TRIS buffered gel stock solution at $\mathrm{pH} 6.8$ or 8.8 (see below) and distilled water. Temed and APS were added to promote polymerisation and crosslinking.

$$
\text { stacking gel resolving gel }
$$

\begin{tabular}{|c|c|c|c|c|}
\hline Concentration\% & 5 & 10 & 12.5 & \\
\hline $30 \%$ Acrylamide* & $2 \mathrm{ml}$ & 8.4 & 12 & (mls) \\
\hline $\begin{array}{l}\text { Tris pH } 8.8 \\
\text { (resolving) }\end{array}$ & $9 \mathrm{ml}$ & 9 & 9 & (mls) \\
\hline $\begin{array}{l}\text { Tris pH } 6.8 \\
\text { (stacking) }\end{array}$ & $3 \mathrm{ml}$ & - & - & \\
\hline Dist Water & $7 \mathrm{ml}$ & 18 & 15 & (mls) \\
\hline $10 \%$ APS $\dagger$ & $100 \mu \mathrm{l}$ & 225 & 180 & ( $\mu l s)$ \\
\hline TEMED & $10 \mu l$ & 15 & 15 & $(\mu l s)$ \\
\hline
\end{tabular}

*30\% Acrylamide with 1\% bisacrylamide (Protogel solution, National Diagnostics, New Jersey, USA)

$\dagger$ APS $=$ Ammonium persulphate (BDH chemicals, Dorset, England), made up in distilled water $10 \% \mathrm{w} / \mathrm{v}$

$\S$ TEMED $=$ NNNN-Tetraethylethalinediamine (Sigma chemicals, Mousourri, USA)

\section{TRIS Stock Solutions for SDS-PAGE Gels}

\subsection{Resolving (Base) Gel}

Comprised 1.5 M TRIS, $0.4 \%$ SDS pH adjusted to 8.8 with $\mathrm{HCl}$

\begin{tabular}{llll}
\hline $100 \mathrm{ml}$ & SDS $0.4 \mathrm{~g}$ & $\underline{500 \mathrm{ml}}$ & SDS $2.0 \mathrm{~g}$ \\
& TRIS $18.16 \mathrm{~g}$ & TRIS $90.9 \mathrm{~g}$ \\
made upto: & $100 \mathrm{ml}$ with D water & & $500 \mathrm{ml}$ with D water
\end{tabular}


3.2 Stacking (Top) Gel

Comprised $0.5 \mathrm{M}$ TRIS, $0.4 \%$ SDS pH adjusted to 6.8 with $\mathrm{HCl}$

$\begin{array}{llll}100 \mathrm{ml} & \text { SDS } 0.4 \mathrm{~g} & \underline{500 \mathrm{ml}} & \text { SDS } 2.0 \mathrm{~g} \\ & \text { TRIS } 6.05 \mathrm{~g} & \text { TRIS } 30.25 \mathrm{~g} \\ \text { made upto: } & 100 \mathrm{ml} \text { with D water } & & 500 \mathrm{ml} \text { with D water }\end{array}$

\section{SDS-PAGE Running Buffer}

This was made up as a ten-times concentrated stock solution and diluted in distilled water prior to use. The electrophoresis apparatus required approximately 2 litres of solution to fill upper and lower resevoirs. The natural $\mathrm{pH}$ of this solution was critical, and if it deviated from $\mathrm{pH} 8.8$ proteins would fail to resolve adequately. It was prepared by mixing reagents in the following proportions.

$\begin{array}{llc} & \underline{1000 \mathrm{ml}} & \underline{500 \mathrm{ml}} \\ \text { glycine } & 144.2 \mathrm{~g} & 72.1 \mathrm{~g} \\ \text { SDS } & 10.0 \mathrm{~g} & 5.0 \mathrm{~g} \\ \text { TRIS } & 30.3 \mathrm{~g} & 15.15 \mathrm{~g}\end{array}$

\section{Coomasie Blue Staining Solution}

This solution was used to stain SDS-PAGE gels to visualise protein. The stain was prepared by mixing constituents in the following proportions.

$\begin{array}{lc}\text { Methanol } & 250 \mathrm{ml} \\ \text { D water } & 250 \mathrm{ml} \\ \text { Acetic acid } & 35 \mathrm{ml} \\ \text { Coomasie blue } & 1.07 \mathrm{~g}\end{array}$




\section{Coomasie Blue De-Staining Solution}

This solution was used to wash non-specific Coomasie blue stain from SDS-PAGE gels. The solution needed to be changed frequently as dye leeched out of the acrylamide. It comprised of $10 \%$ acetic acid, $50 \%$ methanol and $40 \%$ distilled water.
Acetic acid
$300 \mathrm{ml}$
Methanol
$1500 \mathrm{ml}$
D water
$1200 \mathrm{ml}$

During destaining the gel would shrink and was re-expanaded by agitating in distilled water with $7 \%$ acetic acid.

\section{Ten-Times Blotting Buffer for Western Blotting}

A ten-times concentrated stock solution was made from the following reagents.

$\underline{1000 \mathrm{ml}}$

$30.3 \mathrm{~g}$

TRIS

Glycine

$144.2 \mathrm{~g}$

made upto: $1000 \mathrm{ml}$ with $\mathrm{D}$ water $\underline{500 \mathrm{ml}}$

$72.1 \mathrm{~g}$

$500 \mathrm{ml}$ with $\mathrm{D}$ water

Prior to Western blotting $300 \mathrm{mls}$ of ten-times blotting buffer was added to $600 \mathrm{ml}$ of methanol and $2100 \mathrm{ml}$ of $\mathrm{D}$-water to make one-times buffer. The standard blotting tank required 3 litres of reconstituted blotting buffer. 


\section{Appendix 2}

\section{Programmes used for the Analysis of Papillary Muscle Contraction}

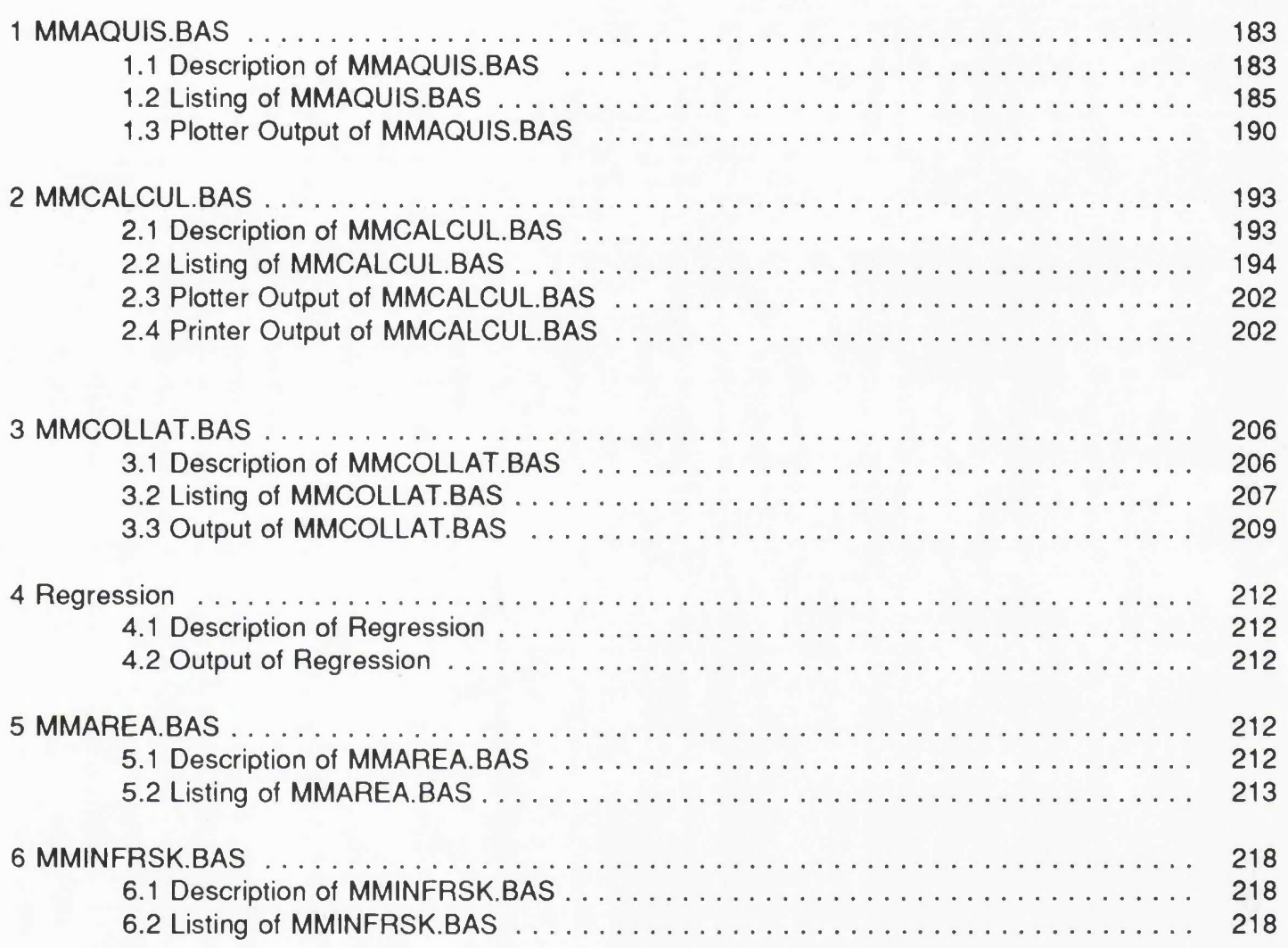

\section{MMAQUIS.BAS}

\subsection{Description of MMAQUIS.BAS}

This program operates in real time to acquire and display data from the $A / D$ converter.

lines

20-40

50

$60-100$

$130-430$

\section{Function}

Request the entry of a file name. The format of this name is (i) first character letter of the alphabet describing the stage of the experiment (ii) the date in 6 figure format (iii) a suffix describing the species being used for the experiment.

Sets the graphic screen size

Interrogate the computer clock to set the time, day, date and month Set up screen windows, graph axes and annotation 
440-580 Inputs data from A/D board via RS232 port, the program loops until output begins, the first 8 bytes are taken as 4 integers ISS, IES, ID1, ID2 that describe steady state, extrasystolic, first post-extrasystolic(1) and second post-extrasystolic(2) coupling intervals, respectively. The next 400 bytes are incorporated into 4,100 character strings describing the tension envelopes of steady state, extra-systolic, first post-extrasystolic and second post-extrasystolic beats.

590-660 Draws the windows at the top of the screen to display force envelopes 670-740 Every second ASC II characters is "peeled" from each of the 4 data strings, inverted and set into an array.

760-820 The ASC II values of the last $64 \mathrm{mS}$ of each of the 4 force envelopes is averaged to give a resting force value.

830-970 Determines the maximal developed force for each of the 4 beats, draws the force envelopes for each of the 4 beats and plots the resting tension on the lower graph axes.

980-1080 Draws maximum developed force for each of the 4 beats on the lower graph axes.

1090-1140 Opens a random access file and writes the beat coupling intervals, ASC II character strings describing the force envelope and the run number to disc.

1160 Controls program flow, until the desired maximal extra-systolic interval is reached, the program loops searching for more data from the A/D converter.

1170-1240 Writes to screen that data acquisition has finished and emits a sound to attract operators attention. (The appearance of the screen at the end of the force interval protocol is shown in Chapter 2 figure 2.7.)

1250-1270 Controls program flow, if the run is successful the program continues, if not it finishes here.

1280-1320 Appends the date and time of the experiment to a log file "datedata" on the floppy disc.

Subroutine 1380 This nested subroutine drives the Epson HI-80 pen plotter. The plotter prints experiment title, date of creation, and force interval points for maximal developed force as well as maximal positive and negative $\mathrm{dF} / \mathrm{dt}$ at each extra-systolic interval. An example of the output of this subroutine is seen in Chapter 3 figure 3.2 and at the end of the program listing.

1350-1360 These lines display the content of the data log file datedata. 
1.2 Listing of MMAQUIS.BAS

1 REM ***Michael Marber 20/12/90***

$5 \mathrm{KEY}(8) \mathrm{ON}$

6 ON KEY(8) GOSUB 1180

7 DIM COORD $(1,3,50)$

10 CLS

20 PRINT "This is the New Data Aquisition Program !":PRINT

30 INPUT "Enter the file name ...New Format...";F\$

40 INPUT"Enter Max coupling interval for interposed beat in $\mathrm{mS}$ ";MIES\%

50 VIEW $(10,1)-(790,210)$

$60 \mathrm{CLS}$

70 DEFINT I-M

$80 \mathrm{~T} \$=$ TIME $\$$

90 MONTH $\$=" J a n F e b M a r A p r M a y J u n J u l A u g S e p O c t N o v D e c "$

100 DEF FNMO =VAL(MID\$(DATE $\$, 1,2)$ )

$110 \mathrm{IR}=1$

120 CLS

130 DIM L(2500),M(50,4),MX(4)

140 GET $(10,5)-(170,200), \mathrm{L}$

$150 \operatorname{VIEW}(10,1)-(790,210), 1$

160 FOR I=1 TO 4

$170 \mathrm{~K}=195 * \mathrm{I}-180$

180 PUT(K,7),L,PSET

190 NEXT I

200 VIEW $(200,230)-(790,380)$

210 WINDOW $(2.1,-20)-(4.5,270)$

220 LINE $(2.2,0)-(2.15,0)$

230 LINE $(2.2,100)-(2.15,100)$

240 LINE $(2.2,200)-(2.15,200)$

250 LINE $(2.2,0)-(2.2,255)$

260 LINE $(2.2,0)-(4.5,0)$

270 FOR $X=200$ TO 1000 STEP 200

$280 \mathrm{Y}=\mathrm{LOG}(\mathrm{X}) / 2.3$

$290 \operatorname{LINE}(\mathrm{Y}, 0)-(\mathrm{Y},-10)$

300 NEXT $X$

310 FOR $X=2000$ TO 20000 STEP 1000

$320 \mathrm{Y}=\mathrm{LOG}(\mathrm{X}) / 2.3$ 
330 LINE $(\mathrm{Y}, 0)-(\mathrm{Y},-10)$

340 NEXT $X$

$350 \mathrm{Y}=\mathrm{LOG}(1000) / 2.3$

360 LINE $(Y, 0)-(Y,-20)$

$370 \mathrm{Y}=\mathrm{LOG}(10000) / 2.3$

380 LINE $(\mathrm{Y}, 0)-(\mathrm{Y},-20)$

390 LOCATE 25,40:PRINT " 1 -SEC

10-SEC";

400 LOCATE 20,19:PRINT "100";

410 LOCATE 17,19:PRINT "200";

420 LOCATE 25,1:PRINT " FILENAME=",F\$;

430 OPEN "AUX" FOR INPUT AS 2

440 A $\$=\operatorname{INPUT} \$(1, \# 2)$

450 IF ASC(A $\$)=0$ GOTO 470

460 GOTO 440

470 INPUT\# 2,ISS,IES,ID1,ID2

$480 \mathrm{D} \$(0)=\operatorname{INPUT} \$(100, \# 2)$

$490 \mathrm{D} \$(1)=\operatorname{INPUT} \$(100, \# 2)$

$500 \mathrm{D} \$(2)=\operatorname{INPUT} \$(100, \# 2)$

$510 \mathrm{D} \$(3)=\operatorname{INPUT} \$(100, \# 2)$

520 LOCATE 15,15:PRINT USING "\#\#\#\#\#\#\#\#\#\#\#\#\#"; IES,ID1,ID2

530 LOCATE 15,10:PRINT ISS

$540 \mathrm{M}(0,0)=\mathrm{ISS}: \mathrm{M}(0,1)=\mathrm{IES}: \mathrm{M}(0,2)=\mathrm{ID} 1: \mathrm{M}(0,3)=\mathrm{ID} 2$

550 M0 $\$=$ MKI $\$$ (ISS)

$560 \mathrm{M} 1 \$=\mathrm{MKI} \$(\mathrm{IES})$

$570 \mathrm{M} 2 \$=\mathrm{MKI} \$(\mathrm{ID} 1)$

$580 \mathrm{M} 3 \$=\mathrm{MKI} \$(\mathrm{ID} 2)$

590 WINDOW

600 VIEW $(10,1)-(790,210)$

610 FOR I=1 TO 4

$620 \mathrm{~K}=195 * \mathrm{I}-180$

630 PUT(K,7),L,PSET

640 NEXT I

650 WINDOW $(0,-30)-(320,300)$

660 LINE $(0,0)-(0,0)$

670 FOR I $=0$ TO 3

$680 \mathrm{~K}=1$

690 FOR J= 1 TO 49 
$700 \mathrm{~A} \$=\mathrm{MID} \$(\mathrm{D} \$(\mathrm{I}), \mathrm{K}, 1)$

$710 \mathrm{M}(\mathrm{J}, \mathrm{I})=256-\mathrm{ASC}(\mathrm{A} \$)$

$720 \mathrm{~K}=\mathrm{K}+2$

730 NEXT J

740 NEXT I

750 SUM $=0:$ MEAN $=0: N N=0$

760 FOR $\mathrm{I}=0$ TO 3

770 FOR $\mathrm{J}=41$ TO 49

780 SUM=SUM+M(J;I)

$790 \mathrm{NN}=\mathrm{NN}+1$

800 NEXT J

810 NEXT I

$820 \mathrm{MEAN}=\mathrm{SUM} / \mathrm{NN}$

830 FOR I=0 TO 3

$840 \mathrm{MX}(\mathrm{I})=0$

$850 \mathrm{~K} 1=(\mathrm{I}+1) * 80-65$

$860 \mathrm{JK}=\mathrm{M}(0, \mathrm{I}+1) / 10$

870 IF I=3 THEN JK=48

$880 \mathrm{IF} \mathrm{JK}>48$ THEN $\mathrm{JK}=48$

890 FOR J=2 TO JK

$900 \mathrm{M}(\mathrm{J}, \mathrm{I})=\mathrm{M}(\mathrm{J}, \mathrm{I})-\mathrm{MEAN}$

910 IF $\mathrm{M}(\mathrm{J}, \mathrm{I})>\mathrm{MX}(\mathrm{I})$ THEN MX(I)=M(J,I)

$920 \mathrm{M}(\mathrm{J}, \mathrm{I})=\mathrm{M}(\mathrm{J}, \mathrm{I})+\mathrm{MEAN}$

$930 \mathrm{~K}=\mathrm{K} 1+\mathrm{J}$

940 LINE (K,M(J,I))-(K,M(J+1,I))

950 CIRCLE(K,MEAN),.02

960 NEXT J

970 NEXT I

980 VIEW $(200,230)-(790,380)$

990 WINDOW $(2.1,-20)-(4.5,270)$

$1000 \mathrm{ES}=\mathrm{IES}$

$1010 \mathrm{X}=\mathrm{LOG}(\mathrm{ES}) / 2.3$

1020 PSET(X,MX(0))

1030 CIRCLE(X,MX(1)),.02

1040 CIRCLE(X,MX(2)),.01

1050 LOCATE 20,1:PRINT "RUN N0=";:PRINT USING "\#\#";IR

1060 FOR J=0 TO 2 
$1070 \operatorname{COORD}(0, \mathrm{~J}, \mathrm{IR})=\mathrm{X} * 2.3: \operatorname{COORD}(1, \mathrm{~J}, \mathrm{IR})=\mathrm{MX}(\mathrm{J})$

1080 NEXT

1090 OPEN "R",\#1,F\$,420

1100 FIELD \#1,2 AS F0\$,2 AS F1\$,2 AS F2\$,2 AS F3\$,100 AS F\$(0),100 AS F\$(1), 100 AS F\$(2),100 AS F\$(3)

1110 LSET F0 $\$=M 0 \$:$ LSET F $\$=M 1 \$:$ LSET F $\$=M 2 \$:$ LSET F $3=M 3 \$$

$1120 \operatorname{LSET~} F \$(0)=D \$(0): \operatorname{LSET} F \$(1)=\mathrm{D} \$(1): \operatorname{LSET} F \$(2)=\mathrm{D} \$(2): \operatorname{LSET} F \$(3)=\mathrm{D} \$(3)$

1130 PUT \#1,IR

1140 CLOSE 1

$1150 \mathrm{IR}=\mathrm{IR}+1$

1160 IF IES=MIES THEN GOTO 1180

1170 GOTO 440

1180 CLOSE

$1190 \operatorname{VIEW}(20,6)-(780,205), 2$

1200 LOCATE 6,35:PRINT"*Finish*":LOCATE 6,40:PRINT;

1210 FOR K=1 TO 2

1220 FOR D=30 TO 2000: SOUND D,1:NEXT D

1230 FOR D=30 TO 2000 STEP 20: SOUND D,1:NEXT D

1240 NEXT K

1250 LOCATE 7,3:PRINT"Okay (y/n) ?";

1260 A $\$=$ INKEY $\$: I F$ A $\$=" "$ THEN GOTO 1260

$1270 \mathrm{~A}=\mathrm{INSTR}(" Y y N n ", \mathrm{~A} \$$ ):IF A =0 THEN GOTO 1260 ELSE IF A >2 THEN PRINT "No.":END ELSE PRINT "Yes."

1280 D $\$=M I D \$(D A T E \$, 4,2)+"$ "+MID\$(MONTH\$,(FNMO-1)*3+1,3)+" "+ $\operatorname{MID} \$(\mathrm{DATE} \$, 7,4)$

1290 REM Record time and date that file was created

1300 OPEN "A",\#1,"datedata"

1310 PRINT \#1,F\$;","T\$;",";D\$

1320 CLOSE 1

1330 GOSUB 1380

1340 END

1350 REM Display datedata $===>$

1360 OPEN "I",\#1,"datedata":WHILE NOT EOF(1):INPUT \#1,A\$,B\$,C\$:PRINT A\$,B \$,C\$:WEND:CLOSE 1

1370 END

1380 REM Plot resitition graphs...

1390 OY $=200$ 
1400 LPRINT "vs1"

1410 A $\$=L E F T \$(F \$, 1)$

1420 A=INSTR("aei",A\$)

1430 IF A>0 THEN LPRINT "si 40,40":LPRINT "di 0,36":GOSUB 1640:LPRINT

"ma 50,1350":LPRINT "la";D\$:OX=660:GOTO 1470

1440 A=INSTR("bfj",A\$): IF A>0 THEN OX=1305:GOTO 1470

1450 A=INSTR("cgk",A\$): IF A>0 THEN OX=1972:GOTO 1470

1460 A=INSTR("dhl",A\$): IF A>0 THEN OX=2640 ELSE OX=668

1470 LPRINT "si 25,25":LPRINT "ma ";OX-600;",";OY:LPRINT "ax $1,120,5,0,250,-50,10,1^{\prime \prime}$

1480 LPRINT "si 20,20":LPRINT "ma ";OX;",";OY:LPRINT "ax 0,750,2,0"

1490 FOR K=200 TO 800 STEP 200:LPRINT "ma ";

OX-5;",";OY+(LOG(K)-4.6)*325.72:LPRINT "dr 10,0":NEXT:FOR K=2000

TO 9000 STEP 1000:LPRINT "ma ";

OX-5;",";OY+(LOG(K)-4.6)*325.72:LPRINT "dr 10,0":NEXT

1500 LPRINT "di 0,36":LPRINT "ma ";OX+30;",";OY+750:LPRINT "la1":LPRINT "ma";OX+30;",";OY+1500:LPRINT "la10"

1510 LPRINT "vs0"

1520 FOR $\mathrm{J}=0$ TO 2

1530 FOR I=1 TO IR-1

1540 LPRINT "am ";2^J;",";OX-COORD(1,J,I)*2.4;

",";OY+(COORD(0,J,I)-4.6)*325.72

1550 NEXT:NEXT

1560 LPRINT "si 30,30"

1570 LPRINT "ma ";OX-550;",";OY+800:LPRINT "la";F\$;" timed at:

";LEFT\$(T\$,5):LPRINT "si 20,20"

1580 LPRINT "ma ";OX-500;",";OY+1000:LPRINT "rm 1,0,0":LPRINT "la = SS"

1590 LPRINT "ma ";OX-450;",";OY+1000:LPRINT "rm 2,0,0":LPRINT "la = ES"

1600 LPRINT "ma ";OX-400;",";OY+1000:LPRINT "rm 4,0,0":LPRINT "la = PES"

1610 LPRINT "si 20,20"

1620 IF OX=2640 THEN LPRINT "ch":CLS:PRINT"Change paper":FOR K=1 TO 3:

FOR F=0 TO 2000 STEP 20:SOUND F,1:NEXT:NEXT ELSE LPRINT "ma $2600,0^{\prime \prime}$

1630 RETURN

1640 REM Title Restitution graphs

1650 CLS

1660 PRINT "Title resitition graphs" 
1670 PRINT:PRINT " Which title do you wish ?"

1680 PRINT:PRINT SPC(5)"1. Control Animal"

1690 PRINT SPC(5)"2. Heat shock Animal"

1700 PRINT SPC(5)"3. No title."

1710 PRINT:PRINT " Option ?";

1720 A $\$=$ INKEY\$:IF A $\$=" "$ THEN GOTO 1720

1730 A=INSTR("123",A\$):IF A=0 THEN 1720

1740 GOSUB 1810

1750 ON A GOSUB $1780,1790,1800$

1760 ON A GOSUB $1780,1790,1800$

1770 RETURN

1780 LPRINT "ma 40,200":LPRINT "laControl ";A\$:RETURN

1790 LPRINT "ma 40,200":LPRINT "laHeat Shock ";A\$:RETURN

1800 LPRINT "ma 0,0":LPRINT "da 0,1000,35,1000,35,0,0,0":RETURN

1810 REM Assign title according to file name

1820 A $\$=$ RIGHT $\$(F \$, 3)$

1830 IF A $\$=" r a b "$ THEN A $\$="$ Rabbit"

1840 IF A $\$="$ rat" THEN $A \$="$ Rat"

1850 IF A $\$="$ fer" THEN $A \$=" F e r r e t "$

1860 IF A $\$=" d o g "$ THEN $A \$=" D o g "$

1870 IF A $\$=" h u m "$ THEN A $\$=" H u m a n "$

1880 IF LEN(A $\$)>3$ THEN RETURN

1890 A $\$="$ animal":RETURN

For an example of the screen output of MMAQUIS.BAS see Chapter 3 figure 3.2 For an example of the plotter output of MMAQUIS.BAS see Chapter 2 figure 2.8 and figure app2.1 in this Appendix.

\subsection{Plotter Output of MMAQUIS.BAS}

An example of the output of this programme is shown in figure app2.1, these force interval curves were derived from the experimental protocol described in Chapter 3. 


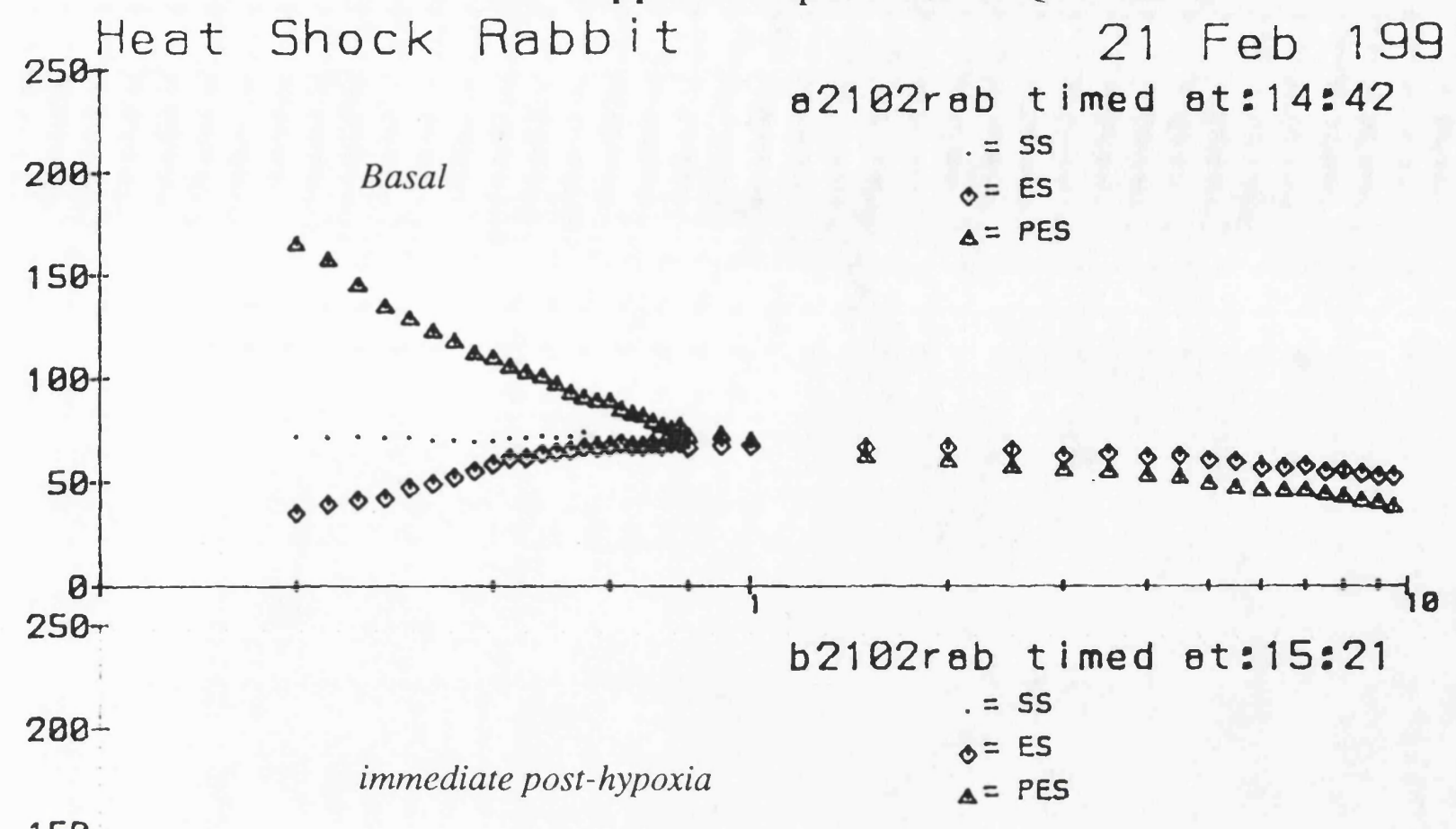

150

100

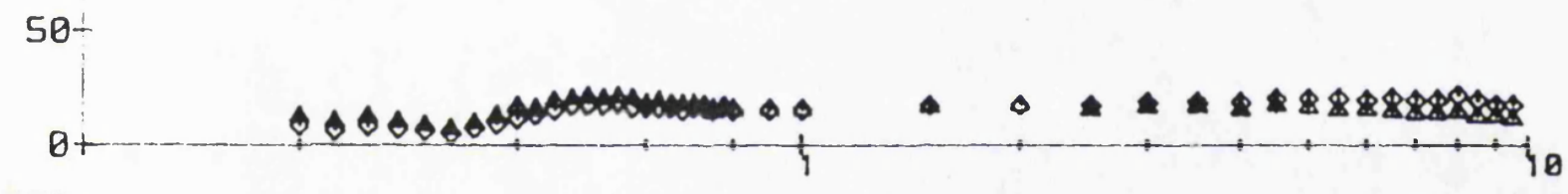

250

c2102rab timed at:15:57

200

30 mins post-hypoxia

. = SS

$0=E S$

$\Delta=$ PES

150

$100-$

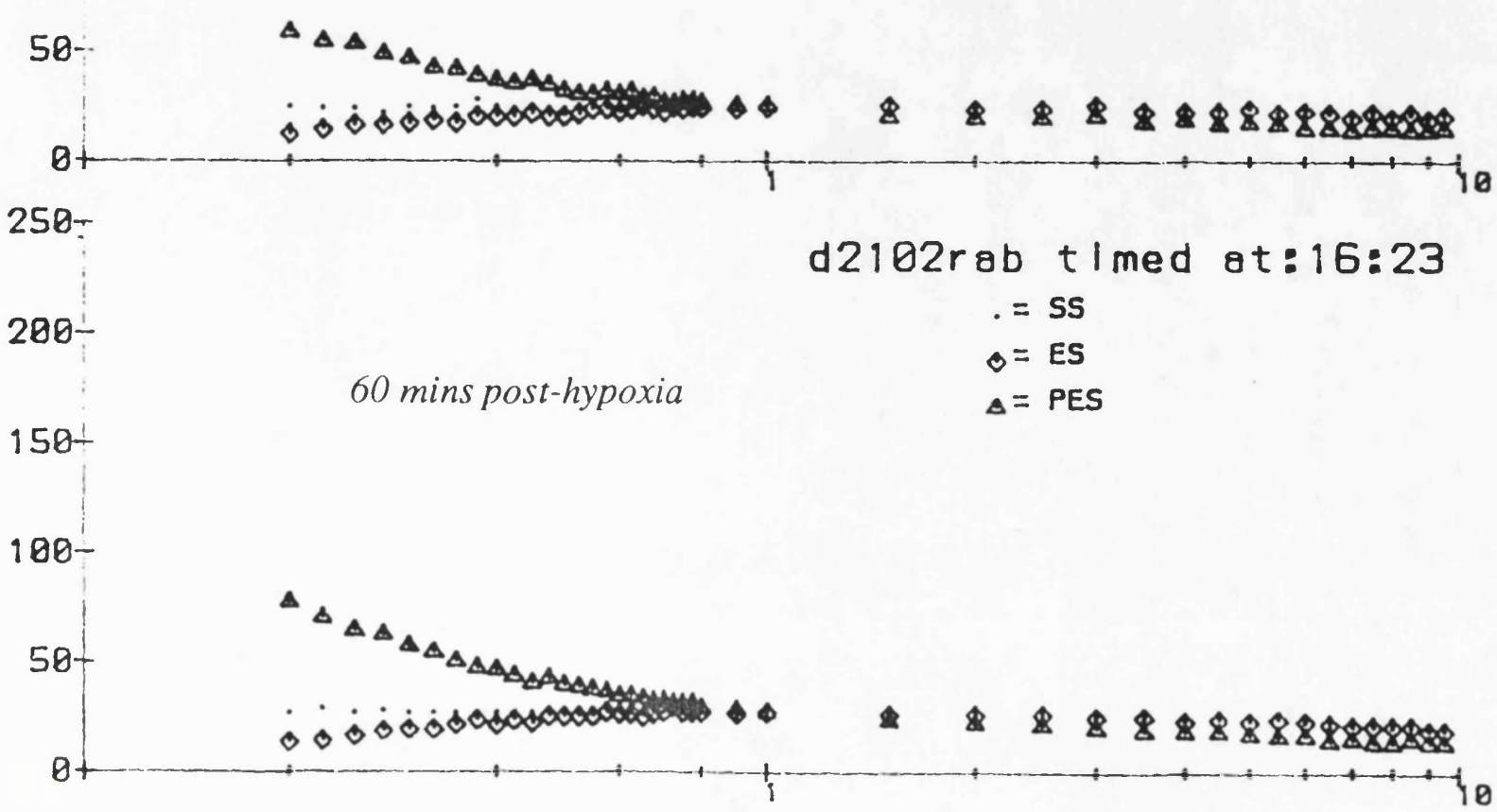




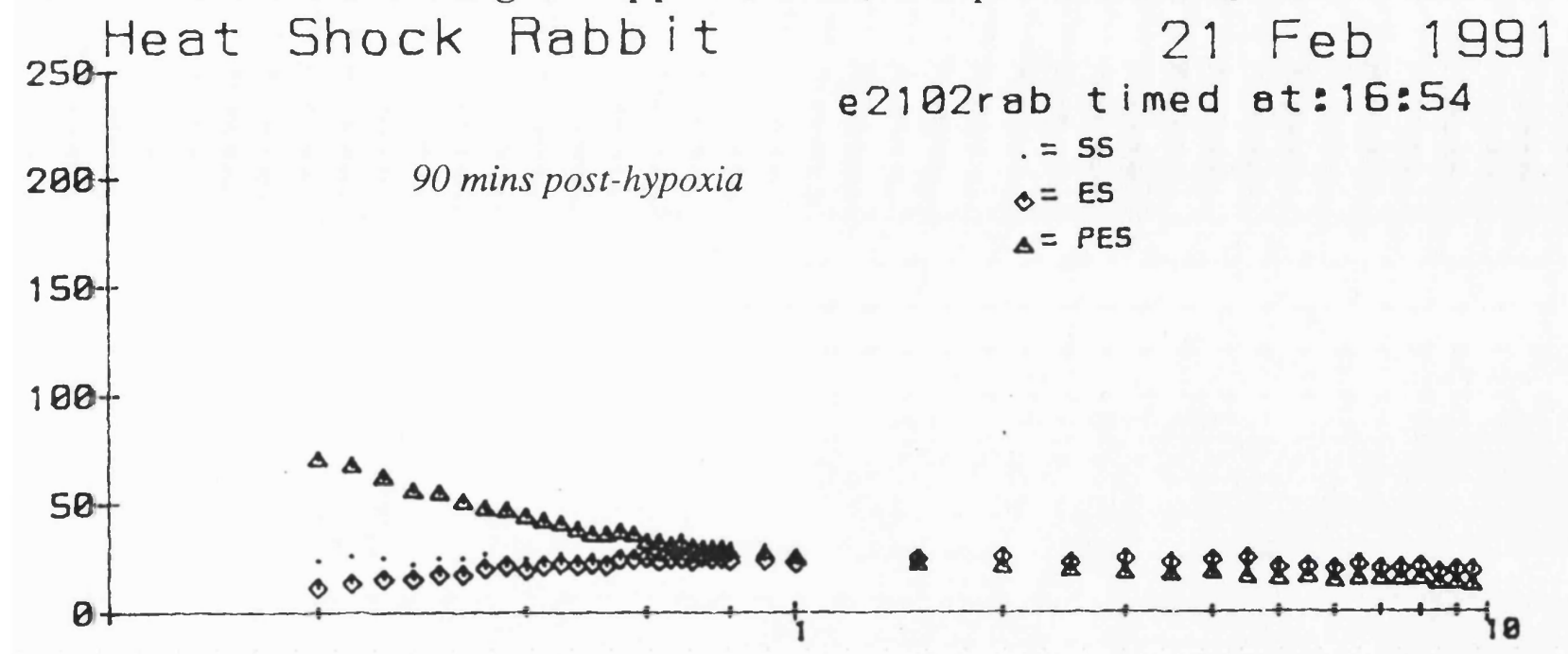

Figure App2.1: The output of MMAQUIS.BAS for the entire sustrate preference protocol (see chapter 4). The $y$-axis of each force-interval curve is in ASC II units, the $\mathrm{x}$-axis in log seconds. Each curve is plotted out in real time at the end of the data collection (when extrasystolic interval $=10 \mathrm{sec}$ ), the steady state beats are averaged and used to calculate developed and resting force. $S S=$ steady state beat, $\mathrm{ES}=$ extrasystolic beat and PES=post- extrasystolic beat. 


\section{MMCALCUL.BAS}

\subsection{Description of MMCALCUL.BAS}

This programme analyses the contraction data saved on disc by MMAQUIS.BAS.

lines function

50-90 Sets all variables to zero and sets the dimensions of the data arrays to be used. Line 60 defines the function used to convert ASC II character values to force in grammes.

160-200 Request information regarding the settings of the bridge amplifier used during the experiment and the name of the data file for analysis.

290 Sets the values for the variables used to convert ASC II to grammes on the basis of the amplifier settings.

Subroutine 1100 Opens the data $\log$ disc file containing the details of date and time the experiment was performed.

Subroutine $1490 \quad$ Plots the axes on the screen for the force-interval relationship. 310-320 Sets the file suffixes to save maximum values for extrasystolic and post extrasystolic force and maximum and minimum values for extrasystolic and postextrasystolic $\mathrm{dF} / \mathrm{dt}$.

330-390 Opens the disc file containing the experimental data and gets data. If the conditions of line 380 are met the file name was incorrect and the program flow is directed to a subroutine 1260 to erase the file. If the conditions in line 390 are correct the end of the file has been reached.

440-560 Examines the data points within the ASC II string for steady state, extrasytolic and postextrasystolic beats. Within the arrays set up for each of these beats the peak force, time to peak force, maximum rate of rise of force, maximum rate of fall of force, resting force are all determined and assigned to their respective arrays for each extrasytolic interval.

570-580 Sets the counters for the number of loops through this part of the programme and sums data for later calculation of means.

subroutine 1190 Time to $90 \%$ relaxation, this subroutine was inactivated since it crashed easily with data glitches and proved difficult to protect with software filters.

585-586 Calculate max and min $\mathrm{dF} / \mathrm{dt}$ and enter values in an array. subroutine $1430 \quad$ Plots force data for on screen restitution curve. 
610 Counts the number of times the disc is accessed, equivalent to the number of runs.

620 Repeats data sorting until conditions in line 390 mean end of file and then the file is closed.

subroutine 1310 This routine calculates means and standard error of the mean for each of the data arrays.

subroutine 1750 This routine ending in line 2120 draws the axes and plots the force interval data on the Epson $\mathrm{HI}-80$ pen plotter, in practise this was very time consuming. An example of the output of the plotter is seen in figure app2.2 at the end of the program listing.

657-780 Prints the data out on a HP Inkjet printer. Control characters are sent to the printer to alter character emphasis. An example of the printer output is shown in figure app2.3 at the end of the program listing.

790-1020 Opens and writes to 6 output files. The data written contains the extrasystolic interval with corresponding values for force for extrasystolic beat, post-extrasystolic beat and positive and negative $\mathrm{dF} / \mathrm{dt}$ for each of these beats.

1030-1090 Output messages to speaker and screen to inform operator that data analysis has ended.

\subsection{Listing of MMCALCUL.BAS}

1 REM $\quad * * * * *$ Michael Marber****** 11/01/91

4 REM MMCALCUL.BAS 11/01/91 CALCULATES PEAK TENSION AND dT/dt IN GRAMS.

5 REM THIS PROGRAM CONVERTS DATA STRING IN FILE F\$ FORMED

6 REM BY MMAQUIS, TO SINGLE PRECISION NUMBER IN FORMAT

7 REM FOR IBM 386 REGRESSION ANALYSIS.

8 REM DATA ARRAYS ARE SET UP AS FOLLOWS/ RTA()=REST TEN; RXT()=t 90\%RELAX

9 REM $\quad$ MX()=MAX TEN; VMND()=MIN dT/dt; VMXD()=MAX dT/dt; TTPT()=t to peak $\mathrm{T}$

40 ON ERROR GOTO 1400

50 DEFSTR F,D,S : DEFSNG E,V :DEFINT C,I,J,R,M,T

60 DEF FNLX $(X)=(((X-172.59) /-128)+B C C *(B A L A N C E-4.2)-Z L C) / L C$

70 DIM M(100,4),MX(3,70),ESI(70),VMXD(3,70),VMND(3,70),RXT(3,70),RTA(3,70)

80 DIM TTPT $(3,70)$ 
$90 \mathrm{IR}=1: \mathrm{COUNT}=0: \mathrm{ET} 1=0: \mathrm{RT} 2=0: \mathrm{RT} 1=0: \mathrm{ET} 2=0: \mathrm{CONST}=1000 / 12$

$100 \mathrm{CLS}$

110 PRINT:

120 PRINT "DATA CONVERSION FROM DATA STREAM TO SNG PRECISION" 130 PRINT "DATA DISC 386-XEN FORMAT MUST BE IN DRIVE B" 140 PRINT "VALUES FOR COUPLING INTERVALS AND TENSION

PARAMETERS TO LPT1"

150 PRINT: PRINT: PRINT

160 INPUT "Enter name of data file";F\$

170 PRINT: PRINT:

180 INPUT "FSD of rec amplifier for this file";MAG

190 IF $M A G<1$ OR MAG>5 OR MAG=3 OR MAG=4 THEN PRINT"Out of range.":

BEEP:GOTO 180

200 INPUT "Balance of recording amplifier for this file";BALANCE

210 REM FILES USING FIRST 4 LETTERS OF THIS NAME

220 REM WITH SUFFIX ECT,DP1,MXDE,MNDE,MXD1,MND1 AND

APPENDED <.DAT>

230 REM WILL BE SENT TO DRIVE B:

240 REM DATA WILL BE IN THE FORM OF ASCI EQUIVALENTS IN THE RANGE

250 REM 0 TO 255 FOR TENSION, AND AS SINGLE PRECISION NUMBER FOR TIME

260 REM EXPRESSED IN SECONDS. CONVERSION OF ASCI TO g BY FNLX

270 REM Calculate conversion constants --->

275 REM $\quad \mathrm{BCC}=$ balance correction constant; $\mathrm{ZLC}=$ Zero load constant;

$$
\text { LC }=\text { loading constant }
$$

$280 \mathrm{BCC}=-34.367 *(\mathrm{MAG}=1)-17.409 *(\mathrm{MAG}=2)-6 \mathrm{MAG}=5)$ :

$\mathrm{LC}=1.47 *(\mathrm{MAG}=1)+.737 *(\mathrm{MAG}=2)+.298 *(\mathrm{MAG}=5)$

290 GOSUB 1100

295 GOSUB 1490

$300 \mathrm{~S} \$=\mathrm{MID} \$(\mathrm{~F} \$, 1,4)$

310 S(1)="B:"+S\$+"ECT.DAT" : S(2)="B:"+S\$+"DP1.DAT" :

$\mathrm{S}(3)=$ "B:"+S\$+"MXDE.DAT"

$320 \mathrm{~S}(4)=" \mathrm{~B}: "+\$ \$+" M N D E . D A T "$ : S(5)="B:"+S\$+"MXD1.DAT":

$\mathrm{S}(6)=" \mathrm{~B}: "+\mathrm{S} \$+$ "MND1.DAT"

$330 \mathrm{~F} \$=" \mathrm{~A}: " \mathrm{~F} \$$

340 OPEN"R",\#1,F\$,420 
350 FIELD \#1, 2 AS F0\$, 2 AS F1\$, 2 AS F2\$, 2 AS F3\$, 100 AS D\$(0), 100 AS $\mathrm{D} \$(1), 100 \mathrm{AS} \mathrm{D} \$(2), 100 \mathrm{AS} \mathrm{D} \$(3)$

360 GET \#1:

370 ISS $=\mathrm{CVI}(\mathrm{F} 0 \$): \mathrm{ESI}(\mathrm{IR})=\mathrm{CVI}(\mathrm{F} 1 \$) / 1000: \mathrm{ID} 1=\mathrm{CVI}(\mathrm{F} 2 \$): \mathrm{ID} 2=\mathrm{CVI}(\mathrm{F} 3 \$)$

$380 \mathrm{IF}$ ISS $=0$ AND I=0 THEN GOTO 1260

390 IF ISS $=0$ GOTO 630

400 REM LOOP TO FIND MAX/MIN AFTER INVERSION, FIRST 5 DATA POINTS EXCLUDED

410 REM Differential at $12 \mathrm{msec}$ ie 3 array point distance.

440 FOR I $=0$ TO 2

$450 \mathrm{RT} 0=0: \mathrm{TOT}=0: \mathrm{EX}=0: \mathrm{MAX}=0: \mathrm{MIN}=0$

460 FOR J= 1 TO 98

$470 \mathrm{M}(\mathrm{J}, \mathrm{I})=\mathrm{ASC}(\mathrm{MID} \$(\mathrm{D} \$(\mathrm{I}), \mathrm{J}, 1))$

$480 \mathrm{M}(\mathrm{J}, \mathrm{I})=(256-\mathrm{M}(\mathrm{J}, \mathrm{I}))$

490 IF J $<5$ THEN GOTO 560

$500 \mathrm{IF} M X(\mathrm{I}, \mathrm{IR})<\mathrm{M}(\mathrm{J}, \mathrm{I})$ THEN MX(I,IR)=M(J,I): TTPT(I,IR)=J*4

505 IF I=0 THEN GOTO 540

$510 \mathrm{MX}=\mathrm{M}(\mathrm{J}, \mathrm{I})-\mathrm{M}(\mathrm{J}-3, \mathrm{I})$

520 IF $M X>$ MAX THEN MAX=MX:TMAX $=\mathrm{J}$

530 IF MX<MIN THEN MIN=MX:TMIN=J

540 IF J>87 THEN RT0=RT0+M(J,I):TOT=TOT+1

560 NEXT

$570 \mathrm{ET} 1=\mathrm{ET} 1+\mathrm{RT} 0: \mathrm{COUNT}=\mathrm{COUNT}+\mathrm{TOT}: \mathrm{RTA}(\mathrm{I}, \mathrm{IR})=\mathrm{RT} 0 / \mathrm{TOT}$

575 IF I=0 THEN RT2=RT2+MX(I,IR):GOSUB 1190:GOTO 600

580 REM GOSUB 1190

581 REM Tally for resting tension \& set resting tension array

$585 \mathrm{VMXD}(\mathrm{I}, \mathrm{IR})=\mathrm{FNLX}(\mathrm{M}(\mathrm{TMAX}, \mathrm{I}))$-FNLX(M(TMAX-3,I))

$586 \mathrm{VMND}(\mathrm{I}, \mathrm{IR})=\mathrm{FNLX}(\mathrm{M}(\mathrm{TMIN}, \mathrm{I}))$-FNLX(M(TMIN-3,I))

590 GOSUB 1430

600 NEXT

$610 \mathrm{IR}=\mathrm{IR}+1$

620 GOTO 360

630 CLOSE \#1

650 GOSUB 1310

655 LOCATE 16,30:PRINT" Title of output >";:INPUT TLE\$ 656 GOSUB 1750 
657 LPRINT CHR\$(1);CHR\$(27);"-";CHR \$(1);CHR\$(1);CHR\$(27);

"E";TLE\$;CHR\$(1);CHR\$(27);"-";CHR\$(0);CHR\$(1);CHR\$(27);"F":LPRINT 660 LPRINT"MMCALCUL output for file ";F\$

670 LPRINT"(";F\$;" was created at ";TSTR\$;" on the ";DSTR\$;")"

680 LPRINT:LPRINT"The following files have been formed from "F\$

690 FOR I= 1 TO 6

700 LPRINT ,S(I):

710 NEXT I

715 P\$=CHR \$(27)+"S"+CHR\$(0)+"+"+CHR\$(8)+CHR\$(27)+"S"+CHR\$(1)+"-"+ $\operatorname{CHR} \$(27)+" T "$

720 LPRINT:LPRINT:LPRINT;"Resting tension during experiment ";P\$;" SE ="; :LPRINT USING "\#\#.\#\#";FNLX(MEAN1);:LPRINT" ";P\$;" ";:LPRINT USING "\#.\#\#\#\#";FNLX(MEAN1+ZD1)-FNLX(MEAN1);:LPRINT" g"

730 LPRINT : LPRINT;SPC(8);"Steady state peak tension ";P\$;" SE =";:LPRINT USING"\#\#\#\#"; FNLX(MEAN0);:LPRINT" ";P\$;:LPRINT USING "\#\#.\#\#\#\#";FNLX(MEAN0+ZD0)-FNLX(MEAN0);:LPRINT"g"

733 LPRINT:LPRINT;SPC(3);"Steady state developed tension ";P\$;" SE =";:LPRINT USING "\#\#.\#\#";FNLX(MEAN0)-FNLX(MEAN1);:LPRINT" ";P\$;:LPRINT USING "\#\#.\#\#\#\#";FNLX(MEAN0+ZD01)-FNLX(MEAN0);:LPRINT" g" 734 LPRINT:LPRINT;SPC(3);"Average time to 90\% relaxation ";P\$;" SE =";:LPRINT USING"\#\#\#\#\#";MEAN2(0);:LPRINT" ";P\$;:LPRINT USING"\#\#.\#\#\#\#";ZD2(0);:LPRINT" msec"

735 LPRINT:LPRINT:LPRINT;SPC(20);"Total number of runs =";IR-1

740 LPRINT: LPRINT

750 LPRINT "FOR EXTRA-SYSTOLIC BEAT"

760 O\$=CHR \$(27)+"S"+CHR\$(0)+"-1"+CHR\$(27)+"T"

770 LPRINT,"Maximum","Time of peak","Maximum","Minimum Relax"

780 LPRINT "ESI(Secs)","tension (g)","tension (ms)","dT/dt g.s";O\$," dT/dt g.s";

O\$;SPC(3);"90\%"

790 OPEN "O",\#1,S(1)

800 OPEN "O",\#2,S(3)

810 OPEN "O",\#3,S(4)

820 FOR J= 1 TO IR-1

830 PRINT \#1,USING"\#\#.\#\#\#";ESI(J),FNLX(MX(1,J))

840 PRINT \#2,USING"\#\#\#.\#\#";ESI(J),VMXD(1,J)*1000/12

850 PRINT \#3,USING"\#\#\#\#\#\#";ESI(J),VMND(1,J)*1000/12 
860 LPRINT USING"\#.\#\#\#";ESI(J);:LPRINT,;:LPRINT USING"\#\#.\#\#\#"; FNLX(MX(1,J));:LPRINT,TTPT(1,J),;:LPRINT USING"\#\#\#.\#\#";VMXD(1,J)*1000/12;:LPRINT,;:LPRINT USING "\#\#\#.\#\#";VMND(1,J)*1000/12;:LPRINT SPC(7);RXT(1,J)

870 NEXT

880 CLOSE \#1:CLOSE \#2: CLOSE\#3

890 LPRINT: LPRINT: LPRINT:

900 LPRINT "FOR POST-EXTRASYSTOLIC BEAT 1"

910 LPRINT,"Maximum","Time of peak","Maximum","Minimum Relax" 920 LPRINT "ESI(Secs)","tension (g)","tension (ms)","dT/dt g.s";O\$," dT/dt g.s";O\$SPC(3);"90\%"

930 OPEN "O", \#1, S(2)

940 OPEN "O", \#2, S(5)

950 OPEN "O", \#3, S(6)

960 FOR J=1 TO IR-1

970 PRINT \#1,USING"\#\#.\#\#\#"; ESI(J),FNLX(MX(2,J))

980 PRINT \#2, USING"\#\#.\#\#\#";ESI(J),VMXD(2,J)*1000/12

990 PRINT \#3, USING"\#\#\#.\#\#";ESI(J),VMND(2,J)*1000/12

1000 LPRINT USING"\#.\#\#";ESI(J);:LPRINT,;:LPRINT USING"\#\#.\#\#";

FNLX(MX(2,J));:LPRINT,TTPT(2,J),;:LPRINT USING"\#\#\#.\#\#\#";

VMXD(2,J)*1000/12; :LPRINT,;:LPRINT USING"\#\#\#\#\#\#";

$\operatorname{VMND}(2, \mathrm{~J}) * 1000 / 12 ;: \operatorname{LPRINT} \operatorname{SPC}(7) ; \mathrm{RXT}(2, \mathrm{~J})$

1010 NEXT

1020 CLOSE \#1:CLOSE\#2:CLOSE\#3

1030 LPRINT: LPRINT: LPRINT:

1040 GOTO 1050

1050 FOR K\%=50 TO 1000:SOUND K\%,1:NEXT K\%

1060 CLS

1070 LOCATE 12,29:PRINT "*** Finished ***"

1080 PRINT:PRINT:PRINT"Bye !"

1090 END

1100 REM Load date data routine

1110 FTR $\$=F \$: T S T R \$=" \_\_$_:DSTR $\$=" \ldots \ldots$ 1990"

1120 OPEN "I",\#1,"datedata"

1130 WHILE NOT EOF(1)

1140 INPUT \#1,A\$,B \$,C\$

1150 IF A $\$=F \$$ THEN TSTR $\$=B \$: D S T R \$=C \$$ 
1160 WEND

1170 CLOSE 1

1180 RETURN

1190 REM Find 90\% relaxation and ave resting tension for ES and DP1

1210 FOR J= TTPT(I,IR)/4 TO 98

$1220 \mathrm{IF} \mathrm{M}(\mathrm{J}, \mathrm{I})<=(\mathrm{RTA}(\mathrm{I}, \mathrm{IR})+.1 *(\mathrm{MX}(\mathrm{I}, \mathrm{IR})-\mathrm{RTA}(\mathrm{I}, \mathrm{IR})))$ THEN RXT(I,IR)=J*4:J=98

1230 NEXT

1235 IF I=0 THEN ET2=ET2+RXT(I,IR)

1240 REM MEAN2(I)=RXT(I,IR)+MEAN2(I)

1250 RETURN

1260 REM SUBROUTINE TO DESTROY INCORRECTLY FORMED RANDOM ACCESS FILE

1270 CLOSE \#1

1275 CLS

1280 KILL F\$

1290 INPUT" INCORRECT FILE NAME, PLEASE RE-ENTER";F\$

1300 GOTO 170

1310 REM Subroutine to calculate Std Error of Means

1311 REM MEAN0=mean peak SS; MEAN1=mean rest $\mathrm{T}$ of SS/ES/DP1;

$\operatorname{MEAN} 2(1)=$ mean $t$

1312 REM to $90 \%$ relax ES; MEAN2(2)=mean $t$ to $90 \%$ relax DP1.

1315 MEAN0=RT2/(IR-1): MEAN1=ET1/COUNT: MEAN2(1)=MEAN2(1)/(IR-1):

MEAN2(2)=MEAN2(2)/(IR-1): MEAN2(0)=ET2/(IR-1)

1320 FOR J=1 TO IR-1

$1330 \mathrm{ZD} 0=\mathrm{ZD} 0+(\mathrm{MX}(0, \mathrm{~J})-\mathrm{MEAN} 0)^{\wedge} 2$

$1340 \mathrm{I}=0$

$1350 \mathrm{ZD} 1=\mathrm{ZD} 1+(\mathrm{RTA}(\mathrm{I}, \mathrm{J})-\mathrm{MEAN} 1)^{\wedge} 2$

$1355 \mathrm{ZD} 2(\mathrm{I})=\mathrm{ZD} 2(\mathrm{I})+(\mathrm{RXT}(\mathrm{I}, \mathrm{J})-\mathrm{MEAN} 2(\mathrm{I}))^{\wedge} 2$

1360 NEXT J

$1370 \mathrm{ZD} 0=\mathrm{SQR}(\mathrm{ZD} 0) /(\mathrm{IR}-1): \mathrm{ZD} 1=\mathrm{SQR}(\mathrm{ZD} 1) /\left(3^{*}(\mathrm{IR}-1)\right)$ :

$\mathrm{ZD} 2(\mathrm{I})=\mathrm{SQR}(\mathrm{ZD} 2(\mathrm{I})) /(\mathrm{IR}-1)$

$1371 \mathrm{ZD} 0 \mathrm{SD}=\mathrm{ZD} 0 *$ SQR(IR-1):ZD1SD=ZD1*SQR(3*(IR-1))

1373 REM SE of Diff MEAN0 and MEAN1=ZD01

$1375 \mathrm{ZD} 01=\mathrm{SQR}(((\mathrm{ZD} 1 \mathrm{SD}) \wedge 2 *(3 * \mathrm{IR}-1)+(\mathrm{ZD} 0 \mathrm{SD}) \wedge 2 *(\mathrm{IR}-1)) /(4 * \mathrm{IR}-2))$

$1377 \mathrm{ZD} 01=\mathrm{SQR}(1 /(3 * \mathrm{IR}-1)+1 /(\mathrm{IR}-1)) * \mathrm{ZD} 01$

1380 RETURN

1400 REM Error trap. 
1410 IF ERR=53 AND ERL=1120 THEN GOTO 1180

1415 PRINT"Error ";ERR;" at line ";ERL

1420 END

1430 REM Plot $\max \mathrm{T}, \max \mathrm{dT} / \mathrm{dt}, \& \min \mathrm{dT} / \mathrm{dt}$

1435 SOUND 50,1

$1440 \mathrm{X}=\mathrm{LOG}(\mathrm{ESI}(\mathrm{IR}) * 10) * 130.29+100$

1450 CIRCLE (X,(90-2.25*VMXD(I,IR)*CONST)+20),2*I

1460 CIRCLE (X,(90-2.25*VMND(I,IR)*CONST)+20),2*I

1470 CIRCLE (X,(180-.72*MX(I,IR))+210),2*I

1475 CIRCLE (X,(180-.72*RTA(I,IR))+210),.5*I

1480 RETURN

1490 REM plot axis

1500 CLS:COLOR 1

1510 PRINT "Differentials and maximal tensions for extra-systolic \&

post-extra-systolic beat";

1520 PRINT SPC(40);"(PES plotted as larger symbols.)"

1530 LINE $(100,20)-(100,200)$ :LINE $(100,110)-(700,110)$

1540 LINE $(100,210)-(100,390): \operatorname{LINE}(100,390)-(700,390)$

1550 LOCATE 2,6

1560 PRINT "+40"

1570 LOCATE 7,9:PRINT "0"

1580 LOCATE 13,6:PRINT "-40"

1590 LOCATE 14,6:PRINT "250"

1600 LOCATE 24,9:PRINT "0"

1610 LOCATE 7,2:PRINT "dT":LOCATE 8,2:PRINT "dt"

1615 LINE $(10,110)-(30,110)$

1620 LOCATE 19,2:PRINT "Max T"

1630 LOCATE 4,71:PRINT "max"

1635 LOCATE 7,71:PRINT "ESI"

1640 LOCATE 8,71:PRINT "time (ms)"

1650 LOCATE 11,71:PRINT "min"

1660 FOR J=200 TO 1000 STEP 200

$1670 \mathrm{X}=\mathrm{LOG}(\mathrm{J} / 100) * 130.29+100$

1680 LINE $(X, 115)-(X, 105): \operatorname{LINE}(X, 390)-(X, 395)$

1690 NEXT J

1700 FOR $\mathrm{J}=1000$ TO 10000 STEP 1000

$1710 \mathrm{X}=\mathrm{LOG}(\mathrm{J} / 100) * 130.29+100$ 
1720 LINE $(X, 115)-(X, 105)$ :LINE $(X, 390)-(X, 395)$

1730 NEXT J

1740 RETURN

1750 REM Routine to plot differential and max. tension graphs

1760 LOCATE 17,40:PRINT "Select Plotter and press a key";

1770 A $\$=I N K E Y \$: I F$ A $\$="$ " GOTO 1770

1780 PRINT " Okay."

1785 LPRINT "em 1"

1790 LPRINT "si 40,40":LPRINT "di 0,36":LPRINT "ma 50,0":LPRINT "la ";TLE\$ 1795 LPRINT "em 0"

1800 LPRINT "si 30,30":LPRINT "ma 100,0":LPRINT "la File ";F\$;" was created at ";LEFT\$(TSTR \$,5);" on the ";DSTR\$

1810 LPRINT "ma 150,0":LPRINT "la This experiment had a FSD of ";MAG;" and a balance of ";BALANCE

1820 LPRINT "si 25,25 "

1890 LPRINT "ma 330,120":LPRINT "ax 1,100,10,0,50,-10,5,1"

1900 LPRINT "ma 830,120":LPRINT "ax 0,900,2,0"

1910 LPRINT "ma 1480,120":LPRINT "ax 1,200,5,0,250,-50,1,1"

1920 LPRINT "ma 2480,120":LPRINT "ax 0,900,2,0"

1930 FOR J=200 TO 800 STEP 200

$1940 \mathrm{X}=\mathrm{LOG}(\mathrm{J} / 100) * 390.88+120$

1950 LPRINT "ma 830,";X:LPRINT "dr 5,0"

1960 LPRINT "ma 2480,";X:LPRINT "dr 5,0"

1970 NEXT J

1980 FOR J=1100 TO 9000 STEP 1000

$1990 \mathrm{X}=\mathrm{LOG}(\mathrm{J} / 100) * 390.88+120$

1995 LPRINT "ma 830,";X:LPRINT "dr 5,0"

2000 LPRINT "ma 2480,";X:LPRINT "dr 5,0"

2010 NEXT J

2012 LPRINT "ma 380,800":LPRINT "la Differential dT/dt (g/sec) verses ESI (msec)":LPRINT "ma 1510,900":LPRINT "la Maximum developed tension (ASCII)":LPRINT "ma 1550,900":LPRINT "la verses ESI (msec)"

2013 LPRINT "ma 420,1200":LPRINT "rm 1,0,0":LPRINT "la = ES":LPRINT "ma 455,1200":LPRINT "rm 2,0,0":LPRINT "la = PES":LPRINT "ma 1590,1200":LPRINT "rm 1,0,0":LPRINT "la = ES":LPRINT "ma $1625,1200 ":$ LPRINT "rm 2,0,0":LPRINT "la = PES":LPRINT "ma 1660,1200" 2014 LPRINT "rm 4,0,0":LPRINT "la = RT" 
2020 FOR J=1 TO IR-1

2030 FOR $\mathrm{I}=1$ TO 2

$2035 \mathrm{X}=\mathrm{LOG}(\mathrm{ESI}(\mathrm{J}) * 10) * 390.88+120$

2040 LPRINT "am ";I;",";830-10*VMXD(I,J)*CONST;",";X

2050 LPRINT "am ";I;",";830-10*VMND(I,J)*CONST;",";X

2060 LPRINT "am ";I;",";2480-4*MX(I,J);",";X

2065 IF I<2 THEN LPRINT "am ";4;",";2480-4*RTA(I,J);",";X

2080 NEXT I:NEXT J

2090 LOCATE 18,40:PRINT "Select Printer and press a key";:SOUND 100,5

2100 A\$=INKEY\$:IF A $\$="$ " GOTO 2100

2110 LPRINT "ch"

2120 RETURN

2.3 Plotter Output of MMCALCUL.BAS

An example of the plotter output of MMCALCUL.BAS is shown in figure $\operatorname{app} 2.2$

2.4 Printer Output of MMCALCUL.BAS

An example of the printer output of MMCALCUL.BAS is shown in figure app2.3. 
acidosis HS cfc0801rab

File $A: b 0801$ rab was created at $13: 30$ on the 08 Jan 1991

This experiment hod a FSD of 2 and o balance of 4.255
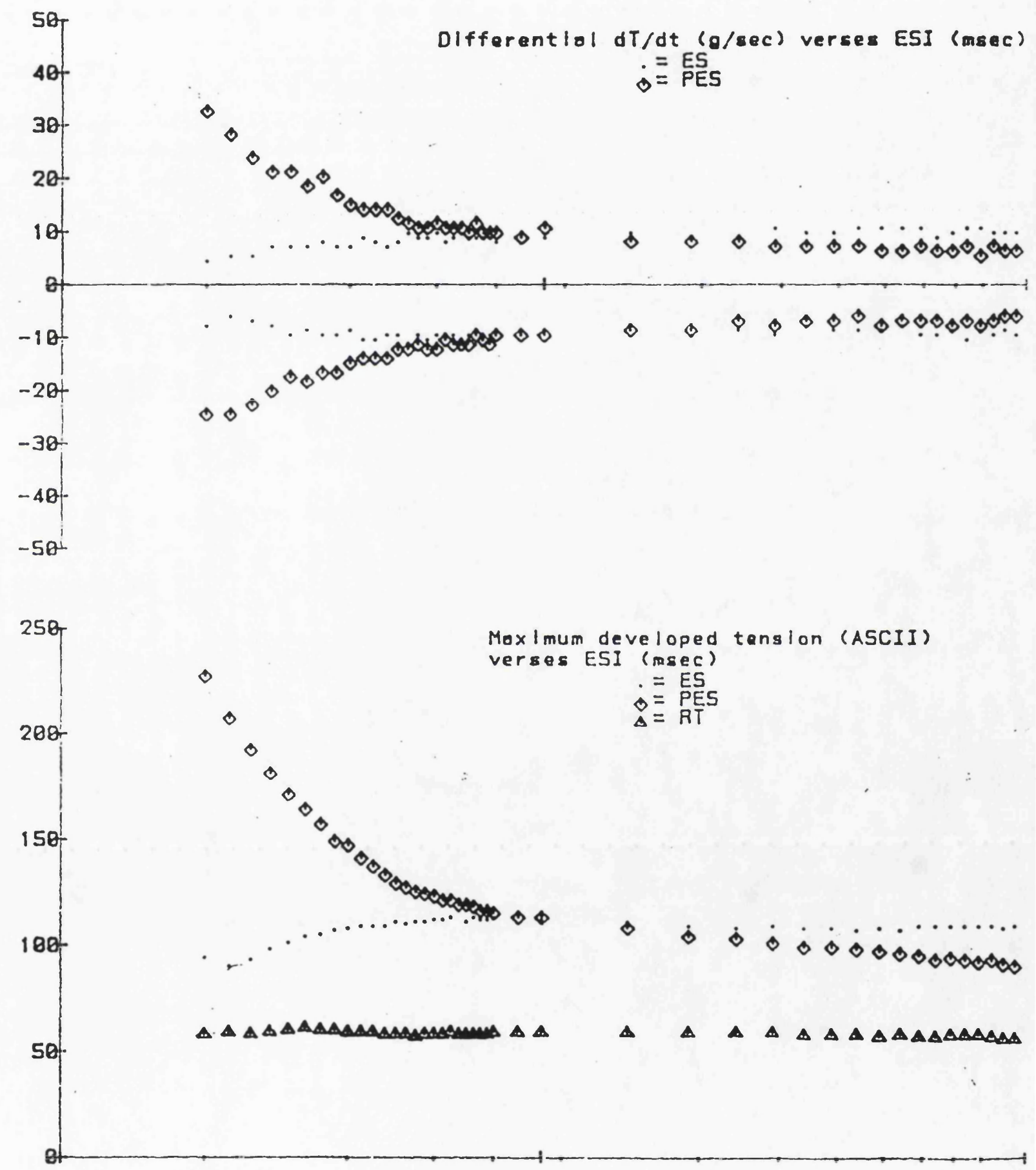

Figure App2.2: Plotter output of MMCAICUL.BAS. Upper trace is of the maximum +ve and -ve first differential of force, at each extrasystolic interval. Lower trace is a classical force interval relationship. $\mathrm{ES}=$ extrasystole, $\mathrm{PES}=$ post extrasystole $\mathrm{RT}=$ resting tension. 


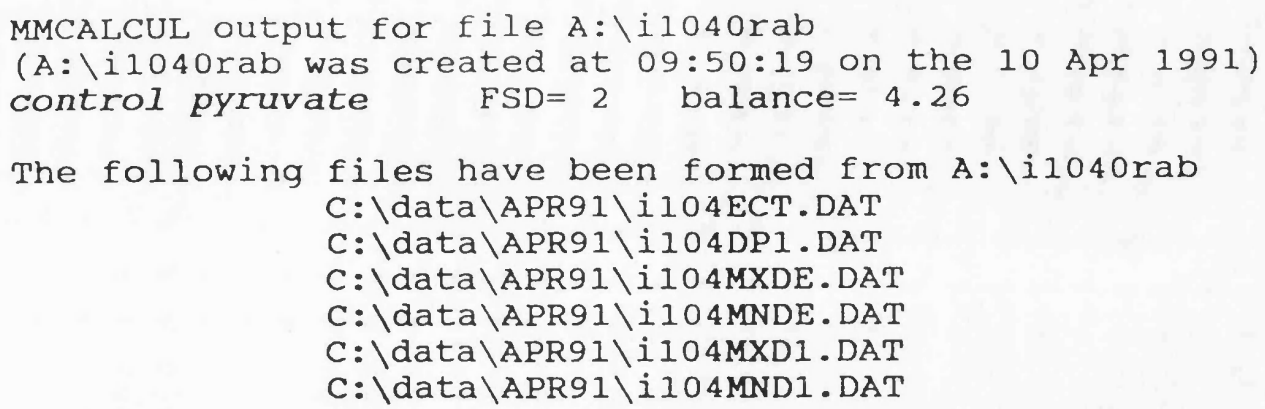

Resting tension during experiment $\pm \mathrm{SE}=0.175 \pm 0.0051 \mathrm{~g}$

FOR STEADY-STATE BEAT

Steady state peak tension $\pm \mathrm{SE}=0.790 \pm 0.0069 \mathrm{~g}$

Steady state developed tension $\pm \mathrm{SE}=0.615 \pm 0.0043 \mathrm{~g}$

Average time to $90 \%$ relaxation $\pm \mathrm{SE}=224.00 \pm 0.816 \mathrm{msec}$

Average $\max$ rate of tension rise $\pm \mathrm{SE}=10.681 \pm 0.119 \mathrm{~g} / \mathrm{s}$

Average min rate of tension rise $\pm \mathrm{SE}=-7.870 \pm 0.096 \mathrm{~g} / \mathrm{s}$

Average time to peak tension $\pm \mathrm{SE}=110.2 \pm 5.7 \mathrm{~ms}$

Total number of runs $=44$

FOR EXTRA-SYSTOLIC BEAT

$\begin{array}{lcc}\text { ESI(SeCs) } & \begin{array}{c}\text { Maximum } \\ \text { tension }(g)\end{array} & \begin{array}{c}\text { Time of peak } \\ \text { tension (ms) }\end{array} \\ 0.200 & 0.466 & 88 \\ 0.225 & 0.392 & 108 \\ 0.250 & 0.382 & 112 \\ 0.275 & 0.403 & 116 \\ 0.300 & 0.424 & 120 \\ 0.325 & 0.456 & 120 \\ 0.350 & 0.488 & 108 \\ 0.375 & 0.488 & 132 \\ 0.400 & 0.498 & 120 \\ 0.425 & 0.562 & 108 \\ 0.450 & 0.530 & 120 \\ 0.475 & 0.572 & 116 \\ 0.500 & 0.572 & 108 \\ 0.525 & 0.657 & 116 \\ 0.550 & 0.604 & 116 \\ 0.575 & 0.625 & 124 \\ 0.600 & 0.636 & 120 \\ 0.625 & 0.636 & 108 \\ 0.650 & 0.625 & 116 \\ 0.675 & 0.636 & 112 \\ 0.700 & 0.625 & 112 \\ 0.725 & 0.625 & \end{array}$

\begin{tabular}{|c|c|}
\hline Maximum & Minimum \\
\hline$\star \star \mathrm{dT} / \mathrm{d} t$ & $g \cdot s^{-1} \star \star \star$ \\
\hline 6.184 & -7.067 \\
\hline 6.184 & -6.184 \\
\hline 7.067 & -6.184 \\
\hline 7.950 & -6.184 \\
\hline 7.067 & -6.184 \\
\hline 8.834 & -6.184 \\
\hline 8.834 & -7.067 \\
\hline 7.950 & -6.184 \\
\hline 9.717 & -7.067 \\
\hline 9.717 & -7.950 \\
\hline 7.950 & -7.950 \\
\hline 8.834 & -7.067 \\
\hline 9.717 & -7.067 \\
\hline 11.484 & -8.834 \\
\hline 9.717 & -7.067 \\
\hline 9.717 & -7.950 \\
\hline 11.484 & -7.950 \\
\hline 10.600 & -7.950 \\
\hline 11.484 & -8.834 \\
\hline 10.600 & -7.950 \\
\hline 8.834 & -7.950 \\
\hline 10.600 & -8.834 \\
\hline 10.600 & -7.950 \\
\hline
\end{tabular}


112

0.604

108

1.000

1.500

0.625

116

0.572

0.583

0.583

0.625

0.572

3.000

3.500

4.000

4.500

0.604

0.615

0.562

5.500

0.572

0.562

6.000

6.500

7.000

0.551

0.541

0.583

7.500

0.530

0.509

0.530

8.500

9.000

9.500
100

104

96

112

96

96

100

100

100

96

96

100

96

100

92

104

104
10.600

11.484

10.600

9.717

10.600

12.367

11.484

11.484

11.484

11.484

13.251

12.367

12.367

11.484

11.484

11.484

11.484

10.600

10.600

9.717

9.717
$-7.950$

$-7.067$

$-8.834$

$-7.950$

$-7.950$

$-7.950$

$-7.067$

$-7.067$

$-6.184$

$-9.717$

$-7.950$

$-6.184$

$-7.067$

$-6.184$

$-7.067$

$-7.067$

$-7.950$

$-7.067$

$-6.184$

$-7.067$

$-6.184$

FOR POST-EXTRASYSTOLIC BEAT 1

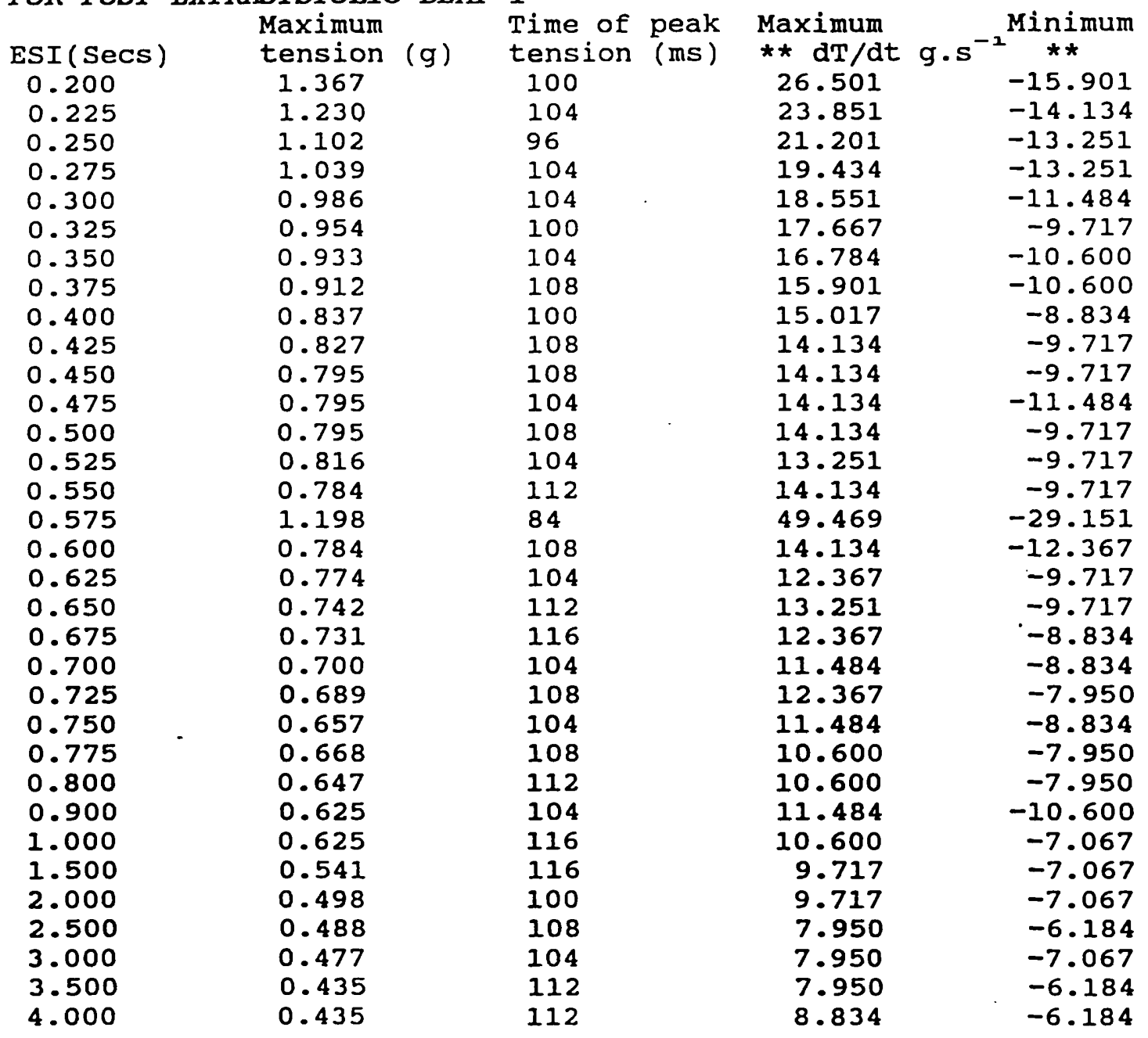

Figure App2.3: Printer output of MMCAICUL.BAS. The parameters decribing the tension enveloppe of the 44 steady state beats are averaged and listed at the start of the output (on the previous page). The remainder of the output lists the changes in these parameters for extrasystolic and post extrasystolic beats at each of the extrasystolic intervals. 


\section{MMCOLLAT.BAS}

This program analysed the output from MMCALCUL. A number of files formed by MMCALCUL would be opened the data at each extrasystolic interval divided by steady state force to give a normalised force, the program then calculated the mean and standard deviation of normalised beat force at each of the 44 extrasystolic intervals for each of the files opened. The means of the normalised beat force at each extrasystolic interval was saved in format for analysis and curve fitting by Regression.

\subsection{Description of MMCOLLAT.BAS}

\section{lines function}

70-90 Request information about the group of experiments to be analysed

100-190 Opens a file containing the file names, steady state developed force and number of initial beats to be excluded, this file could be edited by the DOS text editor if entry error occurred. If the programme had to be restarted after correcting a filename a GoTo line740 was needed at line75.

200-310 Opens each of the experimental files and sets up data arrays of normalised beat force and sets the value of excluded beats to zero.

320-360 Transposes the arrays and calculates mean developed force at each extra-systolic interval.

subroutine 600 Calculates the standard deviation for each of the means at differing extrasystolic intervals.

380-500 Prints the individual normalised values for each experiment together with means and standard deviations. An example of the output is seen in figure app2.4.

510-590 Opens a disc file and writes means, extrasysloic intervals and standard errors to disc.

840-960 Using the data file containing the details of each invidual experiment within the group this routine modifies the file name so that files containing extrasystolic beat force rather than post extrasystolic beat force are examined. 


\subsection{Listing of MMCOLLAT.BAS}

10 REM program to calculate means and spread of a given number of

20 REM beats from differant experiments normalised using steady state paramaters $* * * * * * 01 / 04 / 91 * * * * * *$ Michael Marber

30 REM the DOS line editor is used to correct temp file formed in basic dir

40 DIM DT(100,20),F\$(20),SS(20),MEAN(20),TALLY(20),EXCL(20), SD(100),TAT(100),SUM(100),ESI(100)

50 ON ERROR GOTO 700

60 PION\$=CHR \$(27)+"(s1S"+CHR\$(27)+"(s3B":PIOF\$=CHR\$(27)

$+"(\mathrm{~s} 0 \mathrm{~S} "+\mathrm{CHR} \$(27)+"(\mathrm{~s} 0 \mathrm{~B} "$

70 INPUT "title of experimental series";TITLE\$

80 INPUT "how many experiments to be analysed";NUM

90 S\$="c:ldatal"+MID\$(TITLE\$,1,8)+".fls"

100 OPEN "o",\#1,S\$

110 FOR $\mathrm{J}=1$ TO NUM

120 PRINT J;

130 INPUT "data file name";F\$(J)

140 INPUT "steady state developed tension";SS(J)

150 INPUT "how many of the first data points need to be excluded";EXCL(J)

$160 \mathrm{FS} \$=\mathrm{CHR} \$(34)+\mathrm{F} \$(\mathrm{~J})+\mathrm{CHR} \$(34)$

170 PRINT \#1,FS\$,SS(J),EXCL(J)

180 NEXT $J$

190 CLOSE \# 1

200 FOR J=1 TO NUM

210 OPEN "i",\#1,F\$(J)

220 WHILE NOT EOF(1)

$230 \mathrm{IR}=\mathrm{IR}+1:$ PRINT IR

240 INPUT \#1,ESI(IR),DT(IR,J)

$250 \mathrm{DT}(\mathrm{IR}, \mathrm{J})=\mathrm{DT}(\mathrm{IR}, \mathrm{J}) / \mathrm{SS}(\mathrm{J})$

260 WEND

270 TALLY $(J)=I R: I R=0$

280 IF TOT $<$ TALLY $(J)$ THEN TOT $=$ TALLY(J)

290 FOR X=1 TO EXCL(J):DT(X,J)=0:NEXT X

300 CLOSE \#1

310 NEXT

320 FOR $\mathrm{J}=1$ TO NUM

330 FOR IR= EXCL $(J)+1$ TO TALLY $(J)$ 
340 SUM(IR)= SUM(IR)+DT(IR,J):TAT(IR)=TAT(IR)+1

350 NEXT:NEXT

360 FOR IR= 1 TO TOT: SUM(IR)=SUM(IR)/TAT(IR):NEXT IR

370 GOSUB 600

380 LPRINT PION\$+"COLLATED DATA OUTPUT FOR EXPERIMENTS

"+TITLE\$+PIOF\$

390 FOR IR= 1 TO TOT

400 FOR $\mathrm{J}=1$ TO NUM

410 LPRINT USING "\#.\#\#";DT(IR,J);:LPRINT" ";

420 NEXT J

430 LPRINT:

440 NEXT IR

450 PION\$=CHR $\$(27)+"(s 1 S "+C H R \$(27)+"(s 3 B ": P I O F \$=C H R \$(27)+$

"(s0S"+CHR\$(27)+"(s0B"

460 LPRINT:LPRINT PION\$+"collated data"

470 FOR IR= 1 TO TOT

480 LPRINT USING "\#.\#\#";ESI(IR);:LPRINT " MEAN=";:LPRINT USING

"\#.\#\#";SUM(IR);:LPRINT " STANDARD ERROR=";:LPRINT USING

"\#.\#\#";SD(IR)/SQR(TAT(IR)-1);:LPRINT " NUMBER OF

OBSERVATIONS=";TAT(IR)

490 NEXT

500 LPRINT PIOF\$

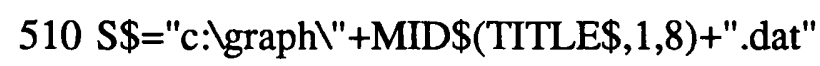

$520 \mathrm{FW} \$=\mathrm{CHR} \$(44)$

530 OPEN "O",\#1,S\$

540 FOR IR= 1 TO TOT

550 PRINT \#1,,ESI(IR),,SUM(IR),,(SD(IR)/SQR(TAT(IR)-1))

560 PRINT ,ESI(IR),,SUM(IR),,(SD(IR)/SQR(TAT(IR)-1))

570 NEXT

580 CLOSE \#1

590 END

600 REM subroutine for SD

610 FOR $J=1$ TO NUM

620 FOR IR=EXCL(J)+1 TO TALLY $(J)$

$630 \mathrm{SD}(\mathrm{IR})=(\mathrm{SUM}(\mathrm{IR})-\mathrm{DT}(\mathrm{IR}, \mathrm{J}))^{\wedge} 2+\mathrm{SD}(\mathrm{IR})$

640 NEXT:NEXT

650 FOR IR=1 TO TOT 
660 IF TAT(IR)=1 THEN SD(IR)=0:GOTO 680

$670 \mathrm{SD}(\mathrm{IR})=\mathrm{SQR}(\mathrm{SD}(\mathrm{IR}) /(\mathrm{TAT}(\mathrm{IR})-1))$

680 NEXT IR

690 RETURN

700 PRINT " file=";F $\$(J)$

710 PRINT "error in line ";ERL,"error code ";ERR

720 RESUME NEXT

730 RETURN

740 REM Wrong input routine to read corrected inputs from disc

750 REM needs insertion of "goto 740" at line 75 to activate

760 OPEN "i",\#1,S\$

770 WHILE NOT EOF(1)

$780 \mathrm{~J}=\mathrm{J}+1$

790 INPUT \#1,F\$(J),SS(J),EXCL(J):

800 PRINT F\$(J),SS(J),EXCL(J)

810 WEND

820 STOP

830 GOTO 190

840 REM Reads disk file with DP1 suffix and converts it to

850 REM ect suffix for analysis of restitution, needs goto 840

$860 \mathrm{REM}$ at line 75 to activate

870 OPEN "i",\#1,S\$

880 WHILE NOT EOF(1)

$890 \mathrm{~J}=\mathrm{J}+1$

900 INPUT \#1,F\$(J),SS(J),EXCL(J):

$910 \mathrm{~W}=\mathrm{LEN}(\mathrm{F} \$(\mathrm{~J})): \mathrm{W}=\mathrm{W}-6: \mathrm{MID} \$(\mathrm{~F} \$(\mathrm{~J}), \mathrm{W})=$ "ect.dat"

920 PRINT F\$(J),SS(J),EXCL(J)

930 WEND

940 TITLE\$=MID\$(TITLE\$,1,5)+"ect":PRINT TITLE\$

950 STOP

960 GOTO 190

\subsection{Output of MMCOLLAT.BAS}

An example of the printed output from MMCOLLAT.BAS is shown if figure app2.4. 
COLLATED DATA OUTPUT FOR EXPERIMENTS HSPBASAL

$\begin{array}{llllllllllllll}1.782 & 1.385 & 1.392 & 1.509 & 1.640 & 1.748 & 1.346 & 1.674 & 0.000 & 1.858 & 2.577 & 1.858\end{array}$ $\begin{array}{llllllllllllll}1.655 & 1.000 & 1.327 & 1.489 & 1.574 & 1.633 & 1.287 & 1.608 & 2.332 & 1.683 & 2.060 & 1.736\end{array}$

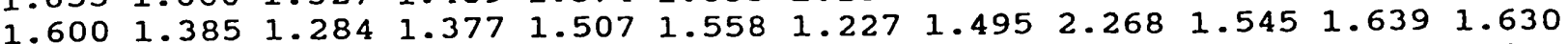

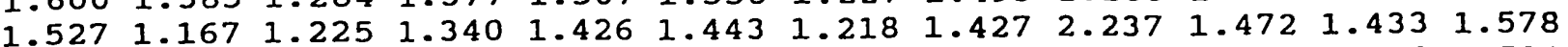
$1.4361 .052 \quad 1.2031 .2831 .4001 .355 \quad 1.168 \quad 1.383 \quad 2.1201 .403 \quad 1.349 \quad 1.508$

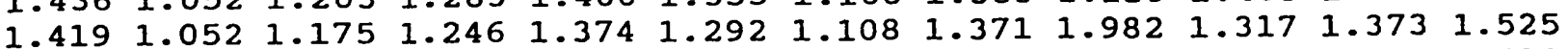

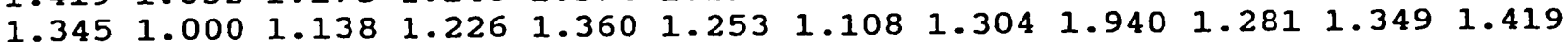

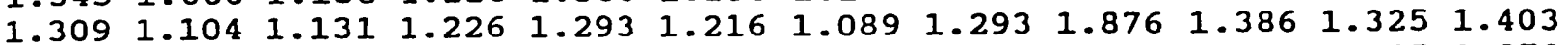

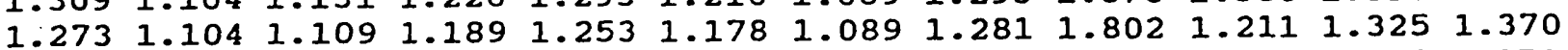

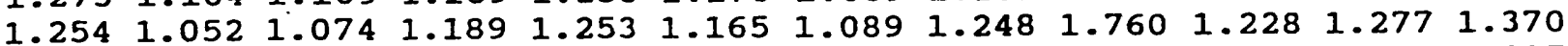

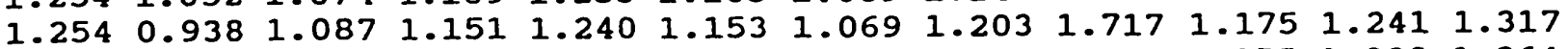

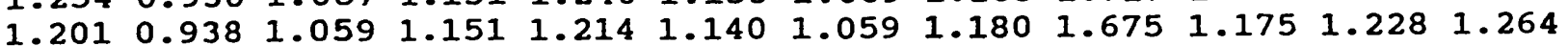

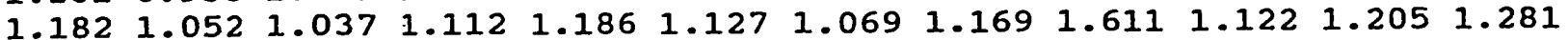

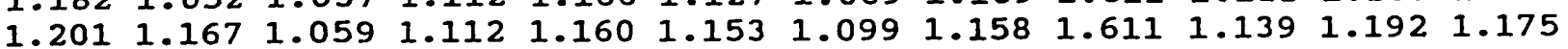
$\begin{array}{llllllllllll}1.127 & 0.885 & 1.022 & 1.075 & 1.186 & 1.115 & 1.108 & 1.124 & 1.558 & 1.122 & 1.168 & 1.191\end{array}$

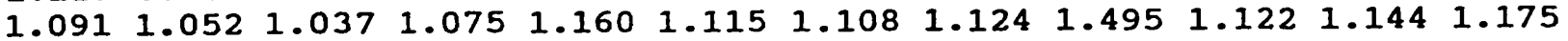
$1.0551 .271 \quad 1.0301 .075 \quad 1.174 \quad 1.090 \quad 1.0891 .1021 .431 \quad 1.122 \quad 1.1441 .191$

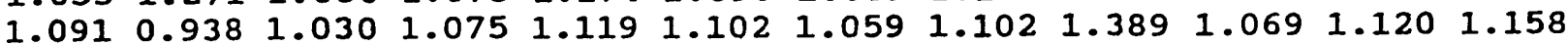

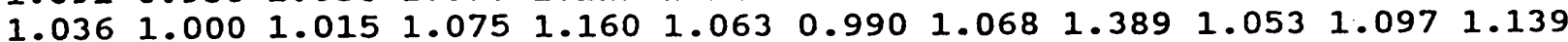
$1.0551 .0001 .0301 .057 \quad 1.107 \quad 1.063 \quad 0.9601 .056 \quad 1.367 \quad 1.053 \quad 1.108 \quad 1.158$ $1.0550 .8331 .0151 .0571 .107 \quad 1.076 \quad 0.9701 .046 \quad 1.3671 .053 \quad 1.0841 .106$ $1.0360 .9381 .0151 .0571 .1191 .051 \quad 1.0591 .034 \quad 1.346 \quad 1.036 \quad 1.084 \quad 1.106$ $1.036 \quad 0.938 \quad 1.008 \quad 1.057 \quad 1.0931 .038 \quad 1.078 \quad 1.034 \quad 1.3251 .0361 .072 \quad 1.106$ $1.0360 .9381 .0001 .018 \quad 1.0671 .038 \quad 1.0001 .046 \quad 1.336 \quad 1.017 \quad 1.084 \quad 1.069$ $1.0191 .0001 .008 \quad 1.0371 .0671 .0130 .9301 .0231 .3251 .0361 .072 \quad 1.158$ $0.9641 .0521 .0081 .0001 .0671 .000 \quad 0.901 \quad 1.0121 .2401 .017 \quad 1.024 \quad 1.036$ $\begin{array}{lllllllllllll}0.945 & 0.885 & 0.993 & 1.018 & 1.053 & 1.013 & 0.880 & 0.989 & 1.187 & 0.964 & 0.976 & 0.964\end{array}$ $\begin{array}{lllllllllllll}0.909 & 1.104 & 0.978 & 0.980 & 0.960 & 0.937 & 0.880 & 0.877 & 1.028 & 0.931 & 0.856 & 0.947\end{array}$ $\begin{array}{lllllllllllll}0.873 & 1.052 & 0.950 & 0.963 & 0.893 & 0.900 & 0.861 & 0.855 & 0.965 & 0.894 & 0.795 & 0.911\end{array}$ $\begin{array}{lllllllllllll}0.854 & 1.219 & 0.958 & 0.963 & 0.867 & 0.886 & 0.880 & 0.831 & 0.943 & 0.858 & 0.759 & 0.858\end{array}$ $\begin{array}{lllllllllllll}0.854 & 1.167 & 0.958 & 0.923 & 0.853 & 0.861 & 0.901 & 0.821 & 0.901 & 0.878 & 0.770 & 0.825\end{array}$ $\begin{array}{llllllllllllll}0.837 & 1.104 & 0.958 & 0.906 & 0.853 & 0.861 & 0.921 & 0.831 & 0.890 & 0.858 & 0.795 & 0.805\end{array}$ $\begin{array}{llllllllllllll}0.818 & 0.938 & 0.986 & 0.923 & 0.826 & 0.873 & 0.930 & 0.831 & 0.880 & 0.842 & 0.783 & 0.805\end{array}$ $\begin{array}{lllllllllllll}0.782 & 1.000 & 0.965 & 0.906 & 0.840 & 0.861 & 0.940 & 0.831 & 0.859 & 0.825 & 0.783 & 0.789\end{array}$ $\begin{array}{lllllllllllll}0.799 & 0.938 & 0.943 & 0.906 & 0.786 & 0.873 & 0.951 & 0.831 & 0.859 & 0.842 & 0.770 & 0.789\end{array}$ $\begin{array}{llllllllllllll}0.799 & 0.938 & 0.936 & 0.906 & 0.786 & 0.861 & 0.930 & 0.843 & 0.837 & 0.842 & 0.770 & 0.789\end{array}$ $\begin{array}{lllllllllllll}0.799 & 1.000 & 0.943 & 0.886 & 0.774 & 0.886 & 0.930 & 0.843 & 0.816 & 0.842 & 0.795 & 0.736\end{array}$ $\begin{array}{llllllllllllll}0.763 & 1.000 & 0.936 & 0.886 & 0.747 & 0.873 & 0.930 & 0.831 & 0.837 & 0.825 & 0.795 & 0.736\end{array}$ $\begin{array}{llllllllllllll}0.782 & 1.000 & 0.950 & 0.868 & 0.747 & 0.873 & 0.921 & 0.821 & 0.816 & 0.842 & 0.783 & 0.805\end{array}$ $\begin{array}{lllllllllllll}0.782 & 0.938 & 0.943 & 0.868 & 0.733 & 0.861 & 0.910 & 0.799 & 0.806 & 0.842 & 0.783 & 0.719\end{array}$ $\begin{array}{llllllllllllll}0.763 & 0.885 & 0.928 & 0.868 & 0.719 & 0.861 & 0.930 & 0.787 & 0.795 & 0.825 & 0.770 & 0.736\end{array}$ $\begin{array}{lllllllllllll}0.746 & 0.885 & 0.928 & 0.849 & 0.707 & 0.823 & 0.901 & 0.775 & 0.774 & 0.825 & 0.759 & 0.736\end{array}$ $\begin{array}{llllllllllllll}0.763 & 0.938 & 0.877 & 0.849 & 0.681 & 0.810 & 0.880 & 0.775 & 0.763 & 0.825 & 0.783 & 0.703\end{array}$ $\begin{array}{llllllllllllllll}0.746 & 1.167 & 0.870 & 0.868 & 0.681 & 0.798 & 0.861 & 0.787 & 0.753 & 0.805 & 0.747 & 0.719\end{array}$

collated data

0.200 MEAN $=1.706$

$0.225 \quad M E A N=1.615$

$0.250 \quad M E A N=1.543$

$0.275 \quad M E A N=1.458$

$0.300 \quad M B A N=1.388$

0.325 MEAN $=1.353$

$0.350 \quad M B A N=1.310$

$0.375 \quad M B A N=1.304$

$0.400 \quad M E A N=1.265$

0.425 KEAN $=1.246$

$0.450 \quad M E A N=1.212$

$0.475 \quad M E A N=1.190$

0.500 MEAN $=1.179$
STANDARD ERROR=0.109 STATDARD ERROR $=0.104$ STAKDARD ERROR=0.080 STANDARD ERROR $=0.083$ STATDARD BRROR=0.079 STANDARD ERROR $=0.072$ STANDARD ERROR $=0.070$ STANDARD ERROR=0.063 STANDARD ERROR $=0.058$ STANDARD ERROR $=0.056$ STANDARD ERROR $=0.057$ STANDARD ERROR $=0.053$ STANDARD ERROR=0.046
NUMBER OF OBSERVATIONS= 11 NUMBER OF OBSERVATIONS $=12$ NUMBER OF OBSERVATIONS $=12$ NUMBER OF OBSBRVATIONS $=12$ NUMBER OF OBSERVATIONS $=12$ NUHBER OF OBSERVATIONS $=12$ NUMBER OF OBSERVATIONS= 12 NUKBER OF OBSERVATIONS $=12$ NUMBER OF OBSERV ATIONS $=12$ NUMBER OF OBSERVATIONS $=12$ NUMBER OF OBSERVATIONS $=12$ NUMBER OF OBSERVATIONS $=12$ NUMBER OF OBSERVATIONS $=12$ 
0.525 0.550 0.575 0.600 0.625 0.650 0.675 0.700 0.725 0.750 0.775 0.800 0.900 1.000 1.500 2.000 2.500 3.000 3.500 4.000 4.500 5.000 5.500 6.000 6.500 7.000 7.500 8.000 8.500 9.000

9.500
$M E A N=1.185$ $M E A N=1.140$ $M E A N=1.141$ MEAN $=1.148$ $M E A N=1.104$ $M E A N=1.090$ $M E A N=1.085$ $M E A N=1.064$ MEAT $=1.073$ $M E A N=1.068$ $M B A N=1.054$ MEAN $=1.057$ $M E A N=1.027$ $M E A N=0.989$ MEAN $=0.949$ MEAN $=0.909$ MBAN $=0.906$ MEAN $=0.893$ MBAN $=0.885$ $M E A N=0.870$ MEAN $=0.865$ MEAN $=0.857$ $M B A N=0.853$ MEAN $=0.854$ $M E A N=0.847$ MEAN $=0.851$ MEAN $=0.832$ $M E A N=0.822$ $M E A N=0.809$ $M E A N=0.804$ MEAN $=0.817$
STANDARD ERROR=0.042 STANDARD ERROR $=0.047$ STANDARD ERROR=0.036 STANDARD ERROR $=0.033$ STANDARD ERROR $=0.032$ $S T A N D A R D E R R O R=0.032$ $S T A N D A R D E R R O R=0.031$ STANDARD $E R R O R=0.036$ STANDARD ERROR $=0.029$ STANDARD ERROR=0.028 STANDARD ERROR $=0.029$ STAWDARD ERROR $=0.030$ STANDARD ERROR=0.024 STANDARD ERROR=0.024 STANDARD ERROR $=0.021$ STANDARD ERROR $=0.020$ STANDARD ERROR=0.034 STANDARD ERROR $=0.030$ STANDARD BRROR=0.025 STANDARD BRROR $=0.019$ STANDARD ERROR=0.022 STANDARD $B R R O R=0.020$ STANDARD ERROR=0.019 STANDARD BRROR $=0.023$ STANDARD ERROR=0.025 STANDARD ERROR $=0.023$ $S T A N D A R D \quad B R R O R=0.022$ STANDARD ERROR $=0.022$ STANDARD ERROR=0.021 STANDARD ERROR $=0.023$ $S T A N D A R D E R R O R=0.038$
NUMBER OF OBSERVATIONS $=12$ NUMBER OF OBSERVATIONS $=12$ NUMBER OF OBSERVATIONS $=12$ NUMBER OF OBSERVATIONS $=12$ NUMBER OF OBSERVATIONS $=12$ NUMBER OF OBSERVATIONS $=12$ NUMBER OF OBSERVATIONS $=12$ NUMBER OF OBSERVATIONS $=12$ NUMBBR OF OBSBRVATIONS $=12$ NUMBER OF OBSERVATIONS $=12$ NUMBER OF OBSERVATIONS $=12$ NUMBER OF OBSERVATIONS $=12$ NUMBER OF OBSERVATIONS $=12$ NUMBER OF OBSBRVATIONS $=12$ NUMBER OF OBSBRVATIONS $=12$ NUMBBR OF OBSBRVATIONS $=12$ NUMBER OF OBSERVATIONS $=12$ NUMBER OF OBSERVATIONS $=12$ NUMBER OF OBSERVATIONS $=12$ NUMBER OF OBSERVATIONS $=12$ NUMBER OF OBSERVATIONS $=12$ NUMBER OF OBSERVATIONS $=12$ NUMBER OF OBSERVATIONS $=12$ NUMBER OF OBSERVATIONS $=12$ NUMBER OF OBSERVATIONS $=12$ NUMBER OF OBSERVATIONS $=12$ NUMBER OF OBSERVATIONS $=12$ NUMBER OF OBSERVATIONS $=12$ NUMBER OF OBSERVATIONS $=12$ NUMBER OF OBSERVATIONS $=12$ NUMBER OF OBSERVATIONS $=12$

COLTATED DATA OUTPUT FOR EXPERIMENTS HSP3OMIN

$\begin{array}{lllllllllllll}1.630 & 1.651 & 1.138 & 2.055 & 1.158 & 2.018 & 0.000 & 2.547 & 2.063 & 0.000 & 3.324\end{array}$

$1.5511 .2081 .0801 .8861 .121 \quad 1.886 \quad 0.000 \quad 2.410 \quad 1.9710 .000 \quad 3.018$

$\begin{array}{llllllllllll}1.474 & 1.208 & 1.116 & 1.772 & 1.158 & 1.772 & 1.578 & 2.233 & 1.851 & 1.240 & 2.715\end{array}$

$\begin{array}{lllllllllll}1.447 & 1.208 & 1.080 & 1.678 & 1.158 & 1.698 & 1.538 & 2.155 & 1.760 & 1.175 & 2.541\end{array}$

$\begin{array}{llllllllllll}1.395 & 1.151 & 1.080 & 1.603 & 1.158 & 1.623 & 1.512 & 2.018 & 1.669 & 1.136 & 2.367\end{array}$

$\begin{array}{llllllllllll}1.342 & 1.057 & 1.058 & 1.546 & 1.200 & 1.566 & 1.447 & 1.861 & 1.669 & 1.175 & 2.215\end{array}$

$\begin{array}{llllllllllll}1.367 & 1.208 & 1.080 & 1.489 & 1.042 & 1.528 & 1.421 & 1.725 & 1.611 & 1.175 & 2.064\end{array}$

$\begin{array}{lllllllllll}1.263 & 1.057 & 1.098 & 1.434 & 1.121 & 1.489 & 1.395 & 1.645 & 1.549 & 1.136 & 1.977\end{array}$

$1.2631 .104 \quad 1.080 \quad 1.4151 .121 \quad 1.415 \quad 1.341 \quad 1.508 \quad 1.5201 .136 \quad 1.912$

$\begin{array}{lllllllllllll}1.263 & 1.000 & 1.058 & 1.377 & 1.079 & 1.377 & 1.329 & 1.431 & 1.486 & 1.208 & 1.869\end{array}$

$\begin{array}{llllllllllll}1.211 & 1.302 & 1.040 & 1.340 & 1.042 & 1.358 & 1.289 & 1.392 & 1.457 & 1.104 & 1.803\end{array}$

$\begin{array}{lllllllllllll}1.211 & 1.057 & 1.058 & 1.301 & 1.079 & 1.340 & 1.289 & 1.372 & 1.366 & 1.104 & 1.738\end{array}$

$\begin{array}{llllllllllll}1.156 & 1.057 & 1.058 & 1.301 & 1.000 & 1.320 & 1.249 & 1.333 & 1.366 & 1.175 & 1.672\end{array}$

$\begin{array}{llllllllllll}1.184 & 1.000 & 1.022 & 1.283 & 1.042 & 1.263 & 1.223 & 1.274 & 1.337 & 1.071 & 1.607\end{array}$

$\begin{array}{lllllllllll}1.156 & 1.000 & 0.982 & 1.246 & 1.042 & 1.226 & 1.223 & 1.235 & 1.274 & 1.032 & 1.586\end{array}$

$\begin{array}{llllllllllll}1.132 & 0.953 & 1.022 & 1.246 & 1.042 & 1.263 & 1.210 & 1.214 & 1.309 & 1.032 & 1.498\end{array}$

$\begin{array}{lllllllllll}1.184 & 1.208 & 1.000 & 1.189 & 1.042 & 1.226 & 1.197 & 1.176 & 1.217 & 1.175 & 1.498\end{array}$

$\begin{array}{llllllllllll}1.156 & 1.057 & 1.000 & 1.169 & 0.958 & 1.189 & 1.184 & 1.137 & 1.217 & 1.000 & 1.477\end{array}$

$\begin{array}{llllllllllll}1.104 & 1.151 & 1.022 & 1.169 & 1.079 & 1.206 & 1.170 & 1.078 & 1.183 & 1.000 & 1.434\end{array}$

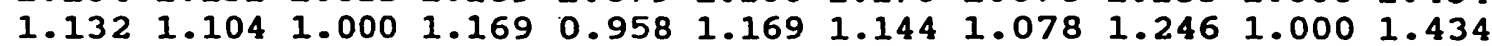

$\begin{array}{llllllllllll}1.079 & 1.104 & 1.022 & 1.151 & 1.000 & 1.169 & 1.132 & 1.018 & 1.154 & 1.071 & 1.369\end{array}$

$\begin{array}{llllllllllll}1.132 & 0.953 & 1.022 & 1.151 & 1.000 & 1.151 & 1.118 & 0.980 & 1.183 & 0.968 & 1.326\end{array}$ $\begin{array}{lllllllllllll}1.079 & 1.057 & 1.022 & 1.112 & 1.000 & 1.132 & 1.132 & 0.980 & 1.183 & 1.071 & 1.260\end{array}$ $\begin{array}{lllllllllllll}1.079 & 1.057 & 1.000 & 1.094 & 0.958 & 1.112 & 1.104 & 0.980 & 1.217 & 1.071 & 1.303\end{array}$ $\begin{array}{lllllllllllll}1.079 & 1.104 & 0.982 & 1.094 & 1.042 & 1.112 & 1.092 & 1.000 & 1.091 & 1.032 & 1.281\end{array}$ $\begin{array}{lllllllllllll}1.025 & 1.057 & 1.000 & 1.057 & 1.000 & 1.057 & 1.078 & 0.941 & 1.063 & 1.032 & 1.129\end{array}$ $\begin{array}{lllllllllll}1.052 & 1.104 & 1.000 & 1.037 & 0.958 & 1.018 & 1.026 & 0.902 & 1.034 & 1.000 & 1.064\end{array}$

Figure App2.4: Printer output of MMCOLLAT..BAS. The matrix at the start of the output on the previous page is from 12 experiments, each column represents a different experiment and each row a different extrasystolic interval. The print in bold type represents the mean and standard error for normalised beat force at a given extrasystolic interval, ie from each row of the matrix. 


\section{Regression}

\subsection{Description of Regression}

This commercial program, which operates in the Microsoft Windows environment, was used to fit force-interval curves to a biexponential function. The data for this program was entered directly from the disc files created by MMCOLLAT.BAS. The program uses an iterative fitting procedure based on the Marquand algorhythm to minimise residual distances between data points and the biexponential function synthesised. The theoretical basis for this method of curve fitting is provided in the software documentation (Blackwell Scientific, Oxford, England).

\subsection{Output of Regression}

An example of the output of this program is shown in figure app2.5.

\section{MMAREA.BAS}

\subsection{Description of MMAREA.BAS}

This program was used to trace the force envelopes recorded at slow paper speed to estimate papillary muscle activity during hypoxia. The envelopes were traced on a bit pad and the stylus location transferred via the RS232 port to a personal computer. This program displayed stylus position on the screen and calculated the area enclosed by the stylus using Simpson's rule.

\section{lines function}

75-148 Defines the scaling functions to transpose stylus position to screen position.

150-178 Sets ports for communication and calculates steady state develped force for normalisation.

180-360 Positional information is entered into an array, the program flow carries on looping until a data point is entered further to the left than the original point. The stylus position is recorded on the screen.

390-470 The area of activity is calculated by Simpson's rule.

475-494 Outputs the actual and normalised activity data to screen and printer 


\subsection{Listing of MMAREA.BAS}

10 REM program to calculate area normalizing for pre-anoxia steady state

20 REM using the suma sketch II set at 9600 baud,

30 REM in ASCII BCD report format, 8 bits no parity.

40 REM (JUMPERS AA IN, AB OUT AND AC OUT)

50 REM ***Downey/Marber*** 25/04/91

60 REM

70 DIM X(250), Y(250)

72 REM Scaling for screen 2

75 DEF FNXC $(X)=(X / 5840) * 650$

76 DEF FNYC $(Y)=(200-(Y / 5840) * 200) * 1.6$

86 PRINT:PRINT

145 CLS

148 SCREEN 2

150 OPEN "com2:9600,n,8,1" AS \#1

160 PRINT\#1, "B":REM Sets pointing mode

170 INPUT "name of experiment"; NAM\$

172 PRINT "click on pre-anoxia SS amplitude"

174 INPUT\#1,XA,YA,A\$:SOUND 1000,2

176 INPUT\#1,XB,YB,A\$:SOUND 1000,2

177 IF A\$="2" THEN PRINT "do you wish to stop pointing?":GOSUB 650: IF Y THEN 492

$178 \mathrm{SS}=\mathrm{SQR}\left((\mathrm{XA}-\mathrm{XB})^{\wedge} 2+(\mathrm{YA}-\mathrm{YB})^{\wedge} 2\right)$

180 CLS:PRINT"!!!start tracing!!!"

182 PRINT "trace out tensions during anoxia"

$190 \mathrm{I}=1$

200 INPUT\#1,X,Y,A\$:SOUND 1500,2

205 IF A $\$=" 2 "$ THEN PRINT "do you wish to stop pointing?":GOSUB 650: IF Y THEN 492

$220 \mathrm{XP}=\mathrm{FNXC}(\mathrm{X}): \mathrm{YP}=\mathrm{FNYC}(\mathrm{Y})$

$221 \mathrm{WP}=\mathrm{FNYC}(\mathrm{Y}+\mathrm{SS})$

225 IF I=1 THEN PSET (XP,WP)

230 IF $X P<0$ THEN $X P=0$

240 IF $\mathrm{YP}<0$ THEN YP=0

250 IF XP $>639$ THEN XP=639

260 IF $Y P>199$ THEN YP=199

270 IF I $>1$ AND $X>X(1)$ THEN LINE -(XP,YP) ELSE IF I=1 THEN PSET(XP,YP) 
$290 X(I)=X$

$300 \mathrm{Y}(\mathrm{I})=\mathrm{Y}$

310 IF $\mathrm{X}(\mathrm{I})<\mathrm{X}(1)$ THEN LINE -(FNXC(X(1)),FNYC(Y(1))):GOTO 370

320 IF $\mathrm{I}=250$ THEN 350

$330 \mathrm{I}=\mathrm{I}+1$

340 GOTO 200

350 PRINT "out of data error"

360 STOP

370 REM analyse data

390 BEEP

400 PRINT I,"!!!points collected!!!"

$410 \mathrm{~A}=0$

420 FOR N=1 TO I- 2

$430 \mathrm{~A}=\mathrm{A}+(\mathrm{X}(\mathrm{N}+1)-\mathrm{X}(\mathrm{N})) *((\mathrm{Y}(\mathrm{N})+\mathrm{Y}(\mathrm{N}+1)) / 2)$

440 NEXT $N$

$450 \mathrm{~N}=1$

470 PRINT:PRINT:PRINT

475 LPRINT:LPRINT:LPRINT:LPRINT "standard activity during ischaemia for ";

NAM\$

481 LPRINT A,"unit secs"

490 PRINT A/SS "standard secs"

491 LPRINT A/SS "standard secs"

492 PRINT "Is there another trace to follow ?":GOSUB 650

493 CLOSE \#1

494 IF NOT Y THEN END

520 GOTO 148

530 RETURN

650 REM yes or no routine

660 Z\$=INKEY\$:IF Z\$="' THEN GOTO 660

670 Z=INSTR("YyNn",Z\$):IF Z=0 THEN GOTO 660 ELSE IF Z>2 THEN PRINT

"No.":Y=0 ELSE PRINT"Yes.":Y=-1

680 RETURN 


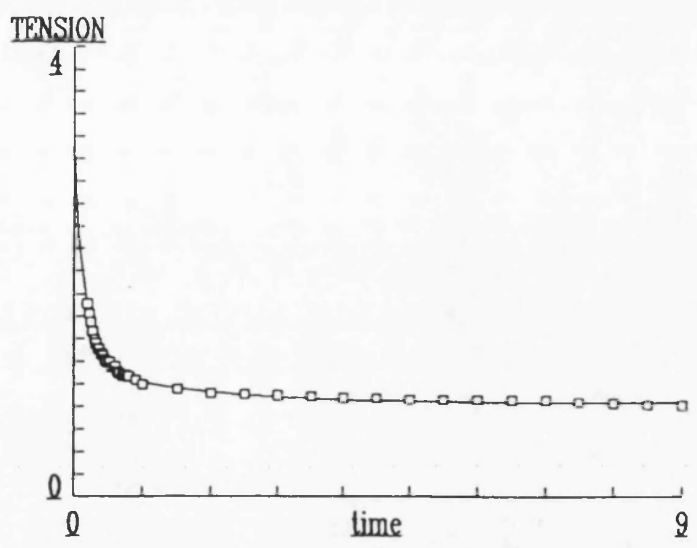

Title Exponential Fall Twin EXP Fit TENSION $=A+B *(\exp (-$ one $/ \mathrm{k} 1$ *time $))+C *(\exp ($-one $/ \mathrm{k} 2 *$ time $))$ Iteration count $=28$

Sum of squares $=0.00947596$

Standard deviation $=0.0157914$

Determination coef. $=0.99573$

A

B

C

k1

$\mathrm{k} 2$

$0.831982+/-0.00690499$

$1.88061+/-0.131076$

$0.337855+/-0.0266514$

$0.165189+/-0.0114523$

$1.61692+/-0.230184$

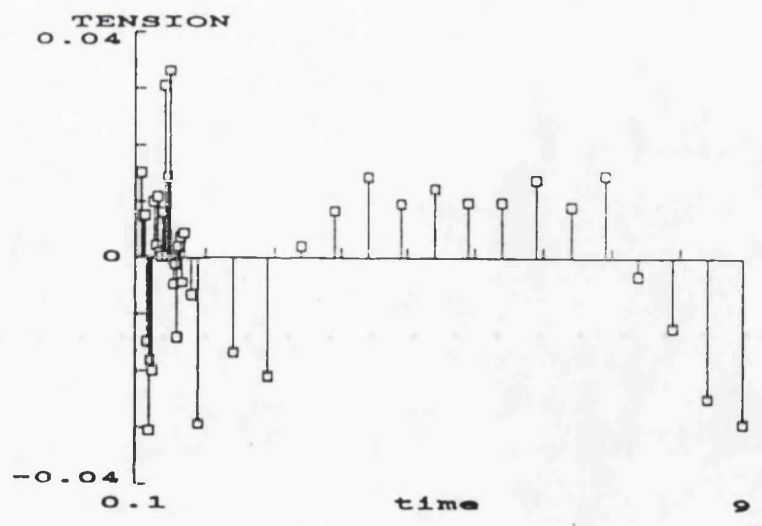

itine, TENSION, Calculated, Residual

Figure App2.5: Printer output of Regression. The upper graph shows the data as squares with the iterative fit as a solid line. The equation used to fit this data is listed in line 2 of the text below the graph. The values for A, B, C, k1 and $\mathrm{k} 2$ together with their errors are given in lines 7 to 11 . The values of $\mathrm{k} 1$ and $\mathrm{k} 2$ are used to compare force-interval relationships (see Chapter 4 figures 4.9 to 4.12 ). The data used to derive this relationship was obtained by MMCOLLAT.BAS and is listed in figure App2.4. The lower graph is of the residuals, ie a graphical representation of how far the fitted line deviates from each of the data points. 
App2.5: Printer Output of REGRESSION (Contd)

$\begin{array}{llll}0.2, & 1.706, & 1.69091, & 0.015089 \\ 0.225, & 1.615, & 1.60763, & 0.00737294 \\ 0.25, & 1.543, & 1.53547, & 0.00753303 \\ 0.275, & 1.458, & 1.47288, & -0.0148772 \\ 0.3, & 1.388, & 1.41852, & -0.0305224 \\ 0.325, & 1.353, & 1.37125, & -0.0182544 \\ 0.35, & 1.31, & 1.33009, & -0.0200863 \\ 0.375, & 1.304, & 1.29417, & 0.00983032 \\ 0.4, & 1.265, & 1.26278, & 0.00222484 \\ 0.425, & 1.246, & 1.23528, & 0.0107244 \\ 0.45, & 1.212, & 1.21113, & 0.00086809 \\ 0.475, & 1.19, & 1.18988, & 0.000119507 \\ 0.5, & 1.179, & 1.17112, & 0.00787719 \\ 0.525, & 1.185, & 1.15452, & 0.0304839 \\ 0.55, & 1.14, & 1.13977, & 0.000234166 \\ 0.575, & 1.141, & 1.12662, & 0.0143816 \\ 0.6, & 1.148, & 1.11486, & 0.0331439 \\ 0.625, & 1.104, & 1.10429, & -0.000291229 \\ 0.65, & 1.09, & 1.09476, & -0.00476274 \\ 0.675, & 1.085, & 1.08613, & -0.0011319 \\ 0.7, & 1.064, & 1.07828, & -0.0142794 \\ 0.725, & 1.073, & 1.0711, & 0.0018974 \\ 0.75, & 1.068, & 1.06451, & 0.00348687 \\ 0.775, & 1.054, & 1.05843, & -0.00443497 \\ 0.8, & 1.057, & 1.0528, & 0.00419735 \\ 0.9, & 1.027, & 1.03372, & -0.00671552 \\ 1, & 0.989, & 1.01843, & -0.029427 \\ 1.5, & 0.949, & 0.965806, & -0.016806 \\ 2, & 0.909, & 0.930063, & -0.0210635 \\ 2.5, & 0.906, & 0.903968, & 0.00203225 \\ 3, & 0.893, & 0.88482, & 0.00818005 \\ 3.5, & 0.885, & 0.870766, & 0.0142344 \\ 4, & 0.87, & 0.86045, & 0.00955049 \\ 4.5, & 0.865, & 0.852877, & 0.0121226 \\ 5, & 0.857, & 0.847319, & 0.00968062 \\ 5.5, & 0.853, & 0.84324, & 0.00976027 \\ 6, & 0.854, & 0.840245, & 0.0137548 \\ 6.5, & 0.847, & 0.838047, & 0.00895278 \\ 7, & 0.851, & 0.836134, & 0.0145661 \\ 7.5, & 0.832, & 0.83525, & -0.00324963 \\ 8, & 0.822, & 0.83438, & -0.0123804 \\ 8.5, & 0.809, & 0.833742, & -0.0247424 \\ 9, & 0.804, & 0.833274, & -0.029274\end{array}$

Figure App2.5: Printer output of Regression (contd). The columns listed above have their headings on the previous page. Extrasystolic interval appears on the left, experimental data in the next column, fitted data in the next column and residuals in the rightmost column. The residuals are squared and summed to give a total measure of goodness of fit this value is given in the upper text on the previous page. At each extrasystolic interval the magnitude of the residuals is small compared to the data values. 


\section{MMINFRSK.BAS}

\subsection{Description of MMINFRSK.BAS}

This program was originally written by Dr James Downey, Dept of Physiology, University of S.Alabama, USA. Their have been some minor subsequent modifications. The program utilizes the bit pad to display and calculate risk areas and areas of necrosis in heart slices. The program flow and logic is similar to MMAREA and will not be described further.

\subsection{Listing of MMINFRSK.BAS}

10 REM program to calculate area

20 REM using the suma sketch II set at 9600 baud,

30 REM in ASCII BCD report format, 8 bits no parity.

40 REM (JUMPERS AA IN, AB OUT AND AC OUT)

50 REM ***James Downey 17 NOV $1990 * * *$

60 REM ***Mike Marber SEPT 1991***

70 DIM X(250),Y(250)

$80 \mathrm{CLS}$

$90 \mathrm{MG}=1.41: \mathrm{TH}=.2^{\prime} * * * * * *$ set defaults here

$100 \mathrm{MG}=\mathrm{MG}^{\wedge} 2$

105 PRINT "magnification now correct (Sept/91)"

110 INPUT "magnification from original (default=1.41)";A $\$$

120 IF A $\$<>" "$ THEN MG=(VAL(A $\left.\$)^{\wedge} 2\right)$

130 INPUT "slice thickness in $\mathrm{Cm}$ (default=.2)"; $\$$

140 IF A $\$<>"$ " THEN TH=VAL(A $\$$ )

150 OPEN "com2:9600,n,8,1" AS \#1

160 PRINT\#1, "B"

170 CLS:PRINT"!!!start tracing!!!":GOSUB 640

180 SCREEN 1

$190 \mathrm{I}=1$

200 INPUT\#1,X,Y,A\$

210 REM PRINT X,Y,A\$:GOTO 120

$220 \mathrm{XP}=(\mathrm{X} / 10)-100: \mathrm{YP}=400-(\mathrm{Y} / 10)$

230 IF $X P<0$ THEN $X P=0$

240 IF $Y P<0$ THEN $Y P=0$

250 IF $X P>639$ THEN XP=639

260 IF YP $>199$ THEN YP=199

270 IF I $>1$ THEN LINE -(XP,YP) : GOTO 290 
280 PSET (XP,YP)

$290 \mathrm{X}(\mathrm{I})=\mathrm{X}$

$300 \mathrm{Y}(\mathrm{I})=\mathrm{Y}$

310 IF $X(I)<X(1)$ THEN LINE -((X(1)/10)-100,(400-Y(1)/10)):GOTO 370

320 IF $\mathrm{I}=250$ THEN 350

$330 \mathrm{I}=\mathrm{I}+1$

340 GOTO 200

350 PRINT "out of data error"

360 STOP

370 REM analyse data

380 SCREEN 1

390 BEEP

400 PRINT I,"!!!points collected!!!"

$410 \mathrm{~A}=0$

420 FOR $\mathrm{N}=1$ TO I-2

$430 \mathrm{~A}=\mathrm{A}+(\mathrm{X}(\mathrm{N}+1)-\mathrm{X}(\mathrm{N})) *((\mathrm{Y}(\mathrm{N})+\mathrm{Y}(\mathrm{N}+1)) / 2)$

440 NEXT $N$

$450 \mathrm{~N}=1$

$460 \mathrm{~A}=\mathrm{A}+(\mathrm{X}(1)-\mathrm{X}(\mathrm{I}-1)) *((\mathrm{Y}(\mathrm{I}-1)+\mathrm{Y}(1)) / 2)$

470 PRINT:PRINT:PRINT

480 PRINT $4 * A /(M G * 152465$ !),"square centimeters"

490 PRINT TH*A*4/(MG*152465!),"cubic centimeters"

500 PRINT "click the pen for another"

510 INPUT\#1, X,Y,A\$ :BEEP

520 IF A $\$=" 2 "$ THEN GOTO 170

530 GOTO 510

$540 \mathrm{Y} 1=\mathrm{Y}(\mathrm{N}-200)$

$550 \mathrm{~N} 1=\mathrm{INT}(\mathrm{X}(\mathrm{N}) / 32)$

$560 \mathrm{X} 2=\mathrm{X}(\mathrm{N})-\mathrm{N} 1 * 32+64$

$570 \times 3=\mathrm{N} 1+32$

$580 \mathrm{~N} 2=\mathrm{INT}(\mathrm{Y} 1 / 32)$

$590 \mathrm{Y} 2=\mathrm{Y} 1-\mathrm{N} 2 * 32+96$

$600 \mathrm{Y} 3=32+\mathrm{N} 2$

610 PRINTCHR \$(Y3);CHR\$(Y2);CHR\$(X3);CHR \$(X2);

620 RETURN

630 SCREEN 3: SYSTEM

640 FOR FT=1 TO 500:NEXT FT:RETURN 


\section{References}

Allen DG, Kurihara S: 1980 . Calcium transients in mammalian ventricular muscle. Eur Heart J. 1(Suppl A):5-15

Allen DG, Orchard CH: 1984. Measurement of intracellular calcium concentration in heart muscle: The effects of inotropic interventions and hypoxia. J Mol Cell Cardiol 16:117-128

Allen DG, Orchard $\mathrm{CH}$ : 1987. Myocardial contractile function during ischemia and hypoxia. Circ Res 60:153-168

Altschuld RA, Ganote CE, Nayler WG, Piper HM: 1991. What constitutes the calcium paradox. Editorial Comment. J Mol Cell Cardiol 23:765-767

Ananthan J, Goldberg AL, Voellmy R: 1986. Abnormal proteins serve as eukaryotic stress signals and trigger the activation of heat shock genes. Science 232:252-254

Andres J, Sharma HS, Knoll R, Sassen L, Verdouw PD: 1992. Enhanced expression of mRNAs encoding ubiquitin and hsp27 in porcine stunned myocardium (Abstract). Circulation 86(Suppl I):I-624

Angelidis CE, Lazaridis I, Papoulatos GN: 1991. Constitutive expression of heat-shock protein 70 in mammalian cells confers thermoresistance. Eur J Biochem 199:35-39

Armitage P, Berry G: 1987. Statistical methods in medical research. 2nd ed. Blackwell Scientific Publications: Oxford

Arrigo AP, Welch WJ: 1987. Characterization of the small 28,000 Dalton mammalian heat shock protein. J Biol Chem 1987 84:15359-15369

Bache RJ: 1984. Can drugs really limit infarct size? In: Hearse DJ, Yellon DM eds. Therapeutic approaches to myocardial infarct size limitation. Raven Press: New York, pp185-208

Bansal GS, Norton PM, Latchman DS: 1991. The 90kd heat shock protein protects cells from thermal stress but not from viral infection. Exp Cell Res 195:303-306 
Barbe MF, Tytell M, Gower DJ, Welch WJ: 1988. Hyperthermia protects against light damage in the rat retina. Science 241:1817-1820

Bardwell JCA, Craig EA: 1987. Eukaryotic Mr 83,000 heat shock protein has a homologue in Escherichia coli. Proc Natl Acad Sci 84:5177-5181

Beaulieu EE: 1987. Steroid hormone antagonists at the receptor level: A role for the heat-shock protein MW 90,000 (hsp90). J Cell Biochem 35:161

Behar S, Reicher-Reiss H, Allmader E: 1992. The prognostic significance of angina pectoris preceeding the occurence of a first acute myocardial infarction in 4166 consecutive hospitalized patients. Am Heart J 123:1481-1486

Benijamali HS, Gao W, MacIntosh BR, ter Keurs HEJD; 1991. Force-Interval relations of twitches and cold contracture in rat cardiac trabeculae effect of ryanodine. Circ Res 69:937-947

Benjamin IJ, Williams RS: 1990. Activation of heat shock transcription factor by metabolic inhibitors in cultured myogenic cells (abstract). Circulation 82 (supplement III):III-351

Benjamin IJ, Kroger B, Williams RS: 1990. Activation of the heat shock transcription factor by hypoxia in mammalian cells. Proc Natl Acad Sci 87:6263-6267

Bennardini F, Wrzosek A, Chiesi M: 1992. AlphaB-crystallin in cardiac tissue. Association with actin and desmin filaments. Circ Res 71:288-294

Berk BC: 1991. The microcirculation in coronary ischemia. Are native anticoagulant mechanisms a path to new therapies? Circulation $84: 439-441$

Bhojani IH, Chapman RA: 1990. The effects of bathing sodium ions upon thr intracellular sodium activity in calcium-free media and the calcium paradox of isolated ferret ventricular muscle. J Mol Cell Cardiol 22:507-522

Bienz M, Pelham HRB: 1986. Heat shock regulatory elements function as an inducible enhancer in the Xenopus hsp70 gene and when linked to a heterologous promoter. Cell 45:753-760

Black AR, Subjeck JR: 1991. The biology and physiology of the heat shock and glucose-regulated stress protein systems. In Jasmin G, Proshek L eds. Stress revisited 
2. Systemic effects of stress. Methods Achieve Exp Pathol, Karger: Basel, vol 15, pp126-166

Black SC, Lucchesi BR: 1993. Heat shock proteins and the ischemic heart. An endogenous protective mechanism. Circulation 87:1048-1051.

Blake MJ, Gershon D, Fargnoli J, Holbrook NJ: 1990. Discordant expression of heat shock protein mRNAs in tissues of heat-stressed rats. J Biol Chem 25:15275-15279

Blanchard EM, Solaro RJ: 1984. Inhibition of the activation and troponin calcium binding of dog cardiac myofibrils by acidic pH. Circ Res 55:382-391

Blatter LA, McGuigan JAS: 1991. Intracellular $\mathrm{pH}$ regulation in ferret ventricular muscle. The role of $\mathrm{Na}-\mathrm{H}$ exchange and the influence of metabolic substrates. Circ Res 68:150-161

Bolli R: 1990. Mechanism of myocardial stunning. Circulation 82:723-738

Brown MA, Upender RP, Hightower LE, Renfro JL: 1992. Thermoprotection of a functional epithelium: heat stress effects on transepithelial transport by flounder renal tubule in primary monolayer culture. Proc Natl Acad Sci 89:3246-3250

Brugge J, Erikson E, Erikson RL: 1981. The specific interaction of the Rous sarcoma virus transforming protein, pp60src, with two cellular proteins. Cell 25:363-372

Brunwald E, Kloner RA: 1985. Myocardial reperfusion: a double edged sword. J Clin Invest 76:1713-1719

Burgman PWJJ, Konings AWT: 1992. Heat induced protein denaturation in the particulate fraction of HeLa S3 cells: Effect of thermotolerance. J Cell Physiol $153: 88-94$

Busch H: 1984. Ubiquination of proteins. Methods Enzymol 106:238-262

Busselen P: 1985. Suppression of cellular injury during the calcium paradox in rat heart by factors which reduce calcium uptake by mitochondria. Pflugers Arch 404:166-171

Calderwood SK, Bornstein B, Farnum EK, Stevenson MA: 1989. Heat shock stimulates the release of arachidonic acid and the synthesis of prostaglandins and 
leukotriene B4 in mammalian cells. J Cell Physiol 141:325-333

Cavallini L, Valente M, Pia Rigobello M: 1990. The protective action of pyruvate on recovery of ischaemic rat heart: Comparison with other oxidizable substrates. J Mol Cell Cardiol 22:143-154

Ceconi C, Curello S, Cargnoni A, Ferrari R, Albertini A, Visioli O: 1988. The role of glutathione status in the protection against ischaemic and reperfusion damage: Effect of N-acetyl cysteine. J Mol Cell Cardiol 20:5-13

Chapman RA, Suleimann MS, Rodrigo GC, Tunstall J: 1991. The calcium paradox: a role for $[\mathrm{Na}] \mathrm{i}$, a cellular or tissue basis, a property unique to the Langendorff perfused heart? A bundle of contradictions. J Mol Cell Cardiol 23:773-777

Chappell SP, Lewis MJ, Henderson AH: 1985. Myocardial reoxygenation damage: can it be circumvented? Cardiovasc Res 19:299-303

Chappell TG, Welch WJ, Schlossman DM, Palter KB, Schlessinger MJ, Rothman JE: 1986. Uncoating ATPase is a member of the $70 \mathrm{kDa}$ family of stress proteins. Cell 45:3-12

Chein KR, Knowlton KU, Zhu H, Chien C: 1991. Regulation of cardiac gene expression during myocardial growth and hypertrophy: molecular studies of an adaptive physiologic response. FASEB J 5:3037-3046

Chevalier B, Callens F, Charlemagne D, Delcayre C, Lompre AM, Lelievre L, Mercadier JJ, Moalic JM, Mansier P, Rappaport L, Samuel JL, Schwartz K, Swynghedauw B: 1989. Signal and adaptational changes in gene expression during cardiac overload. J Mol Cell Cardiol 21:71-77

Chiesi M, Bennardini F: 1992. Determination of alphaB crystallin aggregation: a new alternative method to assess ischemic damage of the heart. Basic Res Cardiol 87:39-46

Chiesi M, Longoni S, Limbruno U: 1990. Cardiac alpha-crystallin. Involvement during heart ischemia. Mol Cell Biochem 97:129-136

Chop M, Chen H, Ho K-L: 1989. Transient hyperthermia protects against subsequent forebrain ischemic cell damage in the rat. Neurology 39:1396-8

Christman MF, Morgan RW, Jacobson FS, Ames BN: 1985. Positive control of a 
regulon for defenses against oxidative stress and some heat shock proteins in Salmonella typhimurium. Cell 41:753-762

Ciocca DR, Fuqua AW, Lock-Lim S, Toft DO, Welch WJ, McGuire WL: 1992. Response of human breast cancer cells to heat shock and chemotherapeutic drugs. Cancer Res 53:3648-3654

Cohen MV, Liu GS, Downey JM: 1991. Preconditioning causes improved wall motion as well as smaller infarcts after transient coronary occlusion in rabbits. Circulation $84: 341-349$

Cornelussen RN, Snoeckx LH, de Bruin LG, van der Vusse GJ, Reneman RS: 1993. Hyperthermic preconditioning improves ischemia tolerance in the hypertrophied and non-hypertrophied rat heart (abstract). J Mol Cell Cardiol;25(SupplI):S56110

Crompton M, Ellinger $\mathrm{H}$, Costi A: 1988. Inhibition by cyclosporin $\mathrm{A}$ of a $\mathrm{Ca}^{2+}$ dependant pore in mitochondria activated by inorganic phosphate and oxidative stress. Biochem J 255:357-360

Currie RW, White FP: 1983. Characterization of the synthesis and accumulation of a 71-Kilodalton protein induced in rat tissues after hyperthermia. Can J Biochem Cell Biol 61:438-446

Currie RW: 1987. Effects of ischemia and perfusion temperature on the synthesis of stress induced (heat shock) proteins in isolated and perfused rat hearts. J Mol Cell Cardiol 19:795-808

Currie RW, Karmazyn M, Kloe M, Mailer K: 1988. Heat shock response is associated with enhanced postischaemic ventricular recovery. Circ Res 63:543-549

Currie RW, Ross BM, Davis TA: 1990. Induction of the heat shock response in rats modulates heart rate, creatine kinase and protein synthesis after a subsequent hyperthermic treatment. Cardiovasc Res 24:87-93

Currie RW, Karmazyn M: 1990. Improved post-ischemic ventricular recovery in the absence of changes in energy metabolism in working rat hearts following heat shock. J Mol Cell Cardiol 22:631-636

Currie RW, Tanguay RM: 1991. Analysis of RNA for transcripts for catalase and SP71 in rat hearts after in vivo hyperthermia. Biochem Cell Biol 69:375-382 
Currie RW, Tanguay RM, Kingma JG: 1993. Heat-shock response and limitation of tissue necrosis during occlusion/reperfusion in rabbit hearts. Circulation 87:963-971

Darley-Usmar VM, O'Leary V, Stone D: 1989. The glutathione status of rat hearts subjected to hypoxia and reoxygenation: the oxygen paradox. Free Radic Res Commun 5:283-289

Daut J, Elzinga G: 1989. Substrate dependance of energy metabolism in isolated guinea-pig cardiac muscle: A microcalorimetric study. J Physiol 413:379-397

De Luca-Faherty C, McKay DB, Parman P, Hill BL: 1990. Uncoating protein (hsc70) binds a conformationally labile domain of clathrin light chain $\mathrm{LC}_{\mathbf{a}}$ to stimulate ATP hydrolysis. Cell 62:875-887

Delcayre C, Samuel JL, Marrote F, Best-Belpomme M, Mercadier JJ, Rappaport L: 1988. Synthesis of stress protein in rat cardiac myocytes 2-4 days after imposition of hemodynamic overload. J Clin Invest 82:460-468

DeWood MA, Spores J, Notske R: 1980. Prevalence of total coronary occlusion during the early hours of transmural myocardial infarction. N Eng J Med 313:897-902

Dietrich DLL, Mast F, Elzinga G: 1991. Myocardial force production and energy turnover in anoxia. Experentia 46:1168-1172

Dillman WH, Mehta HB, Barrieux A, Guth BD, Neeley WE, Ross J: 1986. Ischemia of the dog heart induces the appearance of a cardiac mRNA coding for a protein with migration characteristics similar to heat-shock/stress protein 71. Circ Res 59:110-114

Dillmann WH, Chi S-H, Sayen MR, Mestril R: 1992. Decreased ischemia (Ism) induced injury in rat heart derived $\mathrm{H} 9 \mathrm{c} 2$ cells expressing human inducible heat shock protein 70 (hHSP70i) (abstract). J Mol Cell Cardiol 24(suppl III):S.30

DoH, Department of Health: 1992. Health and personal social services statistics for England. HMSO, London

Donnelly TJ, Sievers RE, Vissern FLJ, Welch WJ, Wolfe CL: 1992. Heat shock protein induction in rat hearts. A role for improved myocardial salvage after ischemia and reperfusion. Circulation 85:769-778

Dorner AJ, Krane MG, Kaufman RJ: 1988. Reduction of endogenous GRP78 levels 
in CHO cells and its effects on secretion (abstract). J Cell Biochem 12D(suppl):276

Downey JM: 1984. Why the endocardium? In: Hearse DJ, Yellon DM eds. Therapeutic approaches to myocardial infarct size limitation. Raven Press: New York, pp125-138

Duncan CJ: 1990. Biochemical events associated with rapid cellular damage during oxygen and calcium paradoxes of the mammalian heart. Experentia 46:41-48

Ellis RJ: 1990. Molecular Chaperones. Semin Cell Biol 1:1-72

Erberli FR, Weinberg EO, Grice WN, Horowitz GL, Apstein CS: 1991. Protective effect of increased glycolytic substrate against systolic and diastolic dysfunction and increased coronary resistance from prolonged global underperfusion and reperfusion in isolated rabbit hearts perfused with erythrocyte suspensions. Circ Res 68:466-481

Fabiato A, Fabiato F: 1978. Effects of $\mathrm{pH}$ on the myofilaments and the sarcoplasmic reticulum of skinned cells from cardiac and skeletal muscles. J Physiol (Lond) 276:233-255

Falk E: 1985. Unstable angina with fatal outcome: Dynamic coronary thrombosis leading to infarction and/or sudden death. Circulation 71:699-708

Ferrari R, Ceconi C, Currello S, Cargnoni A, Ruigrok TJC: 1989. No evidence of oxygen free radicals-mediated damage during the calcium paradox. Basic Res Cardiol 84:396-403

Flack J, Engelman R, Rousou J, Iyergar J, Kimura Y, Prasad R, Das DK: 1990. Preconditioning the heart by repeated stunning improves myocardial salvage (abstract). Circulation 82(suppl III):575

Flaherty KM, DeLuca-Flaherty C, McKay DB: 1990 . Three-dimensional structure of the ATPase fragment of a 70K heat-shock cognate protein. Nature 346:623-628

Flynn G, Chappell T, Rothman JE: 1989. Peptide binding and release by proteins implicated as catalysts of protein assembly. Science 245:385-390

Ford LE: 1990. Mechanical manifestations of activation in cardiac muscle. Circ Res 68:621-637 
Fozzard HA: 1988. Cellular basis for inotropic changes in the heart. Am Heart J $116: 230-234$

Gall JG, Pardue ML: 1971. Nucleic acid hybridization in cytological preparations. Methods Enzymol 21:470-480

Gauduel Y, Menasche P, Duvelleroy M: 1989. Enzyme release and mitochondrial activity in reoxygenated cardiac muscle: relationship with oxygen-induced lipid peroxidation. Gen Physiol Biophys 8:327-40

Gerner EW, Scheider MJ: 1975. Induced thermal resistance in HeLa cells. Nature 256:500-502

Gisolfi CV, Matthes RD, Kregel KC, Oppliger R: 1991. Splanchnic sympathetic nerve activity and circulating catecholamines in the hyperthermic rat. J Appl Physiol 70:1821-1826

Goebl MG, Yochem J, Jentsch S, McGrath JP, Varshavsky A, Byers B: 1988. The yeast cell cycle gene $\mathrm{CDC} 34$ encodes a ubiquitin-conjugating enzyme. Science 241:1331-1335

Goldenberg CJ, Luo Y, Fenna M, Baler R, Weinmann R, Voellmy R: 1988. Purified human factor activates heat shock promoter in a HeLa cell-free transcription system. J Biol Chem 263:19734-19739

Goldschmidt R: 1935. Gen und Ausseneigenschaft. 1.(Untersuchung an Drosophila).Z. Idukt Abstammungs Vererbungsl 69:38-131

Gorge G, Chatelain P, Schaper J, Lerch R: 1991. Effect of increasing degrees of ischemic injury on myocardial metabolism early after reperfusion in isolated rat hearts. Circ Res 68:1681-1692

Gray RE, Grasso DG, Maxwell RJ, Finnegan PM, Nagley P, Devennish RJ: 1990. Identification of a $66 \mathrm{kDa}$ protein associated with yeast mitochondrial ATP synthase as heat shock protein hsp60. FEBS letters 268(1):265-268

Green LAD, Liem RKH: 1989. Beta-internexin is a microtubule-associated protein indentical to the $70-\mathrm{kDa}$ heat-shock cognate protein and clatharin uncaoting ATPase. J Biol Chem 264:15210-15215 
Griffiths EJ, Halestrap AP: 1991. Further evidence that cyclosporin A protects mitochondria from calcium overload by inhibiting a matrix peptidyl-prolyl cis-trans isomerase. Implications for the immunosuppresive and toxic effects of cyclosporin. Biochem J 274:611-614.

Gupta RS: 1990. Mitochondria, molecular chaperone proteins and the in vivo assembly of microtubules. Trends Biochem Sci 15:415-418

GUSTO (Global Utilization of Streptokinase and tPA for Occluded coronary arteries):1993. Interim results presented in Thrombolysis Bulletin. Oxford Clinical Communications KVC108

Gutteridge JMC, Halliwell B: 1990. Reoxygenation injury and antioxidant protection. A tale of two paradoxes. Arch Biochem Biophysics 283:223-226

Hackett D, Davies G, Chierchia S, Maseri A: 1987. Intermittent coronary occlusion in acute myocardial infarction: value of combined thrombolytic and vasodilator therapy. N Engl J Med 317:1055-1059

Hagar JM, Hale SL, Kloner RA: 1991. Effect of preconditioning ischemia on reperfusion arrhythmias after coronary artery occlusion and reperfusion in the rat. Circulation 68:61-68

Hames BD: 1990. One-dimensional polyacrylamide gel electrophoresis. In: Hames BD, Rickwood D eds. Gel electrophoresis of proteins. A practical approach 2nd edition. Oxford University Press: Oxford, pp1-147

Hammond GL, Lai YK, Markert CL: 1982. Diverse forms of stress lead to new patterns of gene expression through a common and essential metabolic pathway. Proc Natl Acad Sci 79:3485-3488

Harboe M, Quayla AJ: 1991. Heat shock proteins: Friend and Foe? Clin Exp Immunol 21:2-5

Havre PA, Hammond GL: 1988. Isolation of a translation-inhibiting peptide from myocardium. Am J Physiol 255:H1024-1031

Heads RJ, Patel VC, Latchman DS, Yellon DM: 1992. Different expression patterns for $65 \mathrm{kDa}$ and $70 \mathrm{kDa}$ stress proteins following ischemic preconditioning (abstract). Circulation 86(suppl I):I-556 
Hearse DJ, Humphrey SM, Bullock GR: 1978. The oxygen paradox and the calcium paradox: two facets of the same problem? J Mol Cell Cardiol 10:641-668

Hearse DJ, Yellon DM, Downey JM: 1986. Can beta blockers limit infarct size? Eur Heart J 7:925-930

Hearse DJ, Tosaki A: 1987. Free radicals and reperfusion-induced arrhythmias: protection by spin trap agent PBN in the rat heart. Circ Res 60:375-383

Henderson AH, Most AS, Sonnenblick EH: 1969. Depression of contractility in rat heart by free fatty acid during hypoxia. Lancet $825-826$

Henderson AH, Craig RJ, Sonnenblick EH, Urschel CW: 1970. Species differences in intrinsic myocardial contractility. Proc Soc Exp Biol and Med 134:930-932

Hershko A: 1988. Ubiquitin mediated protein degradation. J Biol Chem 263:1523715240

Hescheler J, Meyer R, Plant S, Krautwurst D, Rosenthal W, Schultz G: 1991. Morphological and electrophysiological characterization of a clonal cell (H9c2) line from rat heart. Circ Res 69:1476-1486

Heuser JE, Reese TS: 1973. Evidence for recycling of synaptic vesicle membrane during transmitter release at the frog neuromuscular junction. J Cell Biol 57:315-344

Hightower LE: 1991. Heat shock, stress proteins, chaperones, and proteotoxicity. Cell 66:191-197

Hill AV: 1928. Diffusion of oxygen and lactic acid through tissues. Proc R Soc (Lond) 104:39-96

Horwich, A: 1990. Protein import into mitochondria and peroxisomes. Curr Opin Cell Biol 2(4):625-633

Hoshida S, Kuzuya T, Yamashita N, Fuji H, Oe H, Otsu K, Kimura Y, Hori M, Tada M: 1992. Delayed effect of sublethal ischemia on limiting infarct size resulting from sustained ischemia and reperfusion(abstract). Circulation 86(suppl I):I-30

Hoshida S, Kuzuya T, Fuiji H, Yamashita N, Oe H, Hori M, Suzuki K, Taniguchi N, Tada M: 1993. Sublethal ischemia alters myocardial antioxidant activity in canine 
heart. Am J Physiol 264:H33-H39

Howard G, Geoghegan TE: 1986. Altered cardiac tissue gene expression during acute hypoxic exposure. Mol Cell Biochem 69:155-160

Huber SA: 1992. Heat-shock protein induction in adriamycin and picornavirus-infected cardiocytes. Lab Invest 67:218-224

Hunt C, Morimoto RI: 1985 . Conserved features of eukaryotic hsp79 genes revealed by comparison with the nucleotide sequence of human hsp70. Proc Natl Acad Sci 82:6455-6459

Hutchinson EG, Tichelaar W, Hofhaus G, Weiss H, Leonard KR: 1989. Identification and electron microscopic analysis of a chaperonin oligomer from Nuerospora crassa mitochondria. EMBO J 8(5):1485-90

Hutter MM, Sievers RE, Wolfe CL: 1993. Myocardial protection after heat shock pretreatment correlates with the degree of prior stress protein induction (abstract). $J$ Am Coll Cardiol 21:70A

Iida H, Yahara I: 1985. Yeast heat shock protein of $\mathrm{Mr} 48,000$ is an isoprotein of enolase. Nature 315:688-90

ISIS-2 (Second International Study of Infarct Survival) Collaborative Group: 1988. Randomized trial of IV streptokinase, oral aspirin, both, or neither among 17187 cases of suspected acute myocardial infarction. Lancet ii:349-360

Iwaki K, Mestril R, Dillman WH, Chien KR: 1989. Induction of HSP $71 \mathrm{mRNA}$ correlates with arachidonic acid release and the transition to irreversible injury in neonatal rat myocardial cells following ATP depletion (abstract). Circulation 80(suppl II):II-462

Iwaki K, Chi S-H, Dillmann WH, Mestril R: 1993. Induction of HSP70 in cultured rat neonatal cardiomyocytes by hypoxia and metabolic stress. Circulation 87:2023-2032

Izumo S, Nadal-Ginard B, Mahdavi V: 1988. Protooncogene induction and reprogramming of cardiac gene expression produced by pressure overload. Proc Natl Acad Sci 85:339-343

Jaattela M, Wissing D, Bauer PA, Li GC: 1992. Major heat shock protein hsp70 
protects tumour cells from tumor necrosis factor cytotoxicity. EMBO J 11:3507-3512

Jennings RB, Steenbergen C: 1985 . Nucleotide metabolism and cellular damage in myocardial ischemia. Ann Rev Physiol 47:729-749

Jentsh S, McGrath JP, Varhavsky A: 1987. The yeast DNA repair gene RAD encodes a ubiquitin conjugating enzyme. Nature 329:131-134

Johnston RN, Kucey BL: 1988. Competitive inhibition of hsp70 gene expression causes thermosensitivity. Science 242:1551-1554

Jones DB, Hunter NR, Duff GW: 1990. Heat-shock protein 65 as a beta cell antigen of insulin-dependant diabetes. Lancet 336:583-585

Kang P, Ostermann J, Shilling J, Neupert W, Craig EA, Pfanner N: 1990. Requirement for HSP70 in the mitochondrial matrix for translocation and folding of precursor proteins. Nature 348:137-143

Karamazyn M, Mailer K, Currie RW: 1990. Acquisition and decay of heat-shock-enhanced postischemic ventricular recovery. Am J Physiol 259:424-431

Karmazyn M: 1991. Ischemic and reperfusion injury in the heart. Cellular mechanisms and pharmacological interventions. Can J Physiol Pharmacol 69:719-730

Kato K, Shinohara H, Goto S, Inaguma Y, Morishita R, Asano T: 1992. Copurification of small heat shock protein with alphaB crystallin from human skeletal muscle. J Biol Chem 267:7718-7725

Katz AM: 1992a. Mechanism and control of the cardiac contractile process, in: "Physiology of the heart", Raven Press, New York. Ch8, pp178-195

Katz AM: 1992b. Oxidative metabolism, in: "Physiology of the heart", Raven Press, New York. Ch5, pp98-128

Kaufmann SEK: 1989. Heat shock proteins and the immune response. Immunology Today 11:129-136

Keyse SM, Tyrell RM: 1982. Both near ultra violet radiation and the oxidising agent hydrogen peroxide induce a $32 \mathrm{kDa}$ stress protein in normal human skin fibroblasts. J Biol Chem 262:14821-14825 
Keyse SM, Emslie EA: 1992. Oxidative stress and heat shock induce a human gene encoding a protein-tyrosine phosphatase. Nature 359:644-647

Kihara Y, Grossman W, Morgan JP: 1989. Direct measurement of changes in intracellular calcium transients during hypoxia, ischaemia, and reperfusion of the intact mammalian heart. Circ Res 65:1029-1044

Kim IH, Kim K, Rhee SG: 1989. Induction of an antioxidant protein of Saccharomyces cerevisiae by $\mathrm{O}_{2}, \mathrm{Fe}^{3+}$, or 2-mercaptoethanol. Proc Natl Acad Sci 86:6018-6022

Kirkels JH, Ruigrok TJ, Van Echteld CJ, Meijler FL: 1989. Low $\mathrm{Ca}^{2+}$ reperfusion and enhanced susceptibility of the postischemic heart to the calcium paradox. Circ Res 64:1158-1164

Kitagawa K, Matsumoto M, Kuwabara K, Tagaya M, Toshiho O, Hata R, Ureda H, Handa N, Kimura K, Kamada T: 1991. Ischemic tolerance phenomenon detected in various brain regions. Brain Res 561:203-211

Kitagawa K, Matsumoto $M$, Tagaya $M$, Hata $R$, Ueda $H$, Niinobe $M$, Handa $N$, Fukunaga R, Kimura K, Mikoshiba K, Kamada T: 1990. 'Ischemic tolerance' phenomenon found in the brain. Brain Res 528:21-24

Knowlton AA, Brecher P, Apstein CS: 1991a. Rapid expression of heat shock protein in the rabbit after brief cardiac ischaemia. J Clin Invest 87:139-147

Knowlton AA, Eberli FR, Brecher P, Romo GM, Owen A, Apstein CS: 1991b. A single myocardial stretch or decreased systolic fiber shortening stimulates the expression of heat shock protein 70 in the isolated, erythrocyte-perfused rabbit heart. J Clin Invest 88:2018-2025

Koretsune Y, Corretti MC, Kusuoka H, Marban E: 1991. Mechanism of early ischemic contractile failure. Inexcitability, metabolite accumulation or vascular collapse? Circ Res 68:255-262

Koyasu S, Nishida E, Miyata Y, Sakai H, Yahara I: 1989. HSP 100, a 100-kDa heat shock protein, is a $\mathrm{Ca}^{2+}$-calmodulin-regulated actin-binding protein. $\mathrm{J}$ Biol Chem 264:15083-15087

Kucukogklu S, Iliodromitis E, Van Winkle D, Downey J, Marber M, Heads R, Yellon 
DM (abstract): 1991. Protection by ischaemic preconditioning appears independent of stress protein synthesis. J Mol Cell Cardiol 23(Suppl V):S73

Kukreja RC, Batra SK, Loesser KE, Hess ML: 1992. Direct expression of heat shock protein by oxygen radicals in the isolated perfused rat heart (abstract). J Mol Cell Cardiol 24(suppl I):S101

Kukreja RC, Hess ML: 1992. The oxygen free radical system: from equations through membrane-protein interactions to cardiovascular injury and protection. Cardiovasc Res 26:641-655

Kuzuya T, Hoshida S, Yamashita N, Fuiji H, Oe H, Hori M, Kamada T, Tada M: 1993. Delayed effects of sublethal ischemia on the acquisition of tolerance to ischemia. Circ Res 72:1293-1299

Ladzunski M, Frelin C, Vigne P: 1985. The sodium/hydrogen exchange system in cardiac cells: Its biochemical and pharmacological properties and its role in regulating internal concentrations of sodium and internal pH. J Mol Cell Cardiol 17:1029-1042

Lai YK, Havre PA, Hammond GL: 1986. Heat shock stress initiates simultaneous transcriptional and translational changes in the dog heart. Biochem Biophys Res Comm 134:166-171

Landry J, Chretien P, Lambert H, Hickey E, Weber LA: 1989. Heat shock resistance conferred by expression of the human HSP27 gene in rodent cells. J Cell Biol 109:7-15

Langendorff O: 1895. Untersushungen am überlebenden Saugethierherzen. Pflugers Arch 61:291-332

Laszlo A: 1992. The thermoresistant state: Protection from initial damage or better repair? Exp Cell Res 202:519-531

Latchman DS: 1991. Heat shock proteins and human disease. J R Coll Physicians Lond 25:295-299

Lee HC, Smith N, Mohabir R, Chusin WT: 1987. Cytosolic calcium transients from the beating mammalian heart. Proc Natl Acad Sci 84:7793-7797

Lee YJ, Armour EP, Corry PM, Dewey WC: 1990. Mechanism of drug-induced heat 
resistance: the role of protein degradation?. Int J Hyperthermia 6:591-595

Leung DM, Spencer SA, Cachianes G, Hammonds RG, Collons C, Henzel WJ, Barnard R, Waters MJ, Wood WI: 1987. Growth hormone receptor binding and serum binding protein: Purification, cloning and expression. Nature 330:537-543

Lewis MJ, Grey AC, Henderson AH: 1979. Determinants of hypoxic contracture in isolated heart muscle preparations. Cardiovasc Res 13:86-94

Li GC, Hahn GM: 1978. Ethanol-induced tolerance to heat and adriamycin. Nature 274:699-701

Li CG, Werb Z: 1982. Correlation between synthesis of heat shock proteins and development of thermotolerance in chinese hamster fibroblasts. Proc Natl Acad Sci (USA) 79:3218-3222

Li GC: 1983. Induction of thermotolerance and enhanced heat shock protein synthesis in Chinese hamster fibroblasts by sodium arsenite and by ethanol. J Cell Physiol $115: 116-122$

Li CG, Laszlo A: 1985. Amino acid analogs whilst inducing heat shock proteins sensitize cells to thermal damage. J Cell Physiol 115:116-122

Li GC: 1989. in Hyperthermic Oncology, 1988, eds Sugahara T and Saito M. Taylor and Francis , London, Vol 2, pp256-259

Li GC, Vasquez BS, Gallagher KP, Lucchesi BR: 1990. Myocardial protection with preconditioning. Circulation 82:609-19

Li GC, Li L, Liu Y, Mak JY, Chen L, Lee WMF: 1991. Thermal response of rat fibroblasts stably transfected with the human $70 \mathrm{kDa}$ heat shock protein-encoding gene. Proc Natl Acad Sci 88:1681-1685

Li GC, Li L, Liu RY, Rehman M, Lee WMF: 1992. Heat shock protein hsp70 protects cells from thermal stress even after deletion of its ATP-binding domain. Proc Natl Acad Sci 89:2036-2040

Lindquist S: 1986. The heat shock response. Ann Rev Biochem 55:1151-1191

Linquist S, Craig EA: 1988. The heat-shock proteins. Annu Rev Genet 22:631-677 
Linquist-McKenzie SL, Henikoff S, Meselson M: 1975. Localization of RNA from heat-induced polysomes at puff sites in Drosophila melanogaster. Proc Natl Acad Sci 72:1117-1121

Linquist-McKenzie SL, Meselson M: 1977. Translation in vitro of Drosophila heat shock messages. J Mol Biol 117:279-283

Lipsich LA, Cutt JR, Brugge JS: 1982. Association of the transforming protein of Rous, Fujinami and Y73 avian sarcoma viruses with the same two cellular proteins. Mol Cell Biol 2:875-880

Liu GS, Thornton J, Van Winkle DM, Stanley AWH, Olson RA, Downey JM: 1991. Protection against infarction afforded by preconditioning is mediated by $\mathrm{A} 1$ adenosine receptors in rabbit heart. Circulation 84:350-356

Liu Y, Kato H, Nakata N, Kogure K: 1992. Protection of rat hippocampus against ischemic neuronal damage by pretreatment with sublethal ischemia. Brain Res 586:121-124

Liu X, Engelman RM, Moraru II, Rousou JA, Flack JE, Deaton DW, Maulik N, Das DK: Heat shock: 1992. A new approach for myocardial preservation in cardiac surgery. Circulation 86(suppl II):II-358-II-363

Locke M, Noble EG, Atkinson BG: 1990. Exercising mammals synthesize stress proteins. Am J Physiol 258:C723-C729

Loesser KE, Vinnikova AK, Qian YZ, Hess ML, Jesse RL, Kukreja RC: 1992. Protection of ischemia/reperfusion injury by heat stress in rats (abstract). Circulation 86(suppl I):I-557

Löw-Friedrich D, Weisensee D, Mitrou P, Schoeppe W: 1992. Cytokines induce stress protein formation in cultured cardiac myocytes. Basic Res Cardiol 87:12-18

Löw I, Friedrich T, Schoeppe W: 1989. Synthesis of shock proteins in cultured fetal mouse myocardial cells. Exp Cell Res 180:451-459

Löw-Friedrich I, Schoeppe W: 1991. Effects of calcium channel blockers on stress protein synthesis in cardiac myocytes. J Cardiovasc Pharmacol 17:800-806

Löw-Friedrich I, von Bredow F, Schoeppe W: 1991. A cell culture system for the 
detection of cardiotoxicity. J Pharmacol Methods 25:133-145

Lowenstein DH, Chan PH, Miles MF: 1991. The stress protein response in cultured neurones: characterization and evidence for a protective role in excitotoxicity. Neuron 7:1053-1060

Luis AM, Alconada A, Cuezva JM: 1990. The alpha regulatory subunit of the Mitochondrial F1-ATPase complex is a Heat-shock protein. J Biol Chem 265:7713-7716

Martin J, Horwich A, Hartl FU: 1992. Prevention of protein denaturation under heat stress by the chaperonin hsp60. Science 258:995-998

Maseri A, Chierchia S, Davies G: 1986. Pathophysiology of coronary occlusion in acute infarction. Circulation 73:233-239

Maxwell M, Hearse DJ, Yellon DM: 1987. Species variation in the coronary collateral circulation during regional myocardial ischaemia: a critical determinant of the rate of evolution and extent of myocardial infarction. Cardiovasc Res 21:737-746

Maxwell MP, Hearse DJ, Yellon DM: 1989. Inability of desferrioxamine to limit tissue injury in the ischaemic and reperfused rabbit heart. J Cardiovasc Pharmacol 13:608-615

Meerson FZ, Malyshev IY: 1989. Adaptation to stress increases the heart resistance to ischemic and reperfusion arrhythmias. J Mol Cell Cardiol 21:299-303

Meerson FZ, Malyshev IY, Shneider AB: 1991b. Phenomenon of the adaptive stabilization of sarcoplasmic and nuclear structures in myocardium. In: Gulch RW, Kissling $G$ eds. Current topics in heart failure. Steinkopff Verlag Darmstadt: Berlin, pp205-214

Meerson FZ, Malyshev IY, Arkhipenko YV, Vovk VI: 1991a. Adaptive increase in the resistance of the heart to calcium paradox (abstract). J Mol Cell Cardiol 23(suppl $\mathrm{V}): \mathrm{S} 162$

Meerson FZ, Malyshev IY, Zamotrinsky AV: 1992. Differences in adaptive stabilization of structures in response to stress and hypoxia relate with the accumulation of hsp70 isoforms. Mol Cell Biochem 111:87-95 
Mehta HB, Popovich BK, Dillman WH: 1988. Ischemia induces changes in the level of mRNAs coding for stress protein 71 and creatine kinase M. Circ Res 63:512-7

Mestril R, Chi S-H, Sayen RM, Dillmann WH: 1992. Expression of a human heat shock protein in rat heart derived $\mathrm{H} 9 \mathrm{c} 2$ cells confers resistance against ischemic injury (abstract). Circulation 86(suppl I):I-557

Mirault ME, Goldschmidt-Clermont M, Moran L, Arrigo AP, Tissières A: 1978. The effect of heat shock on gene expression in Drosophila melanogaster. Cold Spring arbor Symp. Quant Biol 42:819-827

Miron T, Vancompernolle K, Vandekerckhove J, Wilchek M, Geiger B: 1991. A $25-\mathrm{kD}$ inhibitor of actin polymerization is a low molecular mass heat shock protein. J Cell Biol 114:255-261

Miura T, Yellon DM, Hearse DJ, Downey DM: 1987. Determinants of infarct size during permanent occlusion of a coronary artery in the closed chest dog. J Am Coll Cardiol 9:647-654

Miyata Y, Yahara I: 1991. Cytoplasmic 8S glucocorticoid receptor binds to actin filaments through the $90-\mathrm{kDa}$ heat shock protein moiety. J Biol Chem 266:8779-8783

Moalic JM, Christophe B, Himbert D, Bercovici J, Mouas C, Guicheney P, Baudoin-Legros M, Rappaport L, Emanoil-Ravier R, Mezger V, Swynghedauw B: 1989. Phenylephrine, vasopressin and angiotensin II as determinants of proto-oncogene and heat-shock protein gene expression in adult rat heart and aorta. J Hypertension 7:195-201

Mochizuki S, Neely JR: 1980. Energy metabolism during reperfusion following ischemia. J Physiol 76:805-824

Morimoto RI, Milarski KL: 1990. Expression and function of vertebrate hsp70 genes. In: Morimoto RI, Tissières A, Georgopoulos C eds. Stress proteins in biology and medicine. Cold Spring Harbor Laboratory Press: New York pp323-359

Mosser DD, Kotzbauer PT, Sarge KD, Morimoto RI: 1990. In vitro activation of heat shock transcription factor DNA-binding by calcium and biochemical conditions that affect protein conformation. Proc Natl Acad Sci 87:3748-3752

Muller DW, Topol EJ, Califf RM, Sigmon KN, Gorman L, George BS, Kereiakes DJ, 
Lee KL, Ellis SG: 1990. Relationship between antecedent angina pectoris and shortterm prognosis after thrombolytic therapy for acute myocardial infarction. Thrombolysis and angioplasty in myocardial infarction (TAMI) study group. Am Heart J 119:224-231

Murry CE, Jennings RB, Reimer KA: 1986. Preconditioning with ischemia: A delay of lethal cell injury in ischemic myocardium. Circulation 74:1124-1136

Murry CE, Jennings RB, Reimer KA: 1991. New insights into potential mechanisms of ischemic preconditioning. Circulation 84:442-445

Myers ML, Bolli R, Lekich RF, Hartley CJ, Roberts R: 1985. Enhancement of recovery of myocardial function by oxygen free-radical scavengers after reversible regional ischemia. Circulation 72:915-921

Nagai R, Zarain-Herzberg A, Brandl CR, Fujii J, Michihiko T, MacLennan DH, Alpert NR, Periasamy M: 1989. Regulation of myocardial Ca2+-ATPase and phospholamban mRNA expression in response to pressure overload and thyroid hormone. Proc Natl Acad Sci USA 86:2966-2970

Nakamura Y, Wiegner AW, Bing OHL: 1986. Measurement of relaxation in isolated rat ventricular myocardium during hypoxia and re-oxygenation. Cardiovasc Res 20:690-697

Nakanishi H, Makino N, Hata T, Matsui H, Yano K, Yanaga T: 1989. Sarcolemmal $\mathrm{Ca} 2+$ transport activities in cardiac hypertrophy caused by pressure overload. Am J Physiol 257:H349-H356

Nao BS, McClanahan TB, Groh MA, Schott RJ, Gallagher KP: 1990. The time limit of effective ischemic preconditioning in dog (abstract). Circulation 82(suppl III):271

Norton PM, Latchman DS: 1987. Levels of $90 \mathrm{kDa}$ heat shock protein and resistance to glucocorticoid-mediated cell killing on a range of murine lymphocyte cell lines. $\mathrm{J}$ Steroid Biochem 33:149-154

Nowak TS: 1990. Protein synthesis and heat shock/stress response after ischemia. Cerebrovasc Brain Metab Rev 2:345-366

Ohgoshi Y, Goto Y, Futaki S, Yaku H, Kawaguchi O, Suga H: 1991. Increased oxygen cost of contractility in stunned myocardium of dog. Circ Res 69:975-988 
Oksendal AN, Jynge P, Sellevold OF, Rotevatn S, Saetersdal T: 1985. The calcium paradox phenomenon: a flow rate and volume response study of calcium free perfusion. J Mol Cell Cardiol 17:959-972

OPCS, Office of Population Census and Surveys: 1991. Mortality statistics: cause. London: HMSO (Series DH2 no17)

Opie LH: 1989. Reperfusion injury and its pharacological modification. Circulation 80:1049-1062

Opie LH: 1992a. Cardiac metabolism emergence, decline and resurgence. Part I. Cardiovasc Res 26:721-733

Opie LH: 1992b. Cardiac metabolism emergence, decline and resurgence. Part II. Cardiovasc Res 26:817-830

Opperman H, Levinson W, Bishop JM: 1981. A cellular protein that associates with the transforming protein of the Rous sarcoma virus is also a heat shock protein. Proc Natl Acad Sci USA 78:1067-1071

Orchard CH, McCall E, Kirby MS, Boyett MR: 1991. Mechanical alternans during acidosis in ferret heart muscle. Circ Res 68:69-76

Ostermann J, Horowich AL, Newpert W, Hartl F-U: 1989. Protein folding in mitochondria requires complex formation with hsp60 and ATP-hydrolysis. Nature $341: 125-130$

Paradise NF, Schmitter JL, Surmitis JM: 1981. Criteria for adequate oxygenation of isometric kitten papillary muscle. Am J Physiol 241:H348-H353

Park Y, Kanekal S, Kehrer JP: 1991. Oxidative changes in hypoxic rat heart tissue. Am J Physiol 260:H1395-H1405

Parratt J: 1993. Endogenous myocardial protective (antiarrhythmic) substances. Cardiovasc Res 27:693-702

Pasini E, Cargnoni A, Ferrari R, Marber MS, Latchman DS, Yellon DM: 1991. Heat Stress and oxidative damage following ischemia and reperfusion in the isolated rat heart (abstract). Eur Heart J 12(Suppl):306 
Patriarca EJ, Maresca B: 1990. Acquired thermotolerance following heat shock protein synthesis prevents impairment of mitochondrial ATPase activity at elevated temperatures in Saccharomyces cerevisiae. Exp Cell Res 190:57-64

Pauly DF, Kirk KA, McMillin JB: 1991. Carnitine palmitoyltransferase in cardiac ischemia. A potential site for altered fatty acid metabolism. Circ Res 68:1085-1094

Pelham HRB: 1986. Speculation on the functions of the major heat shock and glucose related proteins. Cell 46:959-961

Perisic O, Xiao H, Lis JT: 1989. Stable binding of Drosophila heat shock factor to head-to-tail repeats of a conserved 5bp recognition unit. Cell 59:797-806

Petersen R, Lindquist S: 1988 . The drosophila hsp70 message is rapidly degraded at normal temperatures and stabilized by heat shock. Gene 72:161-168

Picard D, Slater SJ, Yamamoto KR: 1988. A movable and regulatable function within the steroid domain of the glucocorticoid receptor. Cell 54:1073-1080

Pidgeon J, Noble MIM, Seed WA: 1982. Calcium handling by normal and diseased ventricular myocardium (abstract). Br Heart J 47:193P

Piper CW, Curran B, Davies MW, Lockherat A, Feid G: 1986. Transcription of the phoshoglycerate kinase ene of Saccharomyces cerevisiae increases when fermentative cultures are stressed by heat shock. Eur J Biochem 161:525-31

Polla BS, Mili N, Kantengwa S: 1991. Heat shock and oxidative injury in human cells. In: Maresca B, Lindquist S, eds. Heat Shock. Springer-Verlag: Berlin, pp279-290

Poole-Wilson PA: 1992. Ion movements early in myocardial ischaemia: Relation to arrhythmias, early contractile failure and tissue necrosis. In Myocardial Response to Acute Injury. Eds Parratt JR. Macmillan Press Ltd: London pp83-96

Prasad K, Callaghan JC. 1969: Effect of replacement of potassium by rubidium on the transmembrane action potential and contractility of human papillary muscle. Circ Res 24:157-166

Price BD, Calderwood SK: 1991. Ca2+ is essential for multistep activation of the Heat Shock Factor in permeabilized cells. Mol Cell Biol 11:3365-3368 
Quaife RA, Kohmoto O, Barry WH: 1991. Mechanisms of reoxygenation injury in cultured ventricular myocytes. Circulation 83:566-577

Reimer KA, Jennings RB: 1979. The "wavefront phenomenon" of myocardial ischemic cell death. II. Transmural progression of necrosis within the framework of ischemic bed size (myocardium at risk) and collateral flow. Lab Invest 40:633-644

Riabowol KT, Mizzen LA, Welch WJ: 1988. Heat shock is lethal to fibroblasts microinjected with antibodies against hsp70. Science 242:433-436

Ritossa FM: 1962. A new puffing pattern induced by temperature shock and DNP in drosophila. Experientia 18:571-573

Ritossa FM: 1963. New puffs induced by temperature shock, DNP and salicylate in salivary chromosomes of Drosophila melanogaster. Drosophila Inf Service 37:122-123

Ritossa FM: 1964. Specific loci in polytene chromosomes of Drosophila. Exp Cell Res 35:601-607

Robbins J: 1993. Gene targeting. The precise manipulation of the mammalian genome. Circ Res 73:3-9

Rordorf G, Koroshetz WJ, Bonventre JV: 1991. Heat shock protects cultured neurons from glutamate toxicity. Neuron 7:1043-1051

Rose DW, Welch WJ, Kramer G, Hardesty B: 1989. Possible involvement of the $90-\mathrm{kDa}$ heat shock protein in the regulation of protein synthesis. J Biol Chem 264:6239-6244

Rothman JE, Kornberg RD: 1986. An unfolding story of protein translocation. Nature 322:209-210

Rouleau JL, Juneau C, Stephens H, Shenasa H, Parmley WW, Brutsaert DL: 1989. Mechanical properties of papillary muscle in cardiac failure: Importance of pathogenesis and of ventricle of origin. J Mol Cell Cardiol 21:817-828

Ruigrok TJC, Van Echteld CJA: 1991. The intracellular Na+ concentration prior to the $\mathrm{Ca} 2+$ depletion has no bearing on the occurence of the calcium paradox as originally defined. Editorial Comment. J Mol Cell Cardiol 23:769-771 
Salo DC, Donovan CM, Davies KJA: 1991. HSP70 and other possible heat shock or oxidative stress proteins are induced in skeletal muscle, heart, and liver during exercise. Free Rad Biol Med 11:239-246

Sanchez ER, Meshinchi S, Tienrungroj W, Schlesinger MJ, Toff DO, Prot WB: 1987. Relationship of the $90 \mathrm{kDa}$ murine heat shock protein to the untransformed and transformed states of the L cell glucocorticoid receptor. J Biol Chem 262:6986-6991

Sanchez Y, Lindquist SL: 1990. Hsp 104 required for induced thermotolerance. Science 248:1112-1115

Schaper J, Schaper W: 1988. Time course of myocardial necrosis. Cardiovasc Drugs Ther 2:17-25

Scherer PE, Krieg UC, Hwang ST, Vestweber D, Schatz G: 1990. A precursor protein partly translocated into yeast mitochondria is bound to a $70 \mathrm{kd}$ mitochondrial stress protein. EMBO J 9:4315-4322

Schlesinger MJ: 1990. Heat shock proteins. J Biol Chem 265:12111-12114

Schmid SL, Rothman JE: 1985. Enzymic dissociation of clatharin cages is a two step process. J Biol Chem 260:10044-10049

Schott R, Nao B, Strieter R, Groh M, Kunkel S, McClanahan T, Schaper W, Gallagher $\mathrm{K}: 1990$. Heat shock does not precondition canine myocardium (abstract). Circulation 82(suppl III):III-464

Seed WA, Walker JM: 1988. Relation between beat interval and force of the heartbeat and its clinical implications. Cardiovasc Res 22:303-314

Shaknovich R, Shue G, Kohtz DS: 1992. Conformational activation of a basic helix-loop-helix protein (MyoD1) by the c-terminal region of murine HSP90 (HSP84). Mol Cell Biol 12:5059-5068

Sharif M, Worrall JG, Singh B, Gupta RS, Lydyard PM, Lambert C, McCulloch J, Rook G: 1992. The development of monoclonal antibodies to the human mitochondrial $60 \mathrm{kDa}$ heat shock protein and their use in studying the expression of the protein in rheumatoid arthritis. Arthritis Rheum 35:1427-1433

Sherman MR, Stevens J: 1984. Structure of mammalian steroid receptors: Evolving 
concepts and methodological developments. Annu Rev Physiol 46:83

Shiki K, Hearse DJ: 1987. Preconditioning of ischemic myocardium: reperfusioninduced arrhythmias. Am J Physiol 22:H1470-H1476

Shizukida Y, Mallet RT, Lee S, Downey HF: 1992. Hypoxic preconditioning of ischaemic canine myocardium. Cardiovasc Res 26:534-542

Simpson PC: 1989. Proto-oncogenes and cardiac hypertrophy. Annu Rev Physiol 51:189-202

Smith BJ, Yoffe MP: 1991. A mutation of the yeast Heat Shock Factor gene causes temperature sensitive defects in both mitochondrial protein import and the cell cycle. Mol Cell Biol 11:2647-2655

Snoeckx LHEH, Contard F, Samuel JL, Marotte F, Rappaport L: 1991. Expression and cellular distribution of heat-shock and nuclear oncogene proteins in rat hearts. Am J Physiol 261:H1443-H1451

Snoeckx LH, Cornelussen RN, van der Vusse GJ, Reneman RS: 1993. Post-ischemic electrical stability in the heat-shocked hypertrophied and non-hypertrophied rat heart (abstract). J Mol Cell Cardiol;25(SupplI):S56

Sorger PK, Pelham HRB: 1988. Yeast heat shock factor is an essential DNA-binding protein that exhibits temperature-dependent phosphorylation. Cell 54:841-853

Spradling AML, Pardue ML, Penman S: 1977. Messenger RNA in heat shocked Drosophila cells. J Mol Biol 109:559-587

Steare SE, Yellon DM: 1993. The protective effect of heat stress against reperfusion arrhythmias in the rat. $\mathrm{J}$ Mol Cell Cardiol in press

Steenberg C, Hill ML, Jennings RB: 1987. Cytoskeletal damage during myocardial ischemia: Changes in Vinculin immunofluorescence staining during total in vitro ischemia in canine heart. Circ Res 60:478-486

Stevenson MA, Calderwood SK: 1990 . Members of the $70 \mathrm{kDa}$ heat shock family contain a highly conserved calmodulin-binding domain. Mol Cell Biol 10:1234-1238

Stone D, Darley-Usmar V, Smith DR, O'Leary V: 1989. Hypoxia-reoxygenation 
induced increase in cellular calcium in myocytes and reperfused hearts: The role of mitochondria. J Mol Cell Cardiol 21:963-973

Stratford-Perricaudet LD, Makeh I, Perricaudet M, Briand P: 1992. Widespread longterm gene transfer to mouse skeletal muscles and heart. J Clin Invest 90:626-630 Su C-Y, Dillmann WH, Woods WT, Owen OE: 1992. Heat shock induced oxidative tolerance in muscle cells (abstract). Circulation 84(suppl I):I-33

Subjeck JR, Shyy T-T, Shen JW, Johnson RJ: 1983. Association between the mammalian 110,000 dalton heat shock protein and nucleoli. J Cell Biol 97:1389-1295

Szekeres L, Papp JG, Szilvassy Z, Udvary E, Vegh A: 1993. Moderate stress by cardiac pacing may induce both short-term and long-term cardioprotection. Cardiovasc Res 27:593-596

Tanaka M, Fujiwara H, Yamasaki K: 1992. Is the time course of infarct size limiting effect of ischemic preconditioning bimodal? (abstract). Circulation 86(suppl I):I-343

Tani M, Neely JR: 1990 . Intermittent perfusion of ischemic myocardium. Possible mechanisms of protective effects on mechanical function in isolated rat heart. Circulation 82:536-548

ter-Keurs HED, Gao WD, Bosker H, Drake-Holland AJ, Noble MIM: 1990. Characterisation of decay of frequency induced potentiation and post-extra-systolic potentiation. Cardiovasc Res 204:903-910

Thornton JD, Striplin S, Liu GS, Swafford A, Stanley WH, Van Winkle DM, Downey JM: 1990. Inhibition of protein synthesis does not block myocardial protection afforded by preconditioning. Am J Physiol 259:H1822-H1825

Timerman AP, Altschuld RA, Hohl CM, Brierley GP, Merola AJ: 1990. Cellular glutathione and the response of adult rat heart myocytes to oxidant stress. J Mol Cell Cardiol 22:565-75

Tissières A, Mitchell HK, Tracy VM: 1974. Protein synthesis in salivary glands of Drosophila melanogaster. Relation to chromosome puffs. J Mol Biol 84:389-398

Tuijl MJ, van Bergen en Henegouwen PM, van Wijk R, Verkleij AJ: 1991. The isolated neonatal rat-cardiomyocyte used as an in vitro model for "ischemia".II. Induction of the $68 \mathrm{kDa}$ heat shock protein. Biochim Biophys Acta 1091:278-284 
Tunstall J, Busselen P, Rodrigo GC, Chapman RA: 1986. Pathways for the movements of ions during calcium-free perfusion and the induction of the 'calcium paradox'. $J$ Mol Cell Cardiol. 18:241-54

Ungewickell E, Branton D: 1981. Assembly units of clathrin coats. Nature 289:420422

van Bilsen M, van der Vusse GJ, Snoeckx LHEH, Arts T, Coumans WA, Willemsen PHM, Reneman RS: 1988. Effects of pyruvate on post-ischemic myocardial recovery at various workloads. Pflugers Arch 413:167-173

Van der Heide RS, Sobotka PA, Ganote CE: 1987. Effects of the free radical scavenger DMTU and mannitol on the oxygen paradox in perfused rat hearts. 19:615625

Van Winkle DM, Thorntom J, Downey JM: 1991. Cardioprotection from ischaemic preconditioning is lost following prolonged reperfusion in the rabbit. Cor Art Disese 2:613-619

Varma SD, Morris SM: 1988. Peroxide damage to the eye lens in vitro prevention by pyruvate. Free Rad Res Commun 4:283-290

Vogel WM, Briggs LL, Apstein CS: 1985. Separation of inherent diastolic myocardial fiber tension and coronary erectile contributions to wall stiffness of rabbit hearts damaged by ischemia, calcium paradox and reperfusion. J Mol Cell Cardiol 17:57-70

von Harsdorf R, Schott RJ, Shen Y-T, Vatner SF, Mahdavi V, Nadal-Ginard B: 1993. Gene injection into canine myocardium as a useful model for studying gene expression in the heart of large mammals. Circ Res 72:688-695

Walker DM, Kucukoglu S, Marber MS, Walker JM, Yellon DM: 1992. Heat stress limits infarct size in the isolated rabbit heart. Circulation 86(suppl I):I-557.

Walker DM, Yellon DM: 1992. Ischaemic preconditioning-from mechanisms to exploitation. Cardiovasc Res 26:734-739

Walker DM, Pasini E, Kucukoglu S, Marber MS, Iliodromitis E, Ferrari R, Yellon DM: 1993. Heat stress limits infarct size in the isolated perfused rabbit heart. Cardiovasc Res 27:962-967 
Walker JM: 1983. The contractility of human and guinea-pig ventricular myocardium in vitro. $\mathrm{MD}$ thesis, University of Birmingham

Wall SR, Fliss H, Kako KJ, Korecky B: 1990. Heat pretreatment does not improve recovery of function after no-flow ischemia in isolated working rat hearts (abstract). J Mol Cell Cardiol (suppl 1):S44

Wallenstein S, Zucker CL, Fleiss LJ: 1980. Some statistical methods useful in circulation research. Circ Res 47:1-9

Weich H, Buchner J, Zimmermann R, Jakob U: 1992. Hsp90 chaperones protein folding in vitro. Nature 358:169-170

Weissler AM, Kruger FA, Baba N, Scharpell DG, Leighton RF, Gallimore JK: 1968. Role of anaerobic metabolism in the preservation of functional capacity and structure of anoxic myocardium. J Clin Invest 47:403-416

Welch WJ: 1990. The mammalian stress response: cell physiology and biochemistry of stress proteins. In Stress Proteins in Biology and Medicine. Eds Morimoto R, Tissieres A, Georgopoulos C. Cold Spring Laboratory Press: New York. pp223-279

Welch WJ: 1993. How cells respond to stress. Sci Am 268:34-41

White FP, White SR: 1986. Isoproterenol induced myocardial necrosis is associated with stress protein synthesis in rat heart and thoracic aorta. Cardiovasc Res 20:512-515

Wier WG, Yue DT: 1986. Intracellular calcium transients underlying the short-term force-interval relationship in ferret ventricular myocardium. J Physiol 376:507-530

Wikman-Coffelt J, Stefenelli T, Wu ST, Parmley WW, Jasmin G: 1991. [Ca2+]i Transients in the cardiomyopathic hamster heart. Circ Res 68:45-51

Xiao H, Perisic O, Lis JT: 1991. Cooperative binding of Drosophila heat shock factor to arrays of a conserved 5bp unit. Cell 64:585-593

Yamashita N, Kuzuya T, Hoshido S, Fuji H, Oe H, Kitabatake A, Tada M, Kamada $\mathrm{T}$ : 1992. Relationship between time interval from preconditioning to sustained ischemia and its effects on limiting infarct size (abstract). J Mol Cell Cardiol 24(suppl I):S150 
Yamashita T, Abe S, Arima S, Nomoto K,Miyata M, Maruyama I, Toda H, Okino H, Atsuchi Y, Tahara M, Nakoa S, Tanaka H: 1993. Myocardial infarct size can be estimated from serial plasma myoglobin measurements within 4 hours of reperfusion. Circulation 87:1840-1849

Yellon DM, Downey JM: 1990. Current research views on myocardial reperfusion and reperfusion injury. Cardioscience 1:89-98

Yellon DM, Pasini E, Cargnoni A, Marber MS, Latchman DS, Ferrari R: 1992a. The protective role of heat stress in the ischaemic and reperfused rabbit myocardium. $J$ Mol Cell Cardiol 24:895-907

Yellon DM, Latchman DS: 1992. Stress proteins and myocardial protection J Mol Cell Cardiol 24:113-124

Yellon DM, Iliodromitis E, Latchman DS, Van Winkle DM, Downey JM, Williams FM, Williams TJ: 1992b. Whole body heat shock fails to limit infarct size in the reperfused rabbit heart. Cardiovasc Res 26:342-346

Yellon DM, Latchman DS, Marber MS: 1993. Stress protein- an endogenous route to myocardial protection: fact or fiction? Cardiovasc Res 27:158-161

Yost HJ, Petersen RB, Lindquist S: 1990. Posttranscriptional regulation of heat shock protein synthesis in Drosophila. In Stress Proteins in Biology and Medicine. Eds Morimoto RI, Tissieres A, Georgopoulos C. Cold Spring Harbour Laborotory Press: New York, pp379-409

Ziemiecki AM, Catelli MG, Joab I, Moncharmont B: 1986. Association of the heat shock protein hsp90 with steroid hormone receptors and tyrosine kinase oncogene products. Biochem Biophys Res Commun 138:1298-1307

Zimmerman ANE, Hülsmann WC: 1966. Paradoxical influence of calcium ions on the permeability of the cell membranes of the isolated rat heart. Nature 211:646-647. 


\section{Declaration}

I was the sole investigator of the work described in this thesis. Other collaborative studies involving the techniques and subject matter included in this thesis are listed in the publications following this declaration. The only material included within the thesis is from those publications where I am first author with the co-authors being my supervisors. I recieved some help with the software used for data analysis from Dr J Downey and Mr R Bell.

Some of the results in this thesis have already been presented elsewhere; a list of publications with results in common with this thesis together with copies of relevant manuscripts can be found below and on the following pages.

\section{Publications With Results in Common With This Thesis}

Papers:

Yellon DM, Pasini E, Cargoni A, Marber MS, Latchman DS, Ferrari R. The protective role of Heat Stress in the ischaemic and reperfused rabbit myocardium. J Mol Cell Cardiol 1992;24:895-909.

Yellon DM, Latchman DS. Marber MS. Stress proteins: an endogenous route to myocardial protection? Cardiovasc Res 1993;27:158-161.

Marber MS, Walker JM, Latchman DS, Yellon DM. Myocardial protection following whole body heat stress in the rabbit is dependant on metabolic substrate and is related to the amount of the inducible 70 kiloDalton heat stress protein. J Clin Invest Accepted subject to re-review.

Marber MS, Walker JM, Yellon DM. Heat stress attenuates a sub-maximal calcium paradox. J Mol Cell Cardiol in press 1993.

Marber MS, Walker DM, Eveson DJ, Walker JM, Yellon DM. Rapid atrial pacing does not cause ischaemic preconditioning of the In-situ rabbit heart. Cardiovasc Res 1993;27:597-601

Marber MS, Latchman DS, Walker JM, Yellon DM. Cardiac stress protein elevation following sublethal ischemia or heat stress is associated with resistance to myocardial infarction. Circulation in press 1993.

Walker DM, Pasini E, Kucukoglu S, Marber MS, Iliodromitis E, Ferrari R, Yellon DM: Heat stress limits infarct size in the isolated perfused rabbit heart. Cardiovasc Res 1993;27:962-967

Marber MS, Heads RJ, Yellon DM. Stress proteins, heat stress and myocardial protection. In Kloner RA, Pryzklenk K, Yellon DM, eds. Ischemic Preconditioning. Kluwer Academic Publishers. Due for publication mid 1993

Marber MS. Stress proteins and myocardial protection. Invited editorial for Clinical 
Science. To appear Clinical Science 1993

Marber MS, Yellon DM. The stress response, stress proteins and myocardial adaptation. In Nagano M, Takeda N, Dhalla NS, eds. The Adapted Heart. New York: Raven Press due for publication late 1993

\section{Abstracts:}

Marber MS. Heat stress and myocardial function. Invited lecture to XIIth European Section of the International Society for Heart Research. Leuven, Belgium. J Mol Cell Cardiol 1991;23(suppl V):S35.

Marber MS. Stress proteins and myocardial protection. Finalist for the Glaxo Young Investigator Medal of the Medical Research Society (Association of Physicians). London, England. Clinical Science 1993; In press.

Yellon DM, Pasini E, Ferrari R, Marber MS, Walker JM, Latchman DS. Heat Stress protects isolated perfused rabbit hearts. Br Heart J 1991;66:97.

Pasini E, Cargoni A, Ferrari R, Marber MS, Latchman DS, Yellon DM. Heat Stress and oxidative damage following ischaemia and reperfusion in the isolated rat heart. Eur Heart J 1991; 12S:P1568.

Iliodromitis E, Kucukoglu S, Marber MS, Walker JM, Latchman DS, Yellon DM. Ischaemic preconditioning protects rabbit heart independant of stress protein synthesis. Eur Heart J 1991; 12S:P720.

Kucukoglu S, Iliodromitis E, van Winkle D, Downey JM, Marber MS, RJ Heads, Yellon DM. Protection by ischaemic preconditioning appears independent of stress protein synthesis. J Mol Cell Cardiol 1991;23(suppl V):P51.

Marber MS, Walker JM, Latchman DS, Yellon DM. Heat stress increases the resistance of rabbit papillary muscle to hypoxic and zero calcium perfusion. J Mol Cell Cardiol 1991;23(suppl V):P41.

Marber MS, Heat Stress and myocardial function. Invited lecture to XIIth European Section of the International Society for Heart Research. J Mol Cell Cardiol 1991;23(suppl V):S35.

Marber MS, Walker JM, Latchman DS, Yellon DM. The resistance of rabbit papillary muscle to high flow anoxia is enhanced by heat stress when pyruvate is used as substrate. Circulation 1991;84(suppl II):621.

Marber MS, Walker DM, Yellon DM, Walker JM. Rapid atrial pacing fails to precondition the rabbit heart. J Mol Cell Cardiol 1992;24(suppl I):S92.

Marber MS, Walker JM, Yellon DM. The resistance of rabbit papillary muscle to high flow hypoxia is enhanced by heat stress when pyruvate is used as substrate. $J$ 
Mol Cell Cardiol 1992:24(suppl I):S120.

Marber MS, Walker JM, Yellon DM. Heat Stress attenuates a submaximal calcium paradox in the rabbit. J Mol Cell Cardiol 1992;24(suppl I):S138.

Walker DM, Kucukoglu S, Pasini E, Marber MS, Yellon DM. The effect of heat stress on infarct size in blood vs buffer perfused rabbit hearts. J Mol Cell Cardiol 1992;24(suppl I):S118.

Pantos C, Marber MS, Porter J, Yellon DM, Walker JM. Human atrial trabeculae superfused with human serum are resistant to the acute effects of exposure to $\mathrm{Fe} 3+$. J Mol Cell Cardiol 1992;24: (suppl I) S127.

Marber MS, Walker JM, Yellon DM. Myocardial resistance to calcium overload is enhanced by prior heat stress. Br Heart J 1992;68:72.

Marber MS, Walker JM, Yellon DM. Enhanced myocardial resistance to hypoxia after heat stress is dependant on metabolic substrate. Br Heart J 1992;68;71.

Walker DM, Kucukoglu S, Pasini E, Marber MS, Yellon DM. Preconditioning with heat stress protects the ischaemic myocardium. Br Heart J 1992;68:72.

Marber MS, Walker JM, Yellon DM. Heat stress attenuates the calcium paradox. Eur Heart J 1992;13S:P764. Spain)

Walker DM, Kucukoglu S, Pasini E, Marber MS, Yellon DM. Heat stress protects the ischaemic myocardium. Eur Heart J 1992;13S:2496.

Walker DM, Kucukoglu S, Marber MS, Walker JM, Yellon DM. Heat stress limits infarct size in the isolated rabbit heart. Circulation 1992;86(suppl I):I-557.

Marber MS, Walker JM, Latchman DS, Yellon DM. Myocardial resistance to hypoxia is related to the content of the $72 \mathrm{kDa}$ stress protein. Circulation 1992;86(suppl I):I556.

Marber MS, Walker JM, Yellon DM. Heat stress can protect the rabbit heart by antioxidant-independent mechanisms. Circulation 1992;86(suppl I):I-556.

Marber MS, Walker JM, Latchman DS, Yellon DM. Cardiac 72 and 60-kDa stress protein induction by repetitive ischaemia and heat stress. J Mol Cell Cardiol 1993;25(suppl I):S55.

Marber MS, Walker JM, Latchman DS, Yellon DM. Heat stress enhances recovery following hypoxia and reoxygenation. J Mol Cell Cardiol 1993;25(suppl I):S55.

Marber MS, Walker JM, Latchman DS, Yellon DM. Cardiac stress protein content and recovery following hypoxia/reoxia. J Mol Cell Cardiol 1993;25(suppl I):S56.

Marber MS, Walker JM, Latchman DS, Yellon DM. Repetitive ischaemia and heat 
stress result in delayed myocardial protection. J Mol Cell Cardiol 1993;25(suppl I):S55.

Walker DM, Walker JM, Marber MS, Yellon DM. Attenuation of hypoxic injury to isolated papillary muscles by preconditioning. J Mol Cell Cardiol 1993;25(suppl I):S34.

Marber MS, Walker JM, Latchman DS, Yellon DM. Myocardial 72- and 60kiloDalton stress protein content and resistance to hypoxia/reoxygenation. Br Heart J 1993;69:P74.

Walker DM, Marber MS, Walker JM, Yellon DM. Preconditioning protects isolated rabbit papillary muscles against hypoxic injury. Br Heart J 1993;69:P12

Marber MS, Walker JM, Latchman DS, Yellon DM. Myocardial stress proteins and resistance to stress. Accepted XVth Congress of the European Society of Cardiology, Nice, France. Eur Heart J 1993. In press

Marber MS, Walker JM, Latchman DS, Yellon DM.Ischaemia and heat stress increase the resistance of the in-situ rabbit heart to subsequent infarction. Accepted $\mathrm{XV}$ th Congress of the European Society of Cardiology, Nice, France. Eur Heart $\mathrm{J}$ 1993. In press

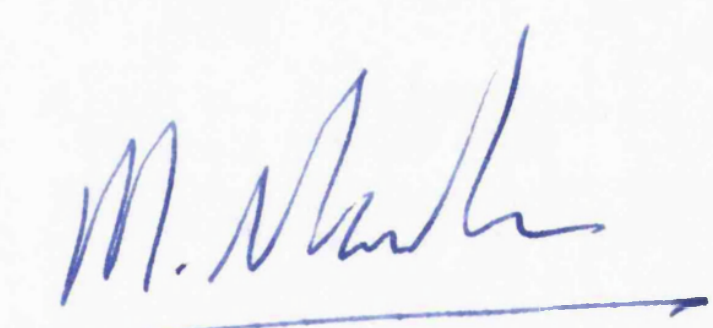

Michael Marber

$30 / 07 / 93$ 


\title{
Cardiac Stress Protein Elevation 24 Hours After Brief Ischemia or Heat Stress Is Associated With Resistance to Myocardial Infarction
}

\author{
Michael S. Marber, MRCP; David S. Latchman, PhD; \\ J. Malcolm Walker, MD, FRCP; Derek M. Yellon, PhD, FACC
}

\begin{abstract}
Background. To test the hypothesis that the heat shock response is associated with myocardial salvage, the heat-stress protein (HSP) content of cardiac tissue was increased by either ischemic or thermal stress. Methods and Results. Rabbits were divided into four groups. Ischemic pretreatment $(n=15)$ comprised four 5-minute episodes of coronary ligation separated by 10 minutes of reperfusion. The corresponding control group $(n=21)$ underwent surgical preparation without coronary ligation. Thermal pretreatment $(n=16)$ involved whole-body temperature elevation to $42^{\circ} \mathrm{C}$ for 15 minutes; corresponding controls $(n=15)$ were treated with anesthetic alone. Twenty-four hours later, hearts were removed for HSP estimation or infarct size assessment after a 30 -minute coronary ligation. Myocardial HSP72 content assessed by Western blotting was elevated by both ischemic and thermal pretreatments $(2.5 \pm 0.2$ units, $n=4$, and $2.8 \pm 0.3$ units, $n=4$, mean $\pm S E M ; P=N S$, respectively) compared with the corresponding control groups $(1.0 \pm 0.3, \mathrm{n}=4, P \leq .01$ and $0.3 \pm 0.1, \mathrm{n}=4, P \leq .01$, respectively). HSP60 was preferentially elevated by ischemic pretreatment. After a $\mathbf{3 0}$-minute coronary occlusion and $\mathbf{1 2 0}$ minutes of reperfusion, ischemic and thermal pretreatments limited infarct size as a percentage of the volume at risk by $28.8 \pm 5.2 \%$ vs $52.0 \pm 5.2 \%, P \leq .01$ and $32.8 \pm 3.8 \%$ vs $56.9 \pm 6.5 \%, P \leq .01$, respectively.

Conclusions. Myocardial stress protein induced by either sublethal thermal or ischemic injury is associated with myocardial salvage. Our findings suggest that stress protein elevation, rather than the nonspecific effects of thermal or ischemic stress, may be responsible for the myocardial protection seen in this model. Our observations may have important implications regarding myocardial adaptation to brief

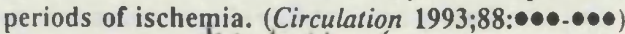

KEY Words heat proteins ick ischemia - myocardium - reperfusion h

$\mathrm{W}$ Then any living cell is exposed to a sublethal elevation of environmental temperature, a series of adaptive changes occur that serve to protect that cell from subsequent increases in temperature. ${ }^{1}$ A group of proteins known as the heat-shock or heat-stress proteins (HSPs) are the major proteins synthesized during such stress and play a pivotal role in providing this protection. ${ }^{2.3} \mathrm{~A}$ similar increase in stress protein synthesis is seen after a variety of nonthermal stresses; for example, in myocardial tissue, stress protein synthesis is increased by ischemic and mechanical injury.4-6 It seems likely, therefore, that stress proteins are involved in recovery from nonthermal as well as thermal injury. This conclusion is supported by the fact that stress proteins raised by one form of sublethal injury seem capable of protecting against a subsequent but different injury, a phenomenon known as cross-

Received October 8, 1992; revision accepted April 30, 1993 From the Hatter Institute For Cardiovascular Studies (M.S.M. J.M.W., D.M.Y.), Division of Cardiology, University College London Medical School, and the Division of Molecular Pathology D.S.L.), University College London Medical School.

Correspondence to Derek Yellon, Director, and Head of of Cardiology, Universty titute for Cardiovascular Studies, Division of Cardiology, University College Hospital, Gower Street, London
WCIE 6AU, England. tolerance. ${ }^{7}$ In keeping with this phenomenon, a number of investigators have demonstrated that whole-body hyperthermia increases myocardial stress protein con. tent and renders the isolated blood-or buffer-perfused heart resistant to subsequent ischemia and reperfusion. ${ }^{8}{ }^{-10}$ However, our attempts to use whole-body heat stress to limit infarction after a 45 -minute coronary ligation were successful in the isolated blood-perfused heart ${ }^{11}$ but unsuccessful in vivo, ${ }^{12}$ leading to the speculation that whole-body heat stress may have deleterious aspects that negate any beneficial effects secondary to myocardial stress protein elevation." In contrast, in the in situ rat heart, Donnelly and coworkers ${ }^{13}$ have demonstrated that whole-body heat stress can reduce infarct size after a 35-minute but not a 45-minute coronary occlusion.

The purpose of this study was to examine in more detail the relation between myocardial stress protein content and the subsequent resistance of the in situ rabbit heart to ischemia. An established protocol ${ }^{4}$ of repetitive short coronary artery occlusions was used to selectively elevate myocardial stress protein content, thereby avoiding whole-body heat stress and its possible deleterious consequences. More importantly, brief coronary occlusions, unlike thermal stress, are a physiologically more relevant stimulus for stress protein induc- 


\begin{tabular}{|c|c|c|c|}
\hline \multicolumn{2}{|l|}{ Group1 ischemia } & \multirow{2}{*}{ Hours roming } & \multirow[b]{2}{*}{120 minutes } \\
\hline 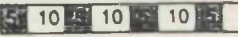 & 24 & & \\
\hline \multicolumn{2}{|c|}{ Group2 Sham Ischemia } & & \\
\hline 50 mins chest open & 24 & Hours 30 minit & 120 minutes \\
\hline
\end{tabular}

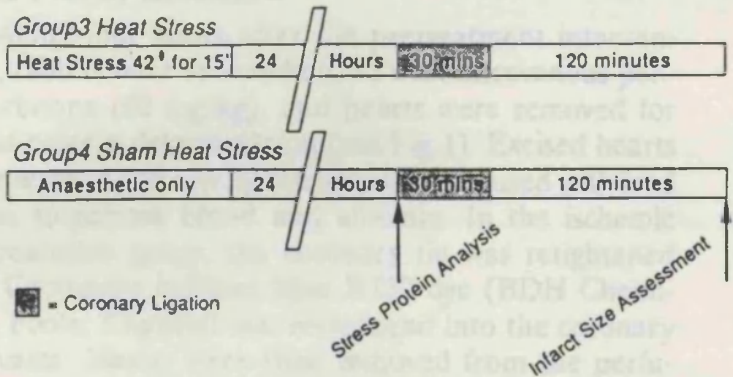

FIG 1. Summary of experimental protocols. Group 1, ischemic pretreatment, was subjected to four 5-minute coronary ligations separated by 10-minute periods of reperfusion. The corresponding sham ischemia group (group 2) underwent identical surgical preparation but without coronary ligation. In the heat-stress group (group 3), after anesthesia, rectal temperature was raised $1042^{\circ} \mathrm{C}$ for 15 minutes by wrapping rabbits in an electric warming blanket. Rabbits then recovered at room temperature. The corresponding sham-heal-stress group (group 4) received anesthesic agent without warming. All groups were allowed to recover for 24 hours before either myocardial stress protein estimation ( $n=4$ for each group) or a 30-minute coronary ligation followed by reperfusion and infarct size assessment ( $n=10$ for each group).

tion, having been likened to the clinical situation during unstable angina. ${ }^{4}$

\section{Methods}

\section{Experimental Protocols and Exclusions}

For the purposes of this study, 67 New Zealand White rabbits $(2.0$ to $3.0 \mathrm{~kg}$ ) were divided into four experimental groups. Within each group, the end point was either stress protein estimation or infarct size assessment (see protocol, Fig 1). Experiments were performed sequentially between the groups, except when an experimental exclusion necessitated repetition. Hemodynamic, temperature, and blood gas data from experiments that were excluded were not used for subsequent analysis.

Group 1, ischemic pretreatment, consisted of 15 rabbits. There was 1 exclusion because of respiratory obstruction and death 2 hours after repetitive ischemia. Four rabbits were used for stress protein estimation and 10 rabbits for infarct size assessment.

Group 2, sham ischemic pretreatment, consisted of 21 rabbits with 7 exclusions. In 3 rabbits, coronary ligation was unsuccessful (no risk area evident after microsphere injection), 2 rabbits failed to reperfuse adequately (as judged by a large area of infarction without intramyocardial hemorrhage), and 2 animals died during the 30 -minute coronary ligation secondary to hypotension and progressive acidosis. Four rabbits were used for stress protein estimation and 10 for infarct size assessment.
Group 3, heat stress, consisted of 16 rabbits, with 2 exclusions. One rabbit died during the 30 -minute coronary ligation, and 1 experiment had to be excluded because of inadequate staining of viable myocardium with triphenyltetrazolium. Ten rabbits were used for infarct size assessment and 4 rabbits for stress protein estimation.

Group 4, sham heat stress, consisted of 15 rabbits. There was 1 exclusion because of inadequate tissue staining. Ten rabbits were used for infarct size assessment and 4 rabbits for stress protein estimation.

\section{Ischemic and Sham Ischemic Pretreatments}

Rabbits were anesthetized with intramuscular fentanyl $100 \mu \mathrm{g} / \mathrm{kg}$ and fluanisone $3 \mathrm{mg} / \mathrm{kg}$ (Janssen Pharmaceuticals, Oxford, UK), followed by intraperitoneal diazepam $2 \mathrm{mg} / \mathrm{kg}$. Anesthesia was maintained by fentanyl and fluanisone administered every 30 minutes. Once the rabbits were adequately anesthetized, they were orally intubated, and limb lead ECG electrodes (Medicotest, Rugmarken, Denmark) were attached. The rabbits were mechanically ventilated with $100 \%$ oxygen at a tidal volume of $5 \mathrm{~mL} / \mathrm{kg}$ delivered at a rate of $1 \mathrm{~Hz}$. A marginal ear vein was cannulated to administer fluids and drugs, and $30 \mathrm{mg}$ of intramuscular amoxycillin was given prophylactically.

The heart was exposed through a median sternotomy, and a coronary artery (usually an anterolateral branch of the circumflex) was underrun with $3 / 0$ silk suture on a tapered needle. The free ends of the suture were passed through a soft vinyl tube so as to form a snare to occlude the artery. Rabbits then received 1000 units of heparin before the first 5-minute coronary artery ligation. Successful ligation was confirmed by myocardial cyanosis with bulging and changes in the amplified ECG signal (ECG amplifier and series 3000 recorder, Gould Inc, Ohio). Reperfusion was confirmed by a myocardial blush and resolution of ECG changes. After four 5 -minute coronary ligations separated by 10 minutes of reperfusion, the vinyl occluder was removed, and the coronary suture was left in situ while the sternotomy was closed by suturing muscle and then skin layers. Animals were given $10 \mathrm{~mL} / \mathrm{kg}$ of $0.9 \%$ saline intravenously, allowed to breathe spontaneously, and eventually extubated.

The surgical preparation for the sham ischemic pretreatment group was identical to that described above. However, although the pericardium was opened, a coronary artery was neither underrun nor occluded, thus avoiding mechanical manipulation of the myocardium, which may in itself act as a trigger for stress protein induction. ${ }^{6}$

\section{Heat Stress and Sham Heat Stress Pretreatments}

Rabbits were anesthetized with pentobarbitone 40 $\mathrm{mg} / \mathrm{kg}$ delivered via a marginal ear vein. In the heatstressed group, rectal temperature was raised to at least $42^{\circ} \mathrm{C}$ for 15 minutes by wrapping the anesthetized rabbit in an electric warming blanket. Animals were then allowed to recover at room temperature.

Sham-heat-stress rabbits were identically anesthetized and wrapped for similar periods with the blanket not turned on. 


\section{Stress Protein Estimation}

Twenty-four hours after the pretreatment intervention, rabbits were reanesthetized with intravenous pentobarbitone $(60 \mathrm{mg} / \mathrm{kg}$ ), and hearts were removed for stress protein determination (see Fig 1). Excised hearts were washed and briefly retrogradely perfused with iced saline to remove blood and albumin. In the ischemic pretreatment group, the coronary tie was retightened and Coomassie brilliant blue R250 dye (BDH Chemicals, Poole, England) was introduced into the coronary perfusate. Hearts were then removed from the perfusion rig, and atria, fat, and right ventricular free wall were removed. In the ischemic pretreatment group, the area without dye (risk zone) was separated from stained (perfused) myocardium. Left ventricular specimens were then rapidly frozen in liquid nitrogen.

At a later date, myocardial specimens were crushed and homogenized in $2 \times$ concentrated sodium dodecyl sulfate-polyacrylamide gel electrophoresis (SDSPAGE) sample buffer (20\% glycerol and 6\% SDS in $0.12 \mathrm{~mol} / \mathrm{L}$ Tris at $\mathrm{pH} 6.8$ ), and protein concentrations were estimated by use of the Pierce BCA reagent (Pierce, Rockford, III) and equalized by further addition of sample buffer where necessary; 2-mercaptoethanol to a final concentration of $10 \%$ was added before boiling. Samples were then centrifuged and stored at $-70^{\circ} \mathrm{C}$. Subsequently, samples were thawed, recentrifuged, and further diluted in sample buffer to allow loading of approximately $30 \mu \mathrm{g}$ of total protein per lane of slab gel.

Proteins were separated by SDS-PAGE on $0.8 \mathrm{-mm}$ thick, $12.5 \%$ acrylamide gels according to Laemmli. ${ }^{14}$ Equivalence of loading and adequacy of sample preparation were determined by visualization of proteins with Coomassie blue stain. When loading conditions were optimal, three identical gels were prepared, each loaded with four heart samples derived from each of the four groups. The proteins on two gels were transferred onto a nitrocellulose membrane (Hybond C, Amersham, Bucks, UK) by Western blotting. The other identical gel was stained with Coomassie brilliant blue to visualize protein for subsequent densitometry.

One nitrocellulose membrane was washed in phosphate-buffered saline with $0.1 \%$ dried skimmed milk powder to block nonspecific binding sites. After washing, the membrane was incubated at $37^{\circ} \mathrm{C}$ for 1 hour with mouse monoclonal IgG cross-reactive to the inducible $72-k D a$ heat shock protein (Stressgen, Sidney, Canada) at 1:1000 dilution. After repeated washing, the membrane was incubated with horseradish peroxidase-conjugated rabbit anti-mouse IgG (DAKO, Denmark) at 1:1000 dilution at room temperature for 1 hour. The filter was then washed and developed by use of enhanced chemiluminescence detection (Amersham, Bucks, UK).

The other identical nitrocellulose membrane was blocked with $3 \%$ skimmed milk powder and after washing at room temperature for 3 hours was exposed to a mouse monoclonal IgG raised against human heat shock protein 60 at 1:2000 dilution. ${ }^{15}$ Subsequent methods were as described above with the second antibody at 1:2000 dilution. The monoclonal used recognizes only the mitochondrial form of the $60-\mathrm{kD}$ a stress protein and does not cross-react with the cytosolic homologue (TCP1). 15

The relative levels of heat shock proteins 72 and 60 were determined using densitometry (model 620 video densitometer with Biorad analyst 2 version 3.1 software, Biorad, Hemel Hempstead, UK), normalizing to the actin band on the Coomassie-stained gel. This procedure adjusts for slight variations in protein loading between samples. ${ }^{16}$

\section{Myocardial Infarction and Infarct Size Assessment}

Approximately 24 hours after the above pretreatments, animals were reanesthetized with pentobarbitone $(40 \mathrm{mg} / \mathrm{kg})$ via the marginal ear vein. ECG electrodes were attached, and the trachea was opened and intubated via a midline cervical incision. Mechanical ventilation was as previously described; however, the tidal volume was reduced to $4 \mathrm{~mL} / \mathrm{kg}$ in keeping with the reduction in ventilatory dead space. The right common carotid artery was cannulated with a short rigid polythene cannula attached to a pressure transducer (P23XL, Gould), for continuous recording of arterial pressure and intermittent arterial blood gas estimations (ABL2, Radiometer, Copenhagen, Denmark). Throughout the procedure, rectal temperature was monitored and maintained between $38.5^{\circ} \mathrm{C}$ and $39.0^{\circ} \mathrm{C}$ by an electric warming pad. The chest was opened (or reopened) via a midline sternotomy, and a coronary artery was identified. Rabbits were given 1000 units of heparin intravenously, and the coronary artery was ligated as described for the ischemic pretreatment group. In the case of the ischemic pretreatment group, the existing coronary tie was reused to ensure that the same coronary bed was rendered ischemic as on the previous day. In the sham ischemia group, identification of a suitable coronary vessel was often difficult, since the surface of the heart was encased in a thin fibrinous exudate.

After a 30-minute coronary occlusion, reperfusion was confirmed by the appearance of a myocardial blush. After 120 minutes of reperfusion, a further 1000 units of heparin was given, and the heart was removed and retrogradely perfused with $0.9 \%$ saline. After blood had been washed out of the coronary vasculature, the coronary snare was re-tightened and fluorescent microspheres infused via the aortic cannula. Demarcation of the myocardial surface at risk (area without spheres) was confirmed under UV light. The heart was then frozen at $-18^{\circ} \mathrm{C}$ overnight. The next day, while still frozen, the heart was sliced at $2-\mathrm{mm}$ intervals at right angles to its long axis. The slices were then incubated at $37^{\circ} \mathrm{C}$ in triphenyltetrazolium chloride $10 \mathrm{mg} / \mathrm{mL}$ of phosphate buffer at pH 7.4. When the viable myocardium had stained, slices were placed in $10 \%$ formalde. hyde solution. Approximately 24 hours later, heart slices were placed caudal surface upward and compressed between two glass plates separated by $2-\mathrm{mm}$ spacers. Risk areas and areas of infarction were traced, photographically enlarged, and planimetered (Summa Graphics, Summa Sketch II, Conn). The area of infarction (no tetrazolium staining) was expressed as a percentage of the area at risk of infarction (no fuorescent microspheres). The volume of myocardial tissue at risk and the volume of intarction were calculated by multiplying 


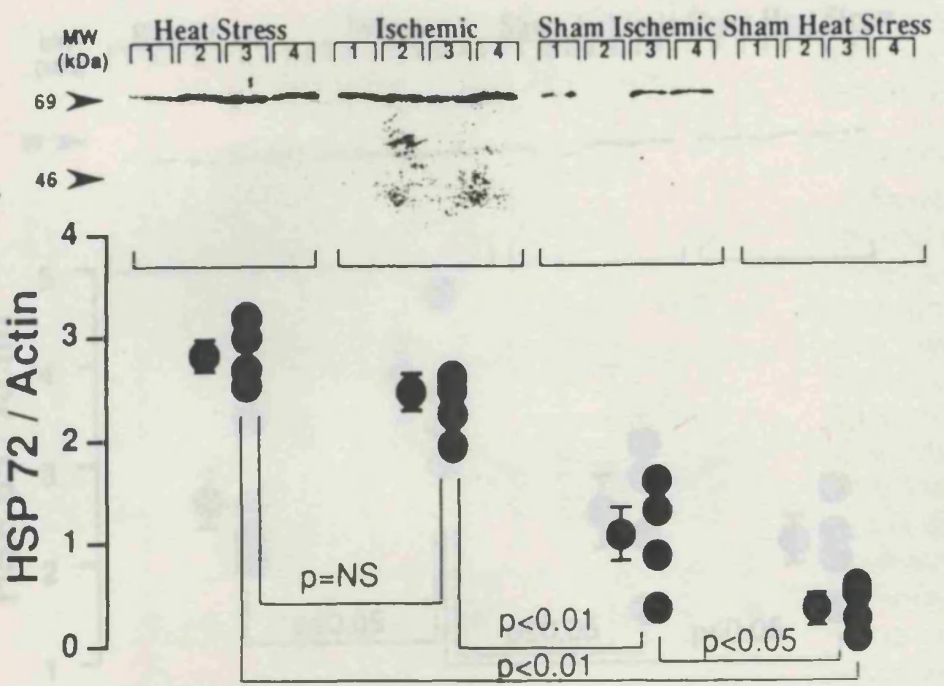

FIG 2. Densitometric assessment of Western blot loaded with four specimens from each of the four intervention groups and probed against heat stress protein (HSP)72. The graded induction of HSP72 between groups seen after immunoblotting (upper portion of figure) becomes more obvious after densitometric assessment (lower portion of figure). On the basis of optical density ratios, heat stress and ischemic pretreatment resulted in a similar level of HSP72 induction $(2.8 \pm 0.3$ units vs $2.5 \pm 0.2$ units, respectively). However, sham ischemia caused a greater induction than sham heat stress $(1.0 \pm 0.3$ units vs $0.3 \pm 0.1$ units, respectively). All comparisons were by ANOVA.

the corresponding areas by the depth $(2 \mathrm{~mm})$ of the tissue slices.

\section{Statistics}

All values are expressed as mean \pm SEM. All comparisons between groups were assessed for significance with a one-way ANOVA, Fisher's Protected Least Significant Difference method being used for comparisons within the ANOVA table. Changes in hemodynamic parameters within groups over the course of the experiment were compared by two-way ANOVA with a post hoc paired $t$ test for comparison of variables at baseline and end of reperfusion. An unpaired $t$ test was used to compare baseline temperatures between heat-stress and sham-heat-stress groups. Association between ratepressure product (RPP) and infarct size was tested by the Spearman Rank Correlation method. Statistical significance was defined as $P \leq .05$.

\section{Results}

\section{Temperature Changes During Heat Stress}

Before warming, there was no significant difference between basal rectal temperature within sham or heatstress groups $\left(39.1 \pm 0.1^{\circ} \mathrm{C}, \mathrm{n}=14\right.$, and $39.0 \pm 0.1^{\circ} \mathrm{C}$, $\mathrm{n}=14, P=\mathrm{NS}$, respectively). After the heat-stress rabbits were wrapped in an electric warming blanket, an average of $42.6 \pm 2.9$ minutes elapsed before the rectal temperature reached $42^{\circ} \mathrm{C}$. The peak temperature recorded was $42.3 \pm 0.1^{\circ} \mathrm{C}$, and the time that the rectal temperature remained above $42^{\circ} \mathrm{C}$ was $17.6 \pm 1.4$ minutes.

\section{Myocardial 72-kD Stress Protein Content}

An initial protocol with only 6 hours of recovery after ischemic pretreatment caused some increase in HSP72, but this was not significantly different'from basal expression and was significantly less than the elevation seen 24 hours after heat stress. The recovery time after ischemic pretreatment was therefore increased to 24 hours.

Fig 2 demonstrates that significant induction of the $72-k D$ stress protein occurred 24 hours after heat stress and ischemic pretreatments. This protein was detected in all myocardial samples examined (including those from the nonischemic area, data not shown). When the blot was examined densitometrically, the graded induction of stress protein with differing interventions became more apparent with thermal pretreatment, resulting in marginally greater induction than ischemic pretreatment. However, the mean stress protein content in each of the pretreatment groups was greater than in corresponding controls. Between the control groups, there was a significant induction in the sham ischemic pretreatment group. The reason for this may lie in the greater surgical trauma occurring with sham ischemic pretreatment, although other differences exist between these two control groups, including the method of ventilation and anesthetic regimens. Compared with the sham-heat-stress group, there was an approximately eightfold HSP72 induction by thermal, sevenfold by ischemic, and threefold by sham ischemic pretreatments.

\section{Myocardial 60-kD Stress Protein Content}

Fig 3 demonstrates that the marked variation seen between experimental groups for the $72-\mathrm{kD}$ stress protein was not apparent when an identically loaded gel was blotted and probed for the $60-\mathrm{kD}$ stress protein. In this model, cardiac HSP60 was not significantly elevated by whole-body heat stress. The only intervention associated with any change in HSP60 content was ischemic pretreatment, which resulted in an approximately 1.5 . to 2 -fold induction compared with other intervention groups. Therefore, differences exist in stress protein inducibility, with HSP72 being more inducible than HSP60, whereas HSP60 is preferentially, though only modestly, induced by ischemia.

\section{Infarct Size}

Risk volume, infarct volume, and percentage of risk area infarcted are shown in Fig 4 . The volumes of myocardial tissue at risk after coronary ligation were not significantly different between intervention groups. However, thermal or ischemic pretreatment resulted in both a significant reduction in absolute infarct volume and a reduction in infarct volume expressed as a percentage of the volume of tissue at risk. The volume of infarction as a percentage of the volume at risk for 


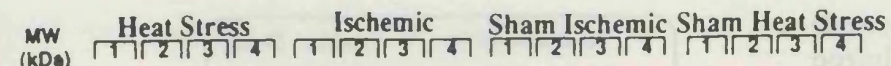

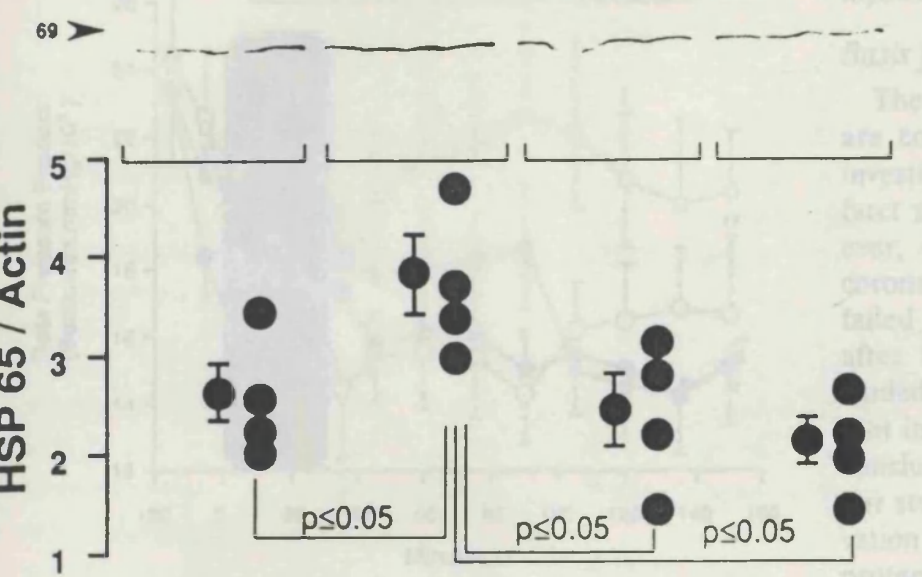

FIG 3. Densitometric assessment of Western blot loaded with four specimens from each of the four intervention groups and probed against heat stress protein (HSP)60. The lane arrangement gel loading conditions and figure layout are identical to Fig 2. HSP60 levels appeared quite similar between groups. The HSP6O band did, however, appear denser in the lanes containing samples from the ischemically pretreated hearts. Densitometry confirmed that ischemic pretreatment was associated with a selective induction of HSP60. Optical density ratios, ischemic pretreatment vs sham ischemia, heat stress, and sham heat stress were $3.8 \pm 0.4$ vs $2.4 \pm 0.3,2.6 \pm 0.3$ and $2.1 \pm 0.2$, respectively. All comparisons were by ANOVA. ischemic pretreatment compared with control was $28.8 \pm 5.2 \%$ vs $52.0 \pm 5.2 \%, P \leq .01$, respectively, and for thermal pretreatment compared with control, $32.8 \pm 3.8 \%$ vs $56.9 \pm 6.5 \%, P \leq .01$, respectively. Therefore, both ischemic and thermal pretreatment resulted in myocardial protection.

In all treatment groups, the infarcted areas exhibited intramyocardial hemorrhage. The absence of any hemorrhage was rare and usually indicative of a failure to reperfuse (see exclusions).

\section{Hemodynamic and Metabolic Data}

Fig 5 describes the changes in RPP that occur before, during, and after the 30 -minute coronary occlusion for each group. There were no differences in RPP between groups before coronary occlusion or during ischemia, implying that myocardial work and therefore oxygen demand was likely to be similar between groups.

During reperfusion, the RPP was consistently higher in the heat-stress group, and at various time points, it was significantly different from all other groups. This preservation of RPP is likely to be independent of infarct size, since a similar reduction in infarct size was seen with ischemic pretreatment but was not associated with any significant advantage in RPP. In an attempt to further elucidate the reason for this apparent advantage, we correlated RPP at the end of reperfusion with infarct volume as a ratio of heart mass (volume : heart weight ratio). As seen in Fig 6, there was no correlation between infarct size and RPP for any group. This finding suggests, surprisingly, that in this experimental model, infarct size does not appear to be the major determinant of cardiac work.

The Table includes the hemodynamic as well as acid/base balance data for each experimental group (the RPP is displayed in graphic form in Fig 5). There were no significant differences between groups in terms of acid/base balance or heart rate.

\section{Discussion}

This study demonstrates that both ischemic and heatstress pretreatments elevate myocardial HSP72 to a similar extent and are associated with a similar reduction of infarct size. This reduction occurs despite similar RPPs before and during prolonged coronary artery occlusion and therefore suggests that the protection is a direct result of enhanced myocardial resistance to infarction. This enhanced resistance to infarction is at

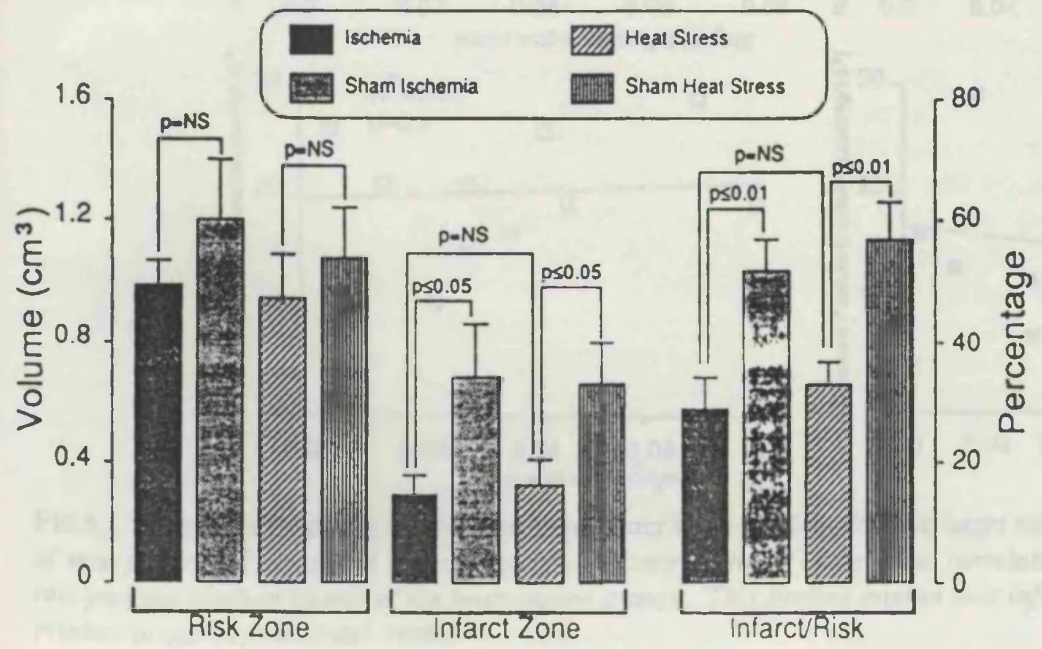

FIG 4. Bar graphs of risk and infarct zone volumes. Risk zones were demarcated by the absence of fluorescent microspheres and infarct zones by absence of staining with triphenyltetrazolium. Both ischemic and heat stress pretreatments were associated with a significant reduction in infarct volume as well as infarct zone as a percentage of risk zone. All comparisons were by ANOVA. 


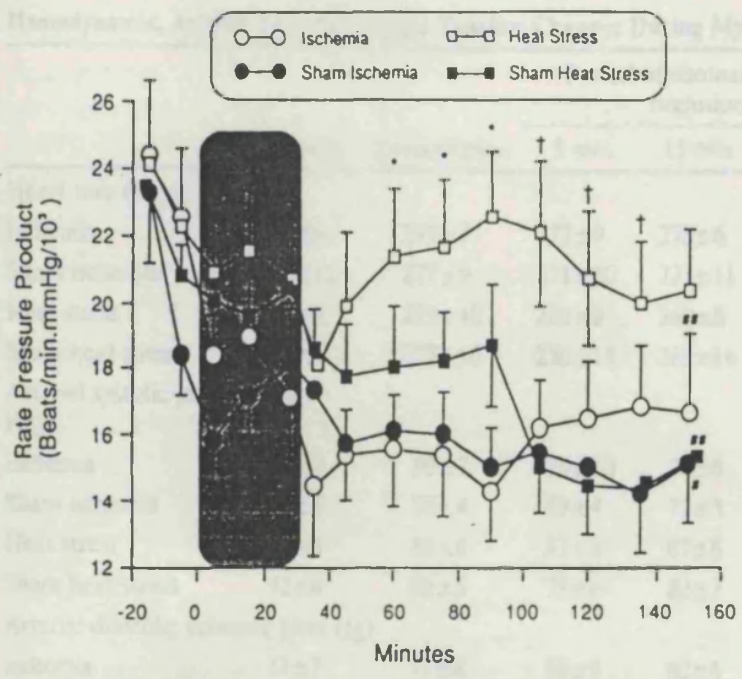

FIG 5. Graph of rate-pressure product before, during, and after 30-minute coronary occlusion. The shaded area represents the period of coronary occlusion. Although no significant differences existed before or during coronary occlusion, upon reperfusion, rate-pressure product was consistently higher in the heat-stress group. Heat stress was the only intervention without a significant drop in rate-pressure product for the duration of the experiment. The minus-15-minute hemodynamic data were collected while the chest was closed, and the minus-5-minute data were collected when the chest and pericardium were reopened. "p $\leq .05$ heat stress vs ischemia and sham ischemia; $\nmid \mathrm{P} \leq .05$ heat siress vs sham isch. emia and sham heal stress; \# $\leq .05$, \#\# $\leq .01$ for each group at 150 minutes vs baseline by ANOVA.

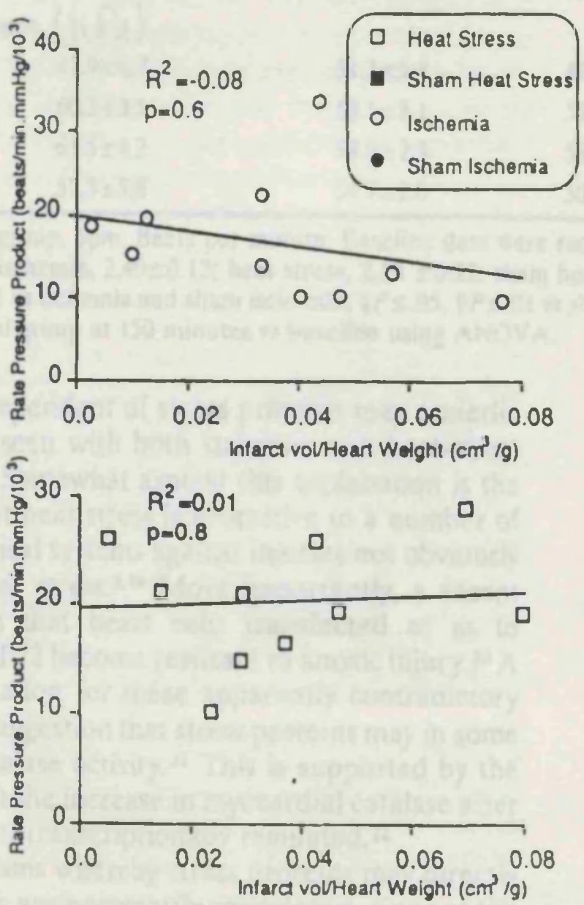

least associated with HSP72, and it is tempting to postulate that it occurs as a direct consequence of myocardial stress protein induction.

\section{Basis for the Reduction in Myocardial Infarct Size}

The design of this study is similar to and the findings are consistent with those of Donnelly et al. ${ }^{13}$ Those investigators were able to demonstrate significant infarct size reduction after whole-body heat stress; however, ischemic pretreatment with a single 20 -minute coronary occlusion failed to reduce infarct size but also failed to increase myocardial HSP72 to the level seen after heat stress. Those investigators, therefore, concluded that the absolute levels of HSP72 may be important in conferring protection from ischemic injury. This conclusion is supported and extended by the findings of our study, which demonstrate that greater ischemic elevation of myocardial HSP72 is indeed associated with protection. Moreover, a modest elevation of HSP72, as occurred with sham ischemic pretreatment, is not associated with protection. In addition, our study suggests that HSP60 induction appears not to be a prerequisite for myocardial salvage, whereas an appropriate level of HSP72 may be. However, the relation between wholebody heat stress and subsequent myocardial salvage is complicated by the fact that in the isolated rat heart, protection seems to involve an increase in myocardial catalase activity rather than an induction of HSP72, since protection can be abolished by inhibition of myocardial catalase with 3 -amino-triazole. ${ }^{17}$ In a preliminary report, endogenous catalase activity was also increased by repeated ischemia and reperfusion ${ }^{18}$; therefore, a common

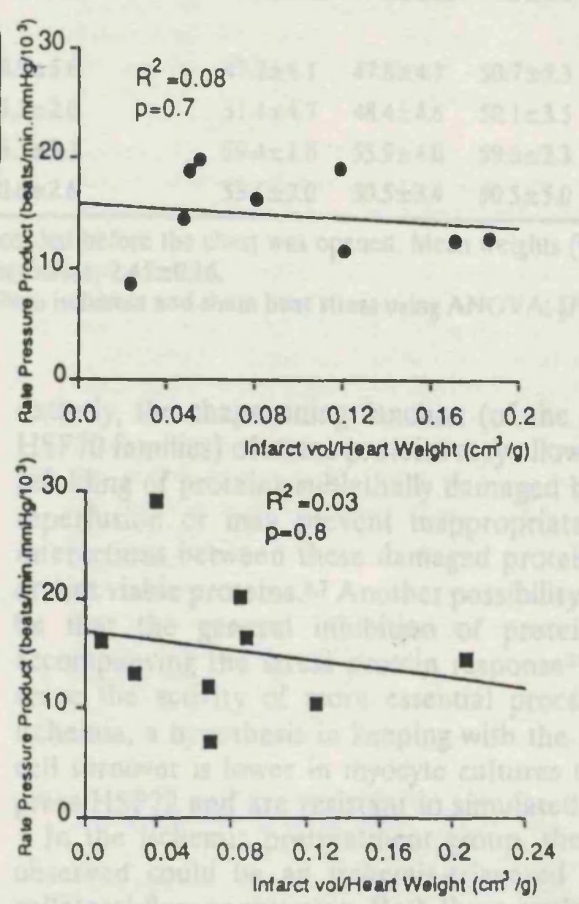

FIG 6. Scatterplots showing relation between infarct volume normalized to heart weight and rate-pressure product at 120 minutes of reperfusion. $R^{2}$ represents the coefficient of determination. There is no correlation between normalized infarct volume and rate-pressure product in any of the intervention groups. This finding implies that infarct volume does not determine rate-pressure product in our experimental model. 


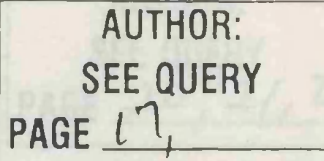

Marber et al Stress Protein and Myocardial Infarction

Hemodynamic, Arterial pH, and Oxygen Tension Changes During Myocardial Infarction

\begin{tabular}{|c|c|c|c|c|c|c|c|c|c|c|}
\hline \multirow{2}{*}{\multicolumn{2}{|c|}{ Baseline }} & \multirow[b]{2}{*}{ Preocclusion } & \multicolumn{3}{|c|}{$\begin{array}{l}\text { Period of coronary artery } \\
\text { occlusion }\end{array}$} & \multicolumn{5}{|c|}{$\begin{array}{c}\begin{array}{c}\text { Period of myocardial } \\
\text { reperfusion }\end{array} \\
\end{array}$} \\
\hline & & & $5 \mathrm{~min}$ & $15 \mathrm{~min}$ & $30 \mathrm{~min}$ & $45 \mathrm{~min}$ & $60 \mathrm{~min}$ & $90 \mathrm{~min}$ & $120 \mathrm{~min}$ & $150 \mathrm{~min}$ \\
\hline \multicolumn{11}{|c|}{ Heart ratc $(\mathrm{bpm})$} \\
\hline Ischemia & $297 \pm 6$ & $277 \pm 7$ & $272 \pm 9$ & $278 \pm 6$ & $270 \pm 5$ & $257 \pm 6$ & $258 \pm 7$ & $255 \pm 5$ & $269 \pm 13$ & $271 \pm 1111$ \\
\hline Sham ischemia & $293 \pm 11$ & $277 \pm 9$ & $271 \pm 10$ & $271 \pm 11$ & $284 \pm 15$ & $267 \pm 10$ & $271 \pm 8$ & $268 \pm 10$ & $270 \pm 11$ & $260 \pm 14 \|$ \\
\hline Heat stress & $273 \pm 9$ & $273 \pm 10$ & $269 \pm 9$ & $269 \pm 8$ & $265 \pm 9$ & $256 \pm 10$ & $259 \pm 10$ & $266 \pm 12$ & $262 \pm 11$ & $264 \pm 11$ \\
\hline Sham heat stress & $281 \pm 7$ & $277 \pm 10$ & $270 \pm 13$ & $265 \pm 14$ & $272 \pm 12$ & $263 \pm 11$ & $266 \pm 11$ & $264 \pm 9$ & $261 \pm 10$ & $260 \pm 99$ \\
\hline \multicolumn{11}{|c|}{$\begin{array}{l}\text { Arterial systolic pressure (mm } \\
\mathrm{Hg} \text { ) }\end{array}$} \\
\hline Ischemia & $92 \pm 7$ & $90 \pm 7$ & $76 \pm 10$ & $77 \pm 6$ & $74 \pm 6$ & $70 \pm 5$ & $70 \pm 6$ & $67 \pm 6$ & $73 \pm 6$ & $74 \pm 8 \|$ \\
\hline Sham ischemia & $91 \pm 6$ & $76 \pm 4$ & $67 \pm 4$ & $72 \pm 3$ & $75 \pm 6$ & $68 \pm 6$ & $68 \pm 5$ & $67 \pm 4$ & $65 \pm 5$ & $71 \pm 6$ \\
\hline Heat $s$ & $99 \pm 6$ & $86 \pm 6$ & $83 \pm 6$ & $87 \pm 6$ & $84 \pm 7$ & $85 \pm 6.4^{\circ}$ & $89 \pm 6$ & $94 \pm 6 \dagger$ & $88 \pm 6 \S$ & $87 \pm 6 \dagger$ \\
\hline Sham heat stress & $92 \pm 6$ & $86 \pm 5$ & $78 \pm 6$ & $82 \pm 7$ & $81 \pm 5$ & $75 \pm 5$ & $73 \pm 5$ & $78 \pm 5$ & $61 \pm 6$ & $63 \pm 79$ \\
\hline \multicolumn{11}{|c|}{ Artcrial diastolic pressurc ( $\mathrm{mm} \mathrm{Hg}$ ) } \\
\hline Ischemia & $73 \pm 7$ & $71 \pm 8$ & $59 \pm 9$ & $60 \pm 6$ & $56 \pm 6$ & $52 \pm 5$ & $52 \pm 5$ & $49 \pm 5$ & $55=$ & $55 \pm 89$ \\
\hline Sham isch & $73 \pm 6$ & $59 \pm 4$ & $53 \pm 4$ & $58 \pm 3$ & $59 \pm 5$ & $53 \pm 6$ & $=5$ & $0 \pm 4$ & 5 & \\
\hline Heat str & $81 \pm 7$ & $73 \pm 7$ & $70 \pm 6$ & & & & & & & \\
\hline Sham heat stress & $78 \pm 7$ & $70 \pm 5$ & $63 \pm 7$ & $68 \pm 8$ & $65 \pm 7$ & $62 \pm 8$ & $57 \pm 7$ & $58 \pm 6$ & $43 \pm 6$ & $49 \pm 68$ \\
\hline \multicolumn{11}{|c|}{ Mean arterial pressure $(\mathrm{mm} \mathrm{Hg})$} \\
\hline Ischemia & $81 \pm 7$ & $80 \pm 8$ & $66 \pm 9$ & $68 \pm 6$ & $63 \pm 6$ & $60 \pm 5$ & $60 \pm 6$ & $56 \pm 5$ & $62=$ & \\
\hline Sham ischemia & $79 \pm 5$ & $66 \pm 4$ & $59 \pm 4$ & $64 \pm 4$ & $65 \pm 5$ & $59 \pm 6$ & $60 \pm 5$ & $56 \pm 4$ & $57 \pm 5$ & $58 \pm 6 \|$ \\
\hline Heat stress & $88 \pm 6$ & $80 \pm 7$ & $77 \pm 6$ & $80 \pm 6$ & $76 \pm 7$ & $77 \pm 7$ & $82 \pm 6^{\circ}$ & $84 \pm 6 \dagger$ & $79 \pm 7 \ddagger$ & $78 \pm 6$ \\
\hline Sham heat stress & $84 \pm 7$ & $76 \pm 4$ & $74 \pm 8$ & $75 \pm 7$ & $76 \pm 6$ & $68 \pm 6$ & $68 \pm 6$ & $71 \pm 6$ & $56 \pm 6$ & $60 \pm 79$ \\
\hline \multicolumn{11}{|l|}{ Arterial blood pH } \\
\hline Ischemia & $7.49 \pm 0.02$ & & $7.50 \pm 0.03$ & & $7.46 \pm 0.02$ & & $7.45 \pm 0.02$ & $7.47 \pm 0.02$ & $7.44 \pm 0.02$ & $7.44 \pm 0.02$ \\
\hline Sham ischemia & $7.53 \pm 0.02$ & & $7.48 \pm 0.02$ & & $7.43 \pm 0.04$ & & $7.44 \pm 0.02$ & $7.43 \pm 0.01$ & $7.40 \pm 0.02$ & $7.39 \pm 0.01 \|$ \\
\hline Heat stress & $7.51 \pm 0.02$ & & $7.53 \pm 0.04$ & & $7.46 \pm 0.02$ & & $7.44 \pm 0.02$ & $7.45 \pm 0.01$ & $7.45 \pm 0.01$ & $7.45 \pm 0.01$ \\
\hline Sham heat stress & $7.46 \pm 0.02$ & & $7.45 \pm 0.04$ & & $7.41 \pm 0.03$ & & $7.42 \pm 0.03$ & $7.42 \pm 0.02$ & $7.38 \pm 0.04$ & $7.38 \pm 0.04 \|$ \\
\hline \multicolumn{11}{|c|}{ Arterial blood oxygen $\left(i f_{b l}\right)$} \\
\hline Ischemia & $47.9 \pm 6.7$ & & $51.3 \pm 5.8$ & 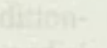 & $48.9 \pm 5.6$ & & $47.2 \pm 6.1$ & $47.8 \pm 4.7$ & $50.7 \pm 5.3$ & $54.2 \pm 4.5$ \\
\hline Sham ischemia & $60.3 \pm 3.1$ & & $59.1 \pm 3.1$ & andisi & $55.1 \pm 2.0$ & & $51.4 \pm 4.7$ & $48.4 \pm 4.6$ & $50.1 \pm 3.5$ & $54.9 \pm 3.1$ \\
\hline Heat stress & $61.5 \pm 4.2$ & & $58.9 \pm 2.3$ & 6402 & $56.3 \pm 3.3$ & & $59.4 \pm 1.6$ & $55.9 \pm 4.0$ & $59.6 \pm 2.3$ & $59.5 \pm 1.7$ \\
\hline Sham heat stress & $52.3 \pm 5.8$ & & $54.7 \pm 2.0$ & & $50.6 \pm 2.6$ & & $53.1 \pm 2.0$ & $50.5 \pm 3.4$ & $50.5 \pm 5.0$ & $55.0 \pm 6.1$ \\
\hline
\end{tabular}

$n=10$ for cach group. bpm, Beats per minute. Baseline data werc recorded before the chest was opened. Mean weights ( $\mathrm{kg}$ ): Ischemia, $2.57 \pm 0.14$; sham ischemia, $2.40 \pm 0.12$; heat stress, $2.58 \pm 0.22$; sham heat stress; $2.45 \pm 0.16$.

$\bullet P \leq .05, \dagger P \leq .01$ vs ischemia and sham ischemia; $\ddagger P \leq .05, \S P \leq .01$ vs sham ischemia and sham heat stress using ANOVA; $\| P \leq .05, q P \leq .01$ for each individual group at 150 minutes vs baseline using ANOVA

mechanism independent of stress proteins may underlie the protection seen with both ischemic and heat-stress pretreatments. Somewhat against this explanation is the observation that heat stress is protective in a number of different biological systems against injuries not obviously involving oxidant stress. ${ }^{2.19}$ More importantly, a recent report suggests that heart cells transfected so as to overexpress HSP72 become resistant to anoxic injury. ${ }^{20} \mathrm{~A}$ possible explanation for these apparently contradictory findings is the suggestion that stress proteins may in some way modify catalase activity. ${ }^{21}$ This is supported by the observation that the increase in myocardial catalase after heat stress is not transcriptionally regulated. ${ }^{22}$

The mechanisms whereby stress proteins may directly limit infarct size are necessarily speculative, since at the molecular level, the key sites of injury during ischemia are poorly understood. The most attractive possibilities involve the stabilization of the cytoskeleton during ischemia by $\alpha \beta$-crystallin (an HSP homologue).23 Alter- natively, the chaperoning function (of the HSP9O and HSP70 families) of stress proteins may allow the correct refolding of proteins sublethally damaged by ischemia/ reperfusion or may prevent inappropriate molecular interactions between these damaged proteins and oth. erwise viable proteins. ${ }^{1,7}$ Another possibility may simply be that the general inhibition of protein synthesis accompanying the stress protein response ${ }^{24}$ helps preserve the activity of more essential processes during ischemia, a hypothesis in keeping with the finding that cell turnover is lower in myocyte cultures that overexpress HSP72 and are resistant to simulated ischemia. ${ }^{20}$

In the ischemic pretreatment group, the protection observed could be an ischemia-triggered increase in collateral flow or stunning. Both these explanations are unlikely, since it is unclear whether the oxygen require. ments and susceptibility to infarction are increased, decreased, or normal in stunned myocardium. ${ }^{25}$ In a previous study, a pattern of ischemic pretreatment 
identical to that used in our experiments produced only a very mild degree of stunning. ${ }^{4}$ In addition, the rabbit is a species deficient in collateral vessels ${ }^{26}$; therefore, any increase in collateral blood flow occurring as a result of ischemic pretreatment would have to be on the basis of new (mitotic) vessel growth, and this is unlikely to occur over a 24 -hour period.

\section{Relation Between Ischemic Pretreatment and Ischemic Preconditioning}

The protocol used in this study and protocols known to trigger "ischemic preconditioning" are identical. 27 Ischemic preconditioning, however, is a short-lived phenomenon with protective benefits waning within 2 hours of reperfusion. ${ }^{27}$ This study therefore raises the possioflayed, but perhaps longer-lasting, second window of protection (a biphasic protective effect). Evidence in support of such a phenomenon exists in neuronal tissue, since the stress protein content of neuronal tissue can be elevated by 2 -minute, daily episodes of ischemia, a treatment that also seems to increase the neuronal resistance to more prolonged ischemia. ${ }^{28.29}$ Preliminary findings from other laboratories confirm a similarly delayed protection at 24 hours after "classic" preconditioning of the dog heart (with repeated coronary occlusions) $)^{30}$ and also 24 hours after rapid pacing of the rabbit heart. ${ }^{31}$ These experimental findings may explain the apparent benefit of a $\geq 7$-day history of angina before myocardial infarction, ${ }^{32}$ although differences in treatment and collateral vessel formation may complicate the issue.

The relation between the early phase of protection after brief episodes of ischemia (as seen in ischemic preconditioning) and the later phase of protection (as seen in this study) is speculative. An obvious inconsistency is the fact that multiple coronary occlusions are as effective as a single occlusion in triggering preconditioning ${ }^{33}$ but differ in their ability to induce myocardial HSP72.4 This inconsistency may explain why protection against ischemic arrhythmia was not seen 24 hours or 72 hours after a single 5-minute coronary occlusion in the rat. ${ }^{34}$

\section{Conclusions}

The results of this study indicate that substantial myocardial HSP72 induction is possible with either sublethal thermal or ischemic pretreatment, whereas HSP60 is preferentially elevated by ischemic pretreatment. This observation is the first indication that myocardial HSP72 may be more important than other stress proteins, since myocardial salvage was seen after a 30 -minute coronary occlusion with both ischemic and thermal pretreatments. In addition to limiting infarct size, whole-body heat stress has other nonspecific effects that act to maintain RPP.

Our results provide evidence for what may prove to be a clinically relevant myocardial adaptive response occurring 24 hours after short episodes of cardiac ischemia that serves to increase myocardial resistance to infarction.

\section{Acknowledgments}

The anti-60-kDa heat-shock protein antibody was a gift from Dr. G.A. Rook, Department of Medical Microbiology,
University College London Medical School. M.S.M. is the recipient of an intermediate fellowship from the British Heart Foundation. We thank the Hatter Foundation for continued support.

\section{References}

1. Hightower LE. Heat shock, stress proteins, chaperones, and proteotoxicity. Cell. 1991:66:191-197.

2. Li CG, Werb Z. Correlation between synthesis of heat shock proteins and development of thermotolerance in chinese hamster fibroblasts. Proc Natl Acad Sci U S A. 1982;79:3218-3222.

3. Sanchez Y, Linquist SL. HSP104 required for induced thermotolerance. Science. 1990;248:1112-1115.

4. Knowiton AA, Brecher P, Apstein CS. Rapid expression of heat shock protein in the rabbit after brief cardiac ischemia. I Clin Invest. 1991;87:139-147.

5. Dillmann WH, Mehta $\mathrm{H}$, Barrieux A, Guth BD, Neeley WE, Ross J. Ischemia of the dog heart induces the appearance of a cardiac mRNA coding for a protein with migration characteristics similar to heat-shock/stress protein 71. Circ Res. 1986;59:110-114.

6. Knowlton AA, Eberli FR, Brecher P, Romo GM. Owen A. Apstein CS. A single myocardial stretch or decreased systolic fiber shortening stimulates the expression of heat shock protein 70 in the isolated, erythrocyte-perfused rabbit heart. I Clin Invest. 1991;\$8: 2018-2025.

7. Yellon DM, Latchman DS, Marber MS. Stress proteins: an endog. enous route to cardiac protection: fact or fiction? Cardiovasc Res. 1ores 1993; 27 i52-161

8. Currie RW, Ross BM, Davis TA. Heat-shock response is associated with enhanced post ischemic ventricular recovery. Circ Res. 1989;63:543-549.

9. Yellon DM, Pasini E, Cargnoni A, Marber MS, Latchman DS, Ferrari $\mathbf{R}$. The protective role of heat stress in the ischaemic and reperfused rabbit myocardium. I Mol Cell Cardiol. 1992;24: 895-907.

10. Liu X, Engelman RM, Moraru II, Lu D, Nath D, Rousou JA, Flack JE, Deaton DW, Maulik N, Das DK. Heat shock: a new approach for myocardial preservation in cardiac surgery. Circulation. 1992; 86(suppl II):II-358-II-363.

11. Walker DM, Pasini E, Kucukoglu S, Marber MS, lliodromitis E, Ferrari R, Yellon DM. Heat stress limits infarct size in the isolated perfused rabbit heart. Cardiovasc Res. La-press. : $5,27.46$. 2

12. Yellon DM, lliodromitis $E$, Latchman DS, Van Winkle DM. Downey JM, Williams FM, Williams TJ. Whole body heat shock fails to limit infarct size in the reperfused rabbit heart. Cardiovasc Res. 1992:26:342-346.

13. Donnelly TJ, Sievers RE, Vissern FL, Welch WJ, Wolfe CL. Heat shock protein induction in rat hearts: a role for improved myocar. dial salvage after ischemia and reperfusion? Circulation. 1992;85: 769-778.

14. Laemmli UK. Cleavage of structural proteins during the assembly of the head of bacteriophage T4. Nature. 1970;227:680-685.

15. Sharif M, Worrall JG, Singh B, Gupta RS, Lydyard PM, Lambert C. McCulloch J, Rook G. The development of monoclonal antibodies to the human mitochondrial $60 \mathrm{kDa}$ heat shock protein and their use in studying the expression of the protein in rheumatoid arthritis. Arthritis Rheum. Inpress. $9942.35 \cdot 1427-14.33$

16. Norton PM, Latchman DS. Levels of $90 \mathrm{kDa}$ heat shock protein and resistance to glucocorticoid-mediated cell killing on a range of human and murine lymphocyte cell lines. J Steroid Biochem. 1987; 33:149-154.

17. Karmazyn M, Mailer K, Currie RW. Acquisition and decay of heat-shock-enhanced postischemic ventricular recovery. Am J Plysiol. 1990;259:H424-H431.

18. Flack J, Engelman R, Rousou J, lyergar J, Kimura Y, Prasad R, Das DK. Preconditioning the heart by repeated stunning improves myocardial salvage. Circulation. 1990;82(suppl III): $111-575$. Abstract.

19. Barbe MF, Tytell M, Gower DJ, Welch WJ. Hyperthermia protects against light damage in the retina. Science 1988:211:1817.1820.

20. Dillmann WH, Chi S-H, Sayen MR, Mestril R. Decreased ischemia (Ism) induced injury in rat heart derived $\mathrm{H} 9 \mathrm{c} 2$ cells expressing human inducible heat shock protein 70 (hHSP70i). J Mol Cell Cardiol. 1992;24(suppl III):S30. Abstract.

21. Kukreja RC, Hess ML. The oxygen free radical system: from equations through membrane protein interactions to cardiovaseu. lar injury and protection. Cardiovasc Res. 1992;26:6+1-655.

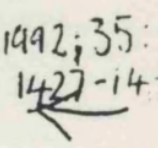




\section{AUTHOR: SEE QUERY}

PAGE

\section{Marber et al Stress Protein and Myocardial Infarction}

9

22. Curric RW, Tanguay RM. Analysis of RNA for transcripts for catalase and SP71 in rat hearts after in vivo hyperthermia. Biochem Cell Biol. 1991:69:375-382.

23. Bennardini F, Wrosek A, Chiesi M. $\alpha \beta$-crystallin in cardiac tissue: association with actin and desmin filaments. Circ Res. 1992;71: 288-294.

24. Havre PA, Hammond GL. Isolation of a translation-inhibiting peptide from myocardium. Am J Physiol. 1988;255:H1024-H1031.

25. Ohgoshi $Y$, Goto $Y$, Futaki S, Yaku H, Kawaguchi O, Suga $H$. Increased oxygen cost of contractility in stunned myocardium of dog. Circ Res. 1991;69:975-988.

26. Maxwell M, Hearse DJ, Yellon DM. Species variation in the coronary collateral circulation during regional myocardial ischaemia: a critical determinant of the rate of evolution and extent of myocardial infarction. Cardiovasc Res. 1987;21:737-746.

27. Walker DM, Yellon DM. Ischacmic preconditioning: from mechanisms to exploitation. Cardiovasc Res. 1992;26:734-739.

28. Kitagawa K, Matsumoto M, Tagaya M, Hata R, Ueda $H$, Niinobe M. Handa N. Fukunaga R, Kimura K. Mikoshiba K, Kamada T. Ischemic tolerance phenomenon found in the brain. Brain Res. $1990 ; 528: 21-24$.
29. Nowak TS. Protein synthesis and heat shock/stress response after ischemia. Cerebrovasc Brain Metab Rev. 1990;2:345-366.

30. Yamashita N, Kuzuya T, Hoshido S, Fuji H, Oe H, Kitabatake A, Tada M, Kamada T. Relationship between time interval from preconditioning to sustained ischemia and its effects on limiting infarct size. J Mol Cell Cardiol. 1992;24(suppl I):S150. Abstract.

31. Szekeres L, Szilvassy Z, Udvary E, Vegh A. Moderate stress by cardiac pacing may induce both short-term and long-term cardioprotection based on different mechanisms. I Mol Cell Cardiol. 1992;24(suppl 1):S120. Abstract.

32. Muller DW, Topol EJ, Califf RM, Sigmon KN, Gorman L, George BS, Kereiakes DJ, Lee KL, Ellis SG. Relationship between antecedent angina pectoris and short-term prognosis after thrombolytic therapy for acute myocardial infarction: Thrombolysis and Angioplasty in Myocardial Infarction (TAMI) study group. Am Heart J. 1990;119:224-231.

33. Li GC, Vasquez BS, Gallagher KP, Lucchesi BR. Myocardial protection with preconditioning. Circulation. 1990;82:609-619.

34. Shiki K, Hearse DJ. Preconditioning of ischemic myocardium: reperfusion-induced arthythmias. Am J Physiol. 1987;22: H1470-H1476. 


\title{
A single five minute period of rapid atrial pacing fails to limit infarct size in the in situ rabbit heart
}

\author{
Michael S Marber, David M Walker, David J Eveson, J Malcolm Walker, \\ Derek M Yellon
}

\begin{abstract}
Objective: Rapid pacing has been shown to precondition the dog heart against ischaemic dysrrhythmia. The aim of this study was to determine whether rapid pacing could also limit infarct size. Methods: Rabbits $(n=5)$ were rapidly paced via the left atrium at $420-480$ beats $\min ^{-1}$. Five min of rapid pacing and 10 min of recovery in sinus rhythm were followed by $45 \mathrm{~min}$ of regional ischaemia and $120 \mathrm{~min}$ of reperfusion. Control rabbits $(\mathrm{n}=9)$ were treated identically without prior rapid pacing. Infarct size was determined in both groups using tetrazolium and expressed as a percentage of the area at risk demarcated by fluorescent miscrospheres. In a separate series of experiments, rapidly paced Langendorff perfused rabbit hearts $(n=9)$ were used to determine coronary flow under perfusion conditions designed to simulate the in vivo situation during rapid pacing. Results: Rapid pacing caused a fall in systolic pressure from 91.4(SEM 4.5) to $47.0(5.9) \mathrm{mm} \mathrm{Hg}(\mathrm{p}<0.01$ ) and diastolic pressure from $67.2(2.9)$ to $23.6(3.2) \mathrm{mm} \mathrm{Hg}(\mathrm{p}<0.01)$. Both recovered within $30 \mathrm{~s}$ of cessation of pacing. During rapid pacing the action potential duration shortened from $192(13)$ to $128(5) \mathrm{ms}(\mathrm{p}=0.01)$ and developed electrical alternans $(n=4)$. Following rapid pacing the ECG showed either ST depression or T wave inversion $(n=4)$. Despite these profound changes, rapid pacing did not reduce infarct size $v$ control $[52.7(4.6) \% v 60.8(9.1) \%$ of the area at risk, respectively]. The in vitro experiments estimated that rapid pacing would result in a reduction in coronary flow to $44 \%$ of that in sinus rhythm without a significant rise in lactate efflux. Conclusions: In our model, pretreatment with rapid pacing fails to reduce infarct size. The most likely reason for this is that rapid pacing at a rate of 480 beats $\mathrm{min}^{-1}$ does not cause myocardial ischaemia of sufficient severity to trigger the preconditioning response.
\end{abstract}

Cardiovascular Research 1993;27:597-601

I recent years the phenomenon of ischaemic preconditioning has received considerable attention.' ? Ischaemic preconditioning is the term used to describe the observation made by Murry and colleagues that short coronary occlusions separated by reperfusion are able to increase the resistance of the heart to a subsequent prolonged coronary occlusion. ${ }^{3}$ Later studies indicated that a single coronary occlusion followed by reperfusion was as effective as multiple occlusions in reducing infarct size. ${ }^{4}$ Although these original descriptions demonstrated myocardial protection in terms of a reduction in infarct size, subsequent studies have shown that identical protocols can reduce ischaemic and reperfusion dysrhythmias. ${ }^{56}$ The protection afforded against either of these endpoints is substantial and has acted as a stimulus for research into pharmacological and other means of inducing the preconditioning response.

Theoretically it may be possible to induce preconditioning by other manoeuvres that alter the balance between myocardial supply and metabolic demand. One such procedure, rapid pacing, reduces myocardial supply (by causing a fall in mean aortic root pressure and increasing the length of time the heart is in systole), while increasing myocardial metabolic demand (by increasing the number of cardiac contractions per unit time). Ischaemia secondary to rapid pacing will differ from coronary artery occlusion in that flow and metabolite washout will continue during the preconditioning insult, and this may prevent the trigger for the preconditioning response. However, it seems unlikely that metabolite accumulation is a prerequisite for the preconditioning phenomenon since regional pretreatment with high flow hypoxia is as efficacious as coronary occlusion in terms of infarct size reduction.

In support of such alternative means of inducing preconditioning, a recent report suggests that short periods of rapid pacing can substitute for "classical preconditioning". Vegh and colleagues found that two brief (two minute) periods of rapid pacing attenuated the dysrhythmias associated with a subsequent 25 minute coronary occlusion in the dog. In addition, these investigators have shown that the degree of protection afforded against arrhythmias by rapid pacing is similar to that of classical preconditioning. The implications of these findings are that it may be possible, by relatively simple means, to precondition the human heart at risk of infarction prior to coronary angioplasty or coronary artery bypass grafting.

In an attempt to address these issues we examined the protective benefits of rapidly pacing the in situ rabbit heart using infarct size as the endpoint. In addition, to examine more closely the effects of rapid pacing on coronary flow and myocardial metabolism we simulated the in vivo effects of rapid pacing by using an isolated perfused heart.

\section{Methods}

The investigations described below were in accordance with Guidance on the operation of the Animals (Scientific Procedures) Act 1986, published by HMSO, London.

In vivo experiments

Rupid pacing group - Male New Zealand White rabbits, $n=5,2.0-2.5$ $\mathrm{kg}$. were anaesthetised with intravenous pentobarbitone $40 \mathrm{mg} \cdot \mathrm{kg}^{-1}$.

The Hatter Institute for Cardiovascular Studies, Division of Cardiology, University College Hospital, Gower Street, London ECIE 6AU, United Kingdom: M S Marber, D M Walker, D J Eveson, J M Walker, D M Yellon. Correspondence to Dr Yellon. 
Further doses were given as required. The trachea was opened via a midline cervical incision after infiltration with $2 \%$ lignocaine solution. Following tracheal intubation animals were ventilated with $100 \%$ oxygen, delivered with a positive end expiratory pressure of $5 \mathrm{~cm} \mathrm{H}_{2} \mathrm{O}$, to maintain anterial $\mathrm{pH}$ in the physiological range ( 7.35 to 7.45 ). A short polyethylene tube connected to a pressure transducer was inserted into the right common carotid artery

The chest was opened via a left thoracotomy and two suction electrodes were applied to the exposed left surface of the heart. The first, an $\mathrm{Ag} / \mathrm{AgCl}$ electrode, was applied over the left ventricle and used to record the monophasic action potential. The second, a platinum bipolar pacing electrode, was placed over the left atrial appendage. In preliminary experiments attempts to pace the right ventricle at rates above $360 \cdot \mathrm{min}^{-1}$ using an epicardial bipolar electrode resulted in profound hypotension and eventual ventricular fibrillation. Rapid pacing, in this study, was achieved by pacing the left atrium at twice the threshold amplitude with a $2 \mathrm{~ms}$ square wave pulse, at a rate of between 420 and $480 \cdot \mathrm{min}^{-1}$. Conduction across the atrioventricular node was confirmed by observing a $1: 1$ relationship between the pacing spike and the upstroke of the left ventricular monophasic action potential. Pacing was initiated at a rate of $420 \cdot \mathrm{min}^{-1}$ and if the systolic arterial pressure remained above $50 \mathrm{~mm} \mathrm{Hg}$ during the first minute, increased to $480 \cdot \mathrm{min}^{-1}$. Following $5 \mathrm{~min}$ of rapid pacing the heart was allowed to recover for $10 \mathrm{~min}$ in sinus rhythm prior to prolonged coronary occlusion, to mimic the reperfusion phase following coronary ligation in classical preconditioning. During the 10 min "reperfusion" period a marginal branch of the left circumflex coronary artery was underrun with $3 / 0$ silk suture on a tapered needle. Five hundred units of heparin were given intravenously, and regional ischaemia was achieved by passing the free ends of the silk suture through a soft plastic tube which was then applied to the surface of the heart to occlude the coronary artery. Successful occlusion was confirmed by cyanosis and bulging of the myocardium. together with shortening of the action potential duration and ST segment elevation on the surface ECG. After $45 \mathrm{~min}$ of occlusion the heart was reperfused by removing the plastic snare occluder. Reperfusion was contirmed by observing a myocardial blush and arrhythmia on the surface ECG. Following $120 \mathrm{~min}$ of reperfusion. a further 500 units of heparin were given. and the heart was removed and retrogradely perfused with $0.9 \%$ saline. After blood had been washed out of the coronary vasculature the coronary snare was retightened and fluorescent microspleres infused via the aortic cannula. Demarcation of the area at risk (areal without spheres) was confirmed under ultraviolet light. The heart was then frozen at $-18^{\circ} \mathrm{C}$ overnight. The following day, while still frozen. the heart was sliced at $2 \mathrm{~mm}$ intervals at right angles to its long axis. The slices were then incubated at $37^{\circ} \mathrm{C}$ in triphenyltetrazolium chloride. $10 \mathrm{mg} \cdot \mathrm{ml}^{-1}$ in phosphate buffer at $\mathrm{pH} 7.4$. Once the viable myocardium had stained, slices were placed in $10 \%$ formaldehyde solution and approximately $24 \mathrm{~h}$ later risk areas and areas of infarction were traced. photographically enlarged, and planimetered.

Comirol group - The control group consisted of nine New Zealand White rabbits treated as described above but without rapid pacing prior to ischaemia.

\section{In vitro experiments}

In an attempt to determine the influence of rapid atrial pacing on coronary artery fiow and myocardial metabolism, a series of in vitro experiments using a Langendorff perfused heart was performed.

Nine New Zealand White rabbits. $2.0-3.0 \mathrm{~kg}$, were anaesthetised with pentobarbitone and heparinised as above. Once the animals had stopped breathing hearts were rapidly excised. placed in iced saline, and within $90 \mathrm{~s}$ were retrogradely perfused with oxygenated Krebs buffer Perfusion was in constant pressure mode with prefiltered (pore size 2 $\mu \mathrm{m}$ ) modified Tyrode's of the following composition (mmol-litre ${ }^{-1}$ ): $\mathrm{NaCl} 118 . \mathrm{KCl} 4.7, \mathrm{MgSO}_{4} 6 \mathrm{H}_{2} \mathrm{O} \quad 1.2, \mathrm{CaCl}_{2} . \mathrm{H}_{2} \mathrm{O} \quad 1.3, \mathrm{NaHCO}_{3} 24$, glucose 10 , pyruvate 2 , and lactate 0.5 , equilibrated with $95 \% \quad \mathrm{O} / 5 \%$ $\mathrm{CO}$, to provide a $\mathrm{pH}$ of 7.35-7.45 and $\mathrm{Po}_{2}$ of $60-70 \mathrm{kPa}$.

The left atrial appendage was removed and a fluid filled latex balloon. connected via a shor length of polyethylene tubing to a pressure transducer, was introduced into the left ventricle by traversing the mitral valse. A thermocouple was placed in the right ventricle by introducing it retrogradely across the pulmonary valve, and the temperature was maintained close $1037^{\circ} \mathrm{C}$ by water circulating in jacketing surrounding the heart chamber and perfusate heat exchanger. Coronary flow was estinated by timed collection of the perfusate leaving the heart.

Hearts were perfused under two different sets of conditions, chosen to simulate the haemodynamics of the in situ heart during sinus rhythn and rapid pacing. In the first set of conditions hearts were paced at 180- $\mathrm{min}^{-1}$ with a coronary perfusion pressure of $75 \mathrm{~mm} \mathrm{Hg}$. The pacing rate and mean coronary perfusion pressure were chosen to correspond to the resting heart rate and mean arterial pressure of rabbits in the rapid pacing group. In the second set of conditions hearts were paced at $48\left(\right.$ ) $\mathrm{min}^{-1}$ with a coronary pertusion pressure of $31 \mathrm{~mm} \mathrm{Hg}$. The pacing rate and meith coronary perfusion pressure were again chosen 10 correspond to the paced heart rate and resulting mean arterial pressure of rabbits during rapid pacing. During both of these sets of experiments the hearts were allowed to stabilise for a $5 \mathrm{~min}$ period before perfusate was collected for estimation of coronary flow $\left(\mathrm{ml} \cdot \mathrm{min}^{-1} \cdot \mathrm{g}^{-1}\right)$ and measurement of lactate efflux. Both sets of conditions were performed with the intraventricular balloon inflated to an end diastolic pressure of $10 \mathrm{~mm} \mathrm{Hg}$ and with the balloon deflated.

Samples of coronary effluent were rapidly frozen and stored for enzymatic lactate determination using a commercial kit (Sigma).

\section{Statistics}

Experimental data between and within groups were compared by unpaired and paired Student I tests respectively. Values are given as mean(SEM). A probability value of less than $5 \%$ was considered significant.

\section{Results}

IN VIVO EXPERIMENTS

\section{Haemodynamic changes during rapid pacing}

With the onset of rapid pacing there was an immediate profound fall in arterial pressure. When pressures were recorded immediately prior to and $4.5 \mathrm{~min}$ after the onset of rapid pacing (fig 1), the mean systolic arterial pressure fell from 91.4(SEM 4.5) to $47.0(5.9) \mathrm{mm} \mathrm{Hg}$, while mean diastolic pressure fell from $67.2(2.9)$ to $23.6(3.2) \mathrm{mm} \mathrm{Hg}$. Following rapid pacing there was a prompt recovery of developed pressure so that at the moment of coronary ligation there was no significant difference in heart rate or arterial pressure between control and rapid pacing groups (fig 2).

During rapid pacing. four out of the five rabbits, developed alternans of monophasic action potential duration and amplitude. Interestingly these changes were not apparent in the surface ECG. In two of the rabbits electrical alternans was associated with alternans of the arterial pressure waveform, with only those heats of long action potential duration being of sufficient force to open the aortic valve and transmit a waveform of adequate amplitude to be recorded in the carotid artery (see fig 3 ).

\section{Haemodinamic changes during coronan ligation and reperfusion}

Figure 2 shows that prior to and during ischaemia, heart rate and arterial pressures were very similar, implying that myocardial oxygen demand and therefore susceptibility to

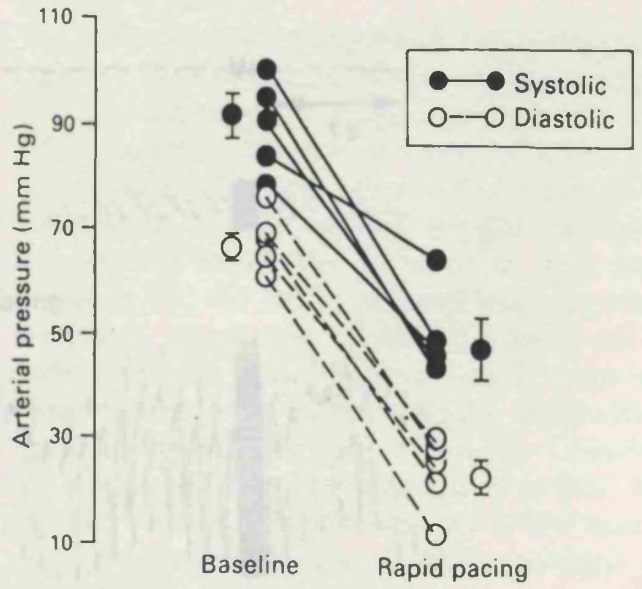

Figure 1 The effect of rapid pacing on systolic and diastolic pressures. The mean systolic pressure fell from $91 \mathrm{~mm} \mathrm{Hg}$ during sinus rhurtm at baseline to $47 \mathrm{~mm} \mathrm{Hg.} 4 \mathrm{~min} 30 \mathrm{~s}$ after the onset of rapid pacing. The corresponding changes in diastolic pressures were from $67 \mathrm{~mm} \mathrm{Hg}$ (1) $24 \mathrm{~mm} \mathrm{Hg}$. Error bars are SEM of mean values. 


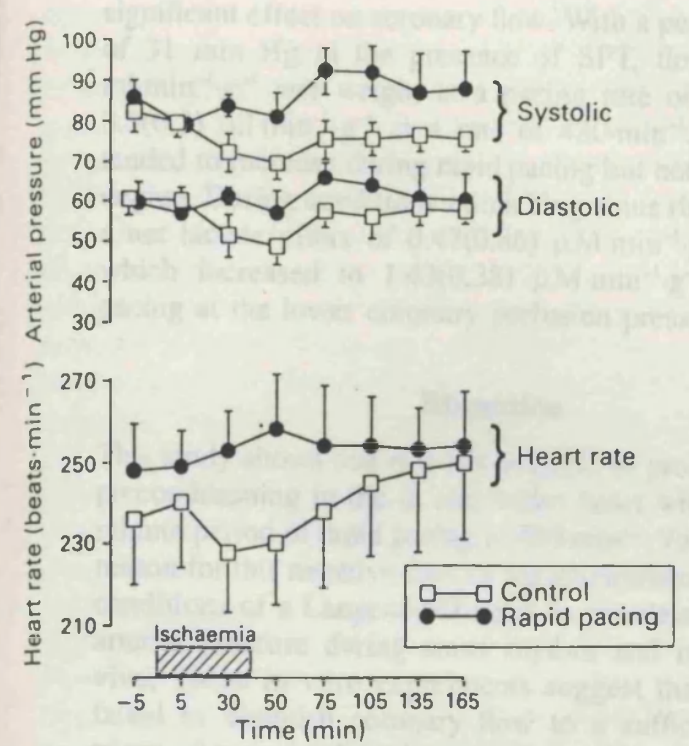

Figure 2 Arterial pressure and heart rate before, during, and fier coronary occlusion in control and rapid pacing groups. Values are means, bars $=S E M$. Although heart rate and arterial pressures vended to be higher in the rapid pacing group, there was no clearly discernable difference. Coronany occlusion was from 0 in $45 \mathrm{~min}$.

infarction should not differ between groups. Upon reperfusion, although no significant differences existed, there was a tendency for heart rate and arterial pressure to be thigher in the rapid pacing group; this may reflect the slightly smaller infarct size in this group (see fig 4).

\section{Assessment of infarct size}

The infarct size was estimated as a percentage of the volume of myocardial tissue at risk. that is, the volume of tissue subtended by the ligated coronary artery. The volume of issue at risk, the volume of tissue infarcted, and the percentage of the volume at risk infarcted were similar in all groups (fig 4).

\section{IS VITRO EXPERIMENTS}

The coronary flow while pacing at $180 \cdot \mathrm{min}^{-1}$ with a coronary perfusion pressure of $75.3 \mathrm{~mm} \mathrm{Hg}$ was $7.3(1.1) \mathrm{ml} \cdot \mathrm{min}^{-1} \cdot \mathrm{g}^{-1}$

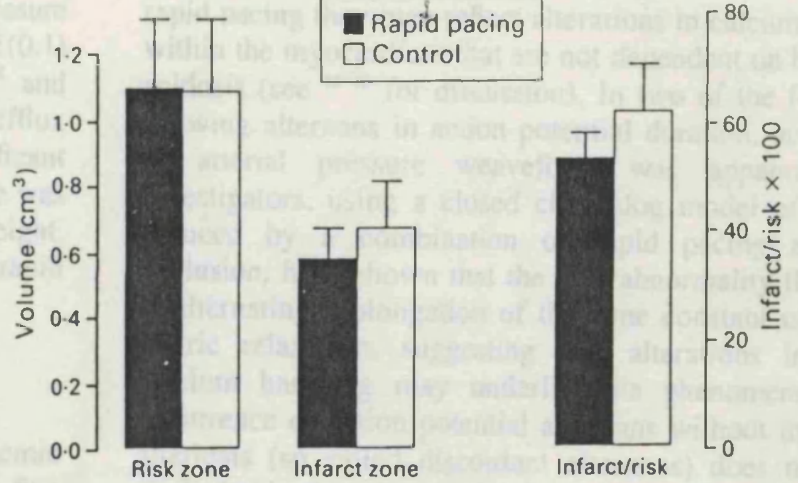

Figure 4 The size of risk and infarction zones in comrol and rapid pacing groups. Risk zones were demarcated by fluorescent microspheres and areas of infarction by incubation in triphenyltetrazolium. Columns are means, bars=SEM. No significant differences exist between groups.

wet weight. After $4.5 \mathrm{~min}$ of pacing at $480 \cdot \mathrm{min}^{-1}$. with a concomitant drop in coronary perfusion pressure to 31.4 $\mathrm{mm} \mathrm{Hg}$, the coronary flow was $3.1(0.4) \mathrm{m} \cdot \mathrm{min}^{-1} \cdot \mathrm{g}^{-1}$ wet weight. These measurements were taken with the intraventricular balloon inflated to give a left ventricular end diastolic pressure of $10 \mathrm{~mm} \mathrm{Hg}$. The coronary flows were little altered when the experimental conditions were repeated with the balloon deflated, with corresponding flows under the two conditions of $7.6(1.5)$ and $3.1(0.5) \mathrm{ml} \cdot \mathrm{min}^{-1} \cdot \mathrm{g}^{-1}$ wet weight respectively. (The changes in developed and end diastolic left ventricular pressures occurring with the onset of rapid pacing have not been presented. as the frequency response of the recording system is not sufficient to record balloon pressure reliably at a heart rate of $480 \cdot \mathrm{min}^{-1}$.)

Interestingly, with a coronary perfusion pressure of 31 $\mathrm{mm} \mathrm{Hg}$, there was little difference in coronary flow with or without rapid pacing $\left[3.1(0.4) \vee 3.4(0.2) \mathrm{ml} \cdot \mathrm{min}^{-1} \cdot \mathrm{g}^{-1}\right.$ wet weight, respectively). To exclude the possibility that coronary flow was being maintained during rapid pacing by the release of adenosine, 8(p-sulphophenyl) theophylline (SPT), at a concentration of $77 \mu \mathrm{M} \cdot$ litre $^{-1}$ (five times the $\mathrm{K}_{\text {i }}$ for the $A_{2}$ receptor), was added to the perfusate. In the four hearts treated with SPT, rapid pacing still did not have a

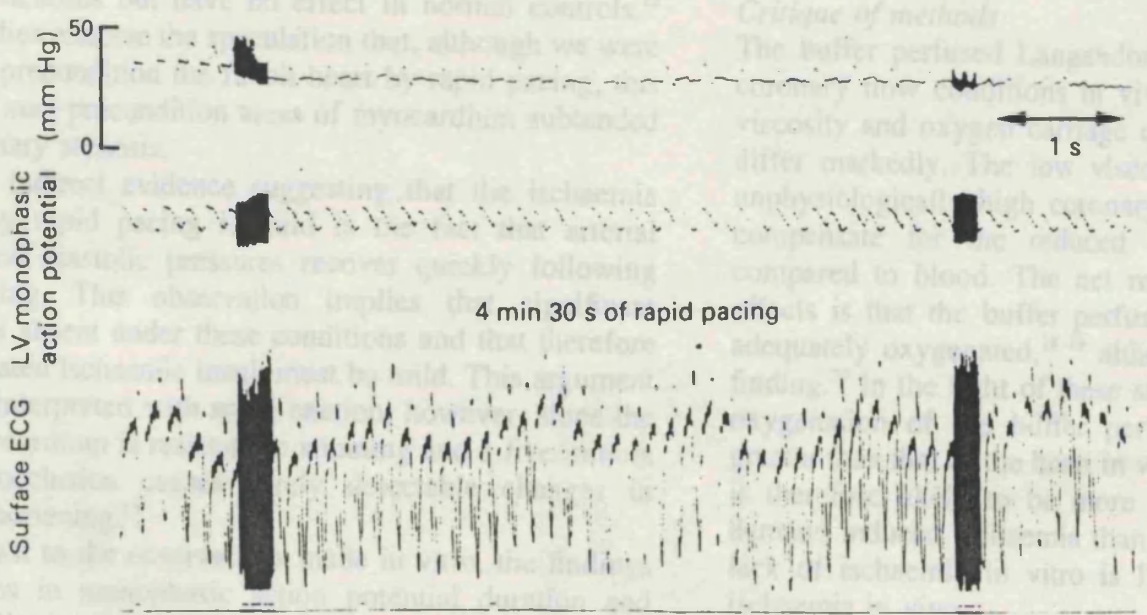

Figure 3 Arterial pressure, left ventricular (LV) monophastic action potential (MAP), and surface ECG towards the cind of the 5 min period of rapid pacing. The record on the far left was obtained at 4 min and that in the centre at 4 min 30).5 after the onset of rapid pacing. There is "concordamt alternans" with cardiac contractions of short MAP duration not giving rise to a significant pressure werceform in the carosid arten. Left atrial pacing spikes are apparent on the MAP record. The alternans see'n in MAP is not appareme on the surface tec: 
significant effect on coronary flow. With a perfusion pressure of $31 \mathrm{~mm} \mathrm{Hg}$ in the presence of SPT, flow was $4.1(0.1)$ $\mathrm{ml} \cdot \mathrm{min}^{-1} \cdot \mathrm{g}^{-1}$ wet weight at a pacing rate of $180 \cdot \mathrm{min}^{-1}$ and $3.9(0.2) \mathrm{ml} \cdot \mathrm{min}^{-1} \cdot \mathrm{g}^{-1}$ at a rate of $480 \cdot \mathrm{min}^{-1}$. Lactate efflux tended to increase during rapid pacing but not to a significant degree. During conditions mimicking sinus rhythm there was a net lactate efflux of $0.47(0.86) \mu \mathrm{M} \cdot \mathrm{min}^{-1} \cdot \mathrm{g}^{-1}$ wet weight, which increased to $1.43(0.38) \mu \mathrm{M} \cdot \mathrm{min}^{-1} \cdot \mathrm{g}^{-1}$ during rapid pacing at the lower coronary perfusion pressure.

\section{Discussion}

This study shows that it is not possible to produce ischaemic preconditioning in the in situ rabbit heart with a single five minute period of rapid pacing at $480 \cdot \mathrm{min}^{-1}$. To investigate the reason for this negative finding we manipulated the perfusion conditions of a Langendorff heart to simulate heart rate and arterial pressure during sinus rhythm and rapid pacing in vivo. These in vitro experiments suggest that rapid pacing failed to diminish coronary flow to a sufficient degree to trigger the preconditioning response.

The mechanisms involved in ischaemic preconditioning are currently a subject of intense investigation. Work from Downey's laboratory suggests that in the rabbit the $A_{1}$ subclass of adenosine receptors plays a central role in the preconditioning phenomenon. ${ }^{4}$ The currently favoured hypothesis, proposed by this laboratory, is that adenosine released during the preconditioning occlusion activates the $A_{1}$ receptor, which, coupled to the inhibitory type of $G$ protein, initiates the preconditioning response. ${ }^{10}$ For rapid pacing to result in preconditioning it would therefore require ischaemia of sufficient severity to cause ATP breakdown and adenosine release. Although the changes in coronary flow in the buffer perfused heart will only approximate to conditions in vivo, we had no direct evidence of substantial ischaemia. In particular there was no significant lactate efflux, or indirect evidence of adenosine efflux in terms of coronary vasoconstriction in the presence of SPT. These findings are in keeping with those of other investigators, who have shown that even in the presence of critical bilateral coronary stenoses the ischaemia induced by rapid pacing is milder than that induced by coronary occlusion. "Similarly, rapid pacing regimens in the human are often capable of inducing objective and subjective cardiac ischaemia in the presence of coronary stenoses but have no effect in normal controls. ${ }^{12}$ These studies endorse the speculation that, although we were unable to precondition the rabbit heart by rapid pacing, this technique may precondition areas of myocardium subtended by a coronary stenosis.

Further indirect evidence suggesting that the ischaemia induced by rapid pacing is mild is the fact that arterial systolic and diastolic pressures recover quickly following rapid pacing. This observation implies that significant stunning is absent under these conditions and that therefore any associated ischaemic insult must be mild. This argument has to be interpreted with some caution, however, since the rabbit myocardium is resistant to stunning and a five minute coronary occlusion causes barely detectable changes in segment shortening. ${ }^{13}$

In contrast to the observations made in vitro, the findings of alternans in monophasic action potential duration and pulse amplitude seen in the in vivo rabbit heart do seem suggestive of ischaemia. However the aetiology of these phenomena is not well understood, and although their appearance at slow heart rates can be indicative of acidosis or ischaemia, ${ }^{14}$ at the faster heart rates occurring during rapid pacing they may reflect alterations in calcium handling within the myocardium that are not dependent on hypoxia or acidosis (see ${ }^{15} 16$ for discussion). In two of the four hearts showing alternans in action potential duration. no variation in arterial pressure weaveform was apparent. Other investigators, using a closed chest dog model of alternans induced by a combination of rapid pacing and caval occlusion, have shown that the first abnormality that occurs is alternating prolongation of the time constant of isovolumetric relaxation, suggesting that alterations in cellular calcium handling may underlie this phenomenon. ${ }^{16}$ The occurrence of action potential alternans without mechanical alternans (so called discordant alternans) does not necessarily imply that changes in action potential duration are an earlier indication of the onset of alternans than mechanical events, since the relationship between these two forms of alternans is far from simple, and is further complicated by open chest preparations where inhomogeneity of temperature may alter regional susceptibility to electrical alternans. ${ }^{15}$

There are two important possibilities to explain the difference in findings between this study and that of Vegh $e t$ $a l .{ }^{8}$ The first is that the degree of cardiac ischaemia induced by pacing was less in our study. However, in Vegh's study the fall in blood pressure at the onset of rapid pacing was in fact less marked than we observed. These investigators showed that on rapid pacing at $300 \cdot \mathrm{min}^{-1}$ (from a resting heart rate of 141), systolic pressure fell from 125 to 87 $\mathrm{mm} \mathrm{Hg}$ and diastolic pressure from 79 to $38 \mathrm{~mm} \mathrm{Hg}$. In comparison, with rapid pacing in our model there was a fall in systolic pressure from 91 to $47 \mathrm{~mm} \mathrm{Hg}$ and in diastolic pressure from 67 to $24 \mathrm{~mm} \mathrm{Hg}$. A less severe ischaemic insult is therefore unlikely to be the explanation for our negative findings. The second and more likely possibility is that the protection seen with preconditioning differs according to whether arrhythmia or infarct size are used as endpoints. For example in a preliminary report Lawson ${ }^{17}$ has shown that a dose dependent relationship exists between the number of preconditioning occlusions and reperfusion induced arrhythmias, while no such relationship exists for infarction. ${ }^{+}$Other possible suggestions to explain the differences between these two studies include species variation, differing preconditioning protocols, and differing lengths of ischaemic insult.

\section{Critique of methods}

The buffer perfused Langendorff heart was used to mimic coronary flow conditions in vivo during rapid pacing. The viscosity and oxygen carriage capacity of blood and buffer differ markedly. The low viscosity of buffer gives rise to unphysiologically high coronary flow rates which partially compensate for the reduced oxygen carriage of buffer compared to blood. The net result of these two opposing effects is that the buffer perfused heart is probably barely adequately oxygenated, ${ }^{18}{ }^{19}$ although this is not a universal finding. ${ }^{20}$ In the light of these studies it seems unlikely that oxygenation of the buffer perfused Langendorff heart is greater than that of the heart in vivo. The Langendorff model is therefore likely to be more prone to flow induced and demand induced ischaemia than the in situ heart and so the lack of ischaemia in vitro is likely to reflect the lack of ischaemia in vivo.

Another possible explanation for our negative findings is that more than one cycle, a longer period of rapid pacing, or a shorter period of more rapid pacing are required for the preconditioning trigger. In previous studies a single five minute coronary occlusion was sufficient to precondition the 
heart even in a well collateralised species such as the dog, ${ }^{4}$ where on average the ischaemic bed will still receive $16 \%$ of mormal perfusion during coronary ligation. ${ }^{21}$ It is therefore posisible that a five minute rapid pacing regime might trigger precconditioning if it reduced global myocardial flow to a similar degree. We were barely able to halve coronary flow with a pacing regime that caused severe haemodynamic changes and it therefore seems unlikely that with normal coronary vasculature the degree of coronary flow reduction required to precondition against infarction can be achieved unliess the period of rapid pacing is drastically extended. For example, Ovize et $a^{22}$ have recently shown that the dog heart can be preconditioned by a $50 \%$ reduction in coronary flow sustained for 15 minutes, while in the same species a similar reduction in infarct size can be achieved with a single five minute period of total occlusion, ${ }^{4}$ a partial effect being evident with a 2.5 minute occlusion. ${ }^{23}$ The question remains as to whether the tendency towards smaller infarcts in the rapid pacing group represents a subtotal preconditioning response which would have become more obvious with repetitive or more prolonged periods of rapid pacing. With a group size of only five in the rapid pacing group it would be possible to "miss" a subtotal preconditioning response but on the basis of power calculations the probability of missing the impressive infarct size reduction seen in classical preconditioning is very remote. ${ }^{24}$

\section{Conclusion}

Rapid pacing for five minutes to a heart rate of $480 \cdot \mathrm{min}^{-1}$ fails to precondition the in situ rabbit heart. despite resulting in profound hypotension. The most likely reason for this failure is that the ischaemia that results during rapid pacing is far less severe than the ischaemia present during coronary occlusion and is insufficient to trigger a preconditioning response.

Dr M S Marber is the recipient of an Intermediate Fellowship from the British Heart Foundation. We are grateful to the Hatter Foundation for its continued support. The MAP electrode used for this study was a gift from Dr Max Lab. Department of Physiology. Charing Cross and Westminster Medical School. London.

Key terms: rapid pacing: ischaemic preconditioning: infarction.

Received 20 August 1992; accepted 30 October 1992. Time for primary review 22 days.

1 Walker DM. Yellon DM. Ischaemic preconditioning: from mechanisms to exploitation. Cardiov'asc Res 1992;26:734-9.

2 Murry CE. Jennings RB, Reimer KA. New insights into potential mechanisms of ischemic preconditioning. Circulation 1991: 84:442-5.

3 Murry CE, Jennings RB, Reimer KA. Preconditioning with ischaemia: a delay of lethal cell injury in ischemic myocardium. Circulation 1986;74:1124-36.
4 Li GC, Vasquez BS, Gallagher KP, Lucchesi BR. Myocardial protection with preconditioning. Circulation 1990;82:609-19.

5 Shiki K. Hearse DJ. Preconditioning of ischemic myocardium: reperfusion induced arrhythmias. Am J Physiol 1987; 253: $\mathrm{H} 1470-6$.

6 Hagar JM, Hale SL, Kloner RA. Effect of preconditioning ischemia on reperfusion arrhythmias after coronary artery occlusion and reperfusion in the rat. Circ Res 1991;68:61-8.

7 Shizukada Y, Mallet RT, Lee SC, Downey HF. Hypoxic preconditioning of ischaemic canine myocardium. Cardiovasc Res 1992;26:534-42

8 Vegh A, Szekeres L, Parrat JR. Transient ischaemia induced by rapid cardiac pacing results in myocardial preconditioning Cardiovasc Res 1991;25:1051-3.

9 Liu GS, Thornton J, Van Winkle DM, Stanley AWH, Olsson RA, Downey JM. Protection against infarction afforded by preconditioning is mediated by $\mathrm{Al}$ adenosine receptors in rabbit heart. Circulation 1991;84:350-6.

10 Thornton J, Downey JM. G $G_{i}$ proteins are involved in preconditioning's protective effect. (Abstract) Circulation 1991: 84(suppl II): 19.

Il Applegate RJ, Walsh RA, O'Rourke RA. Comparative effects of pacing induced and flow-limited ischemia on left ventricular function. Circulation 1990;81:1380-92.

12 Montalescot G, Drobinski G, Maclouf J, el al. Evaluation of thromboxane production and complement activation during myocardial ischemia in patients with angina pectoris. Circulation 1991;84:2054-62.

13 Knowlton AA. Brecher P. Apstein CS. Rapid expression of heat shock protein in the rabbil after brief cardiac ischemia. $J$ Clin lnvest 1991:87:139-47.

14 Orchard $\mathrm{CH}$. McCall E, Kirby MS. Boyell MR. Mechanical alternans during acidosis in ferret heart muscle. Circulation 1991:68:69-76

15 Dilly SG. Lab MJ. Electrophysiological alternans and restitution during acule regional ischaenia in myocardium of anatesthetized pig. J Phwiol (Lond) 1988:402:315-33.

16 Freeman GL. Widman LE, Camphell JM. Colston JT. An evaluation of pulses alternans in closed-chest dogs. Am I P/hsiol 1992:262:14278-84

17 Lawson CS. Dose-dependency for the reduction of reperfusioninduced arrhythmias by ischacmic preconditioning in the isolated blood-perfused rat heart. (Abstract) I Mol Cell Cardiol 1992:24(suppl I):SI48.

18 Paradise NA. Surmitis JM Machall CI O, reserve the left ventricle of isolated, saline-perfused rabbit heart. Am J Phiviol 1984:247: $\mathrm{H} 86 \mathrm{I}-8$.

19 Chemnitius JM, Burger W, Bine RJ. Crystalloid and perfluorochemical perfusates in an isolated working rabbit heart preparation. Am J Phy:siol 1985:249:H285-65.5

20 Murashita T. Kempsford RD. Hearse DJ. Oxygen supply and oxygen demand in the isolated wothing rabbit heart perfused with asanguineous crystalloid solution. Cardiendase Re's |yc). 25:198-206.

21 Maxwell MP. Hearse DJ. Yellon DM. Species variation in the coronary collateral circulation during regional myociadial ischaemia: a critical determination of the rate of evolution and extent of myocardial infarction. Cardioncusc Re's 1987; 21:737-46.

22 Ovize M. Przyklenk K. Kloner RA. Partial coronary stenosis is sufficient and complete reperfusion is mandatory for preconditioning the canine heart. Circ Re's 1992:71:1165-7.3.

23 Ovize M, Przyklenk K, Hale SL, Kloner RA. Preconditioning does not attenuate myocardial stunning. Circulation 1992:85: 2247-54.

24 Altman DG. Practical statistics for medical reseurch. London: Chapman and Hall, 1991:455-60.

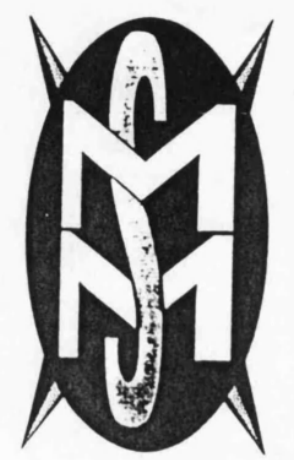




\section{Attenuation by Heat Stress of a Submaximal Calcium}

\section{Paradox in the Rabbit Heart.}

Michael S Marber, J Malcolm Walker, David S Latchman, Derek M Yellon.

From The Hatter Institute For Cardiovascular Studies, Division of Cardiology

(M.S. Marber, J.M. Walker, D.M. Yellon), and the Division of Molecular Pathology

(D.S. Latchman), The University College London Medical School, London, UK.

Correspondence to:

Dr Derek Yellon

Director and Head of Division

The Hatter Institute for Cardiovascular Studies

University College Hospital

Gower Street

London WCIE 6AU. ENGLAND

Tel 071-380-9888 Fax 071-388-5095

RUNNING HEAD: Heat Stress and the Calcium Paradox

Accepted May 1993 for Publication In the J Mol Cell Cardiol 19 pages of text and 5 figures 


\title{
The Protective Role of Heat Stress in the Ischaemic and Reperfused Rabbit Myocardium
}

\author{
Derek M. Yellon, Evasio Pasini*, Anna Cargnoni*, Michael S. Marber, \\ David S. Latchman** and Roberto Ferrari* \\ The Halter Institute for Cardiovascular Studies, Department of Academic Cardiology, University College
Hospital and \\ The Department of Biochemistry, University College $\mathcal{E}^{\circ}$ Middlesex School of Medicine, London, UK, \\ and * The Department of Cardiology, University of Brescia, Italy
}

(Received 1 August 1991; accepted in revised form 13 March 1992)

\begin{abstract}
Derek M. Yellon, Evasio Pasini, Anna Cargnoni, Mighael S. Marber, David S. Latchman, Roberto Ferrari. The Protective Role of Heat Stress in the Ischaemic and Reperfused Rabbit Myocardium. Journal of Molecular and Cellular Cardiology (1992) 24, 895-908. Cells subjected to increases in temperature induce the expression of several proteins known as heat shock or stress proteins. This process enhances the cell's ability to overcome the effects of further stress. In this respect, the effects of heat stress have been reported to protect the hearts of rats following ischaemia and reperfusion. We have confirmed and extended this observation, not only using different indices of myocardial injury but also in another species, namely the rabbit. Animals were anaesthetized and the body temperature raised $1042^{\circ} \mathrm{C}$ for a $15-\mathrm{min}$ period. Controls were treated in the same way but without heating. Twenty-four hours later the rabbits were re-anaesthetized and the hearts removed for either heat stress protein analysis or perfusion with Krebs buffer using an isolated perfused heart apparatus. Hearts were subjected to $60 \mathrm{~min}$ of low flow $(1 \mathrm{ml} / \mathrm{min})$ ischaemia followed by $30 \mathrm{~min}$ of reperfusion. All hearts subjected to heat stress showed an enhanced recovery of function upon reperfusion as measured by improvements in developed pressure $(27.3 \pm 3.6 \mathrm{is} 16.3 \pm 3.0 \mathrm{mmHg})$ and diastolic pressure $(37.3 \pm 7.4$ vs $54.7 \pm 3.1 \mathrm{mmHg}$ ). In addition, creatine kinase release, associated with reperfusion, was significantly reduced in the heat-stressed hearts $(532 \pm 102$ vs $1138 \pm 73 \mathrm{mU} / \mathrm{min} / \mathrm{g}$ wet wt). Myocardial accumulation and release of oxidized glutathione, an index of oxidative stress, was significantly reduced in the heat-stressed group $0.003 \pm 0.003$ vs $0.376 \pm 0.113 \mathrm{nmol} / \mathrm{min} / \mathrm{g}$ wet $\mathrm{wt})$. The improved metabolic status of the reperfused heatstressed hearts was further demonstrated by a significant conscrvation in the levels of ATP $(6.1 \pm 0.9$ vs $2.8 \pm 0.8 \mu \mathrm{mol} / \mathrm{g} \mathrm{dry} w \mathrm{t})$ and CP $(36.9 \pm 6.4$ vs $16.4 \pm 5.1 \mu \mathrm{mol} / \mathrm{g}$ dry $w \mathrm{t})$. Finally, isolated mitochondrial function in terms of respiratory control index (RCI) was maintained in the heat-stressed hearts $(9.2 \pm 0.9$ vs $5.7 \pm 0.2)$ and overloading with calcium was reduced. These data extend the hypothesis that heat stress protects the heart following ischaemia and reperfusion in this in vitro model, in a way as yet undetermined.
\end{abstract}

Key Words: Heat stress; Heat shock; Ischaemia; Reperfusion.

\section{Introduction}

Developing therapeutic means of protecting the ischaemic myocardium has been the goal of cardiologists for over two decades. During this period numerous therapeutic interventions and novel agents have been exhaustively tested but with minimum success (Maroko et al., 1971; Hearse and Yellon, 1984; Hearse $e t$ al., 1986). It is now realized that other than early restoration of coronary blood flow, there is little that can be done in order to salvage the ischaemic myocardium and reduce or limit the ultimate size of an evolving myocardial infarct (Braunwald, 1985; Yellon and Downey, 1990).

It has been demonstrated that hyperthermia induces the synthesis, in the heart and other tissues, of a small group of proteins known as the heat shock or heat stress proteins (HSP) (Currie and White, 1983; Lindquist, 1986). The cells that are subjected to such a stress, and which accumulate these heat stress proteins appear to acquire a transient resistance to further episodes of oxidative stress

Please address all correspondence to: Derek M. Yellon, Director, The Hatter Institute for Cardiovascular Studies, Department of Academic Cardiology, University College Hospital, Gower Street, London WCıE 6AU, UK. 
(Currie el al., 1988). Recent in vitro evidence indicates that this stress response is associated with an enhanced post-ischaemic ventricular recovery of function in the isolated Krebs perfused rat heart (Currie el al., 1988). In addition, Currie and colleagues (1988) also demonstrated that endogenous levels of the anti-oxidant catalase were significantly raised in the heat-stressed animals which they speculate may play a significant role in the overall protective mechanism of the HSPs.

However, as yet, little is understood regarding the exact mechanism of the protection conferred by the expression of these proteins. In addition, no other studies have been undertaken which either confirm or deny these interesting findings. In the light of the above we have repeated the study using an isolated perfused heart model to ascertain if heat stress is indeed associated with a protective effect on the ischaemic/reperfused heart. We have examined this heat stress (HS) response using additional indices of myocardial injury such as the effect HS has on oxidative stress, measured in terms of reduced and oxidized glutathione metabolism; the effect of HS on mitochondrial integrity; the relationship of HS to high-energy phosphate conservation as well as the effect of HS on myocardial function following ischaemia and reperfusion. In addition, these studies of HSP synthesis and its associated cardiac protection, using the ischaemic/reperfusion heart model, were extended to another species, namely the rabbit.

\section{Materials and Methods}

Eighty-two adult male New Zealand white rabbits $(2.5-3.0 \mathrm{~kg}$ body weight) maintained on a standard diet, were used for this study. Of these, 35 rabbits ( 18 control and $17 \mathrm{HS}$ ) were used for the determination of isolated mitochondrial function. A further 35 rabbits (18 control and $17 \mathrm{HS}$ ) were used for the determination of the ATP, CP, GSSG and GSH tissue content and haemodynamic data. Twelve rabbits ( 6 control and 6 HS) were used for the analysis of the synthesis of the HSP70.

\section{Heat stress protocol}

The rabbits were anaesthetized with sodium pentobarbital $(50 \mathrm{mg} / \mathrm{kg}$ iv) and placed on a temperature-controlled heating blanket. Body temperature was allowed to rise to $42^{\circ} \mathrm{C}$ and monitored throughout using a rectal thermometer. The elevated temperature was maintained for $15 \mathrm{~min}$ at $42^{\circ} \mathrm{C}$ following which the animal was allowed to recover to normal physiological temperature. Control animals were treated in the same manner but without heating.

\section{Heart perfusion}

Twenty-four hours after the initial rise in body temperature the rabbits were stunned by a blow to the neck. The hearts were removed and immersed in ice-cold modified KrebsHenseleit buffer. The aorta was then exposed and suspended on a metal cannula. The hearts were perfused using a non-recirculating Langendorff perfusion system using a modified Krebs-Henseleit buffer solution containing $1.5 \mathrm{~mm}$ calcium as previously described (Ferrari et al., 1985). The buffer solution was delivered to the aortic cannula at $37^{\circ} \mathrm{C}$ and at a perfusion pressure of $60-80 \mathrm{mmHg}$, maintained with a Watson Marlow rotary pump (MHRE MK3). The hearts were then paced at 180 beats/min using suprathreshold rectangular pulses of $1.0 \mathrm{~ms}$ duration and perfused under aerobic conditions (coronary flow $22 \pm 0.5 \mathrm{ml} / \mathrm{min}$ ) for a $30 \mathrm{~min}$ equilibration period. Thereafter, the hearts were either perfused under aerobic conditions for a further $90 \mathrm{~min}$, or made ischaemic by reducing the coronary flow from 22 to $1 \mathrm{ml} / \mathrm{min}$ for $60 \mathrm{~min}$. In separate groups of experiments, the hearts, following $60 \mathrm{~min}$ of ischaemia, were reperfused for $30 \mathrm{~min}$ at a coronary flow of $22 \mathrm{ml} / \mathrm{min}$. Left ventricular wall temperature was maintained at $37^{\circ} \mathrm{C}$, irrespective of coronary flow.

\section{Left ventricular pressure}

To obtain an isovolumetrically beating preparation a fluid-filled balloon was inserted into the left ventricle via the left atrium. The intraventricular balloon was connected by a fluid-filled polyethylene catheter to a Statham pressure transducer ( $\mathrm{P} 23 \mathrm{Db})$ for the determination of left ventricular pressure, as previously described (Ferrari et al., 1991). 
Coronary effluent analysis and CPK determination

During each perfusion the coronary effluent was collected in chilled glass vials and assayed, on the same day, for creatine kinase (CK) and reduced (GSH) and oxidized (GSSG) glutathione. CK activity was determined spectrophotometrically by the method of Oliver (1955).

\section{Reduced (GSH) and oxidized (GSSG) gluthathione determination}

GSH and GSSG were analysed by the method of Tietze (1969). The reaction mixture $(1.0 \mathrm{ml})$ contained: $0.05 \mathrm{M}$ potassium phosphate buffer, pH 7.4, $1 \mathrm{~mm}$ EDTA, $0.1 \mathrm{~mm} 5$ 5 '-dithiobis-(2 nitrobenzoic acid) (DTNB), $0.15 \mathrm{~mm}$ NADP and an appropriate volume of sample. After $2 \mathrm{~min}$ of pre-incubation, the reaction was initiated by the addition of $1 \mathrm{U}$ of glutathione reductase and the rate of reduction of the DTNB was monitored continuously at $412 \mathrm{~nm}$; the slope was proportional to the glutathione concentration over the range of $0-1 \mu \mathrm{m}$. GSSG was measured as described above after the preliminary reaction of GSH with $20 \mathrm{~nm}$ N-ethylmaleimide, followed by complete removal of untreated sulphydryl reagent with diethylether.

\section{Isolation of mitochondria}

Mitochondria were isolated at the end of each perfusion by differential centrifugation as previously described (Ferrari and Williams, 1986). Two different isolation media were used. The mitochondria required for oxygen consumption studies were isolated in the medium described by Sordhal et al. (1973) and containing $180 \mathrm{~mm} \mathrm{KCl}, 10 \mathrm{~mm}$ EDTA and $0.5 \%$ bovine serum albumin (BSA). The mitochondria used for the determination of endogenous calcium and of ATP production were extracted in a medium containing $250 \mathrm{~mm}$ sucrose and $5 \mu \mathrm{M}$ ruthenium red (Peng et al., 1977). Ruthenium red, an inhibitor of mitochondrial calcium uptake, was included to prevent calcium accumulation during the isolation procedure (Ferrari and Williams, 1986; Ferrari et al., 1982).
Mitochondrial oxygen consumption measurements

Rates of oxygen consumption were monitored polarographically at $25^{\circ} \mathrm{C}$ using a Clark-type electrode; $1.25 \mathrm{mg} / \mathrm{ml}$ of mitochondrial protein was suspended in $2 \mathrm{ml}$ of an incubation solution containing $250 \mathrm{~mm}$ sucrose, $3 \mathrm{~mm}$ potassium glutamate, $\mathrm{pH} 7.4$, adjusted with Tris and allowed to equilibrate for $1 \mathrm{~min}$. State 3 respiration was initiated by adding $0.5 \mathrm{~mm}$ ADP.

Mitochondrial function was assessed in terms of RCI, QO2 and ADP/O, where RCI (respiratory control index) is the ratio of oxygen used in the presence of ADP to that used in the absence of ADP; QO2 are natoms of oxygen used/mg of mitochrondrial protein/ $\mathrm{min}$ in response to the addition of ADP; and $\mathrm{ADP} / \mathrm{O}$ are $\mathrm{nmol}$ of $\mathrm{ADP}$ used/natoms of oxygen consumed.

\section{Protein determination}

Mitochondrial protein concentration was determined by the method of Bradford (1978) using BSA as standard.

\section{Milochondrial ATP production}

ATP synthesis was initiated by adding $0.5 \mathrm{~mm}$ ADP to the same incubation solution as described for the measurement of oxygen consumption, containing $1.25 \mathrm{mg} / \mathrm{ml}$ of mitochondrial protein

Samples, $200 \mu \mathrm{l}$, were taken before and at 6 , $15,30,45,60,120$ and $180 \mathrm{~s}$ after adding ADP. They were then mixed with $50 \mu \mathrm{l}$ of $10 \%$ perchloric acid on ice.

Precipitated protein was separated by centrifugation and the ATP content of the supernatant was determined enzymatically using the method of Lamprecht and Trautschold (1974). The total amount of ATP produced was calculated as the ATP present in the reaction chamber $15 \mathrm{~s}$ after the transition from state 3 to state 4 respiration.

\section{Mitochondrial calcium content}

Calcium content was determined by atomic absorption spectrometry. Mitochondrial pel-

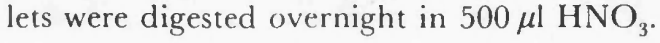


Lanthanum chloride was added to provide a final concentration of $0.1 \% \cdot \mathrm{CaCO}_{3}$ was used as a standard.

\section{Tissue calcium}

At the end of the perfusion period, duplicate samples of left ventricular muscle were taken and digested in $1 \mathrm{ml} \mathrm{HNO}_{3} / \mathrm{g}$ weight tissue. The extracts were diluted in $25 \mathrm{ml}$ of deionized water and the calcium content was assayed as described above. Total tissue water was obtained by drying samples to constant weight at $95^{\circ} \mathrm{C}$.

\section{Tissue ATP and CP determination}

The perfusion of hearts in which ATP and CP levels were determined was terminated by immediate freeze-clamping of the left ventricular apex with aluminium tongs kept at liquid nitrogen temperature. Tissue was stored, ground and mixed with $0.4 \mathrm{~N}$ perchloric acid (approximately $3 \mathrm{ml} / 500 \mathrm{mg}$ ) at $-195^{\circ} \mathrm{C}$. The mixture was transferred from the mortar to a tube and allowed to thaw while stirring on a Vortex mixer. It was further homogenized four times at $0-4^{\circ} \mathrm{C}$ with an Ultra-turrax for $3-5 \mathrm{~s}$, interspaced with $30 \mathrm{~s}$ cooling periods. The homogenate was then centrifuged at $3000 \mathrm{~g}$ for $10 \mathrm{~min}$ at $4^{\circ} \mathrm{C}$. The supernatant fluid was adjusted to $\mathrm{pH} 6.0$ 6.5 with $6 \mathrm{~N} \mathrm{KOH}$. After removal of $\mathrm{KCIO}_{4}$ the extract was used for analysis of highenergy phosphates. A separate aliquot of tissue was dried at $100^{\circ} \mathrm{C}$ to determine dry weight.

ATP and CP were determined with the HPLC method described by Stocchi el al. (1987). The HPLC system included a Waters model $600 \mathrm{E}$ multisolvent delivery system and model 990 photodiode array detector. Separations were performed on a Supelchem C18 $3 \mu \mathrm{m}$ reverse-phase column $(0.46 \times 15 \mathrm{~cm})$. The mobile phase consisted of a gradient of buffer $\mathrm{A}\left(0.1 \mathrm{M} \mathrm{K \textrm {K } _ { 2 }} \mathrm{PO}_{4}, 5 \mathrm{~mm}\right.$ tetrabutylammonium hydrogensulphate, $25 \%(\mathrm{v} / \mathrm{v})$ acetonitril, $\mathrm{pH} 5.5)$ and buffer $\left(0.1 \mathrm{M} \mathrm{KH}_{2} \mathrm{PO}_{4}\right.$, $5 \mathrm{~mm}$ tetrabutylammonium hydrogensulphate, $2.5 \%$ acetonitril, $\mathrm{pH} 6.0$ ). The column was eluted at $0.8 \mathrm{ml} / \mathrm{min}$, usually for $3 \mathrm{~min}$ with buffer A, 2 min with buffer $A$ plus buffer $\mathrm{B}$, increasing to $11 \%$ and $25 \mathrm{~min}$ with a further gradual increase of buffer B to $100 \%$. The column was then equilibrated again for 20 min with $100 \%$ buffer A. Detection was performed at $205 \mathrm{~nm}$ for CP and $260 \mu \mathrm{m}$ for ATP.

$$
\begin{gathered}
\text { Delermination of tissue GSH, GSSG and } \\
\text { sulphydryl groups }
\end{gathered}
$$

For GSH and GSSG determination, $100 \mathrm{mg}$ of tissue was deproteinized with $3 \mathrm{M}$ perchloric acid and the supernatant obtained after centrifugation at $6000 \mathrm{~g}$ for $15 \mathrm{~min}$ was neutralized with $2 \mathrm{M} \mathrm{K}_{2} \mathrm{CO}_{3}$. A sample of the neutralized extract was analysed for GSH and GSSG as described above.

For the determination of protein sulphydryl groups a portion of the left ventricle was homogenized as described by Sedlack and Lindsay (1968), with $20 \mathrm{~mm}$ EDTA pH 7.4, and filtered on nylon mesh. Samples of homogenates were mixed with $0.2 \mathrm{M}$ Tris- $\mathrm{HCl}$, $0.01 \mathrm{M} \mathrm{DTNB}$ and methanol to a final volume of $10 \mathrm{ml}, \mathrm{pH} 8.2$. A reagent blank (without sample) and a sample blank (without DTNB) were prepared in a similar manner. The mixture was incubated for $30 \mathrm{~min}$ at room temperature. Absorbance was read at $412 \mathrm{~nm}$ and a molar extinction coefficient of $13600 \mathrm{~mm}$ / $\mathrm{cm}$ was used. The acid-soluble thiol group content (as an expression of non-protein sulphydryl groups) was determined in the same manner in the supernatant fluid obtained after denaturation of the homogenate with ice-cold 50\% trichloro acetic acid (TCA).

Protein-SH groups were determined by subtracting the acid soluble from the total $\mathrm{SH}$ group content.

\section{Heal stress protein assay}

Samples of myocardium were prepared from apical $(n=10)$ and atrial $(n=2)$ tissue of rapidly frozen hearts by homogenizing approximately $100 \mathrm{mg} / \mathrm{ml}$ (wet wt) in $2 \times$ concentrated SDS-PAGE sample buffer (without 2 -mercaptoethanol). Following the addition of 2-mercaptoethanol (to $20 \%$ ) and bromophenol blue, samples were heated over a boiling water bath for $10 \mathrm{~min}$ and centrifuged at $11500 \mathrm{~g}$ for $5 \mathrm{~min}$. Samples were diluted where appropriate with complete $2 \times$ concentrated sample buffer such that approximately 
$45 \mu \mathrm{g}$ of total protein was loaded per lane.

Proteins were separated by SDS-PAGE on $0.8 \mathrm{~mm}$ thick $12.5 \%$ acrylamide gels according to Laemmli (1970). Two identically loaded gels were prepared with 6 heat-stressed and control samples interposed by molecular weight markers. The proteins on one gel were transferred to nitrocellulose by Western blotting. The other identical gel was stained with Coomassie Brilliant Blue R250 (BDH) to visualize the proteins.

Following transfer the nitrocellulose filters were incubated with mouse monoclonal antisera cross-reactive to the $72 \mathrm{kD}$ HSP 70 (Stressgen) at 1:2000 dilution. Second antibody was horseradish peroxidase-conjugated rabbit anti-mouse antisera (DAKO) at 1:2500 dilution. The filter was developed using enhanced chemiluminescence (ECL) detection (Amersham) and exposed to X-ray film for the appropriate time.

The relative levels of HSP 70 were determined using densitometry (Biorad model 620 video densitometer with Biorad analyst 2 version 3.1 software) normalizing to the actin band on the Coomassie stained gel. This procedure adjusts for slight variations in protein loading between samples.

\section{Experimental compounds and statistical evaluation}

The reagents were of reagent grade quality. All enzymes used for the biochemical determination were obtained from Sigma Company (USA).

The data are expressed as means IS.E.M. of $n$ experiments where each experiment is an individual perfusion or $\mathrm{HS}$ determination For statistical evaluation of the results a multiple group comparison was performed by analysis of variance followed by Student's $t$ test for paired or unpaired data with Bonferroni's corrections, taking $P<0.05$ as the limit of significance.

\section{Results}

Preliminary control studies confirmed our previously reported findings as to the stability of the preparation and indicated that the (30 min) anaesthetization of the animals $24 \mathrm{~h}$ before isolation of their hearts did not pro- duce any alteration in any of the parameters considered in this study.

\section{Mechanical function}

The developed and diastolic pressure for the isolated perfused rabbit hearts are shown in Figures $\mathrm{l}(\mathrm{a})$ and (b), respectively. In control hearts reduction of coronary flow to $1 \mathrm{ml} / \mathrm{min}$ induced a rapid decline of developed pressure. Diastolic pressure began to rise progressively $20 \mathrm{~min}$ after the onset of ischaemia and after $60 \mathrm{~min}$ it had increased to $28.0 \pm 6.1 \mathrm{mmHg}$. Readmission of coronary flow resulted in a rapid, further increase of diastolic pressure, reaching a peak $20 \mathrm{~min}$ after reperfusion with only a $33 \%$ recovery of developed pressure.

Heat shock of the rabbits the day before the experiment did not significantly modify the pressure developed by the isolated heart during the $30 \mathrm{~min}$ before the onset of ischaemia, but it increased the recovery of developed pressure on reperfusion to $71 \%(P<0.01)$. The rise in diastolic pressure during reperfusion was also significantly reduced.

\section{Creatine kinase ( $\mathrm{CK}$ )}

When the hearts were perfused under aerobic conditions for $120 \mathrm{~min}$ only small amounts of CK were present in the coronary effluent (data not shown). In control hearts, due to the severe reduction in coronary flow, ischaemia did not cause any CK release. Reperfusion of these hearts, however, resulted in a marked and sustained exacerbation of the rate of $\mathrm{CK}$ release in to the coronary effluent [Fig. l(c)].

The hearts from heat-shocked rabbits demonstrated a significant and sustained reduction in enzyme release caused by reperfusion as seen in Figure l(c).

\section{Mitochondrial respiration}

Table 1 and Figure 2 summarize the data related to the function of mitochondria isolated from the two groups. Mitochondria harvested from control hearts after $60 \mathrm{~min}$ of ischaemia showed, as expected, a depressed yield, RCI, QO2 but normal ADP/O (near to the expected value of 3 ). Reperfusion of these hearts resulted in a further decline of these indices. 

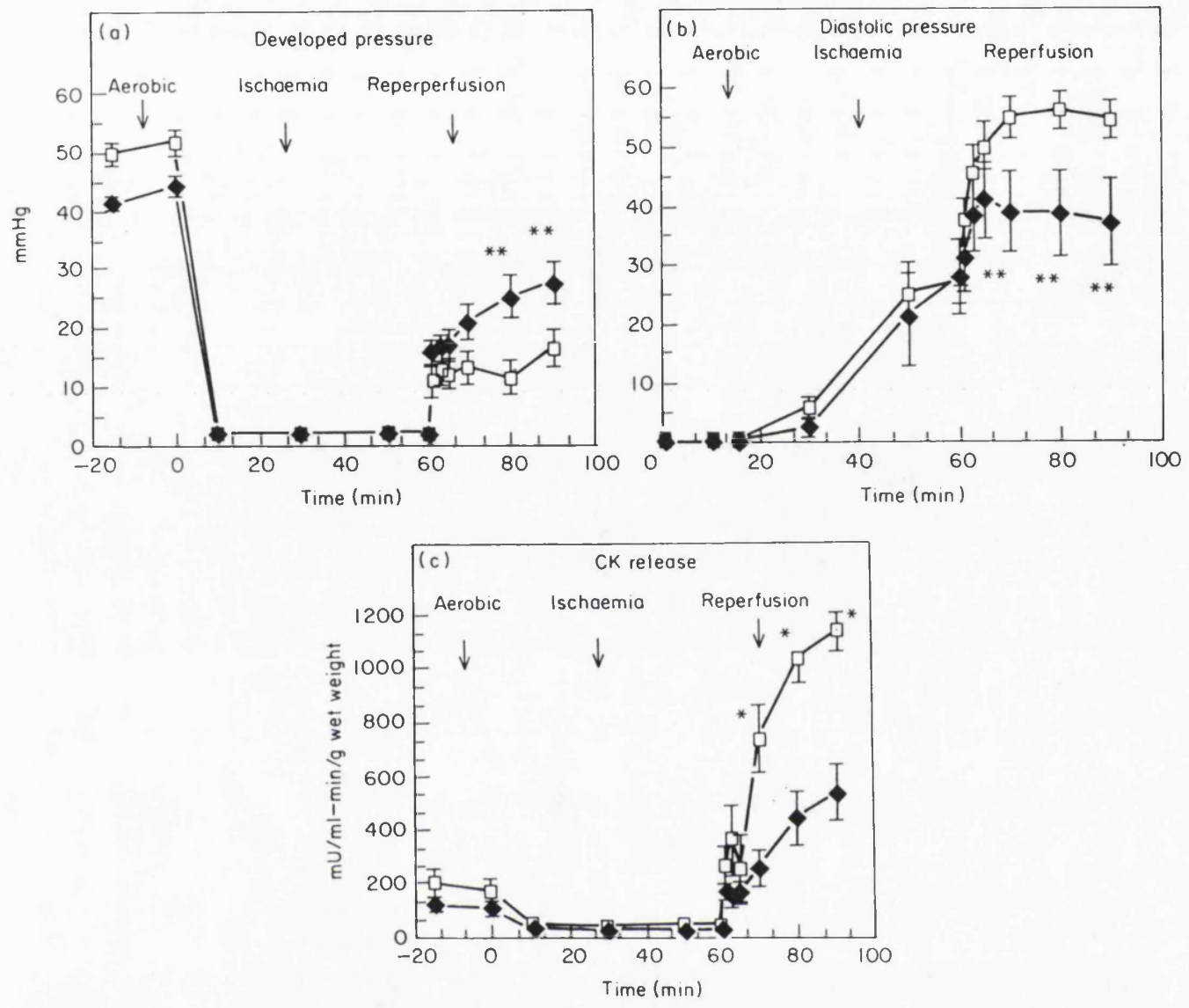

FIGURE 1. (a) and (b) Effect of heat stress on developed pressure. Graphs show both systolic and diastolic pressures of isolated perfused rabbit hearts from both control and heat stressed animals. Each point represents the mean \pm s.E.M. of six hearts from each group. Ischaemia was introduced at $0 \mathrm{~min}$ and maintained for $60 \mathrm{~min}$ and then reperfused for $30 \mathrm{~min}$ as indicated by the arrows. (c) Effect of heat shock on Creatine Kinase (CK) release. Graph showing CK release from isolated rabbit hearts during ischaemia and reperfusion. Each point represents the mean \pm s.E.M. of six hearts. ${ }^{*} P<0.0 \mathrm{I}$ and ${ }^{* *} P<0.05$ from control heart values using Student's $t$ test for paired data. (Control $=$ open symbols and $\mathrm{HS}=$ closed symbols.

Following heat shock the rabbits maintained the oxidative phosphorylating capacity of mitochrondria isolated either after ischaemia or following reperfusion. This protective effect was observed when either glutamate or succinate was used as substrate.

\section{Calcium homeostasis and mitochondrial ATP production}

The data on calcium homeostasis and mitochondrial ATP production are shown in Figure 2. Calcium homeostasis was deter- mined by measuring tissue and mitochondrial calcium in the same heart. For this purpose, the mitochondria were isolated in a medium lacking EDTA and containing ruthenium red.

In control hearts ischaemia caused an alteration of calcium homeostasis, as revealed by a small, non-signficant gain in tissue calcium associated with a significant $(P<0.05)$ increase of mitochondrial calcium content. This was concomitant with a decrease in the mitochondrial ATP producing capacity. Reperfusion of these hearts resulted in a large accumulation of both tissue and mitochondrial calcium with a severe reduction of ATP 
TABLE 1. Effect of heat shock on isolated mitochondrial function

\begin{tabular}{|c|c|c|c|c|c|c|}
\hline \multirow[t]{2}{*}{ Group } & \multirow{2}{*}{$\begin{array}{l}\text { Aerobic } \\
(120 \mathrm{~min})\end{array}$} & \multirow{2}{*}{$\frac{\begin{array}{c}\text { Ischaemia } \\
(60 \mathrm{~min})\end{array}}{\mathrm{RCI}}$} & \multirow{2}{*}{$\begin{array}{l}\text { Reperfusion } \\
\quad(30 \mathrm{~min})\end{array}$} & \multicolumn{3}{|c|}{ Statistics } \\
\hline & & & & $P_{1}$ & $P_{2}$ & $P_{3}$ \\
\hline Control & $23.4 \pm 3.2(6)$ & $8.4 \pm 2.1(6)$ & $5.7 \pm 0.2(6)$ & 0.01 & 0.01 & 0.05 \\
\hline \multirow[t]{2}{*}{ HS } & $21.9 \pm 4.3(5)$ & $16.4 \pm 2.0(6)^{* *}$ & $9.2 \pm 0.9(6) * *$ & N.S. & 0.05 & 0.05 \\
\hline & \multicolumn{3}{|c|}{$\mathrm{QO}_{2}\left(\right.$ natoms $\mathrm{O}_{2} / \mathrm{mg}$ prot $\left./ \mathrm{min}\right)$} & & & \\
\hline Control & $125.4 \pm 5.3(6)$ & $95.1 \pm 3.0(6)$ & $87.3 \pm 4.1(6)$ & 0.05 & 0.05 & N.S. \\
\hline \multirow[t]{2}{*}{ HS } & $145.0 \pm 6.1(5)$ & $139.4 \pm 3.4(6)^{* *}$ & $122.9 \pm 3.3(6)^{* *}$ & N.s. & N.s. & N.s. \\
\hline & \multicolumn{3}{|c|}{$\mathrm{ADP} / \mathrm{O}\left(\mathrm{nmol} \mathrm{ADP} /\right.$ natoms $\left.\mathrm{O}_{2}\right)$} & & & \\
\hline \multirow{3}{*}{$\begin{array}{l}\text { Control } \\
\text { HS }\end{array}$} & $2.7 \pm 0.2$ & $2.6 \pm 0.07(6)$ & $2.6 \pm 0.9(6)$ & N.S. & N.s. & N.S. \\
\hline & $2.6 \pm 0.09(5)$ & $2.5 \pm 0.09(6)$ & $2.6 \pm 0.1(6)$ & N.S. & N.S. & N.S. \\
\hline & \multicolumn{3}{|c|}{ Yield (mg prot/g dry wt) } & & & \\
\hline Control & $10.6 \pm 1.3(6)$ & $7.9 \pm 2.4(6)$ & $6.0 \pm 1.9(6)$ & N.S. & 0.05 & N.S. \\
\hline HS & $12.0 \pm 2.9(5)$ & $11.3 \pm 1.0(6)$ & $9.8 \pm 1.4(6)$ & N.s. & N.S. & N.S. \\
\hline
\end{tabular}

Data obtained using glutamate as substrate and are expressed as means \pm S.E. $P$, relates to the significance of the difference between values obtained after aerobic and ischaemia; $P_{2}$ to those obtained after acrobic and reperfusion; $P_{3}$ to those obtained after ischaemia and reperfusion. ${ }^{* *} P<0.01$; compared with control group).

production during state 3 of mitochondrial respiration.

Heat stress prevented the occurrence of changes in calcium homeostasis during ischaemia and of the large tissue and mitochondrial calcium accumulation on reperfusion. As a consequence of these events, the capacity of the isolated mitochondria to phosphorylate ADP to ATP was also maintained in the HS group.

\section{Tissue ATP and CP reserves}

In control hearts, as expected, ischaemia induced a severe depletion of endogenous stores of $\mathrm{ATP}$ and CP with the readmission of coronary flow failing to restore the tissue content of these high-energy phosphates (Table 2).

Heat stress had no effects on the tissue levels of ATP and CP following $120 \mathrm{~min}$ of acrobic perfusion and failed to reduce the ischaemicinduced depletion of tissue ATP and CP. However, it significantly improved the recovery of $\mathrm{CP}$ and of $\mathrm{ATP}$ following reperfusion, although the restoration of $\Lambda T P$ was not complete.

\section{Oxidative stress}

Oxidative stress was determined by measuring the glutathione status before and at the end of ischaemia, and after reperfusion. In control hearts there was no release of GSH and GSSG during ischaemia (Fig. 3). Despite these findings, the tissue content of GSH and protein$\mathrm{SH}$ following ischaemia was reduced (Table 3 ). Reperfusion of control hearts resulted in a significant increase of GSSG concentration and decrease of protein-SH. These changes were concomitant with a marked and sustained release of oxidized glutathione (Fig. 3 and Table 3 ). Heat shock of the rabbits minimized the occurrence of oxidative stress as the release of GSH and GSSG was reduced and there was also less of an increase of tissue GSSG and protein-SH after reperfusion.

$$
\text { HSP synthesis }
$$

Figure 4 (a) shows the results of the expression and synthesis of the $72 \mathrm{kD}$ HSP from six HS ( 5 ventricular and 1 atrial sample) and six control (5 ventricular and 1 atrial sample) rabbits. Figure $4($ b) also includes the Coomasie stained gel to demonstrate protein loading. 

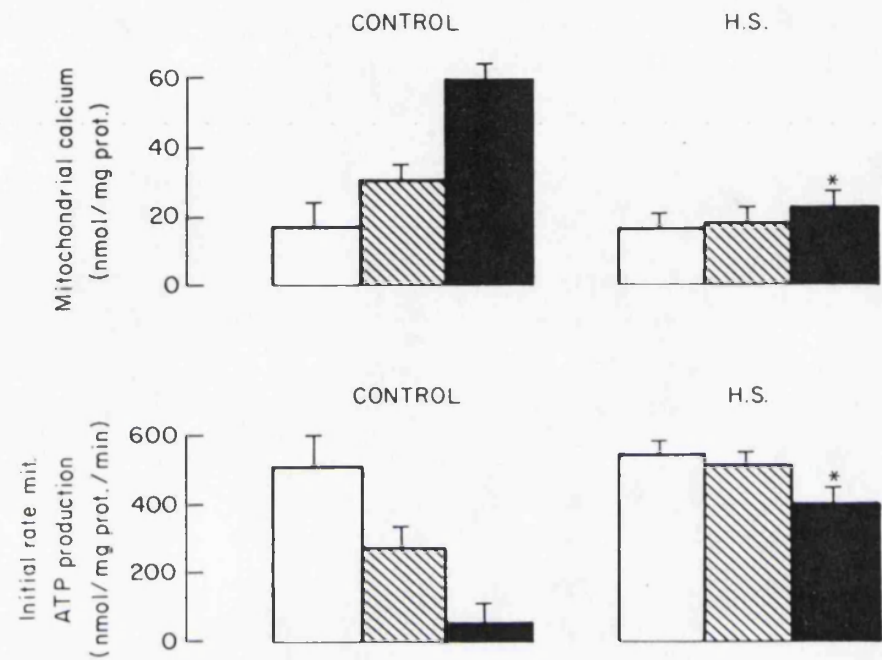

CONTROL

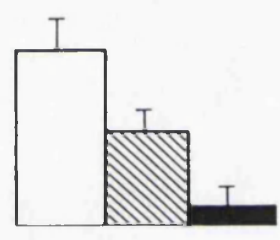

H.S

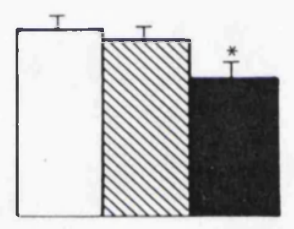

FIGURE 2. Effect of heat shock on calcium homeostasis and initial rate of mitochondrial ATP production. Results are expressed as mean \pm standard deviation of six separate experiments. $P$ relates to the significance of the difference from the control. Open bars, aerobic conditions; hatched bars, end of ischaemia; and black bars, end of reperfusion.

TABLE 2. Effect of heat shock on tissue content of ATP and CP

\begin{tabular}{lcccccc}
\hline Group & $\begin{array}{c}\text { Aerobic } \\
(120 \mathrm{~min})\end{array}$ & $\begin{array}{c}\text { Ischacmia } \\
(60 \mathrm{~min})\end{array}$ & $\begin{array}{c}\text { Reperfusion } \\
(30 \mathrm{~min})\end{array}$ & & Statistics \\
\hline ATP tissue content $(\mu \mathrm{mol} / \mathrm{g}$ dry wt $)$ & $P_{1}$ & $P_{2}$ & $P_{3}$ \\
\hline Control & $19.9 \pm 4.5(6)$ & $3.3 \pm 1.0(5)$ & $2.7 \pm 0.8(6)$ & 0.01 & 0.01 & N.s. \\
HS & $17.6 \pm 4.0(5)$ & $4.0 \pm 1.1(6)$ & $6.1 \pm 0.9(6) * *$ & 0.01 & 0.01 & N.s. \\
\hline \multicolumn{7}{c}{ CP tissue content $(\mu \mathrm{mol} / \mathrm{g}$ dry wt $)$} \\
Control & $49.0 \pm 4.1(6)$ & $15.5 \pm 3.1(5)$ & $16.4 \pm 5.1(6)$ & 0.01 & 0.01 & N.s. \\
HS & $47.3 \pm 3.6(5)$ & $26.2 \pm 2.5(6)$ & $36.9 \pm 6.4(6) * *$ & 0.01 & N.s. & N.s. \\
\hline
\end{tabular}

Data are expressed as means \pm S.E. $P_{1}$ relates to the significance of the difference between values obtained after aerobic and ischaemia; $P_{2}$ to those obtained after acrobic and reperfusion: $P_{3}$ to those obtained after ischaemia and reperfusion. ${ }^{* *} P<0.01 ;{ }^{*} P<0.05$; compared with control group. 


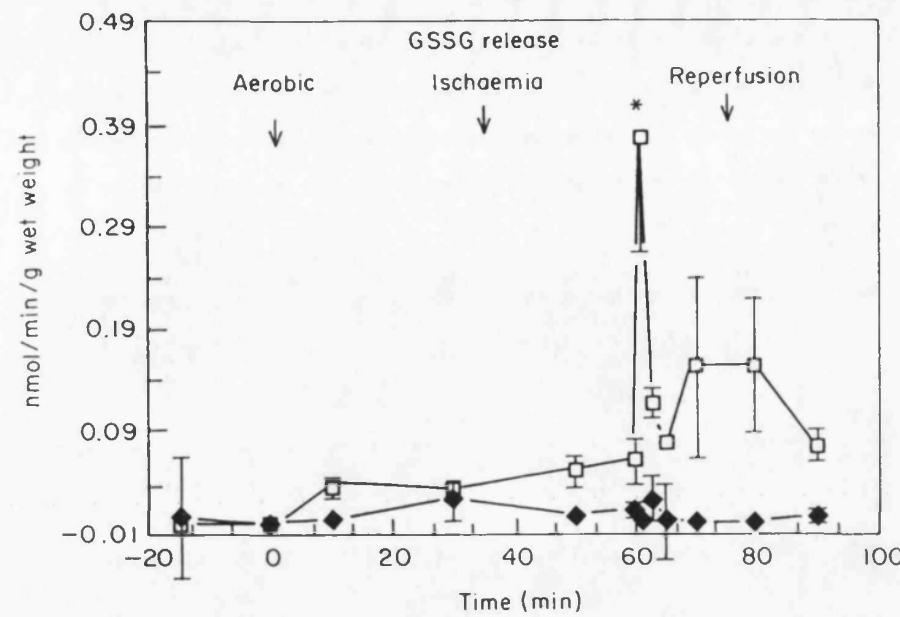

FIGURE 3. Effect of heat shock oxidized glutathione (GSSG) release. Graph showing GSSG release from hearts during ischaemia and reperfusion. Each point represents the mean \pm s.E.M. of six hearts. Description of statistical analysis as per Figure 1 . (control =open symbols and $\mathrm{HS}=$ closed symbols)

TABLE 3. Effect of heat shock on tissue content of reduced and oxidized glutathione and protein-SH

\begin{tabular}{|c|c|c|c|c|c|c|}
\hline \multirow[t]{2}{*}{ Group } & Acrobic & Ischaemia & Reperfusion & & tatistic & \\
\hline & \multicolumn{3}{|c|}{ GSH tissuc content ( $\mathrm{nmol} / \mathrm{mg}$ prot) } & $P_{1}$ & $P_{2}$ & $P_{3}$ \\
\hline Control & $9.6 \pm 0.6(6)$ & $6.0 \pm 0.8(6)$ & $4.07 \pm 0.4(6)$ & 0.05 & 0.05 & N.S. \\
\hline \multirow[t]{2}{*}{ HS } & $10.8 \pm 0.6(5)$ & $6.8 \pm 0.7(6)$ & $6.8 \pm 0.8(6)^{*}$ & 0.05 & 0.05 & N.S. \\
\hline & \multicolumn{3}{|c|}{ GSSG tissue content ( $\mathrm{nmol} / \mathrm{mg}$ prot) } & & & \\
\hline Control & $0.250 \pm 0.06(6)$ & $0.159 \pm 0.03(6)$ & $0.478 \pm 0.04(6)$ & 0.01 & 0.01 & 0.01 \\
\hline \multirow[t]{2}{*}{$\mathrm{HS}$} & $0.248 \pm 0.07(5)$ & $0.142 \pm 0.05(6)$ & $0.132 \pm 0.06(6)^{* *}$ & 0.01 & 0.01 & N.S. \\
\hline & \multicolumn{3}{|c|}{ Protein-SH tissue content ( $\mathrm{nmol} / \mathrm{mg}$ prot) } & & & \\
\hline Control & $320.0 \pm 9.1(6)$ & $95.0 \pm 10.3(6)$ & $188.3 \pm 12.3(6)$ & 0.01 & 0.01 & 0.05 \\
\hline HS & $315.3 \pm 9.4(5)$ & $105.1 \pm 13.3(6)$ & $295.3 \pm 9.9(6)^{* *}$ & 0.01 & N.s. & 0.01 \\
\hline
\end{tabular}

Data are expressed as means \pm s.E. $P_{1}$ relates to the significance of the difference between values obtained after aerobic and ischaemia; $P_{2}$ to those obtained after aerobic and reperfusion; $P_{3}$ to those obtained after ischaemia and reperfusion. ${ }^{*} P<0.01 ; * P<0.05$; compared with control group.

Note that in the control hearts, which were not subjected to hyperthermia, there was only a relatively small expression of the $72 \mathrm{kD}$ stress protein as determined by Western blot analysis. In the heat-stressed group, however, the synthesis of the $72 \mathrm{kD}$ stress protein was signi- ficantly increased when analysed by densitometry. Optical density ratios for the control and HS were $0.50 \pm 0.7$ and $1.9 \pm 0.32$, respectively $(P<0.01$ ) (Fig. 5). In experiments in which the primary, anti-HSP70, antibody was omitted from the reaction the $72 \mathrm{kD}$ band 

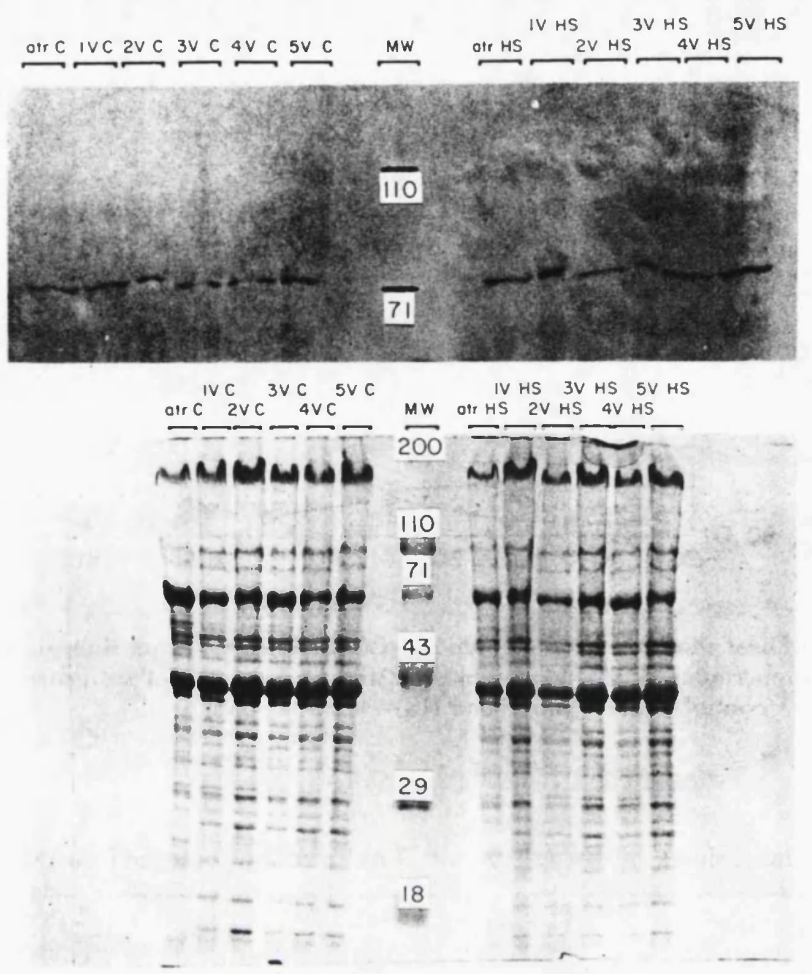

FIGURE 4. The effect of heat stress on the expression of the $72 \mathrm{kD}$ HS protein. This figure shows a Western blot analysis (top) with its corresponding Coomasie stain (bottom) of ventricular and atrial proteins prepared from heat stressed (HS) and control $(\mathrm{C})$ non-heat stressed sham rabbits. Proteins were separated SDS Page.

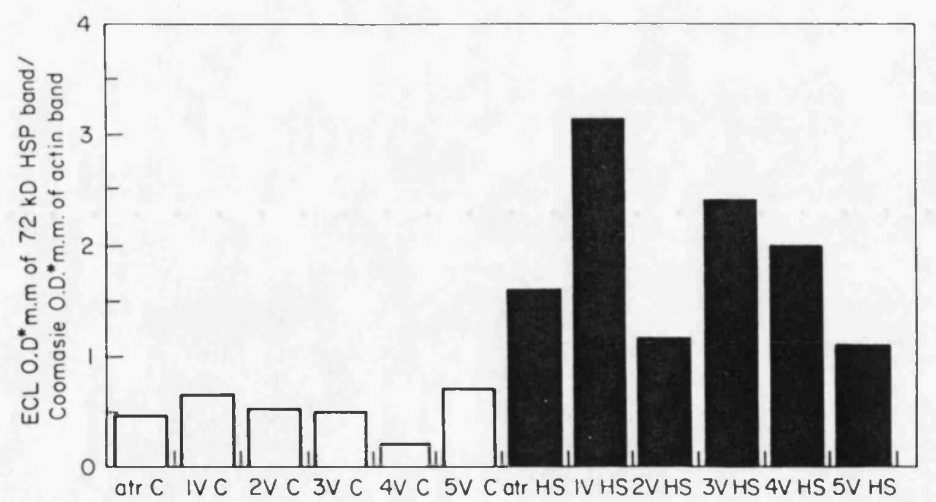

FIGURE 5. Densitometric analysis derived by dividing the optical density of the $72 \mathrm{kD}$ band on the Western blot by the optical density of the Actin band on corresponding Coomasie stained gel. Mean ratios of integrated optical densities for control group is $0.50 \pm 0.07$ and for HS group is $1.90 \pm 0.32 . P=<0.01$. (Controls = open bars and HS= black bars.) 
was not observed, confirming that it was indeed the $72 \mathrm{kD}$ heat stress protein (data not shown).

\section{Discussion}

Our data demonstrate that heat stress with subsequent expression of the heat stress protein confers a significant protection to the isolated rabbit heart subjected to $60 \mathrm{~min}$ of low flow ischaemia followed by $30 \mathrm{~min}$ reperfusion. These findings are in agreement with that of Currie et al. (1988) who were the first to demonstrate the protective role of HSPs in the ischaemic/reperfused rat heart. We have, however, utilized different indices of myocardial damage and show for the first time that heat stress reduces the degree of oxidative stress and the deterioration of mitochondrial function caused by post-ischaemic reperfusion. In addition, by extending these studies to the rabbit we have also shown that the protection exerted by heat stress is not unique to one species alone.

The mechanism for the injury in the reperfused ischaemic heart is still unclear. It is generally thought to be due to either a direct free radical involvement or calcium overload occurring upon reperfusion (for review, see Yellon and Downey, 1990). More specifically, the calcium hypothesis suggests that ischaemia induces a defect in the cell's ability to regulate calcium fluxes such that the cell, and particularly the mitochondria, accumulates toxic levels of calcium on reperfusion (Grinwald, 1982; Ferrari el al., 1988). The free radical hypothesis proposes that cytotoxic oxygenderived free radicals are generated at the time of reperfusion, above the myocardial defence capacity, and exert a deleterious effect on the reperfused hearts (Ceconi et al., 1988). These two mechanisms of injury are not necessarily mutually exclusive. Our finding of an increased production and release of oxidized glutathione indicates that the oxidative stress has occurred on reperfusion which was concomitant with alteration of calcium homeostasis and impairment of mitochondrial function. Heat stress of the rabbits reduced the extent of both these negative events.

Currie et al. (1988) showed that the levels of endogenous catalase, the naturally occurring hydroxyl radical scavenger, were significantly raised in heat-stressed rat heart following similar periods of ischaemia and reperfusion. Inactivating catalase with 3-AT abolished the protective influences seen at 24 and $48 \mathrm{~h}$ postheat stress (Karmazyn et al., 1990). They speculated that the expression of the heat inducible protein itself may not be directly involved in the protective response but hyperthermia, by somehow influencing the catalase activity, could be associated with myocardial protection. Furthermore, Brown et al. (1989) recently reported that endotoxin treatment in rats, $24 \mathrm{~h}$ prior to removal of their hearts, also appeared to be associated with an increase in catalase activity and cardiac protection. Depletion of catalase with 3-AT also resulted in a loss of the protective effect in their model. Certainly an increase in the catalase activity is important and at the myocardial level, where the activity of the enzyme is particularly low, it would scavenge $\mathrm{H}_{2} \mathrm{O}_{2}$ produced from superoxide dismutase, thus reducing as observed the degree of oxidative stress.

Although these studies of catalase and its inhibition are of importance to an understanding of the protective effect of heat stress, one should be careful not to draw too sharp a distinction between HSP synthesis and an anti-oxidant effect of catalase. If catalase is synthesized at increased levels in response to heat it would, by definition, be a heat stress protein, its role as an anti-oxidant providing another aspect of the wide variety of protective roles of HSPs in protein folding and degradation. Similarly, it has been shown that the synthesis of the HSPs themselves, which occurs upon exposure of phagocytes to hydrogen peroxide, is associated with inhibition of superoxide anion generation by these cells following heat shock and it has therefore been speculated that HSPs themselves may have a direct anti-oxidant effect (Polla, 1988).

It should be mentioned that not all studies have been positive. Wall et al (1990) were unable to demonstrate any protection following heat stress in the isolated perfused rat heart with respect to myocardial function. They used a model of 25 min zero-flow ischaemia followed by $30 \mathrm{~min}$ reperfusion which is likely to be too severe to demonstrate any protective effect. The end points that they measured, in which no improvement was noted, were rate pressure product and cardiac 
output. It could be argued that heat-stressed hearts are better able to utilize substrates under low flow conditions compared to control hearts but not when the substrate is absent as in the case with total ischaemia.

The present results are also partially in contrast to that of a very recent study by both Currie et al. (1990) as well as ourselves (Pasini el al., 1991) in which we found that highenergy phosphate levels were no different between heat-stressed and control hearts either during ischaemia or following a period of reperfusion in the rat heart.

In the rabbit study, which we report here, we were able to demonstrate a significantly increased level of ATP and CP at the end of the reperfusion period in the heat-stressed rabbits. The most attractive explanation for this difference is a species difference, between our rabbit study and the two rat studies.

Rouslin et al. (1988) report that much of the ATP used during ischaemia in the rabbit can be attributed to mitochondrial ATPase. Heat stress may act to inhibit this ATPase by acting on a specific ATPase inhibitor protein that exists in most slow heart rate hearts such as that of the rabbit. While mitochondrial ATPase activity is high in the ischaemic rabbit heart it is undetectable in the ischaemic rat heart, due apparently to the lack in situ of mitochondrial ATPase inhibitor function. Thus an inhibition of mitochondrial ATPase would not be expected to alter the rate of ATP utilization in the ischaemic rat heart. Other specifically related differences are also possible such as that mentioned above where Currie $e t$ al. (1990) used a zero-flow ischaemic model compared to our low-flow ischaemic model. Again heat-stressed hearts may be better able to utilize substrate under low flow conditions than control hearts but not when substrate is absent as in the case with total ischaemia.

The higher tissue content of ATP and CP could also be the result of a higher capacity of the mitochondria to phosphorylate ADP into
ATP in the presence of oxygen, as demonstrated by our data on isolated mitochondrial function. Thus the mitochondrial protection which we found in the heat-stressed rabbit could be secondary (1) a general effect on the plasmalemma and/or mitochondrial membranes reducing theit fragility during ischaemia and reperfusion, or alternatively, to a direct reduction of cytosolic calcium accumulation or indeed to at specific inhibition of mitochondrial calcium accumulation, mitochondrial calcium 11:insport and ATP synthesis competing for the same energy source: the transmembrane potential restored on reperfusion by the presence of oxygen

Unfortunately, other than speculation, we are not able to identify the basis for the protection. This could be the result of a direct effect of the expression of the HS protein itself playing a protective role with respect to its ability to cause folding and unfolding as well as translocation of pulypeptides and the assembly and disassembly of protein complexes (for review, see Yellım and Latchman, 1992). Or it could be the result of other indirect effect in terms of oxygen free radical scavenging as suggested by ('urrie el al. (1988). Indeed, we would evell speculate that the constitutive form of the IISP cannot be dismissed as it may itself be involved in protection through post-translilional modification or even translocation briween organelles.

Whatever the case, the encouraging results that we obtained strengthen the view that heat stress and heal shock protein synthesis appear to play a unique role in the cell's overall ability to profect itself from the consequences of ischaemia and reperfusion. Wé believe that by understanding and exploiting the ability of a cell 11 protect itself, be it by heat stress protein synthesis or some other related mechanism, slouuld enable us to investigate new methods and means of protecting the ischaemic and reperfused myocardium.

\section{References}

BRADFORD MM (1978) A rapid and sensitive method for the quantitation of micrograll yuantities of protein utilizing the principle for protein binding. Anal Biochem 72: 248 .

BracNwald EK (1985 Myocardial reperfusion: a double-edged sword? J Clin lnvesi 76: 1713-1719.

Brown JM, Grosso Ma, Terada IS, Whitman gJR, Banerjee A, White CW, Haken ah, Repine JE (I989) 
Endotoxin pretreatment increases endogenous myocardial catalase activity and decreases ischaemia-reperfusion injury of isolated rat hearts. Proc Natl Acad Sci USA 86: 2516-2520.

Ceconi C, Curello S, Albertini A, Ferrari R (1988) Effect of lipid peroxidation on heart mitochondria oxygen consuming and calcium transporting capacities. Mol Cell Biochem $1-5$.

Ceconi C, Curello S, Cargnoni A, Ferrari R, Albertini A, Visioli O (1988) The role of glutathione status in the protection against ischaemic and reperfusion damage: effects of $\mathrm{N}$-acetyl cysteine. J Mol Cell Cardiol 20: 5-13.

Currie RW, Karmazyn MM (1990) Improved post-ischaemic ventricular recovery in the absence of changes in energy metabolism in working rat hearts following heat shock. J Mol Cell Cardiol 22: 631-636.

CuRrIE RW, WhITE FP (1983) Characterization of the synthesis and accumulation of a $71-$ Kilodalton protein induced in rat tissues after hyperthermia. Can J Biochem Cell Biol 61: 438-446.

Currie WR, Karmazyn M, KLoc M, Mailer K (1988) Heat-shock response is associated with enhanced postischemic ventricular recovery. Circ Res 63: 543-549.

FERRari R, Di Lisa F, Raddino R, Visioli O (1982) The effects of ruthenium red on mitochondrial function during post-ischaemic reperfusion. J Mol Cell Cardiol 14: 737-740.

Ferrari R, Ceconi C, Cargnoni A, Pasini E, Boffa CM, Curello S, Visioli O (1991) Effect of propionyl-Lcarnitine on mechanical function of isolated rabbit heart. Cardiovasc Drugs Ther 5; 17-24.

Ferrari R, Ceconi C, Curello S, Guarnieri CM, Caldarera A, Albertini A, Visioli O (1985) Oxygenated mediated myocardial damage during ischaemia and reperfusion: role of the cellular against oxygen toxicity. $\mathrm{J}$ Mol Cell Cardiol 17: 937

Ferrari R, Curello S, Cargnoni A, Condorelli E, Belloli S, Albertini A, Visioli O (1988). Metabolic changes during post-ischaemic reperfusion. J Mol Cell Cardiol 20: 119-133.

FERRARI R, WILLIAms AJ (1986) The role of mitochondria in myocardial damage occurring on post-ischaemic reperfusion. J Appl Cardiol 1: 501 .

Grinwald PM (1982) Calcium uptake during post-ischemic reperfision in the isolated rat heart. J Mol Cell Cardiol 14: $359-365$.

Hearse DJ, Yellon DM (1984) Why are we still in doubt about infarct size limitation? The experimentalist's viewpoint. In: Therapeutic Approaches to Myocardial Infarct Size Limitation, (Eds) DJ Hearse \& DM Yellon. Raven Press, New York: pp. 17-41

Hearse DJ, Yellon DM, Downey JM (1986) Can beta blockers limit infarct size? Eur Heart J 7: 925-930.

Karmazyn M, Mailer K, Currie RW (1990) Acquisition and decay of heat-shock-cnhanced postischemic ventricular recovery. Am J Physiol 259: $\mathrm{H} 424-\mathrm{H} 43$ !

LAEMMLi JK (1970) Cleavage of structural proteins during the assembly of the head of bacteriophage T4. Nature (Lond) 227: 680-685

Lamprecht W, Trautschold E (1974) Adenosine-5'-triphosphate determination with hexokinase and glucose-6phosphate dehydrogenase. In: Method of Enzymatic Analysis, edited by HU Bergmeyer. Academic Press, New York: p. 2021.

Lindquist S (1986) The heat shock response. Ann Rev Biochem 1986; 55: 1151-1191.

Maroko PR, KJekshu's JK, Sobel BE, Watanabe T, Covell JW, Ross J, Braunwald E (1971) Factors influencing infarct size following experimental coronary artery occlusion. Circulation 43: 67-82.

Merta HB, Popovich BK. Dillman WH (1988) Ischemia induces changes in the level of mRNA's coding for stress protein 71 and creatine kinase m. Circ Res 63: 512-517.

Oliver TA (1955) A spectrophotometric method for the determination of creatine phosphokinase and myokinase. Biochem J 61: 116-122.

Pasini E, Cargnoni A, Ceconi C, Curello S, Ferrari R, Yellon DM (1991) Heat stress and oxidative damage after post-ischaemic reperfusion. J . Mol Cell Cardiol (Suppl V): 23

Peng GF, Ranne JJ, Merphy ML, Stracb KD (1977) Abnormal mitochondrial oxidative phosphorylation of ischaemic myocardium reversed by calcium chelating agents. J Mol Cell Cardiol 9: 897.

POLLA BS (1988) A role for heat shock proteins in inflammation. Immunol Today 9: 134-137.

RousLin W (1988) Factors affecting the loss of mitochondrial function during zero flow ischaemia (autolysis) in slow and fast heart rate hearts. J Mol Cell Cardiol 20: 999-1007.

SEDLACK J, LINDSAY RH (1968) Estimation of total protein bound and non protein sulphydryl groups in tissuc with Ellmans reagent. Anal Biochem 25: 192.

Sordahl LA, McCollum WB, Wodd WG, Schwartz A (1973) Mitochondria and sarcoplasmic reticulum function in cardiac hypertrophy and failure. Am J Physiol 244: 497.

Stocchi V, Cucchiarini L, Canestrari F, Piacentini MP, Fornaini (1987) A very fast ion-pair reversed-phase HPLC method for separation of the most significant nucleotides and their degradation products in human red blood cells. Anal Biochem 167: 181-190.

Tietze F (1969) Enzymatic method for quantitative determination of nanogram amounts of total and oxidized glutathione: applications to mammalian blood and other tissue. Analyt Biochem 27: 502-522.

WALL SR, FlisS H, KaKo KJ, KoRecky B (1990) Heat pretreatment does not improve recovery of function after noflow ischaemia in isolated working rat hearts. J Mol Cell Cardiol (Suppl I): S44.

YELLON DM, DOWNEY J.M (1990) Current Research Views on myocardial reperfusion and reperfusion injury Cardioscience 1: 89-98.

YELLON DM, LATChMAN DS 1992) Stress proteins and myocardial protection. J Mol Cell Cardiol 24: 113-124. 


\title{
Heat stress limits infarct size in the isolated perfused rabbit heart
}

\author{
D M Walker, E Pasini, S Kucukoglu, M S Marber, E Iliodromitis, R Ferrari, and \\ D M Yellon
}

\begin{abstract}
Objective: Heat stress, with the expression of heat stress proteins, has been shown to protect the rabbit heart in vitro against global ischaemia/reperfusion injury, though no benefit is apparent in an in vivo rabbit model of infarct size. The aim of this study was therefore to investigate this discrepancy and to discover whether heating itself has any effect which could negate the protection derived from myocardial stress protein synthesis. Methods: (1) To ascertain whether heat stress could limit infarct size in the absence of blood, isolated buffer perfused hearts, with or without prior heat stress, were subjected to $45 \mathrm{~min}$ of regional ischaemia and $120 \mathrm{~min}$ reperfusion, and the resulting infarct size was expressed as a percentage of the risk area (I/R\%). (2) The observations were repeated in an isolated blood perfused heart model in which a support rabbit (heat stressed or control) was used to perfuse the isolated heart. Results: In the buffer perfused heart, prior heat stress reduced I/R from 70.8(SEM 4.4)\%, $\mathrm{n}=10$, in controls to $51.5(5.7) \%, \mathrm{n}=12(\mathrm{p}<0.05)$. In hearts perfused by support rabbits, prior heat stress reduced I/R [from $34.7(3.7) \%, n=16$, to $23.5(3.3) \%, n=15(p<0.05)$ ] only when the perfusing rabbit was a control (not heat stressed). If the perfusing rabbit had been heated, I/R was greater in both heat stressed and control hearts [51.9(7.0)\% and $44.9(3.3) \%, \mathrm{p}<0.05 v$ control support rabbit]. Conclusions: Heat stress limits infarct size in this rabbit model. However it appears to have additional adverse effects, probably on the blood, which may override any benefit associated with myocardial stress protein synthesis.

Cardiovascular Research 1993:27:962-967
\end{abstract}

$\mathrm{S}$ tress proteins have been detected in the heart tissue of a variety of species and their synthesis has been shown to increase with hyperthermia, ${ }^{1-3}$ ischaemia, ${ }^{4}$ ischaemia and reperfusion, ${ }^{5}$ anoxia, ${ }^{6}$ hypoxia, ${ }^{7}$ and pressure or volume overload. ${ }^{8}$ Stress protein induction may therefore play a role in the response of the heart to stress. Cells which are subjected to thermal stress (and accumulate stress proteins) acquire a transient resistance to further episodes of thermal stress, ${ }^{9}$ a phenomenon known as thermotolerance. This has been linked with the expression of stress proteins by the observation that thermotolerance decreases with the decline in stress protein levels ${ }^{10}$ and dependence of the phenomenon on individual stress proteins has been demonstrated using anti-sense RNA ${ }^{11}$ and cell lines with stress protein gene deletions. ${ }^{12}$ It has been suggested, therefore, that pre-elevation of stress proteins might render the heart more resistant to a range of stresses - and in particular to injury from ischaemia and reperfusion. ${ }^{13}$

Evidence in support of this hypothesis comes from in vitro studies in the isolated rat ${ }^{14}{ }^{15}$ and rabbit ${ }^{16}$ heart, examining ischaemia and reperfusion injury. These studies show that prior heat stress is associated with improved postischaemic recovery of function with decreased creatine kinase release, ${ }^{14-16}$ and also improvement in other indices of protection such as preservation of high energy phosphates, reduced oxidative stress on reperfusion, and mitochondrial preservation. ${ }^{16}$

When we extended these investigations to a whole animal model of myocardial infarction in the open chest rabbit, no protective effect on infarct size was seen, in spite of a clear increase in stress protein levels. ${ }^{17}$ The cause of this discrepancy between the in vitro and in vivo studies is unclear, but there are possible explanations. Firstly, heat stress proteins may not be protective against severe forms of injury such as myocardial infarction, and secondly whole body hyperthermia may itself have some adverse effects which negate any beneficial effects that stress proteins might have within the heart.

The two studies described here were designed to examine in more detail whether a severe form of injury such as myocardial infarction could be limited by prior heat stress treatment in the rabbit heart. Initially we extended our previous in vitro observations ${ }^{16}$ to see whether infarct size could be limited by heat stress in the isolated buffer perfused heart. Subsequently, to test whether any additional consequences of whole body hyperthermia were in some way affecting infarct size in the whole animal model, we decided to repeat these experiments in an isolated heart, perfused with blood from a support rabbit. This experimental design allowed us to heat stress either the donor rabbit - so that stress proteins were increased within the isolated heart - or the support rabbit - so that the blood with which the heart was perfused came from a heat stressed rabbit. This enabled us to control for any additional effects of heat stress outside the isolated heart, provided that they were mediated by the blood.

\section{Methods}

These studies were performed acording to the Home Office Guidance on the operation of the Animals (Scientific Procedures) Act 1986, published by HMSO, London.

Stress protein induction

All rabbits receiving heat stress therapy were treated in an identical manner in both experiments. Twenty four hours before an experiment.

The Hatter Institute for Cardiovascular Studies, Department of Cardiology, University College Hospital, Gower Street, London WC1E 6AU, United Kingdom: D M Walker, S Kucukoglu, M S Marber, E Iliodromitis, D M Yellon; Department of Cardiology, University of Brescia, Italy: E Pasini, R Ferrari. Correspondence to Dr Yellon. 
the rabbits were anaesthetised with sodium pentobarbitone $\left(40 \mathrm{mg} \cdot \mathrm{kg}^{-1}\right)$ given via the marginal ear vein, wrapped in a warming blanket, and their core temperatures raised to $42^{\circ} \mathrm{C}$ for 15 minutes. Afterwards all rabbits were allowed to recover. Temperatures were measured rectally with a digital thermometer throughout this procedure. Control animals were treated in the same manner, being anaesthetised and wrapped but not warmed.

\section{Stress protein assay}

Eight rabbits, four heat stressed and four controls, were used for $72 \mathrm{kDa}$ stress protein determination $24 \mathrm{~h}$ after this procedure. After a lethal intravenous dose of sodium pentobarbitone $\left(80 \mathrm{mg} \cdot \mathrm{kg}^{-1}\right)$, the hearts were rapidly removed and frozen. At a later date these specimens were homogenised in double strength Laemmli's sample buffer, ${ }^{18}$ boiled, and centrifuged. Samples were further diluted in buffer to allow loading of approximately $30 \mu \mathrm{g}$ of total protein per lane. Proteins were separated by SDS-PAGE on $0.8 \mathrm{~mm}$ thick $12.5 \%$ acrylamide gels according to Laemmli. ${ }^{18}$ Two identically loaded gels were produced with four heat stress and control samples separated by molecular weight markers. The proteins on one gel were transferred to nitrocellulose by western blotting. The other identical gel was stained with Coomassie brilliant blue R250 to visualise the proteins.

Following transfer the nitrocellulose filters were incubated with mouse monoclonal antisera crossreactive to the $72 \mathrm{kDa}$ stress protein (Stressgen, Sidney, Canada) at 1:1000 dilution. The second antibody used was horseradish peroxidase conjugated rabbit antimouse antiser (DAKO, Denmark) at 1:2500 dilution. The filter was developed using enhanced chemiluminescence (ECL) detection and exposed to $x$ ray film for the appropriate time.

The relative levels of the $72 \mathrm{kDa}$ stress protein were determined using densitometry, normalising to the actin band on the Coomassie stained gel. This procedure adjusts for slight variations in protein loading between samples.

\section{BUFFER PERFUSED ISOLATED HE.ART MODEL}

Isolated heart preparation - Male New Zealand White rabbits (2-2.5 $\mathrm{kg}$ ), prepared as above, were re-anaesthetised with sodium pentobarbitone $\left(40 \mathrm{mg} \cdot \mathrm{kg}^{-1}\right)$ given via the other marginal ear vein. A tracheostomy was performed under additional local anaesthetic $(3 \mathrm{ml}$ of $2 \%$ lignocaine) and the rabbit was ventilated with $100 \%$ oxygen. The respiratory rate was adjusted to keep arterial $\mathrm{pH}$ and $\mathrm{PCO}_{2}$ within normal limits. A thoracotomy was performed in the fourth left intercostal space and the pericardium incised to expose the heart. The left atrial appendage was retracted so that the left coronary anatomy could be identified. A 3-0 silk suture on a small round bodied needle was passed through the myocardium behind the proximal segment of a large anterior arterial branch and the ends were threaded through a smal vinyl tube to form a snare. The rabbits were given 500 units $\cdot \mathrm{kg}^{-1}$ of sodium heparin and the heart was rapidly removed by cutting the great vessels, placed in ice cold saline, and mounted on the Langendorff apparatus. The time from removal of the heart to retrograde perfusion was less than one minute.

Preparation of perfusion apparatus - Perfusate consisted of (in mmol-litre ${ }^{-1}$ ): $\mathrm{NaCl} 118.0, \mathrm{KCl} 4.0, \mathrm{MgCl}_{2} .6 \mathrm{H}_{2} \mathrm{O}$ 1.0, $\mathrm{NaCO}_{3} 24.0$, $\mathrm{NaH}_{2} \mathrm{PO}_{4} .2 \mathrm{H}_{2} \mathrm{O} 0.4, \mathrm{CaCl}_{2} .2 \mathrm{H}_{2} \mathrm{O} 1.8$, glucose 10.0 , pyruvate 2.0 , with bovine serum albumin $2 \%$. Albumin was added to reduce tissue oedema and enable better delineation of infarction with the tetrazolium technique (see below). A standard Langendorff preparation was used in this experiment with an in-line $5 \mu \mathrm{m}$ filter, except that the usual bubble oxygenator was replaced with a thin film oxygenator to avoid excess frothing due to the albumin. The hearts were perfused in constant flow mode in a recirculating system, with recordings taken of coronary perfusion pressure via a side arm in the perfusion cannula. Left ventricular pressures were measured with a fluid filled latex balloon, connected by polyethylene tubing to a pressure transducer and inserted into the left ventricle via an incision in the left atrial appendage. The balloon volume was adjusted to give an initial diastolic pressure of 10 $\mathrm{mm} \mathrm{Hg}$. All hearts were paced from the right atrium at a rate of 180 beats. $\min ^{-1}$

Experimental protocol - Rabbits were alternately either heat stressed or sham treated. The isolated hearts were allowed to stabilise for 15 $\min$, before tightening the coronary snare to induce ischaemia for 45 min and releasing for $2 \mathrm{~h}$ reperfusion. Although there is no cyanosis to help distinguish ischaemia in this model, there was a slight colour change in the ischaemic area which, combined with paradoxical systolic bulging and a rise in coronary perfusion pressure, was sufficient to confirm that the heart had been made ischaemic.

BLOOD PERFUSED ISOLATED HEART MODEL

Isolated heart preparation - This was identical to that described for the buffer experiments.

Blood support rabbit preparation - Male New Zealand White rabbits weighing $3-3.5 \mathrm{~kg}$ were used for this purpose. Rabbits prepared the day before were given anaesthetic (sodium pentobarbitone $40 \mathrm{mg} \cdot \mathrm{kg}^{-1}$ via marginal ear vein), a tracheostomy was performed and the rabbit ventilated as described above. Additional doses of pentobarbitone were given as necessary to maintain anaesthesia. The left femoral artery was cannulated to monitor arterial blood pressure and to facilitate blood gas measurement.

Catheters were placed in the left carotid artery to supply blood to the isolated heart and in the right jugular vein to receive the returning blood. To anticoagulate the circulating blood, the rabbits were given a 1000 unit $\mathrm{kg}^{-1}$ bolus of sodium heparin, with additional doses of 500 units $\mathrm{kg}^{-1}$ every hour. The perfusion circuit consisted of silicon tubing running from the support rabbit's carotid artery, through a roller pump and via a bubble trap to a Teflon cannula from which the isolated heart was suspended. The heart was placed within a chamber and covered with plastic film to help maintain constant temperature. Both the bubble trap and heart chamber were jacketed and circulated with water at a constant temperature of $37^{\circ} \mathrm{C}$. All tubing and glassware had been pretreated with Sigmacote (Sigma Chemical Company, Poole, UK).

The pulmonary artery was cut to allow blood to drip into the heart chamber and from there the blood was returned to the support rabbit by a second roller pump. A thermocouple was inserted through the opening in the pulmonary artery to monitor intracardiac temperature. Coronary perfusion pressure was measured as before and coronary flow was kept constant throughout at $1.2 \mathrm{ml} \cdot \mathrm{min}^{-1} \cdot \mathrm{g}^{-1}$ heart weight. Left ventricular pressures were again measured with a fluid filled latex balloon, with its volume adjusted to give an initial diastolic pressure of between 5 and $15 \mathrm{~mm} \mathrm{Hg}$. The heart was allowed to stabilise for 15 minutes before starting the experiment.

Experimental protocol - Experiments were divided into four groups and were chosen sequentially. In group 1, control donor rabbits provided the isolated hearts to be perfused by control blood support rabbits. In group 2 , heat stressed donor rabbits provided hearts for control blood support rabbits. In groups 3 and 4 , control and heat stressed hearts were perfused by heat stressed support rabbits.

Each experiment consisted of 15 minutes stabilisation, 45 minutes regional ischaemia (by tightening the snare around the coronary artery), and two hours of reperfusion. Ischaemia could be detected by cyanosis and bulging of the affected area and by a sharp rise in coronary perfusion pressure.

\section{Measurement of risk area and infarct size}

After reperfusion in the support animal experiments, the hearts were moved to a second Langendorff perfusion apparatus and perfused with saline at room temperature. After $2 \mathrm{~min}$, the snare was retightened and fluorescent particles (1-10 $\mu \mathrm{m}$ in diameter) infused into the perfusate to outline the area at risk. In the buffer perfused experiments, the isolated hearts were perfused with particles while still on the original perfusion apparatus.

All hearts were then weighed, frozen, and sliced into $2 \mathrm{~mm}$ sections. On thawing, the slices were incubated in 1\% triphenyl tetrazolium chloride (TTC) at $37^{\circ} \mathrm{C}$ for $15 \mathrm{~min}$. TTC stains all living tissues brick red, allowing visualisation of the infarcted areas (white). Tracings were taken of the slices, showing the area at risk (non-fluorescent area under ultraviolet light) and the infarcted area, and these were subsequently measured by planimetry. The volume of tissue was calculated by multiplying by the slice thickness $(2 \mathrm{~mm})$. Infarct size was then expressed as a percentage of the risk zone size.

\section{Exclusions}

A total of 32 rabbits entered the buffer perfused experiments. Ten hearts were excluded, two due to infarction outside risk area (one from each group), two due to arrhythmias in the isolated heart (both from the control group), and six due to poorly defined risk areas (three from each group).

A total of 61 donor rabbits entered the blood support rabbit experiments. Eleven of these were excluded or failed to reach completion. Of these, two hearts had infarction outside the risk area, presumably due to thromboembolic phenomena, three had no clear risk area, four experiments were not completed due to problems with the support rabbit and its extracorporeal circulation, and in two cases there were problems due to ventricular fibrillation in the isolated heart. These exclusions were distributed evenly between the groups. In addition 64 support rabbits were used, seven of which did not complete the protocol due to hypotension (three prior to suspension of the isolated heart).

\section{Data analysis}

All results are expressed as mean (SEM). Statistical analysis between groups in the support rabbit experiments was performed using analysis of variance, followed by multiple comparisons with Fisher's protected least significant difference method. ${ }^{19}$ An unpaired Student's $t$ test was used for comparison between groups in the buffer perfusion experiments and differences within groups were analysed with a paired Student's $t$ test. In all cases $p<0.05$ was taken as the criterion of significance. 


\section{Results}

BUFFER PERFUSED EXPERIMENTS

Haemodynamic data of the isolated hearts - Data collected on developed and diastolic pressures are expressed in figs 1 and 2. Developed pressure changes followed a similar pattern throughout the experiment in both groups, although the observed fall in pressure was only significant in the control group: from 42.6(SEM 3.5) to 27.8(4.6) $\mathrm{mm}$ $\mathrm{Hg}, \mathrm{p}<0.005$ by paired $t$ test. Diastolic pressure tended to rise more in the control group, but this did not reach statistical significance. Initial coronary perfusion pressures are shown in table I.

Infarct size and risk zone size data - Results for risk zone and infarct size (expressed both in absolute terms and as percentages of the area at risk-I/R ratio) are also summarised in table I. The I/R ratio is used for comparisons between groups as this controls for any minor variation in risk area. No correction is made for collateral flow as this is insignificant in the rabbit. ${ }^{20}$ Risk zone size in the control

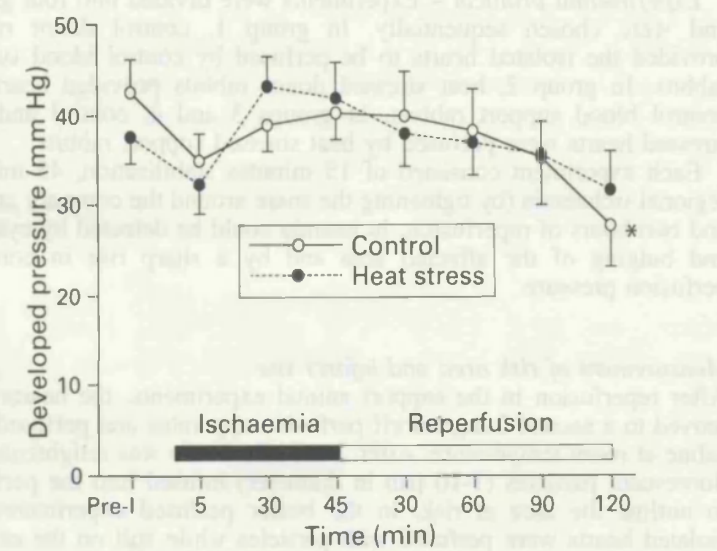

Figure I Changes in developed pressure over the course of the experiment for the two groups of buffer perfused isolated hearts. ${ }^{*} p<0.005 \mathrm{v}$ initial pressure (paired $\mathrm{t}$ test).

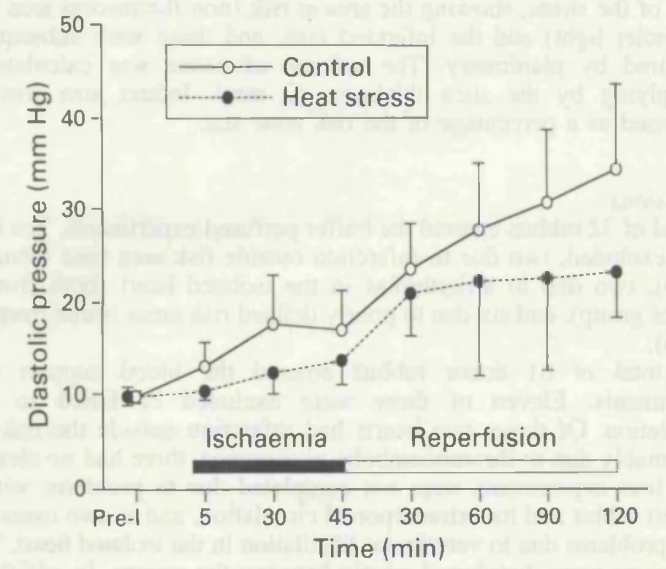

Figure 2 Changes in diastolic pressure over the course of the experiment for the two groups of buffer perfused hearts. group was $1.115(0.150) \mathrm{cm}^{3}(\mathrm{n}=10)$ and it was $0.954(0.127)$ $\mathrm{cm}^{3}(\mathrm{n}=12)$ in the heat stress group (NS). I/R ratio was reduced from $70.7(4.4) \%$ in the control group to $51.5(5.7) \%$ in the heat stress group $(\mathrm{p}<0.05)$. Individual $I / R$ ratios are shown in fig 3.

\section{BLOOD SUPPORT RABBIT EXPERIMENTS}

Haemodynamic data of the isolated hearts - Figures 4-6 show the data collected for developed pressure, diastolic

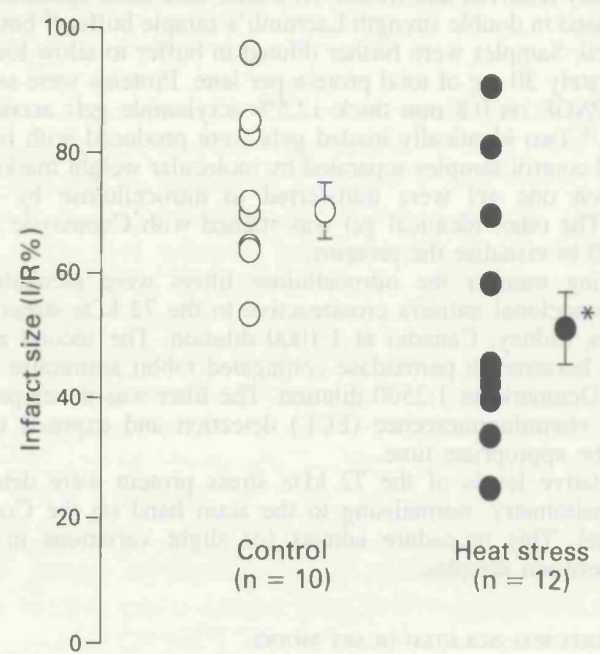

Figure 3 Scatterplot of individual infarct sizes for the two groups of buffer perfused isolated hearts. Means and standard errors are also shown.

$* p<0.05$ v control.

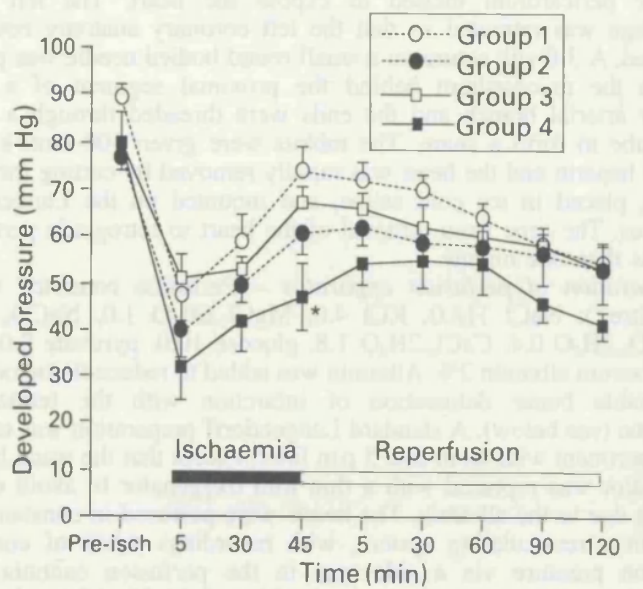

Figure 4 Changes in developed pressure over the course of the experiment for the four blood perfused isolated heart groups. Values are means, bars=SEM. Group I (empty circles) are control hearts perfused by control blood support rabbit; group 2 (filled circles) are heat stress hearts perfused by control rabbits; group 3 (empty squares) are control hearts perfused by heat stress blood support rabbits; group 4 (filled squares) are heat stress hearts perfused by heat stress blood support rabbits. $* p<0.05$, group $4 \mathrm{v}$ group 2.

Table I Buffer perfused experimental data. Values are means (SEM).

\begin{tabular}{lcccc}
\hline & $\begin{array}{c}\text { Coronary perfusion pressure } \\
(\mathrm{mm} \mathrm{Hg})\end{array}$ & $\begin{array}{c}\text { Risk area } \\
\left(\mathrm{cm}^{3}\right)\end{array}$ & $\begin{array}{c}\text { Infarct size } \\
\left(\mathrm{cm}^{3}\right)\end{array}$ & $\begin{array}{c}\text { Infarction } \\
(1 / R \%)\end{array}$ \\
\hline Control $(\mathrm{n}=10)$ & $41(5)$ & $1.115(0.150)$ & $0.793(0.130)$ & $70.7(4.4)$ \\
Heat stress $(\mathrm{n}=12)$ & $35(1)$ & $0.954(0.127)$ & $0.524(0.113)$ & $51.5(5.7)^{*}$ \\
\hline
\end{tabular}

${ }^{*} \mathrm{p}<0.05 v$ control 


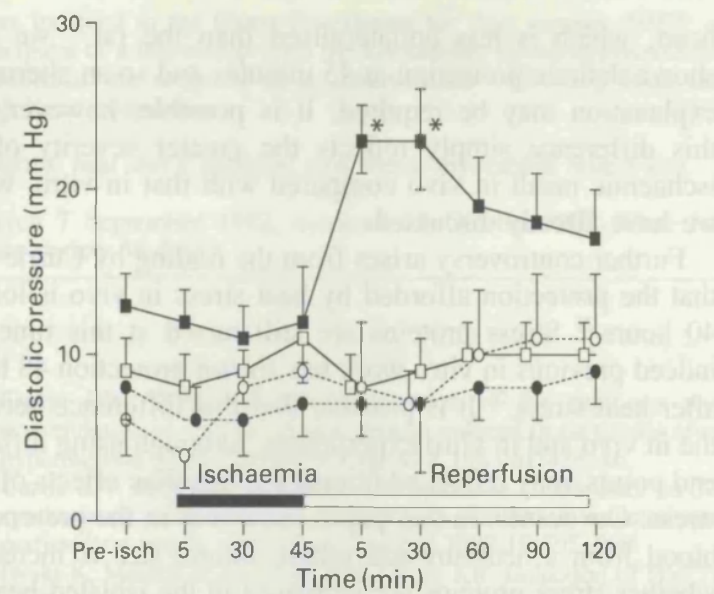

Figure 5 Changes in diastolic pressure over the course of the experiment for the four blood perfused isolated heart groups. For details see legend to fig 4.

$* p \leqslant 0.05$, group $4 \mathrm{v}$ group 2.

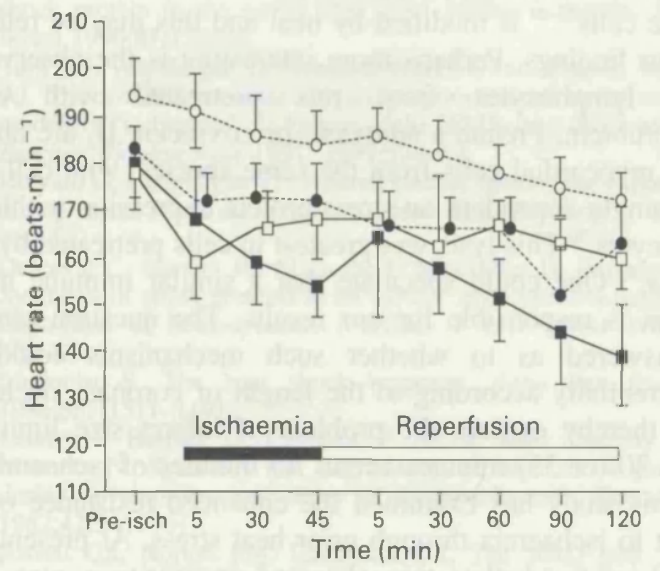

Figure 6 Changes in heart rate over the course of the experiment for the four blood perfused isolated heart groups. For details see legend to fig 4.

pressure, and heart rate respectively. The developed pressures (fig 4) followed similar trends in all four groups, and were not significantly different at either the start or completion of the experiment. However, after 45 minutes of ischaemia the heat stress heart perfused with heat stress blood had significantly worse systolic function than in the control $(p<0.05)$. Similarly with the diastolic pressures (fig 5 ), no significant changes were noted in groups 1-3. However, in group 4, diastolic pressure rose significantly during the early stages of reperfusion $(p<0.05)$, before settling slightly. Heart rates (fig 6 ) decreased slightly in all four groups during the experiment, but were not significantly different from each other at any time point. Initial coronary perfusion pressures are shown in table II.
Risk zone and infarct size data - Table II also summarises the risk zone and infarct sizes, and the I/R ratios for the four groups. With the control blood support rabbits, the risk zone sizes were $0.562(0.053) \mathrm{cm}^{3}$ in the control heart group (group $1, \mathrm{n}=15$ ) and $0.636(0.048) \mathrm{cm}^{3}$ in the heat stress heart group (group 2, $\mathrm{n}=16$ ). Similarly in the heat stress blood support rabbit groups, the risk zone sizes were $0.616(0.059)$ $\mathrm{cm}^{3}$ for the control hearts (group 3, $\mathrm{n}=12$ ) and $0.806(0.123)$ $\mathrm{cm}^{3}$ for the heat stress hearts (group 4, $\mathrm{n}=7$ ). These differences are not statistically significant.

I/R ratio was reduced from $33.7(3.7) \%$ in the control hearts (group 1) to $23.5(3.3) \%$ in the heat stress hearts (group 2), when perfused with blood from a control support rabbit $(\mathrm{p}<0.05)$. With a heat stressed support rabbit, I/R ratios were significantly higher in both groups: $44.8(3.3) \%$ for the control hearts (group 3) and $51.9(7.0) \%$ for the heat stress hearts (group 4) $(\mathrm{p}<0.05$ compared with groups 1 and 2 , respectively). Although group 4 had larger infarcts than group 3, this did not reach statistical significance. Individual $\mathrm{I} / \mathrm{R}$ ratios for the four groups are shown in fig 7 .

\section{Stress protein determination}

The $72 \mathrm{kDa}$ protein was seen in all specimens examined (fig 8 ). There was an obvious increase in its expression after heat stress, which can be seen with optical densitometry to be approximately sevenfold.

\section{Discussion}

Our results show that prior heat stress can limit infarct size in the isolated perfused rabbit heart. However the perfusate

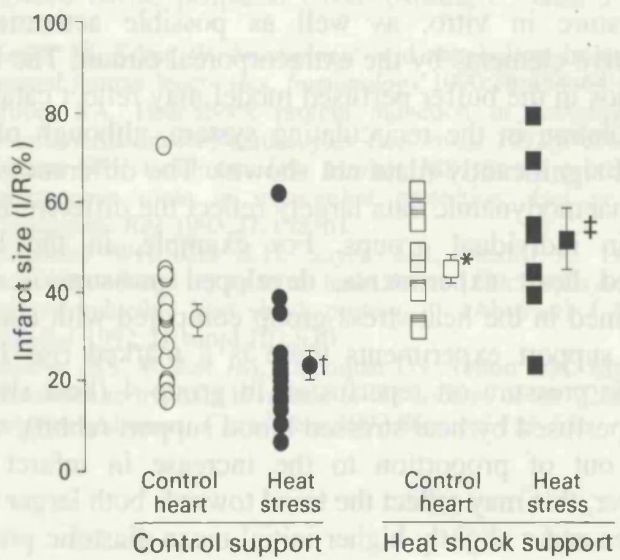

Figure 7 Scatterplot of individual infarct sizes for the four blood perfused isolated heart groups. Means and standard errors are also shown.

${ }^{*} p<0.05$ v control heart perfused by control blood support rabbit; $\dagger p<0.05$ v control heart perfused by control blood support rabbit; $\mp p<0.01 \vee$ heat stress heart perfused by control blood support rabbit.

Table II Support rabbit cxperimental data. Values are means (SEM).

\begin{tabular}{lccc}
\hline & $\begin{array}{c}\text { Coronary perfusion pressure } \\
(\mathrm{mm} \mathrm{Hg})\end{array}$ & $\begin{array}{c}\text { Risk area } \\
\left(\mathrm{cm}^{3}\right)\end{array}$ & $\begin{array}{c}\text { Infarct size } \\
\left(\mathrm{cm}^{3}\right)\end{array}$ \\
\hline Group $1(\mathrm{n}=15)$ & $51(1)$ & $0.562(0.048)$ & $0.202(0.036)$ \\
Group 2 $(\mathrm{n}=16)$ & $50(2)$ & $0.636(0.048)$ & $33.7(3.7)$ \\
Group 3 $(\mathrm{n}=12)$ & $48(1)$ & $0.616(0.059)$ & $23.5(3.3) \dagger$ \\
Group 4 $(\mathrm{n}=7)$ & $54(5)$ & $0.806(0.123)$ & $0.276(0.024)$ \\
\hline
\end{tabular}

Group 1 are control hearts perfused by control blood support rabbits; group 2 are heat stress hearts perfused by control blood support rabbits; group 3 are control hearts perfused by heat stress blood support rabbits; group 4 are heat stress hearts perfused by heat stress blood support rabbits. $\dagger \mathrm{p}<0.05 v$ group $1 ;{ }^{*} \mathrm{p}<0.05 v$ group $1 ; \neq \mathrm{p}<0.01 v$ group 2 . 


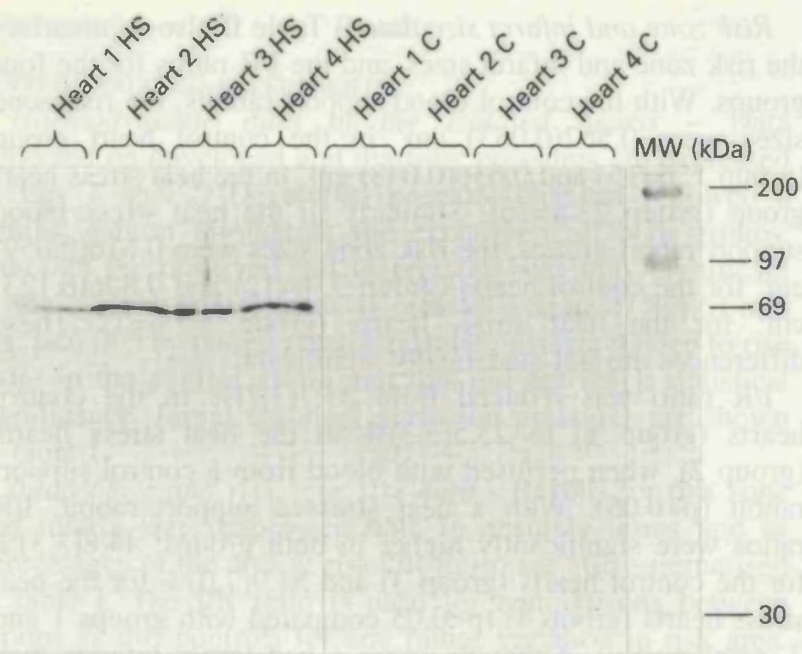

Figure 8 Western blot of extracts from four heat stressed (left four lanes) and four control (right four lanes) rabbit hearts. Densitometric quantification reveals an approximately sevenfold induction of the $72 \mathrm{kD}$ a stress protein: heat stress 2.83(SEM 0.154) units v control $0.412(0.113)$ units $(p<0.01)$.

used is clearly critical, as protection is lost when the heart is perfused with blood from a heat stressed rabbit. Where blood from a control support rabbit is used, a similar degree of protection can be seen in both the buffer perfused and blood perfused models, although infarct sizes are greater in the former. It is interesting to note that the blood perfused model gives smaller infarct sizes than seen in vivo, possibly due to a combination of factors such as the constant flow system used, reduced cardiac work, and a lower operating temperature in vitro, as well as possible activation of vasoactive elements by the extracorporeal circuit. The larger I/R ratios in the buffer perfused model may reflect catabolite accumulation in the recirculating system, although $\mathrm{pH}$ did not fall significantly (data not shown). The differences seen in the haemodynamic data largely reflect the different infarct sizes in individual groups. For example, in the buffer perfused heart experiments, developed pressure is better maintained in the heat stress group compared with controls. In the support experiments there is a marked rise in the diastolic pressure on reperfusion in group 4 (heat stressed hearts perfused by heat stressed blood support rabbit), which seems out of proportion to the increase in infarct size. However, this may reflect the trend towards both larger mean risk area and a slightly higher initial mean diastolic pressure which has occurred by chance in this group (these differences did not reach statistical significance).

Although 24 hours after heat stress we were unable to show any limitation of infarct size following 45 minutes of ischaemia in the in vivo rabbit model, ${ }^{17}$ recent evidence from both Currie et $a l^{21}$ and our own work ${ }^{22}$ has shown that heat stress can limit infarction after 30 minutes of ischaemia in the same model. This somewhat surprising discovery that the length of the ischaemic time is so critical also appears to be true for the rat, where heat stress protects against a 35 minute coronary occlusion but not against 45 minutes of occlusion. ${ }^{23}$ Here the authors have suggested that this reflects a delay in irreversible myocardial injury. ${ }^{23}$ Such a situation may also exist in ischaemic preconditioning, in which protection is lost as ischaemia is prolonged and infarct size tends towards $100 \%$ of the risk area. ${ }^{24}$ However in the rat, infarct size following 45 minutes of ischaemia is only about $60 \%$ of the risk area. ${ }^{25}$ Furthermore, in our studies in the isolated rabbit heart, which is less collateralised than the rat, ${ }^{20}$ we have shown definite protection at 45 minutes and so an alternative explanation may be required. It is possible, however, that this difference simply reflects the greater severity of the ischaemic insult in vivo compared with that in vitro, which we have already discussed.

Further controversy arises from the finding by Currie et al that the protection afforded by heat stress in vivo is lost by 40 hours. ${ }^{21}$ Stress proteins are still raised at this time and indeed previous in vitro work has shown protection 48 hours after heat stress. ${ }^{26}$ It is possible that this difference between the in vivo and in vitro experiments, although using different end points, may reflect additional extracardiac effects of heat stress. Our results in this paper show that in the presence of blood from a heat stressed rabbit, infarct size is increased, whether stress proteins are increased in the isolated heart or not. This provides evidence in favour of such an extracardiac effect. Indeed these results also suggest that any deleterious effect of heat stress on the ischaemic/reperfused myocardium may be mediated by the blood. There is some evidence that the function of blood constituents such as platelets ${ }^{27}$ and white cells ${ }^{28} 29$ is modified by heat and this may be relevant to our findings. Perhaps more interesting is the observation that lymphocytes from rats pretreated with either doxorubicin, Freund's adjuvant, or coxsackie $B_{3}$ are able to lyse myocardial cells from the same species, with cell lysis seemingly dependent on stress protein expression within the myocytes. ${ }^{30}$ This lysis was greatest in cells pretreated by heat stress. ${ }^{30}$ One could speculate that a similar immune mechanism is responsible for our results. The question remains unanswered as to whether such mechanisms could act differentially according to the length of coronary occlusion and thereby explain the problem of infarct size limitation with 30 (or 35 ) minutes versus 45 minutes of ischaemia.

This study has examined the enhanced resistance of the heart to ischaemia through prior heat stress. At present it is not known whether this observed resistance comes from stress proteins themselves or alternatively is mediated by some other aspect of heat stress. ${ }^{31}$ For example, Currie and co-workers $^{26}$ found that myocardial catalase, an endogenous antioxidant, is increased twofold by heat stress. When heat stressed rats were pretreated with 3-amino-1,2,4-triazole to deplete catalase, the benefits of heat stress on ischaemia/ reperfusion were abolished in their model. It is possible, therefore, that heat stress works entirely by increasing catalase activity, with stress proteins being an epiphenomenon. However the evidence for acquired thermotolerance resulting from increased levels of stress proteins is more conclusive ${ }^{10-12}$ and a preliminary study by Dillmann et $a^{32}$ has shown that induction of $70 \mathrm{kDa}$ heat shock protein by plasmid transfection of embryonal rat heart cells leads to cellular protection in a model of ischaemia. Further circumstantial evidence that the protection associated with heat stress may be stress protein mediated comes from isolated papillary muscle studies where resistance to hypoxia has been positively correlated with the content of the $72 \mathrm{kDa}$ stress protein. ${ }^{33}$ Therefore there may be a direct protective role for stress proteins in ischaemia, in addition to the effects on catalase activity.

In conclusion, we feel that prior heat stress is protective to ischaemic myocardium, although these studies cannot link this causally to stress proteins. In view of the difficulties we have discussed in attempting to limit infarct size with heat stress, it is important to develop aiternative less abusive methods of stress protein induction within the heart to further evaluate their possible protective role. 
We are indebted to the Hatter Foundation for their support. DMW is the recipient of a fellowship from the Rockefeller Research Foundation and MSM is the recipient of an Intermediate Fellowship from the British Heart Foundation.

Key terms: heat shock proteins; heat stress; myocardial infarct size.

Received 7 September 1992; accepted 29 October 1992. Time for primary review 36 days.

1 Currie RW, White FP. Characterization of the synthesis and accumulation of a 71-kilodalton protein induced in rat tissues after hyperthermia. Can J Biochem Cell Biol 1983:61:438-46.

2 Currie RW. Effects of ischaemia and perfusion temperature on the synthesis of stress induced (heat shock) proteins in isolated and perfused rat hearts. J Mol Cell Cardiol 1987;19:795-808.

3 Iwaki K. Mestril R, Dillman WH, Chein KR. Induction of HSP7I mRNA correlates with arachidonic acid release and the transition to irreversible injury in neonatal rat myocardial cells following ATP depletion. (Abstract) Circulation 1989:80(suppl II):Il-462.

4 Mehta HB, Popovich BK, Dillman WH. Ischemia induces changes in the level of mRNAs coding for stress protein 71 and creatine kinase M. Circ Res 1988;63:512-7.

5 Knowlton AA, Brecher P, Apstein CS. Rapid expression of heat shock protein in the rabbit after brief cardiac ischemia. J Clin Invest 1990;87:139-47.

6 Tuiji MJ, van Bergen en Henegouwen PM, van Wijk R, Verkleij AJ. The isolated neonatal rat-cardiomyocyte used as an in vitro model for "ischaemia". Induction of the $68 \mathrm{kDa}$ heat shock protein. Biochim Biophvs Acta 1991; 1091:278-84.

7 Howard G, Geoghegan TE. Altered cardiac tissue gene expression during acute hypoxic exposure. Mol Cell Biochem 1986;82: $460-8$

8 Delcayre C, Samuel JL, Marrothe F, Mescadier JJ, Rappaport L. Synthesis of stress proteins in rat cardiac myocytes 2-4 days after imposition of hemodynamic overload. J Clin Invest 1988;82: $460-8$.

9 Lindquist S. The heat shock response. Annu Rev Biochem 1986:55:11511-1191

10 Landry J, Bernier D, Chretien P, Nicole LM, Tanguay RM, Marceau N. Synthesis and degradation of heat shock proteins during development and decay of thermotolerance. Cancer Res 1982;42:2457-61.

11 Bansal GS, Norton PM, Latchman DS. The 90kD heat shock protein protects cells from thermal stress but not from viral intection. Exp Cell Res 1991;195:303-6.

12 Sanchez Y, Lindquist SL. HSP104 required for induced thermotolerance. Science 1990):248:1112-5.

13 Yellon DM, Latchman DS. Stress proteins and myocardial protection. J Mol Cell Cardiol 1992;24:113-24.

14 Currie RW, Karmazyn M, Kloc M, Mailer K. Heat shock response is associated with enhanced postischemic ventricular recovery. Circ Res 1988:63:543-9.

15 Pasini E, Cargnoni A, Ferrari R, Marber MS, Latchman DS, Yellon DM. Heat stress and oxidative damage following ischaemia and reperfusion in the isolated rat heart. (Abstract) Eur Heart $J$ 1991;12(suppl):306.

16 Yellon DM, Pasini E, Cargnoni A, Marber MS, Latchman DS, Ferrari R. The protective role of heat stress in the ischaemic and reperfused rabbit myocardium. J Mol Cell Cardiol 1992;24: 895-907.

17 Yellon DM, Iliodromitis E, Latchman DS, ef al. Whole body heat shock fails to limit infarct size in the reperfused rabbit heart. Cardiovasc Res 1992;4:342-6.

18 Laemmli UK. Cleavage of structural proteins during the assembly of the head of bacteriophage T4. Nature 1970:227:680-5

19 Snedecor GW, Cochran WG. Statistical methods. Iowa: Iowa State University Press, 1980:215-33.

20 Maxwell MP, Hearse DJ, Yellon DM. Species variation in the coronary collateral circulation during regional myocardial ischaemia: a critical determinant of the rate of evolution and extent of myocardial infarction. Cardiovasc Res 1987;21: $737-46$.

21 Currie RW, Tanguay RM, Kingma JG. Heat-shock response and limitation of tissue necrosis during occlusion/reperfusion in rabbit hearts Circulation 1993:87:963-71.

22 Marber MS, Latchman DS, Walker JM, Yellon DM. Cardiac stress protein elevation 24 hours following brief ischemia or heat stress is associated with resistance to myocardial infarction. Circulation (in press).

23 Donnelly TJ, Sievers RE. Vissern FLJ, Welch WJ, Wolfe CL. Heat shock protein induction in rat hearts. A role for improved myocardial salvage after ischemia and reperfusion? Circulation 1992:85:769-78

24 Murry CE, Jennings RB, Reimer KA. New insights into potential mechanisms of ischemic preconditioning. Circulation 1991:84: $442-5$.

25 Yellon DM, Alkhulaifi AM, Browne EE, Pugsley WB. Ischaemic preconditioning limits infarct size in the rat heart. Cardiovasc Res 1992;26:983-7.

26 Karmazyn M, Mailer K. Currie RW. Acquisition and decay of heat-shock enhanced postischemic ventricular recovery. $A m J$ Physiol 1990:259:H424-31.

27 Gader AMA, Al-Mashhadani SA, Al-Harhy SS. Direct activation of platelets by heat is the possible trigger of the coagulopathy of heat stroke. Br J Haematol 1990;74:86-92.

28 Cooper J, Kochevar DT, Rowlands C. Elevation of heat shock protein 70 by moderate heat stress enhances mitogenesis in isolated bovine peripheral blood. (Abstract) FASEB J 1990;4: A2224.

29 Koller M. Konig W. Arachidonic acid metabolism in heat shock treated human leucocytes. Immunology 1990;70:458-64.

30 Huber SA. Heat-shock protein induction in Adriamycin and picornavirus-infected cardiocytes. Lab Invest 1992;67:218-24.

31 Yellon DM, Latchman DS, Marber MS. Stress proteins: and endogenous route to myocardial protection: fact or fiction? Cardiovasc Res 1993;27:158-61.

32 Dillmann WH, Chi S-H, Sayen MR Mestril R Decreased ischemia induced injury in rat heart derived $\mathrm{H} 9 \mathrm{c} 2$ cells expressing human inducible heat shock protein 70. (Abstract) $J \mathrm{Mol} \mathrm{Cell}$ Cardiol 1992;24(suppl III):S30.

33 Marber MS, Walker JM, Latchman DS, Yellon DM. Myocardial resistance to hypoxia is related to the content of the $72 \mathrm{kDa}$ stress protein. (Abstract) Circulation 1992;86(suppl I):I-556.

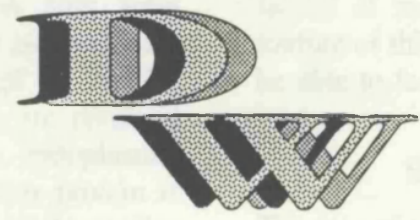




\title{
Viewpoint
}

\section{Stress proteins - an endogenous route to myocardial protection: fact or fiction?}

\author{
Derek M Yellon, David S Latchman, and Michael S Marber
}

A $\mathrm{n}$ increase in whole body temperature, in both the rabbit and rat, renders the heart resistant to subsequent ischaemia. ${ }^{1-3}$ The cause for this enhanced resistance is unknown but all published studies have shown that a rise in temperature induces cardiac stress protein synthesis. The question of interest is whether these stress proteins determine the observed enhanced resistance of the heart to ischaemia, or whether this is mediated by some other aspect of heat stress. The aim of this article is to speculate on the relationships between heat, stress proteins and myocardial protection.

\section{Function and properties of stress proteins}

To understand the possible protective benefits of stress proteins a brief description of their function is necessary. The stress proteins are a group of highly conserved proteins that are synthesised in response to varying stresses. ${ }^{4}$ Such proteins may be either constitutive, in that they are present in the unstressed state but are preferentially synthesised during stress, or inducible, being present only in the stressed organism. They consist of families of proteins with differing functions (see for review) and their nomenclature is according to molecular weight. The $90 \mathrm{kDa}$ family binds actin, a phenomenon that is modulated by both calcium and calmodulin. ${ }^{5}$ Their location is predominantly cytoplasmic and they are thought to act as chaperones, ${ }^{4}$ being able to transport molecules through their interaction with actin filaments while also offering protection against inappropriate or premature molecular interactions. Proteins of the $70 \mathrm{kDa}$ family bind ATP and, among other functions, are involved in post-translational import of proteins into endoplasmic reticulum and mitochondria. ${ }^{6}$ The $72 \mathrm{kDa}$ stress protein is inducible and the most frequently used marker of the cardiac stress response. Proteins of the $60 \mathrm{kDa}$ family are predominantly mitochondrial and facilitate the necessary refolding of proteins imported from the cytoplasm. Numerous other lower molecular weight stress proteins exist, the most highly conserved being ubiquitin, which by binding to proteins targets them for degradation.

\section{Speculations on the mechanisms of stress protein protection against ischaemia/reperfusion}

Following ischaemia and reperfusion one can hypothesise that stress proteins could both prevent cellular damage by maintaining protein structure despite the de-energised state and enhance recovery by promoting degradation of denatured proteins while assisting relocation of newly synthesised proteins.

The key proteins that are damaged during ischaemia and reperfusion have not been fully characterised, and the exact mechanisms by which stress proteins may influence the damage that occurs are unknown. Changes in cytoskeletal proteins are thought to occur during ischaemia. ${ }^{8}$ Interestingly one such protein, $\alpha$ crystallin, which is homologous to the low molecular weight stress proteins, is concentrated at the $\mathrm{Z}$ disc and may protect the cytoskeleton during physiological non-ischaemic stress. ${ }^{9}$ However since $\alpha$ crystallin aggregates within 10 minutes of the onset of ischaemia, ${ }^{9}$ it is an unlikely candidate for the protection during ischaemia and reperfusion. The important reduction in fatty acid oxidation that follows ischaemia has been partially attributed to oxidation of thiol groups within the branch point enzyme carnitine palmitoyltransferase. Following ischaemia and reperfusion this key enzyme is thought to undergo a conformational change (rather than proteolytic cleavage), ${ }^{10}$ which alters its affinity for malonyl CoA. Since the $60 \mathrm{kDa}$ family of mitochondrial proteins is involved in the initial folding of this mitochondrial protein, these proteins may also be able to facilitate its correct refolding.

\section{Stress proteins and thermal injury}

The role of stress proteins in the limitation of thermal injury is less controversial. Acquired thermotolerance describes a phenomenon whereby the resistance of tissue to a lethal temperature rise can be enhanced by a preceding sublethal temperature rise. The first episode of thermal stress results in stress protein production and as a direct result of the synthesis of these proteins thermotolerance occurs. Hence

University College and Middlesex Hospital Schools of Medicine, University College Hospital, Gower Street, London WC1E 6AU, United Kingdom - The Hatter Institute for Cardiovascular Studies, Division of Cardiology: M S Marber, D M Yellon; Division of Molecular Pathology: D S Latchman. Correspondence to Dr Yellon. 
thermotolerance follows the accumulation of stress protein temporally. "In addition, thermotolerance can be abolished by the use of stress protein anti-sense RNA ${ }^{12}$ or cell lines with heat stress protein gene deletions, ${ }^{13}$ and artificial constitutive expression of an inducible stress protein can confer thermotolerance without the need for a preceding sublethal temperature rise. ${ }^{14}$

\section{The phenomenon of crosstolerance}

Is it possible to induce thermotolerance by elevating stress proteins by means other than heat? Such a possibility is suggested above, ${ }^{12-14}$ and has been confirmed by the finding that stress proteins synthesised as a result of exposure to ethanol and sodium arsenite are able to confer thermotolerance. ${ }^{15}$ This phenomenon which is related to acquired thermotolerance is known as crosstolerance. A conceptually interesting extension to these observations could be termed reverse crosstolerance. Thus imagine that a particular non-thermal stress is associated with stress protein production: will tissue with thermally pre-elevated stress protein have an enhance tolerance to this stress? Clearly this is speculative, but logic dictates that this is the case.

Since permanent coronary occlusions as well as short occlusions with reperfusion result in stress protein synthesis, ${ }^{16} 17$ investigators following the above line of reasoning have been prompted to examine the protective benefits of heat stress with respect to myocardial ischaemia. As expected, hearts with thermally pre-elevated stress protein content do seem more than ordinarily resistant to ischaemia, ${ }^{1-3}$ although there are some preliminary reports that do not support these conclusions. ${ }^{18}{ }^{19}$ If the phenomenon of reverse crosstolerance were to apply generally then prior heat stress should render the heart tolerant to subsequent volume or pressure overload, ${ }^{20}$ exposure to cardiotoxic drugs, ${ }^{21}$ high doses of inotropic drugs, ${ }^{22}$ mechanical stretch, ${ }^{23}$ and anoxia, ${ }^{24}$ since all these stresses are associated with cardiac stress protein synthesis. The effects of prior heat stress on these subsequent stresses has not to our knowledge been examined.

\section{Are stress proteins the mediators of the protection following heat stress?}

Apart from the above relationship between heat stress, stress protein synthesis, and protection against ischaemia and reperfusion, is there any direct evidence that heat stress protects via an elevation in cardiac stress protein content?

Currie and coworkers were the first investigators to show the protective benefits of heat stress in the isolated rat heart.' In these studies the myocardial content of the $72 \mathrm{kDa}$ stress protein and the resistance of the heart to ischaemia and reperfusion follow the same time course. ${ }^{125}$ However, the same investigators also demonstrated an approximately twofold increase in myocardial catalase activity following heat stress. ${ }^{25}$ When heat stressed rats were pretreated with 3-amino-1,2,4-triazole, myocardial catalase activity was diminished to half that in the control group. Such pretreatment abolished the benefits of heat stress, but interestingly did not worsen the ischaemic injury in control hearts. ${ }^{25}$ The results of these studies suggest that heat stress may act via an increase in endogenous catalase activity, stress proteins being an epiphenomenon. Further studies by the same workers have shown that the increase in catalase activity following heat stress is not associated with any change in levels of mRNA coding for catalase, suggesting heat may alter translational or post-translational control processes. ${ }^{26}$ Interestingly a preliminary report from other workers has shown that in the pig heat stress induces cardiac (72 kDa) stress protein and increases endogenous superoxide dismutase activity but does not affect catalase. ${ }^{27}$ It seems quite feasible that heat stress, by enhancing the heart's endogenous antioxidant defences, may be able to protect against reperfusion injury. In keeping with such a hypothesis we have been able to demonstrate that oxidant stress (measured indirectly by glutathione status, protein-SH groups and mitochondrial function) is greatly attenuated following ischaemia and reperfusion of the heat stressed rabbit heart. ${ }^{3}$

Investigators have also attempted to use ischaemia followed by an eight hour reperfusion period to induce stress proteins. The protective benefits of ischaemic and heat induced stress protein pre-elevation in an infarct size model ${ }^{2}$ were than compared. In this study only heat pretreatment resulted in protection. The authors concluded, however, that the ischaemic pretreatment was not nearly as effective as heat in raising the cardiac content of the $72 \mathrm{kDa}$ stress protein and it was for this reason that no protection was seen. An alternative explanation would be that, unlike heat stress, ischaemia did not increase endogenous myocardial antioxidant defences. In addition to the enhanced antioxidant defences that follow heat stress, it has recently been shown that prior heat stress protects the myocardium against a calcium paradox, ${ }^{28}$ an injury in which oxidant stress is not thought to play a major role. ${ }^{29} 30$ Thus it could be argued that some portion of the heat stress response represents a form of cardiac adaptation capable of limiting myocyte injury independent of antioxidant mechanisms.

The strongest evidence to date of a direct protective role for heat stress protein in anoxic injury is in the form of a preliminary report by Dillmann and colleagues. ${ }^{31}$ These investigators have shown that successful transfection of an embryonal rat cell line with plasmids expressing the human inducible $70 \mathrm{kDa}$ heat shock protein resulted in resistance to anoxia and reoxygenation injury.

\section{Stress proteins and ischaemic preconditioning}

Since ischaemia, both with ${ }^{17}$ and without $^{16}$ reperfusion, is known to increase stress proteins, one could surmise that the synthesis of stress proteins as a consequence of such ischaemia might be expected to minimise the damage caused to the heart during a subsequent more sustained ischaemic episode. In view of this hypothesis there has been intriguing speculation on the involvement of these proteins in another method of endogenous cardiac protection, the phenomenon known as ischaemic preconditioning. ${ }^{32}$ In this process a series of short coronary artery occlusions followed by reperfusion renders the heart resistant to a subsequent sustained period of ischaemia. ${ }^{33}$ Although the mechanism of ischaemic preconditioning is unclear, the involvement of stress proteins appears an attractive possibility. In agreement with this idea, Knowlton et al ${ }^{17}$ showed that brief bursts of ischaemia, such as occur in ischaemic preconditioning, can induce the expression of the mRNA and accumulation of the $72 \mathrm{kDa}$ stress protein within the heart. These investigators ${ }^{17}$ found that the $72 \mathrm{kDa}$ stress protein only became noticeably raised after two hours of reperfusion and remained increased for at least 24 hours after the ischaemic insult. In contrast the protective effect of ischaemic preconditioning has been shown to diminish beyond about 60 minutes. ${ }^{34}$ The involvement of protein synthesis in ischaemic preconditioning has 
also been questioned by the finding that the protective benefits of preconditioning can still be observed under conditions where de novo protein synthesis has been almost entirely inhibited ${ }^{35}$ (although stress protein was not measured directly). In view of the above, doubt is cast on the role of stress proteins in the protection observed during ischaemic preconditioning. It should be emphasised, however, that the limited amount of work examining the relationship between stress proteins and ischaemic preconditioning has focused almost entirely on the $72 \mathrm{kDa}$ stress protein, ignoring the other stress proteins. Until other such families of stress proteins are examined in more detail their involvement in this protective phenomenon cannot categorically be ruled out.

Although the protective benefits of classical preconditioning begin to wane approximately 60 minutes after coronary occlusion, ${ }^{34}$ the stress protein content of the heart continues to rise ${ }^{23}$ offering the theoretical possibility of a delayed, but perhaps longer lasting, "second window of protection". Evidence in support of such a phenomenon exists in neuronal tissue, as the stress protein content of neuronal tissue can be increased by daily two minute episodes of ischaemia, a treatment that also seems to increase the neuronal resistance to more prolonged ischaemia $\left(\mathrm{see}^{36}\right.$ for review). Preliminary findings suggest that a similarly delayed protection occurs 24 hours after "classical" preconditioning of the dog heart ${ }^{37}$ and also 24 hours after rapid pacing of the rabbit heart. ${ }^{38}$ The ramifications of these unsubstantiated findings are immense and may explain the apparent benefits, despite more severe coronary artery disease, of a history of angina prior to myocardial infarction. ${ }^{39}$

\section{Conclusion}

In conclusion we believe the cell's inherent ability to produce specific stress proteins to help protect itself against thermal injury is not in question. At present, however, there is little direct evidence to suggest that the myocardial protection that follows heat stress or ischaemia is related to the expression of these proteins. The key question that stil needs to be answered is whether an increase in stress proteins by non-thermal means will prove effective in limiting myocardial injury. Although preliminary evidence suggests that this may be the case, ${ }^{31}$ until studies are designed which can either limit stress protein expression or conversely cause expression of the proteins via a nonabusive procedure (ie, specific pharmacological therapy or gene tranfection), their importance in myocardial protection, although attractive, must remain uncertain.

Received 5 February 1992; accepted 11 August 1992.

MSM is the recipient of a British Heart Foundation intermediate fellowship.

Key terms: stress protein; heat sress; ischaemia; myocardial protection.

1 Currie RW, Ross BM, Davis TA. Heat-shock response is associated with enhanced postischemic ventricular recovery. Circ Res 1989;63:543-9.

2 Donnelly TJ, Sievers RE, Vissern FLJ, Welch WJ, Wolfe CL. Heat shock protein induction in rat hearts. A role for improved myocardial salvage after ischemia and reperfusion? Circulation 1992:85:769-778

3 Yellon DM, Pasini E, Cargnoni A, Marber MS, Latchman DS,
Ferrari R. The protective role of heat stress in the ischaemic and reperfused rabbit myocardium I Mol Cell Cardiol 1992:24: 895-907.

4 Linquist S, Craig EA. The heat shock proteins. Anmu Rev Genet 1988:22:631-77.

5 Koyasu S, Nishida E, Yoshihiko M, Sakai H, Yahara I. HSPI00. a $100-\mathrm{kDa}$ heat shock protein, is a $\mathrm{Ca}^{2+}$-calmodulin-regulated actin-binding protein. $J$ Biol Chem 1989:264:15083-7.

6 Deshaies R, Koch B, Werner-Washburne M. Craig E, Scheckman R. $70 \mathrm{kD}$ stress protein homologues facilitate translocation of secretory and mitochondrial precursor polypeptides. Nature 1988:332:800-5.

7 Cheng MY, Hartl FU, Martin J, et al. Mitochondrial heat-shock protein hsp60 is essential for assembly of proteins imported into yeast mitochondria. Nature 1989;337:620-5.

8 Steenbergen C. Hill ML, Jennings RB. Cytoskeletal damage during myocardial ischemia: changes in vinculin immunoflourescence staining during total in vitro ischemia in canine heart. Circ Res 1987;60:478-86.

9 Chiesi M, Longoni S, Limbruno U. Cardiac alpha-cystallin. Involvement during heart ischemia. Mol Cell Biochem 1990:97:129-36.

10 Pauly DF, Kirk KA, McMillin JB. Carnitine palmitoyltransferase in cardiac ischemia. A potential site for altered fatty acid metabolism. Circ Res 1991;68:1085-94.

$11 \mathrm{Li}$ CG, Werb Z Correlation between synthesis of heat shock proteins and development of thermotolerance in Chinese hamster fibroblasts. Proc Natl Acad Sci USA 1982;79:3218-22.

12 Bansol GS, Norton PM, Latchman DS. The $90 \mathrm{kD}$ heat shock protein protects cells from thermal stress but not from viral infection. Exp Cell Res 1991;195:303-6.

13 Sanchez Y, Linquist SL. HSP104 required for induced thermotolerance. Science 248:1112-5.

14 Angelidis CE, Lazaridis I, Papoulatos GN. Constitutive expression of heat-shock protein 70 in mammalian cells confers thermoresistance. Eur J Biochem 1991;199:35-9.

$15 \mathrm{Li} \mathrm{GC}$. Induction of thermotolerance and enhanced heat shock protein synthesis in Chinese hamster fibroblasts by sodium arsenite and by ethanol. I Cell Physiol 1983:115:116-22

16 Dillmann WH, Mehta H, Barrieux A. Guth BD, Neeley WE, Ross J. Ischemia of the dog heart induces the appearance of a cardiac mRNA coding for a protein with migration characteristics similar to heat-shock/stress protein 71. Circ Res 1986:59:110-14.

17 Knowlton AA. Brecher P, Apstein CS. Rapid expression of heat shock protein in the rabbit after brief cardiac ischemia. J Clin Invest 1991:87:139-47.

18 Wall SR, Fliss H, Kako KJ, Korecky B. Heat pretreatment does not improve recovery of function after no-flow ischaemia in isolated working rat hearts. (Abstract) J Mol Cell Cardiol 1990 (suppl 1):S44.

19 Schott R, Nao B, Stieter R, et al. Heat shock does not precondition canine myocardium. (Abstract) Circulation 1990:82(suppl III): III 464 .

20 Chevalier B, Callens F, Charlemagne D, et al. Signal and adaptational changes in gene expression during cardiac overload. $J$ Mol Cell Cardiol 1989;21:71-7.

21 Low I, Fiedrich T, Schoeppe W. Synthesis of shock proteins in cultured fetal myocardial cells. Exp Cell Res 1989;180:451-9.

22 White FP. White SC. Isoproterenol induced myocardial necrosis is associated with stress protein synthesis in rat heart and thoracic aorta. Cardiovase Res 1986:20:512-5.

23 Knowlton AA, Eberli FR, Brecher P, Romo GM, Owen A, Apstein CS. A single myocardial stretch or decreased systolic fiber shortening stimulates the expression of heat shock protein 70 in the isolated, erythrocyte-perfused rabbit heart. I Clin Invest 1991:88:2018-25.

24 Tuijl MJM, van Bergen en Henegouwen PMP, van Wijk R. Verkleij A. The isolated neonatal rat-cardiomyocyte used is an in vitro model for "ischemia". Induction of the $68 \mathrm{kDa}$ heat shock protein. Biochim Biophy:s Acta 1991;1091:278-84.

25 Karmazyn M. Mailer K. Currie RW. Acquisition and decay of heat-shock-enhanced postischemic ventricular recovery. Am J Physiol 1990;259:H424-31.

26 Currie RW, Tanguay RM. Analysis of RNA for transcripts for catalase and SP71 in rat hearts after in vivo hyperthermia. Biochem Cell Biol 1991:69:375-82.

27 Liu X, Engelman RM, Maulic N. Heat shock: a new approach for myocardial preservation in cardiac surgery. (Abstract) Circulation 1991:84(suppl II):II-687.

28 Marber MS, Walker JM, Kucukoglu S, Yellon DM. Heat stress attenuates a submaximal calcium paradox in the rabbit. (Abstract) J Mol Cell Cardiol 1992;24(suppl I):S138.

29 Duncan CJ, Biochemical events associated with rapid cellular damage during the oxygen- and calcium- paradoxes of the mammalian heart. Experientia 1990;46:41-8 
30) Ferrari R, Ceconi C. Curello S, Cargnoni A, Ruigrok TIC No evidence of oxygen free radicals-mediated damage during the calcium paradox. Basic Res Cardiol 1989:84:396-403.

31 Dillmann WH, Chi S-H, Sayen MR Mestril R Decreased ischemia (Ism) induced injury in rat heart derived $\mathrm{H} 9 \mathrm{c} 2$ cells expressing human inducible heat shock protein 70 (hHSP70i). (Abstract) J Mol Cell Cardiol 1992:24(suppl III):S30.

32 Walker DM. Yellon DM. Ischaemic preconditioning - from mechanisms to exploitation. Cardiovasc Res 1992:26:734-9.

33 Murry CE, Jennings RB, Reimer KA. Preconditioning with ischemia: a delay of lethal cell injury in ischemic myocardium. Circulation 1986;74:1124-36.

34 Van Winkle DM. Thornton JD, Downey JM. Cardioprotection from ischemic preconditioning is lost following prolonged reperfusion in rabbits. (Abstract) Circulation 1991;84(suppl II): II-432.

35 Thornton J, Striplin S, Liu GS, et al. Inhibition of protein synthesis does not block myocardial protection afforded by preconditioning. Am J Physiol 1990):259(Heart Circ Physiol): H1822-5.

36 Nowak TS. Protein synthesis and heat shock/stress response after ischemia. Cerebrovasc Brain Metab Rev 1990:2:345-66.

37 Yamashita N, Kuzuya T, Hoshido S, et al. Relationship between time interval from preconditioning to sustained ischemia and its effects on limiting infarct size. (Abstract) $J$ Mol Cell Cardiol 1992;24(suppl I):S150.

38 Szekeres L, Szilvassy Z, Udvary E, Vegh A. Moderate stress by cardiac pacing may induce both short-term and long-term cardioprotection based on different mechanisms. (Abstract) J Mol Cell Cardiol 1992;24(suppl I):S120.

39 Muller DW, Topol EJ, Califf RM, et al. Relationship between antecedent angina pectoris and short-term prognosis after thrombolytic therapy for acute myocardial infarction. Thrombolysis and angioplasty in myocardial infarction (TAMI) study group. Am Heart J 1990;119:224-31.

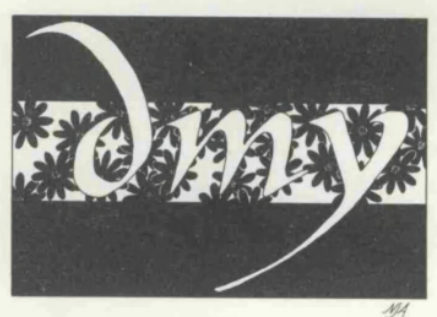

